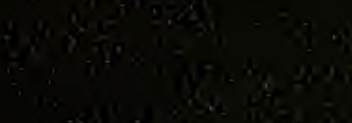




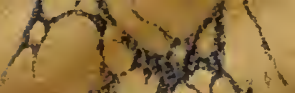

if $i$

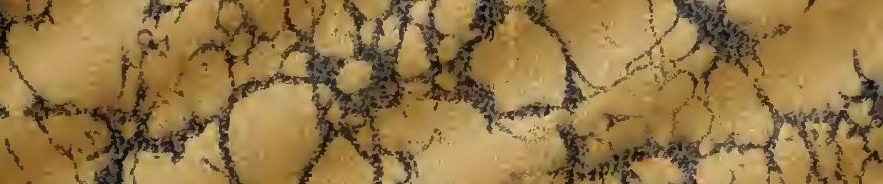

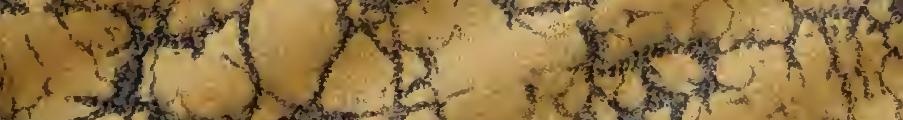
(1)

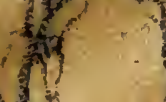

(द.

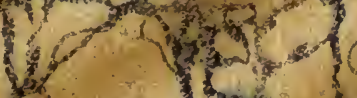

in

1 is का है

है.

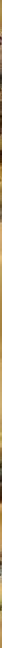

30?

(3)

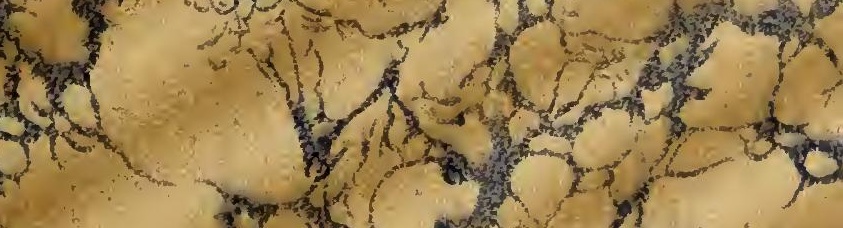
( $x$ - 20 
Digitized by the Internet Archive in 2009 with funding from NCSU Libraries 


ENCYCLOPÉDIE CADÉAC

\section{I I}

\section{SE M I O L O G I E}

DES MALADIES

DES ANIMAUX DOMESTIQUES.

TOME II 
Recherches expérimentales sur la morve (en collaboralion aree M. Mallet). (Récompensé par l'Acatémie dos sciences et par l'Académie de nédecine.) Paris, 1886, 155 jages.

Mémoire sur la contagion miasmique du charbon, de la chvelée, de la morve el de la tubereulose (en collaboration avec M. Mallet). (Couronné par l'Académie de médecine de France.)

Recherches expérimentales sur les essences. - Ltude des liqueurs d'absintle, d'arquebuse. de l'eau de melisse des Carmes, de Garus (en collaboration arec le Dr Meunier). Paris. 1891. (Ouvrage couronné par l'Acatémie des seiences el récompensé par l'Académie de médecino de france.)

Pathologie générale des animaux domestiques. ¿eédition, 1904. 1 vol. in-1s jésus, ilvec 3 a figures. Paris, J.-B. Baillice el fils.

Sémiologie et diagnostic des maladies des animaux domestiques. 2e édilion, 1904. I vol. in-1s jésus, avec ligures. Paris, J.-B. Baillicre et fils (Éncyclopédie Ćadéac).

Pathologie interme des animaux domestiques. 8 vol. in-18 jésus, aver ligures. Paris, J.-B. Bailliere et fils, 1896-1899 (Encyclopédie Cadéac). Ourrage récompensé par l'Institut, en 1901 .

Anatomie pathologique des animaux domestiques. Qe édilion, 190:i. 1 vol. in-1s jésus, aree fig. Paris, J.-13. Bailliere et fils. 


\section{ENGYGLOPÉDIE VÉTÉRINAIRE}

Publiée sous la direction de C. CADÉAC

\section{SÉ M IOLOGIE ET DIAGNOSTIC}

I) ES MALADIES

DES ANIMALX DOMESTIQUES PA R

\section{G. GADÉAC}

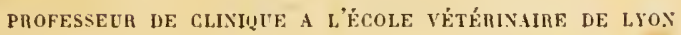

\section{DEUXIËME ÉDITION}

TOME II

Appareil génital. - Mamelles et Lactation. - Appareil de l'innervation. - Orgmes des sens. - Ippareil locomoleur. Température. - .volution iles maladies.

biagnostic el pronostic.

\section{Avec 129 figures}

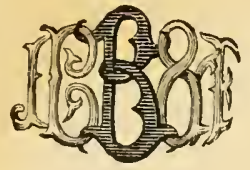

\section{PARIS}

LIBRAIRIE J.-B. BAILLIERE ET FILS

19, rue Haulefeuille, près du boulerard Saint-fiermain

1905

Tous irouts réservás. 



\section{SÉMIOLOGIE}

\section{SÉMIOLOGIE DE L'APPAREIL GÉNITAL}

PREMIERE SEGTION

ORGANES GÉNITAUX DU MALE

\section{I. - FOURREAU ET PÉNIS.}

Topographie. - Le fourreau est constitué par le repli cutané qui entoure normalement la portion libre du pénis. Quand celui-ci entre en érection, sa partie libre fait saillie au dehors et le repli s'efface.

Le pénis est implanté sur l'arcade ischiale par deux racines; il descend entre les cuisses, passe entre les deux sacs dartoĩques et se termine un peu plus en arant, par une extrémité libre.

L'extrémité de la verge ou gland est renllée chez le cheval, très effilée chez le taureau, allongée chez le chien. Chez tous les animaux, le pénis est constitué par un tissu fibreux érectile; chez le chien, la portion antérieure présente un os.

Exploration. - Cheval. - L'exploration du fourreau se fait sur l'animal fixé, debout ou couché. Sur l'animal debout, l'opérateur, placé en devant de la croupe, introduil dans le fourreau la main qui correspond au côté où CADÉac. - Sémiologie, $2^{e}$ édit. 1I. -1 
il se tronve; il saisit le pénis qu'il tire el fail saillir completement au dehors.

Chez les animaux entiers, on peut provouper l'érection en l'approcliant d'une jument en rut.

Sur l'animal couché, on pơrte le membre postérieur superficiel eu avant; l'explorateur, placé derrière la croupe, saisit de la main gauche (le cheral élant conché à droite) le fourreau qu'il tire en arrière; de la main droite intro-

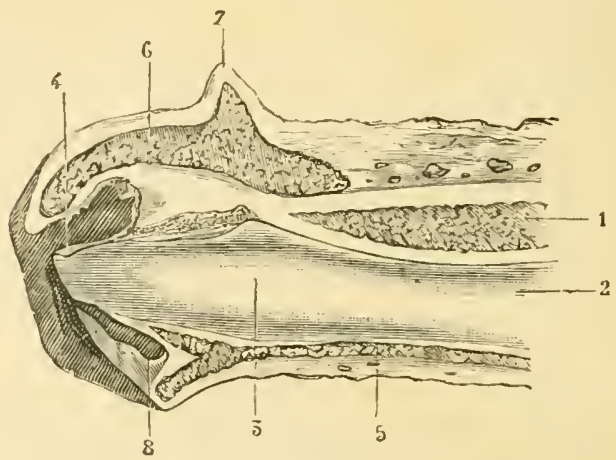

Fig. 1. - Coupe longitudinale de l'extrémité lilıre du péuis du cheval à l'état de relielıement.

1, lissu érectile du corps caverneux. - 2, canal de l'urètre. - 3, fosse navieulaire. - 4 , tolie urélral. - 5, tissu ́rectile du eanal de l'urèlre.6 , tissu érectile du glan $1 .-7$, couronne du gland. -8 , sinus urétral.

duite lans le fourreau, il tire le pénis : le prépuce peut ritre ainsi complètement relourné el mis à découvert.

Pour l'exploration du fourreau et du pénis des aulres animaux, voy. Organes urinaires.

Modificalions pathologripues. - Les altérations du fuunke u consistent dans des éruplions diverses vésiculeuses ou pustuleuses, dans des exulcérations conséculives a leur déliscence (dourine el horsepox), dans un écomlement douloureux de matière d'apparence puru- 
lente (acrobustitc), dans un rétrécissement du fourreau qui empèche le pénis de sortir (phimosis).

On peut renconlrer sur le fourreau des végétations polypeuses (surcomes, fluromes), des fics ou verrues (particulièrement chez les solipèdes), des tubercules, des rarites, des adimes énormes chez le buat:

Les neoplasio's de nature sarcomateuse ou fibreuse (fics, cormes) atteignent quelquefois des dimensions telles qu'elles mettent obstacle à l'accomplissement dı coït.

Le pÉsıs, à l'état normaI, reste dans le fourreau; il peut pendre inerte au dehors (maladics adynumiques, anemies, lésions de la moclle, paralysie du pénis).

L'inllammation du pénis (balanite) le fait augmenter de volume et l'empèche de rentier dans le fourreau (paraphimosis).

On y rencontre de la tıméfaction, des tubercules, des vésicules, des pustules daus le horsepox, la dourine, la morre, la yourme, des végétalions polypeuses, surtout cliez le chicu, des néoplasmes (fibromes, can'roüdes).

On observe aussi des érect ons pernianentes el répétées du pénis ( riapisme) dans le cours du sutyriusis, pendant la rage, les affections de la moelle, l'inflammation du cerveau el le télanos.

Quelquefois le pénis présente des anomalies de conformation : impcrforation de l'uretre, ouverture au bord inférienr dn pénis (hypospadias), au bord clorsal (épispadius).

\section{II. - TESTICULE ET GIINE VAGINALE.}

Exploration (1) - A la place de la malière sébacée qui recomre nommlement les enveloppes testiculaires, on pent observer une poussiere grisitre daus les maladies ehroniques, ancmiques et eacheetiques.

l.es rourses sont plissées el les teslicules ritractés pen-

(1) Voy. Castratiun, Manuel opératoire in Encyclopédie vilérinaire. 
dant les grands froids, dans les affections douloureuses les plus diverses, dans le volvalus, l'invagination, l'étranglement herniaine, la fourbure, l'orchite, l'épididymite.

Les allections les testicules produisent souvent un œdème de la région; les bourses sont augunentées de volume et domsent au toucher une sensation molle, lluctuante, généralement indolore dans l'hydrocèle, pàteuse et douloureuse dans la hernie inguinale et la raginalite.

Les testicules eux-mèmes sont plus volumineux et douloureux dans l'inflammation traumalique ou spécifique (morve, farcin); un testicule peut avoir subi une hypertrophie compensatrice; les deux peuvent ètre atrophiés prar altération des vaisseaux (bistournage) ou sous l'influence d'une infection (pyohémie) el d'altérations diverses (surcocélc, orchite).

I.a région testiculaire peut présenter des cicatrices consécutives à la castration, des anomalies; il y a ectopie quand le lesticule s'est arrèté dans sa migration normale; on peut constater la polyorchidie, la cryptorchidie, la monorchidie, l'anorchilic.

La polyorchidic ou l'existence de plus de deux testicules est très rare; il y a presque toujours eu confusion, on a pris la queue de l'épididyme pour un testicule.

On désigne sous le nom de synorchidie la coalescence des deux testicules.

L'incersion des testicules est fréłuente. Le cordon testiculaire peut être tuniéfié, enflammé (chompignon, funiculite); les veines du cordon peuvent avoir subi une dilatation variqueuse (curicocèle) ou être le siège d'un étrangrlenent.

Clıez le bwuf, il se produit quelquefois après la castration une déchirure périlonéale située soit entre le cordon testiculaire et le bord antérieur de l'ilium, ou plus souvent, sinon exclusivenent, entre le canal déférent et le faisceau vasculaire ì travers le méso qui mit ce canal à la paroi du hassin. L'étranglement de l'anse intestinale 
qui s'engage dans cetle ourerture porte le nom de hernie pelvienne.

On peut ohserver de la spermatorrhée dans les maladies de la moclle épinière, quand l'organisme est épuisé.

\section{DEUXIÈME SECTION}

ORGANES GÉNITAUX DE LA FEMELLE

\section{I. - VULVE.}

Topographic. - La vulve est l'orifice extérieur du vagin ; c'est une fente allongée verlicalement, située dans la région périnéale, présentant deux comıissures, deux lèvres, une cavité contenant le méat urinaire et le clitoris.

Exploration. - Cet orifice est facilement explorable avec les doigts de la main droite : on emploie rarement le spéculum; les Allemands recommandent l'emploi du miroir de Polansky et Schindelka.

La vulve est chaude, rouge foncé et phlegmoneuse dans Ia septicémie puerpérale; elle sécrète une matière purulente et présente quelquefois des fausses membranes de nature diphtéritique.

Dans l'éruption pustuleuse des raches et des juments (coupox, horsepox), la rulve, également tuméfiée, rouge, chaude, présente des pustules et des vésicules.

On peut voir aussi des pustules au niveau de la vulve, dans le cours de la fiéure aphteuse, de la peste bovine. Dans l'hydropisie générale, la vulve esl odémateuse; la nymphomanic sarcompagne de mouvements fréquents du clitoris.

\section{II. - VAGIN.}

Topographie. - Le vagin est un organe situé dans la cavité pelvienne, qu'il traverse horizontalement d'avant 
en arrière, en rapport en lıat avec le reclum, en bas avec la vessie, de chaque còté avec les mretères; il fait suite à lit vulve el se contimue par l'utérus.

Exploration. - On l'explore, le plus souvent, chez les grands animaux, avec les Joigts de la main droite, quelquefois avec la main tout entière. Souvent, il est ıécessaire d'examiner la muqueuse varinale; on emploie alors le spéculum de dimensions variables, quon a le soin d'enduire d'une couche de vaseline.

Il s'éconle du vayin, à l'état physiolngique, pendant les clıaleus, un liquide séreux, sangumolent qu'on ne loit pas confondre avec un écoulement pathologique.

L'écoulement que l'on peut olsserver est de nature très variable: séro-purulent, muco-purulent, daus la raginitc et l'endometrite catarhales ou purulentes; jaune sale, rougeître: brunître, infect Jans l'endométrite septique (septicémie puerpèrale).

Il est de nature et de colorations variées dans la peste borine, la tuberculose le l'utérus, le cancer utrin; enfin le vagin pent donner écoulement à du sang (métrorragie).

Quelquefois il reste, dans cet organe, des masses muqueuses, purulentes, boueuses, sanguinolentes, qui proviennent du rein, le la vessie et du bassinet (yruvelle).

Les tumeurs du vagin (myxomes et sareomes, papillones) sont assez fréquentes, particulièrement chez la chienne.

Le renversement du vagin s'observe avant et après le part, surtout chez les grandes femelles et notamment chez la vache.

Le vagin peut être le sirge de diverses éruptions conLagieuses.

Il peut présenter :

$1^{\circ}$ Un exanthème coütul qui s'olserve dans les espèces chevaline, borine, orine, caprine et porcine, et qui a été décrit sous les noms d'exanthème contagicux des oryunes 
genitaux externes, de vaginite contagieuse chez la vache et de bulunite chez le taurean.

La B.ıLıxıte débute par une congestion interne de la verge, qui devient rouge foncé ; puis par l'apparition, au bout de quelques jours, de petites élevures à peine grosses comme la tête d'une épingle, qui ne tardent pas à devenir blanchâtres et à s'excorier. La muqueuse pénienne, alors dépourvue de son épilhélium, se recouvre d'une abondante é épaisse couche de pus blanc crémeux, sans odeur marquée, ct sécrèle unc notable quantité de liquide lactescent, qui s'écoule goutle à goulte par l'ouverture du fourreau. L'érection est accompagnée de soutlrances violentes. Si l'on abandonne cetle halanite à elle-mème, elle dure environ un nois et disparaît saus laisser de traces (Lucet) (1).

La viginite se manifeste dans les premiers jours qui suivent la saillie; les lèrres de la vulve s'engorgent, deviemnent volumineuses; la muqueuse vaginale se congestionne, se tuméfie, puis se recouvre d'un cnduit blanc jannàtre plus ou moins abondanl, sans odeur, s'éteudant sur les parois du varin, depuis la commissuro inférieure jusquaux deux tiers supérieurs environ; eu mème temps, un liquide trouble, lígèrement jaunàtre, s'écoule goutte à goutle au dehors.

Si l'on enlève cet exsudat purulent, la muqueuse apparait privée de son épithélium, vivement cnflammée el légèrement hourgeomnante. Cette vaginite évolue en trois semaines à un mois. Ou ignore la cause spécifique de cette raginite $(2)$.

$2^{\circ}$ Le vagin est quelquefois le siege d'une inflummation maligne, contagieuse, accompagnée de nécrose de la muquense (Ellinger) (3).

(1) Lucet, Recueil de méd. vét., 1889, 1. 730.

(2) Mathis. Journal de l'École véterinaire de Lyon, 1899, p. 198.

(3) Ellinger, Berliner Thierar. Worhenschr., 1923, p. 25. 
Les symptòmes débutent, vers le troisième jour qui suil l'accouchement, par de la fièvre, une légère tuméfaction du vagin, avec efforts expulsifs el fréquentes envies d'uriner. Un écoulement vasrinal gris verdatre ou couleur chocolat au lait, d'odeur infecte, ne tarde pas à se produire.

La maladie se termine souvent par lit métrite ou une septicémie rapidement mortelle. La muqueuse vaginale devient rouge foncé ou rouge brun; elle se modifie parfois et se recourre d'un enluit plus ou moins épais. Les escarres sont superficielles ou profondes; quelquefois la nécrose intéresse la lotalité de la membrane muqueuse.

Toutes ces manifestations ont été attribuées au bacille de la nécrose; mais il est probable qu'on a affaire à la septicémie puerpérale associée à une infection secondaire par le bacille de la nécrose.

$3^{\circ}$ Le vagin est quelquefois le siège de gramulations, de nodosités ou de régetations rerruqueuses symptomaliques d'une affection chronique contagieuse, décrite sous les noms d'éruption noduleuse du ragin des raches, de vaginite verruqueuse, de vagino-métrite !ranuleuse contagieuse.

Cette éruption débute par le gontlement de la vulve et une rougeur inllammatoire de la muqueuse vaginale qui se recouvre d'un dépôt blance grisàtre, muco-purulent, puis la muqueuse revêt une coloration jaunâtre typique et l'on voit apparaitre un grand nombre de nodosités rapprochées, groupées ou disposées en série, grosses comme une tète d'épingle ou une cerise, qui font légèrement saillie sur la muqueuse vaginale. Ces nodosités résultent de l'hypertroplie des follicules lymplatiques enflammés et offrent une teinte foncée; leur surface est lisse, leur consistance ferme; elles deviennent avec le temps jaunatres ou d'un rose clair et paraissent transparentes; elles sont très nombreuses dans le vestiluale et autonr du clitoris. 
On observe en mème temps un flux vaginal, vitreux, alhumineux, parfois crémeux ou mélangé de flocons purulents et toujours inodores. Les animaux se campent frétuemment pour uriner et font de grands efforts expulsifs suivis tpuelquefois d'avortement.

Ces manifestations persistent quelquefois plus de six mois; les femelles sont en état de rut permanent et sont stériles, utilisables seulement pour la boucherie.

Patuugénie. - Cette infection vagrinale est déterminée par un diplocoque ou court streplocoque souvent incorporé dans le protoplasma des cellules purulentes; il a été isolé par Ostertag. Comme preuves de sa spécificité on invoque: $1^{\circ}$ sa présence conslante dans toutes les varialions morbides; $2^{\circ}$ la possibilité de déterminer un catarrhe typique avec sa culture pure introduite dans le vagin.

L'affection se transmet aux hovins de tout àge par lintermédiaire des litières infectées: les aulres espèces ne penvent être infectées par ce microbe (1).

$4^{\circ}$ On peut rencontrer dans le vagin de la rache, de la chienne, des régétations papillomateuses analogues à celles qui sont connues chez l'homme sous le nom de créles de coq; elles ont élé signalées chez la rache par Lavirotte (2), qui a constaté qu'un tamreau dont la verge en était recouverte a contaminé plus de 60 vaches. Ces végétalions transmissibles sont une cause de stérilitŕ, car, après leur excision et leur cautérisation, plusieurs vaches considérées comme stériles ont été fécondées.

Cadéac ( 3 ) a démontré l'inoculabilité des végétations du vagin et du pénis des chiens; les condylomes se propagent comme les verrues et les papillomes.

(1) Nathis, Journal de Lyon, 1902, p. 37.

(2) Lavirolte, Journal de Lyon; 1892, p. 577.

(3) Cadéac. Sur la transmission expérimentale des papillomes des diverses espèces (Societé des sciences vét. de Lyon, 1901, p. 280). 


\section{III. - UTÉIRUS.}

Topographie. - L'utérus est un sac memliraneux situé dans la cavité abdominale, dans la région sous-lombaire, anquel on reconnaît un corps et deux cornes. Il est suspendu à la région sons-lombaire par deux lames membraneuses, les ligaments larges, lesquels soutiennent en mème lemps les ovaires et les oviductes.

Exploration. - L'exploration de l'utérus se fait par le loucher rectal (grandes femelles), louchers rectal et alodominal combinés (pelites femelles).

L'ulérus peut présenter une augmentation de volume. lue à l'accumulation dans sa cavité de produils morbides liquides ou solides (môles, môles-myxomes, hydromètre, métrite chronique).

L'utérus est tuméfié, douloureux, dans le cas de metrite, métro-péritonite. On peut observer la déviation de l'utérus, la heruie de l'utérus (hysterrocèle!, le recul ou la torsion de l'utérus gravide ou son renversement, constater la rigidite du col (spasme), son envahissement par des tumeurs ou son oblitérution. La malrice pent se rupturer longitudinalement ou transversalemenl; il pent y avoir rétention du foetus, non-déliviance.

On peut confondre l'exagération de volume de cet organe (hyllopisies, tumeurs) a vec la gestation.

Pour différencier ces étals d'une façon sùre, il suffit de faire l'exploration rectale de la matrice; si l'on sent les mouvements du foetus, on est fixé immédiatement.

Ou peut igalement ausculter les hruits du coeur du foetus, à traver's les parois de l'utérus et đle l'abdomen. On ne les entend guère que dans les deux ou trois derniers mois de la gestation; leur nombre est très variahle.

Diverses circonstances, telles que l'éloignement du foetus de la paroi abdominale, l'interposition d'une portion de l'intestin, empèchent de percevoir ce choc cardiaque. 
Modifications pathologiques. - L'utérus demeure généralement à l'abri de toute infection tant que la femelle est viergé; mais il est exposé à des accidents nombreux, soit pendant la gestation, soit après l'accouchement. Cet organe acquiert alors une importance capitale. Son volume, ses besoins nutritifs pendant la grestation, son action mécanique et physiolorrique sur les organes enviromnants lui assurent alors une prépondérance indiscutable.

Après le part, l'utérus peut être le siège d'un grand nombre d'infections locales susceptibles de se généraliser (fierre puerperale).

$1{ }^{\circ}$ Accidents pendant la gestation. - Sous l'influence de la gestation, l'utérus acquiert un tel développement qu'il peut gèner les organes environnants el produire ainsi de la constipation, des coliques, affaisser les vaisseaux pelviens, d’où résulte l'udème des extrémilés el de la parlie inférieure de l'abdomen, comprimer les nerfs oblurateurs et engendrer des phénomẻnes de parésie, de paralysie ou de paraplégie (paraplégie ante partum).

On peut constater en mème temps du pica par insuffi. sance de la nutrition, des crampes par arrêl de la rotule, conséquence de l'amaigrissemenl, de l'amaurose passagère, de l'albuminurie dont l'importance chez la femme est telle qu'elle permet par son intensilé de préroir les accidents éclamptiques. Règle générale, on ne constate l'éclampsie que chez les femmes albuminuriques. L'albuminurie, considérée comme fréquente chez la rache par Franck, n’a pas été observée par Bournay el Besnoit, qui l'ont recherchée. Quoi qu'il en soit, la présence du fœtus modifie la nutrition de la mère; il peut mème se produire une véritable auto-intoxication gravidique résultant de la rélention dans loorganisme des poisons issus des combustions et dont l'élimination peut être entravée par un fonctionnement insuffisant du rein. 
J'expérimentation a d'ailleurs permis de constater que vers la fin de la grossesse, chez la femme, l'urine perd son pouvoir toxique, de sorte que l'organisme est à ce moment plus ou moins saturé de poisons (Ghambrelent et Laulanié).

L'éclampsie résulte de cette intoxication.

Les infections qui menacent la matrice et le fotus sont plus redoutables yue les intoxications.

L'arortement sporadique ou enzootique est la conséquence la plus fréquente de ces infections. L'avortement de la vache peut résulter de maladies microbiennes et d'intoxications de la mère propagées an fotus par le placenta, de maladies du fortus; mais le plus souvent l'avortement est l'expression d'une infection vaginale ascendante.

Cette infection est la règle dans l'avortement épizootique, qui doit être considéré comme le résultat d'un calarrlıe utérin spécifique, produit par une bactérje déterminée (Bang). L’importation d'une vache pleine infectée est suivie de l'infection du local; elle peut même contaminer ses voisines avant d'avorter. Les agents pathogènes parvenus à la vulve au contact d'une litière infectée cultivent sur Ia muqueuse, gagnent le vagin, pénètrent dans l'utérus (1).

L'avortement épizootique de la jument comme de la vache se propage par contagion. L'agent infectieux se trouve, dans lous les cas, dans le foetus expuIsé et dans l'écoulement vaginal qui suit l'avortement, car son lieu d'élection est la matrice gravide; les cheraur. hougres et les étalons n'offrent aucune manifestation lémoignant d'une infection susceptible d'être considérée comme le résullat du contage de l'avortement.

L'infection s'effectue ordinairement par les fumiers, les déjections solides ou liquides répandues sur le sol.

Cette infection est tantót bénigne et se traduit par une

(1) Bournay, Obstêtrique vétérinaire in Encyclopédie, p. 322. 
léçère tuméfaction de la rulve et de la muqueuse vaginale, puis par quelques coliques sourdes suivies de lexpulsion du fœtus âgé de quatre à sept mois au plus, dont la jument se débarrasse comme d'un produit excrémentitiel; lantòt on a affaire à la forme maligne qui se manifeste à partir de sept mois de gestation; l'avorlement est suivi de non-delivrance qui peut entraìner la métrite puerpérale, compliquée à son tour d'arthrite, de tendinite et de synorite infectieuse, de fourbure ou d'hémoglobinurie avec paraplégie (1).

20 Accidents consécutifs à l'accouchement. - L'accouchement est fréquemment suivi de traumatismes plus ou moins graves (excoriations, plaies simples, plaies contuses, déchirures, rupture du périnée, fistule rectovaginale, thrombus de la vulve et du vagin, suppuration de l'utérus, métrorragie plus ou moins intense).

L'accouchement est souvent le point de départ d'accidents infeclieux qui ont un grand intérêl en obstétrique vétérinaire.

Ces infections des nouvelles accouchées se présentent avec des formes très nombreuses commandées par l'étendue des lésions traumatiques qui les rendent possibles, la réggion des organes génilaux alleinte, la virulence de l'agent palhogène et surtout la résistance de l'organisme. Tantòt cette infection s'accuse par une inflummation cutarrhale superficielle accompagnée d'une lièvre légère; tantòt par la formalion de foyers purulents, la mortification des tissus et une fière intense; tantòt, enfin, cette infection se généralise à tout l'organisme, produit une hyperthermie considérable et lue rapidement la femelle.

Cette infection des organes génitaux est facile et fréquente. Elle est favorisée:

(1) Guillerey, Journal de l'École vétérinaire de Lyon, 1901, p. 544. 
10 Par la conformalion des organes gomitaux qui forment une cavilí close, véritable lıallon de culture fréquemment lubité par des microbes indifférents (Iignières) (1) et qui au noment de laccouchement, par les lochies, les exsudats sóro-sanguinolents qui s'y forment, fournit ahri, nourriture et tempéralure à des microbes divers apportés dı delıors par les mains de l'opérateur, les instruments, etc. L'infeclion s'établit sur les parois d’un tel organe avee la plus grande facilité.

2 Par la disposilion anatomique du placenta qui, suivant les esjèces, favorise davantage la pénétration des microorganismes clıez les femelles caruivores, qui, après la mise-bas, présentent une plaie physiologínue à la face interne de l'utérus, que chez les herbivores, dont la muqueuse nlírine ne présente, à ce moment, qu'une simple exfoliation épidermique.

$3^{\circ}$ Par la dystocie qui, par la fatigue qu'elle impose à la femelle, les opérations et les traumatismes qu'elle entraine, constitue la cause la plus à craindre et la plus fréc|uente des infections des organes génitaux.

L.esinfections qui atteignentles organes génilaux après l'accoucliement sont surtout celles que nous avons en vue ici. On les désigne d'un nom générique, eınprunté à l'obstétrique humaine : ce sont les infections puerpérales.

Selon la virulence des microorganismes qui les occasionnent, elles se montrent sous la forme phlogogène, sous la forme pyogène ou sous la forme septique ou pulrile. Alors que les symptimes locaux sont surtout accusés laus les formes pyogènes, les symplòmes géuéraux, stupeur, fièvre, adýnamie, constituent la dominante le la srine morbide lors d'infection septique.

La firre de lail (2) de la femme est actuellement considéríp comme l'expression d’infections allénnées des

(1) Siraus (.t Sanchez Toledo n'y ont pas trouse de microbes (Journal de Lyon, 188s, p. 325). Lignières, Bull. de la Soc. centr., 1896, p. 55.

(2) Wallt ard, Semaine médicale, 1895, p. 195. 
organes génilaux qui se produisent an moment oủ la sécrétion lactée s’établit; cependant, chez certaines femmes, la fluxion mammaire peut s'accompagner de céplıalée, d’accélération du pouls el mème d’une légère hyperthernie; ce fait sohserve surtout lorsque la femme 11 allaite pas (1).

Chez les fenelles domestiques, hors les cas de part Jahorieux, oì, par suite du surmenage, la température peut subir une légère élévation, toute hyperthermie doit ètre considérée comme un indice d’infeclion génilale post partum.

Les iufections puerpérales donnent lieu, tantòt à des lésions localisées sur les organes génitaux, tantôt à des lésions géuéralisées, c’est-à-dire dépassant la sphère gŕnitale. Les principaux éléments de cetle infection sont représentés par la vaginite, la mótrite, la septicémic te parturition. Cetle dernière, appelée encore métro-péritunile infectieuse, métro-peritonite puerpèrale, septieémie puerperale, représente la forme la plus redoutable des infectious post partum.

La septicémie de parturition résulte de l'infection des organes génitaux et nème de l'organisme culier par des microbes doués dune grande virulence et provoquant rapidement I"intoxication de la femelle.

Alors que dans la raginite et la metrite simples ce sont les symptómes locrux qui prédominent, ici, au contraire, ce sont les symptômes ginerrax uni frappent l'observateur. La maladie se caractérise, en effet, par une évolution rapide, une fière intense, un abattement prononcé, et une inllammalion plus ou moins accentuée des organes génitaux.

La contagiosilé de la septicemie puerpérale de la femme a élé signalée dès 184 par Sernmelweiss. Soutenue frar Tarnier, elle a été expérimentalement lémontrée par Coze et Felı, Pasteur, Doleris, Mazini, el enfiu par Chau-

(1) Kibemonl-Bessaignes et Lepage, Précis d'obstélrique, p. 584 et 1295 
reau el Alloing. I'après ces clerniers auleurs, la maladie serait due au streptococcus puerperulis, ou streptocoque du pus, qui, "suivaut l'état de sa virulence el suivint les conditions de milieu qu'il rencontre, peut être plus particulièrement : pyorgène, phlogogène ou septique " (Arloing $r$ ).

Des reclierches récentes montrent que le streptocoque du pus n'est pas le seul nicroorganisme qui, cliez la femme, puisse donner najssance à la septicémie puerpérale; le staplıylocoque, le Bacterium coli commune peuvent en faire autant. Il est mème des cas oì la maladie résulte d'une infection polymicrobienne : streptucoques, slaphylocoques, cocci, etc. (1).

Chez les femelles domestiques, la bactériologrie de la seplicémie de parturition est très peu étudiée (2).

Colin (3) a apercu, dans l'exsudat pleural d'une lapine morte de septicénie de parturition, des " corpuscules irréguliers, påles », et a provoqué la mort de plus en plus rapide de liois lapins par l'inoculation de cet exsulat. Brusasco (' $x$ ), chez la chèvre, a trouvé, dans le sang, dans l'exsudal péritonéal, etc., le Bacterium septicum puerperiele.

Lucet a isolé des lésions de la septicémie de parturition, chez la vache, des microorganismes divers et pense avec raison que, cliez cette femelle, il existe plusieurs sortes de septicémie puerpèrale.

Cuillé, chez la vache, a trouvé le streptocoque du pus, à l'état de pureté, dans le sang du caur. Besnoit, chez la mème femelle, a observé un cas de septicémie puerpérale produite par le colibacille.

(1) Voy. Arloing, Leẹns sur la tuberculose et certaines septicémies, chap. $x, \mathrm{p}, 488$.

(2) Ribemont-llessaignes et [,ppage, Précis dobstétrique, 1897, 1\%. 1300 ct 131 .

(3) Colin, Sur une altération du sang liéc aux accidents de la parlurilion (Académie de mídecine, 14 octobrc 1879, el Arch. vèt., 1879, p. 831 ).

(4) Brusasco, Journal de Lyon, 1884, p. 327; Il medico Veterinario, 1883. 
Il se dégage naturellement des faits cités pour l'obstétrique Lumaine et pour l'obstétrique vétérinaire, que la septicémie de parturilion peut être provoquée par des microbes divers, dans toules les espèces animales.

\section{IV. - CASTRATION.}

Les testicules et les ovaires ont sur l'ensemble de l'organisme une influence indirecte, lointaine. Cette action, yui s'exerce par l’intermédiaire des produits de désassimilation ou de sécrétion interue, est désignée sous le nom de corrélation.

Les modifications du milieu intérieur produites par la vie des cellules ou par les sécrétions internes sont susceptibles dagir sur les autres cellules; elles peuvent agir aussi sur le système nerveux et retentir ensuite indirectement sur d'autres organes.

Effets de la castration. - La castration modifie: 10 le caractère; $2^{\circ}$ la forne du corps; $3^{\circ}$ la proslate; $4^{\circ}$ l'utérus. La caslration exerce une grande influence sur les instincts génésiques, qu'elle ne supprime pas toujours; elle retentil sur les mamelles et la sécrétion lactée et influe sur la nutrition.

$1^{\circ}$ Caractère de l'animal. - Le caractère subit de profondes modifications sous l'influence de la castration.

L'étalon farouche ou indomptable, rétif ou méchant, devient tranquille, doux, maniable, docile au frein el facile a utiliser; le taureau dangereux devient un bouf placide.

Tous les animaux castrés sont plus faciles à dresser.

Ces effets sont surtout prononcés quand l'animal est castré de bonne heure, c'est-à-dire avant que les testicules soient en pleine activilé.

La castration modifie aussi le caractère et le limbre 
de la voix. Certains animaux, comme le chapon et le cheral hongre, deviennent silencieux. Mans tous les cas, il y a diminution de la force, de la sonorité, de l'ampleur et du vibrant de la voix chez les animaux émasculés.

Le chien devient paresseux; il perd une grande partie de son activité, mais il ne perd pas son intelligence.

La castration modifie en elfet surtout le caractère, sans affaiblir l'intelligence.

Les castrats huminins sont habiluellement pusillanimes, couards, sans viguenr, mais non sans intelligence. Aristomène, crénéral de P’olémée, Ali, grand vizir de Soliman, le philosophe Phavoriuus prouvent que l'homme dépourvu de testicules peut avoir de grandes qualités intellectuelles. l.es chirns ont moins d'entrain à la chasse, mais il ne semble pas qu'ils oublient ou perdent les qualités qu’ils ont acıuises par le dressage. Ils deviennent très apathiques et engraissent comme les eumuques.

Les castrats acquièrent de bonne heure mu embonpoint exagéré et leur visage flétri prend l'aspect vieillot.

lat rache et la truie castrées sont également plus calmes et ont mue grande tudance ì l'engraissement.

2) Forme du corps. - La forme du Corps des animaux castrós diffire sensiblement de celle des animanx entiers. la castration inllue surtout sur le diveloppement des mîles.

Sous cette influence, la nufrition générale prend un autre essor. Elle se ralentil dans les parties antértieures, dont le développement dome à l'organisme achevé les formes caractéristipures lu lype de l'espèce à laquelle il appartient; elle concentre an contraice son énergie sul les parties postérieures, qui devienneutplus larges et plus étolfées (II. Bouley).

Quelle différence entre l'encolure, la crinière de l'étalon ou le train antérieur et le bassin llu tinureau, et l'état de ces régions cliez les animaux castrés de homne heure! 
Les phanères, crinicre et queue chez le cheral, chignon chez les borjus, sont plus fouruis chez le màle que che\% la femelle et, dans les espèces pourvues de comes, on troure des parlicularités intéressantes. Les cornes dn tanurean, larges à la base, fortes et puissantes, se développent en épaisseur comme en largeur, tandis que celles de la rache sont toujours moins volumineuses; la laine du mouton se rapproche de celle de la brebis; chez le chapon, les plumes de la quene, au lieu de se relever et de se recourber en faucille, restent horizontales; les cornes ne poussent pas chez le mouton castré; elles deviennent beaucoup plus longues et moins grosses chez les hovills.

Les animaux castrés, comme les femelles, ont des formes plus fines et plus élégantes. la tête est plus légère, l'encolure moins nusclée, les membres plus grèles, le train antérieur et le train postérieur s'égalisent.

Toutes proportions gardées, la verge du cheral hongre et du brinf est moins longue et moins grosse que celle de l'étalon et du taurcan; mais la masse cérébrale du cheval hongre l'emporte sur l'étalon et sur la jument.

Si l'on compare les différents organes composant la masse encéphalique, on constate que la conséquence la plus remarquable de l'émasculation est l'augmentation absolıe et relative dı poisls du correlet el la diminution des hémisphères cérébraux el de l'isthme (Colin).

La castration du lapin détermine un allongement des os, des fémurs, des tilias, dı sacrum avec alténuation des courbures el agrandissement du canal médullaire (Poncet). Les eunuques aussi ont les épaules étroiles, le bassin large, les formes arrondies, empitées; l'élargissement du larynx ue se produit pas; il ne leur vient point de poils an menton, ni aillenrs; le pénis reste petit; les femmes privées d'ovaires avant la puberté conservent le type infantile.

Les sujets chàtrés sont des neutres; les différences 
qui existent entre les sexes s'effacent; l'homme àgé n'a plus le caractère, les groùts, la voix, l'aspect de l'homme viril; les femmes yui ont cessé d’avoir leurs menstrues prennent quelquefois une voix grave et l'on observe la pousse des poils à la lèvre supérieure ou au menton. Ces femmes-hommes étaient appelées viragines par Jes Romains. Quand les hiches sont devenues stériles, on voit parlois leur têle s'orner de hois comme les cerfs, et chez les oiseaux il est fréquent de rencontrer de vieilles femelles ayant cessé de pondre qui alors prennent la livrée des mâles.

3o Prostate. - La prostate el le testicule subissent un développement parallèle; la prostate demeure petile chez les animanx castrés.

Quand l'évolution du testicule est arrêtée, la prostate ne se développe pas. Cet organe s’atrophie mème lorsqu'on supprime le testicule après son développement complet. Cette atrophie secondaire a élé constatée expérimentalement chez les animaux. Les embryologistes ont élabli l'existence de rapports intimes entre les testicules et la prostate; les pathologrstes ont confirmé le fait.

Ainsi, lans la monorchidie, le lobe correspondant de la prostate est lıbituellement seul alrophié, alors que l'atrophie prostatique est totale dans la cryptorchidie. Les expérimentateurs ont fourni la démonstration complète des relations fonctionnelles entre la prostate et les testicules.

Kirley constate que l'extirpation totale des resticules est rapidement suivie de l'atroplie de la prostate; fuinard vout la castration dı chien s'accompaguer de la réduction de volume de la prostate hypertrophiée; Ginyon et Legueu castrent deux chiens el conslatent au bout de cinq mois que la prostate a diminué des deux tiers; chez un troisième, l'atrophie est déjà appréciable au hout de deux mois et demi. Cette atrophie 
débute rapidement, elle est déjà très nette à l'examen histologique un mois et deni à deux mois après la castration.

De là est née l'idée d'employer la castration pour combattre les hypertrophies de la prostate. On avait conslaté que l'ovariotomie amène quelquefois la disparition des fibromes de la matrice et l'atrophie de cet organe, et l'on a pensé que la castration peut exercer une influence analogue. Cette opération est actuellement recommandée contre les complications du prostatisme. Mais il est clair que les néoplasmes de la prostate ne sauraient disparaîlre sous l'influence de la castration.

Il convient de rechercher si l'extirpation des lesticules est indispensable et si la ligature ou la section des canaux déférents ne suffit pas pour déterminer la dimiuution de volume de la prostate.

On aurait ainsi l'avantage de conserver les testicules avec l'action tonifiante qu'ils exercent sur l'organisme, toul en produisant une action salutaire sur la prostate.

$4^{\circ}$ Utérus. - L'UTÉRus suljit diverses modifications à la suite de la castration, qui pourtant dans bien des cas ne supprime pas les règles quand elle est pratiquée chez la femme après la puberté : l'utérus s'atrophie complèlement par corrélation ovarienne; cette atrophie est déjà manifeste au bout de vingt jours, ce qui démontre que l'utérus peut ètre considéré comme l'annexe de l'ovaire.

Cette dégénérescence atrophique est analogue à celle qui survient chez la femme à l'époque de la ménopause. Elle se traduit par une diminution de volume de l'ulérus, par le raccourcissement et le rélrécissement du vagin.

Cette dégénérescence a élé étudiée chez la lapine par Buys et Vanderbelve, en 1894. Elle consiste en un processus atrophique sans stíatose, le tissu conjonctif de l'endométrium se transforme en tissu fibreux cicatriciel et l'épi- 
thélium des frlandes muqueuses est frappé de dégénérescence el de nécrose. Quand les règles persistent chez la fenme ovariotomisée, c'est que, selon loute probabilité, l'extirpation des ovaires a élé faite d'une manièrc incomplete.

;0 Les instincts génésiques sont-ils constamment supprimés par la castration? - Assurément la castration pratiquée sur les animaux jeunes fait disparaître les appétits sexuels et mẻme la faculté d'entrer en érection; mais les sujets opérés à l'àge adulte peuvent conserver ces aptitudes pendant un temps variable.

Les sujets émilsculés et les femelles ovariolomisées ont quelquefois des besoius sexuels aussi impérieux, des arleurs génitales aussi vives que les sujets possédant les testicules ou les ovarres.

Cerlains castruls lumnains présentent des ardenrs sexuelles excessives, comme en témoignent des observatious de Teinturier, de lichet, de Princetean; ils peuveut avoir des éjaculatious véritables et terminer le coïl par une émission de liquides sécrétés par les grlandes annexes de l'appareil gémital.

Un voil également des chevaux castrés, privés de leurs deux testicules, conserver dans une mesure plus ou moins grande leur aptitude au coït avec toutes ses manifestalions physiologiques. Checal hongre n'est donc pas toujours synonyme de cheval indiffirent, au point de vue de la vie sexuelle.

A quelle eanse faut-il attribuer la persistunee des instincts genisiques apris la cutrution? Le cheval semble pouvoir conserver l'allure du chevill entier après la eastralion, losspu'il y a triorehidie ct que l'opération ne porte que sur deux testieules ou yuatul l'opération est incompléte.

"Dans le cas de triorchidie, le testicule supplémentaire entrotiout les inslincts gémésiques el, paur les fitre cusser, il suflit de pratiqler l'ablation de celte troisiène 
glande génilale. Le fait a été signalé plusieurs fois. Delaforge a enlevé trois testicules à un poulain àgé de six à dix mois. Dans les autres cas, publiés par Létard, Nocard, Labal, Friez, Gallier, il s'agit d’animaux qui, à la suite d'une castration régulière, comportant l'ablation de deux testicules, ont présenté, plusieurs mois plus tard, un troisième testicule qu'il a fallu enlever pour modifier le caractère de l'unimal (1).

La valeur de ces observations est nettement conlestée par Jacoulet. Cet auteur pense, avec raison, croyons-nous, qu'ilne s'agit dans tous ces cas - comme le dit Gallier' luinême au sujet de celui qu'il a observé - que d'une casträtion incomplèle comme l'a observé Bournay; la section du cordon testiculaire, qu'il s'arisse des casseaux ou de la torsion bornée, peut ètre pratiquée trop bas, trop près du testicule, sur la glande elle-mème, el le troisième testicule ne serait autre chose que l'extrémité hypertrophiée du moignon formé par l'épididyne et très probablement par une partie du testicule. Il ne faut pas oublier cependant qu'un testicule peut se dédoubler. Newcomb a châtré un cheral porteur de quatre testicules; chaque testicule normal s'était divisé en deux. Lors de cryptorchidie, le sujet conserve le tempérament du cheval entier, lursque la castration, pour le testicule caché, - l'autre étant supposé enlevé, - ne porte que sur une partie de l'épididyme engagée dans le trajet inguinal. Le testicule indemne reste dans l'abdomen.

La disparition des instincts génésiques s'oblient en enlevant le testicule retenu, par les procédés ordinaires de castration des cryptorchides (Bournay). Celle uquestion de la persistance des instincts génésiques après la castration est particulièrement intéressante quand des sujets, normalement conformés, sont opérés par les procédés de

(1) Bournay, Fersistance des inslincts génésiques el rélivité chez un cheval chitré. Extirpation du cordon lesliculaiıe Gicioson (lievue rét., 1899, p. 333 ). 
castration les plus suivis, les casseauxà leslicules couverts et la torsion bornée.

Des faits très curıeux ont été rapportés par Mauri (1), qui a publié un mémoire sur ce sujet.

Ou y trouve notamment l'histoire d'un cheval de quatre ans qui avait été chàtré et qui, malgré cela, avait conservé toutes les allures d'un étalon. Il était sans cesse tourmenté par l'instinct génésique et faisait entendre des liennissements et des ronflements qui devenaient surtout signilicalifs à l'approche d'une jument. Quand on lui en présenlait une, il la flairait, la mordillait, entrait eu érection et, brusquement, se cabrait sur elle pour la saillir enfin avec une grande vigueur. L'acte terminé, il éprouvait très manifestement le sentiment de lassitude, de défaillance momentanée que présente tout étalon dès que la saillie est effectuée et, à la sortie du pénis des voies géuitales de la femelle, on pouvait voir s'écouler encore un liquide visqueux, frlant, un peu moins épais que du sperme ordinaire. Ce liquide ne renfermait pas un seul spermatozoïde. Malgré toutes les recherches faites sur cet animal, Mauri ne découvrit aucune glande génitale.

Les vétérinaires des dépôts de remonte ont constaté que 2 à 3 p. 100 en moyenne des jemnes chevaux hongres entretenus dans les annexes conservent des instincts génésiques, effectuent la saillie, émettent du liquide spermatique sans jamais féconder les juments qui les recoivent.

Ordinairement ces chevaux ne hennissent pas et ne recherchent guère les juments; ils opèrent seulement la saillie quand elles sont en chaleur et à la suite d'atlouchements nombrenx. Mais quand ils sont en train, ils peuvent l'accomplir plusient's fois en très peu de temps et avec la vigueur d'un étalon. Sendrail a vin un cheval hongre qui, placé entre deux juments en rul, les a saillies

(1) Mauri, Contribution à l'étude de l'influence du testicule et des ovaires sur les instiucts génésiques (Kevue vét., 189i, p. 473). 
yuatre fois en l'espace de deux heures, allant alternativement de l'une à l'autre.

Dans ces cas, la persistance des instincts génésiques semble résulter de la présence d'une masse plus ou moins considérable de substance lesticulaiı, restée à l'extrémité du cordon, par suite d'une application défectueuse du casseau ou de la pince à torsion, el, comme l'a démontré Bournay, pour supprimer ces inslincts, il n'y a qu'à extirper le cordon testiculaile en le sectionnant audessus de l'épididyme.

Lovariotome ne fait pas toujours disparaitre les appétits sexuels chez la femme; il y en a qui sont aussi passionnées après l'opération qu'avaıt.

La suppression des ovuires chez la femme exagère leur irritabilité; elles éprouvent souvent au début des bouffées de chaleur, avec sensation d'oppression, d'étouffement. L'hystérie n'est guère modifiée par la castration. D'ailleurs, l'o variotomie pratiquée chez les femelles domestiques pour combattre la nymphomanie ne donne très souvent que des résultats médiocres ou nuls. Les instincts génésiques persistent après l'opération, chez la jument comme chez la femme, avec toute leur ardeur.

Il résulte des observations de Degive, Traslot, Cadiot, Delamotte, Cadéac, Flocard, etc., que les juments nymphomanes chàtrées demeurent aussi méchantes, aussi excitables qu'avant l'intervention. Thomassen a fait remarquer avec juste raison que la nymphomanie caractérisée exclusivement par un désir exagéré de l'acte vénérien est seule améliorée par la castration; cette opéralion n'a, au contraire, ancun effet sur la nymphomanie marquée par des défauts de caractère.

D'ailleurs, il faut reconnailre que l'ovariotomie ne fait pas constamment cesser les chaleurs; on a constaté que, sur trois vaches parfaitement clıàrées, deux manifestèrent encore des chaleurs après l’opération.

Cadéac. - Sémiologie, 2e édit.

11. -2 
La persistance des chaleurs est parfois observée chez des truies après l'ovariotomie; mais il ne me paraît pas absolument établi qu'on n'a pas dans ces cas laissé la moindre Irace d'ovaire. Cependant, il existe quelques faits très probants.

" Un châtreur de profession opère trente-huit petites truies àgées de quarante-cinq à soixante jours. Malgré cetle opération, ces bètes, à partir de l'ìge de trois à huit mois, entrent eurutmensuellement comme celles quin'ont pas élé chàtrées.

"L'autopsie démontra cependaut que l'opération avait été bien faite, que cornes de la matrice, oviductes et ovaires avaient été enlevés. " (Barthéleny.)

Bassi a recueilli des faits coufirmatifs de ceux de Barthèlemy. l.es uns se rapportent à l'espèce bovine et les autres a l'espèce canine. De deux vaches nymplomanes chitrées par Bassi, l'une s’engraissa cousécutivement et ne manifosta plus l'ardenr, l'autre continua ì être en rut. Une autre, chàtrée à l'école vétérinaire de Turin, et lrien chàtrée, ainsi que l'apprit l'autopsie qu'on en lit, manifesta encore des chaleurs après l'opération.

Bassi opéra à l'àge de deux mois une chiemue gravide qui ne manifesta jamais de chaleurs. Il fit la mème opération sur une chienue de même race. Elle avait à ce moment trois ans et avait léjà donné deux portées. Cela ne l'empêcha point d'entrer en clıaleurs dans la suite et de se laisser couvrir, sans résultats, d'ailleurs. Abattue deux ans après pour cause d'épilepsie, on put voir à l'autopsie yu'elle avait été, bien chàtrée et qu'il ne restait aucun vestige des ovaires. It ne faut done point, conclut Bassi (1), dire que si des chaleurs apparaissent après la castration e'est que nécessairement l'opération a été mal faite. Il ne faut pas davaulage dire, avec quelques physiolugistes, que les chileurs sont toujours la traduction exté- 
rieure du phénomène de l'ovulation; elles peuvent avoir d'autres causes.

En somme, malgré l'importance capitale des ovaires dans l'ovulation et le réveil des instincts génésiques, on est forcé de reconnaîlre que, mème en dehors d'eux, les instincts peuvent persister et se manifester, chez la fenme, par des désirs et des sensations voluptueuses, chez les l'emelles par la persistance de chaleurs.

Les centres nerveux génito-spinaux peuvent entrer en fonclion indépendamment des testicules et des ovaires et accomplir ainsi les actes physiologiques qui entrent daus leurs attributions ordinaires (Guinard) ( 1 ).

\section{$6^{\circ}$ Influence de la castration sur la sécrétion lactée.}

La castration augmente la sécrétion lactée chez les raches ovariolomisées; la sécrétion lactée reste à un taux égal, sinon supérieur, à celui du vêlage pendant une moyenne de vingt à vingt-ımalre mois.

II résulle des observations d'un grand nombre de praticiens (Levrat, 1834; Clırlier, 1848; Flocard, 1895), qu'une vache ovariolomisée donne au moins dans l'année qui suit l'opération 1300 à 1400 litres le lait le plus que si elle n'eût pas subi d'opération. Il arıive même que la sécrétion lactée est prolongée pendant plusieurs années. Flocard a vu des vaches ne descendre à 8 litres de lait par jour que sept ans après la castration; d'autres ont mis cinq ans pour descendre à 12 litres; une autre trois ans ponr descendre à 18 lilres. Cette dernière a donc fourni près de 7000 litres de lait par an pendant trois ans.

La castration modifie non seulement la quantité de lait, mais elle a une grande influence sur sa composition. Sa qualité s'améliore beaucomp, la proportion de beurre peut doubler; la caseine, les sels, la lactose angmentent également; mais, au bout de deux mois plus tarl, la richesse

(1) Guinard, Castration (Dict. de rhysiologie de Richet, t. 11, p. 491). 
du lait diminue graduellement pour sarrèter cependant i un chillre toujours supérieur au chiffre inilial (Flocard). Eu somme, le lait des vaches châlrées est plus riche en beurre et en casime. Enfin, quand la quantité de lait diminue, on constate une grande tendance à l'engraissement, de sorte que la castration offre de grands avantages pour la production de viande comme pour la production de lait.

$7^{\circ}$ Influence de la castration sur la nutrition. - La Nutrition générale est modifiée par la castration; les sujets privés de leurs glandes génilales paraissent manquer d'un stimulant utile à l'activité de leurs échanges nutrilifs. Pöhl a démontré directement le pouvoir oxydant de la spermine; il y a augnentation de l'urée el diminution des leucomaines urinaires. Par la castration, on prive l'organisme d'un tonifiant, on mobilise les forces vitales de l'organisme du côté des fonctions individuelles, on ralentil les échanges, on favorise l'accumulation de la graisse.

Par contre, le développement normal des glandes génitales amène le développement parallèle des glandes mammaires, de sorte qu'on ne s'explique pas que la suppression des premières puisse exagérer la sécrétion des srcondes.

\section{V. - OVAIRES.}

Topographie. - Ce sont les organes essentiels de la ginération chez la femelle. Ils sont situés dans la cavité alıdominale, altachés à la région sous-lombaire, un peu nil arrière des reins.

Exploration. - L'exploration se fait par le rectum (grandes femelles), par l'ablomen (petites femelles).

Eille peut révéler la tuberculose des ovaires, leurs diverses dégénérescences, leur intlamnation (ovarite), les kystes simples ou dermoüdes et diverses autres causes de nymplomunie. 


\section{VI. - NYMPHOMANIE.}

Définition. - On désigne ainsi une exacerbation anormale des fonclions génitales chez nos femelles domestiques.

Les raches atteintes de cette affection sont désignées sous le nom de taurelières, le terme nymphomane étant réservé pour les jumeuts.

Ce trouble, observé fréquemment cliez la rache, la inment, rarement chez la chiemne el la chatte, est mal connu chez la brebis, la chèrre et la trivie; c'est plutòt un symptòme dépendant de causes variables qu'une affection spéciale (1).

Pathogénie. - L'alimentation abondante, l'áge arancé semblent être les causes principales de ce trouble. Dix vaches taurelières autopsiées par Schmidt n'ont présenté aucune altération génitale.

La prédisposition à la nymphomanie est héréditaire. Une rache devenue laurelière après un quatrième velagre engendre une génisse qui devient également laurelière après sa seconde gestation. Les chaleurs non satisfaites, le contact répété du mâle, les attouchements vulvaires, favorisent sou apparition (Detroye).

Cependant, la nymphomanie est souvent déterminée par une altération des organes génitanx (inflammation, déçénérescence kystoïde, hypertrophie, tumeurs cancéreuses, sarcomateuses el tuberculeuses des ovaires, vaginite chronique, endométrite chronique, obstructions du col, tumeurs, changements de rapport, atrophie et hypertrophie de l'utérus). On peut constater la persistance de la nymphomanie après la castration de la rachc et de la jument. On peut mèıne voir la nymphomanie apparaitre après la castration. French enlève à une chatte les deux

(1) Voy. Nimpomane in Obstétrique de l'Encyclopédie vciérinaire, par Bournay, p. 175. 
ovaires, les oviductes et tout l'utérus qui contenait six fœtus. Un mois après, elle manifestait un appétit sexuel, qui persisla pendant quatorze mois.

Caractères. - Chez la rache, cet état se traduit pardes chaleurs fréquentes et par la non-conception, malgré les coïts répétés; l'animal est inquiet, gralte le sol ; il pousse des beuglements (malde beuglement), monte sur les autres vaches, parfois présente de véritables accès furieux. Le lait diminue considérablement.

Chez la jument, on ohserve des chaleurs fréquentes à manifestations très bruyaules. Au moindre attouchentent de l'éperon, de la main ou des traits, la jument pousse un cri aigu, le clitoris fait saillie, un jet d'urine s'échappe et une vigoureuse ruade termine ces manifestations (jument pisseuse).

A la longue, on peul observer l'amaigrissement, la diminution de la sensibilité générale, comme dans l'immobilité (tic ovarique).

\section{VII. - SATYRIASIS.}

Définition. Garactères. - C'est un trouble géuital qui répond chez les mâles à la nynıhomanie des femelles.

On l'observe chez le clicon, l'ćtalon, le tanreau, le bone. Cel état s'accuse par des érections fréquentes et prolongées, une surexcitation très grande à la vue des femelles.

Parfois, quand l'ardeur n'e: 1 pas satisfaile, l'excitation s'exagère et peut devenir rabiforme. Les animaux maigrissent, devienuent tristes; on a constaté chez le cheval un étal analogue à l'immobilité. Souvent ce trouble se termine par la cachexie (lic testiculaire).

Dans l'espèce chevaline, les monorchides et les cryptorchides sont fréquemment affectés de satyriasis.

Ce trouble a élé observé également dans la rage du chicn; enfin, il peut être le signe d'un trouble nerveux mal délini. 
I.e régime débilitant, les exercices violents et la castration calment les bêtes nymplomanes on affectées de salyriasis.

\section{VIII. - ONANISME.}

Définition. - On désigneainsi “l'ensemble des moyens employés par l'un et l'autre sexe pour produire artificiellement l'orgasme vénérien, en dehors des conditions du coït normal " (Christian).

Certains auleurs ont désigné ces manœuvres sous le nom d'autosburbation.

Les animaux, comme l'homme, semblent s'y livrer pour satisfaire le mème sentiment de volupté. On a vu des étalons se maslurber alors que, près des femelles, ils n'éprouvaient aucunc excitalion.

Le satyriasis, la cryptorchidie, les dèsins vénériens non salisfaits, le voisinage des femelles en chaleur sont les causes les plus fréquentes de ce trouble.

Caractères. - Les étalons, pour accomplir cet acte, se frappent le ventre avec la verge; les juments se froltent contre tous les obstacles qu'elles peuvent rencontrer; les ânes prenneut la verge avec les lèvres. Le cliten et le bélier sont les animaux qui se masturbent le plus fréquemment.

Plassio (1) a observé l'autosturbation sur une dizaine de chevanx à la fois; mais les animaux nutilisent pas le mème procédé que les innes pour éjaculer.

Quand l'animal veut effectuer cet acte, il demeurc immobile, les yeux fixes; la verge sort du fourrean et arrive à un élat de demi-éreclion. A ce moment, par la contraction des muscles ischio-caverne $1 x$, la verge opère des mouvements de haut en bas qui complètent l'érection, puis, celle-ci étant prononcée, le pénis vient toucher la face inférieure de l'abdomen, les mouvements deviennent

(1) Plassio, Giornale di med. vet. mil., 1988. 
plus rapides, il se produil des frémissements musculaires et l'éjaculation a lieu.

Pour combattre cette habitnde qui délermine l'épuisement des animaux, il est nécessaire de pratiquer la castration de l'îne et le placer autour des reins des cheraux une ceinture large de 10 à 13 centimètres et munie à la partie médiane de la paroi abdominale de trois rangées de clous dont la poinle est libre. Jès que l’érection est complèle, la verge vient se piquer sur ces clous et une rapide rétraction se produit.

\section{X. - PERVERSION GENITALE.}

Le chien cherche quelquefois à s'accoupler avec des espèces différentes. I.es gros terre-neuve, les saint-bernard se jettent quelquelois sur les jeunes filles et les renversent en manifestant un vif désir d'effectuer l'acte de la copulation.

II en est d'autres qui recherchent les poules. Villemin (1) rapporte à cet égard une observation curieuse.

Un chiea de rue de deux mois cherchait constamment à s'accoupler avec des poules, et, pour les retenir, il les mordait au cou ou les étranglait.

Châtré à l’àge de dix mois, il continua à manifester la même manje; une seule poule supportait avec résignation les actes du jeune chien.

Cet animal, amené par un marinier de passage, va retrouver ses chères poules et essaie de recommencer chaque fois qu'il en a l'occasion.

Certains étalons ayant loutes les apparences de la santé, de la force et de la viguenr, placés près de juments en claleur, n'entrent pas en érection.

Pour la faire apparaitre, dans un cas de ce genre, il était absolument indispensable de faire d'alord claquer

(1) Villemin, Bulletin de la Société des sciences vétérinaires, 1903. 
un fouet autour de lui, puis de lui en faire sentir quelque peu la lanière dans les jambes. Au fur et à mesure de ces manouvres, le gonflement de la verges'opérail et l'étalon, une fois préparé, accomplissait aussi prestement le coït que tout autre cheral entier; il n'était pas stérile.

Le cas de ce cheval rappelle celui des humains pervers qui recourent à des flagellations pour se nıeltre en état d'éréthisme génital.

Le bruit du fouet provoque la sensation, celle-ci amène l’image, l'image fait apparaitre l'idée (Cornerin) (1).

\section{X. - IMPUISSINGE.}

L’impuissance est un état particulier empèchant le coït de s'accomplir. On peut l'observer dans les deux sexes, lien que l'ardeur génésique soit très déreloppée et l'animal fécond.

Ce trouble résulte ordinairement de maladies des organes génitaux (phivzosis, volume anormal de la verge), de lésions articulaires empèchant le cabrer ou de lésions médullaires empèchant l'érection.

\section{Xi. - STÉRILITÉ.}

La stérilité est un état qui n'entrave pas l'accomplissement du coït, mais ‘ui s'oppose à la procréation d'êtres nouveaux.

Elle reconnait un grand nombre de causes. C'est ainsi que chez le mâle elle peut iésulter de l'arrêt des testicules dans l'abdomen (cryptorchidie, monorchidie, etc.).

L'orchite, l'inflammation de l'epitidyme et les tumeurs diverses qui peuvent se localiser dans le testicule aboutissent au mème résultat.

La stérilité peut encore tenir à l'arrèt de sécrétion du

(1) Cornerin, Journal de l'École vitsinaire de Lyon, 1395, p. 578. 
sperme (aspermie), à l'absence de spermatozoïles dans le liquide éjaculé (azoospermic) ou bien à la destruclion des spermatozoïdes par un liquide purulent el acide. Soixante vaches saillies par un laureau qui portait des végétations (papillomes) sur le pénis sont demeurées infécondes et ont présenté de nombreux papillomes sur la muqueuse utérine (Lavirotte) (1).

l.es sarcomes de l'extrémité du pénis peuvent jouer le même rôle.

Les poils feutrés et disposés en anneau autour de la verge peuvent rendre l'érection du taureau si douloureuse qu'il peut refuser de saillir (Bonillon) (2).

Chez la femelle, l'arrèt de développement des organes génitaux internes, les affections de l'ovaire (kystes, tumeurs), des trompes ntérines, de l'utérus, du col utérin et du vagrin (sérétions acides), sont les causes les plus fréquentes de la slérilité (Urbain André) (3).

Sur un tiers des ulérus des vaches stériles, Fiorentiıi a trouvé de l'endométrite catar hale, avec dégénérescence de l'épithélium et prolifération de cerlains points de la 'muqueuse, qui présente un aspect verruqueux. Le liquide contenu dans la cavilé utérine renferme un microorganisme de forme ova'e, présentant une cetlaine amalogie avec le $B$. coli. Dans les autres cas, il y a des méliites interstitielles, glandulaires, sepliques, tuberculeuses, etc , qu'il faut comballre par de fréquents lavages anliseptiques de cette cavité Jans les jours qui suivent la parturition (4).

La stérilıté est quelquefois due à des dérangements anatomiques ou fonctionnels du vagin, du canal, du col utérin et de l'ouverture de la matrice, tels que llexion ou constriction du canal, hypertrophie des lèvres de la

(1) Lavirotte, Journal de méd. vét. et de zootechnie, 1892.

(2) Bouillon, Journal de Lyon, 1898, p. 91.

(3) Urbain André, Annales de méd. vet.. 1890, p. 299.

(4) Consulter Obstétrique de l'Encyclopédic vét., par Bournay, p. 179. 
vulve, conformation anormale du col utérin, contraclions spasmodiques ou manque de puissance musculaire.

Pour y remédier, on conseille d'introduire les spermatozoïdes dans les organes générateurs de la femelle, c'est-à-dire l'utérus.

Voici la laçon de procéder conseillée par M. Heape.

On fail saillir la jument. On recueille lans son vagin le sperme au moyen l'une pelite seringue préalablement tenue à la température du corps. On introduit le boul de la seringue dans le col utérin et l'on pousse le liquide clans l'utérus. Il faul éviter l'introduction d'air dans la matrice. On peut mettre à la seringue un ajutage en caoutchouc pourvu d'un bout d'un centimètre et demi de diamètre. Le sperme est plus facilement puisé par la seringue si l'on déprime la face inférieure du vagin avec les doigts. Certains auteurs proposent de recueillir la liqueur séminale, d'en remplir des capsules de gélatine qu'on introduit dans la matrice; la gélatine se dissout el les spermatozoïdes se trouvent libres dans la cavité utérine.

Pour M. Heape, le système de la seringue est préférable. Il cite les résultats remarquables qui ont étẻ obteuns en Anérique et en Australie.

Il est certain que cette fécondation artificielle devrait être tentée plus souvent el qu'on ne derrait retirer définitivement les juments de la reproduction, quand on les croit stériles, quaprès avoir essayé ce moyen (The V'eterinarian, avril 1898).

\section{XII. - ATTENTATS CRIMINEL.}

A diverses époques, on a signalé la mort de poules, de borins et de solipèdes résultant d'actes bestiaux, sadiụıes ou d'actes criminels.

$1^{\circ}$ Bestialité. - Les relations bestiales ne peurent guère aroir de conséquences pathologiques que chez les poules. Les effets de ces rapports ont été décrits par 
Guillebeau (1). "L'attentat de bestialité sur" les poules a pour effet une rupture du foie suivie d'une liémorragie interne souvent mortelle."

Les os sont quelquefois fracturés. Dans les cas de mort subite, il peut y aroir distension du cloaque; mais cette distension ne peut être utilisée pour le diagnostic qu'avec circonspection, car les poules, à l'état normal, ont souvent un cloaque très spacieux.

La présence de spermatozoïdes de mammifère sur la muqueuse de cette cavité peut èlre constatée; le diagnostic est alors certaiu. En ellet, les cas où le chien introduit des spermatozoïdes dans le cloaque de la poule sont très rares. L'examen microscopique permet de différencier très facilement les spermatozoïdes de l'homıne, qui ont une lète renflée, de celux du coq, qui ont une tête très allongée.

$2^{\circ}$ Sadisme. - Les désirs génésiques associés à des instincts cruels incitent l'homıne dont la raison est faible à commettre de véritables assassinats pour se procurer une volupté complète.

Ces dégénérés utilisent habituellement une tigge plus ou moins acérée qu'ils introduisent dans le rectum ou le vagin des ruminants ou des solipèdes. Ils ne se contentent pas de produire une seule blessure; la vue du sang, les angoisses de l'agonie sont la source de leur jouissance; ils multiplient les piqùres, ils fourragent à plusieurs reprises et ils pénètrent très profondément.

Ils n'épargnent pas ordinairement un animal de l'étable; le nombre de blessures diminue seulement avec leur ardeur. Alors que la première victime reçoil quinze atteintes, les dernières assaillies n'en reçoivent qu'une ou deux effectuées arec moins de vigucur, de telle soite que l'épuisement graduel de la frénésie se décèle dans un

(1) Guillebeau, Des blessures faites aux animaux domesliques par des persommes atteintes de psychopathie sexuelle (Journal de l'École véterinaire de Lyon, 1899, p. 1). 
lıorrible graphique, iuscrit dans les clıairs vivantes des pauvres auimaux (Guillebeau).

Les altentats criminels commis ainsi sur des animaux et bien reconnus sont assez nombreux.

Historique. - En 18z̈̈, Mazzini (1) constate, dans les environs de Novare, quatre cas de mort sur des bovilés ì la suite de blessures profondes du rectum par un instrument piquant.

Des faits semblables sont observés par Micellone (2) chez deux cheraux de troupe et chez un cheral appartenant à une compagnie d'omnibus.

Perroncito (3) rapporte que dans une ferme où dixhuit raches avaient succombé en moins d'une année, il a conslaté, à l'aulopsie de l'une des viclimes, des plaies et des perforations intéressant a la fois le rectum et le vagin avec pénétration d'un corps étranger dans la cavité abdominale et perforations viscérales profondes.

Guillebeau a décril toute une série d'attentats de mème nature.

A Lanjuau, en 1891, sept raches présentent successivement des hémorragies vaginales abondantes.

Chez toutes on remarquait, le plus souvent sur l'hymen, quelquefois seulement à l'extrémité orale du vagin, d'étroites fistules s'avançant à une profondeur variable et s'arrêtant, dans la majorité des cas, avant d'avoir attein le péritoine ou le rectum. Trois succombent, une doit ètre abattue, trois guérissent.

L'auteur' soupçonné tiest pas inquiété.

A Obersteg, dix raches ou génisses et dix chèv'es succombent dans la première moitié de 1896 ; l'origine du mal estétablie, on constate des blessures vaginales et des perforations profondes jusqu'à la lıuitième còte. Le coupable est un dégénéré el un sadique; il est considéré comme irresponsable.

(1) Mazzini, Gioruale di med. veter., 1847, p. 208.

(2) Micellone, Giornale di vetcrin. milit., 1892, p. ว2.

(3) Perroncito, Giornale della R. Societa reter., 1898, p. 674.

CAdÉAc. - Sėmiologie, ?e édit.

11. --3 
A Wolfikon, luit vilches sont blessées simultanément en mars $189 . ;$ quatre succombent, les autres sont ahattues quinze jours plus tard, denx autres raches sont frappées; l'une meurt, l'autre est abaltue. On croit à ume contagion redoulable et les animaux survivants sont livrés en bàteà la boucherie, surle conseil du vétérinaire sanitaire. Weux mois plus tard, le malheurenx propriétaire achète deux raches qui succombent presque aussitôt. 1l lente d'entretenir quelques chirres; celles-ci meurent an hout de quelques jours.

Désespéré, il abandonne son domaine, et l'autent de tous ces crimes, un garcon de lerme, va se placer à Schwarzenbach, où il continue ses exploits; il tue en une amnée une gúniss', une chirro et quatre boufs.

Renroyé à tonveau, il entre chez un cultivateur de lickenbach; en quelques jours, quatre raches sont tuées par pertoration du rectum ou du vagin. l.e malfaiteur est enrore considéré comme irresponsable.

En 1897, dans l'étable de lickenbach, déjà dépeuplée, uu luanf succombe à des perforatious multiples du rectum; le coupable est un jeune vacher, qui, obéissint à une impulsion irrésistible, a voulu reproduire les actes erininels q̨ui lui ont íté racontís.

Labat rapporte qu'en 1891 une série d'accidents mortels est conslatée sur des bètes bovines daus une métairie d'Auterive (fiers : huil lietes sur dix succombent (deux Jurnls, trois raches, deux ginisses, un veaul, dans l'espace d'un mois et demi.

Une surveillance sévère fut organisée et l'on fint par surprendre une vieille femme (la belle-mère du métayer) en train de fouiller brutalement avec un manche de fourche dans le vagin d'une géniss" nourellenient arivée. Elle a aroué aroir procédé de la mène manière sur les autres liêles borines qui ont succombé.

Ln $189 \%$, labat et I.eclainche (1) constatent des accidenls

(1) Jahat et Leclainche, Revue vèt., 189?, p. 521. 
de même nature chez un propriélaire qui a perdu dans l'espace de quatre ans vingt-cinq chevanx on mulets. Le coupable, armé d'une tige longue et acérée, placé derrière la victime, introduit le corps vulnérant dans le rectum, puis il fouille lorutalement darrière en avanl; la main est mainterue assez basse, car toutes les blessures siègent vers le plancher du bassin, ce qui implique mne direction de bas en haut et d'arrière en avant.

"En certains cas, le criminel a perforé le culon flottant et déterminé une péritonite aigruë rapidement mortelle. En d'autres, il a lacèré cu traversé les parois lu rectum, crensan (destrajets fistuleux dans le tissu conjonctif du bassin. "

Des attentats semblables on été recomnus par Leclainche chez les borims d'une ferme de Taru-et-Garonue; les plaies siégeaient sur le plancher du rectum, parce que les animaux étaient en contre-bas dans l'étable.

Symptômes. - Les błessures profondes du rectum sont accompagnées chez les solipídes de coliques el de rejet de sang par l’anus; puis des signes de péritonite aiguë. Chez les borins, on constate des elforts le défécation avec rejet de matières sanguinolentes et parfois de véritables hémorrağies suivies de péritonite.

Les blessures du vagiu s'accompaguent d'lrémorragie primitive ou secondaire, d'efforts expulsifs qui commencent à partir du premier, du deuxième ou du troisieme jour; ils sont accompagnés de pertes sauguines modérées dues au détachement des escarres. Si, dius le cours le la maladie, il survient un avortement, lhémorragie est alors assez abondante pour derenir presque toujours mortelle.

Les efforts expulsifs, la voussure du dos, la raidenr des membres postérieurs sont consécutifs à la douleur traumatique el s'établissent du prenier au troisième jour. On conslate en mème temps du météorisme, de l’iurumination et yuelquefois de la péritonite. Quand celle-ci ne se produit pas, la guérison survient habituellement au bout de huit à quinze jours de maladie 


\section{SEMIOLOGIE DES MAMELLES ET DE LA LACTATION}

\section{I. - MIMELLES.}

1. Topographic. - Les mamelles sont inyumules, rentrales ou pectorules. Exclusivement inguinales chez la jument, la rarhe, la hrebis et la chirre, elles sont simultanémeut inguinales, ventrales et pectoraleschez la truic,

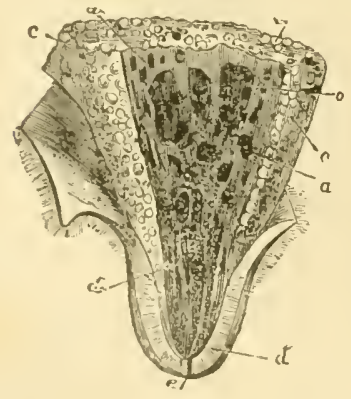

Fis 2. - Mamelon ou liayon de vaçe. llaussa cavité, aboulissent 'n $a$, a les principaux combuts galaclophores. la chivune, la chatte et la lipline.

Yormalement, la mamelle a une consistance molle, élaslique; elle est coinposée, chez la vache, de glandes séparées, en quelque sorte indépendanles, auxquelles on donue communément le nom de quartiers. Ceux-ci sont d'aulant plus saillants que la sécréliou laclée est plus active et la mamelle plus pleine. Chaque quartier éracue son produit de sécrétion par un canal (cunal guluctophore) onvert à l'extrémité d'un appendice plus ou moins dérelopué appelé mamelon (lig. : .

Chez li riche, la mamelle ou pis présente quatre quartiers, dont les mamelons atteignent un développement considérable. 
Chez la jument, la mamelle est globuleuse et généralement composée de deux quartiers; ses mamelons sont courts et percés de deux ouvertures; ils sont peu apparents, même pendant la période de lactation.

Chez l'anesse, les mamelons possèdent ordinairement à leur extrémité trois orifices.

Chez la brebis, la mamelle est globuleuse et composée de denx quartiers; chaque tetine possèle une ouverture unique. Le repli mammaire, situé à la face interne des cuisses, forme une poche riche en glandes sébacées et sudoripares.

Chez la cherre, elle a une forme variable, ressemblant à celle de la brebis chez certains individus; elle prend, chez certains antres, une apparence cylindripue.

Chez la truie et la chienne, les mamelles sont dautant plus nombreuses que la race est plus commune; elles sont appelées, suivant leur siège, mamelles inguinules, ventrales, pectorales; elles sont d'autant plus volumineuses qu'elles sont plus postérieures.

Chez les trnies, le nombre des glandes qui sécrètent est ordinairement en rapport avec le nombre des petits; on en compte ordinairement dix (quatre inguinales, deux abdominales, quatre pectorales); les mamelons sont toujours très apparents et possèdent de cinu à dix orifices.

La mamelle est exposée soit au début, soit pendant la laclation, it un grand nombre d'infections.

L'extrémité des trayons est, pendant le fonctionnement de la glande, une voie pour ainsi dire constamment onverte à l'infection; ces organes trainent sur le sol, ils sont souillés par le fumier et penvent ainsi recueillir soit des germes spéciliques ou de simples saprophytes susceptibles de devenir dangereux au moment de la parturition, quand lo pis est le siège d'une congestion très intense. Le lait est lui-même un excellent milieu de culture. La plupart des germes, streptocoques, bactéridie charhonneuse, elc., 
injectés dans les canaux galactophores, s'y développent admirablement.

Or, tonte culture microbienne faite en grand comme dans la mamelle, comparable à cel égard à un immense ballon, s'accompanne d'une production abondante de toxines, dont les effets peuvent se localiser à la mamelle (m’mumites streptococciques), produire la gangrèue de la mamelle avec le conconrs Je ces microhes (araignée de la brohis) on devenil le point de départ de phénomènes toxiques gẻnéraux caractérisés par des phénomènes pạrabyliques (fievere vitulaire).

Cette maladie est essentiellement une intoxication. Résulte-t-elle des prouluits de destruction cellulaire ou des produits de sécrétion microbienne, on même de l'associatiou de ces deux sources de toxiques?

Gratia (1) s'est prononcé en faveur de la première manière de voir; il considère la fiève vitulaire comme la conséfuence de la transformation des cellules ulanduIirires en colostrum.

"On peut se demander, en effet, si le nombre prodigrienx des cellules glandulaires nourelles qui subissent la transformation en colostrum - dont une parlie seulement est aliminéc par les trayons -- ne livrent pas à la résorption, et en dose massive, des produits de déchets dont l'accumulation dans te sang pourrait donner lien à des accilents toxiques analogues à ceux de l'urémic. Toutes choses égales d'ailleurs, la quantité de ces substances rigressives, de ces leucomaïnes, si l'on veut, élant pro. porlionuelle au rolume et ì l'achivité des mamelles, on pourrait concevoir ainsi les prédilections de la maladie pour les bonnes lailières. Les poisons lésorbés dans le pis par les reines et les lymphatiques ne leviendraient pentitre loxiques que par excès, en raison d'un développemont glandulatre exceptionnel el peut-être aussi à raison

(1) Gralia, Ammales de médecine rétérinaire. 1896. 
de l'insuffisance simultanée de leur élimination par les urines."

- On s'explique ainsi la prédisposition spéciale des vaches bonnes laitières à la fièvre vitulaire; mais il n'esı pas absolument étalili que l'infection microbienne est absolument étrangère à son développement, c'est-à-dire à cette inloxication.

Qnoi qu'il en soit, l'injection à 1 p. 100 d'une solution de 230 grammes environ d'iodure de potassium dans chaque quartier de la mamelle suffit pour guérir promptement la vache malade alteinte de fièvre vitulaire (Schmidl).

On obtient également la guérison, avec la même facilité, par des injections d'eau boriquée, d'eau salée et de fluorure de sodium on simplement par des insufflutions d'air; il suffit de presser la mamelle, de la malaxer et de faciliter l'expulsion des poisons qui s'y sont déreloppés pour obtenir la diminution des phŕnomènes paralytiques, comme on détermine la chute de la température en facilitant l'écoulenient des matières pyrétogènes d'un abcès par un débridement, d'une plaie par le renouvellement d'un pansement antiseplique.

La technique des injections mammaires est très simple. On utilise un cathéter ou un lube trayeur relié par un lube de caoutchouc à un flacon de 2 litres dont le bouchon, solidement fixé, porte deux tubes de verre coudés à leur sortie; l'un plonge jusqu'au fond du vase et reçoit l'extrémité libre du caoutchouc qui porte le cathéter; l'autre, qui s'arrête à quelqques millimètres au-dessous du bouchon, est fermé par un tampon l'ouate à la partie libre et reçoit le tube d'une poire en caoutchouc. L'appareil fonctionne conme un pulvérisateur à main.

On peut se contenter d'un entonnoir maintenu à une hauteur convenable et relié au cathéter par un lube de caoutchouc. 
$4 \frac{1}{2}$ SEMMOLOGIE DES MAMELLES ET DE LA LACTATION.

Pour les injections d'air, on peut utiliser les pompes à bicyclettes.

II. Exploration. - $a$. Inspection. - L'inspection met en ividence:

$1^{0}$ Les changements de rolnme : fluxion, congestion, inflammation, adène d'un ou de plusieurs yuartiers. Les mamelles sont petites chez les femelles vierges; les réservoirs galactophores fout défaut; elles grossissent à mesure qu'on se rapproche de l'époque de la partırition;

20 Les changements de coloration [rongeur de la congestion, taches violarées des maladies intectieuses, teinte noiratre de la mammite gangreneuse des brehis laitières (Nocard), gangrène du mamelon];

$3^{\circ}$ Les éruptions : phlyctèues, vésicules de la fière aphteuse, pusiule du corpox, du horscpox et du roryzu gangreneux ;

$4^{\circ}$ L'écoulement anormal du lait galactorrhee);

亏o La présence de mamelles supplimentaires qu'on observe fréquemment chez la rache et la brebis, et l'atroplie d'un ou de plusieurs quartiers à la suite de mammiles aiguës ou chroniques.

b. Palpation. - La palpation dénonce:

Jes épaississements culanés (anasarque, cicatrices, éruptions), la durelé de la mamelle dans le cas de mummite, sour empâtement daus le cas d'œdème ;

La présence de tuneurs situées plus ou noins profondément dans l'épaisseur de l'organe (sarcomes, fibromes. enchondiomes, carcinomes); des abces, des fistules, et l'atrophie scléreuse de la mamelle; les nodosités iuflamnatoires daus le cas d'infections microbiennes localisées;

Jes foyel's tuberculcux dont le contenu se mélangre frépuemuent au lait.

c. Sondage. - Lesondage peutrévéler des olıstructions, des calculs, et faciliter l'écoulement du lait ou des produits 
anormaux (sang, pus) accumulés dans la mamelle, indiyquer le trajet des fistules el la profondeur des abcès.

La sécrétion lactée, normalement intermittente, peut se manifester en dehor's dela gestation (galuctose anormale); elle pent éliminer des principes qui changent les caractères physiques ou chimiques du lait; elle peut ètre supprintée (agalaxie); elle peut être exagérée (polygalaxie); clle peut ètre pervertie (lait sunguinolent, purulenı, etc.).

\section{I1. - OBLITERITION DES TRIYONS.}

Les oblitérations des trayons sont congénifales ou acquises et ont une gravité sn rapport avec leur siegge et leur étendue.

Les oblitéralions ccnjénitales s'observent au moment de la première lactation; elles sont généralement limitées a l’exlrémilé de l’organe; mais elles peuvent aussi s'étendre plus ou moins profondément. Les oblitéralions acquises résultent d'une tumélaction du trayon, d'un épaississement de la muqueuse et de son épithélium converti en un cordon dur, épais comme une plume d'oie à l'intérieur de la tetine; mais ce sont les néoplasies fibreuses, les papillomes de la muqueuse qui en sont les causes les plus communes. I.es tumeurs fibreuses siègent le plus sourent an tiers inférieur du trayon; elles sont arroudies et gênent ou empèclrent totalement le passage du lacit.

sirebel (1) a constaté l'ublitération des trayons par des membranes en diaphragrme placées plus on moins hant.

Pour empêcher une olstruction nouvelle par la cicatrice, il faut toujours opérer aseptiquement, pouctionner le lrajou non ouvert à l'aide d'une sonde ou d'un petit trucart; une petile bougie trempée laus la glycériné et introduite dans le canal empèche lout rétrécissement ultérieur.

(1) Strebel, Schweizer Archiv, 1901.

3. 


\section{III. - GALAGTOSE ANORMALE.}

Définition. - Sous le nom de gralactose anormale, ou de lartation hétérochrone, on désigne la sécrétion laclée se manifestant indépendamment de toute gestation. Eıle se manifeste quelquefois dès la naissance chez la pouliche, lis gúnisse, la cherrette comme chez l'enfant, ou à l'àge alulle chez les femelles vierges ou iyant deja mis bas.

On a pu recueillir chez une chevrette nouveau-née io centimètres cubes de lait; cette quantité atteignail 10: centimetres cubes an boul de quarante-cinq jours.

l.es chiennes, les chattes, les lapines non couvertes otlient souvent une poussée de lait au moment oủ elles devaient accoucher. Il en est mème qui présentent rérulièrement ce phénomène.

Celle laclation hétérochrone résulte quelquefois d'un tic: les génisses qui ont l'habitude de se teter (autosucrion) ou de se faire teter arrivent à présenter du lait.

Les animaux adultes ou vieux (jmment on rache) parviennent quelquefois à donner ainsi une assez grande quanlité de lail pour nourrir un jeune sujet.

On lait cesser cette sécrétiun de lait en supprimant la telée et toutes les causes d'excitation de la glande mammaire.

Celte sécrétion anormale de lait a été signalée quelquefois cliez le bonc.

\section{IV. - IGALAXIE.}

Définition. - Laymlaxie ou aqualaclie, c'est-à-dire. l'absence complète de la sécrétion lactée, n’a jamais ét: observée cliez les animaux.

On il l'habitude de désigner ainsi la diminulion de cetle sćcrćlion; ce phénomène n’est pas très rare chez les vaches primipares quiperdent leur lait dès qu'on les prive de leur venu vaches bomnes meres; exceptionnellement 
les raches ne donnent pas une goutte de lait après leur premier reau (Lafosse).

Lagalaxie est quelquefois dorigine infectieuse (Voy. Maladies des mamelles!.

Le plus sourent l'agalaxie est produite par une alimentalion insulfisante ou résulte de maladies locales (mammites), de maladies fébriles générales (péripneumonie, fierre aphteuse ou d'empoisonnements belladone, stramoine, colchique d'automne, ombellifères, grande ciguë; les préparalious jodées el tous les déplétifs (saignée, purgutifs, antipyrine) produisent le même résultat.

La pilocarpine n'a pas d'influencesur la quantité de lait sícrélé (Cormerin. Le chlorate de polasse exagère au contraire sa sécrétion. L'agalaxie se traduit par l'affaissement des mamelles et par l'amaigrissentent et l'affaiblissement du petit: il peut mème mourir de faim si on ne lui donne pas une autre nourrice ou une autre alimentation.

Une alimentation aqueuse (betteraves, carottes, soupes) et des émollients appliqués sur les mamelles combaltent ce trouble sécrétoire s'il n'est pas engendré par une maladie grave et prolongée. Les traites fréquentes el le massage ramènent la sécrétion lactée.

\section{V. - POLYGILACTIE OL POLYGALAXIE.}

Définition. - On désigne ainsi une production abondante d'un lait séreux, c'est-à-dire pauvre en crème et riche eu eau.

Signification. - Celte anomalie est quelquefois indiriduclle, indépendante de toute maladie; il y a des bêtes à lait paurre comme il $\mathrm{y}$ en a d'autres à lait riche en principes nutritifs.

La polygalaxie est symptomalique de l'anémie, de la tuberculose; elle est également produite par une alimentation aqueuse, par le séjour dans des étables humides. 
L.e lait est légèrement bleuàtre ou moins blanc; il est moins épais, car il renferme moins de beurre, et il est moins inutritif. De bons aliments (fourrages secs et "zrains) peuvent triompher de celte perversion de la sécrélion lactée quand elle est due à une mauvaise alinentation ; elle est réfractaice à tous les modificateurs hyqiéniques comme à lous les médicaments quand elle est liée à une malarlie chronique ou inhérente à la bète elle-mème.

\section{VI. - G.I.ICTORRIÉE OL GALACTIRIRÉE.}

Définition. - Ce trouble sécrétoire consiste dans l'écoulement du lait eu dehors de foute mulsion.

Ce phénomène est la conséquence d'une paralysie passagere ou permanente du sphincter des trayous.

La paralysie passagère succèle à une réplétion exagéıée de la manelle par suite d'un retard accidentel ou intentionnel apporté à la mulsion ou à la tetée.

On observe la galuclirhe chez les vaches non traites pour les faire paraitre meilleures laitières et qui demeurent longtemps exposées sur le champ de foire; chez les vaches lonnes laitières, quand vient l'heure de la tetée, ou chez les lemelles auxquelles on a supprimé leur petit et qu'ou fait teter plus rarement.

Le lait contenu dans les réservoirs galactoplores funit par vainere la résistance du sphincter des trayous et par séchapper au dehors. Ce phénomène est alors sans signification.

La paralysie permanente peut résulter d'un réritable épuisement procédant de l'anémie par alimentation insuffisante, on d'une alfection constitutionnelle. On peut lobserver chez la jument pendant la convalescence de la fière typhoüle; elle a été signalée cliez de jeunes hêles privées dis tibres musculaires qui constituent le sphincler (furstenlerg), clez des animaux porteurs de verrues situées à l'extrémilé du mamelon. Les vaches 
vieilles présentent souvent de li parésie des sphincters et laissent écouler le lait d'une manière intermittente.

La galactorrhée est menacée de se compliquer de mammite par suite de la contamination du lait qui s'écoule à l'extrémilé du trayon; ou de gulaclocèles par rétention forcée du produit de sécrétion.

\section{VII. - PERVEIRSION DE LA SÉCRÉTION LACTÉE.}

Le lait peut présenter à sa sorlie de la mamelle diverses altérations résultant d'une infection des canaux galactophores ou d'une affection mamnaire; il peut être sangunolent, purulent ou cailleboté, graveleux, visqueux, amer, acide ou virulent.

\section{VIII. - LAIT SANGLINOLENT.}

Caractères. - Le lait sauguinolent est caractérisé par la présence de globules rouges dans le lait. Ce liquide est plus ou moins rouge au moment où il sort du pis suivant sa richesse en globules sanguins. Si on le laisse reposer, dans un vase, il pàlit graduellement ou perd entièrement sa teinte rouge parsuite du dépòt des hématies. Ce lait est toujours riclıe en albumine; il se coagule en grumeaux brunatres, sous l'inlluence de la chaleur; il est peu appétissant, mais il n'est pas nuisible.

Signification. - Le saug mélangé au lait provient d'une lyyperdiapédèse ou de la rupture de vaisseaux capillaires qui déversent direclement les globules dans les acini et les canaux excréteurs. Ces phénomènes sont symptomatiques de la conyestion mammaire, de traumatismes (contusions, plaies) ou des diverses formes de mummiles.

\section{IX. - LAIT PURULENT.}

Caractères. - Le lait purulent ou cuilleboté est plus ou moins cliargé d'élémenls pyogéniques, qui forment quel- 
(quefois un dépòt abondaul au fond dı vase; la couche purulente se différencie nettement du lait normal par sa coloration blanc jaunitre et grumeleuse.

Signification. - Le lait purulent s'observe dans lous les accidents pyogéniques susceptibles d'atteindre les acini, tels que les abcès du pis qui sont en communiealion avec les conduits collecteurs, ou les mammites traumatiques ou spécifiques compliquées de suppuration.

I'n tel lail est nuisible en raison des toxines el des germes qüil renferme; de plus, il est répugnant et ne poul ètre consommé à l'élal crus.

\section{X. - I.IT GRIVELEUX.}

I.e lait graveleux est celmi qui renferme des concrétions de dimensions, de nombre et de nalure variables. On peut recomnaitre trois soltes de concrétions: lactées : les calculs veritables, les faux calculs et les conerements.

"La grossenr cles premiers va du grain de millet au haricot; leur forme est sphérique ou allongée, ieur couleur blanche ou grise et leur surface lisse ou rugueuse. A leur intérieur se trouve un noyan qui a servi de centre d'attraction et autour duquel se sont disposés des sels terreux en couchesconcentriques. I'une densité noyenue de 2J86, ils contiennent surtout du earbonale de chaux, des pliosphates lerreux, des malières grasses et quel ques autres subslances organiques, de la caséine notamment, qui joue le ròle de ciment.

"L'aspect extérieur des faux culculs rappelle celui des vrais, mais, n'ayant pas de noyau cristallin, ils consistent simplement en un coagulal extérieur recouvert d'une croùle saline.

"Les concrements ne présentent pas de stratification. Ce sont des masses amorphes à surface rude, d'apparence crayeuse, se ramollissant dans l'eau et d'un poids spécifique de $211 \%$. Chez eux, ce sont les phosphates de chaux 
et de magnésie qui dominent, puis viennent le carbonate de chaux et les substances organiques.

"Si les concrétions sont petites et nombreuses, l'écoulement dı lait continue à se faire, mais le trayon donne à la pression une sensation qu'on assimile à celle qu'il produirait s'il y avait dans son intérieur quelques grains de sable. Quand ces concrétions s'agglomèrent ou deviennent rolumineuses, le trayon s'obstrue complètement et l'évacuation du lait est impossible. " (Cornevin.)

L'origine de cesconcrélions estinconnue. La précipitation des sels terreux doit résulter de l'action de certains microbes, car on ne saurait invoquer pour fournir une explication plausible l'absorption d'un excès de sels produisaut une sursaturation du lailen sels de chaux.

\section{XI. - LAIT VISQUEUK.}

Définition. - On désigne sous le nou de lait visqueux, fuant, albumineux ou mucilagineux, une altération caractérisée par un élat aøgglulinalif accompagné de la formation de filaments qui rendent le lail comparable au mucilage.

Sa réaction est acide, son odeurbutyrique; il se coagule plus rapidement que d'habitude, mais le coagulum est visqueux et manque de cohésion; entin ce lait est paure en beurre et offre un mauvais gout.

Tantôt cette altération se manifeste immédiatement après la mulsion; tantòt vingl-quatre ou quarante-huit heures après. Dans le premier cas, le lait sort visqueux de la mamelle; ce phénomène est alors symptomatique d'uue mammitelatente ou très bénigne que rien ne décèle extérieurement. Les agents qui rendent le lait visqueux sont peu virulents ou n'exercent qu'une action fermentative. Cette transformation visqueuse se produit ordinairement après la traite sous l'influence de ferments variés dont l'étude est faite en hygiène. 


\section{XII. - IAIT IMER.}

Le lait amer au sortir du pis indique généralement une infection des canaux galactophores. Ce fait est d'autant plus certain gu il $y$ a des vaches qui donnent du lait normal par un ou deus trayous et dont les autres fournissent du lait jui est amer.

Ordinairement l'amertume du lait résulte de sa contamination après la traite par des germes spéciaux.

\section{XIII. - LAIT ALIUE.}

Le lail acide est caractérisé par sa réaction franchement acide en sortant du pis et par sa coagulation spontanée en masse ou en grumeaux. D'ailleurs, on peut constater pendant la mulsion mème qu'il s'ícoule de petils coagrulums blancs ou légèrement jaunàtres. L'acidité du lait est symptomatique de l'iufection de la mamelle par des streptocoques. Ce phénomene est très marqué dans la manmite streptococcique, il peut mème contriluner à dépister les formes larvées de cette atrection.

\section{XIV. - LAIT VIRILENT.}

La mamelle et le lait penvent servir de milieu de culture a un grand nombre de germes qui font coaguler le lait ou le rendent incoagulable, visqueux, amer, imbarattable, purulent ou putride, etc.

l'infection peut s'effectuer : 10 par effraction directe ; $2^{\circ}$ par l'orifice du trayou; $3^{\circ}$ par la roie sanguine ou par la voie lympluatigue.

L'effraction de la pean peut engendrer une lymphangite seconlaire qui intéresse secondairement le tissu glandalaire ou de préférence le tissu interstitiel.

Les Iramatismes plus pénétrants peuvent inoculer 
directement la mamelle et ètre le point de départ de foyers de suppuration ou de phlegmons septiques. Les ouvertures des trayons peuvent, en sünfectant all contact de la litière, contaminer de proche en proche les canaux jusqu'aux acini. Ces infections ascendantes se réalisent encore assez fréquemment. Le sang peut déverser à son tour dans la mamelle tous les microbes qu'il charrie accidentellement ou qui sont élaborés dans un point de l'organisme et qui sont entraînés journellement de ce foyer dans la circulation sanguine.

Les expériences qui ont été faites à ce sujet démontrent que la glande mammaire est une puissante voie d'élimination des microbes conme des toxines.

Le lacille de la tuberculose peut ainsi se retrouver parfois dans le lait, quoique la mamelle soit absolument indemne de lésions tuberculeuses.

Qu'on iujecte le bacille pyocyanique dans le sang de femelles en lactation, ce microbe se retrouve dans le lait quelques heures après. On peut combattre ces infections mammaires par des injeclions antiseptiques pratiquées par l'ouverture des trayons quand les orifices ont un diamètre assez considérable pour permettre l'introduction de l'extrémité des sondes ou des canules. On utilise à cet effet cliez la rache el chez la chirre les solutions boriquées a \& p. 10n, les solutions de fluorure de sodium à 2 p. 1000, l'eau iodée à 2 p. 100.

A l'intérieur, on peut administrer l'iodure de potassium ou les essences antiseptiques qui s'éliminent par la glande mammaire. 


\section{SENIOLOGIE DE LAPPAREIL DE L'INNER YATION}

Topographie. - Physiologie. - Les divers appareils nécessaires au bon fonctiomnement et à l'entretien de l'organisme sont commandés et dirigés par l'appareil de l'imnervation.

Il comprend une partie centrale de l'axe cérébro-spimal et une partie périphérique, les nerfs qui partent de la partie centrale pour se distribuer dans les diverses parties du corps. L'axe cérébro-spinal renferme lui-mème le cerveu et la moelle réunis entre eux par l'isthme encéphaliqu. L'ensemble de ces organes constilue liustrument des relations de l'animal avec son milieu et des rapports fonctionnels des viscères entre eux.

Les impressions procédant d'un changement dı milieu extérieur (iufluences physiques, chimiques, mécaniques) sout transmises aux centres nerveux où elles sont converties en sensalions; elles sont suivies de mouvements connus sous le nom de mourements réflexes.

On les désigne ainsi parce ıue l’impression extérienre qui les produit ne parvient aux muscles quaprès avoir traversé un foyer particulier de la substance grise encéphato-médulluire pour se rélléchir vers la périphérie. La sensation et le mourement sonl les deux termes extrèmes du cycle parcourn par les excitations periphériques (fig. 3).

Cette chatne comporte une voie centripète ou sensitive. un centre gris de réception, une voie centrifuge motrice et un organe réactionnel: mnscle, glande, ctc. 
SEMIOLOGIE DE LAPPAREIL DE L'INNERVATION. כ̈

Les nerfs couduisent donc toules les impressions sen12 silives qui vont de la phériphérie ver's les \& les ordres élaborés par les centres. L'extrémité de chaque fibre nerveuse part d'une cellule ou aboutit à une cellule.

Éléments du système nerveux. - L'élément fondamental du système nerveux réside dans le neurone avec tous ses prolongements. Chaque neurone présente deux pòles et émet deux sortes de prolongements : les prolongements protoplas miques et le prolongement eylindraxile.

Les PROLONGEMENTS PHO-

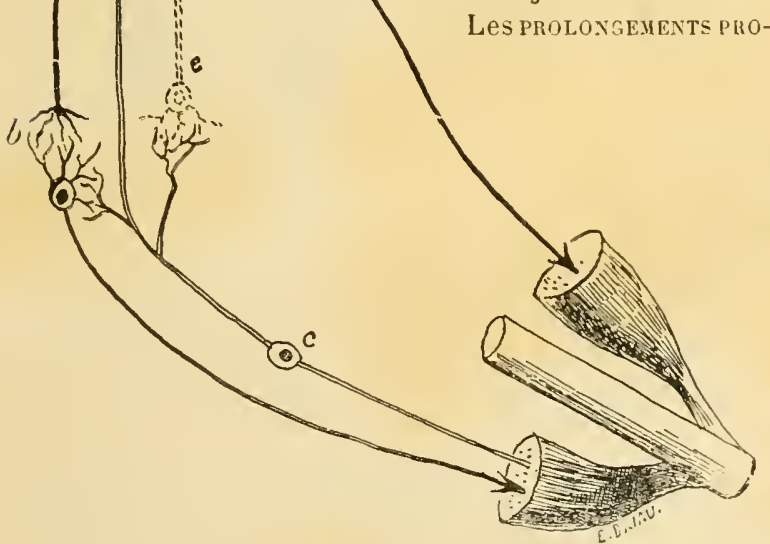

Fig. 3.

$a$, neurone moteur central; $b, \mathrm{~N}$, moteur périphérique; $c$, N. sensilif périphérique; $d, \mathbf{N}$. sensitil central ; $e, \mathrm{~N}$. intercalaire (sensitivo-moteur) $; b^{\prime}, \mathrm{N}$. moteur périphérique (du muscle antagoniste). (Thèse de Gerest.)

TOPLISMQUES offrent de nombreuses ramifications (den- 
56 SÉMIOLOGIE DE L'APPAREIL DE L'INNERVATION.

drites), de plus en plus déliées, terminćes par une exłrémité libre.

Le cyliudraxe ou prolongement cylindruxile, appelé

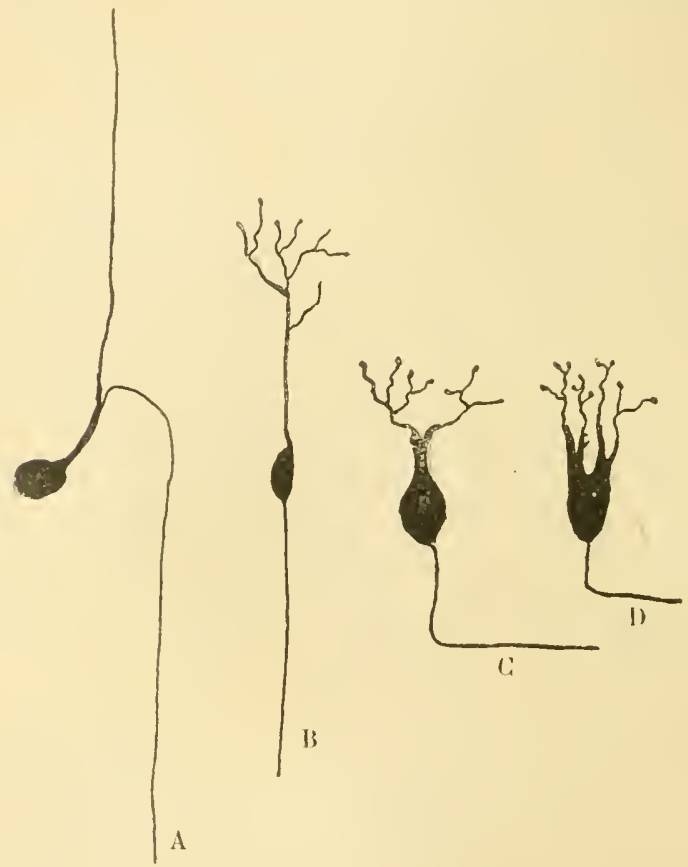

lïg. 4. - Cellules ganglionnaires fdapres von Gehuchten!.

A, laclile (ganglionnaire spinal): I; olfactive (cellule olfaclise de la piluilaire); C ef D, optiques deux tyjes de eellules ganglionnaires de la rétine;. (Thise de Cierest.)

axone, qui forme les nerfs on la sulstance blanche, émet dans le névraxe un grand nombre de branches collatérales qui constituent comme lui une arborescence dont les branches seterminent également par une extrémité libre. 
Au point de vue fonctionnel, les prolongements dits protoplasmiques sont toujours cellulipètes: l'influx nerveux les parcourt en marchant vers la cellule; le prolongement cylindraxile est toujours cellulifuge, en ce sens que l'influx nerveux qui le traverse part de la cellule.

Le neurone tout entier peut ètre assimilé à un arbre dont les racines représenteraient les prolongements, et dont le tronc avec sa frondaison représenterait l'axone el ses arborisations termiuales; l'influx nerveux suil la marche ascendante de la sève dans les plantes (1).

Les neurones sont indépendants, c’est-à-dire que les ramifications terminales du neurone envoyeur ne sont qu'arliculées avec les branches dendritiques du neurone récepteur.

Chaque ARC Riflexe comprend un neurone sensitif et un neurone moteur périphèrinues.

Au niveau de la moelle épinière, le neurone sensitif est formé par une cellule du ganglion rachidien corresponlant cs qui émet un prolongement ramifié en T; l'unedes branches forme le cylindraxe d'une fibre sensitive qui recueille les impressions tactiles de la périphérie (fig. 6).

(1) Laulanié, Élémen/s de physiologie, p. i70. 
La deuxieme brunche constitue une des fibres radiculaires posterieures, pénètre dans la moelle et se divise en doux branches, en branche ascendante et brauche descendante énettant des collutérales trunsversales dont les extrimités s'articulent avec les dendrites d'un neurone moteur. Le neurone à son lour par l'un de ses prolongements se termine dans un muscle on dans une glande; le premier am eau, l'arc réflexe, est constitué; le deuxiène anneau, l'ure cérébral, est formé aussi de deux neurones centraux, un neurone sensitif el un neurone moteur.

Le neurone sensilif central NSG est constitué par une des cellules des noyaux situés au niveau du bulbe rachilien (noyuux de Goll et de Burdach).

les prolongemenls cellulipètes s'articulent avec les arborisations terminales d'un neurone sensitif périphéxique. Son prolongement cylindraxile traverse le plan médian et ra se terminer dans l'hẻmisphère du còté opposé en s'articulant avec les dendrites d'une cellule pyramidale de l'écorce.

Le NeURone motrun central est constitué par une cellule pyramidale de la zune psychomotrice. Les clendrites s'articulent avec l'extrémité d'un neurone sensitif rentrul ou d’un neurone venant d'une aulre région le l'écorce; son prolongement cellulifuge va directement jusqu'au bulbc où il traverse le plan uédiau pour aller s'articuler avec les dendrites d'mu nensone moteur périphiriquc.

Chaque neurone moteur périphérique s’articule au moins avec deux nenrones: un meurone sensitif pripherique qui lui communi jue les excitations cxtérieures, et un weurone moleur contral qui lui communique les excilations motrices volontaires.

II existe trois ordres de chaines ou ares simples: les ares r'efres médullaires ou nésencéphaliipues, les ares córébrux et les arcs cérebelleur. Ces deux deruicrs sunt placés en dérivation sur les ares réllexes. Chaque arc est enfin complété par des neurones d'ussuciation; il y a des 


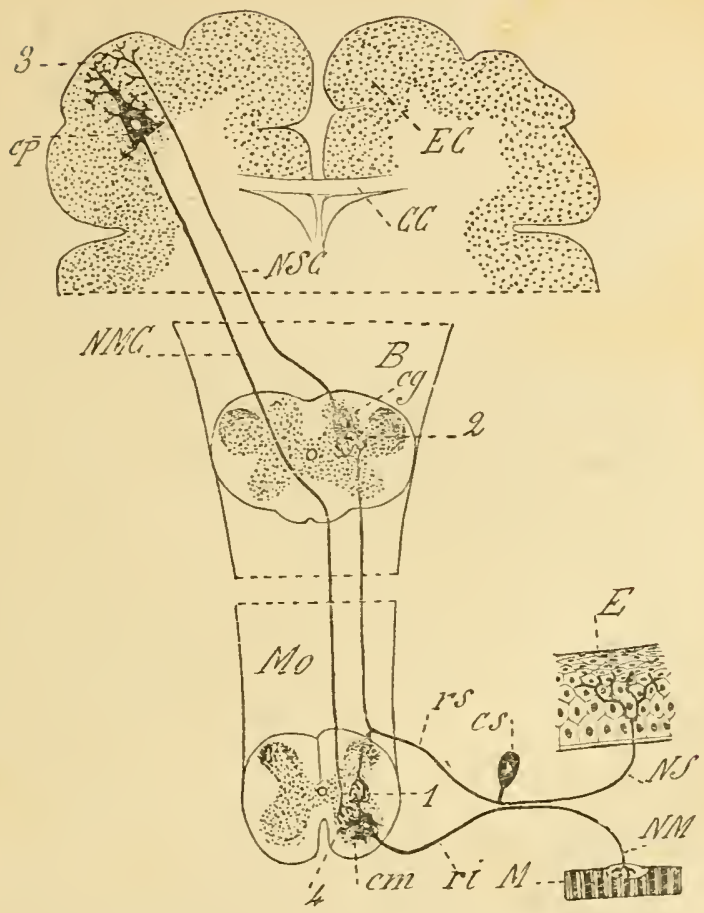

Fig. 6. - Schéma d'un arc réflexe mélullaire et d'un arc cérébral (d'après Mathias Duval).

E, surface sensible (épitlélium): XS, nerf sensitif; $c s$, cellule du gangliun spinal ; $r s$, racines supérieures; 1 , collatérale renant s'articuler arec la cellule radiculaire $\mathrm{cm}$, dont le prolongemeut cylindrasile, passant par la racine inféricure $r i$, puis par le nert moteur $\mathbf{X I I}$, vient actionner la fibre musculaire. $\mathbf{l}$; 2, articulation du neurone sensitif périplıérique $c s$, arec un neurone selsilif central cg, lequel va lui-mème s'articuler en 3 avec un neurone psichomoleur ou moleur central $c p$, dont le prolongement nerveux vient actionner en 4 le neurone moteur périphérique $\mathrm{cm}$; $\mathrm{Mlo}$, moelle; $\mathrm{B}$, bulbe; $\mathrm{EC}$, écorce cérébrale; LC, corps calleux; XSC, çlindrase du neuroue sensitif central ; NHC, cylindraxe lu neurone moleur central. 
neurones d'association médullaires, des neurones d'association cérébraux et des neurones d'association cérébelleux qui assurent les enchainements nécessaires au développement des actious uervenses les plus complexes.

Modifications des neurones. - Les neurones éprouvent des changements morphologiques et des changements chimiques. Normalement, les cellules olfactives, џui sont de vérilables neurones, présentent des oscillations de leurs prolongements comme s'ils allaient a la recherclie des particules odorautes; les bitonnets et les cones se rétractent dans l'obscurité de telle sorte que l'épaisseur de la rétine diminue sensiblement; le proloplasma des cellules ganglionnaires de cette membrane se déplace sous l'influence de la lunière du corps cellulaire vers les prolongements.

Dis lors, il est probable que les contacts qui s'établissent an niveau des articulations entre les prolongements cylindruxiles et les prolongements protoplasmiques sont tantòt relàchés, tantòt intimes, selon 'jue ces prolongements s'étirent, se rétractent et interrompent plus ou moins les communications.

Cette théorie de l'umiboïsme a permis à Matlıias Duval d'expliquer le sommeil, la mémoire, l'association des idées. La chaine des neuroues pent présenter des ruptures passagères, leurs prolongements peuvent marcher les uns vers les autres ou se rétracter, rélablir des cuntacis interrompus, en créer de nouveaux, etc.

l)ans le sommcil provoqué par la morphine, Demoor a constaté que les prolongements dendrilipues des cellules cércibrales se rétractent et prenment l'aspect moniliforme. Ils semblent alors formés d'une série de grains protoplasmiques réunis par des filaments très grèles.

C. lte contractilité des neurones a été également misc en évidence dans l'électrisution du cerveau dans l'électrocution, dans l'aspluyxic, dans l'insomnie. 
Odier a constaté que les prolongements des neurones moteuıs périphériques se rétractent et disparaissent en quelque sorte dans le corps de la cellule sous l'influence des courants induits longtemps prolongés.

\section{I. -... NERFS.}

Division. - Fonctions. - Les nerfs sont des organes de Iransmission; ils comprennent des nerfs moteurs, des nerfs sensitifs el des nerfs mixtes. [n nerf est moteur parce qu'il se termine dans un miscle; un nerf est sensitif quand il est relié par une chaîne de neurones à un point de l’écorce cérébrale capable d'éprouror une sensation.

La sensitivite des nerfs mixles réside dans les racines postérieures; leur molricité réside dans les racines antérieures; la sensibilité et la motricité ont des roies conductrices autonomes.

La coxocctibicité est cette fonction par laquelle les excitations parcourent directement une fibre nerveuse jusqu’à l'extrémité lerminale sans se propager aux fibres roisines.

L’Excitabilıté des nerfs est mise en éridence par des excitants mécaniques (pincements, percussions), chimiques (sel marin, alcalis, acides, glycérine, alcool, bile), physiques (électricilé).

La section des nerfs, la ligature, la conıpression, l'écrasement, larrachement, les agents toxiques suppriment les fonctions des nerfs soumis à ces influences sans faire disparaitre complètement la sensibilité dans les régions qư̈ls innerraient.

Sensibilité récurrente. -- On désigne ainsi la sensibilité qu'on peut éveiller' par l'escilalion des racines antérieures ou de leur bout périphérique.

Découverte par Magendie, elle fut étudiée par Cl. Bernard et bien établie par Schiff en 1850 .

$$
\text { Cauéac. - Sémiolngie, ae édit. II. - }
$$


II constata qu'après la section des rucines posterieures on trouve an bout de plusieurs jours des fibres digénérées dans les racines unterieures correspondantes.

In 1867, Richet, en triniani un cas de section du nerf médian, olserva que la sensibilité persistait dans tout le terriloire animé par le neff coupé. Les rétérinaires euxmêmes avientremarquué quela névrotomie plantuire unilatŕrale faite au-dessus dı boulet n'anesthésie pas complètement la moilié du doigt, et Moreroft tentait d'expliquer ce fitit surprenant en invoquant la solidarité fonctionnelle des nerfs planlaires el la possibilité de leur suppléance.

Alloing et Tripier ont mis en évidence l'existence de la sensibilité récurrente sur le bout périphérique de tozs les norfs sectionnès. Il a démontré que cette sensibilité du hout périphérique est due à des fibres récurrentes provenant des nerfs voisius. En effet, quand on sectionste un nerf, on trouve peu de jours après, dans le bout périphérique, au milieu des fibres dégénérées, un certain nombre de filres suines.

Réciproquement, le bout central contient un certain $110 \mathrm{mbre}$ de fibres dégénérées. Il est clair que les libres saines dı bout périplérique sont dememées en relation arec leurs centres trophiques el qu'elles ne peurent gagner ces centres que par l’utermédiaire d'm nerf voisin. Il s'elfectue ainsi, entre les divers nerfs qui se rendent dans une région, des íchunges le fibres passant de l'un à l'anlle au niveau de leurs extrénités terminales, remonlaul Jlus ou moins haut el linissant par disparaître à une hauteur varialife.

Les nerfs empiètent réciproquement sur les territoires d"leurs voisins. On comprend ainsi la persistance ou le retour prématuré de la sensilililé dans des régions privées complètement en apparence de leurs nerfs sensitifs.

Modifications pathologiques. - Les nerfs peuvent ètre alteints directement on indirectement par l'intermi- 
diaire de leurs centres trophiques. les agents physiqucs, chimiques ou toxiques que l'on tépose sur les nerfs produisent des altérations inflammatoires ou dégénératives de ces cordons.

Les lésions nervcuses consécutives aux lésions des centres trophiques font partie intégranle des maladies des centres uerveux.

Ces névrites d'origine centrale olrrent des lésions péripluériques d'une intensité proportionnelle à celle des lésions des centres trophiques. Telles sont les nérrites peripherriques de la tremblunte du monton dont l'étendue est subordonoée aux lésıons des grosses cellules des cornes antérieures (Besnoit et Morel)(1).

Il y a desnévrites el par conséquent des dégénérescences de cause iuterne produites par des agents toxiques minéraux ou par des toxines microbiennes; elles sont fréquemment multiples en raison de la diffusion dans tont l'organisme de l'agent provocateur.

Sous linfluence des sections, des traumatismes, des substrunces chimiques déposées surles nerfs ou dans leur voisinage, des distensions, des déchinums, des arrachements, des intoxications (arsenic, mercure, phosphore, plomb, etc.), des infections (gourme, maladie dı jeune àrge), des altérations nombreuses se produisent: les unes sont primitives; elles intéressent les nerfs; les autres sont secondaires; elles intéressent parfois lescentres nerveux, les organes innerrés, les muscles, les os, le tissu conjonctif, etc.

\section{I. - Mltérations primitives.}

Les nerfs sont susceptilles d'éprouver des phénomènes de dégénération, de régénération el diverses altérations variables suivant la cause provocatrice de la névrite.

(1) Voy. Maladies de la moelle in Pathologie interne, t. VIII de l'Encyclopédie vétérinaire. 
DC̈génćralion. - Après la section d'un nerf (1), le bout périjhériøue dégénère : une heure après, la myéline a alısorbé une parlie du plasma circumvoisin et subi un gonflement; elle est disposẻe en boules et filaments environnés de glolules rouges et de cellules lymphatiques arrondies ou irrégulières auxyuelles se joignent bientot les cellules comrctives revennes à l'état embryonnaire; tous ces bléments absorbent la myéline.

Finyt-quatre heures apres la section, les tubes nerreux ¿ myéliue sont profondément modifiés, comme en témoiguent les impréguations à l'acile osmique, "les noyaux des senments iuterannulaires sout légèrement lıypertrophićs; le protoplasma, particulièrement autour des noyaux et des incisures, est. plus abondant qu’à l'élat normal.

"Ces allérations s'accentuent les jours suivants; les noyaux devienueut plus volumineux; leurs nucléoles s'accroisseut et se divisent; les noyaux prennent alors la forme de bissac et subissent également la division; le protoplasma prend un développement considérable, il refoule au niveau du noyau et de quelques incisures la gaine de myéline el le cylindraxe, il remplit en certains points tout le calibre du tube; il se clıarge de graisse qui se colore par l'acide osmique en jaune brunâtre, ainsi que de gouttes de myéline qui, sous l'influence du mène réactif, premuent une teinte gris bleuitre.

"I.a gaine de myéline est aunsi divisée dans chaque seguent interannulaire en fraguents jus ou moins nombreux et de dimensious diverses, séparés les uns des autres par des proints protoplasmiques au niveau desquels le cylindiaxe a également disparı. Le cylindraxe est en effet

(1) La seclion d'une racine molrice amèue la dégénération périphérique; la section d'une racine sensilive a pour conséquence la dígénéralion de celle porlion de la racine qui est en rapport avec la moelie. - Les fibres a myéline des cordons sympathiques dégénèrent a la suite d'une section, comme les fibres à myếline de̊s norfs cérúbro-spinaus. 
détruit comme la gaine de myéline par le protoplasma en voie de déreloppement. " (Babinski.)

Les tules nerveux sont finalement représentés par la gaine de Schwann; les noyaux, la substance protoplasmique et les amas de myéline, les cylindraxes ont disparu.

Ces modifications apparaissent d'abord à l'cxtrémité péripherique des tubes nerveux, au voisinage des plaques motrices et descorpuscules sensitifs; elles ne se déreloppent pas avec la mème rapidité dans bien des lubes nerveux; certaines tibres éprouvent en deux jours plus de transformation que d'autres en six jours.

Viuggt-quatre heures après la section, les noyaux de la plapue motrice elle-mène sont sphériques, volumineux, et font une saillie plus considérable à la surface du faisceau musculaire.

Le nerf perd son excitabilité électrique dès que le cylindraxe a subi une solution de continuité dans une partie quelconque de son parcours, ce qui arrive généralement chez le lapin deux jours après la section, quatre jours après chez le rlizen (kanvier).

Les fibres de Reyak présentent des modifications beaucoup moins prononcées.

Quatre jour's après la section du nerf, les noyaux de ces fibres sont lyypertrophiés et possèdent plusieur's nucléoles ; quant aux fibres elles-mèmes, on y observe des vacuoles a la place desquelles apparaissent vers le septième jour des granulations graisseuses.

Les cellules conjonctives du lissu intrafasciculaire, les cellules endothéliales des vaisseaux capillaires, des artérioles et des veinules, el enfin les cellules de la gaine lamellense contiennent dans leur protoplasma, à partir du ringt-cinquième jour environ qui suil la section, des goultelettes graisseuses, qui, selon toute probabilité, sont des produits de transformation de la myéline, dialysés par la membrane de Schwann. 
La chégénéralion des nerfs est un phénomène actif: les segments interamnulaires perdent leur différencialion analomique etreviennent à l'état embryonnaire, les noyaux se multiplient, le protoplasma se développe, d'où ı́esulte la clisparition de la myéline et du cylindraxe; les transformations s'opèrent d'autaut plus vite que l'animal en expérience est plus jeune, plus robuste, doué d'une plus grande vitalité.

Les altérations du bouticentral du nerf sectiomé s'arrètent parfois au premier étranglement annulaire; le cylindıaxe est conservé et subit simplement des modifications superficielles; il devient moniliforme, s'hypertrophie par places el sa constitution fibrillaire devient plus apparente, les cellules lympluatiques digèrent peu à peu la myéline.

Régénération des nerfs. - Les deux abouts du nerl' sectionué, mis en conlact, ne tardent pas à se souder; la continuité du cordon nerveux se rétablit rapidement; il n'en est pas de mème des fonctions physiologiques.

La réapparition de la seusibilité dans une région immédialement après l'affrontement des bouts d'un uerf seclionné, olsservée par quelques chirurgiens, est un fait contraire, quelle que soit l'explication qu'on en donne (récurrence, suppléance, arrêt de l'inhibition ou dynamosénie), à l'expérimentation plıysiologique et à l'analyse histologique. Le lissu conjonctif dı nerf et les cellules migratrices mises en liberté à la suite de la section sont les seuls éléments aux dépeus desquels s'opère cette cicatrisation.

Les cylinlraxes divisés ne se réunissent jamais d̀ d'autres cylindraxes. Le retour des fonctions exige tonjours une véritable régcuération, c'est-à-dire la néoformation de tubes nerveux.

Celte néformation réclame trois mois au minimum pour s'accomplir. L'extrémité du bout ceutral s'hypertrophie, les cylindraxes présentent une striation longitu- 
dinale et chaque nowveau cylindraxe formé par la segmentation longitudinale le l'ancien est l'origine d'un nouveau tube nerveux; il se forme ainsi un renflement blanchàtre ou grisâtre adhérent aux tissus, enveloppé de tissu cicatriciel; c’est le bourgeon le régénération. Les cylindraxes nouveaux, au nombre de 20 à 40 par cylindraxe ancien, se développent par bourgeounement périphérique, pëuèlrent dans le tissu cicatriciel, le traversent, s'engagent dans les gaines de Schwann du segment périphérique ou entre ces gaines el, a la faveur de ces conducteurs de la régénération, tendent à alteindre les organes iunerrés par le nerfsectionné.

"Les fibres de nourelle formation, dépourvues d’abord de myéline, s'entourent de cellules, se transforment en noureaux segrments interammulaires, qui sont, au délıut au moins, lien plus ténus, bien plus minces que les se ments auciens. On trouve parfois, en dissociant le hout périplıérique d'un uerf en roie de régénération ou complètement régénéré, d’anciens tubes nerveux dont la myéline n'existe plus que par places sous forme d'amas Granuleux el dans liutérienr desquels sont réunies plusieurs libres à myéline grèle de nouvelle formation. " (Bal,inski.)

Quand il existe un espace de deux il trois centimètres entre les bouts du nerf sectionné, le tissu cicatriciel qui comble cet espace peut servir de conducteur à la régénération.

Les jeunes fibres qui traversent ce tissu finissent par alteindre les gaines du bout périphérique dégénéré; mais heaucoup de ces fibres s'égarent et s'atrophient, de sorle que la régéuération est incomplète.

Les régious innervées peureul ne récupérer que partiellement la sensitilité et la motricité.

Quand la distunce à franchirest plusconsidérable, quand les résections nerreuses dépassent sept centimètres, les fibres issues du bout central ne parriennent pas à atteindre 
celles du hout périphérique dégénéré et la régénération devient impossible, car le conducteur indispensable fait défaut.

La régenérution s'ellectue généralement dans l'espace de cinr a six mois; elle est plus rapide chez les animauo jeunes el vigou!eus que chez les animaux vieux et débilités; elle est enfin plus rapide quand la résection es! faite à ıne petite distance de l'extrémité périphérique du nerf : la régénération du médian est plus difficile que celle du nerf plantaire.

Signification. - La dégénération et la régénération des nerfs ont une signification très nette : la paralysie dans le premier cas, le rétablissement des fonclions nervenses dans le second.

La parulysie locale, diffuse ou genéralisée trahit en somme l'altération nerveuse de la manière la plus saisissante. Si tou; les muscles, si tous les organes peuvent ître frappés de paralysie, on constate cependant de réelles prédispositions: les nerfs rackidicns sont plus souvent altérés que les nerfs bulbaires.

l'armi les uerfs rachidiens, le nerf fémorul et le nert radiul sont le plus souvent atteints de névrite ou de dégénérescence. Les muscles auxquels ces nerfs se distribuent perdent Jour tonicilé; ils s'atrophient pendant que leurs untugonistes s'liypertrophient. On peut constater en mème temps dans les nerfs et les muscles des modifications de l'excitubilité électriqne.

l. ExcitimLt: Électmole des nerfs moleurs est augmentéc au lébut de la névite; mais celte augmentation est tres lingice.

On pent constater la diminution ou la disparition de cette excitirbilité.

Sous le nom de Rlaction de blicénéreschince, on désigne lensemble des réactions électrịues fournies par les nerfs et les muscles dans certains élats patlrologiques. 
Ces modifications de l'excitabilité des nerfs moteurs el des muscles ont une certaine importance relative au point. de vue du diagnostic et du pronostic des paralysies chez l'homme; mais chez les animaux elles en ont beaucoup moins, à cause de leur impressionnabilité et de leur indocilité.

On se sert, pour les rendre manifestes, d'appareils spéciaux, machinesvoltaïques ou machines voltafaradiques, en employant la mélhode unipolaire. Dans cette méthode, on applique sur le muscle ou le nerf que l'on se propose d'exciter une seule électrode (l'électrode diffirente), jamais les deux à la fois. L'autre électrode (l'indifférente) s'applique sur une région quelconque du corps.

Normalement, à des courants d'intensité connue correspondeut des réactions physiologiques; les variations quantitatives ou qualitalives de l'excitabilité des nerfs moteurs et des muscles constituent ce que Erb a appelé réaction de dègénèrescence. Ses caraclères ont été constatés dans les paralysies expérimentales. On a observé, dans leur état complet de développement, que :

$1^{0}$ Le tronc du nerf n'est plus excilable ni par les courants faradiques, ni par les courants voltaïques;

$2^{\circ}$ La contraclilité faradique des muscles est abolie, tandis que la contraclilité voltaïque est conservée et mème exaltée; le muscle est alors facilement tétanisable.

Ce syudrome se présenle avec des formes particulières. Parmi les syndromes de dégénérescence différents entre eux, citons: les syndromes succédant aux sections nerveuses, à la section ou à la destruction de la moelle, à l'intoxication par le curare, par la strophantine.

Bien que la réaclion de dégénérescence perde dans notre médecine sa grande valeur pronostique à cause de l'irritabilité de nos sujets, il importe de signaler les phénomènes qu'on peut observer dans le cas de régénération. Les cylindraxes de nouvelle formation se meltent en rapport arec les fibres musculaires : l'excitabilité 
faralique et l'excilabilité voltä̈que du nerf reparaissent.

Les premiers indices peuvent se reconnaîlre dans la portion du nerf siluée près de la lésion. Les muscles, de leur côté, sont plus lents à recourrer leurs réaclions normales, et proglessivement la conlracliiité électrique rederient normale.

liref, ces manouves permettent de différencier les polynévrites des paralysies dorigine centrale où la contractilité électro-musculaire est conservée.

Quand ces paralysies sont incomplètes, elles s'accompasuent quelquefois de tremblements. Cadéac a signalé les tremblements des muscles de la face et de la langue dans un cas de paralysie labio-glosso-laryngee.

(1I peut constater (n même temps des troubles de la sensibilité, des zones d'anesthésie et des zomes d'hyperesthésie, des troubles des riflexes qui sont ordinairement dimiuués ou abolis ( 1 .

\section{II. - Lexions serondinires.}

L.es lésions nerveuses se propagent yuelquefois vers les centres; elles entrainent aussi deslésions des organes dans lesquels ces nerfs se distriliuent.

Lésions de la moelle consécutives à la section des racines postérieures. - Les fibres nervenses des racines postérieures ayant pour centres trophiques les cellules des galiglions spinaux, c'est le lout en connexion avec la moelle qui dégénère après la section de ces racines.

La dénénération se propage aux fibres ralirulaires de la moelle et a un grand nombre de tubes qui constituent les corduns postérieurs.

Lésions centrales consécutives à certaines altérations des nerfs craniens et rachidiens. - l.es recherches de

I) Cadéar, Jomrnal de l'École rélérinaire de Lyon, 18n7. 
Hayem, Tiesler, Yégas et Joseph Gudden, Mendel, Erlitzky, llomen, Redlich Forel, Bregmann, Ranvier ont démontré que les lésions nerveuses sont quelquefois suivies de lésions centrales. IIayem a observé, à la suite de l'arrachement du sciatique, une myélite cicatricielle correspondant au trajèt des racines postérieures à travers les faisceaux postérieurs. Les altérations varient d'intensité: il peut y avoir soil une simple atrophie des cellules nerveuses correspondant au nerf, soit une myelite aigmë entrainant la mort.

La subslance grise de la corne postérieure s'alrophie, la corne antérieure perd ses saillies et sesangles et ne contient que peu de grandes cellules; le cordon postérieur est moins volumineux et la partie postérieure des cordons latéraux légèrement atrophiée.

Gudden et ses élèves ont observé que l'arrachement du facial daus le canal de Fallope amène la disparilion de ses fibres radiculaires et de son noyau inférieur.

Forel a arraché des nerfs eraniens (fucial, hypoylosse, trijumeau, vugue, glosso-pharyngien) chez des animaux adultes et a constaté des lésions dans leurs noyaus bulbaires.

Dans d'antres expériences, Darlischewitch, employant la méthode de coloration de Ilarchi, constate yue l'arraclnement d'un nert' est suivi d'altérations dans son bont central et ses noyaux d'origine; que les lésions dues à l'arrachement d'un nerf rachidien sont moins prononcées que celles dues à l'arrachement d'un nerf cranien.

Bregmann a étudié les altérations du bout central consécutives à l'arrachement des nerfs cruniens chez des adultes. Le bout central dégénère; au début l'altération se montre seulenent sur quelques fibres, puis la lésion s'étend et la dégénération envahit mème la racine.

Leslésions centrales se développent plus facilement quand on fait choix d'animaux nouveau-nés, et qu'on opère sur un nerf cranien lout près de son point d'origine. 
Hayem prétend que la simple section du nerf sciatique peut amener des altérations des centres nerveux; mais il est probable que dans ses expériences l'infection de la plaie n'a pas été évitée.

Le mème auteur a développé des lésions spinales en irritant des nerfs par le contact de cristaux de bromure de potassium ou la piqùre avec une aiguille trempée dans la nicotine: les altérations étaient analogues à celles observées dans la myélite aíyuë de l'homme; la pie-mère et l’araclınoïle étaient aussi dégénérées. Hayem pense que l'irrilation se transmet du nerf à la moelle par l'interméliaire du lissu conjonctif et des cylindraxes.

Avant lui, Tiesler avail appliqué des substances irritantessur les nerfs sciatiques du lapin. "Lin de ces animaux devint paraplégique et succomba trois jours plus tard; l'autopsie fit découvrir dans l'épaissenr du nerf sciatique, sur le point où l'irritalion avait été produite, un foyer purulent et un autre foyer dans le canal vertébral autour des racines du nerf, plès de leur émergence. La moelle épinicre, dans la région correspondante, était ramollie et infiltrée de corps granuleux et de leucocytes. La partie du nerf comprise entre les deux foyers purulents paraissait parfaitement saine (1).

Dans ce cas, l'infection de la plaie n'est pas contestable, mais il est probable que les microbes sont parrenus dans la moelle par une autre voie que le systeme circulatoirc.

l'itres et Vaillard n'ont pas constaté d'altérations ascendantes des nerfs, mais seulement des nérrites nécrosiques localisées, en injectant des substances irritantes a॥ roisinage des troncs nerveux des membres.

Si l'on sort du domaine expérimentul pour entrer dans celni de la clinique, on constale que lit propagation de l'inflammation de la périphérie d'un nerf vers ses parties centrales est rare. 
Tanlòt l'inflammation se propage par le cylindraxe jusqu’à la cellule, tantit elle atteint le système nerveux

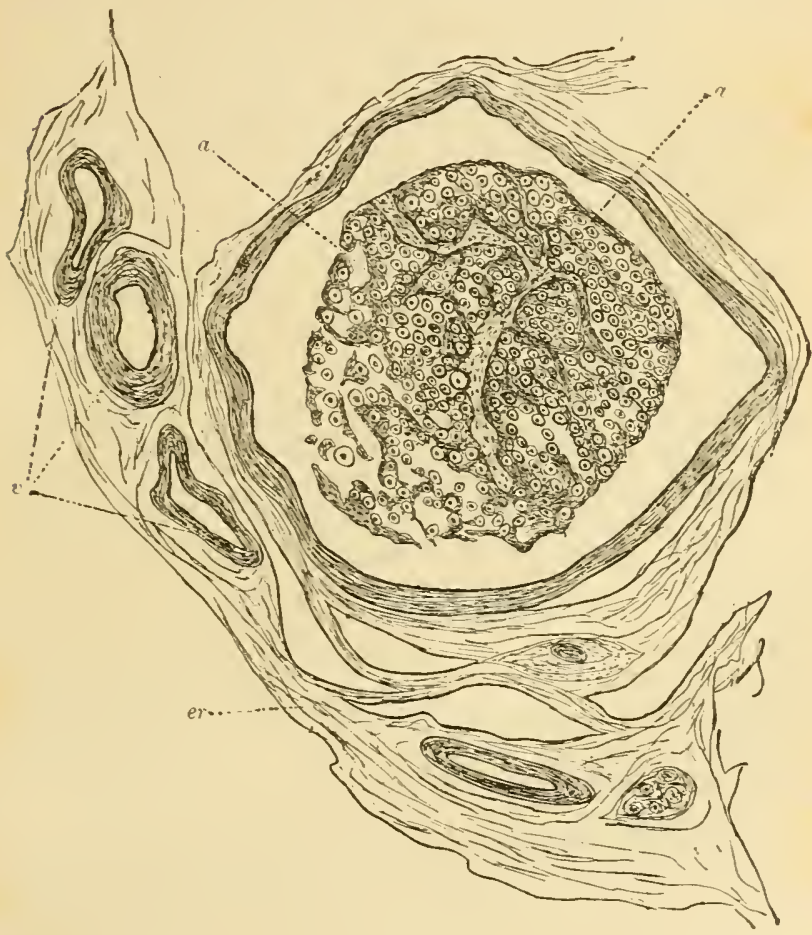

Fig. 7. - Verf plantaire (coupe Iransrersale). - Hypertrophie du tis-u conjonctif intrafasciculaire (Pécus).

$a$, tissu conjonclif intrafasciculairc; er, tissu conjonctif iuterfasciculaire lyy ertrophié ; $v$, raisscaux du lissu conjonclif périfasciculaire.

central par l'intermédiaire du tissu conjonctif périfasciculaire et intrafasciculaire: elle est parenchymateuse d ins le premier cas, interstitielle dans le second.
CAdÉAc. - Sémiologie, $2 e$ édit.
11. -5 
La ıévrile parenchymateuse ascendante est motrice ou sensitive.

La nèrrite motrice ascerrdante est une lésion qui, après avoir pris naissance dans un nerf moteur, remonte vers les racines antérieures et les cornes antérienres de la moelle.

la nétrite sensitive ascendante consiste dans une lésion intéressant un nerf sensitif et tendant à gagner les ganglions spinaux correspondants (1) (fig. 7).

La plupart des destructions nerveuses s'accompagnent exclusivement d'une dégénération du bout périphérique; le bout central demeure sain ou ne s'altère qu'au voisinaçe immédiat de la lésion; mais cette régle n'est pas absolue. I'inflammalion d'un nerf moteur est susceptible de se propager jusqu'à la moelle, et Traslıot a signalé la nevrite motrice ascendante chez le clieval. Nous allons résumer les principaux faits relatifs à celle question.

Les lésions qui fonl parlie intégrante des névrites ascendantes intéressent les nerfs, les ganglions rachidiens, les racines et la moelle.

Névromes. - Ia portion terminale des nerfs sectionnés est fréquemment, cliez le chevaluévrotomisé, le siège d'uu nirrome constitué par des fibres nerveuses grêles à Irajet sinueux et englobées dans une gangrue de tissu conjonctif épais et lense.

Ces fibres gî̂les proviennent du bourgeonnement du bout central des tubes nerveux qui ont conservé leur vitalité après la section. Le tissu conjouctif dense qui comprime les fibres nerveuses est généralement le résultat de l'infection microhienne.

La production du nérrome est souvenl le résulat d'une insuftisince d'asepsie. Quand on enlève deux à trois centimètres de nerf saus précautions asépliques, on peut prédire presyle à coup sùr la production d'un névrome douloureux.

(1) Pécus, Nérrile olservie chez un cheval affecté de maladie naviculaire double (soc. des sciences vel., 1901, 1. 150). 
Yous arons pratiqué comparativement deux séries de névrotomies d'après deux méthodes: l'une, qu'on peut qualifier de timide et de sale en raison du peu de longueur du nerf sectionné et du peu de soins apporlés dans l'exécutiou de l'opération; l'autre, qu'on peut appeler hardic el propre puisquon fait une grande incision et une résection nerveuse de près de dix centimètres de lon"r et qu'on obtient la cicatrisation par premiere intention. Les animaux de la première série sont presque constamment redevenus boiteux; ceux de la seconde continuent, depuis plusieurs années, à bénéficier de l'action curative de l'opération.

La suppuration excrce une influence désastreuse sur les suites de la névrolomie. On a l'occasion de le constater dans les névrotomies basses ê doubles. Le côté interne, opéré le premier, est quelyuefois infecté au moment où l'animal est retourné et où l'on prépare la région externe. Quelques mois plus tard, le còté interne qui a suppuré est le siège d'un névrome très douloureux à la pression et l'animal boite; au contraire, le cóté externe dont la cicatrisalion a été immédiate, est, au-dessous de la section, d'une insensibilité parfaite. Dans ces cas, on a une guérison durable d'un còté, passagère de l'autre; l'extirpation du névrome - opération obligée — vient réparer la faute d'asepsie ou d'antisepsie qu'on avait commise.

Le lissu fibreux cicatriciel exerce une compression douloureuse sur les lubes nerreux du bout central; il semble servir de conducteur pour les cylindres d'axe en voie de bourgeonnement; la régénération du nerf est rapide ê l'animal est bientòt atteint de nouveau d'une claudication plus prononcée qu'avant l'opération.

Conséquemment, névrotomiser un animal signilie: résection d'une graude étendue de nerf, six centimètres au ninimum, et réunion de la plaie par première intention.

La névrite ascendante se déclare quelques jours après l'infection de la plaie opératoire; le bout central gui 
baigne dans le pus s'enllamme. Il s’agit généralement d'une périncivrite. Quand cette névrite est évitée, le bout central du nelf sectionné est généralement plus grẻle que son congénère.

Les lésicns histologinnes sont les snivantes: "un nombre plus on moins grand de fibres nerveuses ont subi des modifications; quelıues-unes sont plus grìles ıu’à l'état normal et présentent les caractères de l'atrophie simple; il y en a dont le contour manque le netteté et tous les éléments qui les constituent, cylindraxes, segments interannulaires, paraissent intimement confondus; il s'agit lá, si les observations des autenrs qui ont sigualé cette altération sont exacles, d'une sorte de nécrose, de dégénération spéciale qui a pour conséquence la destruclion de la fibre nerveuse; enfü, dans certaines fibres on trouve une multiplication des noyaux de la gaine de Sclıwann.

Ces altérations tubulaires du noyau central constituent ce que nous avous appelé la nérrite purenchymatense ascendante."

I.a cause de l'atrophic des fibres qui appartiennent an hout central du nerf sectionné n'est pas comme; suivant Forel, les cellules gangliomaires avec leurs fibres, forment des éléments conıparables à ceux qui représentent les animaux inférieurs, ces éléments se modilient comme eux quand on supprine une partie de la masse constituée.

Friedländer el Krauss soutiennent que les fibres sensitires qui se terminent dans les corpuscules de Meissner et de l’accini sont les seules parties du segment central qu dégénèrent, parce cułil y a interruption entre leur bout central et lenr organe terminal; Marie prétend aussi que certaines filires du bout central ont leurs noyaux d'origine, leurs véritables centres trophiques à la périphérie. Ces hypothèses ne sont nullement justifiées par l'expérimentation.

l.es cellules des ganglions rachicliens sont normales ou légèrement atrophices. 
Les cordons postéricurs s'atrophient, les cornes postejricures perdent leurs saillies et leurs angles et ne renfermont que peu de grandes cellules, on constale quelquefois de la myélite transverse avec foyers de suppuration.

Des troubles très élendus peuvent ètre ainsi mis sur le compte de la nér ite ascendante.

On constate quelquefois les doulemrs lancinantes provoquées par la pression exercée le long du nerf blessé ou sectionné.

Des tronbles lricomoterms accompanent ces doulenrs. Nous arons ru des chevanx présenter de l'incoordination motrice après la névrotomie.

Nous avons vu notamment un cheval, dont le membre nérrotomisé, soulevé convulsivement dans la marche, renait à chaque pas toucher ou croiser le genou du memlire opposé.

Trasbot a observé nne hémiplégie conséculive à une compression du plcxus brachial déterminée par la posilion déculitale.

Cinq jours plus lard les phlyctènes formées sur l'épaule et l'avant-bras droits, à la suite de la friction d'essence de térébenthine qui avait été faite ou consécutivement aux troubles trophiques, se déchirent.

Deboul, le membre postérieur droit repose sur le sol par la région de la pince seulement, et la douleur assez vive dont il est le sière s'exagère quand on lui imprimail des secousses en divers sens. On conslate un peu plus tard les caractères de l'atrophie des muscles de l'épaule droite et du bris.

Le postérieur droit fortement aedématié est complètement paralysé. La douleur de ce membre a fait place à une anesthésie complète; vingt-cinq jours après, lihemiplegie est atténuée; elle finit par disparaitre complètement.

(1) Trasbot, Archives vétérinaires, 1s53, p. 201. 
Lésions des muscles. - Les lésions musculaires commencent quinz jours après la suppression de l'innervation et se développent plus ou moins rapidement " suivant l'espèce animale, l'àge de l'animal, son état de santé, les condilions hygiéniques dans lesquelles il se trouve; elles apparaissent flus vite dans les muscles ronges que dans les muscles blancs.

Au bout de trois ou quatre semaines, l'atrophie devient macroscopiquemeut apparente et on peut la reconnailre à la vue ou à la ralpation. Elle s'accentue de plus en plus et, après plusieurs mois, les fibres musculaires dégénèrent presque completement, quoique pendant longtemps il reste encore dans quelques fibres de la substance contractile, ce que démontre l'électrisation dı nuscle mis à nu.

Le muscle ainsi modifi est notablement réduit de volune (Babinski). Il peut récupérer son volume et ses fonclions si le tissu musculaire n’est pas trop altéré.

Histologiquenext, on constate d'abord dans certaines fihres la disparition de la striation transversale et un aspect granuleux (dégénération gramuleuse); d'autres ont simplement diminué de volume (atriphic simple). Ia substance contractile est diminuée; les noyaux dı sarcolemme sout plus nombreux.

L'atrophie de la substance contractile marche de pair avec la tuméfaction de la substance proloplasmique non différenciée; la substance contractile est absorbée par le protoplasma. Les muscles éprouvent des modificalions analogues à celles qui se produisent dans le bout périphérique d'un nerf sectionné.

On connaît les conséquences de ces altérations; nous y reviendrous en nous occupant des amyotrophies el des purulysies en général.

Lésions des os. - l.es os subissent fréı̧uemment des altérations à la suite de nérrites et de la section des nerfs qui s'y distribuen!. 
On a signalé la raréfaction du lissu osseux. Latrophie du tissu osseux peut résulter des lésious centrales, médullaire, ou perriphèriques.

On a constaté que dans la chorie du chien comme dans la paralysie infantile, il y a une atrophie manifeste de tous les systèmes de la région atteinte. Les muscles, les nerfs, les os en particulier sont réduits à leur plus simple expression.

Goubeaux relate un cas de chorée dans lequel le squelette du membre antérieur gauche pesail 103 grammes, alors que son congénère frappé de chorée n’alteignait que le poids de 38 grammes.

l'atrophie porte non seulement sur le volume, mais encore sur la longueur des rayons osseux; les différences de longueur ainsi observées sont environ de trois à quatre centimètres. Il en est de mème pour le diamẻtre transversal.

Mais parfois dans la paralysie infantile, on constate 1\%yuertrophie des os du corps, du radius et du cubitus sans dystrophie, c'est-à-dire sans altération du tissu osseux.

Après la contusion du plexus brachial chez l'homme, on a constaté à la fois l'hypertrophie des ongles et l'atrophie des phalunges qui deviemnent noueuses au niveau des articulations; les doigts sont en forme de coins, mais parfois, ils sont effilés en forme de cierges.

Ogle et Blum ont signalé la raréfaction osseuse après la section du nerf median; Mnrat a observé un mal perforant des deux premiers orteils après la section du sciatique poplité externe.

A la suite de sections nerveuses, Vulpian a obtenu tantòt de l'atrophie, tantòt de l'hypertrophie. Schiff constate, après section du nerf dentaice inférieur, un accroissement considérable de la màchoire.

Après la section des nerfs périphériques, il n'est pas rare de constater cliniquement des troubles atrophiques des os préexistants et des troubles hypertrophiques consis- 
tant dans l'ossification des tissus environnant le périosle. la fracture dı radius avec lésion du médian peut être suivie d'un cal rolumineux, de douleurs nérralgiques, d'ulcérations de l'index et du médius (Paget).

Sous l'influence de compressions nerveuses, déterminées par des sabots étroits et courts, Jaboulay, Pollosson

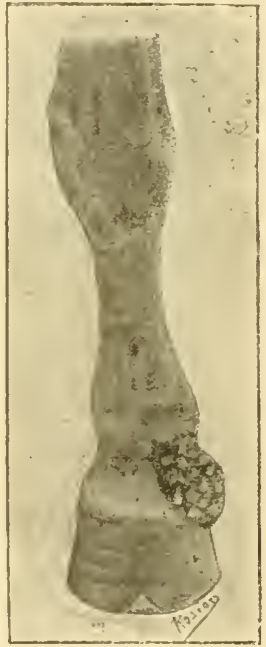

Fig. *. - Ilypertrophie de la forme après la névrolomie.

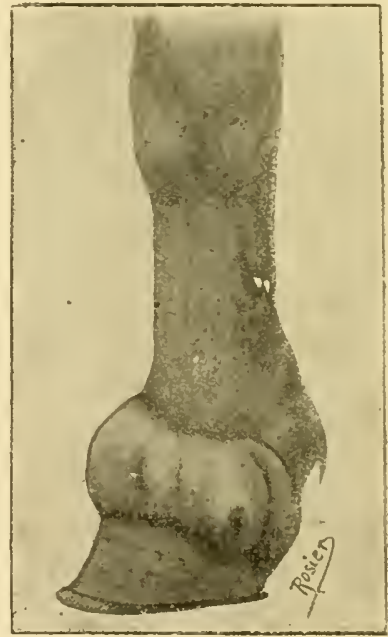

Fig. :. - La forme a donblé de rolume.

ont constaté la disparilion de la première phulunge du gros orteil; elle est remplacée par une coque osseuse irrégulière d'un volume plus considérable que le squelette primitif.

l.es altérations atropliqques consécutives a des réseclions nerveuses pratiquées chez le cheval cousistent essentiellement dans un rumollissement du tissu osseux qui s'effrite, s'éuiette, arec la plus grande facilité; les vaisseaus s'y raréfient; il se laisse entamer par la rénette ou le bistouri et présente une teinte jaunìtre. 
Celte atrophie porte principalement sur les os ancions du squelette et particulièrement sur le centre de los; la moelle devient plus diffluente; le tissu osseux en se raréfiant a plus de tendance à se fracturer, mais les tissus périphériques semblent, au contraire, avoir une tendance à végéter (fig. 8, 9).

La suppression de l'innervation dans une région tend a favoriser l'atrophie de la partie centrale de l'os el liypertroplie de la partie périplérique. Les lissus environnants se laissent plus facilement envahir par le tissu osseux. C'est probablement pource motif que les fractures se consolident plus facilement chez les aliénés àgés que chez les personnes àgées saines.

Comment se comportent les néoformations osseuses en présence de la névrotomie? - Les considérations qui précèdent lendent à établir que la disparition des formes carlilagineuses ou périostiques ne peut guère résulter de la névrolomie. Les fails signalés par Nocard, Mollereau, Magnin, lémoignant de l'atrophie des formes sous l'influence de la névrotomie, dímontrent seulement l'irrégularilé des troubles dystrophiques consécutifs à cette opération.

La névrotomic lend généralement, quand ses elfets sont rendus durables par l'extirpation d'une grande élendue de nerf, à produire un double résultat : atrophique au centre, hypertrophique à la périphérie par ossilication des tissus parostaux.

L'auguentation de rolume des formes après la nérrolomie a été signalée dès 1830 par Villate fils, puis par Renault, Peuch et Cadéac (fis. 10, 11).

On peut roir les formes décupler de volume après la névotomie sans qu'il soit possible de faire intervenir linjection pour expliquer ces hypertrophies.

Les formes les plus iégétantes, celles qui offrent l'aspect madréporique le plus prononcé après macération, celles qui ont l'évolution le plus nettement stalactiforme sont 
82 SĖMIOLOGIE DE L'APPAREIL DE L'INYERVATION.

celles yui ont été combattues par la nérrotomie. Celte opiration gorge ou tend à gorger de sucs le tissu conjonclif péricartilagineux el périosseux et facilite ainsi l'expansion du tissu osseux d'origine périostique.

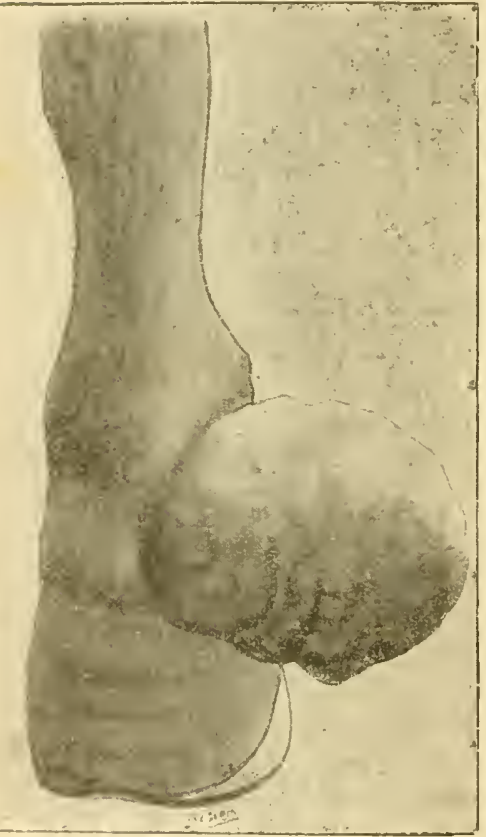

Fig. 10. - Dimensions de la forme au moment de l'abatage el trois ans après Ia névrotomie.

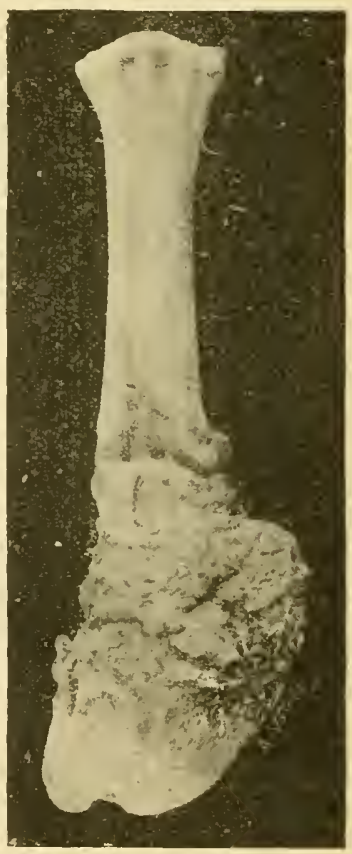

Fig. 11. - Squeletle de la forme précédente (.11. Blanc).

On sait en effet que les nerfs vuso-moteirs jouent un rỏle capilal dans la production de ces odèmes. Les membres frappés d'hémiplégie s'vedématient et celte lésion se limile exactement au còté malade. Ce phéuomène peut apparailte quelques heures après des lésions médullaires frac- 
tures de la colonne vertébrale, poliomyélites infantiles)

Par contre, la ligature de la reine cuce inférieure n'est pas suivie d'épanchement sous-cutané; mais si l'on sectionne le sciatique d'un còté, un épanchement se produit du côté privé d'innervation : tandis que le côté intact est épargné.

Ces infiltrations des tissus sous-cutanés ont été maintes fois constalées à la suile de plaies, de contusions portant sur les netfs périphériques.

Comment se produisent ces lésions trophiques? - Selon Morat, les lésions trophịues sont le résultal d'une cessation de conduclion nerveuse, d'une paralysie des éléments nerveux qui se rendent aux tissus soit pour en diriger les fonctions (motricité, sécrétion), soit pour en recueillir les impressions nécessaires aux actes réflexes par lesquuels es entretenue l'innervation de ces tissus, soit enfin pour y gouverner des fonctions plus intimes et qui échappent à l'analyse.

En résumé, il parait exister dans la moelle des centres d'où partent des fibres centrifuges qui, d'une facou non définie, tiendraient sous leur dépendance la trophicité, et qui seraient indépendantes de la sensibilité, de lamotricité et de la vaso-motricité.

Tous les troubles trophiques observés chez l'homme se retrouvent chez les chevaux névrolomisés; mais on ne les observe que le loin en loin, ce qui explique l'insuccès de ceux qui ont essayé de les reproluire.

La distribution des fibres nerveuses dans les tissus et leur récurrence nétant régie par aucune loi, on concoit que les troubles trophiques consécutits aux névrotomies ne puissent ètre reproduits d'une manière certaine.

I)ailleurs, les troubles osseux atrophiques comme les amyotrophies elles-mèmes n'exigent pas pour se produire la suppression de l'innervation. En effet, si l'on détermine expèrimentalement chez des chicns et des lapins une arthrite double, on voit l'atrophie se produire seulement 
du côté on le nerf centripète n'a pas été sectionné (Onanofl' et Deroches) (1).

L'alrophic parait ètre de nature réflexe et dépendre du retentissement de la lésion locale sur la moelle. Daus toute arthite, le systeme osseux sutrophie comme le systeme museulaire, el c'est parce que l'éparvin se résune ordinairement dans une ou plusieurs arthrites '[u'on rencontre si communément cette altération caractérisée par une asymétrie de la croupe et de l'ilium.

P'arfois l'infection s'ajoute au trouble nutritif et produit des periostites, des escarres; les os se nécrosent elı même temps que les parties molles.

Lésions des vaisseaux et du tissu conjonctif. - Après la sectiou du sympathique cervical, l'oreille et la moitié correspondante de la tète deviennent le siègo d'une congestion intense et d'une forte élévation de température. b'autre part, la ligiture de la ceine fémorale an-dessous de l'anneau crural n'est suivie d'cedème que lorsqu'on associe a cette opération la section du scialique. Cet oolème est dù à la solution de conlinuité des fibres vaso-motrices contenues daus le sciatique.

l.es nerrites et la dégénérescence du nerf sont suivies de troubles vaso-moteurs qui sont quelyuefois très prononcés, comme en témoignne une observation clinique de Caléac et Raymond sur la paralysie de la queuc et des splineters, accompagnée d'un adème prononcé de la base de la queue et du pourtour des sphincters (2).

1] faut reconnaitre aussi que des lésions vasculaires importantes, comme l'endophlébite (fig. 12), des lésions nerveuses (nèvrites interstiticlles) et osseuses (ostéites) peuvent se déveloper antérieurement à toute névrolomie comme en témoignent les rechesches de l'écus (3).

(1) Bulletin de la Societé des Sciences vét., 1900, p. 270.

(2) Voy. l'uralysie de la queue et des sphincter's, in l'athologie interne, t. VIII.

(3) Jécus, Troubles vascutaires et nerveux dans un cas de maladie naviculaire (Swc. des sciences rét., 1901, p. 140). 
Lésions de la cornée et des téguments. - La section du trijumeau rend la cornce insensible; puis cette membrane devient opaline, opaque dès le troisième jour, lépithélium

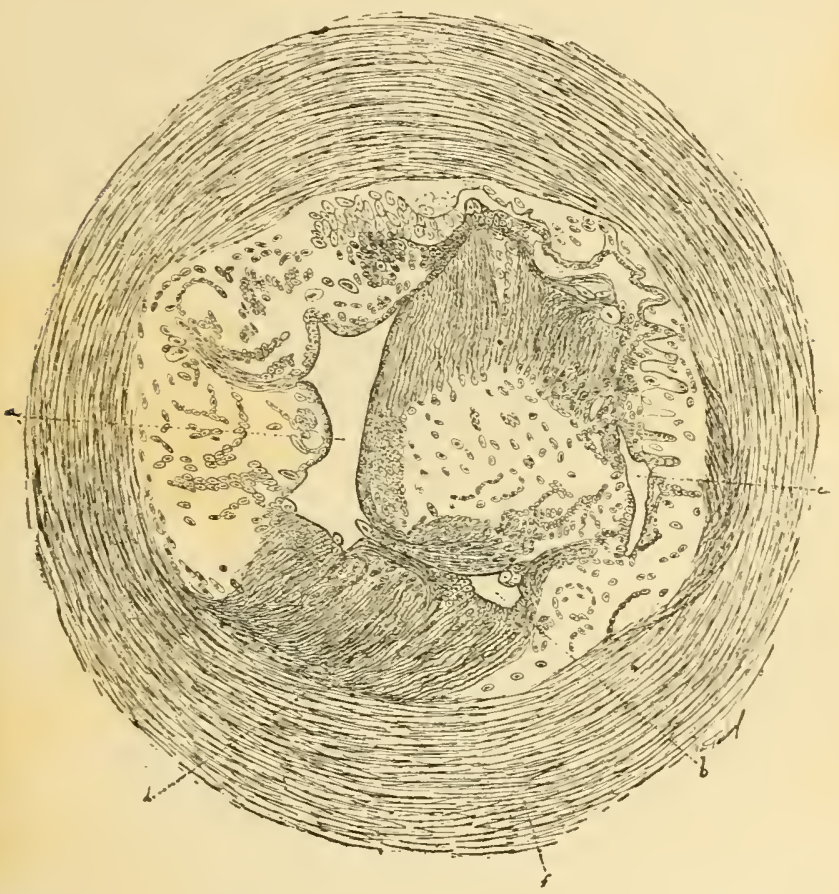

Fị̆. 12. - Endophlébile presque entièrement oblilérale et périplslébile de la reine plantaire externe antérieure dıoile, lio diamèlres (Pécus).

$a, b . c$, vestigges de la lumière du vaisseau; $d$, endohhẻlium végélant fibreux ; $f$, tunique externe artérialisée.

qui lapisse la face anterieure de la corne tombe, les cellules lymphatiques l'infiltrent dans toute son épaisseur, se convertissent en globules de pus et l'œil subit la fonte purulente. 
Les recherclıes pliysiotogiques ont dëmontré que les lésions corncemes consécutives à la section dı trijumean ne sont pas dues a la suppression d'un influx trophique, mais sont liées à l'abolition de la sensibilité de l'organe.

Sans attribuer aux troubles trophiques l'ensemble des altérations qui se jroduisent dans l'ail aprés la section du trijumeau, il parait cerlain que la cornée séparée des centres nerveux par la section du trijumeau est plus vuluérable qu'à l'état normal.

D'autre part, les altérations tramaliques dont elle est le siège facilitent l’infection qui engendre la suppuration et la perte de l'ceil. Les gernes infectieux pénètrent sans doute plus facilement dans la cornée et les milieux de l'(eil à la suite de la suppression de liullux trophique.

Diverses altérations se développent dans la patte d'un animal après la section des uerl's du membre correspondant.

La section du scialique cluez le lapin ou clıez le chien est snivie, quelques semaines après cette opération, d'une série d'accidents qui se manilestent au niveiu de la région la plus exposée aux frottements. Il se produit une simple nicrobiose des tissus qui se complique fré puem. ment d'une iuflammation phlegmoncuse. L'ulcération des parties superficietles gagne les parties profondes, atteint les os et provorpue une periostite et une ostiite.

Quitud on se borne à sectionner les racines sensitives, on ne constale aucun trouble trophique, d'où Ranvier conclut que des ulcérations qui succèdent à la section du sciatique ne sont dues ui à la smppression d'mn influx trophiıue, qui provienulrait des yanglions spinuu.c correspondanls, ni à l'abolition de la sensibilité.

II liaudrait en conclure que les rroubles trophiques oblenus dépendent de la section des filets vaso-moteurs contenus dans le tronc du sciatique. Les troubles vascuJaires dus a la paralysie vaso-motrice diminuent la résistance des tissus el préparent l'infection qui joue un grand 
ròle dans la production des lésions inflammatoires phlegmoneuses el gangreneuses.

Des troubles trophiques peuvent se produire conséculivement à la nérrotomie. Ils consistent dans un eczèma limité, dans le décollement partiel du sabot au niveau du

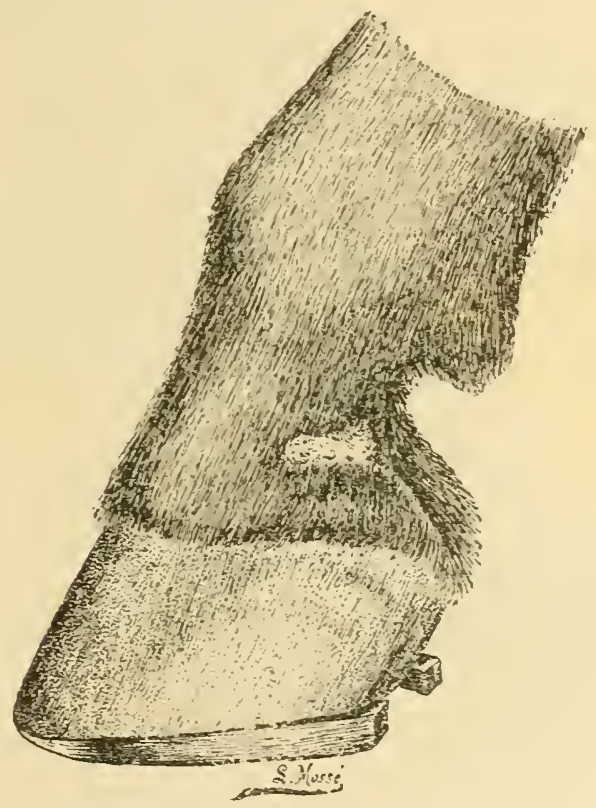

Fig. 13. - Eczéma limité conséculil à la section du nerf plantaire externe.

boumelet, dans la nécrose de l'os du pied et parfois dans la chute du sabot avec engorgement diffus de tout le membre (lig. 13, 1't).

L'EczÉM. survient ordinairement un mois après l'opération; il se développe toujours au niveau de la couronne, sur les parties latérales correspondant aux régions privées de nerls. Réduit généralement à une simple 
plaque, cet ecsému alfecte quelquefois les caractères d'une éruption résiculeuse ou pusluleuse suivie de petites plaques eczémaleuses multiples réparties, semble-t-il, sur le trajet de trois branches du nerf plantaire sectionné.

Nous arous eu l'occasion d'observer des désordres tro-

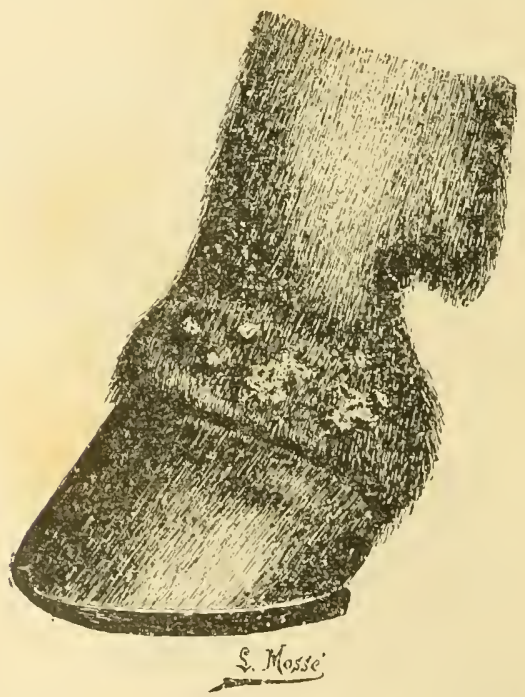

Fig. 11. - Petites plaques eczémateuses multiples.

phíques intéressant la troisiome phalange chez un cheral de gros trait, affeclé d'une forme externe très volumineuse, traitée par la nérrotomie plantaire unilatérale (fig. 15!. Nons avons conslaté un suintement au niveau du bourrelet, avec décollement du sabot et nécrose de la troisième phalangre; l'os s'effritait, il semblait privé de toute vascularisation ef offait une teinte jaunatre.

la chute du sanot he s'observe jamais chez les chevaux affectés de formes on de maladie nariculaire qu'à la suite de la névrotomie haute et double. Celle terminaison, à 
peu près fatale quand on pratique la nerrotomie haute et double chez les chevaux fombus, peul se produire quand

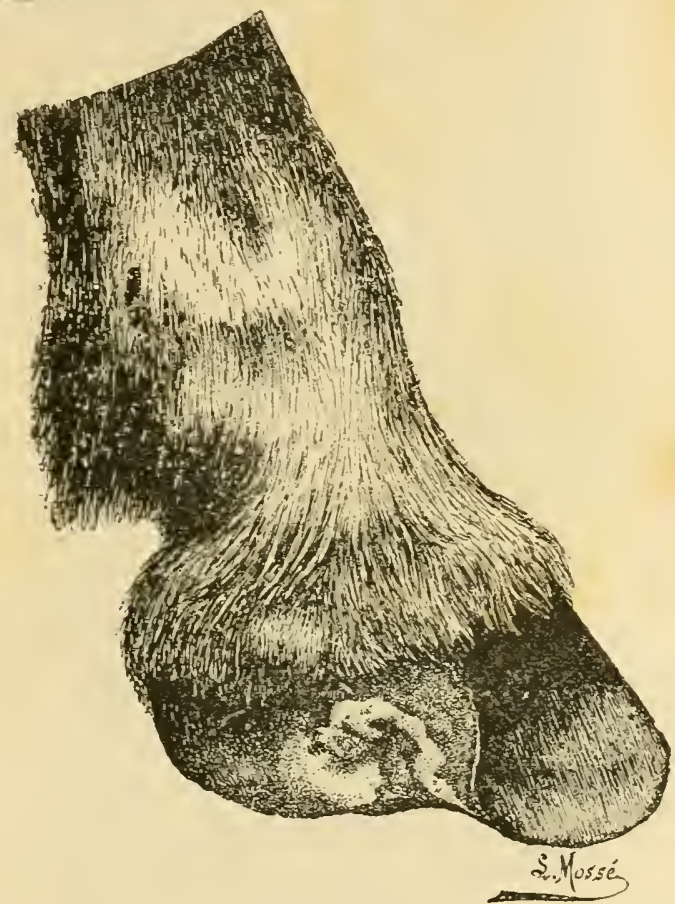

Fig. 15. - Nécrose sćclse de la troisième phalange (còté inlerne) da la nérrotomie plantaire haute chez un cheral all'ceté d'une forme cartilagineuse.

on sectionne uniquement les branches postérieures des nerfs plantaires fig. 16 et 17 .

Les troubles circulatoires engendrés par la fourbure sont exagérés par la section des filels sympatliques vaso-moteurs contenus dans les nerfs plantaires ( 1 .

(1) Cadeac, Sur la nérrotontic et les troubles trophiques consécutifs it cette operalion (Journal de méd. vétér. et de zootech., 1879, p. 139). 
90 SÉMOLOGIE DE L'APPAREIL DE L'IXNERVATION.

Les poils subissent aussi des altérations après la section des nerfs; la section du scialique provoque presque

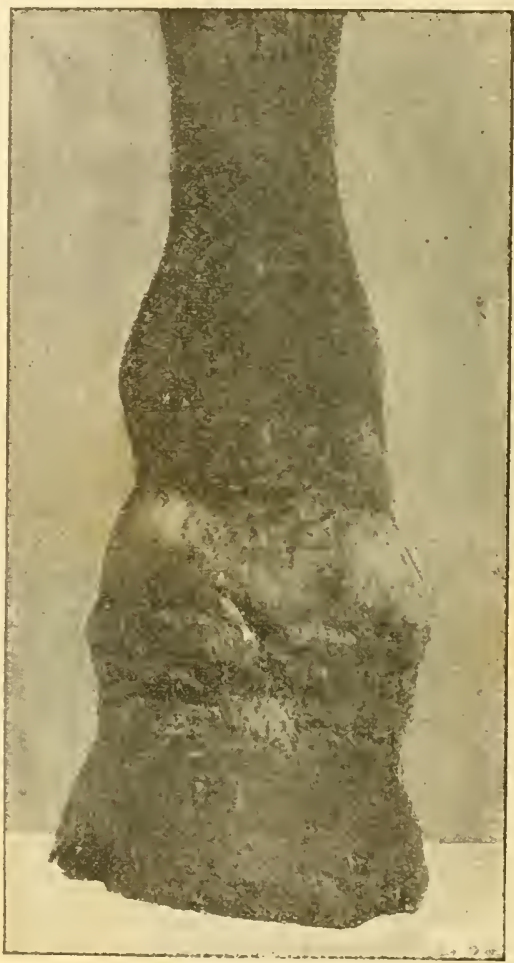

Fig. 16. - Déformation de l'extrémité inférieure du membre compliqué de cliute du sabol après la uevrotomie.

toujours la chute des poils dans un terriloire plus ou moins étendu, mais ceux-ci repoussent assez rapidement, mème quand le nerf ne se régénère pas.

(1) Cadéac, Sur les accidents conséculifs i la neirrotomic (Soc. des sciences vét., 1003, p. :2z). 
La section des premieres paires cervicales avec destruction de leurs gunglions ruchidiens est suivie, chez le chat el le lapin, de l'apparition de plaques alopéciques dans le territoire des nerfs occipilaux.

Chez les petits amimaux les ongles se délachent quelquefois des orteils et chez les solipédes on constate quelquefois la chute du subot.

Lésions pulmonaires. - La section des deux pneumogastriques rédnil de moitié, quelquefois darantage, le nombre des nouvements respiratoires; elle détermine dans le poumon de l'emphysemr, de la congestion,

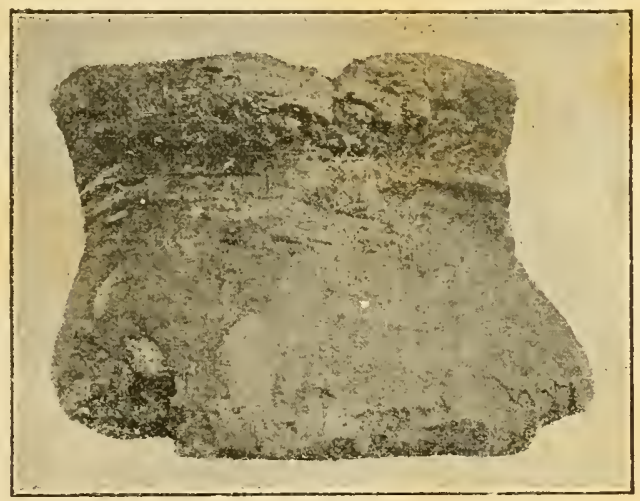

Fig. 17. - Écrasement et déformation du sabot.

des noyaux d'hèmorragie, de splénisation el d'hèpatisation; en un mot, toutes les lésions de la tronchopneumonie.

Ces altérations sont encore ici le résultat des troubles d'origine nerveuse qui affaiblissent le terrain et pernettent aux microbes contenus normalement dans les cavités respiratoires de s'y greffer, de végéter, de donner nais- 
sance à lles altérations locales et mème de pénétrer par les roies Iymphatiques ou sanguines dans tout l'organisme. Cette diminution de la résistance à la suite de la section nerveuse est pronvée par une expérience élégante uqui consiste à sectionner un pneumogaslrique chez le lapin ou le robare dans le péritoine duquel on introduit de la substance tuberculeuse; il se développe généralement dans le poumon du coté de l'opération des lésions untrerculeuses beaucoup plus prononcées que chez les animaux qui servent de témoins.

L'hémiplégic peut se traduire par du cornage; la respiration est dyspnéique, le larynx frappé d'anestlésie ne protegre plus les voies aériennes contre les corps étrangers qui infectent le poumon.

Lésions cardiaques. - On sait que la section des pneunogastriques provoque l'accélération des battemenls curdiarues. Eichhorst soutient que chez les oiseaur la section des pmeumogustriques ou mème d'un seul de ces nerfs a pour résultal de provoquer une dégénérescence graisseuse aiguë du caur.

\section{II. - MOELIE.}

Fonctions. - La moelle sert d'intermédiaire entre le cerveau et la périphérie; elle transmet les ordres du cerveau et lui apporte les impressions périphériques; cette louble fonction nécessite deux sortes de voies: les voies senstlives et les voies motrices. La moelle possede en outre des centres directeurs de cerlains actes de la vie de relation.

Serlionne; la moelle transversalement; toutes les parties situées en arrière de la section sont paralysécs de la sensibilité et du mouvement; mais les excitations porlées sur les régions paralysées déterminent des 
mourements reftcxes. La moelle est donc un conducteur et un centre.

Rôle conducteur. - A cet égard, la moelle offre à étudier les roies sensitives et les voies motrices.

$1^{\circ}$ Les roles sexsitives qui se constituent à partir des fibres radiculaires poslérieures embrassent le faisceau de Goll, le faisceau de Burdach et très probablement le faiscean latéral profond.

La destinée de ces fibrcs radiulaires est très complexe; elles peurent atteindre le bulbe et sarticuler par leurs extrémités avec les prolongements des cellules assemblées dans les noyaux de Goll et de Burdach; elles se terminent aussi dans la substance grise de la moelle ; mais toute fibre radiculaire émet un grand nombre de collatérales et toute collatérale s'articule dans la substance grise avec un neurone qui étend sa sphère d'influence.

Ces collatérales peuvent : $1^{\circ}$ se terminer sur les neurones le la corne postèrieure du même còté; $2^{\circ}$ passer par la commissure grise et se terminer dans la corne postérieure du còté opposé; $3^{\circ}$ se terminer sur les cellules des colonnes vésiculaires de Clarke et se relier par leur intermédiaire avec le cervelet; $4^{\circ}$ gagner la téte de la corne unterieure du mème còté et se terminer sur les neurones moteurs péripliériques.

Au point de vue fonctionnel, ces neurones centraux reliés aux fibres radiculaires conduisent la serisibilité jusqu’à l’écorce cérébrale (neurones médullaires sensitifs), jusquan cervelet par le faisceaı cérébelleux (neurones cirébelleux), ou président à l'exécution des mouvements réflexes (neuronés cordonaux de Cujal, neurones d'ussociation inter-sensitico-motrice de Mathias Duval).

Au collet dubulbe, le cordon postérieur subit la décussation et forme du còté opposé, avec le faisceau latéral profond, le ruban de Reil au niveau des tubercules quadrijumeaux pour aboriler à la capsule interne de concert 
avec les fibres venant des noyaux dits sensitifs des nerfs craniens.

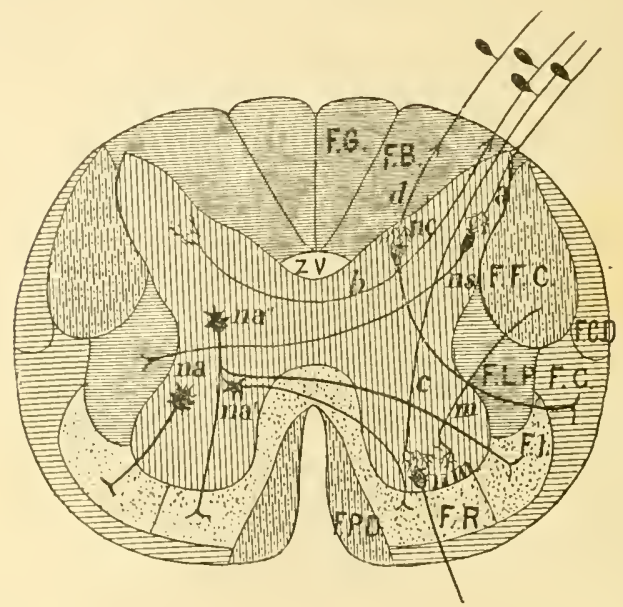

Fig. 18 .

(F.F, cordon de Goll.......... Végínérescence ascen Toies $\quad$ F.B. cordon de Burdach........ diute a long trajet. sensitics. \{ F.L.P, faisceau latéral profond (dégénérescence aścendante à court terme).

Voice fE.P. L, faisceat pyramidal croisé. \{ Dégénérescence descenmolrices. F.t'It, fitisceaı pyramiditl direct. daule à long trajet.

loies ,F.C.I), faisceau cérébclleus direct. ( Dégénérescence ascen cirébelleuses. F.fi, faisceau de Gowers........ dante a long trajel.

F.I, faisceau intermédiaire (dégénérescence descendante à Voies 1 court trajet).

rillexes. F.R, faisceall radiculaire (dégénérescence mixle à court trajet).

$a$, collatérale courte. $-b$, collatérale commissurale. $-c$, collatérale réllixe. - $d$, collatírale cérébelleuse. - $n s$, nenrone sensilif médullaire jetaut son axone dans le fuisceau latéral profond du côté opposé. nc, neurone cérébelleux jelant son axoue dans le faisceau de Cioners. $n a, n i^{*}, n \iota^{\prime}$, neurones corlonaux jetant lenr anone dans les diverses régions du faisceau fondamental (1'après Mathias Duval).

Là, les fibres du faisceau scnsilif se terminent et s'articulent avec de nouveaux nemrones gui en reprennent 
les fonctions et en prolongent le cours jusquau cerveau. Le faisceau sensitif ainsi reconstitué pénètre dans la couronne rayonnante ou se dispose en érentail pour aller se terminerdans les divers foyess sensitifs de l’écorce cérébrale.

2o Voles yotrices. - Les voies motrices sont constituées dès leur origine par les neurones de la zone psycho-

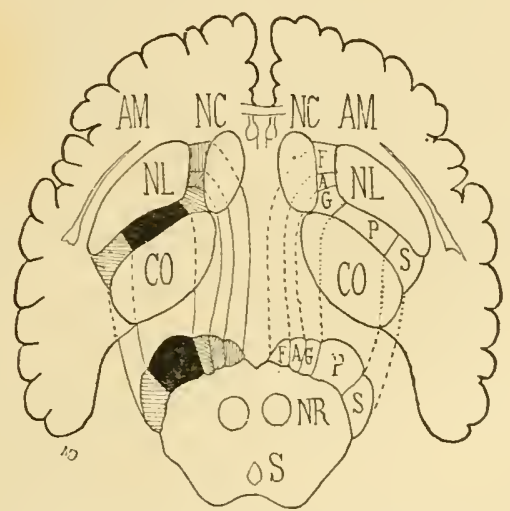

Fig. 19. - Place des nireaus sensilits et moteurs au nireau de la capsule interne, d'après Mathias Duval.

motrice de l'écorce dont les grandes cellules pyramidales forment le corps cellulaire. Elles s'avancent daus la couronne rayonnante, pénétrant dans la capsule interne dont elles forment la région antérieure ou lenticulostriée.

A ce niveau, le fuisceau motour comprent : le fuisceu géniculé G, ainsi désigné à ra'son de sa place dans la capsule et dont les fibres passent du cólé opposé pour se terminer sur les noyaux moteur's des nerfs bulbaires; le faisceau de l'aphasie (A, le fuisceau frontal $(\mathbf{F})$, dont la fonction est encore mal déterminée; le faisceau pyir midal (P), qui forme l'élément essentiel des voies 
motrices dans les rérgions sous-jacentes; il forme les pyramides antérieures dı bulbe; le faisceau myramilal croise et le faisceau pramidal direct ou de Tïrck de la moelle.

Toutes les grandes cellules motrices de la moelle sont reliées aux cellules pyramidates de la zone psycho-motrice.

\section{Movens utilisés pour différencier ees voies. -} Ces délerminations ont été fatites : $1^{\circ}$ par la mélhode vallérienne; 2o par la méthode cmbryologique; $3^{\circ}$ par la méthode expérimentale qui permet d'exciter les diffèrents faisceaux de la moelle.

$1^{\circ}$ Méthode vallérienne. - Quand les fibres nerveuses sont séparées de leur centre trophique, elles dégénèrent; c'est-à-dire quelles éprouvent un ensenble d'allérations qui les rendent reconnaissables et pernettent de les suivre dans tout leur trajet. Les fibres yui ront vers l'encéphale subissent liz dégénérescence ascendante; celles qui se dirigent vers la périphérie subissent la dégénérescence descendınte. Ces dégénérescences systématiques sont réalisées par diverses maladies qui intéressent exclusivement un faisceau et laissent les autres indemnes.

On connaît ainsi les mýálites localisées à la substance grise des cornes anlérieures (poliomyélites), à la substance blanche (leucomyslites), aux grosses cellules des cornes antérieures (tremblante du mouton), au faisceau pyramidal ou cordon de Goll, etc. (nous y reviendrons plus loiu).

20 Méthode embryologique. - Flesclısig, en étudiant le développement les centres nerveux, a constalé que la myélinisalion des fibres nerveuses n'a pas lieu en mème temps dans loutes les parties du névraxe; elle se produil dans certaius faisceaux avant de commencer dans les autres; cetle méthode analytique a la mẻme signification que la đégénérescence vallérienne. 
$3^{\circ}$ Méthode expérimentale. - On détermine l'excitabilité des diverses régions de la moelle, puis on sectionne les divers cordons; on achève de préciser ainsi le ròle sensitif ou moteur dévolu à chacun d'eux.

La substance grise de la moelle est directement ine $x$ citable; mais elle pent ètre excitée par cerlains excitants naturels ou médicamenteux; les cordons postéricurs sout doués d'une sensibilitẻ extrêmement vive, comme en témoignent les cris et les mourements de défense qui suivent chaque excitation. Ils sont plus excitables à la superficie que dans la profondeur. Si l'excitation porte sur le condox dE GoLl, on a des résultats excito-sensitifs; si elie intéresse le voisinage des racines nerveuses, on obtient des phéuomènes exclusivement réfle.ts.

Les cordons INFÉRO-LATÉRAUX sont liés à la motricité, mais ils sont très médiocrement excitables.

Chauveau nie absolument leur excitabilité; Vulpian prétend qu'arec des excitations fortes, il a pu constater leur excilabilité.

La conductibilité cle la moelle a été établie par la section successive des divers cordons médullaires.

La sectıon dEs condons pastérıuns qui sonl si sensibles nabolit pas la sensibilité; elle s'exalte même daus tous les points du corps situés en arrière de la section. Quon détruise toutes les parlies de la moelle excepté les cordons postérieurs, on supprime la sensibilité. Ces résultats, paradoxaux en apparence, tiennent à ce qu'on a envisagé la sensibilité en bloc. Or, il existe divers modes de sensibilité consciente qui ont des foyers listincls dans l'écorce cérébrale.

On distingue la sensibilité tactile et le sens musculaire d'une part, la sensibilité ì la donleur et la sensibilité thermique d'autre part.

Ces sensibilités, qui ont des centres corlicaux distincts, sont recueillies à la périphérie par les nerfs sensilifs;

$$
\text { Cadéac. - Sémiologie, } 2^{\text {édit. }} \text { II. }-6
$$


pent-être par des fibres différentes, et se propagent dans la moelle par des voies spéciales.

Ces cordons postèrieurs constituent les voies de la sensibilité tactile et de la sensibilité musculaire, comme on témoigne l'expérience suivante :

Quand toute la moelle du chien est seclionnée hormis les cordons postérieurs, la sensibilité à la douleur n'existe plus; il ne crie pas si on lui pince fortement la patte; mais il possède encore la sensibilite taclile; il dresse les oreilles, soulève la tête et se relourme chaque fois qu'on agit sur sa peau à rebrousse poil ou qu'on produil des frôlements (Schiff).

Par contre, si l'on sectionne les cordons posterieurs, l'animal conserve la sensibilité aux impressions doulonreuses, mais il a perdu la sensibilité musculaire et la sensibilité tactile.

On peut en conclure que la substance grise est indispensable ì la transmission des impressions douloureuses el des impressions thermiques.

la pathologle réalise des destructions de faisceaux avec des dissociations de sensibilités équivalentes ou mème plus neltes que celles qui résultent de l'expérimentation.

Dans la syringomyélic qui signifie moelle creuse, affection marquée par la destruction de la substance grise dlans une rígion médullaire plus ou moins étendue, on constate la thermoanesthésie el l'analgésie ou insensibilité à la clouleur coüncidant avec la conservation de la sensibilité lactile.

On peut piquer, pincer, déchirer la peau; on peut également la brûler ou entourer les membres de glace sans provoquel la moindre réaction. Il est sourent difficile chez les animanx d'apprécier l'existence de la sensiliilité tactile qui est conduite par les cordons postėrieurs, yuand la sensibilité lluermique et la sensibilité a la douleur (conduites à travers la substance grise) ont complèle- 
ment disparu. On peut cependant recourir à divers attouchements et au chatouillement pour mettre la sensibilité tactile en évidence.

Dans l'utaxie locomotrice progressive de l'homme caraclérisée par une sclérose des cordons postérieurs, la sensibilité musculaire est abolie ou diminuée, les mouventents sont incoordonnés, ils dépassent le but ou ne l'atteignent pas comme chez les animaux auxquels on a sectionné ces cordons.

L'hémisection de la moelle du chat à droite, au niveau de la région dorsale, s’accuse par des troubles analogues.

Six mois après celte opération la sensibilité musculaire arait complètement disparu du còté de la section. En effet, si lon tire la patte postérieure gauche, celle-ci se dérobe par une rapide flexion, tandis que la droite ne produit aucune réaction. Si l'animal est couché sur le bor d'une lable, le membre gauche se fléchit brusquement et prend pied sur le bord de la table, tandis que le membre droit continue de pendre inerte (Herzen).

Les hémisections de la moelle pratiquées dans la région dorsale sont suivies de la disparition de la sensibilité du còté opposé; elle est non seulement conservée du côté correspondant, mais elle s'aiguise progressivement à tel point que peu de jours après lopération, les excitations les plus légères provoquent des accès épileptiformes.

Les roies de transmission de la seusibilité douloureuse sont cruisées; les voies de la sensibilité tactile et musculaire sont directes dans la moelle.

Les voies motrices subissent dans la moelle un entrecroisement partiel, comme en témoignent les hémisections pratiquées dans la région cervicale et l'excilation des cordons antérjeurs par des piquires pratiquées à l'aide d'une forte épingle; on constate que les mouvements les plus énergiques ont lieu du còté de l'excitation; mais les muscles du còté opposé se contractent aussi, quoique bien moins énergiquement. 
Injections anesthésiques. - Les injections intraarachnoüliennes et épidurales de solutions de cocuïne ont été utilisées pour insensibiliser des régions étendues du corps. Udier, puis Carini ont cocaünisé directement la moelle du clien; Tuffier a adopté la solution au cinquantième sans déterminer aucune altération histologique persistante.

Cuillé et Sendrail (1) ont appliqué celte méthode chez le rheval, le bauf et le chien. On se sert d'un trocart de $0^{m}, 10$ de longueur, $0^{m}, 00 t \tilde{y}$ de section chez les deux premières espèces et d'une aiguille de Pravaz de $0^{\text {m }}, 06$ de loug avec $0^{\mathrm{m}}, 001$ de diamètre chez le chien. On fait pénétrer l'instrument au point de croisement du milieu le la colonne vertébrale avec une ligne réunissant le sommet des angles ilianues internes.

Les doses convenables paraissent ître de 1 à 3 centimèlres cubes de la solution de cocaïne à 2 p. 100 suivant la taille pour le cheval et le bœuf. On ne doit pas dépasser un centimètre cube chez le cliien. Lanalgésie est parfaite pendant une heure environ, sans modification sensible de lit motilité, sauf dans le traiı postérieur oì elle est affiiblie.

Une injection faite chez le chien dans l'espace occipitoallö̈dien, au niveau du bulbe, a respecté la tète et a provoqué une démarche rampante sur le sternum.

Iu point de vue clinique, on a constaté à la suite de ces injections la disparition de la douleur pendant un laps de temps plus on moins considérable.

La moelle est un centre nerveux. - La moelle préside aux réflexes; cliaque muscle contient les deux exlrémilés de l'axe réflexe, c'est-à-dire les terminaisons des fibres motrices et des terminaisons sensitives disséminées qui sout. les agents ou les dépositaires périphé-

(1) Cuillé el Sendrail, Rerue vit. de Toulouse, 1901, p. \$?. 
riques du sens musculaire. Les réflexes tendineux et la tonicité sont les deux manifestations de la sensibilité musculaire.

Les RÉFLEXES TENDINEUx sont les mouvements produits dans un muscle par la percussion de son tendon : réflexe rotulien, etc.

La toxicité Musculalle est l'expression d'une action réflexe permanente qui maintient les muscles dans un état de tension active continue. En supprimant les nerfs, on supprime celte tonicité : la paralysie du facial est suivie de la dériation de la face vers le còté sain; les muscles inertes obéissent à la tonicité constante des muscles du cỏté opposé.

Celle tonicité nusculaire assure l'occlusion des sphineters (pylore, anus, col de la vessie); ils ne peuvent mème se relàcher que sous l'influence d'une intervention inhibitoire.

La moelle possède un grand nombre de centres des mouvements réflexes des muscles de la vie organique:

Le cextre excitateur de la fonction glycémique, silué près de l'extrémité supérieure de la moelle cervicale entre lebulbe rachidien et l'orignine de la quatrième paire spinale.

Le centra cilio-spinst qui produit la dilatation de l'iris; il siège dans la partie inférieure de la moelle cervicale et entre en activité sous l'influence des excitations des racines postérieures, des cordons postérieurs, etc.

Les CEXTREs accÉlérateurs du Ceur, situés entre les trois dernières paires cervicales et les cinq premières paires dorsales.

LES CEXTRES VASO-MOTEURS SUDORIPARES existent surtout dans l'axe gris.

Les CExtres de Flexion ET d’extexsion sont situés sur toute la longueur de la moelle.

Les CENTRES DES RÉFLEXEs RESPIRATOIRES sont reliés aux centres supérieurs, mais ils peuvent fonctionner d'une manière indépendante. 
10:) SÉmologie de L'APPAREIL DE L'INNERYATION.

Le centre ano-spinal, situé dans la moelle lombaire, agil sur le sphincter de l'anus et recoit les excitations des nerfs de la muqueuse rectale. La section bilatérale des nerfs sensilivo-moteur's de l'anus n'entraîne pas l'incontinence ni des fèces, ni de l'urine. l'élasticité des sphincters supplée à leur tonicité quaud celle-ci fait défaut.

Le centre vésico-sprnat, situé au-dessus du précédent. est formé de deux moitiés dont l'une agrit sur le sphincter à fibres lisses et ne recoil que des excitations centripètes, tandis que la seconde, placée sous la dépendance de la rolonté, agit sur le muscle de Wilson.

Le centre génito-spinal comprend: $1^{\circ}$ le centre des mouvements de l'utérus et du vagin qui est mis en activité par tontes les excitatious partant de l'utérus. Ainsi une chicnne paralysée par la section de la moelle dorsale peut entrer en chaleur, s'accoupler et mettre has uormalement.

2 Le centre de l'rection, excilé par les centres nerveux et par les nerfs sensitifs de la pean, du pénis et de la muqueuse du gland.

$3^{\circ}$ Le centre de l'ejuculation siegge au niveau de la quntriène vertèbre lombaire chez le lapin; il agit sur les canaux déférents et le muscle bulbo-caverneux.

Modifications pathologiques. - La moelle peut être le siège d'hémorragies, d'inflammations, de dégénérations secondaires.

\section{I. - IÉmorragres.}

Définition. - Les liémorragries médullaires consistent dans des extravasitions de sang au niveau du plexus brachial, du plexus lombaire ou dans le voisinage du bube.

Elles sont méningées hématorachis) ou médullaires (hemalomyilic). Elles s'effectuent à la périphérie de la moelle, à l'intérieur clu canal de l'épendyme ou dans l'épaisseur de la substance médullaire; elles sont solitaires 
ou multiples et procèdent habituellement dans ces cas d'une altération générale des raisseaux.

Signification. - Les hémorragies médullaires sont l'expression, cliez le cheval, des traumatismes, des fractures de la colonne vertébrale, de la goume qui produit fréquemment des hémorragies capillaires disséminées, de l'hemoglotinurie qui s'accuse quelquefois par des extrarasations sanguines siluées en arrière durenflement lombaire au niveau des cordons nerveux dont l'ensemble forme la queue de cheval, de la dourine dont les lésions hémorragiques sont si prononcées qu'on en a fait pendant longtemps une myélite hémorragique (Tanholler) et de la pachyméningite.

Les hémorragries sont symptomatiques, chezle chicn, de contusions, de tranmatismes, de grains de plomb el de la muladie du jeune áge qui revendique l’immense majorilé des hémortagies médullaires observées. Tantò la moelle est enrobée dans un caillot, tantòl la régrion des cornes antérieures est le siège de petiles hémorragies capillaires disséminées.

\section{II. - Myélites.}

Définition. - Caractères. - Les inflammalions de la moelle sont tantòt uiguës, tantôt chroniques, tantòt diffuses (myélites gourmeuses, myélites de la maladie du jeune àge), tantòt localisées à un court segment (myélites transverses du chien) marquées par un foyer de ramollissement.

On distingue encore des myélites intéressant les méninges el la superficie de la moelle (méningo-myélites, des myélites centrales syringomyélie). Elles peuvent se cantonner à la substance grise des cornes antérieures (poliomyélites) qu'on observe sonvent chez le chien à la suite de la maladie du jeune àge; à la substance blanche (leucomyélites des cordons latéraux, elc.) (Voy. Dégénérescences), aux racines antérieures et postérieures el aux 
grosses cellules des cornes antérieures comme la tremblante du mumton (Benoist el Morel).

Parfois l'inflammation est si limitée qu'elle ne produit qu'une monopligic; parfois elle est si profonde qu'elle entraine la paraplégie et qu'elle se complique de troubles cérébraus, bullaaires et protuliérantiels.

Dans les myilites aiguës, on constate un ramollissement plus ou moins prononcé du tissu médullaire accompagné ou non d'hémorragies; dans les myélites chroniques, on conslate fréufuemment une sclérose vasculaire on une sclérose systématique du cordon intéressé on des dégénérescences. Ilabituellement les lésions se concentrent sur les granules eellules des eornes anlérieures qui éprouvent une dégénérescence granuleuse, une altération du protoplasma et du noyan, un élal vacuolaire de la cellule el de ses prolongements. Il en est ainsi dans la tremblante du monton, la maladie dn jeune àge du chien, etc.

Pathogénie. - Les myélites se rangent en deux classes : les myélites par infection et les myélites par intoxication; mais les myclites infeetieuses sont en dernière analyse des inyélites toxigues.

Les agents susceptibles de déterminer des myélites sont très nombreux.

Le bacille fyocyanique, la toxine diphtéritique, le bacille de la tubereulose aviaire ou humaine, le staphylocoque doré, le colibacille, le bacille lyphique, le streptocoque, le bacille de la septicémie de la somris, le microbe de la maladie du jenne age, le microbe de la gourme, les intoxications d'origine alimentaire, comme cela est probable dans la tremblante du monton, sont autant de causes susceptibles de provoquer des myélites.

La plupart de ces myélites peuvent ètre obtenues, soit en inoculant dans les vaisseaux des cultures vivantes; c'està-dire les microbes eux-mèmes, soit en inoculant les produits solubles de ces microbes, c'est-à-dire leurs toxines. 
Quand l'expérience est faite avec les cultures vivantes, on peut trouver les microbes palhogènes au milieı des lésions médullaires; mais ils peuvent faire entièrement défaut.

La présence ou la colonisation sur place d'un microbe n'est donc pas une condition essentielle à la détermination des lésions; il est mème démontré que la plupart des microhes pathogènes agissent surtoul par leurs toxines : lésions médullaires provoquées par la toxine pyocyanique, la toxine diphtéritique, la toxine streptoeoccique.

Le microbe peut avoir disparu et sa toxine pathogène persister; le microbe pathogène peut avoir étẻ cultivé en un lieu fort éloigné de la moelle et sa toxine vient néanmoins adultérer l'axe bulbo-spinal (diphtérie, tétanos).

Daus la maladie des pulpes, la paraplégie succède à l'injection dans le sang du liquide filtré et stérilisé de pulpes de betteraves. La myélite de la maladie alu jeune age évolue souvent au moment de la disparition des troubles digestifs et des troubles respiratoires.

Les accidents paralytiques chez les animaux en expérience survienuent à des époques indéterminées; ils sont précoces ou tardifs; parfois la paralysie survient comme première nianifestation apparente de l'infection, l'animal ne paraissant nullement impressionné par les agents infectieux quon lui avait inoculés; de telle sorte que la myélite qui se montre plus tard a les apparences d'une maladie spontanée.

\section{III. - Dégénénations secondaires.}

Pathogénie. - Toutes les compressions ou seclions plus ou moins complètes de la moelle sont suivies de dégénérescences secondaires de cet organe.

Ces dégénérescences sucèdent, chez le cheral, à des compressions déterminées par des tumeurs (sarcomes melaniques, sarcomes encephalö̈des, sarcomes fasciculès, 
papillonies, cholestéatomes, abcès), à des lésions vertébrales (fractures, entorses, exostoses, périostoses), à des lésions des méninges (pachyméningite spinale chronique ou à larves d'vestres) (Dieckerhoff), à une sclérose du tissı conjonctif résultant d'une artérile spinale médiane d'où résulte la dégénérescence ou l'atrophie de la caudı equina.

Chez le bruf, les tubercules, chez le chicn, les plaques osseuses de la pachyméningite ossifianle sont des canses imporlantes de compression et de dégénérescence.

Caractères. - On appelle dégénérations ascendantes celles qui se produisent entre le point où porle la lésion primilive et le cerveau, dégénérations descendantes celles qui se développent entre le point où porte la lésion primitive et les régions inférieure ou postérieure de la moelle. La région de la moelle située en avant de l'altération porle le nom de supérieure; celle qui est située en arrière est appelée inférieure; la face rentrale de la moelle est appelée antirieure el la face dorsule est appelée postérienre.

Ces dégénérescences connues chez le chien, le sont très incomplètement chez les autres animaux qui périssent ou sont sacrifiés comme inutilisables avant que les lésions aient eu le temps de se développer.

I. Dégénérations descendantes. - Elles sont conséculives aux lésions cérébrales ou aux lésions médullaires.

A. Lésions cérébrales. - Ces dégénérations atteignent exclusivement le faisceau pyramidal. Il n'est pas inulile de rappeler que ce faisceau part des circonvolutions motrices, descend dans la cupsule interne dont il occupe les deux tiers antérieurs du segment postérieur, puis il gagne la partie moyenne de l'étage inférieur du pédoncule, la protubérance el enfin la pyramide du bulbe; il forme après 
décussation, le faisceau pyramidal croisé, une autre portion continue son trajet le long du sillon antérieur sous le nom de faiscenu pyramidal direct.

Les tument's cérébrales (les échinocoques du cerveau, Dollar) petıvent détruire ce faisceau à son origine d'un seul cùté de sorte qüil se produit généralement une himiplégie incomplète accompagnée de contracture, c'est une hémiplégie à tendance spasmadique avec incoordination motrice accompagnée généralement de la paraJysie du facial (1).

B. Lésions médullaires. - Les dégénérations descendantes de la moelle cousécutives aux lésions transversales

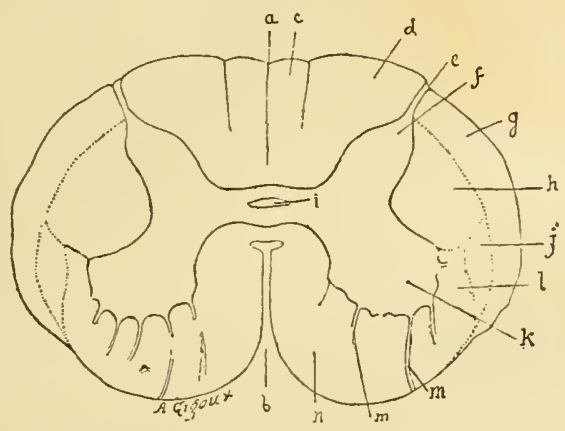

Fig. 20. - Coupe trausversale de la moelle épinière (schématique) (Dexler).

$a$, sillon longiludinal postérieur. - $b$, sillon longiludinal antérieur. $c$, faisceau de Goll. $-d$, faisceau de Burdaclı. - $e$, racine postérieurc. $f$. corve postérieure. - $g$, faisceau cérébelleux direct latéral. - $h$, fạisceau pyranidal. $-i$, canal central, $-j$, faisceau de Gowers. $-k$, corne antérienre. $-l$, faisceau latéral profond. - $m$, racine autérieure. $-n$, cordon antérieur.

de la moelle peuvent alteindre : $1^{\circ}$ le cordon antéro-latéral, 2 les cordons postérieurs.

(1) Voy. t. VIII de la Pathologie interne, $x \mathrm{p}$. 
$1^{\circ}$ Cordon antéro-latéral. - Composé du faiscean pyramidal, du faiscean intermédiaire du cordon latéral et du cordon antèrieur, le cordon antéro-latéral peut êlre altéré dans ses diver's éléments; sa destruction entraine une himiplegie spinale.

Les fibres du faisceau pyramidul dégénèrent moins facilement chez le clien que la portion médiane d ci cordon antérieur. Schultz a observé la dẻgénérescence du cordou

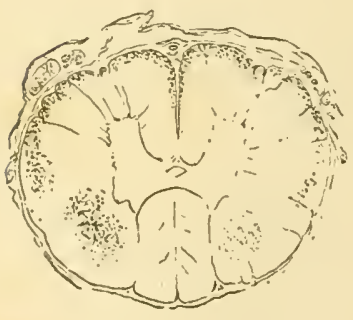

Fig. 21.-Chien. - Troisic̀ne dorsale; la dégénération desceudante porle sur le faisceau pyramidal croisé: sur les fibres de la région sulco-marginale et en outre surle faiscean intermidiaire (Dexler).

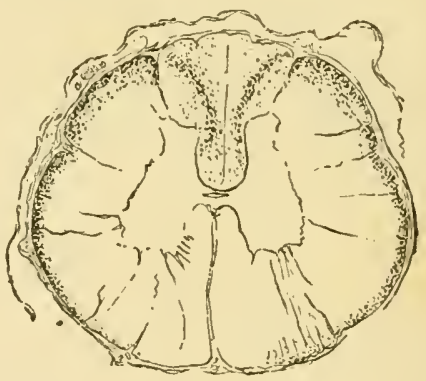

Fig. 22. - Chien. - Première cervicale. La presque lotalité du cordon postérieur est dégénérée. L'altération du faisceau cérébelleux direcl s'élend en avaul presque sur les bords du silion anléricur (Hexler!

pyramidul direct; Dexler, celle du faisceau pyramidal croisé et celle du faisceau intermédiaire du col'don latéral (Lïwenthal), sans que ces denx fọers de dégénéresccnce fussent réunis.

20 Cordon postérieur. - Cette dégénérescence a été étıdiéc pa. Schifferdeclier qui a constaté des dégénérescences traumatiques diffuses de la moelle d'un chien; les lésions intéressaient les cordons postérieurs et latéraux ainsi que les racines correspondantes qui étaient atrophiées. Celte dégénérescence a élé étudiée aussi par Dexler, Gombault et I'hilippe, Flechisig, Berkout. 
II. Dégénérations ascendantes. - Cordons postérieurs. - Les dégénéralions ascendantes intéressant les racines postérieures ou les cordons postérieurs peuvent Jans ce cas se limiter au faisceau de Burlach ou au faisceau de Goll ou affecter ces deux faisceaux à la fois. L'atrophie de ces faisceaux a été bien suivie par Dexler.

Toutes ces dégénérescences sont généralement de quelyues centimètres seulement.

Les altéralions de la’ sensibilité consécutives à ces

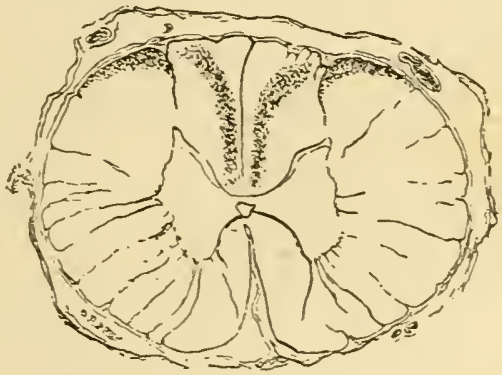

Fig. 23. - Chien. - Partie supéricure de la région cervieale. immédiatemenl au-dessous de l'enlrecroisement dans le cordon postérieur; la dégénérescence est presque enlièrement limitce au rạon situé entre ies faisceau de Goll et de Burdaclı; quant au faisceau cérébelleux direct, les parties antérieures périphériques du corton laléral devienneut de plus en plus libres (Dexler).

compressions médullaires sont très variées; on peut constater :

$1^{\circ}$ De la douleur due à la compression et à l'irritation des racines des uerfs rachidiens (pseudo-nérralgies); cette yyperalyésie est généralement bilatirale ttrigionule, elle intéresse la pean mais non les muscles.

20 Des zones danesthesie disposées en plaques dans le domaine des nerfs affeclés; mais ces plaques ne sout jamais bien limitées chez les animaux, mème quand les foyers de ramollissement médullaires sont bien délimités :

Cadéac. - Sémiologie, qe édit. 11. - i 
l'iusensibilité des testicules est très fréquente chez le chien.

Lasensibilité musculaire persiste souvent dans les parties péripliériques après rupture tolale ou partielle de la moelle du chien avec section complète des cordons postérieurs.

Les chiens opérés portent les pattes postérieures d'une manière beaucoup plus défectueuse que les pattes antérieures (Rosenbach et Schtscherback). Dexler a coustaté

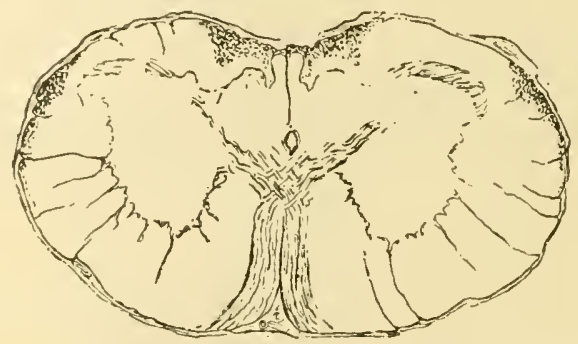

Fig. -t. - Chien. - P'artic inlérieure du quatrième ventricule; dans le bulhe, le faisceau dégenéré du corion postérieur s'approche du noyau de Burdach et de Goll; le faisceau cérébelleux dıreet en dígẻnération est plus court que dans la région cervicale (Les contours de loutes les figures sont

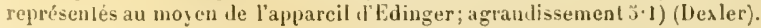

qu'un chien affecté d'une compression de la moclle dorsale tléchissail sur les membres en descendant, il marchait normalement en marchant.

Apres quelques semaines, on vit apparailre des oscillations du train postéricur; "11 autre affecté de lésions plus jononcées, appuyait avec lasurface palmaire; il éprouvait des flexions subites qui indiquaient, outre l'altération du seus musculaire, la perte de la conicité musculaire.

Un conslate en mème temps une exargération des réflexes.

Cordon antéro-latéral. - Les fibres atteintes par la dégénération ont leur principale origine daus la moelle. l)exter a constaté nettement les dégénérescences du 
BULBE.

fuisceau cérebelleux et l'altération de diverses parties du cordon latéral ainsi que du faisceau de Gowers.

L'étude des fonctions du cervelet nous renseignera sur l'importance et la signification de la dégénération du faisceau cérébellcux.

\section{III. - BULBE.}

Le bulbe est un lieu de passage pour les excitations centrifuges el centripètes; il est en mème temps un centre important d'actes réflexes ou automatiques.

Conductibilité. - Les hémisections du bulbe pratiquées au-dessus de la décussation entraîuent une hémianesthésie et uue hémipléyie croisées.

Chez le chien mène, la pyramide fournit un faisceau uou seulement pour le cordon latéral du còté opposé, mais aussi pour celui du côté correspoudant, de telle sorte que chez cet animal, une lésion corticale unilalérale culraine souvent ou mène habituellement une dégénéralion bilatérale des cordons laléraux.

Chez le thien, chaque lıémisphère est donc habituellement en rapport avec les deux moiliés de la moelle. Il en est de mème chez le chat et chez le singe.

Horsley et Schaffer ont monıré que clıaque hémisplı̀̀re est en comnexion avec les muscles du tronc de l'un et de l'autre còté, de sorte que l'ablation de l'uu des centres corticaux ne produit pas sur ces muscles l'ellet notable el qu'une destruclion bilutérale est nécessaire pour les paralyser.

Lentrecroisement des impressions sensitives au niveau du bulbe est également inconılete; tous les conducteurs de la sensibilite des membres ne franchissent pas la ligne médiane ponr converger vers la moitié opposée du bulbe; la diminution de la sensibilité peut être peu marquée; il faut donc admettre qu'il y a dans la moelle allongée des conducteurs sensitifs non entrecroises. 
Une des conséquences de l'ućmsectiox, c’est une hyperesthésie du cólé de la lésion; on l'attribue à une irritation des éléments sensitifs en arrière de la section.

Quand la section du Lulbe est faite au niveau du collet; c'est-à-dire daus l'étroite zone où s'opère la décussation, les effets paralytiques sont bilatéraux.

\section{Connexions du bulbe et de la moelle avec le cervelet.-} Le bulbe el la moelle ont des connexions importantes avec le cervelet. Cet organe est mis en rapport avec la périphérie par des conducteurs centripétes el des conlucteurs centrifuges.

Les conducteurs centripètes passent presque entièrement par le corps restiforme ou pédoncule cérebclleux inférieur. Ces roies comprenuent :

1 "Le raisceau cénébelleux pinect de la moelle ou fuisceau de Flechsig qui nail des colonnes de Clarke de la moelle, clsemine à la face externe du pédoncule cérébelleux inférieur et aboutit à la partiedorsale du vermis supérieur.

2o Le faIsceau de Burdach, parti des noyaux bulbaires des cordons postérieurs, forme les fibres arciformes externes el postérieures du bulbe et se reud daus le pédoncule du nnême côté.

30 Le rascrau de Golf part aussi d'un noyau du bulbe: c'est le noyau de Goll qui donne naissance à deux sortes de fibres: les unes directes faisant partie des fibres arciformes externes el postérieures, les autres croisées; cellesci, après décussation, sortent par le sillon médian antérieur, coutournent horizontalement la pyramide antérieure pour se reudre aux fibres du pédoncule cérébelleux opposé.

Du bulbe envisagé comme centre. - Le bulbe, qui est le centre d'origine d'un grand nombre de nerls craniens moleurs et sensilif's, lient ainsi sous sa dépendance un grand nombre d'actes tels que la succion, la mastication, la déglutilion, la respiration, etc. (fig. 23̈). 
$1^{\circ}$ Centre respiratoire. - De nombreux expérimentateurs ont constaté que la destruction du nœud vital ne supprime pas les mouvements de la respiration dans ie thorax. C'est qüil existe une série de noyaux gris dans les cornes antérieures de la moelle et dans la substance grise molıice du bulbe échelonnés sur des niveaux

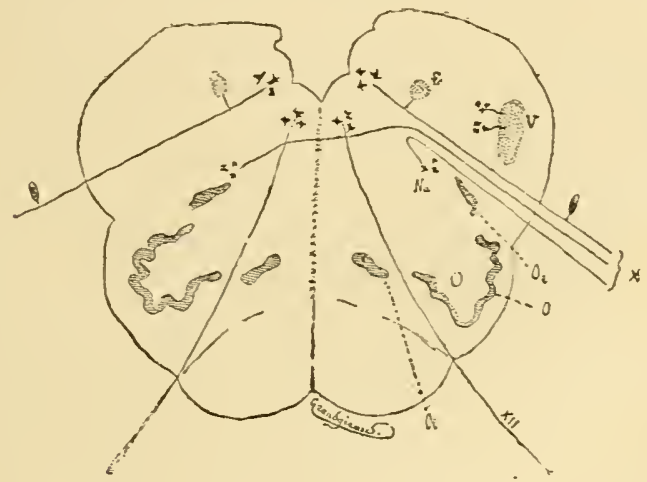

Fig. 25. - Coupe du bulbe ver- la partie postérieure du calamus.

I, pneumogastrique. - Xll, hypoglosse. - Xa, uogau accessoire moleur de la dixième pairc. - Fr, faisceau respiratoire (raciuc ascendante de $\mathbf{X}$ el $\mathbf{X}$. - $\mathrm{V}$, racine descendante du Irijnmeau. - 0 , olive. - Oi, olive accessoire inlerne. - Oc, olive accessoire externe.

différents et qui forment les centres segmentaires des muscles respiratoires de telle sorte que si lon sectionue le bulbe en arant du nœud vital, on n'aholit que les mouvements respiratoires de la face.

En dehors de ces centres secondaires, il existe un centre respiratoire proprement dit qui réside dans le bulbe el qui assure la synergie régulière des différents groupes musculaires. Ce centre, qui peut ètre subdivisé en deux autres, l'un expirateur, l'autre inspirateır, reçoit des incitations réflexes ou volontaires qu'il transmet, en les réglant, aux muscles de la respiration. 
Les gaz du sang (oxygène et acide earbonique) sont les principaux excitants des centres respiratoires.

$2^{\circ}$ Centre de la déglutition. - La déglutition s'effeclue encore après l'extirpation du cerveau et après la section du bulbe sur un plan prenant immédiatement en arrière de la protubérance; c'est que les nerfs glosso-pharyngien, pneumogustrique et spinal qui prennent naissance dans le bulbe jouent un ròle important daus la déglutition. Mais c'est l'excitation initiale qui prend naissance dans l'isthme du gosier qui est le point de dipart de lous les mouvements réflexes qui se succèdent daus la déglutition, les mouvements péristaltiques de l'usoplıge sont entièrement subordonnés à cette excitation.

$3^{\circ}$ Centre de la mastication et de la succion. - Ces deux actes réllexes sont wouvernés principalenent par liı branche motrice du trijumeau, le facial et les cervicaux pour la voie centrifuge et exclusivement par le trijumeau pour la voie centripète.

40 Centre du clignement. - Ce réflexe a le trijumeau pour voie centripèle et le facial pour voie centriluge.

$5^{\circ}$ Centre de la phonation. -- La moelle allongée contient les noyaux d'origrine de tous les nerfs qui agissent sur les organes périphériques le la voix $\left(10^{\circ}, 11^{\mathrm{e}}\right.$ et $12^{\mathrm{e}}$ paires) Elle renferme anssi le centre de la proluction des sons articulés. En effel, si l'on sectionne le bube en arriere de la protubérance sur un animal, celui-ci pousse un cri bref et sans expression.

l.e hulbe conlient encore le centre romitif, le centre tussiparc, le centre de l'étermuement, le centre irido-pupillaire, le centre glycogénique, placé à égale distance du nerf acoustique et du pneumogastrique, le centre pour les mouvements de luterulite des yeux, les centres earliaques et 
vasculaires (centre modératenr du cour, centre accélérateur du cour); les centres vaso-moteurs (centre rasoconstricteur, centre vaso-dilatateur), les centres sécréteur's des sécrétions salivaire, gastrique, pancréatique.

Modifications pathologiques. - Le bulbe peut ètre le siège :

10 D'hémorragles produites par des chocs, des heurts, des contusioxs, etc., et suivies de mort immédiate ou de phénomènes paralytiques très complexes accompagnés crénéralement le troubles respiratoires et circulatoires.

20 D'ALTÉRATION DES NOYAUX yOTEURs caractérisée par la paralysie des muscles des lèvres, de la langue, du roile du palais, du plarynx, du larynx et connue sous le nom de paralysie labio-glos:o-larynyée (1)

$3^{\circ}$ D'a Bcès consécutifs à la gourme, à la suppuration de l'oreille interne, à des Iraumatismes.

$4^{\circ}$ De tumeurs diverses (sarcomes) et de parasites (canures, œstres, sclérostomes, etc.).

\section{IV. - PROTUBÉrince ANNULAIRE.}

La protubérance annulaire ou pont de Varole se dessine en saillie Iransversale sur la face inférieure de l'isthme entre le bulbe rachidien el les pédoncules cérébraux.

Elle est un conducteur et un centre.

Conductibilité. - La protubérance est un lieu de passage pour les fibres nerveuses réunissant les noyaux bulbaires et médullaires aux centres supérieurs. Les cordons se rendant aux centres bulbaires s'y entrecroisent, tandis que les cordons moteurs des membres s'entrecroisent plus en arrière.

(1) Voy. Pathologie interne, t. VIII, p. 933. 
116 SÉmlOLOgIE DE L'AIPAREIL DE L'INNERVATION.

Il s'ensuit que toute lésion protubérantielle unilatérale entraine une paralysie du mème còté dans le territoire innervé par des nerfs craniens el une paralysie du cûté

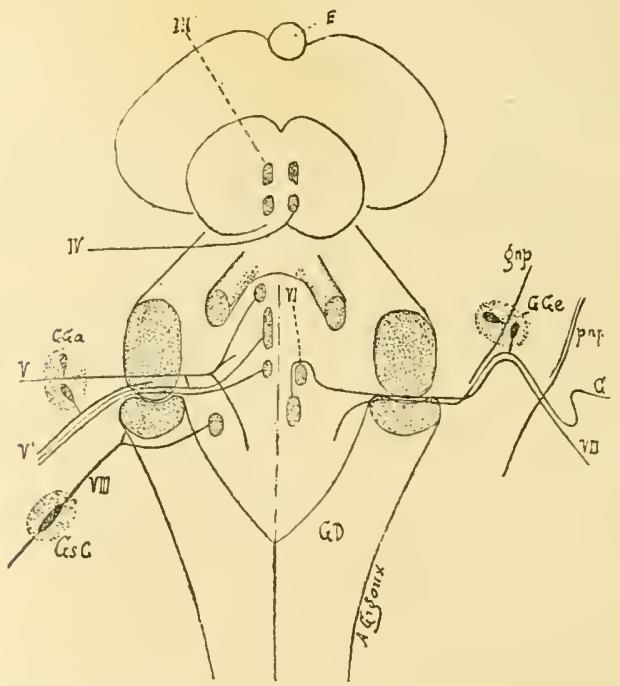

Fig. 20. - Origines réelles des nerfs eraniens de III à Vill.

IV, V, Y', I'l, VIl, WIIl, paires nerveuses cranienues. - qmp, grand nerl pétreux; - pnp, petit nerf péfreux. - $\mathrm{C}$, corle du tympan. - [II. oculomoleur emmmun. - IV, nerf pathétique. - V, racine seusitive du trijumeau. - I', racine motrice du trijumeau (uerf maxillaire inférieur). - VI, oculomoleur externe. - Vll, nert facial. - VIll, acoustique. - GGa, ganglion de Gasser. - GGe, ganglion génieulé. - GsC, ganglion spiral de Corti.

opposé dans les régions innervées par les nerfs rachidiens. Tel est le syndrome de Millard-Gubler ou himiplégie alterne.

Excitabilité. - Les piquires pratiquées sur la face antéricure atteignent le plus souvent le faisceau moteur et 
provoquent des mouvements convulsifs très violents. Sur les parties latérales, elles atteignent la racine ascendante du trijumeau et déterminent une douleur intense ujue l’animal exprime par des cris perçants.

Sur la face posterieure ou bulbaire de la protubérance l'aiguille peut atteindre le coude du facial ou le noyau de la $6^{\mathrm{e}}$ paire (oculo-moteur externe), d'où résulte le strabisme unilatéral externe.

Un lien existe entre les noyaux de la $6^{\mathrm{e}}$ paire et de la $3^{e}$ paire des nerfs craniens; il est formé par des fibres anastomotiques situées entre les deux noyaux et fonctionnant à la manière de la double guide dans un attelage à denx chevaux: l'excitation du noyau de l'oculo-moteu' externe ou sa destruction produisent la déviation conjuguée des deux yeux.

Centres nerveux. - Envisagée comme centre, la protubérance donne naissance à des nerfs craniens; clle possède le centre réllexe de la mimique et de l'expression confondu avec le noyau du facial, le centre de la mastication et de la succion situé dans le noyau du trijumeau, le centre de nouvement des yeux (Laborde et Mathias Duval), le centre d'équilibration, le centre épileptique, le centre sensorio-moteur ou coordinaieur des mouvements émotiounels.

Modifications pathologirfues. - La protubérance peut être le siège d'hémorragries, de ramollissement et de tumeurs.

Les HÉMORRAGIES avec ou sans RAMOLLISSEMENT procẻdent d'altérations athéromateuses des vaisseaux, d'hémophilie, de parasites et s'accompagnent d'hémiplégie alterne (paraIysie faciale d'uı còté, avec paralysie des membres dı còté opposé).

Les tureurs sont des mélanomes, des sarcomes, des fitromes, des endothcliomes; elles sont accompagnées de 
mouvement de rotation, de défaut d'íquilibre, d'hémiatrophie et d'hemianesthésie de la face.

\section{PEDONCULES CÉREBRALX.}

Les pédoncules cérébraux constituent la grande voie de communication motrice etsensitive entre la moelle épinière et les parties plus élevées de l'encéphale qu’ils semblent supporter.

Excitabilité. - On admet quils sont excilables dans presque toutes leurs parties à condition d'atteindre le ruban de Reil (fuisceau sensitif) ou le faisceau moteur de l'étagne inférieur. Dans le premier cas, on obtient les manifestations de la sensibilité la plus vire; dans le second la piqûre détermine des mouvements dans les muscles du côté opposé et des mouvements en manége. On 11 Y trouve pas de centres spéciaux.

Conductibilité. - Les pédoncules cérébraux sont conducteurs. Les hémisections du pédoncule cérébral produisent une lıẻmianesthésie el une hémiplégie croisées. On constale souvent une hémiplégrie alterne supérieure ou le symbrome de Weber.

i.e SYXDROME DE WEBER consiste dans la combinaison d'une paralysie du moteur oculaire commun du còté de la lésion avec une hémiplégie croisée, tolale ou non, motrice el quelquefois sensitive.

Modifications pathologiques. - Les pédoncules cérébraux peurent ère le siḱge dhemorrugies d'origine infectieuse (gourme, mennonie, maladie dujeune âge), de ramollissement résultant de thromboses, d'enbolies ou d'lıémorragries, de tumeur's diverses. 


\section{VI. - TLBERCLLES QLADRIJLMEALX.}

Les tubercules quadrijumeaux sont en relation arec les bandeleltes optiques, par l'intermédiaire du corps senouillé externe, avec le ruban de Reil, arec les nerfs auditifs et les nerfs optiques: l'énucléation de l'œil, l'atrophie des yeux, des cheraux atteints de fluxion périodique. sont suivies infailliblement de l'atrophie des couches opti,jues et du corps genouillé externe.

Chez les mammiferes, l'entrecroisement des nerfs optiques est partiel; les fibres du tiers externe de la rétine sont directes, celles des deux tiers internes sont croisées: la destruction des tubercules quadrijumeaux antérieurs entraine une cécité partielle dans les deus yeux, l'hémiopie ou l'hémianopsie latérale homonyme.

Les tubercules quadrijumeaux reçoivent les impressions visuelles el tiennent sous leur dépendance les mourements réflexes altachés à ces impressions. Ces mourements intéressent l’iris, les yeux et la tète.

Le RÉFl.EXE RÉTINO-PCPILLAIRE provoque le resserrement de la pupille el le myosis lans lesdeux yeux; la destruction des tubercules quadrijumeaux est suivie de la dilatation permanente de la pupille.

Les voies centrifuges de ce réflexe sout conslituées par les filores iridiennes du neif oculo-moteur commun dont le noyau est relié aux tubercules quadrijumeaux par des fibres commissurales.

Les mourements des yeux, élévation, abaissement, dévialion, convergrence, sont des mouvements réflexes sous la dépendance des lubercules quadrijumeaux.

Couches optiques. - On considère les couches optiques comme le foyer où arrivent toutes les impressions: Meynert leur accorde certaines propriélés motrices.

Ce sont des centres traversés par loutes les fibres sensitives qui, de la moelle, se rendent à l'écorce céré- 


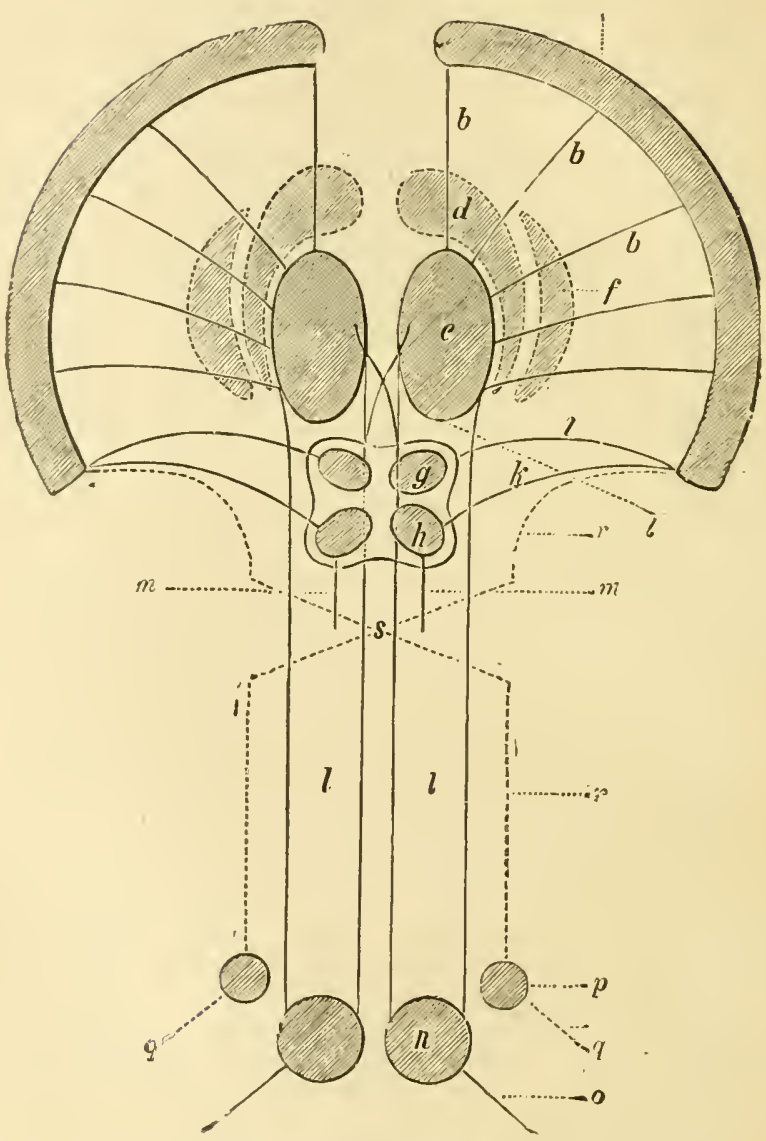

Fig. 27. - Scliéma des rapports et connevions des couches optiques et des tubercules quadrijumeaux.

$a$, écorce grise des hémişulı̀res. - $b$, couronue rayonnante de la coucle optique. $-c$, couche oplique. $-d$, corps strié. $-f$, noyau lenticulaire. - g, tubercule quadrijumeaı antérieur. - $h$, tubercule quadrijumeau postérieur. - i, bras du tubercule quadrijumeau antérieur ou couronne rajon- 
brale; c'est la dernière étape qu'elles franchissent. Les couches optiques, les corps opto-striés sont des relais moteurs pouvant jusqu'à un certain point suppléer chez les animaux l'écorce cérébrale.

Toutes les impressions qui n'arrivent pas aux couches optiques ne peuvent provouger que des réflexes.

Les exlirpations partielles qu'on peut réaliser sur ces foyers gris sont suivies de troubles visuels résultant de linterruption de la continuité des fibres optiques et d'hemianesthesie croisé, car la plupart des fibres sensilives de la capsule interne s'interrompent dans la couche optique.

\section{VYI. - CORPS STRIÉS.}

Corps striés. -- Ce sont deux masses grises interposées sur le trajet des pédoncules céréliraux.

Les fibres médullaires les traversent pourgagner l'écorce grise cérébrale, et les divisent en deux portions : l'externe appelée noyau extra-ventriculaire ou lenticulaire, l'interue appelée noyau intra-ventriculaire ou cunde.

On n'étallit pas de distinction physiologique entre ces deux parties. L.eur destruction produit l'hémiplégie croisée et curable par suppléance de la moitié opposée lu corps, comme celle des couches optiques produit l'hémianesthèsie de la région opposée au còté de la lésion.

Tolhnagel et Beaunis ont affirmé l'existence dans ces corps d'un centre dont la piqùre pousse l'animal à courir et qu'ils ont appelé nodus cursorius.

nante de ce tubercu'e. - $k$, bras du tubercule quadrijumeau postérieur. $l$, système de projection du second ordre. Fibres appartenant à la couclue optique (région de la calotte, ou élage supérieur du pédoneule cérébral). $m$, sysleme de projection du second ordre, provenaul des tubercules quadrijumeaux el se réunissant aux faiscesux de la calotte. - $n$, subslance grise le la nınelle (cornes antéricures). - o, nerfs moteurs périphériques (racines sfinales antérieures). $-p$, substance grise de la moelle (cornes postérieures). $-q$, nerfs sensitifs périphériques (racines spinales yoslérjeures). - $r$, laisceaux sensitifs allant directement (sans interruption) jusqu'à la substance grise corticale des hémisphères (Huguenin). 


\section{SÉMIOLOGIE DE L'APPAREIL DE L'INNERYATION.}

Carville et Duret en détruisant le noynu caurle chez le clicn ont observé des mouvements en manège accompa-

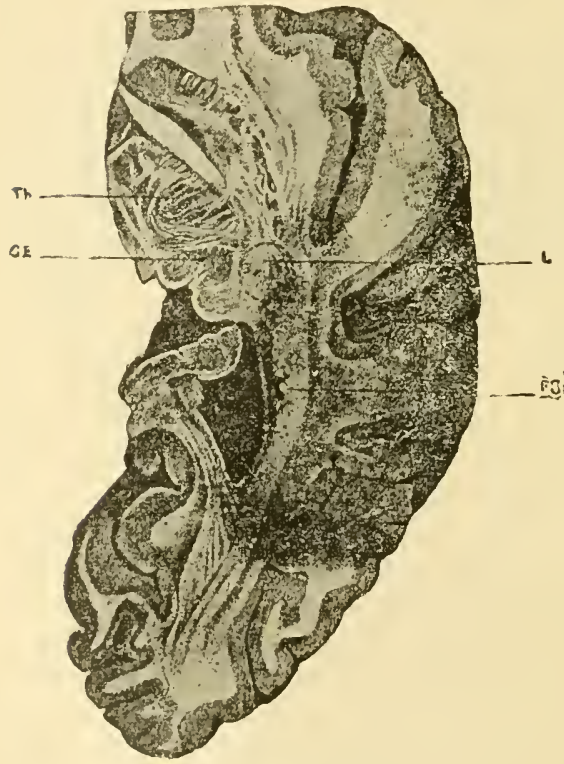

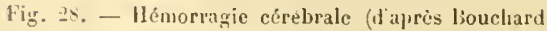
et (harcol).

Th, thalamus. - CE, corps grennuille externe. FS, faisceau sensitif. -- 1, lésion de l'hémianesthésic dans le carrefour sensilif. dite lemliculo-strièe entraine par sa destruction une himiplegie croisce et incurable accompagnée de contracture; elle conprend donc des fibres motrices en grante partie.

Ces deux parties se réunissent sur un angle conslituant le genou de la capsule; la loranche anterieure ou lenticulostriee est motrice, Ja branclıe postérieure ou lenticulooptique est sensitive: Ie faisceau sensitif de la capsule 
interne occupe la partie externe de la branche postérieure de cetle lame nerveuse; c'est le carrefour sensilif.

Les rccherches de Charcot ont démontré que les foyers capsulaires occupent la partie la plus postérieure du segment postérieur de la capsule interne, c'est-à-dire le carrefour sensitif. L’hémianesthésie sensorielle indique presque toujours une lision cupsulaire et non un foyer cortical.

L'hémianesthèsie d'origine corlicale se borne ordinairement à la sensibilité générale et respecte la sensibilité sensorielle.

\section{VIII. - CERVELET.}

Rapports. - C'est un organe très important supporté par l'isthme et séparé du cerveau par la tente du cervelet.

Le cervelet est en relation avec les diverses parties du système nerveux par une série d'arcs cérébelleux placés en dérivation sur les ares réflexes.

On y rencontre : $1^{\circ}$ Les neurones centrifuges dont le corps cellulaire est formé pir les grandes cellules de Purkinje et dont laxone va se terminer sur les grandes cellules motrices et la moelle; $2^{\circ}$ des neurones centripètes en relation avec la moelle, les noyaux de Goll, de Burluch, la protubérunce anmulaire, la couche optique.

Les relations du cervelet sont représentées daus la figure ci-dessous.

Une grande incertilude règne encore sur les fonctions du cervelet. Sans entrer dans le délail des nombreuses théories émises à ce sujet par Flourens, Longel, Vulpian, Ferrier, Luciani et autres, nous dirons que les auteurs accorlent an cervelet une action sur le système musculaire et qu'en somme les opinions ne diffèrent que sur la nature de l'action.

Si, pour les uns, il règle la tonicité musculaire, il va jusqu'à coorclonner les mouvements pour les autres.

On comprend dès lors que les lésions cérébelleuses se 
traduisent surtout par une anomalie dans la marche et l'attilude générale.

Le cervelet est un centre. - Le cerveletest donc surtout un centre qui agit sur le système musculaire; ce sont les

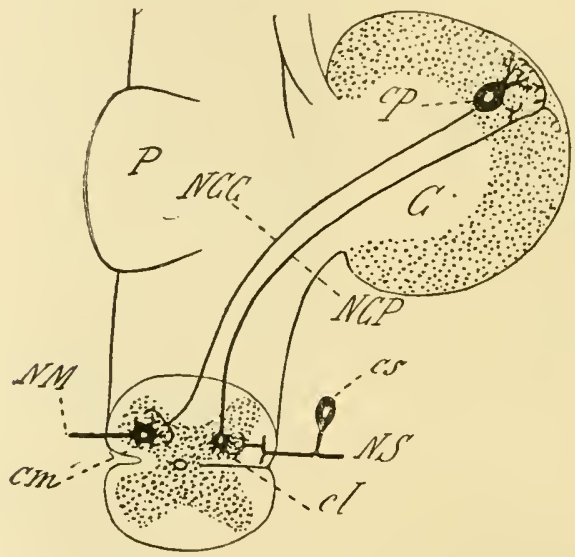

Fig. -9. - Schéma d'un arc cérébellenx (d'après Mallias l)usal).

P, protubėance. - $\mathrm{C}$, cervelel. - NS, nerf sensilif, - cs, corps du neuroue sensilif périphérique. - NM, netf moleur. $-\mathrm{cm}$, corps du uenrone motenr périphérique. $-c l$, NCP, neurone cérébellenx périphérique (dont le corps $c l$ siège diıns la colonne de Clarke de la moelle). - cp, NC.C, neurone cérébelleux central (dont le corps $c p$ n'est antre quine cellule de f'urkinje de l'écorce du cervelet).

troubles de ce dernier appareil qui révèlent les lésions du cervelel.

Quelquelois, aucun pliénomène anormal ne se fait remarquer pendant la vie, et à l'ouverture de la boite cranienne, on trouve une altération plus on moins prononcée du cervelet. Nothnagel a fait observer que, dans ces cas, l'altération atteint presque tonjours un liémisphère cérébelleux complet, le second hémisphère commandant alors 
seul à tout l'organisme. Uais il n'en est plus de mème quand le vermis est touché.

Alors a pparail un phénomène capital, essentiel : l'ataxie cérébelleuse, l’incoordination cérébellense, la titubation, et c'est la lésion du vermis, seule, qui peut produire cette titubation.

Mais si la lẻsion ne s'attaque qu'à mne partie circonscrite de l'orqáne, alors apparaissent des signes d'asthénie, d'affaiblissement de la force musculaire, et consécutivement des mouvements incoordonnés spéciaux.

L'un des plus caractéristiques, chez le chien surtout, est le roulement de l’animal, qui, placé sur le sol, effectue une série de tours sur son axe longitudinal, autrement dit, "il roule ". On verra plus loin comment se fait ce roulement. Aussi nous pourons dire avec Poincaré que le cervelet esı une pièce imporlante du sys ṡème locomoteur encéphalique, représenté par les corps oplo-striés, la protuberance, le bulbe et lui. C'est le centre del'équilibre.

Les recherches de Thomas ont mis ce firit en ividence d'une manière indiscutable.

Après l'ablatiox totale ou partielle du cervelet chez le chien, l'animal est incapable de garder l'équilibre et il reste couché.

Si l'ablation est complète, elle proroque de l'opisthotonos arec extension des membres, si elle est incomplete et unilutérale, le corps de lopéré est déformé par du yleurothotonos à concavité tourıée vers la lésion et il est parfois entrainé dans un mourement le rotation qui s'effectue autour de l'axe longitudinal et du cóté de la lésion.

Au bout de quatre à cinq jours, la contracture diminue et l'opéré est capable d'effectuer quelques mourements de locomotion. Il se lère d'abord et ne se tient debout qu'à la condition de placer ses membres dans l'abduction et d'élargir ainsi sa base de sustentation.

Si on l'y sollicite, il consent et parrient à se déplacer; 
mais sa démarche est incertaine, litubante comme celle de l’ivresse et arrêtée par des chutes fréquentes; la tèle de l'animal est agitée par des oscillations et des tremblements que tout mouvement exagère; il n'aboie pas, il ne peut prendresa nourriture; il marche péniblement et se fatigue beaucoup, car il est obligé de faire de grands efforts volontaires pour corriger le désordre de l'automatisme.

L'innimal s'habitue progressivement à marcher; mais tous les mouvements qui ne lui sont pas familiers, comme l'ascension on la descente d'un escalier, font reparaitre les mêmes troubles de la locomolion; il culbute et dégringole; il est obliqé d'apprendre tous les mouvements pour substituer son activité rolontaire à l'automatisme cérélielleux.

A alıcun moment, on ne constale, ni trouble de la sensibilité, ni amoindrissement de la force musculaire.

Excitabilitè. - L'excitation électriçue du cervelet provoque des monvements forcés des yeux, de la tète et du corps. L'excitation des lobes latéranx est suivie de la déviation des yeux, celle de la partie antérieure du vermis fait baisser la tète; celle de la partie postérieure détermine son élération.

Selon Thomas, le cervelel. est le centre réflexe des mouvements correcteurs qui maintiennent l'équilibre.

Modifications pathologiques. - Le cervelet est fréquemment le sipge d'hémorrayies observées clıez le chien, á la suite le la maladie du jeune àgre; chez le cheral, à la suite de tumeurs; elles s'accompagnent des troubles caractéristiques de l'atarie cérébelleuse. On y constate aussi quelquefois des abcés.

\section{X. - CEIRVEIL.}

Fonctions. - Le cerreau est le siège des phénomènes psychiques, sensation, pensée, volition); il reçoit toutes 
les impressions conscientes el distribue fous les ordres. Cet organe se compose de substance blanche et de substance grise, mais, à l'inverse de ce qui existe dans la moelle, la substance blanche on centre ovale se trouve au centre, la grise à la surface, formant l'écorce grise cérébrale à laquelle se rendent toutes les fibres de la capsule rayonnante.

Circonvolutions. - La surface du cerveau offre un plissement qui augmente la surface saus augmenter le volume.

Les parties saillantes contournées ou droites portent le nom de circomolutions; le pli est une partie de circonrolution; on distingne des plis de subdirision qui accusent l. dédoublement d'une circonvolution, des plis d'amastomose. de passage ou de communication unissaut des circouvolutions. Celles-ci sont siparées par des scissures profondes. dessillons, des incisuresou des nervures, c'est-ì-dire par des divisions le plus en plus superficielles. On appelle manteau de l'himisphere, l'ensemble les circonvolutions; il reconvre tout l'hémisphère excepté le hile; c'est-à-dire l'espace, qui reçoit l'extrémuté dı pédoncule cérébral. Le borl de cette entrée ou limbe est entouré d'une circonvoIution, euserrant le corps calleux, l'extrémité du pédoncule cérébral et le trigone appelée grand lobe limbique.

Localisations cérébrales. - L'étude anatomique des circonvolutions cérébrales qui vient d’ètre faite par Lesbre (1) fait connaitre la topogra phie du cerveau de chaque espèce animale, mais nous laisse dans lignorance des fouclions dévolıes aux diverses circonvolutions reconnues et décrites dans le manteau de l’hémisphère. D’ailleurs la topographie des centres n'est pas liée à la morphologie des circonvolutions.

Cequi importe au clinicien, au pathologiste par-dessus

(1) Lesbre, Étude des circonvolulions cérébrales dans la série des mammifères domestiques (Sociëte des scienccs vétèr., 190 i, p. 72). 
tout, c'est deconnaitre larépartition des centres elleur siège dans l'écorce cérébrale. Les recherches de Fritz et Hitzig ont mis en évidence dès 1870 la sensibilité de certains points de l'écorce cérébrale. Depuis, les travaux de Ferrier, Horsley, Munck, Charcot et Pitres, Franck, Arloing ont établi l'existence à la surface du cerveau de deux calégories de centres nerveux : $1^{\circ}$ les centres psycho-moteurs qui assurentl'exécution des mouvements volontaires; 2 - les centres pyscho-sensitifs qui président aux sensations correspondant aux divers sens et qui provoquent les mouvements atlachés à l'exercice de ces organes.

10 Centres psycho-moteurs. - La topographic de ces centres est constante chez les animaux de mème espèce, mais elle varie d'une espèce à l'autre de telle sorte que deux circonvolutions homologues répoudent à des centres différents.

La détermination de ces centres a élé faite par la méthode des excitations et des ablations de manière à préciser le rôle de chaque centre et à suivie les effets de leur suppression.

Chez les solipèdes, la distribution des centres moteurs a fait l'objet d'un travail de M. Arloing qui a remarqué que chaque hémisphère a une fonction bilatérale, de sorte qu’un liémisphère pent être détruit sans que la santé soit troublée.

En expérimenlant sur l'ame (lig. i6 et 47 ), il a trouvé une zone (1) dont l'excitation oblige l'animal à ramener ses nembres sous le trouc; ce mouvement est plus prononcé du cóté opposé à l'excitation. Si l'on excile le foyer (2), on obtient le rapprochement des mâchoires, accompagué d'un mouvement de diduction de la màchoire inférieure.

L'excitation du foyer (3) proluit des mouvements des naseaux el de la lèvre supérieure.

Quand on excite le point (4), on provoque les mouve- 
ments de la langue et des joues; au niveau du point ( 30 on provoque l'écarlement des màcloires avec la flexion el l'iıclinaison du cou.

L'excitation portée au niveau du point (6) est suivie d'un mouvement de clignement de l'cil du còté opposé à lexcitation.

Si l'on porte l'excilant au foyer ( $\boldsymbol{\imath})$, on obtient l'acclusion de la fente palpébrale du côté opposé avec des cou-

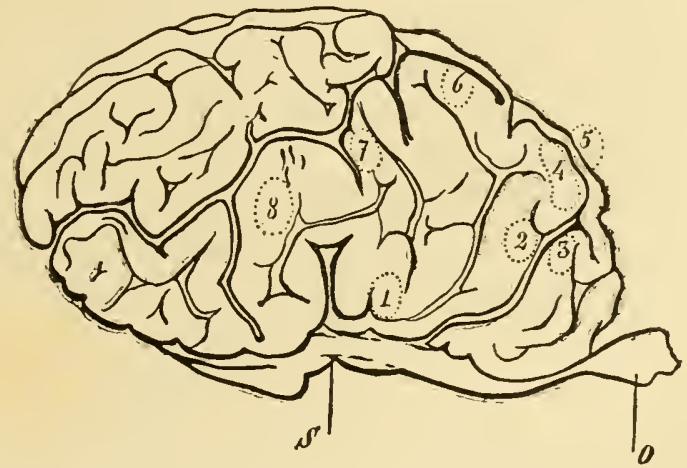

Fig. 30. - Face latérale de l'hémisphère cérébral de l'ìne (d’après Arloing).

0 , lobule olfaclif; $\mathrm{S}$, vailée de Sylvius; $1,2,3,4,5,6,7,8$, voir dans le texte.

rants faibles; si on emploie des ccuranls forts, le mouvement se propage à l'autre œil.

Eı excitant le point (8), on obtient l'élévation de la paupière supérieure et l'adduction de l'oreille du còté opposé avec des courants moyens; si on se sert de courants forts, on a le mème mouvement, mais moins prononcé du còté excité.

l.e gyrus sigmoïde est demeuré inexcitable.

Chez le chien, les centres psycho-moteurs ont été établis par les travaux des premiers expérimentateurs, Fritz, Hitzig, Ferrier et Munck. 
Ces centres, locilisés par Hitzig et Ferrier (fig. i), sont: le centre des muscles de la nuque $(a)$, le centre des

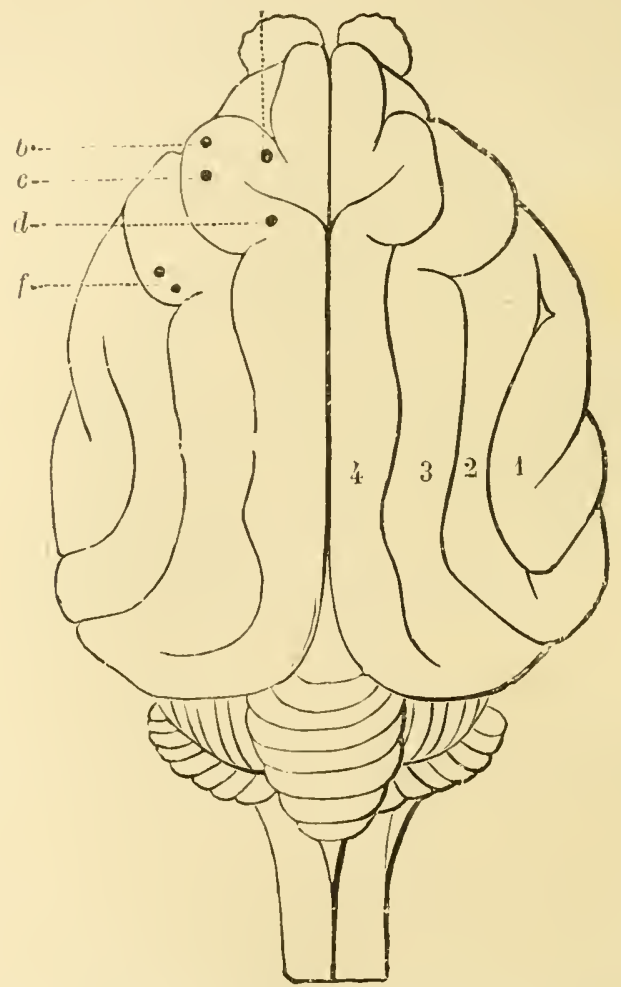

Fig. 31. - Centres moteurs corticaux de l'hémisphìre gauche chez le chius (Hıtzig et Ferrier).

cxtenseurs et adducteur's du membre antérieur (b) vers l'extrémité extérieure du gyrus sigmoïde, le centre des flechisseurs et rotuteurs de ce mème membre (e), le centre moleur des nuscles du membre postérieur en arrière et 
en dedans du centre des mouvements lu membre antérieur $(d)$, le centre des mouvements de la face $(f)$.

11. Arloing a observé sur un King's Charles présentant une blessure à la région frontale gauche de la tète, une destruction de la couche superficielle du gyrus sigmoïde déterminant des troubles du bipède latéral droit consistant dans un affaiblissement de ce bipède, surtout lorsque l'animal voulail courir. La sensibilité tuctile était diminuée du côté droit du corps.

On peut y relrouser d'autres centres localisés par Ferrier, tels que le centre des mouvements de la queue à l'angle de réunion de la soissure longitudinale et du crucial ch arrière de ce dernier; centre de rétraction et d'extension du membre antérieur situé à la partie postérieure du gyrus sigmoüde; centre d'éléválion de l'épaule el d'extension du membre antérieur entre les centres $b$ el $c$; le centre des mouvements des yeux, des paupiẻres et de la pupille, situé en avant du centre $f$ sur la partie antérieure de la deuxième circonvolution.

L'ablation de la troisième circonvolution chez le chien lui fait perdre la faculté d'aboyer (Durel).

Le fail capilalà retenir c'est que chez le chien l'exploration électrique du gyrus sigmoide met en évidence une série de points excitables provoquantla contraction d'un groupe défini de muscles toujours les mèmes et situés du còlé opposé. Ces foyers constituent autant de centres. Ou trouve aiusi parmi ces centres principaux ceux qui répondent aux muscles du tronc, de la unque, de l'extréınilé antérieure, de l'extrémité postérieure, le la íace, des yeux et des creilles. Chaque rentre se subdivise à som tour en foyers déterminant des mouvements de flexion, J'extension, d'abduction, ou d'adductiou, selon l'intensilé du courant el la place assignée aux électrodes.

Chez le singe, les travaux de Ferrier et d'Horsley ont fisé la topographie des centres psycho-moteurs d'une manière très complète. 


\section{Effets de l'excitation des centres psycho-moteurs.}

Ces excitations se Iraduisent, suivant leur intensité et leur durée, par des secousses, des contractions cloniques ou des convulsions épilentiformes (ípilepsie corticale). Le fonctionnement des centres moteurs est indépendant des corps striés et des couches optiques.

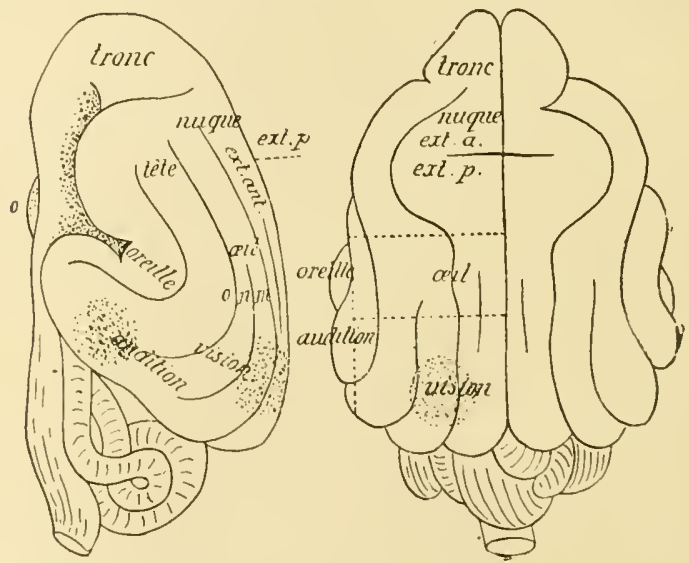

Fig. 32. - Topographic des centres psycho-moleurs el des centres psychosensilifs sur le cerveau du chien (d'après Munk).

Lablation de ces centres entraine des troubles importants de la motricité et de la sensibilité; l'animal effectue bien les mouvements d'une manière maladroite; il a perdu la sensibilité tactile et la sensibilité musculaire, dans la région qui correspond au centre détruit. Si on a enlevé le centre d'un membre antérieur, on peut donner à ce membre les atiitudes les plus anormales, il les conserve toutes : ce membre esl anesthésié, ces troubles peuvent s'atténuer avec le lemps; mais il suffit de faire marcher l'animal les yeux bandés pour les voir se reproduire. 
20 Centres psycho-sensitifs. - On a réussi a déterminer expérimentalement des centres sensitifs ou psycho-sensoriels.

Centre de la vision. - Ainsi le centre cortical de lu iision réside dans la région occipilale : l'ablation du lobe occipilal des deux còtés détermine la cécité complète;

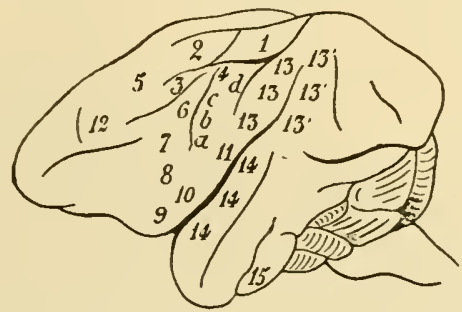

Fig. 33. - Topographie des centres corticaux sur le cerreau du singe (d'après Ferrier).

1, mouvement en arant de l'extrémité postérieure; 2, molvements combinés de l'extrémité postérieure pour saisir un objet ou pour gratter la peau du ventre; 3 , mouvements de la queue; 4 , adduclion du bras; 5 , extension du bras el de la main; 6 , supination et flexion de lavant-bras; 7 , rétraction de la commissure des lèvres; 8 , relèvement de l'aile du nez et de la lèvre supérieure, abaissement de la lèrre inférieure; 11, rétraction de la commissure des lèvres et contraclion du peaucier cervical faisant lourner la tèle du còtè opposé; $1 \nexists$, déviation conjuguée des yeux et de la tête qui se portent du cóté opposé. - $a, b, c, d$, mouvements des doigts. - 13, 13'. mouvements des reux et des paupières combinés avec des mouvemenls de tout le corps.

l'ablation unilalérale détermine des effets variables suirant la proportion des fibres optiques entrecroisées qui est des deux liers chez l'homme et chez le singe et des trois quarts chez le chien.

Chaque rétine est conséquemment en rapport arec les deux hémisphères. Il résulte de cette disposilion que la moitié drojte du champ visuel se projelte sur l'hémisphère gauche el, réciproquemen!, la moilié gauche du champ visuel se projelte sur l'hémisphère droit. Quaud CADÉAC. - Sémiologie, ze édit. II. - 8 
13' SEHIOLOGIE DE L'APPAREIL DE L'INNERVATION.

on ferme un ceil en regardant un objet, la moitié de cet objet est vue par un hémisphère et la seconde moitié pat l’atutre hémisphère.

Les extirpalions partielles du centre oplique déterminent dans le champ risuel des taches obscuresappelées scotomes. Quand l'animal est atteint de scotome central, la vision

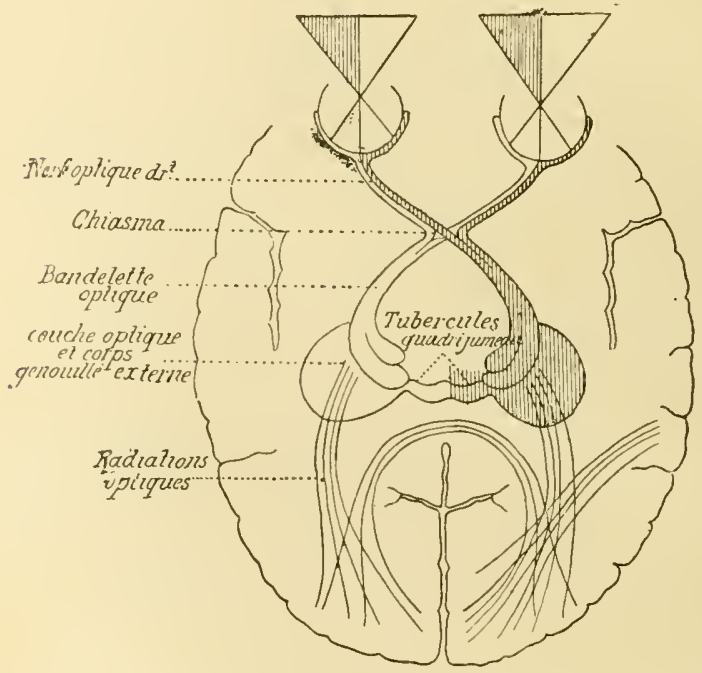

Fıg. 34. - Schèma des radiations opliques depuis la réliue jusqu'aux liẻmisplì̀rès cérébraux (imilé de Dèjerine).

dislincte est abolic; il ne semble pas areugle, il peut se diriger, mais il ne distingue pas les oljets et ne peut pas les recomnaitre.

L'exeitution électrique du centre visuel produit un mouvement de latéralité des yeux du còté opposé et une contraction des deux pupilles; Ferrier consilère ces mouvements comme réllexes et dus à l'excitation par une sensation visuelle suljjective. 
Centre psycho-acoustique. - Il occupe le voisinage de l'extrémité supérieure du lobe temporal, son ablation détermine la surdité du còté opposé, son excitation proroque des mourements associés de l'oreille et de la tète pareils à ceux qu'effectue l'animal qui devient attentif.

Centres gustatifs et olfactifs. - Les centres gustatifs et olfactifs sont encore imparfaitement determinés; Ferrier

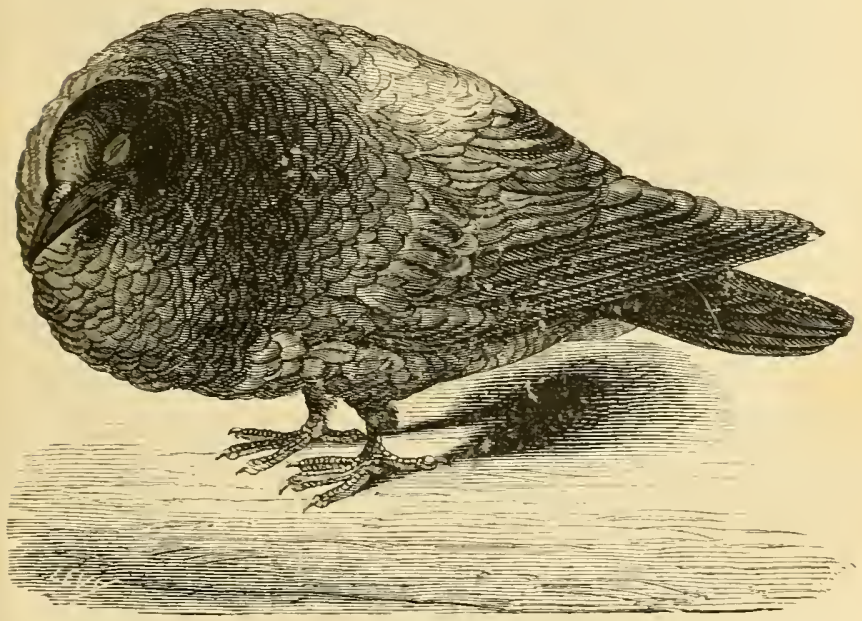

Fig. 33. - Pigeon après l'ablation des lobes cérébraux (Dalton).

eur assigne l'extrémité inférieure de la circonvolution de 'hippocampe.

Les localisations corlicales des fonctions organiques sont rop incertaines pour qu'il y ait lieu de les indiquer ici.

Les centres psycho-moteurs et les centres psycho-sensitifs qui sont excitables forment les centres de projection; ils sont complétés par les centres psychiques inexcitables qui constituent les centres dissociation. 
Modifications pathologiques. - Les maladies du cerveau peuvent supprimer totalement ou partiellement ses fonclions.

Suppression totale. - Ia destruction totale a été réalisée expérimentalement.

Cliez le pigeon (fig. 3ä) l'ablation les lobes cérébraux est suivie d'un état soporeux, J'une sorte de sommeil.

Pourtant, il se tient en équilibre, la tète infléchie et placée sous l'aile; il n'abandonne son immobilité que sous l'influence d'excitations extérieures; mais il n'exécute plus que des acles automatiques. Les réactions correspondent exactement anx excitations.

Chez le chien, l'extirpation complète du cerveau a été réalisée par Goltz, en procédant partiellement et snccessirement, afin de donner à l'animal le temps de se rétablir de chaque traumatisme.

Ce chien sans cerveau se réveillait mieux sous l'influence des impressions tactiles que sous celle du liruit; il répnndait par des mouvements appropriés à toutes les provocations sensorielles. Si on le tirail brusquement de sa cage, il entrait en fureur, abnyait et uordait autour de lui, et mordait surtout yuand il n'avait pas recu de nourriture depuis longtemps; il possédait, au bout d'un an, la sensibilité musculairé beaucoup mieux qu'un chien auquel on a enlevé récemment une partie du cerveau.

Il avait le sens de la température, car ce chien retirait. vivement les patles d'une écuelle remplie d'eau froide; le sens du toucher était fortement émoussé; mais il n'élait. sensible à aucune caresse; toute expression de joie faisait défaut; les pupilles se contractaient vivement à la lumière; il n'était donc pas absolument aveugle.

Ce chien mangeait fortbien ce qu'on lui avait mis dans la gueule, mais il etait incupable de rechercher de luiméme sa nourviture; il ne la trouvait pas, mème lorsqu'il aurait pu la prendre tout à fait à proximité; l'instinct sexuel faisait complètement défaut. 
Selon Goltz, la lésion de déficit la plus importante qu'on observe après l'ablation du cerveau, c'est la perte de tontes les manifestations ou expressions d'où nous inférons la raison, la memoire, la réflexion et l'intelligence de l'animal. Les déments, les aliénés sont des hommes sans cerveau comme le chien décérébré.

Chez les solipèdes, les tumeurs de la boîte cranienne (ostéomes, carcinomes, mélunomes), les tumeurs des méninges (sarcomes, papillones), les tumeurs des ventricules (cholestèatomes), affaiblissent considérablement toutes les facultés sans les supprimer entièrement. (Voy. plus loin Immobilité.)

Chez le bour, la tuberculose, les tumeurs cérébrales peuvent également détruire progressivement le cerveau, qui, réduit à sa plus simple expression, sinon pétrifé comme l'ont prétendu divers auteurs, n'empèche pas les animaux d'aller aux pâturages; ils mangent l'herbe des prés comme les grenouilles décérébrées de Schrader prenaient les vers.

Exploration. - Inspection. - L'inspection du cràne permet de constater l'asymétrie de cette région.

Bassi a constaté l'asymétrie du cràne chez neuf chevaux épileptiques.

L'asyınétrie n'était pas limitée aux os pariètaux, mais elle s'étendail encore à la partie correspondante du frontal; elle consistait en une proéminence d'une moitié latérale du cràne, mais sur les uns c'étail la droite el sur les autres, c'était la gauche.

A mesure que l'épilepsie fait des progrès et que les attaques deviennent plus fréquentes, l'asymétrie cranienne se prononce et une moitié de la lête proémine sur l'autre. Mais X. Bassi incline à penser que cetle proéminence n'est qu'apparente et résulte plutôt de ce que l'autre moitié est déprimée. Il a eu l'occasion de faire préparer la tète d'un cheval épileptique et il a pu consla- 
ter très nettement que l'asymétrie élait le résultat d'une dépression notable du parietal el de la partie supérieure du frontal droit. L'hémisphère cérébral droit était de moindre volume que le gauche et sa pulpe plus consistante comme si elle avait subi une compression.

Trinchera a constamment rencontré cette asymétrie chez les chevaux epileptiques. Elle constitue un moyen de différencier l'épilepsie du vertige.

Palpation. - Elle permet de constater une ilévation de la température dans la congestion active du cerveau, dans l'encephalite du chien, dans le coryza gangrencux du boruf, dans la méningite cérébro-spinale, dans la fievre charbonneuse, dans l'insolation, dans le tournis du monton.

Percussion. - Elle met en évidence une sensibilité excessive des parois craniennes chez les montons affectés de tournis; la pression révèle la méme modification au niveau de la région lombaire quand la moelle est enllanımée.

\section{X. - ROLE DU SYSTEME NERVECX.}

IRéction nerveuse. - Le rỏle du système nerveux en pathologie est capital; la réaction nerveuse accompagne tous les processus : congestions, odìnes, hémorragies, inflammations, troubles trophiques, etc.

Par l'intermédiaire des vaso-moteurs, le système nerveux influence la répartitiou du saug dans les tissus, fait varier l'apport des mafériaux nutritifs, augmente ou diminue le fonctionnement des organes, agit aussi sur la calorification, les sécrélions glandulaires, dirige la diapédese. Toutes ces modifications résultent d'actions vasomotrices qui peuvent se produire dans tous les points de l'organisme. Les vaso-moteurs qui président au relachemenl et à la dilatation des vaisseaux comprennent 
ies nerfs raso-constricleurs et les nerfs vaso-dilatateurs.

La section du sympathique au cou met le ròle des vasoconstricteurs en évidence : les vaisseaux répandus dans la moitié correspondante de la tête et de l'encolure se dilatent, leurs fibres lisses sont paralysées; la pression augmente dans les artères, diminue dans les veines; les incisions pratiquées de ce côté sont suivies d'hémorragies bien plus abondantes que du còté opposé; la peau se couvre de sueur el l'hémisphère cérébral correspondant se congestionne et s'échauffe.

Si au lieu de sectionner le sympathique cervical, on l'excite, des phénomenes inverses apparaissent; les vaisseaux contractés s'effacent, la peau pâlit, la température s'abaisse.

Les Nerfs vaso-coxstricteurs répandus dans tout l'organisme viennent de la moelle en suivant les trajets des racines anterieures et des rameaux communicants pour se jeter dans le grand sympathique et gagner les divers plexus vasculaires. II existe sous le bulbe un centre principal de ces nerfs; mais des centres secunduires sont échelonnés dans la moelle; ils sont subordonués au centre bulbuire, car toute section médullaire qui les en sépare est suivie d'une paralysie temporaire des vaisseaux.

Les ganglions du grand sympathique remplissent enfin les fonctions des centres vaso-constricteurs.

Ces centres out une action continue, ils maintiennent la tonicité des vaisseaux.

Les nerfs vaso-DlLatateurs produisent la dilatation des vaisseaux. On démontre leur existence en excitant le bout périphérique du filet sectionné; on obtient une rasodilatation immédiate arec tous les phénomènes inhérents : exagération des sécrétions glandulaires, écoulement abondant du sang par les plaies accidentelles ou artificielles.

On constate des nerfs dilatateurs dans tous les vaisseaux. Le lingual emprunte ses fibres vaso-dilatatrices à la 
corde du tympan; les nerfs érecteur's produisent ainsi une vaso-dilatation active; le maxillaire supérieur et le buccal renferment les fibres dilatatrices des muqueuses nasale, buccale, gringivale et labiale, etc.

Le fonctionnement des appareils nerveux vaso-moteurs entre en jeu sous l'influence d'un grand nombre de causes qu'il faut passer en revue.

Congestions. - L'excitation d'un nerf centripete, qu'il soit sensitif, sensoriel ou viscéral, provoque une série de modifications vasculaires. On peut observer quatre orlres de phénomènes différents, suivant qu'il y a excitation ou paralysie des vaso-constricteurs, excitation ou paralysie des vaso-dilatateurs.

On constate généralement une vaso-constriction au début suivie hientòt d'une vaso-dilatation; si l'excilation est très énergique, la vasn-constriclion peut passer inapercue.

Les phénomènes vaso-moteurs sont généralement symétriques. Quand on plonge une main dans l'eau froide, l'autre se refroidit; c'est là un exemple frappant d'aclion vaso-molrice bilatérale et symétrique. On peut constater des réflexes symétriques dissemblables : quand les vaisseaux de l'un des reins se dilatent, ceux de l'autre rein se rétrécissent.

Ce résultat peut ètre rapproché des eflets produits par l'arrachement du ganglion ccrical supérieur; l'opération entraine la congestion auriculaire du còté opéré et un rétrécissement des vaisseaux du côté sain.

[il véritable balancement peut se produire ainsi entre la circulation superficielle et la circulation profonde ou viscérale.

Le froid qui anémie la peau congestionne les poumons, le foie, le rein, etc. jusqu'à produire une hémorragie; mais ces congestions réflexes favorisent la diapédèse, la phasgocytose. Quand on inocule le streptocoque de l'érisy- 
péle sous la pean de l'oreille du lapin, on constate que l'affection demeure bénigne quoique la lésion locale soit plus intense si l'on arrache le ganglion cervical supérieur du sympathique. Chez les hémiplégiques, on constate fréquemment une élévalion de température des memlures inerles par suite de la paralysie des vaso-constricteurs.

Des composes chimiques d'origine organique ou d'origine exlérieure agissent sur les appareils vaso-moteurs et congestionnent les organes.

L'urée provoque la dilatation des vaisseaux du rein et élève la pression artérielle; certaines matières albuminoides telles que les protéoses exercent une action vasodilatatrice très marquée.

Les substances qui se produisent dans les muscles sous l'influence de la fatigue, troublent la respiration, élèvent la pression sanguine et produisent des dilatations vasculaires. En iıjectant à un chien du saug défilıriné provenant d'un aulre chien préalablement soumis à un travail musculaire prolongé, on obtient des effets analozurs.

La suppression des glandes à fonction antitoxique comme les corps thyroüdes est suivie de congestions viscírales.

I.es grandes brilures agissent aussi sur les vaso-moteurs par les produits toxiques retenus ou formés dans l'organisme; des produits délerminent une paralysie vasomotrice profonde accompaguée de congestion et d'hémorragies viscérales.

Les produits des glandes à sécrétion interne agissent spécialement sur les vaso-motears. On connait l'action vaso-constrictive de l'extrail de glande pituitaire et de l'adrinaline ou extrait médullaire des capsules surrénales du boeuf et du mouton et des extraits aqueux ou glycérinés du rein, l'action vaso-dilatatrice de l'extrait thyroidien et de l'extrait de thymus, de foie, de pancréas, d'oraires, de testicules, de poumon, de parotide, de sous-maxillaire du chien. 
Toutes ces substances raso-motrices qui se trouvent. dans les extraits sont-elles régulièrement déversées à l'état physiologique par ces organes dans la circulation?

Cette question importante est anjourdhui ì peu près résolue pour le rein et les capsules surrénales. En recueillant en effet le sang veineux de ces organes, en le défibrinant et en l'injectant à dose variable à les lapins, à des rllats el à des chiens, on constale une vaso-constriction évidente avec élévalion de la pression arlérielle et ralentissement du cueur.

Toutes les glandes, et non pas seulement celles qui sont dépourvues de conduit excréteur, livrent au sang quelque substance ulile au fonctionnement de lorganisme. Cette substance exerce une action vaso-motrice plus ou moins prononcée et disparait ensuile très rapidement par oxylation.

\section{Y a-t-il antagonisme entre les deux groupes d'extraits} vaso-constricteurs et vaso-dilatateurs. - Les expél'iences qui ont été faites a ce sujet démontrent la suprématie du zroupe vaso-constricteur. Quand on a déterminé une vaso-dilatalion avec l'extrait thyroidien, l'injection d'extrait surrénal ou l'iujection dn mélange des deux extraits délermine l'élévation de la tension artériełle, c'est-à-dire le resserrement des vaisseaux.

In excès de rénine ou d'extrait rénal dans le sang peut avoir pour conséquence l'hypertrophie du coeur.

I) 'autre part, les appareils vaso-moteurs sont prodigieusement influencés par les produits microbiens. La tuberculine, la malléine, la toxine diphteritique, etc., exercent une aclion vaso-dilatatrice très marquée; mais cetle deruière ne produit cet effet que plusieurs lieures après l'injection, comme si la toxine devait élaborer des poisons qui agissent seuls sur les vaso-dilatateurs et principalement sur le cour dont les systoles sont affaiblies.

Parmi les produils microbiens, il y a peu de substances vaso-constriclives. 
Ilémorragries. - Si une réaction nerveuse modérée suffit pour déterminer la congestion d'une région ou d'un or rane, une excitation plus vive peut ètre suivie de ruptures vasculaires. Les expériences de Vulpian ont permis de constater la dilatation énorme des vaisseaux abdominaux à la suite de la section des tubercules quadrijumeaux; celles de Brown-Séquard ont établi que la piqüre des divers centres nerveux est suivie 'd'hémorragies viscérales; la piqùre de la moelle au niveau de la sixième dorsale amène une hémorragie des capsules surrénales, celle du bulbe provoque des foyers sous-péricardiques, celle du corps restiforme entraîne des épanchements sanguins dans l'oreille du même còlé. L'ablation du plexus solaire est suivie d'épanchements sanguins daus la cavité intestinale.

Un bain froid, une douche penvent produire par action réllexe des épistaxis, de l'apoplexie pulmonaire, etc. Dans les cas d'usphyxie encéphulique par compression des carolides, on constate aussi des hémrorragies sous-cutanẻes et sous-miqueuses.

L'asphycie, d'une manière générale, provoque une dilatation des vaisseaux de la peau et en même temps une contraction des vaisseaux des viscères.

OEdèmes. - L'exsudation séreuse ou l'œù̉me est. une conséquence dela congestion. L'œdème se trouve ainsi inlimentent lié aux troubles vaso-moteurs. Il survient dans les mèmes conditions que les modifications sécrétoires déterminées dans les glandes par les actions vaso-dilatatrices.

Les cedèmes se produisent aussi dans l'échauboulure, dans l'anusarque, etc. (Voy. Anatomie pathologique).

Infanmations. - Quand un corps étranger a été introduit dans les tissus, l'excitation du système nerveux qui ne tarde pas à se prorluire engendre la clouleur et 
secondairement des phénomènes de vaso-dilatalion d'où découlent les aulres symptòmes de l'iuflammation (rougeur, chaleur, tuméfaction).

En dehors de ces phénomènes locaux vaso-moteurs, on constáte d'autres acles réflexes qui retentissent sur la nutrition de l'organisme; il en résulte une élévation de la température générile, une exagération daus la production des matières excrémentitielles et de l'acide carbonique.

Manifestations dystrophiques. - Le système nerveux assure la nutrition des diverses parties de l'organisme. Les troubles tropliques consécutifs à sa suppression ne dépendent donc pas simplement d'une absence de fonctionnement, mais principalement d'une modilication profonde de la nutrition.

Si l'on coupe la corde du tympan, les papilles du còté correspondant perdent rapidenent leurs caractères spécifiques, partout les éléments différenciés se dépouillent de leurs caractères histologiques, un tissu de remplissage se développe et la sraisse vient remplacer les parties atrophiées. C'est dans les muscles que ce remplacement apparait de la manière la plus frappante.

Les altérations articulaires produisent celte atrophie misculaire en Irois ou quatre jours; il suffit de sectionner les racines postérieures pour prévenir cetle atrophie, car en interrompant le réllexe, on empèche l'atrophie musculaire consécutive aux entorses ou aux lésions des articulations.

Choc nerveux, - Les excitations nerveuses sont susceptibles de produire trois variétés de syncopes: syncope cardiaque, syncope respiratoire, syncope des ichanges ou mieux syncope nutritive. C'est celle-ci qui caractérise le choc nerveux. Cet accident peut survenir sous l'influence de traumatismes peu intenses. Il n'existe aucun 
rapport constant entre l'intensité de la cause et la gravité de ses effets.

Les traumatismes des centres nerveus (cerveau, bulbe, moelle), les impressions morales, les excitations sensorielles, emotionnelles, les excilations des nerfs sensitifs par irritalion des nerfs de la peau, des nuscles, des tendons et surtout du périoste et de l'os sont des causes de choc nerveux. Les traumatismes déterminent le choc quand un viscère (rein, utérus, ressie, intestiı, testicule) est atteint.

La suppression des échanges qui caractérise le choc nerveux s'accompagne toujours d'un abaissement rapide de la température : $\mathbf{x}$ à J̆ deırés eu quelques heures chez l'homme: \pm à 6 degrés chez les cobayes en 30 minutes; la putréfaction des sujets qui ont succombé au choc est très

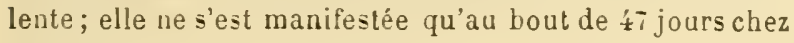
un chien.

\section{Réactions nerveuses dans les intoxications.}

- Qu'un poison soit introduit dans l'organisme, il se produit dès son entrée dans la bouche des réflexes qui exagèrent la salivation d'où résulte la dilution du produit avalé; en mème temps le goût désagréable du poison irrite les terminaisons nerveuses et amène le rejet d'une autre partie avant la déglutilion. Puis le vomissement, la diarrhée combattent l'absorption; il s'établit des contrecourants suivis de transsudations. Toutes ces manifestations résultent d'une excitation nerveuse. Gràce au système nerveux, tous les appareils sünsurgent contre les agents nocifs.

Les réactions protectrices de l'appareil respiratoire contre les intoxications sont l'étemuement, la toux qui tend à rejeter tous les produits délétères, l'exsudation qui les incorpore; celles de la conjonctive et de l'ail sont le larmoiemenl et l'occlusion des paupières; celles de la peau sont la cangestion el l'œè̀me; celles des organes internes

$$
\text { CADÉAC. - Sémiologie, ?̨e ėđit. II. - } 9
$$


sont analogues. Il se produit dans le poumon, dans les reins, des phénomènes de congestion el d'exsudation ou d'hémorragie qui tendent à diluer les poisons et à faciliter leur élimination, à ralentir leur absorption et à atténuer leurs effets.

Quand les réactions dépassent le but, il se produit des phénomènes inhibitoires susceptibles de produire la mort immédiatement par syncope. Les réflexes, salutaires dans l'immense majorité des cas, peuvent devenir mortels.

En dehors des réflexes, le système nerveux répond aux intoxications par des manifestations délirantes, par des convulsions (strychnine, téniasis, elc.).

Certains toxiques enfin sont susceptibles de paralyser les arcs réflexes : la curare empêche l'excitation centrifuge de parvenir aux muscles; l'atropine suspend l'action inhibitoirc des pneumogastriques sur le cœur; les anesthésiques et les hypnotiques diminuent le pouvoir réactionnel du système nerveux. Contre les intoxications chroniques, les réactions du système nerveus sout principalement d'ordre psychique; elles consistent dans la diminution ou la disparition des sentiments affectifs, de la volonté, de la mémoire et de l'intelligence.

Rénctions nerveuses dans les infections. - Les infections les plus graves sont celles qui résultent de germes ou de toxines qui entravent ]e pouvoir réactionnel du système nerveux, c'est-à-dire celles qui s'opposent aux congestions réflexes, à la diapédèse, à l'exsudation. Les affections qui tendent le plus vers la guérison sont celles qui stimulent les terminaisons sensitives et provoquent un réflexe vaso-dilataleur entraînant une congestion locale, premier acte de défense de l'organisme. L’expérience suivante en fournit une démonstration éclatante.

Qu'on inocule le streptocoque sous la peau des deux oreilles d'un lapin; une des oreilles est Iaissée in lacte; on coupe les nerfs sensitifs (auriculo-cervicaux) de l'autre; 
on constate que de ce còté l'érysipéle est plus grand que du còté sain; la section nerveuse entrave la guérisou.

Chez un autre lapin inoculé de mème, on arrache le ganglion cervical supérieur du sympathique (nerf centrifuge), on constate que de ce còté la réaction inflammatoire est plus intense; mais la réparation est également plus rapide. Les substances qui agissent sur les centres vaso-dilatateur's favorisent la guérison par le mème mécanisme. La paralysie vaso-motrice relarde la dissémination des bactéridies charbonneuses, injectées sous la peau, tandis que la section des nerfs seusitifs accélère l'évolution du processus infectieux.

La vaso-dilatation est d'autant plus efficace qu'elle est plus précoce; elle hâte la guérison en augmentant la diapédèse et en exagérant la phagocytose; il y a quarante à cinquante fois plus de cellules rondes du còté où l'on arrache le ganglion cervical supérieur du lapin que du côté sain.

Les invasions microbiennes des muqueuses sont suivies de phénomènes réflexes vaso-dilatateurs qui exagèrent les sécrétions dont les produits (salive, mucus, larmes) balaient les produits microbiens. Partout, le système nerveux constitue un appareil avertisseur et un moyen de défense contre les infections : la section des pneumogastriques permet l'inflammation du poumon.

Ces faits montrent que le système nerveux dirige un grand nombre de phénomènes morbides, qui interviennent dans le développenent des lésions locales et des troubles viscéraux comme ses symptơmes généraux (fièrre, hypothermie). Si le chien anquel on veut prendre la température s’agite, sa température s'élève rapidement; la réaction nerveuse détermine presque subitement de l'hyperthermie.

On va étudier successivement les troubles de l'instinct et de l'intelligence (délire, hallucinations, vertiges, coma), les troubles de la motilité (paralysie, ataxie, convulsions, 
tremblements), les troubles de la sensibililé générale (douleur, hyperesthésie) et spéciale (vision, audition).

\section{XI. - INTEILIGENCE.}

Considérations générales. - L'intelligencedes animaux est aujourd'hui universellement reconnue. Les travaux d'Alix, de Joly et surlout de C. Chomel et Fermand Rudler, comme les observations journalières qui sont faites par tous ceux qui aiment et qui étudient les chiens et les chevaux, démontrent la multiplicilé de leurs facultés intellectuelles.

Les animaux, conme l'homme, possèdent des centres psycho-sensibles étroitement liés aux centres psycho-moteurs, les premiers sont coordinateurs des seconds. Il en résulte que les facultés intellectuelles qui u'ont certainement aucun siège particulier dans le cerveau, paraissent être le fruit de l'intervention de tous les centres sensibles: le cerveau étant le rendez-vous de l'action de tous les organes. Les principales manifestations de l'intelligence du cheral sont, dit-on, la sensation, la perception, le souvenir, l'attention et partiellement le jugenent, le raisounement, l'imagination qui constituent l'entendement.

Facultés intellectuelles. - Les facultés du cheval ont été résumées par Chomel et Rudler de la manière suivante :

"Le cheval est attentif a tout ce qui l'entoure, et cette attention va souvent jusqu'à l'ubservation, la persévérance, l'entêtement.

Il a une grande mémuire des lieux et le sens spécial de la direction ou sens de l'espace ou de l'orientation, qui n'esl, semble-t-il, qu'une forme de mémoire. 11 peut apprécier les faits, les juger et jusqu’à un cerlaiu point raisonner, comme en témoignent divers actes rapportés par Joly (1).

(1) Joly, De l'intelligence du cheval, 1890. 
Il peut encore présenter des phénomènes d'activilé psychique (contagion de la peur, rèves, états hypuotiques, délire, hallucinations, etc.).

Les fonctions cérébrales du cheval se rapportent encore aux sensations, aux mourements, aux facultés d'expression, aux instincts, an caractèrc, aux passions.

Le cheral est sujet comme l'homme aux sensations tactiles (de contact, de pression, thermique, sensibilité musculaire, sensations douloureuses, soif, faim, fatigue, etc.), gustatives, olfactives (celles-ci très développées) et visuelles. On admet généralement, sans que cela soit démontré, qu'il est d’autant plus actif et plus intelligent, que ses sens sont meilleurs, c'est-à-dire plus perfectionnés.

Habituellement, les sens relatifs à la connaissance sont plus parfaits chez l'homme et ceux relatifs à l'appetit, plus parfaits chez l'animal; ces derniers seraient mème plus développés chez l'animal qui vient de naître, que chez l'enfant nouveau-né.

Chez le cheval, on trouve plus ou moins le sens du mouvement " qui lui permet d'apprécier le meilleur emploi á faire de ses membres pour développer sa force, ses moyens, en rue de l'exécution des différents mouvements, notamment du saut, des exercices de manège ou de haute école " (Jacoulet et Chomel). Il y a lá uıe série d'opérations mentales complexes, créant les idées de causalité et de comparaison.

Toutes les sensations animales paraissent tributaires de la loi psycho-physique de Fechner disant que: "les sensations croissent proportionnellement aux logarithmes des excitants ".

Les chevaux offrent des mouvements souvent compliqués, ayaut " un haut degré de généralisation et decoordination ", qui sont une preave de la puissance de la moelle comme conducteur nerveux, de la multiplicilé des acles réflexes, de la prépondérance du cerveau dans son 
rôle coordinateur, régulateur, c’est-à-dire dans sa fonction psychique.

Les facultés d'expression du cheval sont multiples et complexes; elles se traduisent par une mimique, le hennisscment sous l'influence de l'attention, de la peur, de la joie, de l'impression des odeurs, etc. M. Joly a cherché à grouperces divers mouvements expressifs suivant les trois principes énoncés par Jarwin (1). Par un langaqe élémentaire (dia, ho, hu) ou une phonomimique, on se fait très bien comprendre du cheval. Le développenent de son intelligence paraît d'ailleurs ètre en rapport avec la compréhension du langage.

Le caractere et les passions du cheval sont d'ordre extrêmement varié et Buffon avait assigné, avec raison, à cel animal toutes les nuances des passions. Cliez lui, les sentiments affectifs, sociaux se rencontrent fréquemment. "Il est susceptible d'affection, de reconnaissance, de haine, de rancune, de colère. Il a de la joie, de la tristesse, du clagrin, de l'amour, de la jalousie, beaucoup d'émulation, de l'ambition, voire mème de l'orgueil (2)" ; il peul simuler une vive douleur par des lancinations ou une boiterie qui disparaissen

La dominante du caractère chez le cheval est la douceur, la soumission, la sensibilité. " Déjà sociable à l'état saurage (où les chevanx vivent en tribus), le cheval se nontre très doux, naturellement craintif, peureux, en domestication (3)."

Enfin les instincts sont pour le cheral comme pour l'homme une faculté psychique. Il y a chez lui l'instinct de préscriation, l'instinct sexuel. Le besoin de mouvement est aussi fort développé, de mème que l'instinct d'imitation se caractérise par ses tics (4). Parmi les instiucts du cheval

(1) Darwin, Expression des émotions chez lhomme et l'animal, 1872.

(2) Jacoulet et Chomel, Loc. cit., I. I, p. 193.

(3) Jacoulet et Chomel, Loc. cit., p. 194.

(i) Du moins pour le lic de l'ours (Rudler et Chomel). 
il faut placer aussi les aptitudes spéciales qui ne sont qu'une mémoire héréditaire organique.

L'échelle intellectuelle des animaux dont on parle tant ne peut guère se comprendre que dans la même espèce; appliquée à tous les animaux comparés entre eux elle devient une fantaisie.

Elle serait bien autrement intéressante dans l'ordre pathologique. Il est probable que les principaux troubles psychiques des animaux suivent plus ou moins exactement les grandes divisions de leurs facultés intellectuelles.

Pour M. Joly (1), "l'héredité des qualitès morales fut démontrée plus ou moins scientifiquement par Pline, Buffon, Girou de Buzareigne, etc. D'après Charlton Bastian (2), pour tout ce qui touche aux perceptions, émotions et facultés motrices, la doctrine de l'acquisition héréditaire peutètre regardée comme solidement prouvée par les observations et les expériences de Douglas et Spalding ".

Hérédité et érolution sont jointes dans les faits psychiques comme dans les autres faits organiques, la première représentanl le progrès, la seconde, la conservation (Spencer).

Il en est de mème de l'automatisme des mouvements complexes résultant du dressage qui exige l'intervention de la volonté; il provient d'un long apprentissage et représente un réel facteur héréditaire.

Dans l'habitude, il faut voir un effet de la mémoire organique ou une " mémoire inconsciente des mouvements associés, combinés en vue d'un but ".

Toute la vie intense du cheval relève d'une bonne organisation cérébrale sans qu'il ait cependant l'idée accusée du temps, l'entendement, l'imagination, ni la notion de

(1) Joly. Loc. cit., p. 153.

(2) Ch. Bastian, Le cerveau et la pensée. 
152 SÉMIOLOGIE DE L'APPAREIL DE L'INNERVATION.

l'avenir. Elle est le résultat progressif de l'éducation acquise et transmise.

Buffon avait considéré pour la première fois, dans les animaux, l'éducation comme cause du développement de leurs facultés, ce qui l'arail conduit à démontrer la haute supériorité de l'homme basée sur sa faiblesse mème (Chomel et Rudler).

\section{XII. - DÉGÉNÉRESCENCES.}

Définition. - L'idée de la dégénérescence chez l'animal a fait son apparition en médecine vétérinaire avec un important: travail de M.l. Chomel el Rudler (1), auquel nous avons emprunté les matériaux de cetle élude.

La dégénérescence est caractérisée chez l'animal par la constatation de stigmates physiques et psychiques dits de dégénérescence. Ces stigrmates consistent dans des malformations, des troubles intellectuels, un état de déséquilibration particulier, etc.

La dégénérescence n'appartient pas à un état pathologique particulier. C'est un vice de développement, une manière d'ètre anormale el permanente qui se traduisent à la fois par des caractères morphologiques (signes physiques) ainsi que par le fonclionnement vicieux de l'organisme (signes psychiques).

L'animal dégénéré est un ètre physiquement ou intellectuellement anormal. Les antécédents psychopathiques jouent un rôle considérable dans la dégénérescence des sujets.

En fait, la ressemblance est le caraclère fondamental de l'hérédité physiologique, la dissemblance est au contraire la caractéristique de l'hérédilé palhologique.

Chomel el Rudler admettent comme facteurs de cette

(1) C. Chomel et Fernand Rudler, Iléréditè morbide et dégénérescence. Des stigmales physiques et psychiques dits de dégénérescence chez l'animal et en parliculier chez le cheval. Ourrage communiqué. 
dissemblance des conditıons héréditaires natırelles, artificielles on accidentelles. Le terme de dégénérescence employé par les auteurs diffère totalement d'ailleurs de la dérradation de la race (Buffon), de la dégéuération du type (Blumenbach), car alors il y a plutòt une transformation continue et un perfectionnement graduel (Sanson).

L'exposé fait par les auteurs des méthodes sootechniques d'amélioration dans leurs rapports avec l'hérédite et la dègènérescence vient à l'appui de cette thèse que la race se conserve simplement on bien qu'elle se détruit et s'éteint s’il existe des causes de dégénérescence. Or ces causes sont fort nombreuses; il y a un très fort déchet dans la fabricalion du cheval par exemple en tant que dégénérescence. Celle-ci existe aussi bien dans l'ordre psychique que dans l'ordre physique. La "vie intense " du cheval relève "d'une bonne organisation cérébrale " et l'organisme animal " peut n'ètre plus sain el devenir anormal sans être malade $»$.

Les dégénérescences ont leur source dans des modifications lréréditaires physiques ou psychiques, comme en témoignent un grand nombre d'expériences et d'observations.

Dégénérescence expérimentale. - Les lésions artificielles et accidentelles de l'embryon, les accidents et traumalismes du jeune àge, les intoxications, les infections, le surmenace, les conditions de vie imposées aux animaux (milieu, éducation, dressage, etc.) ont permis de créer diverses dégénérescences.

Brown-Séquard a établi l'hérédité de l'épilepsie produite expérimentalement par sections el irritations de la moelle vers la $10^{\circ}$ dorsale; Luciani, celle de l'épilepsie corticale. De mème, des chiens, devenus épileptiques à la suite de traumatisnes cérébraux, peuvent engendrer des épileptiques (Cadéac).

Aprés la section partielle du bulbe rachidien, Brown- 
Séruard a encore observé une exophtalmie chez les parents et une exophtalmie identique chez les descendants.

Les intoxications (alcoolisme, etc.) créent également la prédisposition héréditaire pouvant aller jusqu'à la dégénérescence (Mairet el Combemal). Il parait en ètre de même encore pour certaines infections microbiennes (gourme, fièvre typhoïde, etc.) et peut-être aussi pour les auto-infections (surmenage chronique, affections gastrointestinales résultant de tic, elc.).

Les troubles pendant la vie foetale ou l'ovulation (Charrin, Delamarre, Moussu), les traumatismes de tout genre (Dareste), les émotions, les frayeurs, les maladies du foetus sont des causes très fréquentes de dégénér'escence.

Les conditions qui influent sur le jeune sujet peuvent agir dans le même sens à un degré moindre (nourriture défectueuse, défaut d'hygriène, travail prématuré, etc.). M.I. Chomel et Rudler citent l'hérédité des instincts, des tics, des troubles intellectuels.

L'influence héréditaire se fait neltement sentir sur les instincts des animaux qui sont éminemment transmissibles. Voisin les considère comme les mobiles inférieurs de l'intelligence, susceptibles de se modifier par l'éducation el dont les modifications elles-mêmes se transmellent héréditairement.

On pourrait en dire autant des tics, notamment du tic de l'ours, trouble psycho-moteur, dont l'étude délicate a été récemment faite par Chomel et Rudler.

Les stigmates phy'siques dits de dégénérescence s'observent également dans les stéréotypies de léchage (1) L'bérédité du tic a d'ailleurs été constatée et démontrée par Farges, Weber, Collin, Joly, etc.

L'lıérédité des facultés intellectuelles est difficile à bien préciser, mais du moins, pour le cheval, les fonctions cérébrales paraissent assez étroitement liées aux sensations et

(I) F. Rudler et C. Chomel, Nouvelle Iconogr. de la Salpêtrière, $1904, n^{\circ} 6$. 
perceptions, c'est-à-dire à l'organisation des illees. Par les sensations, on pourra se rendre un compte plus ou moins exact des phénomènes de dégénérescence.

La mémoire acquise est hér'éditaire, elle implique l'idée de temps, de lieu et de choses vues et comprises. Par elle on explique laptitude progressive de certains cheraux au saut, aux mouvements compliqués. La maladresse persistante de certains chevaux qui préfèrent la chute à un effort de volonté est une sorte de mémoire organique inconsciente.

Au contraire, la mémoire héréditaire donne la clef des aptitudes spéciales.

La rolontè se manifeste par des mouvements sériés allant jusqu'à la plus haute culture des attitudes.: Par contre, la rétivité, la méchanceté, le refus d'obéissance (état sauvage), la peur sont alliés le plus sourent à l'hérédité.

Très souvent les idées de défense relèvent des troubles de la sensibilité générale.

La transmission héréditaire des caractères psychologiques acquis par le chien de chasse, explique clairement l'hérédité incontestable des troubles psychiques.

Chez l'homme, elle peut faire admettre la tendance au vol, aux obsessions, l'impulsion au suicide, etc.

Suirant Mosso (1), il faudrait compter trois ou quatre générations pour que les chevaux perdent leurs instincts sauvages.

Des faits où une rétivité évidente s'est perpétuée de l'ascendant au descendant ont été cités par Pierquin (2), Joly (3), etc.

Les peurs morbides sont assez fréquentes chez les animaux.

Si le sens musculaire mesurant la distance et l'étendue des objets, la marche automatique sont le fruit d'opérations

(1) Mosso, La peur.

(3) Pierquin, Traité de la folie des animaux.

(3) Joly, Loc. cit., p. $18 \%$. 
cérébrales complexes, il peut y avoir, d'autre part, retard de la marche, incoordination motrice, effort exagéré pour un but simple (action de trottiner) qui résultent du développenent incomplet du sens musculaire ce quiréalise en somme la dégénérescence.

Enfin les aterrations des sens, la perversion du goùt, de l'odorat, l'hyperexcitabilité cutanée de certains chevaux témoignent également des troubles nerveux : chevaux ayant l'habitude de manger de la terre, chiens à odorat très développé se roulant en avalant des excréments, gloutonnerie, voracité, etc. (Chomel et Rudler).

En somme, le terrain psychopathique préparépar les legs héréditaires et les acquisitions indixiduelles se caractérise chez l'animal comme chez l'homme par des malformations physiques, et l'inégalité de développement des centres psychiques, psycho-moteurs et psycho-sensoriels. Le terme héréditaire implique une notion étiologique; le terme dégénéré, une notion symptomatique.

Pour éludier la dégénérescence, il faul rechercher avec soin les antécédents héréditaires de l'animal, établir les caractères de son individualité physique et psychique, inspecter la forme du corps, les altitudes, les mouvements, les actes, et même à la rigueur recourir à l'expérimentation (psychométrie, hypnotisme, etc.). C'est à l'aide de ces diverses méthodes que Chomel et Rudler ont constaté chez le cheval de nombreux stigmates physiques et psychiques dits de déqénérescence, soit dans les dégénérescences héréditaires, soit dans les dégénérescences d'évolution. Nous allons rapporter ici très succinctenent ces divers signes.

Stigmates physiques. - Les malformalions physiques constituent non une preuve, mais une simple présomption de dégénérescence. Les signes morphologiques viennent à l'appui des symptômes psychiques pour caractériser la dégénérescence, pour ıui fournir une 
expression analomique : ils rérèlent un développement défectueux de l'organisme.

Les stigmates physiques comprennent, d'après Chomel et Rudler, les malformations de la tète (crâne, face, oreilles, yeux, nez, bouche), du tronc et des membres.

$1^{\circ}$ Cràne. - L'élude de ces anomalies est encore peu avancée. Un connait cependant :

1. Des ayomalies de volume : microcéphalie (cràne plus petit chez quelques chevaux) et l'hydrocéphalie (cràne volumineux, en forme de boule chez les poulains).

$2^{\circ}$ Des anomalies de FORME : plagiocéphalie (cràne aplati fortement asymétrique), scaphocéphalie (saillie de la suture frontale), naticéphalie (déformation du crâne avec sillon médian et saillies des bosses frontales).

Les déformations artificielles comprennent les compressions accidentelles qui résultent fréquemmenl, chez le cheval, de contusions ou de chutes. Quelques chevaux sont dits cornus parce qu'ils présentent au front des restiges de cornes.

Le cheval Androclès (par Dollar et Alabama) avait sur le fronl, entre les oreilles et les yeux, deux rudiments de cornes comme Dutch Skater; cette particularité se trouve donc deux fois clans la descendance de The Flying Dutchman. L'asymétrie cranienne se retrouve chez. la plupart des chevaux épileptiques (Bassi, Trinchera).

20 Face. - L'asymétrie faciale a été mise en évidence par Chomel et Rudler daus leur étude des tics. On la constate de la manière suivante :

Par L'inspectios, on peut remarquer, en se plaçant devant le cheval, s'il y a abaissement d'un œil, déviation du nez à droite ou à gauche.

Eı soulevant les lèvres et en se plaçant de face ou de profil, on observe la dentition irreguliere, le defaut d'affrontement des incisives (prognathisme, brachygnathisme), le chevuuchement des dents, la présence d'incisives supplémentaires, une usure dentaire anormale caractérisant le tic à l'appui. 
La palpatiox de la face permel de reconnaitre si l'asymétrie provient des parties molles ou des parties osseuses.

La mexsuration de la face fournil desindications sur sa forme et sur son développement. La meilleure manière dejuger de l'asymétrie de la face consiste à mesurer au décimètre et comparativement de chaque côtéla distance comprise entre l'angle interne de l'ceil et l'extrémité de clıaque apophyse zygomatique (Chomel et Rudler).

Oreilles. - Le cheval méfiant, hargneux, couche souvent ses oreilles; celui qui est peureux, craintif, atteint de cécité, les a constamment en mouvement; l'animal sourd les tient immotiles.

Yeux. - Parmi les stigmates physiques fournis par les yeux, il faut citer : l'inégalité pupillaire, les yeux inéganx, les anomalies de coloration ( les cataractes précoces (Chomel et Rudler).

Nez. - La lèvre supérieure déviée à droite ou à gauche, la lève inférieure pendante sont considérées par les auteurs précités comme des stigmates de dégénérescence.

Les ditlormités du nez sont le plus souvent acquises; mais la déviation latérale signalée dans les tics par Chomel et liudler est congénitale; il en est de même pour la dévialion de la cloison.

Bouche. - L'asymétrie des arcades dentaire, l'usure inégale des dents, l'accumulation constante des aliments entre les joues et les màchoires (animal qui fait magasin), le défaut de parallélisme des arcades dentaires, les dents supplémentaires, les anomalies de siège, deèruption, sont des manifestations de dégénérescence.

Tronc et membres. - MM. Chomel et Rudler ont mis en relief un certain nombre de stigmates physiques: malformations et dépressions thoraciques; asymétrie de l'épaule, de la hanche; genou brassicourt; étranglé à la base; genou de veau; genou renvoyé; mauvaise conformation des jarrets; défectuosités du boulet; la 
plupart des tares osseuses; panardise; cagnardise du membre; cryptorchidie; etc., etc. Ces auteurs citent de très nombreux exemples de transmissions observés sur les chevaux de sang.

Symptômes somatiques. - Ils comprennent des troubles du système nerveux, des fonctions génésiques, digestives, de l'appareil urinaire.

Dans ce groupement on trouve : les troubles de la motilité (cabrer fréquent, lancer, ruade, elc.), les allures irrégulières non acquises (amble, Iraquenard, elc.), l'instabilité motrice, l'action de trottirer, les attinudes spèciales (décubitus fréquent, etc.), l'exagération du réflexe lombaire, le peu de résistance du dynamomètre caudal au soulèrement, les phinomènes d'anesthésie ou d'hyperesthésie, l'hypersécrétion sudorale, le satyriasis chez l'étalon, la nymphomanie chez la jument, l'aérophagie, les perversions digestives, le cornage et le surmenage chroniqurs, elc.

Le lercement latéral, l'action de lillarder, de faucher, de se couper ou de s'alteindre, etc. sont des défectuosités temporaires dont la signification est moins grande que laction de trottiner qui est fréquente chez les dégénérés!

Stigmates psychiques. - Le cheval, disent Chomel et Rudler, peut exécuter toute une série d'actes qui pour ètre très simples et susceptibles seulement d'un perfectionnement très limité, ne sont pas moins facilement reconnaissables et se distinguent nettement des actes purement réflexes d'origine bulbo-nédullaire. Dans le tic de l'ours par exemple, ils ont noté desactes volontaires, des actes d'imitation, phénomènes psycho-moteurs qui impliquent nécessairement la mise en jeu des centres supérieurs d'association et de coordination et qui ne peuvent s'expliquer que par la participation de l'écorce cérébrale.

Les troubles psychiques de l'animal consistent dans des 
troubles lu caractère (nervosisme, émotivité, impressionnabilité, phobies visuelles et auditives) dans des perturbations del'intelligence, de la volonte. La tare psychique chez l'animal, qu'elle soit due à la diminution de la synthèse psychique, à la prédominance des instincts, ou à lou te autre cause, coüncide souveut avec une hérédité accumulée et arec des stigmales physiques de dégénérescence.

Les stigmates psychiques se rapportent:

10 Aux troubles de la manue ou troubles psychiques sensitifs qui s'expriment chez le cheval par de l'agitation, de l'égarement, de la défiance, des phobies visuelles et auditives, de l'apathie et du dégoùt pour l'exercice. Autant le cheval bien équilibré est doux, obéissant, autant le déırénéré psychique oppose au contraire des résistances, une attitude menaçanle.

$2^{\circ}$ A Ux actes rmpulsifs, à la condition d'ètre fréquents, sans utilité et irrésistibles : aclion de ruer, de trottiner, de s'emballer au mépris de tout danger, etc. Certains chevauxtiqueurs sont très impatients, ils présentent des trépiguements, des hochements de tète, ils fontdes bonds iutempestifs, ils s'emballent et s'atfolent saus motif. Parmi ces chevaux, ils en est même qui se livrent à de véritables accès de férocité!

$3^{\circ}$ A L'HÉRÉDité Nerveuse accumulée, capitalisée, allant jusıu'au nervosisue. A ce sujet, M. Chomel et Rudler distinguent fort justement le tempérament nerveux des chevaux de sang du déséquilibre nerveux des dégénérés.

$4^{\circ}$ AUx Troubles de La volonté, DU CARACTĖRe, résultant de * l'affaiblissement du pouvoir gónéral de la synthèse psychique " (aboulie ou dimiuntion de la volonté, hésitation, paresse, impuissance dans les actes, caraclère inégal, intermittent, fantaisiste, défaut de courage, etc.). Certains chevaux linueurs s'isolent difficilement diune colonne, d'autres fléchissent brusquenent sur leurs membres sous le coup d'une émotion vive.

$\breve{3}^{\circ}$ Aus phobies, hallucivatoxs (sensorielles, visuelles ou 
molrices) (craintes, répulsions, cheral emballé, etc.). $6^{\circ}$ A LA MÉCHANCETÉ ET A LA RÉTIVITÉ PRÉCOCES qui sont souvent héréditaires.

La dégénérescence est souvent la conséquence d'une aggravation progressive de génération en génération des tares transmises. Gràce à la sélection qui se fait chez les animaux, la race animale est moins dégénérée que l'homme. On ne saurait comparer l'intelligence des idiots avec l'intelligence des animaux, car l'animal a ordinairement un développenent intellectuel normal. Quand l'étude des sens et des sensations qui s'y raltachent (vue, ouïe, goût, sens musculaire, sensibilité générale, etc.) sera plus avancée chez les animaux, il deviendra facile d'apprécier leurs troubles psychiques. Parmi les animaux dégénérés, on pourra reconnaître des visuels, des auditifs, des sensilifs, des moteurs. 0n dressera l'échelle des diverses dégénérescences. On associera les signes psychiques aux signes physiques pour avoir toute leur signification; car on sait que des troubles psychiques isolés peuvent s'observer sur de très bons chevaux. Les signes nıorphologiques et psychiques n'ont de valeur que par leur accumulation et à la condition d'ètre très accusés, trẻs prononcés, sinon il y a seulement présomption de dégénérescence.

On s'efforcera enfin, non seulement par la sélection, mais encore par l'éducation, l'influence de l'entrainement, d'enrayer la dégénérescence et de produire une Iransformation saisissable. Les travaux de Chomel et Rudler ne peuvent que faciliter les progrès de l'élevage, de la zootechnie comme des études cliniques.

\section{XIII. - DÉLIRE.}

Pathogénie. - Le délire est une perversion des fonc- 
tions psychiques (1) résultant d'un trouble matériel des circonvolutions cérébrales : congestion, anémie, lésion de nutrition, intoxications, variations de Ja température, etc.

La congestion, l'inllammation des méninges et de l'encéphale, les thromboses des artères cérébrales (Siegen) peuvent le provoquer.

Les maladies infectieuses (pneumonie, fiève typhoïde, tuberculose du bouf, peste bovine, coryza gangreneux, rage, morve aiguë) déterminent le délire en altérant le sang (anoxyhémie, asphyxie), en provoquant la fixation des microbes infectieux dans le cerveau ou en produisant une congestion cérébrale; en empêchant la désassinilation par suite de l'altération du filtre rénal, en exagérant la dénutrition par suite de l'élévation de la température organique. Le délire des maladies infectieuses est donc un délire toxique.

Les poisons (belladone, jusquiame, mercure, plomb, strychnine), administrés à haute dose, sont une source de délire; les essences provoquent, suivant leur nature, un délire furieux ou un délire tranquille (2).

Les chiens soumis à l'action du chloral, du chloroforme et de léther poussent des hurlements plaintifs ou des cris furieux, des gémissements bruyants comme s'ils étaient cruellement martyrisés. Cette agitation frénétique du chien chloroformé ressemble beaucoup à un élat convulsif.

Quand le chloral est donné à doses assez fortes pour annuler la motilité volontaire, les chiens rêvent encore; ils ahoient légèrement comme fout parfois les chiens endormis de sommeil naturel; parfois ils font entendre de longs et plaintifs gémissements bien qu'ils ne subissent aucune opération.

(1) Labat, Deux cas de délire aigu sur deux juments (Revue vitérinaire, 1893, p. 13. - Ducasse, Attitude delirante d'un cheval au cours d'une preumonie (Képertoire de police sanilaire, 1903).

(2) Cadéac et Heunier, Recherches expérimentales sur les essences Paris, 1892. 
Dans la période post-chloroformique, des grenouilles sont prises l'accès de délire véritable; elles ont des hallucinations et se précipitent sur les objets voisins pour les mordre en supposant probablement que ce sont des proies qui leur sont offertes.

Les animaux en inarition ne présentent jamais un délire semblable à celui de l'homme qui meurt de faim.

Le rếce lui-même peut être considéré comme une sorte de délire. Les chiens, les chats rèvent, aboient, se plaignent, etc.

On ne constate pas chez eux de somnanibulisme et les états analogues à l'hypnotisme sont caraclérisés par la stupeur sans délire.

Caractères. - Ce symptôme est dénoncé par une surexcitation extraordinaire des animaux.

Les cheraux se dressent sur les membres, montent sur la crèrbe, se cabrent, se renversent, frappent des pieds, se moldent, mordent les personnes, prennent des attitudes impulsires: ils ont perdu le sentiment de tout dançer; ils se bi isent quelquefois la tète contre les murs.

Les bêtes borines poussent des beuglements effrayants, trappent du pied et de la corne, bavent et grincent des dents; les moutons se livrent à des sauts très grands; les porcs grognent, s'agitent, courent, se heurtent, grattent le sol arec les pieds, le groin et s'enfoncent dans la litière; les chiens sont inquiets, ils font entendre des aboiements plaintifs, tournent en cercle, en tonneau, mordent la paille, les barreaux, les portes, etc., et sont en proie à un délire furieux.

L'homme sous le coup d'une intoxication psychique perd graduellement la puissance directrice de l'attention et de la voionté. L'homme ivre, l'aliéné, l'épileptique ne s'appartiennent plus, ils ne peuvent se ressaisir, ils sont envahis par des idées dontils ne peuvent arrèter le débordement et c'est cette absence de frein qui constitue le délire. 
164 SÉMIOLOGIE DE L'APPAREIL DE L'INNERVATION.

On sent bien, au début de l'ivresse, que cette influence inhibitrice dirigeante est sur le point de nous échapper; et on fait de grands effor's pour essayer de la garder.

Ainsi l'état de saine raison parait ètre constitué par deux phénomènes fondamentaux : d'abord la notion complète de la réalité, notion qui est analogrue et presque identique à celle qu'ont les autres hommes; ensuile le pouroir directeur, inhibiteur qui constitue l'attention et la volonté.

Au contraire, l'état de délire est un état psychique dans lequel la notion de la réalité est nulle (rêve), incomplète (ivresse), différente de celle qu'ont les autres hommes (aliénation), et dans lequel aussi le pouvoir d'altention est diminué ou aboli.

Autrement dit encore, la perversion fonclionnelle de lintelligence porte avanl tout sur l'appareil de coordination, de direction et de régulation des idées (Richet).

\section{NIV. - IALLLCINATIONS.}

Définition. - C'est un trouble psychique caractérisé par une sensation sans objet.

Ce délire ressemble beaucoup au réve qui se produit pendant le sommeil physiologique. Il est dû à la mise en activité involontaire de la mémoire et des centres cérébraux sans qu'ils aient reçu d'excilation périphérique. Pendant l'hallucination, l'animal reproduit tous les actes qu'il accomplit dans le fonctionnement normal de l'organisme; lousles mouvements sont parfaitement coordonnés et exécutés.

Signification. - C'est un symptỏme d'états morbides plus ou moins complexes (rage, charbon)(1). Tous les animaux sont capables d'hallucinalions; c'est chez le chien qu'elles sont surtout fréquentes et faciles à constaler.

(1) Voy. ces maladies: Pathologie des maladies contngieuses de l'Encyclopédie vétérinaire. 
On a divisé les hallucinations en psychiques et sensorielles, très difficiles à différencier chez les animaux.

Chaque espèce d'intoxication entraine une hallucination spéciale.

L’imprégnation des cellules cérébrales par des essences

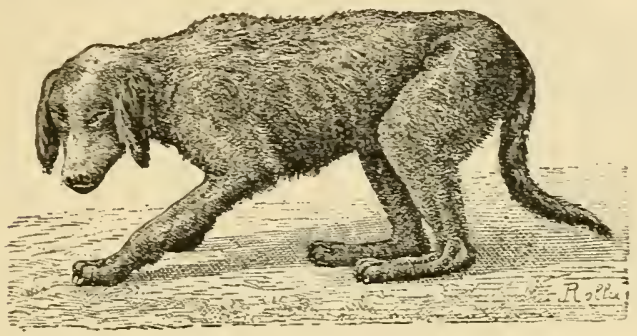

Fig. 36. - Hallucination caractérisée par la vision d'un obslacle, délerminée par l'essence de basilic.

excito-stupéfiantes produit des hallucinations de la vue à caractère terifiant. Avec la sauge, l'hysope et l'absinthe, les animaux sont en proie à des visions effray antes; mais au lieu de reculer, ils se défendent; au lieu de fuir, ils attaquent. Ils ont toujours un aspect courroucé; ils aboient, cherchent à mordre, ils deriennent furieux el mème féroces.

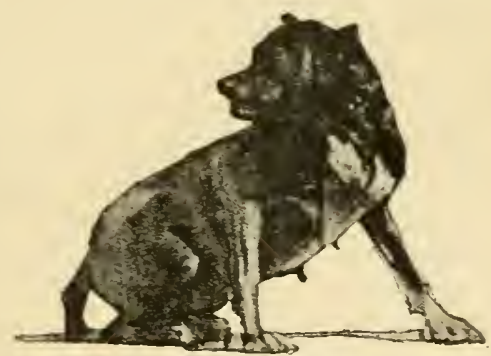

Fig. 3:- - Hallucinations agressires déterminées par l'cssence de sauge.

\section{Le chien court} effrayé avec une rapidité extrème et il s'arrète brusquement comme devant un obstacle insurmontable, les jarrets fléchis, l'oreille tendue, il fait volte-face, retourne en arrière, puis reprend sa course folle en avant et fran- 
chit d'un saut puissant ce qui l'avait arrèté en premier lieu. Il revient en arrière plusieurs fois en faisant un coude pour éviter le même obstacle et ressaute chaque fois à la mème place. Il exécute ainsi une sorte de rêve bien systé-

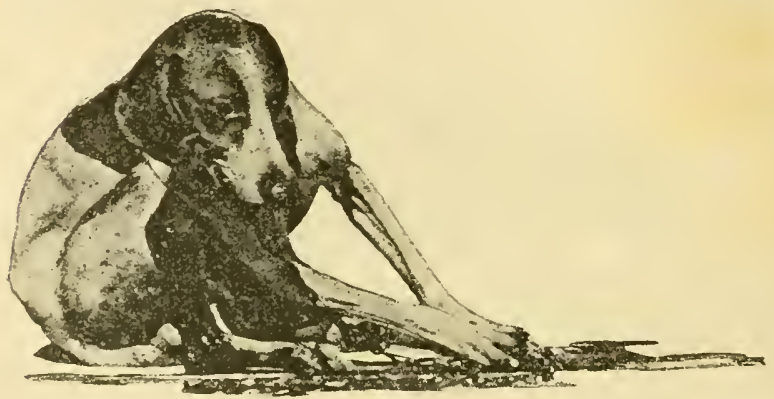

Fig. 38. - Hallucinations terrifiantes déterminées par l'essence de fenouil.

matisé qui implique une certaine logique dans sa réalisation.

La lavande, l'angélique produisent des visions qui glacent les chiens d'épouvante el leur enlèvent toute idée de se défendre. La menthe et l'origan déterminent des hallucinations de l'odorat; l'animal se met à quêter, à flairer; il lève le nez, cherche le vent, marche avec hésitation, l'œil fixe; il chasse un gibier imaginaire (1).

L'essence de fenouil produit des hallucinations terrifiantes, mais les animaux se rejettent brusquement en arrière pour éviter un péril imaginaire (fig. 38).

On observe parfois des hallucinations de la sensibilité générale dans l'intoxication par le vulnéraire; à tout instant, le chien se mord la queue, le train postérieur, le flanc comme s'il voulait se lébarrasser d'insectes dont il croit sentir les piqûres. Il bondit même parfois pour atteindre la mouche imaginaire à laquelle il attribue, sans doute, les fourmillements toxiques produits par les essences.

(1) Cadéac et Mleunier, Recherches exp'rimentales sur les essences. Intoxication par l'eau d'arquebuse, Paris, 1892. 
Les anesthesiques modifient aussi les fonctions cérébrales; le chien qui, soumis à l'inlluence du chloroforme, s'agite violemment, aboie, croit chasser, est la dupe d'hallucinations. Il en est de même de l'étalon qui, pendant l'éthérisation, hennit, sort le pénis et exécute tous les mouvements de la copulation (Bouley).

Sarradet a signalé le cas d'un bceuf qui, au travail, s'arrètait brusquement, les yeux hagarıls. A l'étable, il poussait des beuglements terribles et s'enfuyait tremblant couvert de sueur (1).

Chez le chieu, Thirion a observé des hallucinations de la vue consécutives à la maladie du jeune ige. Cet animal manifestait une impressionuabilité particulière à l'égard de la lumière; il fixait sans cesse une partie d'un mur blanc éclairé par le soleil; la lumière déterminait chez lui une hallucination coustante.

\section{XV. - ACCÈS RABIFORMES.}

Définition. - On désigne ainsi un ensemble de troubles sensitifs et moteurs analogues à ceux qu'on observe dans la rage, mais indépendants de l'action du virus rabique sur les centres nerveux.

Leur importance est considérable parce qu'on les observe particulièrement chez le chien et qu'ils peuvent simuler la rage au point de rendre le diagnostic de cette maladie très difticile.

Des états symptomatiques analogues ont été constatés chez l'homme, le cheval, les grands ruminants.

Pathogénie. - Ces fausses rages sont déterminées par des causes très variées:

Chez l'homme, par des émotions, des excitations, l'hystérie, le delirium tremens; clıez le clieval par le vertige;

(1) Sarradet, Revue vétérinaire, 1876, p. 221.

(2) Thirion, Recueil de méd. vét., 1898. 
chez les grands ruminants par l'ail (Pascault), par la fièvre vitulaire, les troubles gastriques (Detroye) ou sous l'influence d'une cause inconnue, comme la rage atténuée se terminant par la guérison (Repiquet); chez le chien

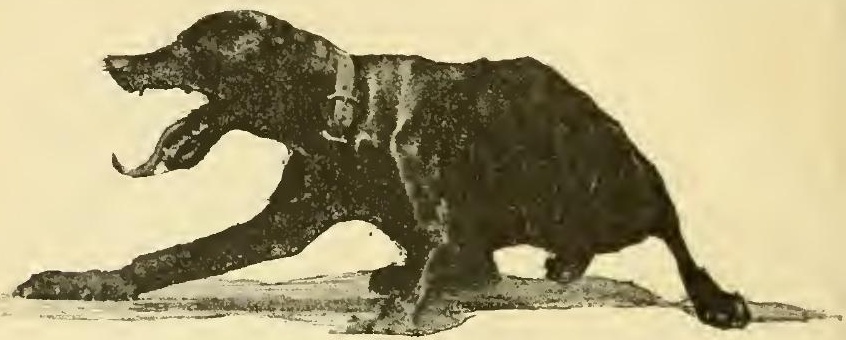

Fig. 65. - Caractère agressif d'un chien après l'absorption veineuse de 25 centigrammes d'esseuce d'hysope (Cadéac).

par l'épilepsic, la gastro-entérite, les empoisonnements (tanaisie), la maladie du jeune áge et surtout par l'arrét de corps étranger's dans ie pharynx, l'es tomac ou l'intestin, et par les affections vermineuses du tube digestif.

Cette relation entre les affections vermineuses, les corps étrangers dutube digestif et les manifestations rabiformes a été nettemenl établie par diverses observations (1).

En effet, les uns ont vu les symptômes disparaître après l'administration d'un purgatif, d'un vomitif ou d'un vermifuge qui chassait le colps étranger ou le parasite, d'autres ont trouvé à l'autopsie des chicus pris d'accès rabiformes des corps étrangers ou des vers accumulés dansl'intestin et n'ont puproduire la rage parl'inoculation du bulbe de ces animaux (Cadéac) (2).

Des symptòmes rabiformes sont quelquefois consécutifs

(1) Repiquel, Société des sciences vét., 1899, p. 91.

(2) Voy. Corps étrangers du tube digestif in Pathologie interne de l'Encyclopédie vétérinaire. 
à la gestation extra-utérine (Barzoff, ou à une fausse gestation (1).

Caractères. - Sous l'influence des causes énumérées ci-dessus, on peut roir se manifester lous les symptòmes qui caractérisent la rage. Les animaux deviennent tristes, taciturnes, leur caraclère est complètement changé; ils présentent une sensibilité, une impressionnabilité anormales, une altération des sens et de la voix. Tantót ils deviennent furieux, la bouche remplie de bave, les yeux élincelants et manifestant des envies de mordre; tantòt ils restent plongẻs dans la torpeur, se cachent, fuient quand on les approche. Ils peuvent être pris de convulsions, d'accès épileptiformes accompagnés de mourements convulsifs des màchoires, de salivation abondante, etc. Parfois la déglutition est impossible (corps étrangers dans le larynx), l'animal reste la gueule béante, avec écoulement de bave, regard triste, etr.

Tous ces symptómes peuvent être engendrés par des produits loniques, notamment par des essences.

Comment reconnaitre si l'animal qui présente ces symptômes est enragé ? - On peut recourir à l'inoculation intracranienne du bulbe de l'animal suspect, pratiquer l'examen histologique des ganglions cérébro-spinaux et sympathiques, rechercher le sucre dans ses urines.

$1^{\circ} L^{\prime}$ inoculation intracranienne du bulbe de l'animal sus. pect lève tous Jes doutes quand on obtient un résullat positil; mais un résulıal négatif ne permet pas d'affirmer absolument que l'animal n'était affecté que de symptòmes rabiformes. Des chiens qui ont communiqué la rage par des morsures et qui ont été tués pendant l'évolution

(1) Sbraggia, Il Nuoro Ercolani, 1901. Une chienne qui nasait pas été courerle depuis deux ans arait la manie de ta malernité; elle s"èlail bloltie dans une espèce de couchette qu'elle s'était creusée dans la lerre et cherchait à mordre; elle avait montré un an aupararant un accès semblable de pṣ̣cho. pathie.

C.ADĖAC. - Sémiologie, 2e édit.

II. -10 
rabique possèdentquelquefois un bulbe dépourvu de virulence parce que les germes de cetle affection progressant par la voie nerveuse n'ont pas encore atteint cet organe.

$2^{\circ} L^{\prime}$ cxamen histologique des gunglions cèrébro-spinaux donne-t-il des résultats caracteristiques? Van Gehuchten et Nélis, en 1900, signalèrent des lésions spéciales dans les ganglions cérébro-spinaux et sympathiques du chien mort de la rage. Certaines capsules endothéliales de ces ganglions renferment, à la place d'une cellule nerveuse, un grand nombre de petiles cellules tassées les unes contre les autres; dans certaines capsules, on apercoit encore une portion de la cellule nerveuse. Ces auteurs déclarèrent que ces lésions permettent le diagnostic post-mortem de la rage.

Ces faits furent recherchés par de nombreux observateurs et furent confirmés.

Vallée, notamment, formula les conclusions suivantes : "La lésion signalée par Van Gehuchten et Nélis est constante chez les animaux qui meurent de l'évolution naturelle de la rage. En ce qui concerne les animaux sacrifiés, on peut conclure avec certitude à l'existence de la rage si l'on trouvela lésion ganglionnaire; mais l'absence de celle-ci n'implique point la non-existence de la rage chez l'animal suspect. "

Belitzer est arrivé à des conclusions analogues.

La méthode de Nélis permet un diagnostic rapide chez tous les chiens morts de la ragre et chez la plupart de ceux qui ont été abattus au cours de la maladie.

Chez le cheral, le bouf, le monton et le porc les lésions du ganglion plexiforme sont moins significalives et elles ne permettent un diagnostic sir que chez les animaux morts et non chez les animaux abaltus.

Le ganglion cervical supérieur est, en général, moins alléré yue le plexiforme.

Les lésions des ganglions commencent dans la rage, nou par une prolifération de l'endothélium des capsules 
des cellules nerveuses, mais par une infiltration des cellules du ganglion par le tissu conjonclif. Cette infiltration est déjà visible assez souvent chez les chiens sacrifiés prématurément et chez les autres animaux morts de la rage. La proliféralion endothéliale est consécutive à l'atrophie des cellules nerveuses.

Ces lésions sont-clles spécifques? - Les vieux chiens présentent des lésions fréquentes de neurophagie de sorte que, suivant Vallée:

"La lésion des ganglions cérébro-spinaux et srompathiques est constante chez les animaux qui meurent de la rage; mais si le chien suspect est déjà vieux, la seule constatation de ces lésious est insuffisante, en l'absence d'autres signes rabiques, pour conclure à l'existence de la rage. "

$3^{\circ}$ La présence du sucre dans l'urine ou la glycosurie n'a aucune signification diagnostique; elle n'implique nullement que l'animal est enragé.

\section{XVI. - IIPNOTISIE. - CATALEPSIE.}

Définition. - Lihypnotisme est considéré comme une névrose ou un état particulier dı système nerveux déterminé par des manouures artificielles.

Cet hypnotisme ou grand hypnotisme de Charcot est dénoncé nar une hyperexcitabililé neuro-musculaire telle que la pression qui, au niveau d'un tronc nerveux, produit les mênes effets que l'électrisation. Cette forme d'hypnotisme n'intéresse pas à proprement parler la médecine vétérinaire.

Il en est tout autrement du petit hypnotisme, c'est-à-dire de la suggestion qui a pour liut de développer un état de suggestibilité à des degrés divers. L“idée suggérẻe peut devenir sensation, imagre, acte ou mouvement ou, inversement, elle peut neutraliser un mouvement (Bernheim), et engendrer la lethargie et la catalepsie. 
Le petit hypnotisme a été utilisé en thérapeutique humaine : la suggestion médicamenteuse, l'électrisation, le massage, les frictions cutanées, la métallothérapie, la dosimétrie, la magnéthothér'clpie ou traitement par l'application des aimants, l'homœopathie, le galvanisme, etc., sont des traitements qui s'adressent au moral des malades.

Les Procédés d'hypnotisation d'homme à homme sont très nombreux (1). On utilise général ement les anciennes pratiques des magnétiseurs ou les passes magnétiques avec l'hypnose par' suggestion verbale.

Ces passes sur la tête, le front, les épaules, consistent à faire des mouvements uniformes de haut en has, audevant des yeux, comme si en abaissant les mains, on pouvait faire fermer les paupières.

D'ailleurs l'hypnose et ses différentes phases (léthargie, cutalepsie, somnambulisme) peuvent s'obtenir d'emblée, l'un ou l'autre, suivant les sujets, par fixation du regard.

La léthargie se transforme en calalepsie par l'ouverture des yeux des sujets; la catalepsie redevient léthargie par l'occlusion des yeux ou l'obscurité. Les deux se transforment en somnamoulisme par friction légère du vertex du sujet et le somnambulisme devient de nouveau léthalgie par compression légère des globes oculaires. L'état de léthargie est rebelle aux suggestions (Bernheim).

En résumé, l'hypnotisme est un sommeil provoqué, la catalepsie une des formes de ce sommeil, caractérisé par une abolition du sentiment et de l'entendement, en même temps que chaque partie du corps conserve la position qu'on lui avait donnée antérieurement.

Il n'y a pas seulement la calalepsie du mouvement. On peut au lieu de laisser un membre dans la position dans laquelle on l'a fixé, l'agiter, lui faire exéculer tel ou tel mouvement et alors ce mouvement se continue, et cela

(1) Chomel, Le maynétisme animal. - Répertoire de police sanitaire, 1903, p. $10^{\circ} 2$. 
sans relàche, sans fatigue, et aussi sans conscience. La cutalepsie et l'automatisme ont ainsi des traits communs.

La catalepsie a été observée chez le chcral(Hering), le bauf (Landel), le loup (Liesering); l'hypnotisme a été provoqué chez les chats, les poules, les grenouilles, les cochons d'Inde, les écrerisses, les serpents, comme chez l'homme.

Les diverses espèces animales ressentent dilléremment l'influence hypnotique et dans chaque espèce, chaque individu est frappé avec une intensité variable. Les animaux très jeunes et très vieux paraissent complètement réfractaires (1).

Animaux susceptibles d'être hypnotisés. - Les faits de sommeil hypnotique naturel ou provoqué sur les animaux sont nombreux.

En particulier l'art de charmer les serpents par la musique est attesté par différents auteurs que cite M. Joly (2). Les sons bruts et monotones, produiraient chez ces animaux une sorte d'extase, de charme extatique (Edw. Tylor (3), Virey, etr.), el les sons brusıues et forls, des attaques épileptö̈des. C"est pourquoi les petits oiseaux tombent parfois foudroyés aux premières détonations du canon (Trasbot) (4), de mème que le type humain entre en catalepsie au bruit subit d'un tam-tam, d'une explosion et Inème, dans des cas plus rares, au lic-tac d'une montre. Il est vrai que chez lui l'attouchement de cerlaines régions du corps, dites zones hypnogénes (5), produit le même effet qui n'a jamais été signalé chez les animaux.

(1) Dr Bernheim, Hypnotisme, suggestion, psychothérapie. Procédés de la Salpétrière,

(2) Joly, Le magnétisme animal étudié chez les animaux. Manuscrit communiqué.

(3) Edw. Tylor, La civilisation primitive, 1876. - Ur Virey, Mours et instincte des animaux. - Dumeril et Bibron, Erpétologie générale, t. $1 \mathrm{~V}$.

(4) Remarque faite à l'École vétérinaire d'Alfort les jours de tir au canon sur le poiygone de Vincennes.

(5) D'après Pitres, il existerait des zones hypnogènes simples, à effets successifs, à effets incumplets. 
Aulant el plus que la musique, la lumière vive produit une véritable action fascinatrice (sur les poissons, les écrevisses, les mouches, les oiseaux).

"Des milliers d'oiseaux, dit M. Joly (1), trouvent la mort au sommet du phare de la Liberté devant New-York.

Le cheval du Paragruay, l'âne africain, accourent aux feux des campements.

Les dauphins sont attirés par les feux des pècheur's (2). "

Laction somnifère d'un miroir en rotation est constante chez les allonettes. La vue d'un autre animal peut d'ailleurs exercer ce même pouvoir fascinateur (Thomas) (3). La caille et la perdrix sont stupéfiées au nez d'un chien d'arrêt; le crapaud, les petits oiseaux sont fascinés parfois par les yeux étincelants du serpent (4).

Le crapaud attire aussi la belette; le serpent à sonnette, l'écur'euil (5), et de cette facon, les grands rapaces, immobilisent leur proie qui ne peut s'enfuir. "On prétend, dit M. Joly, que tous les ophidiens, depuis les énormes serpents d'Amériøue jusqu'aux vipères, ont le privilège de paralyser les batraciens et certaius oiseaux. On assure qu'à force d'altacher leurs regards étincelants sur la grenouille et le rossignol, par exemple, ils les plongrent dans le relâchement musculaire le plus complet et forcent l'oiseau chanteur à descendre de branche en branche jusqu’à terre. " Enfin il est des effets de suggestion lyppotique identiques obtenus par des excitations trusques et fortes, par la compression de certaines parties du corps (Lîte, nuque, nreilles), par le refroillissement ou par la peur (Richet, Foveau de Courmelles).

La perdrix, les cerfs, les eherreuils, très faiblement

(1) Joly, Op. cil.

(2) Foveau de Courmelles, Les facultés mentales des animanx.

(3) Communicalion verbale de II. Thomas a M. Joly sur les mours d'une araighée africaine à reflel métallique, atlirant ainsi des moucherons.

(4) Anédée Dupau, Leltres sur le magnétisme animal, 18:6. - Dumeril et Bibron, Loc, cit.

(5) D’après Ménault. Voir Thomson : Passions des animaux, p. 118. 
atteints par le plomb du chasseur, tombent brusquement pour se relever bientòt et s'enfuir au plus vite (1). Les compressions exercées de chaque côté de la tète des petits oiseaux, dit M. Joly, au niveau des oreilles, ou mème des commissures du bec, les laissent inmobiles et inertes dans la main. D'après Richet (2), une grenouille plongée dans de l'eau à $36^{\circ}$, sera immobile, inerte, comme anesthésiée; elle ne fera aucun mouvement et cependant le cœur battra encore et les muscles seront encore excitables. Les cheraux, soulevés de terre, sont encore en catalepsie (Preyer).

Azam rapporte que dans les foires du Midi, les bateleurs clarment les coqs en leur plaçant le bec sur une planche et en traçant sur cette planche une ligne noire ou un trait de craie passant entre les deux pattes de l'animal. En quelques instants, le coq tombe en catalepsie. Dès 1646 , le Père Kïcher employait un procédé analogue pour endormir les poules.

Czermack avait institué diverses expériences scientifiques. Il opéra sur des écrevisses, de nombreux oiseaux, des pigeons; répéta les expériences de fircher sur la poule, vit l'inmobilité persister après l'effacement du trait de craie, eut recours à des procédés divers (pression sur les oreilles ou dans l'angle de la mâchoire, objet devant les yeux ou sur la tête).

Il suffit même pour ol,tenir des effets analogues de pla. cer sous l'aile la tète d'un canard ou d'une poule après l'avoir quelque temps balancée. L'aninal à moitié endormi ne veut pas ou ne peut se déplacer; le cerveau paralysé ne peut commander aux muscles, qui deviennent calaleptiques.

Alix a endormi des chats en les regardant fixement dans Ies yeux après les avoir immobilisés. Les paupières sont ouvertes ou demi-closes et cependant le clignement

(1) Preyer, Die Kataplexie, Iéna, 1878.

(9) Ch. Richet, Essai de psychologie générale, p. 38. 
n'est pas totalement aboli; les mourements de déglutition et de respiration sont intacts. Il y a paralysie du mouvement volontaire des muscles de la vie animale ou plutòt, paralysie du pouvoir de relàchement des muscles.

Tarchanoff a noté sur les grenowilles chloroformées de curieux phémomènes qui ressemblent à la catalepsie. Au moment où "commencent à se dissiper les effets du chloroforme, les grenouilles ont des attitudes bizarres et, pendaut un temps assez court, elles présentent un état cataleptique très net. On sait qu'alors elles ont aussi des hallucinations.

Joly a repris la plupart des expériences antérieures ; il s'est efforcé de déterminer les meilleurs procédés d'hypnotisation sur les ponles, les cochons d'lnde, les oiseaux, les grenouilles, les crapands. Il a remarqué qu'un animal est plus facilement catalepsié en le plaçant sur un linge blanc, en lui faisant entendre le tic-tac d'une montre, en le faisant jeùner quelque peu, en le maintenant en captivité, etc..., et il a constaté de très grandes différences individuelles entre les espèces et mème de sujet à sujet. Il cite l'influence des caresses de la main et de la voix sur la louve, le chacal et montre que la pression progressive oculaire invite tout particulièrement le chien au sommeil. Et il conclut qu'on pourı peut-ètre utiliser un jour l'hypuotisme comme un agent de dressage ou d'anesthésie (1).

On peut admettre que les résultats obtenus par les célèbres dresseurs de chevaux (Pluvinel, La Guérinière, Rarey, Powel), comme les " tours » du cirque et la plupart des "figures ou airs " de manège étaient le fruit de la suggestion directe créant un automatisme cérébral, puis l'automatisme des mouvements. "C'est dans le dressage du cheval, dit M. le Dr Rouhet, que la volonté émer-

(1) Alix, L'Esprit de nos bêtes. - Joly, Guenon, L'Intelligence des animaux. 
gique de l'homme peut obtenir ses plus puissants effets, qui peuvent aller jusqu'à la suggestion (1). "

Le fameux Lœffler, dentiste américain, en abordant les chevaux les plus méchants (Galaor), opérait aussi vraisemblablement par la suggestion. De nos jours, les lords anglais pratiquent le sussurement des lèvres pendant le pansage du cheval, méthode qui rappelle un peu le procédé de domptage de lord Sullivan (par Eclipse, Arc-enciel) et qu'on avait, de ce fait, surnommé le Chuchoteur. Toujours et partoul, l'influence psychique a fait diminuer l'activité et la spontanéité au profit de la passivité et de l'aulomatisme. Et cet automatisme animal, étudié dans ses causes, dans ses effets, va fournir bien cerlainement l'un des chapitres les plus intéressants de la psychologie animale (Chomel).

L'état des facultés est différent suivant le degré du sommeil; il y a d'abord perte de la volonté, puis la conscience des choses extérieures disparait et au réveil le sujet a perdu le souvenir de ce qui s'est passé pendant le sommeil.

L'élat cataleptique est provoqué par l'impuissance du cerveau à résister aux excitations réflexes transmises mécaniquement aux muscles. Le mouvement et le relàchement volontaire sont également paralysés.

Fröhner cite un cas de catalepsie chez le chien; il a constaté des troubles de la motilité, des troubles psychiques, des troubles de la sensibilité, les divers organes étaient comme anesthésiés, il y avait perte de la vision, de l'odorat, de l'ouïe.

L'intelligence, la volonté et la conscience étant moins développées chez les animaux que chez l'homme, on conçoit que les procédés hypnogéniques soient souvent

(1) Voir les expériences tentées avec le cheval Germinal. Gazette hippique de 1901). - Consulter aussi Marcel Baudoin : Les intellectuels athletes (Gaz. méd. de Paris, no 4, p. 52). 
sans résultat sur eux, aussi la question est-elle encore très obscure (1).

Aruch a observé chez le clien trois cas de paralysie psychique ressemblant aux paralysies hystèriques connues cliez l'homme :

Premierement sur uu petit terrier : au moment où sa maitresse lui mettait son pardessus, on remarquait une faiblesse des extrémités postérieures, un changement dans la voix, une sorte de paraplégie et la production de réflexes sur toute la surface du corps. Le deuxième cas sur un chien maltais, la maladie lui était survenue au moment où sa maîtresse prenait son enfant dans les bras, l'animal paraissait très abattu. Un troisième cas sur un chien âgé de onze ans ayant subi une correction; il rentra dans sa niche, se coucha et présenta des convulsions des extrémités en mème temps que ses yeux offraient un strabisme divergent.

Les manifestations des états hypnotiques sont en somme t:ès variables. D'ailleurs la définition précise du sommeil hypnolique n'est pas encore établie à l'heure actuelle. Liebeault établit deux sortes de sommeil: le sommeil légor (divisé en 4 degrés) et le sommeil profond ou somnambulique (à 2 degrés). Pour Bernheim, le sommeil léger comprend 6 degrés et le sommeil profond, avec amnésie au riveil, comporte 3 degrés.

D’après la théorie de Bouchard, le sommeil serait dú à l'accumulation d'une substance narcotique résultant d'un travail de désassimilation. Suivant la thérie histologique, le sommeil résulterait de la rétraction du neurone (cellule nerveuse avec son prolongement cylindraxile de Deilers et ses prolongements protoplasmiques). Cette théorie permet d'expliquer l'état de veille et la brusquerie du réveil au milieu d'un lourd sommeil.

(1) Bystirie chez lechien (The Veterinarian, 1858, p. 577). - Accès spasmodiques chez le chien (Berliner, 1892, p. 177). - Hylmotisme, lype convulsif, nevralgie (.Monashefte, 1892, p. 519). 
Les etats hypnotiques ont pour base l'impressiomnabilité psychique, l'extrème fragilité du réflexe psychique, le plus délical de tous les réflexes. Aussi la suggestion hypnotique n'est pas seulement l'art d'endormir les malałles par la persuasion; c'est plutòl l'art de créer une psychopathie artificielle et passagère, sans sommeil, avec sommeil ou avec l'illusion du sonmeil. Bien qu'on ait pu déterminer, chez l'homme, par la parole, des suggestions de motilité, de sensibilité, des suggestions d'actes, des illusions et des hallucinations, tous les phénomènes hypnotiques ne relèvent pas uniquement de la doctrine de la suggestion.

Les causes prédisposantes en sont toujours l'hérédité, le surmenage, les émotions vives, les troubles psychiques, les maladie anéniantes. Le résultat est l'état d'automatisme du sujet aboutissant à des actes de psycho-dynamie (Chomel).

Et nous devons constater que les manifestations psychomotrices ne sont pas quantité négligeable dans la vie animale, car elles peuvent aboutir à une " psycho-biologie et à une psycho-thérapeulique » (Bernbeim).

\section{XVII. - PEUR.}

Définition. - On qualifie de peureux ou d'ombrageux l'animal qui s'effraye à la vue de quelque objet qui le sulprend, lui parail dangereux ou menaçant.

L'animal est peureux par hatitude : c'est alors un vice individuel, ou bien il est peureux par accident et, dans ce cas, la peur peul se communiquer à un grand nombre d'animaux (panique gènérale).

Lanimal de naturel peureux ou ombrageux manifeste son effroi à la moindre influence provocatrice : un bruit soudain, un mourement brusque, un objet qui se montre subitement comme un train, une locomotive le font camper, pointer, faire des écarts et rien ne peut le calmer, et l'empècher de fuir. 
Ce qu'il $y$ a de remarquable, c'est qu'un cheval, par exemple, qui aura eu peur en un lieu quelconque ne repassera en ce lieu qu’avec un sentiment de crainte.

La peur est un véritable vice, car les animaux yui en sont affectés deviennent de plus en plus peureux, et semblent souvent chercher un objet dont ils pourraient avoir peur. Ils deviennent presque inutilisables ou causent des accidents nombreux.

Remarquons qu'une vue basse peut rendre un animal peureux; mieux vaut un cheval aveugle qu'un cheval myope.

Les animaux peureux par accident sont très nombreux; tous les jours, sur les champs de foire, on roit des paniques, qui ont quelquefois des conséquences désastreuses. Sur les champs de bataille, mème, on a vu des régiments de cavalerie en entier pıis subitement de panique, de sorte que l'armée a été privée d'une de ses ressources les plus précieuses.

La panique est un affolement complet des animaux réunis en masse, accompagnée d'une débandade générale, d'une fugue effrénée, sans direction, sans but, sans destination (llugues). La panique est la fuite subite, imprévue, affolèe d'un grand nombre d'animaux rassemblés.

Chez les moutons, animaux très peureux, les paniques sont très communes : il est vrai que cliez eux, il suffit qu'un seul mouton donne le signal pour que tous les autres suivent.

"Sous l'influence de circonstances qui agissent sur la vue ou l'ouie des aninaux, l'impression se propage d'un animal à l'autre, de proche en proche, mais avec une grande rapidite; quelquefois cette impression se produit avec une soudaineté extraordinaire sur tous les poiuts du champ de foire.

"Le bourdonnement quui accompagnne les grandes réunions d'hommes, le tintamarre des saltimbanques et de leurs bruyants instruments, le sifflement de la vapeur des 
machines, le hruil, d'une scie opérant avec des mouvements rapides, l'influence d'une luttequisurgit entre deux ou plusieurs sujets mal contenus : telles sont les causes capables d'égarer les animaux, surtout quand leur système nerveux est déjà surexcilé par ces grandes réunions auxquelles ils ne sont pas habitués " (Abadie) (1).

Les borins peuvent ètre pris aussi de panifue; mais après leur premier mouvement de fuite, ils ont quelquefois un retour sur eux-mêmes qui les fait revenir sur l'obstacle ou l'objet qui les a d'abord effrayés.

Pour tous les animaux, la peur est d'ailleurs en rapport avec leurs moyens de défense, et les bovins sont par là mieux partagés que les ovins ou les équidés. On a souvent attribué les paniques des boufs à la présence de mour hes : de là ce nom qui leur est donné.

Dans l'cspice clievaline, on voit très souvent de ces paniques sur les champs de foire ou dans les troupeaux au bivac.

Certains auteurs attribuent ces paniques à la présence de bètes fauves, de serpents. D'autres, comme Decroix, invoquent une aclion electrodynamique. Les changements brusques de température, l'arrivée de la pluie, les orages, un bruit produit par le bourdonnement d'un insecte, le combat de rhiens, de deux taureanx, ou la brisure d'une branche d'arbre peuvent aussi amener ces accilents.

\section{XXIII. - RËTIVITÉ.}

Caractères. - La rétivité est un vice caractérisé par le refus d'obéir. Les animaux rétil's sont inutilisables à la selle comme au trait (rétivite absolue), ou ne sont rebelles qu’à un de ces services (rétivité relutive).

Les variélés le rélivité relative sont trẻs nombreuses :

(1) Abadie, Panique ches les animaux (Rerue vét., 1877, 1. 496 ).

Cadéac. - Sémiologie, 2e édit. I1. - 11 
chevaux rétifs pour to cavalier, pour le trait, pour la voiture lourde; clevaux rétifs quand ils sont seuls et qui vont bien en paire.

Il en est d'autres qui refusent de suivre un chemin, de passer derant certains obstacles, devant une maison, ou qui s'arrêtent brusquement et se laissent baltre indétiument plutôt que de se déplacer.

Le clueval rétif est donc celui qui ne vent pas obéir à celui qui le monte ou qui le conduil. Docile, tranquille à l'écurie ou au pâturage, il n'a de caprices (fu'au travail.

les manifestations de la rétivité sont très différentes. Suivant qu'elle est active on passive, le cheval s'agite ou reste immoliile.

Quand il s'agite, l'animal est intraital,le. Il cherche à se débarrasser de sou cavalier par de brusques ćcarts, des sauts de mouton, en se cabrant et en ruant. S'il est attelé à une voiture, il recule, brise les traits et est insensible aux coups commeaux caresses de son maitre. Dans cet état, l'animal présente une respiralion accélérée, la sueur est abondante, le cour bat d'une façon désordonnée, la tète est expressive, les naseaux el l'oil sont largement ouverts. La pliase d'excitation est plus ou moins longue; la fatigue a raison de sá rétivité. A cette phase succède alors un abatlement parfois considérable, qui rend l'animal maniable.

Quand il reste immobile, l'animal, pour une raisou souvent ignorée, ne veut pas arancer, il se campe sur ses membres et u'écoute ni la voix de son conducteur, ni les menaces ni les comps, mais, remarque curieuse, si on lui fail rebrousser chemin, il se conduit comme un animal docile.

Bien souvent, cette rétivité, caractérisée par le refus d'avincer et de reculer, s'observe chez des animaux altelés à des voitures plus clıargéos que d'ordinaire sans l'ètre au delà du poids qu'ils peuvent traìuer.

Le mulet présente très souvent cette rétivité passive 
qui peut se montrer aussi bien à l'écurie que dans les sentiers difficiles et escarpés des montagnes, rétivité presque toujours constatée quand il est seul.

Pathogénie. - L'àne et le mulet sont très fréıuemment rétifs; leur entêtement est proverbial. L.es chevaux nerveux, de race distinguée, sont plus sujets à ce vice. C'est quelquefois un symptôme des affections des centres nerceux (méningo-encéphalite chronique et tumeur du cervcau), des altérations des barres, d'une hyperesthésie du tégument engendrée par la pression des harnais (selle trop sanglee, collier trop étroit, mal de garrot, d'encolure, etc.).

Les chaleurs et la nymphomanie chez la jument, les parasites intestinaux, contribuent à engendrer la rétivité. On a mẻme cherché à établir une relátion entre la rétivité des cheranx et les altérations profondes des principaux viscères de l'organisme. Siegen a constaté chez des animaux rétifs le volvulus intestinal, la rupture du diaphragme; l'hypertrophie du caur; l'invagination du cæcum dans le côlon. Mais il faut reconnaitre que la volonté de l'animal a une large part dans les manifestations de ce vice : le cheval n’est jamais rétif quand il se dirige vers son écurie. Les signes de la rétivité n’apparaissent que lorsqu'il doit faire un travail pénible, suivre un chemin inconnu on accidenté.

Le caractère entèié des animaux; l'excès de fatigue, le changement de conducteur sont des causes de rétivité. Des animaux très doux, très obéissants deviennent rétifs et mécliants en changeant de conducteur.

\section{XIX. - MÉcIIATCETÉ.}

Par méchanceté, on entend l'habitude ricieuse qu'ont les animaux de faire usage de leurs moyens de défense

(1) Siegen, Annales de méd, vét., 1879, p. 64t. 
18.' SÉMIOLOgIE DE L'APPARELL DE L'IXNERVATION.

pour altaquer l'homme qui veut les approcher, les monter, les atteler, les panser ou les ferrer.

Caractères. - Les modalités de ce vice sont très variées : chevaux mordeurs, rueurs, ou serrant les persomnes qui les abordent ou les soignent contre les parois de la stalle ou qui se cabreut et se renversent. Beaucoup le chevaux sont chatouilleux; ils couchent les oreilles, crient, montrent les dents quand on les approche; ils ne sont pas méchanls.

Le cheval mordeur se jette indifféremment sur l'homme et les animaux ou exclusivement sur une seule personne : il montre les dents en couchant les oreilles dès qu'on l'approche, ou mord sournoisement. Parfois, il s'acharne sur sa victime qu'il foule aux pieds avec férocité.

Le cheval rueur donne un coup de pied en vache quand il projette un piet de derrière en avant el de cóté; il lance un coup de pied en arrière ou projette avec force les deıx pieds; quelquefois, il ne rue qu'a la vo.ture ou quand il est en cheville; parfois il frappe du devant, se cabre et projette brusquement les membres antérieurs sur les personues ou les chevaux voisins; d'autres ne se livrent à ces monvements désordonnés que lorsquon veut les seller, les brider, les monter ou les atteler; d'autres s'emportent, s'emballent quand ils sont attelés et brisent tout.

l'athougénie. - La méchanceté est quelquefois une manifestation lıéréditaire, ou le résultat d'une impressionnabilité excessive.

Le dressage insuffisant, les mauvais truitements, la douleur déterminéc par les harnais ou par des blessures, l'excitation produite par les piquires de mouches, laons, hippobusques, la nymphomanic chez la jument, peuvent lui donncr naissance.

Certaines femelles ne deviennent méchantes qu'après 
la mise-bas; beaucoup de chevaux entiers très méchants deviennent très doux quand ils ont subi l'opération de la castration (1).

La rélivité et la méchanceté sont quelquefois liées à une castration incomplète, comme en témoignent deux observations de Bonrnay; l'extirpation des cordons testiculaires a suffi pour rendre les animaux très loux.

On peut maitriser les animaux par suggestion et par des moyens mécaniques comme la bride Norton, etc. (2).

Celte bride d'entrainement consiste en une longe tressée en lissu extrèmement souple (corde de coton) et portant trois anneaux. L'anneau 1 est à une extrémité de la longe; l'auneau 2 est à environ 70 centimèlres de l'anneau 1; l'anneau 3 est à environ 16 centimètres de l'anneau 2.

l.a portion comprise entre les anneaux 2 et 3 est mise dans la bouche du cheval à la facon d'un mor's de filet, l'anneau 2 étant ì droite de la bouche.

La portion comprise entre les anneaux 2 et 1 passe en tìtière par-dessus la tête du cheval et vient relomber à rauche de la tète, l'ameau 1 à hauteur de l'ail.

Ceci fait, passer l'extrémité de la longe (qui a son point d'insertion à l'anneau 3) dans l'anneau 2, tirer pour que la longe fasse gournette, puis passer l'exllémité libre de la longe par-dessus l'encolure, l'engager dans l'anneau 3, puis dans l'anneau 1, puis enfin dans l'annean 3, el tirer à soi pour ajuster le toul.

Dans ces condilions, et tonjours sous la léserve que la longe est firite d'un tissu très souple, chaque fois que le cheval résiste, il se prend lui-mème dans un triple noud conlant.

Au contraire, chargue fois qu'il cède en se portant en

(I) Voy. Méchanceté et Rétivité in Médecine légale de l'Encyclopirlic vitivinaire, par Gallier

(2) Aureggio, Diessage des chevan. michants, menrs, vicienr, froploms, nerveux, ombrageux, ne se laissant ferrer, ni monter, ni atteler (société de Sciences véterinaires, 1902). 
186 SÉMIOLOGIE IE L'APPAREIL DE L'INNERVATION.

avant, il haisse la lète, et les nouds se desserrent d'euxmêmes.

On comprend donc très bien qu'un cheval qui a ressenti plusieurs fois la douleur inlligée par ce triple noeud coulant, n'ait plus qu'une idée: s'y souslraire, et pour s'y soustraire, soit obligé de se porter en arant. Cette bride nous rend de très grands services à la clinique; les animaux les plus difficiles et les plus méchants sont maìrisés en quelques minutes.

\section{XX. - IMMUBHLTÉ.}

L'immobilité est un syndrome caractérisé prar divers troubles moteurs engendrés par une dépression cérébrale.

Pathogénie. - L'inmobilité apparait dans l'hydrocéphalic chronique, l'encéphalite; elle est quelquefois la conséquence le tumeurs intrurentricultires, de tumeurs osseuses de la boîte cranienne, d'hypertrophic, de liystes et de concrétions des plexus choroides (myxomes, cholestéatomes).

En résumé, toules les influences qui diminuent progressivement la capacité de la cavité cranienne et le fonctionnemeut de l'encéphale eıgendrent ce complexus symptomatique appelé immobilité. Que ces causes agissent de dehors en dedans, ou de dedans en dehors, elles exercent leur influence sur toutes les parties de l'encéphale en raison de l'inextensibilité de la boite cranienne.

Ancune parlie du cerveau n'est soustraite à cetle inlluence déprimante. L’angmentation de la masse cérébrale, c'est-à-dire l’accroissement du contemu pour un contenant imrariable, engendre, en vertu du principe de l'ascal, un surcroît de pression qui se fait également sentir sur tous les organes renfermés daus la cavité cranieune.

Les circonvolutions s'aplatissent, le liquide sous- 
arachnoïdien reflue dans le rachis, la circulation de retour est ralentie ou arrêtée : de là, la congestion passive, des adèmes, de l'hydropisie ventriculaire, de l'ischẻmie ou mème des hémorragies passives, lous phénomènes qui dépendent exclusivement et fatalement d'une expansion excessive de la masse intracranienne.

La compression est et demeure toujours le phénomène essentiel. La torpeur, la somnolence, l'allaiblissement intellectuel, la diminution de l'activité bulbaire sont la conséłuence de cette action mécaniıue. L'irritation déteruninée par les agents de compression peut manquer ou passer inapercue; elle est toujours secondaire; la substance cérébrale étant refoulée et non létruite, les fonctions sont obscurcies, et non supprimées.

Exceptionnelle chez le bouf, le mouton et le por'c, l'immobilité est fréquente chez le cheval. Cette fréquence résulte de la diversité des affections susceptibles de l'engendrer. Elle serait plus commune chez le cheval hongre que chez Iétalou; on la rencontre plus souvent cliez les animaux des races du Nord que chez ceux qui appartienuent aux races du Midi.

Caractères. - Les elfets de cette compression lente du cerveau se traduisent par un ensemble de troubles sensitivo-moteurs, sensoriels et psychiques significatifs quoique disparates. De fait, il n'existe pas de symptòme constant.

L'AFFaiblissemext intellectuel est le plus fixe de tous. Ce signe s'accuse par des troubles variés de la physionomie, de l'attitude du corps, de la démarche et par l'incapacité à vouloir et à agir. Le stimulus de l'activité normale est diminué ou éteint; la lenteur de toules les opérations cérébrales est le phénomène dominant.

La physionomie reflète généralement cet étal de dépression et de torpeur. D'autres fois, la tète est relevée d'une manière anornale; l'œil est hagard, brillant, grand ouvert, imbécile; les oreilles exécutent les mouvements variés 
188 SÉMIOLOGIE DE L'APPAREIL DE L'INNERVATION.

et sont portées irrégulièrement; l'animal tressaille au moindre bruit : on peut le croire en proie à une excitation anormale, mais ses impressions ne durent pas: l'indifférence, la passivité reprennent ordinairement le dessus.

L'attitule exprine parfois l'inertie, l'immobilité, l'inpuissance; l'animal semble avoir perdu la faculti de réagir; il deneure dans les attitudes anormales qu'on lui a données on yu’il a prises. Parfois, il commence à se déplacer; mais il oublie de continuer et sa tète, tournée vers la croupe, inclinée de côté ou fortement relevée, trahit l'arrêt ou l'impuissance de sa volontí. L'activité psychique volontaire est remplacée par une activité iout automatique, d'où résulte une iudécision dans toutes les sensations. La perception est affaiblie et manque de netteté.

La demarchc accuse tantòt cette délaillance de la volonté, tantòt une sorte d impulsivité que rien ne peut maitriser. Daus le premier cas, l'animal se laisse conduire sans résistance; il marche lentement, automatiquement; dans le second, il ne subit aucune influence; tel cheval refuse absolument d'aller à droile, tel autre à gauche; il va sans rien voir; il ne reconmait pas les obstacles qu'il rencontre sur son chemin; nous avons vu un cheval qui allait butter contre un arbre ou contre le mur chaque fois qu'on voulait l'empècher de rentrer à l'écurie. La mémoire est obnubilée; le cerveau est incapable de grouper et de coordonner ces sensations dont l'empreinte est toujours très superficielle; l'animal ne distingue pas un fossé d'une ornière.

L'affaihlissement de sa sensibilite l'empêche d'obéir; la pression du mors ne l'arrète pas quand il est lancé; les coups d'éperon ne le font pas avancer yuand il tend à reculer ou à se renverser. On peut le voir se jeter de cóté, franchir les parapets qui horılent les routes, s'arrèter brusquenent saus pouvoir avancer, 
parce que, sous l'influence de la dépression des actions vitales, il a perdu lå faculté de vouloir et rien ne peut le pousser à l'action. Cette incapacité de la volonté est quelquefois partielle. Dupuy rapporte qu'un cheval qui ne pouvait prendre le trot allait facilement au pas et au galop.

fieneralement il ne peut reculer; ce signe se manifeste d'emblée ou après un certain temps d'exercice au trot : parfois l'animal effectue quelques pas de recul, puis les membres antérieurs ne sont plus soulevés et le recul devient impossible. D'antres fois, l'animal ne perd la faculté de reculer que lorsquil a un poids à déplacer; il commence par labourer le sol de ses sabots, puis il se jette de còté ou se renverse lans les brancards. A ces symptòmes s'ajoutent quelques troubles inconstants que nous allons indiquer.

Les convulsions ÉPILEPTIFormes apparaissent quelquefois à la suite de compressions rapides; elles sont généralement partielles (Voy. Tumeurs cérébrales); elles sont localisées à la tête ou à la partie supérieure du corps; lanimal ne tombe pas; elles peuvent se montrer à l'écurie, mais elles se manifestent le plus souvent après un exercice plus ou moins violent.

Les Accés de vertige peuvent se produire dans le cours de cette maladie, sous l'influence d'une congestion cérébrale consécutive à la compression. "A l'écurie, tantôt ils poussent au mur avec tant d'énergie qu'ils s'excorient la peau du front et des orbiles; lantòl ils se redressent sur leur train de derrière et restent quelyues instants dans l'atlitude du cabrer (Dupon), les sabots antérienrs appuyés sur le fond de la mangeoire ou mème engagés entre les barreaux lu ràtelier; d'autres fois, enfin, ils lirent au renard sur leurs longes, se renversent et se livrent par terre à des mouvements désordonnés. Quand ces troubles se manifestent au moment où les animaux sont altelés, ils deviennent immaitrisables, soit qu'ils se 
portent en avaut, soil qu'ils se jetteut de côté, soit que, s'agilant sur place, ils se cabrent et se renversent (II. Bouley) (1). I'autres vont en cercle dans une direction déterninée.

L'HYPERESTHÉSIE CUTANėe est quelquefois très prononcée ; il y a des chevaux affectés de compression cérélurale qui sout méchants, ombragenx et inutilisables.

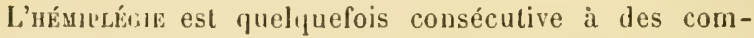
pressions unilatérales occasionnées par des tumeurs. signalons encore des troubles cardiaques, les paralysies de la langue, des michoires, du pharynx, le nystagmus et le plosis (Dexler). Dans quelques cas d'immobilité, on remarque de l'amaigrissement musculaire localisé, des anesthésies cutanées limities, des monoplégies (Dexler).

L'amaurose a été quelquefois signalée.

Conditions susceptibles de modifier l'intensité des symptòmes. - Le travail est une cause d'aggravation de tous les symptònes; certaius clsevaux dont la santé semble alsolument parfaite au repos manifestent, après une course, des signes prononcés d'immobilité.

La respiration et l'lématose sont alors plus difficiles; la teusion sanguine est augmentée, le cerveau se congestionne; la pression est augmentée dans les capillaires et l'oxygùne n'arrive plus en quautité suffisante pour assurer l'activité complète des zones motrices el des zones sensitives.

Pendant les ehaleurs de l'éle, les symptòmes s'exagèrent; crpendant Wolstein prétend qu'on a beaucoup exagéré les effets de cette cause. On constate généralement une allénuation de tous ces signes dès le commencement de l'automne jusqu'au printemps.

la dicte el une nourriture pauvie atténuent les symptomes; la prélıension de grains les exaqère. Les

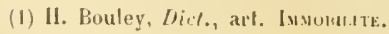


observations de Dieckerioff ne corroborent pas cette opinion: les chevaux immobiles, peu nourris, auxquels on donne une grande quantité d'avoiue, sont excités; ils sont plus maniables et supportent mieus le travail.

Chez la jument, l'époque des chuleur's est une cause certaine d'aggravation des symplônes. L'élal de gestation, au contraire, les atlénue. Dieckerlıoll a également constaté que la sécrétion lactée fait office de dérivatif; les symptômes sont moins appréciables; ils augmentent d'intensité pendant la première semaine du sevrage du poulain; puis le sang se répartit plus régulièrement et l'exagération des troubles disparait.

La plupart des maladies aiguës (catarrhes aigus ou chroniques, pneumonies, entérites, affections du foie) exagèrent la pression intracérébrale el aggravent tous les symiptòmes.

Les maurais traitements exercent une action analogue; mais les modificalions symptonualiques produites par celte cause sont passagères. Ces soltes de paroxysmes n'ont aucune inlluence sur la marche générale de la maladie (Dieckerhoff).

La marche des troubles enı́ndrés par la compression du cerveau n'offre rien de fixe; elle dèpend de l'évolution de la cause provocatrice : méningite, paclıyméningite, tumeur, elc. Que l'évolution soit lente ou rapide, le sujet est incurable; il peut mourir subitement par paralysie dn cerveau, par hémorragie, ou à la suite d'une chute par commotion cérébrale ou choc du liquide céphalo-rachidien. Ordinairement, il devient intitilisable; il est livré à la boucherie ou meurt dans le marasme, par insuffisance de nutrition ou d'alimentation.

Diagnostic. - Le diagnostic de l'insuffisance du fonclionnement cérébral, qu'on désigne sous le nom d'immolilité, présente souvent des difficultés presque insurnontables 
Le degré d'intelligence varie chez le cheval comme chez l'homme. Indéperdanment de toute altération cérébrale acquise, certains chevaux sont peu intelligents ou imbéciles, ils ne sont jamais véritahlement immobiles; ce sont des dégénérés, mais non des malades. Leur cerveau a senlement éprouvé un arrèt de développement. Leur tête est lourde, parfois mal conformée, les yeux iniutelligents, hébétés, hagards; les oreilles ont un jeu inégal; les membres sont posés en dehors de la ligne d'aploml, trop en avant ou trop en arrière; les réactions contre les piqûres sont nulles ou insignifiantes; on peut leur donner et leur faire conserver me attitude anormale.

Beaucoup te ponlains de race commune ou de clievan. de quatre à cinq ans peuvent ètre regardés comme immobiles; mais il est à remarquer que ces animaux reculent, se nourrissent normalement et ne croisent pas les membres antérieurs. Cependant, nous avons vu un cheval parfaitement sain dont les deux membres antérieurs étaient constamment croisés au repos.

D’autres causes que le défaut d'intelligence peuvent faire croire à l'immobilité. A signaler le lymphatisme, la fatigue, les affections typhoüdes, les affections le l'oreille, les collections purulentes des sinus sphénoïdaux et du labyrintlre elhmoidal, la congestion cérébrale simple, la méningite cérébrale, l'cmpoisonnement par les narcotiques et loutes les affections susceptibles d'empêcher l'animal de reculer (tour de rein, maladics des jurrets).

Il convient d'ajouter que les chevaux vieux el avenyles tienuent la tète basse et accusent une indifférence profoude pour tout ce qui les entoure; mais ils ne croisent pas les nembres comme les chevaux immobiles.

Les signes les plus nets de l’immobilité sont l'héhétude, l'inconscience; à l'écurie, le cheval n'a pas l'instinct le prendre le foin: si on lni en présente une poignée, il la saisit, la mâche quelque peu, puis la laisse tomber; si on lui offre à boire, il plonge la tête au fond du seau 
et ue la retire brusquement que lorsque l'isphyxie est imminente.

En allure, il est mou, indécis, il recule difficilenent, tourne avec peine, quelquefois avec plus de difliculté d'un còté que de l'autre. Lui déplace-t-on mu membre? il le laisse dans la position, quelquefois pínible, qu'on lui a fait prendre.

Parfois il arrive que ces signes, très intenses pendant une période variable, s'atténuent peu à peu pour réapparaitre ensuile (Mauri) (1).

Le syndrome immobilite étant reconnu, il faut clıercher à déterminer la cause provocatrice de ces troubles : tumeurs, liydropisie des ventricules. La constance de l'affaiblissement intellectuel est le signe le plus caractéristipue de l'hydropisie ventriculaire; l'excitation, le iertige, les troubles épileptiforınes dénoncent une tumeur du cerveau, des méninges, ou de la paroi cranienne.

\section{XXI. - MPOPLEXIE.}

Définition. - L'apoplexie est un symptôme caractérisé essentiellement par la perte subite des fonctions de la vie de relation.

Dans l'apoplexie, il y a, en elfet, abolition brusque et simultanée de toutes les fonctions cérébrales, intelligence, sersibilité, motilité volontaire sans modification essentielle de la respiration et de la circulation. Elle mène rapidement à la mort, malgré la persistance momentanée des fonctions de la vie végétalive.

Sou invasion est soulaine; les symptòmes ont la brusquerie d'une attuque; le sujet s'afliaisse, tombe inerte dans un coma profonl (lig. 40). Toutes les fonctions nerveuses (motrices et sensitives) sont suspendues; la respiration et lat circulation se ralentissent; la température s'abaisse ;

(1) Voy. Pathologie interne de l'Encyclopédie vétérinaive, t. VIII, et Immobulité in Jurisprudence de l'Encyclopédie vétérinaire. 
194 SÉMIOLOGIE DE L'APPAREIL DE L'INNERVATION.

le pharynx, le larynx et le voile du palais sont souvent paralysés; il y a des accès de suffocation, des troubles de la déglutition, de la constipation et enfin de la grlycosurie et de l'albuminurie transıtoire. A ces phénomènes de la première heure succède fréquemment une accélération rapide des fonctious respiratoire et circulatoire, prélude d'une mort prochaine.

Pariois le sujet récupère peu à peu les fonctions nerveuses, mais il y a loujours une moilié du corps plus

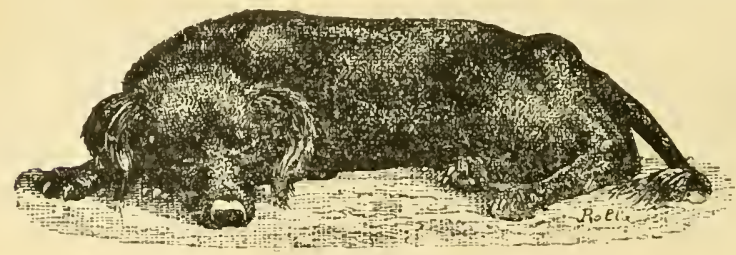

Fig. Ho. - Etal apopleclique ches le chien.

affectée que l'autre (hémiplégrie), comme les réllexes provoqués l'indiquent; la sensibilité et la motilité demeurent longlemps asymélriques.

Signification. - L'état apoplectique résulte d'une action d'arrèt exercée par l'irruption brusque de sang dans l'encéphale.

On l'observe daus les hemorragies círébrales ou míchllaires qu'on qualifie justement d'apoplectiformes en raison de la rapidité d'évolution des troubles qu'elles produisent; l'apoplexie apparait plus tardivenent dans l'oneéphalite, la myélite, la míningite.

Les embolies qui suppriment hrusquement la circulation dans certains territoires nerveux déterminent l'apoplexie; le couy de chaleur, congestiomant les centres nerveux, aboutit an même résultat; les frictions trop irriluntes sur 
la tige dorso-lombaire ou sur le crâne amènent des symptònes apoplectiformes.

Certaines maladies infectieuses produisent ce phénomène; les unes, grâce à des localisations microbiennes dans les centres nerveux; les autres, grìce à des toxines qui agissent sur certains centres. Ainsi dans le charbon bactiridien, le rouget $d u$ porc, la fievere typhoüde, le cholira des oiseaux, certains empoisonmements comme celui qui est produit par le bleu de Prusse, aloutissent à l'apoplexie.

Certaines affections du corur et des vaisseaux, en prirant plus ou moins de sang les centres nerveux, donnent le mème résultat. C'est la brusquerie du début des accidents (chute de l'animal, perte de conuaissance, etc.), qui caraclérise l'attaque apoplectiforme.

Sa cause intime réside dans l'anémie corticale produite par l'abaissement de la pression artérielle, conséquence do la rupture vasculaire, dans le choc hémorragique et la compression de la surface entière des centres nerveux et enfin dans l'inhibition des centres nerveux. L'apoplexie ne mérile ce nom que si elle est instantanée; la perte de connaissance graduelle constitue le coma; la syncope s'en différencie par l'affaiblissement du cœur et de la respiration; l'usphyxie, par la cyanose el le refroidissement des extrémités; l'cipilepsie, par le retour rapide de l"̈ntégrité fonctionnelle.

\section{XXII. - COMA.}

Le coma est un syndrome caraclérisé essentiellement par la perte du mourement, de la counaissance et de la sensibilité, les fonctions de circulation et de respiration continuant à s’accomplir. Lá dépression cérétrale, la somnolence, la stupeur en constituent les différents degrés. Dans tous ces cas, la perte de conmaissance cst incomplète. 
Pathogénie. - Toutes les alfections du cerveau, des múninges et les tranmatismes craniens peuvent le produire. On l'observe également dans l'ruilepsie, le tournis, les malarlies infectieuses, certaines maladies hépatiques, dans les coliques gastro-intestinules par surcharge alimentaire, l'mimie, l'intoxication par le jlomb, l'oxyde de carbone, l'alcool.

Le coma est dì à l'épuisement des cellules nerveuses (coma succédant an vertige) ou à l'imprógnation des cellules par des substances toxiques, microbienues (charbon, gourme, seplicémic, fierre lyphoüde, coryza gangreneu.e lu bouf, pestc borine), par des poisons de l'organisme (maladies du foie, du rein), par des poisons externes (plomb, oxyde de carbone, opium, solanés, champignons).

Dans quelques cas, il résulterait de l'unémie rérébrale ou d'une hématose incomplèle (maladies du poumon, du cour).

Il est. quelquefois déterminé par des conlusions du cràne qui déterminent l'inhibition des centres sensitivo-moteurs; le coma est une manifestation de l'insolalion.

Caractères. - Là tète de l'animal est abaissée et appuyée sur un objet quelconque, le plus souvent sur la mangeoire; on observe de la sommolence, de l'lébétude, une perte de conscience évidente. Le sujet ne fait nulle atteution à ce qui l'entoure; il ne réagrit à aucune excitation; si on lui présente des aliments, il ne les prend pas, et si on essaye de les lui faire prendre le force, ils passent souvent dans les voies respiratoires et jrovorguent la toux.

Le coma doit être distingué du sommeil; il ollre les caractères objectifs du sommeil, mais c'est un sommeil morbide, c'est-á-dire lourı, profond, continuel. Les excitations extérieures sont impuissantes à réveiller les facultés abolies, landis que dans le sommeil, ce rércil est facile. 


\section{XXIII. - TICS MOTEURS.}

Définition. - L'étude des tics des animaux est peu avancée, malgré leur fréquence clıez les équidris, les horillés et mème les suidés.

On les connaîl so:ıs le nom de muruaises habitudes, d'habitudes vicieuses, de tics coordonnés. I.a plupart d'entre eux consistent dans des mouvements involontaires, qui se répètent fréquemment sous forme d'accès. C'est la répétition inévitable d'un même mouvement, de la mème contraction musculaire qui distingne les tics.

Nous les divisons en tics actifs (tics de ruer, de mordre, tic aérophagique, tic de lécher, etc.), et en tics passifs, caractérisés exclusivement par une atlitude passive (lic d'appuyer un pied postérieur sur l'autre).

Cette classification est évidemment appelée à subir de nombreuses modifications à mesure que nos connaissances sur l'origine des lics se compléteront.

Les uns et les autres sont comparables aux tics de l'homme; ils consistent en effet en un trouble psychomoleur.

"Le trouble psychıque est caraclérisé par une imperfection de la volonté liée à un arrèt partiel du développement psychique.

"Le trouble moteur est, au début, une réaction motrice provorquée, tantòl par une incitation venue de l'extérieur, tantòt par une incitation corticale, une idée.

"Dans le premier cas, l'acte moteur est une réponse corticale à une incitation périphérique, un geste logiquement exécuté dans un but défuni. Par la répétition, ce geste devient habiluel et acquiert ainsi l'automutisme. Mais l'incitation provocatrice ayant disparu, il continue i se reproduire sums cause et sans but, constituant une "manifestation motrice excessive et intempestive.

" Jans le second cas, l'acle moteur est directement 
commandé par une idée; celle-ci disparaissanl, le mouvement persiste, et, eu vertu du mème mécanisme pathogénique, il continue à se répéter sans cause et sans but, excessif, intempestif.

"Le trouble moleur est un moucement convulsif, de forme clonique ou tonique (1). (Meige et Feindel.)

Le cheval n'est pas un ètre " purement spinal ". Il possède une écorce cérébrale dont l'action ou les réactions retentissent sur ses actes moteurs. Il a des centres psycho-moteurs; il est capable de volonti, de coordination ; il peut imiter; il peut répéter; il peut prendre des habitudes.

J'autre part, les anomalies physiques ou psycliques ne sont pas rares chez le cheval. Il a ses tares corporelles; il a ses caprices, ses manies, ses habitudes vicieuses (Chomel et Rudler).

1. Ties actifs. - Ils compremnent des tics convulsifs et des liabitudes vicieuses.

\section{$1^{0}$ Tices par habitule ou habitudes vicieuses.}

- Ces tics sont les suivants: $1^{\circ}$ tic de mordre les couvertures; $2^{\circ}$ lic d'ouvrir les porles et de se délicoter; $3^{\circ}$ tic le tirer au renard; $4^{\circ}$ tic du cabrer $; 5^{\circ}$ tic de ruer $6^{\circ}$ tic de frapler du devant; $7^{\circ}$ tic de mordre $8^{\circ}$ lic de reculer; $9^{\prime \prime}$ rétivité; $10^{\circ}$ tic de refuser de se laisser panser; $11^{\circ}$ tic, de s'emporter ou de s'emballer.

Pour empêclıer les animaux de manger les couvertures on a conseillé l'usage du collier à chapelet, du bàton fixé au licol et au surfaix, d'une bavette faisant gouttière et dépassant légèrement l'extrémité inférieure de la tête et les convertures, de la muselière, etc., etc.

Nous préconisons aujourd'hui un moyen facile à

(1) Chomel et Rudler. Tie de lours chez le cheval et les lics drimitaton shez l'homme (Bulletin Société centrale, 1903, p. 449). 
employer, peu conteux et d'une grande efficacité; c'est le suivant :

On adapte dans les parties de la couverture accessibles aux dents du cheval, un certain nombre de punaises à dessin, disposées en quinconces et dislantes les unes des autres d'environ 8 centimètres. Les punaises sont maintenues en place à l'aide de quelques petites bandes de toile cousues à la face interne de la couverture.

On conçoit aisément ce qui se produira chaque fois que l'animal voudra mordre ou lécher sa couverture : il se piquera le bout du nez et. renoncera bientòt à son habitude.

2u Tics convulsifs. - Ils se résument dans un mourement convulsif, coordonné, normal, primitivement volontaire, localisé à un département musculaire ou même à un seul muscle. Ces tics peuvent siéger à la têle, anx membres ou être mixtes.

\section{A. - TIC: CUNVULSIF DE LA FMCE.}

Définition - C'est $11 n$ monvement convulsif conscient et habituel caractérisé par la contraction involontaire des muscles de la face. Il n’a guère été ohservé que chez le "/hich, où il succède à la chorée. C'est une véritable névrose, qui se différencie par son origine centrale des tics qui procodent de l'irritation d'un nerf moteur ( $i$ ic symptomalique), ou de l'irritation d'un nerf sensitif (tic reflece).

I.e tic essentiel consiste dans des secousses instantanées qui se manifestent seulement sur l'un des côtés de la liıce, inlíressanl les joues et les paupières.

Cerlains rhic'ns affectés de la chorée ne présentent qu'un lic de la michoire inférieure cousistant dans un écartement et un rapprochement brusque de celle-ci.

Ces mouvements convulsifs se généralisent quelquefois à loute la tète et mème à la langue parésiće et faisant sail- 
lie en dehors de la bouche comme j'ai eu l'occasion de l'observer: la langue, rejelée du côlé droit de la bouche, pent inerte; elle a conservé son volıme normal, mais elle est molle, légèrement cyanosée, recouverte de terre; les piquures faites sur cet organe décèlent une grande atlénuation de la sensibilité.

On remarịue que, rel organe ectopié est le siège de mourements comrulsifs, saccadés, rythmiques, relativement pen accentués, il est vrai, mais insuftisants pour permptre it la langue de rentrer complètement dans la cavité huccale.

Le còté droil de la face présente des mouvements convulsifs, intéressant surtout le muscle crotaphyte, l'orbiculaire des paupières; les muscles de l'oreille participent également à ce mouvement cloréique. L'oreille droite est soulevée et abaissée alternativement, l'œil s'ouvre el se ferme de mème.

La paralysie de la langrue est lue á une altération du nelf moteur grand hypoglosse, le lic choréique de la face est dì à une lésion du noyau du nerf facial el en particulier des rameaux se dirigeant vers l'oreille externe et le crolaphyte

Chez le cheval, Bartolucci a observé un tic convulsit spécial : l'animal accomplit des mouvements rapides, désordonnés dans toute la moitié gauche du corps, comme si celle-ci agissait sous l'impulsion d'un courant électrique. En outre il y a soulèvement de la commissure ganche des lèrres, el déplacement correspondant de l'aile au nez, clignement de l'ocil gauclie, mouvements obliques des màchoires avec grincements de dents. Ensuite l'animal baisse la tête à grauche comme pour saisir quelgue clıose, pliant le cou arec des frúmissements musculaires superficiels du cou et du thorax. Linfin, le membre postírieur frauche se fléchit, puis s'étend comme pour ruer.

Ces pliénomènes se succèdent avec la rapidité de l'éclair'; ils se répètent tonjours daus la mème forme, par accès 
isolés, séparés par des intervalles plus ou moins longs de calme parfait.

\section{B. - TICS DE LA LANGUE.}

Caractères. - Ils consistent dans une attitude anormale de cet organe ou daus des mouvements particuliers ou bizarres qui se reproduisent intempestivement, mais qui s'éloignent toujours des mouvements de la vie ordinaire : animal qui double la langue en dessous, la replie audessus du mors; tic de la langue serpentine dénoncé par un mouvement rapide de la langue qui entre et qui sort continuellement de la bouche en imitant le mouvement d'ondulation des serpents.

\section{C. - TICS IDE LÉCIIAGE.}

Caractères. - En dehors du pica qui incite les animaux à ingérer des corps étrangers, ì se lécher ou à léclıer leurs voisins d'une manière passagère ou permanente et que nous avous décril plus haut (1), il existe un tic nerveux bien étudié chez le cheval par MM. Chomel et Rudler. Ce tic est un véritable trouble psycho-moteur, une habitude motrice désignée sous le nom de stéréotypie.

Les stéréotypies sont des attitudes, des mouvements, des actes de la vie végétative, qui sont coordonnés, n’ayant rien de convulsif, qui ont au contraire l'apparence d'actes intentionnels ou professionnels, conscients tet volontaires au début et qui deviennent plus tard automatiques et subconscients par le fait même de leur longuc durée et de leur répétition $(2)$.

" $1^{\circ}$ Phénomènes moteurs. - Le cheval atteint de cette habitude vicieuse lèche ses voisins en suenr; il lèche

(1) Sémiologie, p. 22. Aberration du goù, malaciu, pica. Encyclopialie vétérinaire.

(2) Moige et Feindel, Les lics et lewr traitement. P'aris, 1902, 
aussi les murs, les mangeoires. La bouclse entrouverte, les joues légèrement creusées, les yeux demi-clos, la tète indifféremment haute ou basse, le sujet sort ou rentre altermativement la langue dans un mouvement régulier presque rythmé.

La durée des accès de léchage est généralement de quinze à vingt minules.

On les observe de préférence à l'écurie, au retour du travail ou pendaut les haltes.

Ce tic est arrèté par la distraction; il s'exagère sous l'influence de la fatigue et se complique quelquefois de mordillement et de màchonnement.

" 20 Troubles psychiques. - Le lécheur est nerveux, émotif, impressionnable; il s’isole difficilement des chevaux qui sont près de lui; il est très sensible aux excitations et s'affole, ou est très impatient.

" $3^{\circ}$ Troubles de la sensibilité. - La plıpart des chevaux léeheurs offrent une anesthésie du bout du nez, mue sensibilité inégale des barres. Ces animaux suent facilement et abondamment.

"Stigmales physiques, - Les animaux affectés de ce tic du léchage présentent des asymétries faciale et corporelle : abaissement d'un ail, déviation du nez et plus spécialement hypertrophie de la parolide, épaississement et induration de la muqueuse des lèvres, hypertrophic des commissures labiales, inflammation chronique de la muqueuse de la lèvre supérieure, érosions gingivales, carie dentaire, saillie des barres.

C'est bien là un trouble psycho-moteur, sans phénoniène convulsif, que, pour ce motif, Chomel el Rudler ratlachent au genre des steréotypies, voisin des lics.

les sujets qui en sont affectés sont des déséquilibrés sujets à des aecès ‘d'impatience et à des phobies et yui 
contractent l'habitude de lécher quand ils présentent des lésions de la bonche ou des dents" (Chomel et Rudler).

\section{1). - TICS DIVERS.}

11. Tic de frotter les dents contre la mangeoire. Quelquefois les chevaux perdent cette habitude, quand on les attache à un poteaı, la tête relevée.

$b$. Tic d'agiter la lèvre inférieure. - Consiste dans un mouvement continu, régulier, saccadé, rythmique et rapide de la lèvre inférieure qui s'éloigne el se rapproche de la lèvre supérieure en produisant souvent un bruit disgracieux. Certains chevaux passent rapidement la langue sur Ies lèvres.

c. Tic du menton. - Les sujets appliquent le bout du nez contre la paroi murale de l'auge et se livrent pendant de longues heures à un mouvement de va-et-vient, d'avant en arrière, ou d'un còté à l'autre.

d. Tic de grincer des dents, douvrir et de fermer alternativement la bouche. - Les muscles masséters sont le point de dejpart de ces tics.

$e$. Tic de saisir l'une des branches du mors avec la lèvre inférieure ou avec les dents. - Les animaux affectés de ces tics sont difficiles à conduire (1).

$f$. Tic d'encenser. - La tète s'élève et s'abaisse perpendiculairement avec la régularité du balancier d'une pendule. Ces mouvements alternatifs d'extension et de fiexion se manifestent quand l'animal est altelé ou monté. Ce tic n'est que la reproduction inlenpestive d'un mouvement réflexe, automatique, procédant d'une don-

(1) Consulter l'article Tic du Dictionnaire de M. Bouley et Reynal, par Cadéac. 
leur vive des barres, d'une pression désagréable, gènante ou donloureuse du mors, d'un étrunglement intestinal (hernie etranglèe inguinule, etc.).

\section{E. - TIC IE LOURS.}

Définition. - Le tic de J'ours consiste dans un balancement de la tête et de l'encolure accompagné ou non d'oscillations analogues du corps sur les membres antérieurs. Ce balancement est habituel, rythmé, oscillatoire et symétrique; les mouvements se succèdent, en effet, toujours dans le même ordre suivant un plan ëtabli ; ils sont nettement coordonnés et reviennent sous forme d'accès; ils ressemblent à ceux qu'exécute l'ours en cage.

Ce tic a fait l'objet d'un travail important de MM. Cihomel et Rudler dont nous reproduisons ici les parties essentielles.

Les manifestations de ce tic se traduisent par des attitudes spéciales et des mourements anormaux qui se produisent très souvent le jour, parfois la nuit, de préférence à l'écurie dans les périodes de repos, pendant les pansages, dans les intervalles des repos, à tous les ìges de la vie, en toute région, en toute saison.

Les troubles qui les caractérisent comprennent: $1^{\circ}$ des phénomènes moteurs; $2^{\circ}$ des phénomènes psychiques; $3^{\circ}$ les troubles de la réflectivité, de la sensibilité et des troubles trophiques $; 4^{\circ}$ des stigmates physiques. Il faut les passer en revue.

" $1^{\circ}$ Phénomènes moteurs. - Dans le mourement de balancement qui caractérise le tic de lours, les menlores antérieurs sout toujours écartés et déviés en dehors de la ligne d'aplomb dans l'attitude du cheval trop ourert du decluns et punurd. l.e poser des pieds se fait successivenent sur loute la surface plantaire ou sur la pince el la ma* 
melle interne du pied au fur et à mesure des oscillations dı corps.

Chez cerlains chevaux, pendant le tic, un pied anlérieur est alternativement soulevé et quitte le sol à chaque déplacement du corps, avec le geste de commencer un mouvement de danse. Toutefois, si cette posilion des membres el pieds anterrieur's ne varie guère, il en est autrement pour les pieds et membres postèrieurs. Cenx-ci sont tautòt rapprochés (cheval trop serré du derrière, clos ou crochu), tantòt écartés (cheval trop ouvert du derrière), tantôt placés l'un devant l'autre, mais rarement soulevés durant le mouvement de tic, sauf cependant pour le pied situé en avant qui reste attaché au sol par la pince.

Quelquefois, l'appui ne se fait que sur trois pieds dont um postèricur au poser (sans pour cela que le tic cesse). On note également l'intervention fréquente des pieds postérieurs à lappui et au soutien, et l'oscillation du menbre postérieur à l’appui.

Le mourement doscillation ou de translation du corps est tantôt limité à l'avant-main, tantôt conımuniqué à tout le corps au point de déterminer mème un déplacement de la queue. Il débute par la tẻte, avec la nuque en avant comme si le cheval voulait donner un coup de tète, ou encore avec le bout du nez en avant. Quelquefois, ce mourement est tout d'une masse, la tête étant verticale, et le cheval parait alors pivoter autour de la croupe. Ilabituellement, c'est le train antérieur qui se déplace tandis qu'au train postérieur ou arrière-main ce n'est plus qu'un mouvement communiqué. Chez un cheval, on remarque que la tête se déplace pour son compte, entraînant ensuite le déplacement de l'avant-main. Chez un autre, il existe pendant le tic un michonnement fréquent, et, de plus, l'animal couche parfois les oreilles au moindre bruit. Habituellement Ia tête est maintenıe à hauteur normale, et son mouvement latéral se trouve limité par le licol et la longueur de la chaine d'attache.

CADÉAC. - Sémiologie, $2^{\mathrm{e}}$ édit. II. -12 
Dans le mouvement de balancement itendi à tout le tronc, le corps du cheval est incurvé tautôt à gauche, tantòt à droite et inversement. Ce mouvement d'oscillation est relativement fréquent, on peut compter quarante déplacements réguliers, rythmés à la minute.

Le tir: cle l'our's s'observe de préférence à l'écuric, à l'instant de la distribution d'avoiue ou dans les intervalles des repas, et au delıors pendant les pansages ou encore pendant une manœurre dès qu'il y a un repos prolonge. Le cliangement de sol ne modifie pas le tic. Sur certaius clsevaux le tic parait plus fréquent la nuit.

Chez tous il disparaît habituellement sous l'infuence de l'attention, de la gourmandise, pendant la miction, la mastication, le travail.

Le phénomène du balancement se remarque sur des clıevaux spécialisés dans les services les plus divers (tráit, selle, service d'éclaireur), ayant de bonnes, moyennes on mauvaises allures. Mais tous ces animaux ont des membres particulièrement tarés (molettes, vessigous, hygromas, éparvius).

"20 Phẻnomènes psychiques. - Ils sont naturcllemeıt très difficiles à saisir clıez le cheval en raison dı développement incomplet de l'intelligence, de la volonté el de la conscience dhez cet animal. Mais on ne saurait mécomnaitre chez lui l’aclivité collicale. Celte activité corticale est troublée de différentes façous chez les chevaux liqueurs. Ils sont plus ou moins émotifs, attentifs, nerveux, impressionnables, irritables, impatients, furieux, difficiles au dressage ou au travail, alfectueux ou mṕchants pour les autres chevaux el pour l'homme; il en est qui se livrent à des manifestations folles, qui offrent une hyperesthísie cutanée extrême, qui ruent ou mordent, qui sont difficiles ả seller, à brider, à ferrer, à atteler. D'autres sont tellement impatients qu'ils ne penvent demeurer en place, qu'ils mordillent contiuuellement 
la chaîne d'attache. Tous présentent des manifestations impulsives ou abouliques, un certain déséquilibre de la volonté, de l'instabilité motrice, etc.

Celte irritabilité, ce nervosisme particulier, cette agitation insolite signalée par Cadéac a été bien éludiée par Chomel et Rudler.

" $3^{\circ}$ Troubles de réflectivité et de sensibilité. - Chomel et Rudler ont examiné chez ces tiqueurs la flexion du rein, l'épreuve du dynamomètre caudal, la sensibilité du nez à la piqùre, les réllexes de l'encolure et du pied, la rétlectivité pupillaire.

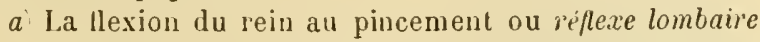
est très exagérée chez les chevaux tiqueurs; la plupart manifestent une grande irritation ou une flexion outrée.

6) L'épreuce du dynamomèlre cuudal (queue soulevée par les crins, à une certaine distance de sa base) indique au contraire que la résistance au soulèvement de la queue cst fortement diminuée cliez ces sujets. Ce lynamomètre caudal, qui est peu influencé par l'àge et beaucoup au contraire par l'état maladif, donne assez bien dans l'état sain une indication sur la résistance musculaire du cheval. Il est constant que la faible résistance s'observe normalement chez tous les liqueurs de l'ours.

c) La sensibilité du nez à la piquire a été trouvée exagérée, sans pourtant devenir anormale chez une jument qui offrait par contre un froncement excessif des muscles du bout du nez.

d) Le réflexe de lencolure (caresses avec la main produisant le va-et-vient des oreilles) est géméralement normal ou peu accusé.

e) Le réflexe du pied cherché de différentes façons (pied brusquement élendu par la pince, nembre lléchi vivement au genou, tapotement sec tes tendons, etc.) n'a domné aucun résultat appréciable.

f) La réflecticité pupilluire juil alternativement fermé et 
ouvert) est difficile à saisir, sauf en pleine lumière. Elle a paru normale che\% tous nos tiquenrs.

Eufin signalons la réaclion du mastoüdo-haméral au pincement (à sa sortie de l'épaule), laquelle esl négrative dans le tic de l'ours, mais parait donner quelıues résultats pour les aurres tics.

" $4^{\circ}$ Stigmates physiques. - Les anomalies asymétriques du squeletle, des muscles de la peau, des poils friquents chez les dégénérés et les liqueurs humains se retrouvent chez les chevaux affectés lu lic de l'ours.

Tous ou presque tous sont asynétriques ou dyslıarmonịpes. On peut constater l'asymétrie du cràue, l'asymétrie de la lace, l'abaissement d'un ceil, la déviation du nez à droite ou à gauclıe, l’asymétrie de l'épaule marquée par une dépression des muscles sous-épineux et long abducteur du bras et l'asymétrie de la hanche caractérisée par ın affaissentent de l'angle externe de l'ilium. Tous ces stigmates sobservent isolément ou réunis en nombre plus ou moins considérable chez ces chevaux liqueurs.

En somme, le tic de l'ours est constitué chez le clıeval jar un phéuomène moteur, neltement caractérisé et par un élat psychopatlıique spécial quon peut rapprocher de celui des tiqueurs humains. "Chomel el Rudler.)

\section{F. - TICS DES MEMBIRES.}

Caractères. - Certains "hevilux ont l'habilude de lever sans cesse un membre postérieur; d'autres se bulancent sur les hamehes en les portant suecessivement à droite et a Enache; d'autres grattent continuellement le sol de leus jiels anlericurs, conme s’ils étaient affectés de coliques. Il en est qui trottent à l'écurie; certains se roulent en putrant a l'écurie.

11. Tir.s passifs. - Ce sont : 1" le tic de la langue pendante; $2^{\circ}$ le lic de secoucher en vache $3^{\circ}$ le lic d'appuyer 
un pied postérieur sur l'autre. Jes conséquences de ces diverses habitudes sont trop cannues pour qu'il soit nécessaice de sy appesantir.

\section{XXIV CONTULSIONS.}

Définition. - Le syndrome convulsions est une exagération de la motilité, caractérisée par les contractions involontaires des muscles de la vie de relation, fréquemment accompagnées de troubles nerveux, respiratoires, circulatoires. C'est une hyperkincive.

La conrulsion est all mouvement ce qu'est le délire à lintelligence, li douleur à la sensibilité.

On a confondu convulsion et spasme, mais le nol convulsiun doil ètre réservé aux troulıles siégeant sur l'appareil moteur de la vie de relation, le mot spasme s'applique aux troubles convulsifs qui se manifestent dans la sphère organique. Dans

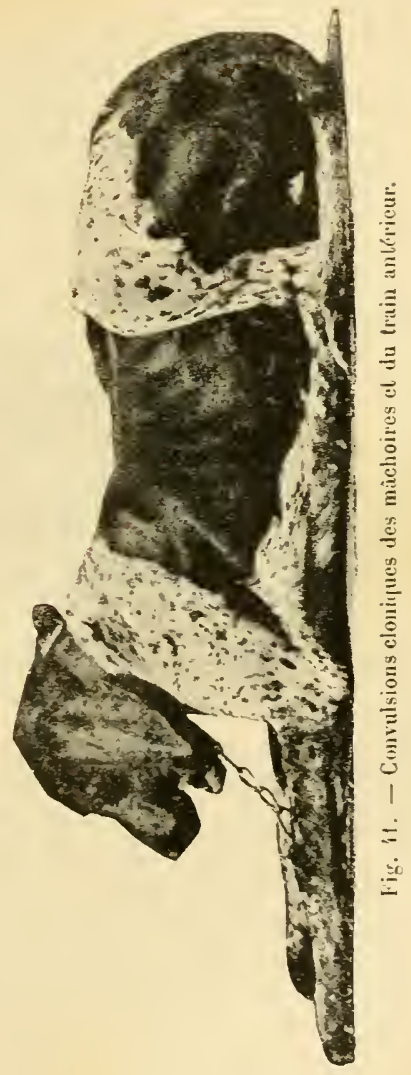
quelques organes, il est vrai, les éléments de la vie organique et de la vie de relation ajoutent leurs efforts ; spasmes et convulsions se confondent aussi.

On divise les convulsions en partielles (vomissement) 
210 SÉMIOLOGIE DE L'APIAREIL DE L'INNERVATION.

et en générales, suivant qu'elles affectent un muscle (chorée), un groupe musculaire (tic) ou l'ensemble du système musculaire (épilepsie). On distingue encore des convulsions toniyues, cloniques et mixtes.

Dans la convulsion toniquc, il y a rigidité pernanente, tension continue des éléments contractiles; les seconsses sout d'aulant plus limitées que la contraction est plus prolongée (tétanos).

La convulsion clonique est composée par une succes-

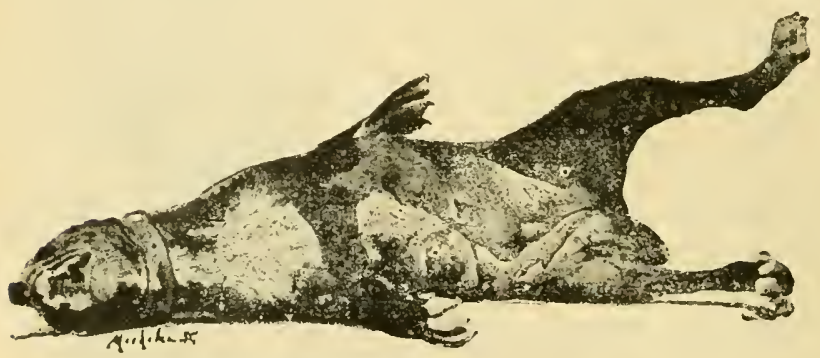

Pig. 42. - Phase tonique de la crise d'épilepsie déterminće par l'essenre de romarin.

sion plus ou moius irrégalière de secousses élémentaires, séparées par des phases d'immobilité el de résolution musculaire (chor'é), deuxième phase de l'épilepsiej (Iig. 41 .

Pathogenie. - Les contractions musculaires sont commandées par le systime nerveux et sont dues à des excitations fournies par les centres encéphaliques bulhaires et médullaires. Il fiut envisager le ròle de l'élément musculaire et celui de l'élément nerveux.

11. Muscle. - L'étude physiologique de la contraction musculaire a montré que son élément est la secousse, c'est-á-dire un raccourcissement brusıute des fibres musculaires suivi d'un relàclement immédiat. Une excitation 
isolée, instantanée produit une secousse. La contraction est un phémonnène complexe, composé d'une série de secousses si rapides qu'elles se fusionnent, ce qui entraine l'élat de rigidité du muscle (télanos plıysiologique).

Les nouvements volontaires sont donc letaniques: il en est de mème de la convulsion lonique; elle est due à une excitation qui se répète comme les décharges d'une machine électrique et maintient la rigidité musculaire.

La convulsion clonique, comparable à la secousse, a aussi

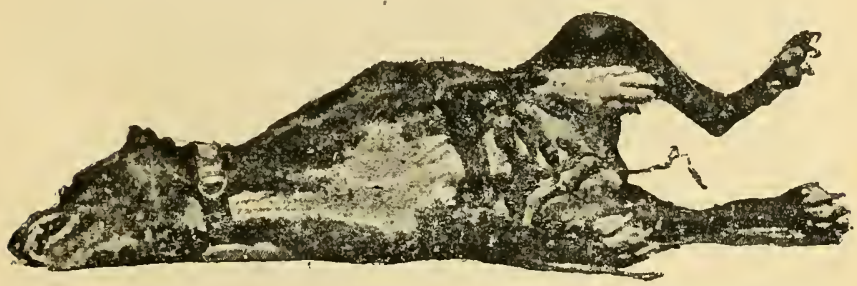

Fig. 43. - Altaque d'épilepsie accompagnée d'expulsion d'urine qui forme un véritable jet, chez. une chienne de 12 kilogrammes, après l'injection dans la saphène de 25 centigrammes d'essenę d'hysope.

pour cause une excitation brusque, puissante, isolée, instantanée, mais qui se répète à divers intervalles.

Il n'y a qu'une différence de degré entre la convulsion clonique, la convulsion tonique el la contraction normale; celle-ci répondant à un état intermédiaire, c'est-à-dire à des excitations moins puissantes, mais successives. Les seconsses musculaires sont dissociées dans la convulsion clonique, fusionnées dans la contraction normale, et renforcées dans la convulsion tonique.

b. Système nerveux. - Les causes des convulsions ont été groupées selon qu'elles agissenl sur les organes périplériques ou sur les centres nerveux.

$1^{\circ}$ Nerfs. - Le nerf est un conducteur : il transmet des excitalions reçues sur son trajet ou parties des centres. 
Suivant leur succession, leur intensité, ces excitations déterminent la contraction normale ou les diverses convulsions.

Un nerf moteur peut être excité directement dans certains cas; il en résulte des convulsions limitées à la région iunervée par ce nerf.

C'est ainsi que des traumatismes, contrsions, érrasement, dilacération, déchirure, compressions, névromes, tiraillements de fitets nerveux par des cicalrices, peuvent amener des convulsions. La compression déterminée par un polype, dans les cavilés nasales et les sinus, avaitrendu un cheval épileptique (1) et l'extirpation de cette lumeur avait fait disparaitre les convulsions.

Les maladies de l'oreille fotites murulentes, otites parasilaries) sont suivies de convulsions (épilepsie des chicus de meute). Cette maladie d'apparence mystérieuse est caractérisée par l'apparition de crises épileptiformes qui frappent tout à coup le malale, sans qu'aucun prodrome n’ål pu en révéler l'existence; au chenil, à l'état de repos, rien ne peut faire supposer l'existence du mal : les animanx atteints sont aussi gais, aussi ardents, en aussi bon ćtat que les autres. Ils chassent avec autant d'ardeur que les aulres, puis tout a coup, au bout d'une heure on quelquefois moins, l'animal lance un cri violent plus aigu, plus perçant; c'est lá l'indice du début d'une crise. Lientól, en cffét, le malade, affolé, bondit à travers les taillis, l'oil hagard, l'écume à la gueule, hurlant quand il rencontre un obstacle; puis après avoir tourné deux ou trois fois en cercle, il tombe, en proie à une véritable atlaque d'épilepsie. Si l'altaque n'a pas été trop violentc, si le mal n'est pas trop ancien, le chicn peul se relever après un quart d’heure, hébété, épuisé de fatigue; il peut alors parfois se remettre à chasser avec ardeur comme si rien ne s'était passé.

(1) Voy. art. P'olype du Dictionnairevétérinaire de II. Bouley, par M. Cadéac. 


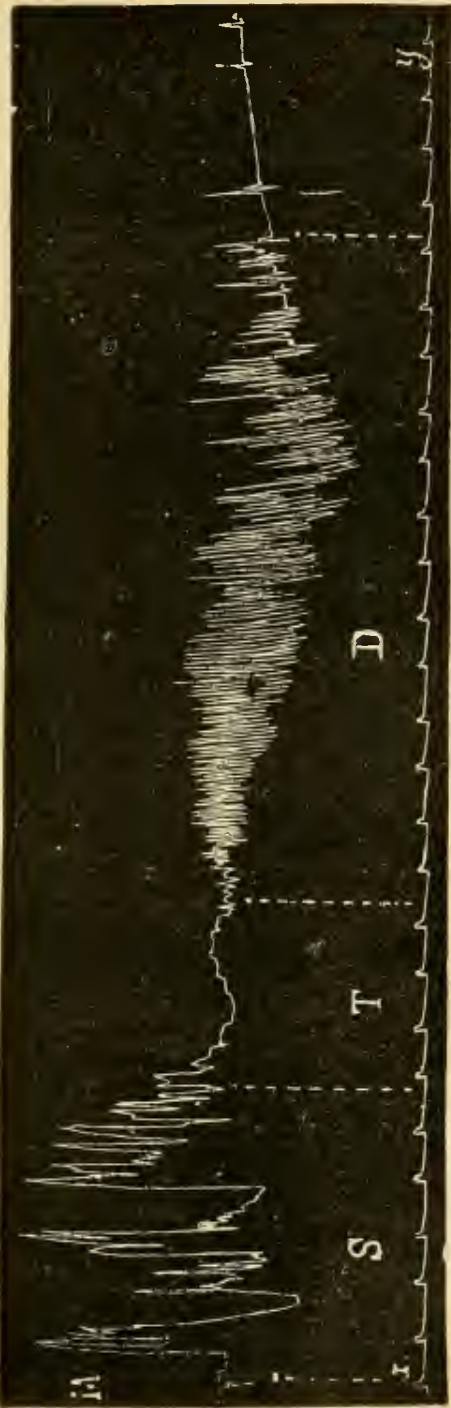

官

$\because$ in

$\stackrel{0}{0}$

$\stackrel{\nu}{\varrho}$

$\div \pi$

E-

응

놀

$\therefore$

$\vec{\Xi}$

을

巳

क क

它

\&

क्ष

: क

$\because \quad 0$

ए हू

ก है

อ क छ

क 0

इ छี

o $\omega \infty$

1 ह ङ

- ${ }^{\circ}$ है

$\leftleftarrows$ 管

$\therefore$ ० 
Mais il arrive souvent que l'on n'assiste pas à toules les phases de l'accès; après le premier hurlement, l'animal disparaît alors pendant une demi-heure ou une heure. puis rejoint ses compagnons, sans que l'ou sache ce qu'il est devenu pendant cet intervalle.

Pendant longtemps, rien ne distingue l'animal malade des animaux sains, mais quand la maladie est ancienne, et que les accès épileptiformes sout fréquents, les malades deviennent plus sombres, plus sauvages qu à l'orlinaire, ils se retirent, susolent de leurs compaguons; plus tird encore, les accès sont si fréquents el si graves, qu'à peine en action, les malades sont frappés, et quaprès l'attaque ils refusent de se remettre en marche, quand ils ne meurent pas sur place. Toujours l'accès se montre pendant la chasse; quand il n'est pas en action, le chien malide peut devenir conılètement abruti, impropre à son service, parfois complètement sourd; mais au chenil, au repos, il n'a jamais d'altaque.

En examinant minutieusement des têtes de chiens morts ou abattus et alteints de cette maladie, Nocard (1) ne trouva d'abord aucune lésion de l'encéplate et dı bulbe; mais il trouva an fond du conduit auditif externe el des deux côtés une masse assez considérable de cérumen, de couleur chocolat, refoulant la membrane du tympan qui faisait une saillie convexe du còté de l'oreille iuterne. L'examen microscopique de cette matière montra une grande quantité d'acares à tous les degrés de développement appartenant au genre choriopte (symliote de Delafond) et à l'espèce appelée par Mégnin, Chorioptes culudatus. Hering, Zïrn (1874), Guzzoni (1877), Mégnin (1881), étudièrent ces acares.

En introduisant de cette matière cérumineuse dans l'oreille de rhiens sains, on a pu produire che\% ceux-ci la maladie; de mème yu'on a pu arrêter la malalie dans

(1) Nocard, Ln maladie ipileptifurme des chiens de meute (Acariase auriculaire\}, in Arrh. vetér., 1-82, p. 201. 
sa marche, en tuant dans le conduit auditif mème, les acares qui s'y trouvaient.

C'est également par excitation réflexe que les rers intestinaur, les traumatismes des muscles, des tendons, les douleurs intenses provoyuent des convulsions. Ces convulsions d'origine périphérique (excitation du sciatiyue) peuvent ¿̀tre produiles expérimentalement (Brown-Séquard); on peut en rapprocher les tics nerveux, déterminés par les irrilations des noyanx d'origine des ner's de la face ou des membres (lic convulsif de la face, chorée du chien).

20 Centres nerveux. - Dans la plupart des cas, les convulsions sont dues à une altération anatomique des centres nerreux qui échappe souvent à l'autopsie, mais d'où découle une irritabililé trop grande, un pouvoir réflexe trop considérable, de sorte que les réactions motrices ont une intensité anormale. On s'explique ainsi l'influence de l'héédilé, de l'áge, du sexe, des ultérations dusang, dans le développement des convulsions.

L’épilepsie, l'éclampsie, certains tics sont liéréditaires, Luciani a observé deux fois cette transmission; cinqchiens jeunes moururent d'épilepsie quatre jours après leur naissance.

L.es sujets jeunes, les chiennes qui viennent d'accoucher sont prédisposés à l'éclampsie.

Le sang chargé de poisons minéraux, gazeux, organiques, microbiens, plomb, mercure, acide carbonique, oxygène sous pression, urémie et toxines de la rage, des fières érup. tives, du tétanos, du charbon, produisent des convulsions.

$3^{\circ}$ Moelle. - la moelle épinière est le centre des excitations molıices réflexes : l'exagération de celle activité est une source de convulsions partielles ou générules. Si l'excitation est trop considérable, elle ert transmise à des centres plus élevés : une généralisation des convulsions en est la conséquence. 


\section{SÉMIOLOGIE DE L'APPAREIL DE L'INNERVATION.}

On peutexpérimeutalement augmenter le pouvoir réflexe de la moelle en la séparant de l'encéphale qui contiendrait un centre modérateur (Sestchenow) des fonclions médullaires.

Certains médicamenls ont un effet analogue : ce sont des poisons convulsivants strychnine, thibaine, nicoline, brucine, etc.).

Les convulsions d'origine spinale peuvent ille dues à des excilations anormales, à des lésions du tissu mélullaire. Les traumatismes rachiliens, les tumeurs qui comprimeut la moelle, les myélites, méningites, méningo-myélites s'accompagnent fréquemment de convulsions.

La chorée des animaux qui olfre le type des convulsions cloniques est généralement une affection médullaire (Clıauveau, Cadéac et Guinard,

\section{$4^{\circ}$ Bulbe et protubérance. - Aux excitations périplıé-} riques anormales, le bulbe répond par des réactions motrices violentes, des convulsions généralisées. C'est dlonc le centre convulsivant par excellence.

Nothnagel a voulu détermiuer la situation exacte de ce centre, qu'il a nommé région des crampes. C'est le siège principal de la modificalion physiologique qui traduit l'attaque convulsive de forme épileptique, comme le démontre la coïncidence des troulıles généraux, respiratoires, circulatoires.

Le bulbe est donc un centre épileptogène. Ce centre est mis en action par des excitations diverses, par les essences d'absiuthe, de sauge, d'hysope, de romarin, de fenouil, d'ail, etc. (Cadéac et Meunier), par des poisons, le plomb, le mercure, l'alcool.

L'excitation des nerfs spinaux (Brown-Séquard), leur compression par des tumeurs amène des altaques d'épilepsie qui cessent après l'extirpation de ces tumeurs.

$ð^{0}$ Cerveau. - Les circonvolutions cérébrales sont le 


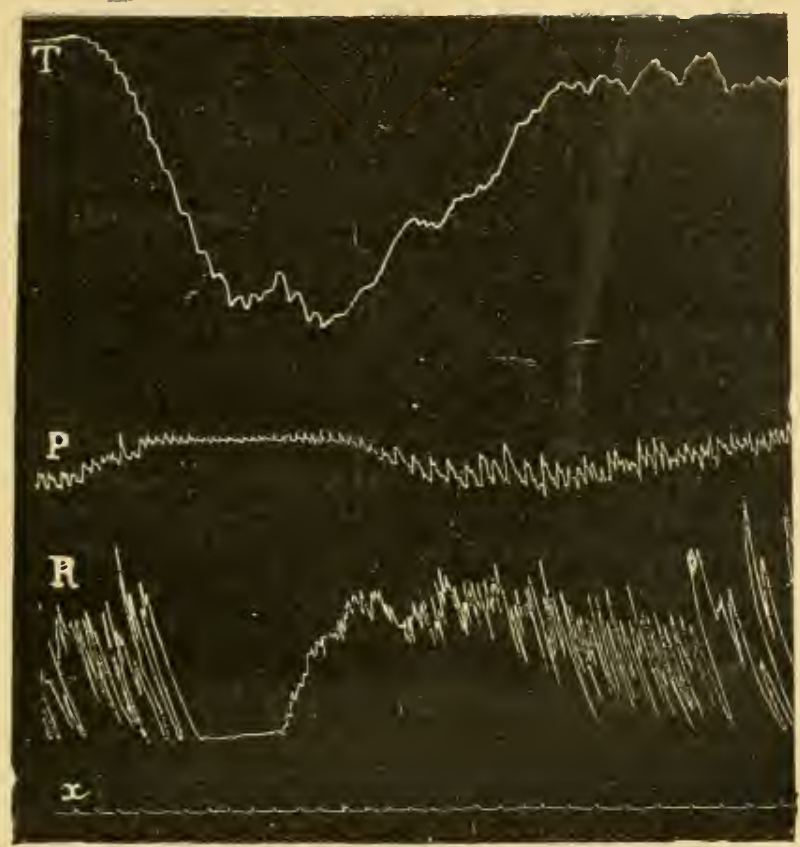

Fig. 15.

I'endant la periorle lonipue [', Ies pulsalions devieunent si petiles qu'elles sont à peine visibles, les petites collines qui les représenlent deviennent ensuile plus saillantes qu'à l'ćlat normal. On observe ainsi un affaiblissement. eonsidérable des systoles pendant la pririode tonique et une exagération de leur amplitude pendant la période clonįue. Pendatt la période lonique de l'altaque, la tension artériclle $T$ subit une dépression qui corresponul â la contracture cardiaque. $R$, respiration enregistrée a l'aide d'une ceinture pourve d'un cylindre en caoutchouc placé autour du thorax. On observe l'abord des secousses eloniques des nuscles intereoslaux suivies de la tétanisation de ces muscles el de l'arrêt te la respiration en inspiration forcée; l'attaque ayant commencé à la fin de l'espiration, la plume trace une ligne horizontale qui se relève pour marquer des vibrations qui augmentent graduellement d'amplitude pendant la plase elonique. - $x$, ligne les secondes.

$$
\text { Cisté.c. - Sémiologie, ?e élit. } 11 .-13
$$


point de départ des convulsions épileptiformes et d’attaques d'épilepsie essentielle.

L'épilepsie franchement cérébrale est héréditaire, elle s'observe sur les animaux, bu:l', rhicm, cheral. L'attaque se produit sous l'inlluence d'excilations périplıériques de nature variable : vue d'un objet quelconque, d'un drap, d'une cigogne, comme on l'a observé chez le chu'ral; de traumatismes de l'encéphale, d'un bruit intense (coup de sifllet, déclıarges de mousqueterie, etc.).

Ia ligature des carotiles, des saiynés abondantes amènent des convulsions par suite de l'auémie cérébrale (épilepsic ischèmique).

On est parvenu à reproduire expérimentalement l'épiIppsie corticale chez les animaux ( $/$ hich, $1 / h i l)$. Ferrier, Frauck et Pitres qui ont réalisé ces expériences, ont monlré que des excitations électriques, mécaniques, chimiques, pour ètre efticaces doivent être portées sur les zones motrices: celles-ci sont donc les seules zones épileptogìnes.

Ces auteurs supposent que les convulsions sont dues aux décharges des cellules nerveuses de l'écorce qui se comporteraient comme des condensateurs susceptihles d'accumuler les excitations et de les mettre en lilierté quand la tension a acquis un degré suffisant. Dans une première phase, celle mise en liberté serait rapide, continue ct régulière, l'épuisement survenant progressivement, les excitations se succéderaient bientòt d'une manière irréğulière, par à-coups, ce qui expliquerail les caractères de la crise d'épilepsie.

A son début, elle est caractériséc, en effet, par la rigidité des muscles, par les convulsions toniques, auxquelles succèdent bientôt des convulșions cloniques de plus en plus espacées el la résolution musculaire.

Les maladies du cerveau, la miningite, l'encéphalite, les hémorragies cérébrales, sont souvent accompagnées dr ees phénomènes convulsifs. 
Caractères des convulsions, - Les caractires des convulsions varient suivant la cause provocatrice; les poisons végélaux et microbiens, qui se diffusent dans toute l'économie, déterminent des convulsions générulisées (épilepsie); les irrilations, les intoxications, les dégénérescences locales s'accusent par des convulsions partielles (chorée). Les convulsions partielles ne diffèrent souvent des con* vulsions généralisées que par leur élendue; elles peurent en différer aussi par leur caraclère tonigue comme dans le tétanos, clonique dans la chorée et le tic nervercx ou successivement tonique et clonique comme daus l'épilepsie.

Les convulsions toniques sont exlrêmement fréquentes et présentent entre elles une grande parenté. Le système nerveux intoxiqué n'a pas des manières différentes de réagrir, de sorte que les poisons convulsivants les plus divers ont des effets presque identiques. Toute intoxication se traduit par de l'excitation ou de la dépression. Si c'est l'excitation qui lomine la scène, le poison est dit convulsivant; si, an contraire, la dépression clomine, le poison est prralysant ou anesthexique. D'ailleurs, tout poison dépressif est excitant au début de son action, tout poison convulsif létermine à la lin de son action des effets paralysants. Ia strychnine administrée à forte dose à un animal dont on privient l'asphyxie par la respiration artificielle détermine de la dépression; les sels ammoniacaux et la vératrine se comportent de mème.

Quand on intoxique un chicu par une dose convulsivante d'essence de sutuce, l'hysope, de romarin, d'alsinthe, etc., on détermine des convulsions à caractère epileptiforme qui se déroulent habituellement de la manière suivante:

Presque immédiatement après l'injection, le chien remis en liberté, s'arrète brusquement; il reste immobile, les membres postérieurs écartés, le regard fixe et plein d'inquiétude ou d'étonnement, la pupille est très dilatée; le sujet frissonne. Bientòt, de petiles secousses contrac- 
tent hrusquement les muscles du con et impriment à la tète des mouvements saccadés de négation el d'approbation; en même temps, les paupières clignotent, la face grimace, puis ces contractions spasmodiques gagnent les muscles de l'épanle el dı dos; toute la partie antérieure du corps est soulevée sur place par saccades, ou portée en arrière dans un monvement de recul (fig. 46 ).

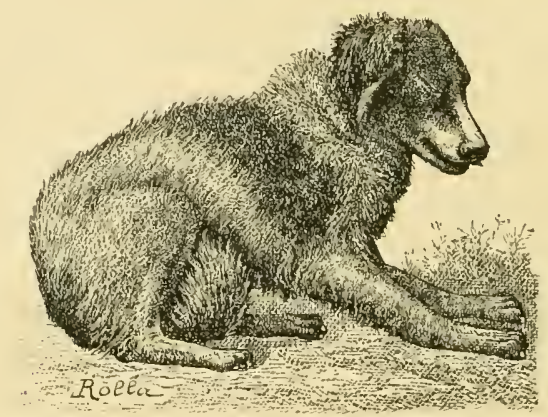

lig. 40.-Début de laaltaque d'épilepsie determinée par l'essence d'hysope.

L'animal se ramasse sur lui-mème, se cramponne pour résister à ces sorles de décharges électriques; il tombe tout à coup. Les membres se raidissent, les postérieurs s'écartent, les antérieurs s'étendent le long du corps, la tète se renverse sur l'encolure, le corps se courbe en arc pour produire tour à tour de l'emprosthotonos, de l'opisthotonos et du pleurothotonos, parfois même, il est souleré toul d'une pièce comme mû par un ressort, et il frappe violemment le sol de lout son poids.

Les mâclıoires, un moment resserrées, s'écartent brusquement et s'entrechoquent, la sensibilité est complètement éteinte; on peut piquer l'animal sans modifier en rien la crise.

A celte phase tonique, succède bientòt la phase cloniqu'. 
I'ne salive épaisse, souvent sanğuinolente, s'écoule des commissures; urine et matières fécales s"ćchappent involontairement; l'ail est procident et la respiration, un moment suspendue, devient stertoreuse. Avant de tomber daus un relàchement musculaire plus ou moins prononcé, l'animal bat l'air de ses membres et se roule clans tous les sens.

L'attaque dure une minute environ. La crise passée, lanimal sc relève vivement, part lıélété et tourne avec une vigueur extrême comme un inconscient autour du

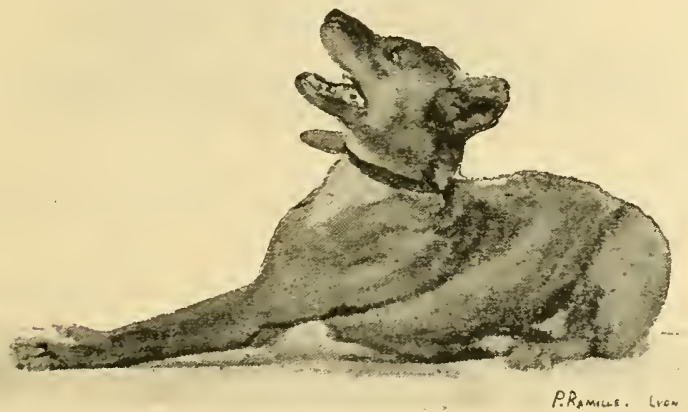

Yig. 47. - Physionomie du chien affeclé d'une attaque d'épilepsie dèterminée par le Calamus aromaticus.

point d'attache de sa chaine. Un obstacle, un contacl, une simple provocation extérieure, une cause excitatrice quelconque suffit pour déterminer un nouvel accès dont l'exaltation est variable, mais il est semblable comme forme au premier et souvent plus intense.

Les attaques épileptiques sont mème fréquemment subintrantes. Quand l'animal résiste à ces convulsions, l'excilabilité morbide s'atténue progressivement, les accès ultérieurs sont de moins en moins forts et séparés les uns des autres par des intervalles de plus en plus longs. 
Puis, les attaques d'épilepsie cessent, malı̣ré des excitilious assez vives; celles-ci ne produisent plus que des nouvements convulsifs partiels limités de préférence aux muscles du cou et de la tèle, amenant ainsi cette sorte d'aura iéphalique qui annonce an clébut de ces expériences, les grandes crises épileptiques (fig. 48).

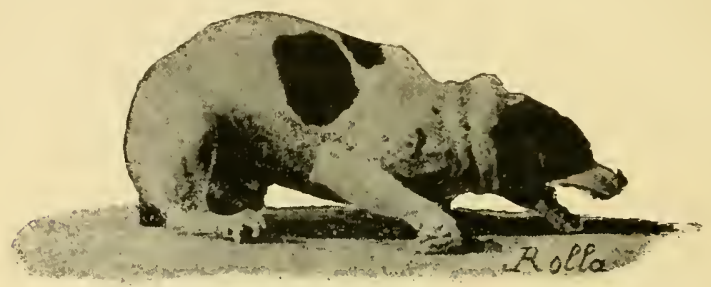

fig. is. - Itlaque comiliale thez uu chicn qui reste debout pendant une partie de la première période; les onglons sont crispés; les mâchoires convulséos (Cadéac).

La vorT résulte de l'asphyxie produite par la tétanisation des muscles iuspirateurs qui empêchent totalement le renouvellement de l'air dans les poumons. On peut y remédier daus l'empoisommement strychnique par une insufllation vigoureuse d'air dans la trachée.

La mort est aussi le résultat de l'hyperthermie déterminée par l'exagération des contractions musculaires. Ivec la cocüne, la vératrine et les sels ammoniacaux, on peut, en pratiquant la respiration artificielle, délerminer la mort des animaux en expérience uniquement par l'hyperthermie; les animaux convulsés et refroidis ne succombent pas.

Signification. - L'épilepsic est un mode de réaction du syslème nerveux qui caractérise non seulement une maladie essentielle, mais encore l'attaque corticalc déterminée expérimestalement par l'excitation électrique de l'écorce cérébrale, l'attaque d'épilepsie produite par les essences 
et l'attaque d’épilepsie, strychnirue. Qu'il s'agrisse d'une excilation dynumique ou d'une excitation tonique, le système nerveux répond de la mème manière, par unc succession des périodes tonique el clonique.

L'excitation convulsive est un des processus par lesquels passe la cellule nerveuse avant de mourir.

Dans l'ANÉME, dans l'HÉMORKAGIE, ces convulsions sont très fréquentes et constituent un des signes précurseurs de la mort. L'anémic des artères encéphaliques entraîne des convulsions.

Les himorragies profuses sont suivies chez le chicn de convulsions épileptiques. Si l'on anémie le cerveau d'un chien, soit en injectant de l'air dans une carotide, soit en tetanisant le cœur, on voit, dix à vingt secondes après, survenir une violente convulsion tonique qui s'arrète bientòt par épuisement de l'animal, de sorte que l'excitation convulsive nous apparaît en dernière analyse, comme une des phases de la mort des cellules nervenses motrices, que ce soit par l'anémie, ou l'hémorragie, ou par l'action d'un poison (Richel).

Les méningites produisent moins des convulsions que des contractures; les tumeurs cérébrales sont une cause fréquente de manifestations épileptiformes.

Si les poisons convulsivants portent leur action sur tous les éléments de l'axe cérébro-spinal, ils ont une action de prédilection pour tel ou tel centre.

Les essexces et la picrotoxine portent le maximum de leur action sur le bulhe; la cocaine, la santonine sur l'écorce cérébrale; la strychnine sur toutes les cellules nerveuses motrices.

L’unéme détermine quelquefois des convulsions à caractère épileptoïde.

L'ÉcLAMPsı, maladie produite par une unto-intoxication fracidique, c’est-à-dire par la rétention de produits toxiques dans lorganisme, se traduil aussi par des accès de spasmes toniques et cloniques plus ou moins nombreux : 
22' SÉMIOLOGIE DE L'AIPAREIL DE LIXNERVATION.

les màchoires s'écartent et se rapprochent en faisant entendre un claquement sec ef loruyant; la salive s'écoule mousseuse, la dyspnée est profonde, la chienne ouvre largement la bouche comme si elle venait de faire une grande course; elle manifeste une angoisse profonde.

Les yeux sont saillants et pirouettent quelquefois dans leur orbite, la conjonctive est injectée, la pupille dilatée.

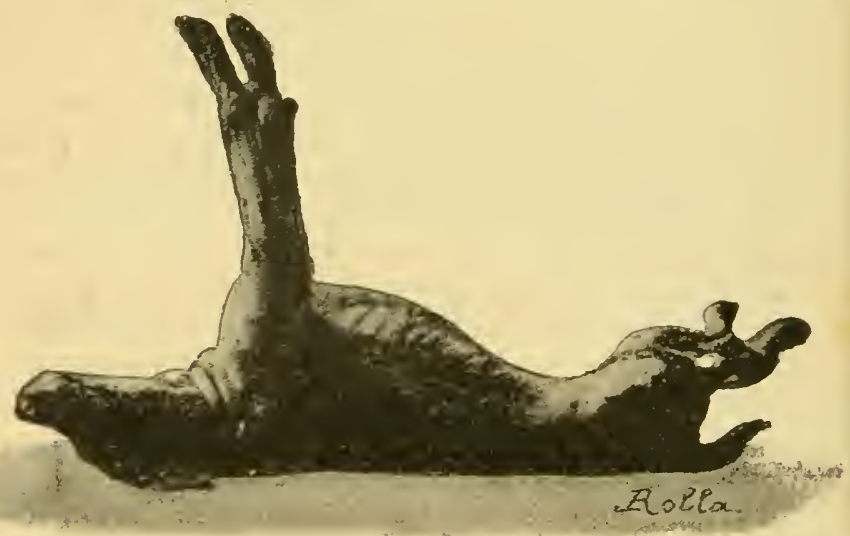

Fig. 49. - Intoxication du chien par l'essence de calament; létanisation des membres antérieurs, les spasmodịues dúterminent un changement du côtó du sujet (Cadéac).

La marche est pénible ou impossible, l'animal tombe et a le la peine à se relever; il est extrèmenent agité, ses membres se raidissent ou s'agilent dans différents sens, les lèvres se contractent et découvient les dents qui se heurtent violemment et déchirent la langue.

Les accès éclamptiques diffèrent des accès épileptiques par la perte de conuaissance qui accompagne ces deruiers. La chienne atteinte d'éclampsie est consciente tant qu'elle vit, car la mort peut survenir par paralysie générale ou aspliyxie. 
La FIETRE VITULAIRE qui aboutit à la paralysie de certains organes, est également une affection convulsivante, produite aussi par une auto-intoxication. Son apparition est dénoncée par une période d'excitation passagère suivie de coma ou par des accés éclamptiques loujours accompagués d'une période de coma. On observe donc toujours et partout la phase d'excitation et la phase de dépression. La vache mugit, cherclie à donner des coups de corne; jette la tête à droite et à gauche, son ail est lıagard ; on constate quelquefois des mourements convulsifs dans certains groupes musculaires; l'encolure offre des contractions spasmodiques; la vache màchonne, fait cntendre un bruit de succion ou se lèche avec frénésie sur quelque point du corps, au grassel, à l'avant-bras. Mais les poisons stupéfiants prédominent généralement et l'animal prend bieıtôt une physionomie comateuse.

Les accès sont moins intenses que daus les affections convulsivantes qui précèdent; ils sont beaucoup plus longs; leur durée varie de une heure à onze heures; les accès dépilepsie qui sont si violents s'éteiguent souvent en moins d'une minute; les accès déclampsie, moins intenses, offrent une durée intermédiaire. Tous ces accès sont composés d'un mélange de convulsions toniques et cliniques.

Le TÉr.sios, qui se résume également dans une intoxication, est caractérisé par une contraction spasmodiuue permanente des muscles striés : la tête est tendue sur l'encolure qui est fortement relevée, les oreilles sont droiles, la queue horizontale ou redressée; les membres sont raides el écartés, les muscles durs et saillants, le corps clignotant recouvre plus ou moins le glole oculaire; on constate le rapprochement des arcades dentaires par contraclure des masséters (trismus).

Parfois la tète, l'encolure, le tronc sont placés sur la mème ligne (orthotonos); la lête peut être relevée, renversée en arrière, la colonne dorso-lombaire concave 
(opisthotonos). On peut observer une incurvation opposée, la tèle abaissée, la colonne dorso-lombaire voussée en contre-haut, e'est l'emprosthotonos; on a enfin signalé une inclinaison latírale (pleurothotonos) (Voy. Télanos, in Pllhologie chirurgicale, tome 1, p. 139).

Les affections épileptorrines, éclamptiques ou tétaniques, sont d'autaut plus graves que la température est plusèlevér. L.es hautes températures caractérisent le tétanos frave; les hautes températures ront de concert avec les convulsions violentes. Il en est ainsi lans lontes les maJadies infectieuses.

L'hypothermie engendre le coma de la fievre ritulaire: les convulsions se manifestent au début quand la température est élevée; l'hypothermie qui se manifeste d'emblée avec un coma profond est le prélude de la mort; l'absence de convulsions et l'hypothermie aceusent lintoxication complète de l'organisme sans réaction possible; les forles doses de strychnine et d'essence de sange ne déterminent pas des convulsions, la vie étant entretenue par la respira. tion artificielle; les animaux sont immobiles; les muscles et les glandes sont inertes; il ne peut se produire, ni chaleur, ni convulsions.

La tempéruture a une grande action sur le résultat de toutes intoxications. l'inlluence exercée par les toxiques est d'autant plus intense yue la température est plus élevée.

La cocüme se prète admirablement a cette démoustration. Une élévation de température de trois degrés diminue de moitié la dose de chlorlıylrate de cocainne nécessaire ponr amener les convulsions. L'abaissement de température entraine non senlement une diminution de sensibilité aux convulsions, mais clle modifie encore la forme de ces convulsions.

Ainsi, chez les chiens refroidis, les attaques convulsives prennent une forme toxique, avec contraclures prolongées, qui diffère de l'attaque épileptoüde, suivie de secousses 
clouiques violentes, laqquelle caractérise la forme des conrulsions chez les chiens échauffés. Le froid rend la convulsion plus longue et leaucoup moins violente, en mème temps qu'il rend nécessaire une dose plus forte de poisorr. C'est le cercle ricieux par excellence : l'hyperthermie angmente les convulsions, et les convulsions augmentent l'hyperthermie.

La chorée est le type des manifestalions conrulsives cloniques. Ciest chez le chien qu'on pent le mieux les étulier; elles sont si connues qu'il est inutile d'y insister (Voy. Clıorée, in Pulhologie interne, tome VIII).

Chez le brut, les manifestations choréqques ont été étudiées chez deux animaux par Besnoit. "Elles consistent dans un cas, dans les contractions spasmodiques rythmées du membre postérieur gauche et synchrones avec les mourements respiratoires; elles coincident très exactement arec la fin de l'inspiration. Elles siègent sur tous les museles de la moilié supérieure fítucle du train postérieur, mais paraissent intéresser particulièrement le triceps crural, le fessier superticiel, l'origine de l'iliospinal, le sphrincter anal et les muscles coccygiens du eòté gauche.

Pendant la station cleboul, les mourements choréiques déterminent un soulèvement brusque et violent du membre postérieur ganche par une flexion convulsive des rayons articulaires les uns sur les antres; ils provotguent, en outre, une brusque déviation de la base de la quene vers la gauche et une constriclion énergique de l'anus, qui s'efface subitement et s'enfonce profondément.

Dans le décubitus, l'effet produit est de mème ordre mais moins marqué; les rayons supérieur's ne se flí chissent plus; la partie supérienre du membre est simplement soulevée en bloc par la convulsion.

La sensibilité parait abolie à la partie supérieure du

(1) Hesnoil, Revue vétérinuire, 190ะ, ए. 29:. 
membre postérieur gauche, sauf au niveau des muscles rotuliens, où elle est cependant très atténuée : l'animal ne réagit pas aux piquures d'épingle failes dans les régions supérieures du membre; il se défend au contraire énergiquement quand on explore les régions inférioures et notamment quand on touche le canon.

Au membre postéricur droit, la sensibilité est normale; l'animal chasse les mouches qui se posent sur ce menibre.

Mais les manifestations nerveuses ne tardèrent pas à s'aggraver.

Cinq jours plus tard, des contractions cloniques apparaissaient sur le membre postérieur droit, intéressant uniquement le demi-tendineux et le demi-membraneux. L'étal général était loujours bon, mais la malade était désormais dans l'impossibilité absolue de se tenir debout.

Les jours suivants, les secousses convulsives augmentent encore d'intensilé, aussi bien à gauche qu'à droite. Celles de gauche restent toujours synchrones avec.les mouvements de la respiration; à droite, au contraire, le synchronisme fait défaut entre les mouvements respiraloires et choréiques : ces lerniers, tout en étant très rythmés, se répètent à intervalles un peu plus longs que les premiers.

A partir de ce moment, l'état général s’altère rapidement; l’appétit disparaîl, la diarrhée survient, les grandes fouctions s'effectuent irrégulièrement. Puis, les secousses choréiques, toujour's rylhmées, s'accélèrent pendant que disparaît le synchronisme des contractions lu côté gauche avec les mouvements respiratoires.

L'animal est alors considéré coume définitivement perdu : on se décide à le sacrifier.

L'autopsie, comme il était à prévoir, n'a donné aucun résultal (Besnoit). "

Parfois les convulsions allectent, chez les porcelets comme chez les enfants, un caractère spécial; les phéno- 
nomènes convulsifs constituent toute l'affection. Yous croyous devoir les décrire ici parce qu'on n'est pas encore lixé sur' leur cause provocalrice.

\section{XXY. - CONVULSIONS DU PORCELET.}

Définition. Historique. - On désigne ainsi un enseınble de troubles nerveux; c'est-à-dire un syndrome susceptible d'apparaitre sous des influences éliologiques nulliples. C'est Besnoil (1) qui a altiré l'attention sur ces troubles, qui a colligé les olservations antérieures et qui en a donné une description complète.

Ces convulsious sont appelées Gisclä̈re dans le Tarn, Gingle dans la Dordogne, vertigc dans les départements du Centre: le terme conuulsious, qui a une signification symptomatique, est préférable, car il ne préjuge rien de la wature du mal qui paraît spécial au porcelet. Les observations de Fouriaud (2), de Loucou, de Coslelet (3), de Besnoit témoignent de la fréquence de ces manifestations.

Caractères. - "Les couvulsions du porcelet présentent une grande analogie avec les convulsions de l'enfant; il existe cependant des différences qui mérilent d'ètre signalées.

Chez l'enf'ant, l'attaque convulsive, parfois précédée de prodromes (agitation générale, cris anxieux, fixité du regard...), éclate le plus souvent d'une façon soudaine. Brusquement, la face pàlit, le corps se roidit el l'enfant perd connaissance. Puis, à celle période de couvulsion Lonique succèdentrapidement des secousses qui débutent à la têle, puis se généralisent au tronc et aux membres. La tèle est étendue en arrière, en avant ou laléralement;

(1) Besnoil, Revie vétérinaire, 1901, 1. 331.

(2) Fouriaud, De l'éclampsie des jeunes porcs (Rovue vét., 18:9, p. (i6).

(3) Coslelet, De l'éclampsie chez le porcelet (Piogrès rét., 1900, 1. I, p. $\$ 01)$. 
les globes oculaires, agilés de violentes saccades, pirouelleut en lous sens; la face est grimaçante, les commissures des lèvres, tirées en dehors, découvrent les dents et laissentécouler une écume blanclictre, quolquefois sanguiuolente lorsque les mâchoires, serrées par un trismus, continu ou intermittent, ont blessí la langue. Lesmuscles du tronc, d'abord railis, sont bientòt agités de secousses convulsives. Le bras, l'avant-bras, les doignts s'élendent el se fléchissenl alternativement.

la respiration est gûnée et bruyante par suite du spasme dı larynx el du draphragme. Parfois mèmeil y a menace d'asphyxie; les lèvres sout blanchàtres, la face cyanosée, la respiration stertoreuse.

Pendant tout le temps que dure l'attaque, il y a perte complète de la connaissance el de la sensibilité cutanée.

Au bout d'une a ciny minutes, quelquefois yuinze, rarement davantage, les contractions loniques cessent, la respiration devient plus régulière, la face pàlit et la cyanose disparait. L'altayue est lerminér, laissant après elle du coma et de la somnolence.

Elle peut se répéter à intervalles jlus ou moins longrs, et on pent compler jusqu'it dix ou vingt acès par jour.

La mort peut survenir par asphyxic au cours d'une attaque. En général, les accès disparaissent sans laisser de traces; exceptionnellement, cependant, ils peuvent ètre suivis d'accidents divers: paralysies, contractures passagères ou permanentes, strabisme... dus saus doute a la stase sanguine les centres nervenx consécutive ì l'attaque.

Chez le porcrlel, l'attaque revît habituellement une physionomie un pen différente. Elle n'est jamais précédẻe d'aucun prodrome; l'animal commence par présenter, lıuit à dix minutes environ après le lébut du repas, quelques tremblements à peine perceplibles dans les membres postérieurs.

Ces tremblements s'accusent rapidement, deviennent 
très violents et se transforment bientòl en véritables secousses, qui ébranlent tout le corps. En mème temps, le malade piéline des membres postérieurs; la physionomie anxieuse, crispée, il se dresse sur les onglons postérieurs et parait éprouver des sensations très douloureuses; il continue cependant à manger avec une certaine avidité. Mais les secousses s'aggravent encore et, deux on trois minutes environ après l'apparition des premiers tremblements, elles sont devenues tellement violentes que l'animal perd l'équilibre et tombe sur le sol, où il reste allongé, les membres postérieur's élendus sous l'abdomen. Aussitòt, les tremblements cessent, et la physionomie reprend toute sa sírénité; les violentes douleurs que paraissait éprouver le malade quelques secondes avant, semblent avoir subitentent disparu.

Le porcelet reste encore abruti pendant quelques instants, puis, après vingt, quarante, soixante secondes, rarement davantagn, il se releve et continue son repas interrompu par la clute.

L'acculmie est d'ailleurs de courte durée. Quelques minutes plus lard survient une seconde attaque. Elle débute comme la précédente, mais les tremblements se gónéralisent. Les membres antérieurs sont à leur tour agités par des tremblements d'abord légers, puis violents el bientôt les quatre membres sont secoués par de violentes convulsions. A claque instant, le malade est sur le point de perdre l'équilibre; lorsque la chute est immineute, il s’éloigne le la mangeoire, se dresse sur ses onglous, piétine avec rage el piyue le groin contre le sol comme pour prendre uı point d'appui supplémentaire, mais ce secours est insufitisant, il tombe et aussitôt les secousses convulsives cessent.

Le repas est repris de nouveau; il n'est enfin terminé qu'après une ou plusieurs attaques semblables aux précédentes. Ces altaques successives peuvent présenter quelques légères variantes; en fin de compte, elles abou- 
tissent loujours à la perte de l'équilibre el à la chute sur le sol.

Au moment précis où la chute se produit, les membres sont raidis, comme tétanisés, contracturés. Ils paraissent transformés en piquets rigides, impuissants à maintenir l'équilibre constamment compromis par les oscillations convulsives imprimées au corps, par les trépignements continuels et par les tentatives de déplacement du malade.

Pendant le décubitus, le porcelet reste tantòt complètement étendu, tantòt assis, mais dans les deux cas, les memlres postérieurs sont complètement allongés sous l'abdomen.

Parfois, au début des attaques, quand les tremblements apparaissent, on observe une sorte de gêne de la déglulition: après avoir pris des aliments, l'animal lève le nez et tend la tète par saccades lorusques comme pour favoriser la déglutition.

Souven aussi, l'altaque s'accompagne d'une gêne de la respiration qui est accélérée et bruyante.

A aucun moment, pendant les accès, on n'observe de salivation, ni de pirouettement des yeux, ni de perte de connaissance. La sensibilité est également conservée: l'auimal réagit aux piqûres d'épingle. On peut noter dans certains cas, à la suite des accès: de l'hémiplégie, de la paraplégie et des contractures variées. Parfois, on constate la mort par asphyxie au cours de crises épileptiformes survenant au moment du repas.

I! u'y a, en somne, entre les convulsions de l'enfant et celles du porcelet, que des différences d'intensité; mais exceptiomellement, l'attaque peut avorter cliez l'enfant et prendre la forme habituelle de l'atlaque du porcelet, de mème qu'on peut voir l'attaque du porcelet acquérir toute la gravité de celle de l'enfant.

La symptomatologie est donc identique et vraisemblablement de "mème origine " (Besnoit). 
Pathogénie. - L'aige a une grande influence; les convulsions frappent les porcelels pendant la première année, de préférence même pendant les six premiers mois de l'existence.

L'hirédité n'est pas étrangère ì leur apparition chez l'culiunt, chez le chien; il est probable qu'il en est de même chez le porcelet.

Ces accidents se manifestent particulièrement chez les animanx rachitiques.

l'helminthiase intestinale 'jui joue un ròle important dans les convulsions du chien, n'engendre pas celles du pore.

Les intoxications par les toxines de la pneumo-enterite, du rouyet paraissent être les seutes causes occasionnelles (Besuoit).

On combat ces convulsions à l'aide des antispasmodiques et des anesthésiques.

\section{XXVI. - CONTRACTURES.}

Définition. - La contraclure consiste dans une conIraction permanente et soulenue des fibres musculaires. Elle diffère de la convulsion tonique par sa durée.

Les masses musculaires dures, rigides, insensibles à la pression, dimintent de longueur, ce qui amène une déviation des rayons ossenx.

Ce qui caractérise la vérilable contracture, c'est que le muscle n'est pas altéré dans sa structure. Que si, par un moyen quelconque, on fail cesser l'excitation nerveuse qui maintieni le muscle en état de contracture, comme, par exemple, si l'on anesthésie profondément le malade, la vraie contracture disparait, tandis que la rélraction musculaire, qui dépend d'une cause anatomque el non d'une cause physiologique, ne disparail pas par la chloroformisation.

On met à profit cette propriélé de disparaitre sous l'in- 
23. SEMHOLORIE DE LALPAREIL DE L'INNERVITION.

fluence de l'anesthésie pour réduire les vieilles huxations ou pour provoquer le relàchement ales sphincters (rechum, vayin, etc.).

Les crimpes succèdent à des lésions des muscles, des nerfs. Les contractures qui surviennent à la suite de l'oblitération des artères iliaques, de l'artère bracliale, sont de fausses contractures.

On les a vues, che\% le $\cdot$ ln 4 al, suecéder à l'introduction du doigt dans l'oreille ou à la pression exercée au voisi-

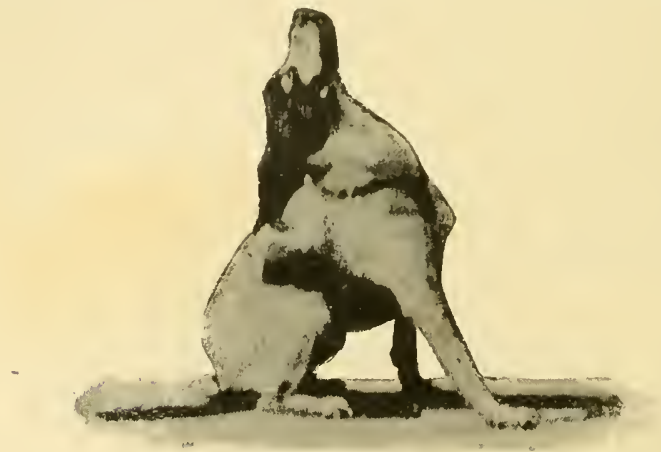

Fig. io. - Contraclure du cou délerminée par l'essence de sauge.

nage par la bride, le membre postérieur de ce còté prenait immédiatement une posilion horizontale; Dycer a vu les muscles tléchisseurs de la tête tellement contraclurés que les naseaux touchaient le sternum; Heisemann a signalé des contractions spasmodiques des muscles d'un cólé de l'encolure el du membre antérieur correspondant; Moller, celle des muscles olécraniens. Carougeau a chservé les spasmes sous une forme généralisée an début de tous les mouvements volontaires.

Caractères. - "Lol'squ'on excite le cheval pour le faire avancerou reculer, il se produit tout à coup une rigidité 
générale pour ainsi dire tétanique qui, pendant quelques secoudes, reud tout mouvement absolument impossible. C'est le systeme musculaire tout entier qui se met ainsi subitement en contracture.

Les muscles de l'encolure, des membres antérieurs, des membres postérieurs se contractent brusquement, uri déplacement en avani est ébauché et immédiatement arrèté; tous les muscles reslent tendus. On observe aussi ces troubles au niveau des muscles de la tète: on voit les lèvres, les naseaux, les paupières, les oreilles s'agriter par quelques oscillations, puis rester fixes. Il en résulte une impression d'angoisse frappante, profonde.

Tous les mouvements sont donc très gênés. Celte gène ne dure pas longtemps, vingt à trente secondes, puis la contracture cesse; les mouvements deviennent possibles. L'animal se met en marche, les premiers pas sont irréguliers, les membres sont jetés en avant et en dehors comme dans les cas dataxie locomotrice.

Enfin, au bout de quelques pas, en trois à quatre minutes, tout rentre dans l'ordre, tout spasme disparail, les nouvements redeviennent normaux.

Si le malade est arrêté, laissé all repos un instant, le corlège symptomatique qui vient d'ètre décrit reparait à une nouvelle excitalion.

Chaque fois que l'animal arrêté est sollicité pour se mouvoir, les contractures se reproduisent.

Ce sont surtout les excitations brusques, violentes, par surprise, un coup de fouet, un claquement fort qui déterminent les accès les plus intenses.

Jans aucuu cas l'animal ne menace de lomber à terre, jamais il ne cesse de percevoir ce qui se passe antour de lui.

Les accès ne sout pas douloureux; si pendaul la crise la physionomie est antroissée, cela tient uniquenent à la contracture des nuscles de la fare; ce masque disparait aussitòt que les mouvememts redeviennent libres et le 
malade a l'allure indolente d'un cheval àgé habitué à travailler au pas.

Quelle que soit la frétuence des crises, elles se reproduisent toujours avec la même intensité.

Il en résulte une fatigue réelle lorsquon rend les contractures trop fréquentes; c'est ce qui explique (avec l’ìge) la maigreur du sujet.

Pendant la contracture, les muscles sont durs, saillants. Ils semblent plus excitables à la piqùre avec une épingle, au choc. Cet ensemble symptomatique ne correspond pas à une maladie bien définie, il ne s'igit pas d'épilepsie incomplète. Jamais, en effel, ce cheval n'est tombé et n'a eu perle de connaissance ou de sensibilité au moment des accès, pas de salivation " (Carougeau).

C'est donc une névrose différente de l'épilepsie. Elle est d'ailleurs également incurable.

Les antispasmodiques se sont montrés aussi inefficaces que les excitanls; le bromure de potassium que la strychuine. Cette maladie est peut-ètıe comparable à la maladie de Thomsen chez l'homme. Ces phénomènes sont ordinairement passagers.

Pathogénie. - Les causes intimes des contractures sont très obcures; on les a attribuées à une augmentation de la tonicité du muscle, à une coagulation de la myosine comme dans la rigidité cadavérique. On a aussi admis des contractures d'origine nerveuse, et ce serait la moelle qui jouerait le ròle essentiel dans la production de ce symptôme.

Toutes les contractures traics reconnaissent une seule et mique cause, c'est une excitation des centres nerveux non volontaire, laquelle délermine alors la contraction prolongée de la fihre musculaire.

Les contractures reflexes sont provoquées par un stimulus de la péripbérie; les contractures non réflexes sont provoquées par une excitation des centres nerveux. 
Le type des contractures reflexes, cest la contraction des sphincters consécutive à une excitation traumatique ou pathologique de la muqueuse qui recourre les sphincters.

Ln traumatisme de la cornée détermine la contracture de l'orbiculaire, une fissure à l'anus détermine le ténesme rectal; les lésions articulaires entrainent parfois dans les muscles des membres des contraclures plus ou moins marquées.

Les lésions du système nerreux central (cerceau, bulbe, moelle) peureut engendrer des contractures. Une piqùre légère de l'istlıme encéphalique amène anssitut la contracture de plusieurs groupes musculaires. On obserse des contractures dans les inflammations des centres nepreux ou de leurs enreloppes.

Lne hèmisection de la moelle à la région cerrico-dor. sale augmente la rétlectivité médullaire, à tel point que la percussion réitérée du tendon rotulien provoque alors un vérilable élat de contracture qui dure plusieurs minutes.

De même, ell exagérant par de faibles doses de strychnine, le pouvoir excito-moleur de la moelle, chez des chiens alteints de dégénérescence descendante à la suite de lésions de la zone motrice, on provoque la contracture réflexe dans le membre correspondant au còté dégénéré, tandis que la percussion du tendon rotulien, de l'autre còté, ne proroque que des secousses simples dans le membre du mêrne cỏté.

Il y a beaucoup de cas de paralysie du chien accompagnée de contracture; on observe également ce phénomène dans la chorée.

Les contractures ont une durée trẻs rariable, elles sont parfois douloureuses, mais soureut indolores.

Cher. les fretus, la contracture des muscles de l'encolure et des membres ne résulte pas d'une position anormale prise par le fotus et conservée longtenıps , H. Bouley), ni d'une maladie nerreuse, mais d'une maladie de lamnios. 
Dareste a dímoutré que che\% les oiseaux, les anomalies de disposition du tronc et des membres sont dues à des compressions de l'amnios, qui enserre l'emlıryon à une époutue où les éléments anatomiques le celui-ci ne sont pils dilférenciés et il a pensé que les choses se passent le mème chez les mammifères.

La contracture est souvent le terme ultime de l'excitalion motrice produite par les essences de sause, d'absintlue, d'hysope, de fenouil, etc., à faible dose.

Les sujets sont envaluis par les contractures parlielles

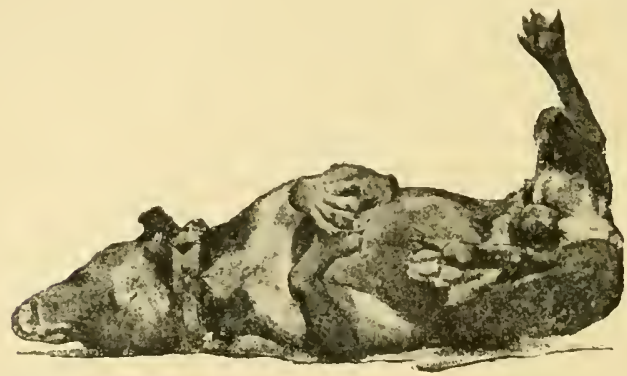

Fig. d'opilepsie dólerminée par l'essence de romarin; relichemenl des aulres memlires (Cadéac).

ou générales qui peuvent empècher tout nouvement, ou bien ils rampent péniblement, les memlures postérieurs lléchis, les antérieurs allonırés en avant, parfois les muscles du cou sont éfalement pris, la tîte est fortement étendue en arrière ou ramenée vers le thorax ou le sternum.

l.e tétanos s'accompagne de contractures partielles ou zénérales dues à l'action des toxines du bacille de Nicolaier.

I)ans l'ergotisme, dans le slrychnisme, c'est une substance toxique qui provoque cette excitabilité médullaire. Les lésions du corps thyroüde peuvent également pro- 
duire des contractures par intoxication. Quelle que soit la cause provocatrice, le muscle contracluré n'íprouve pas

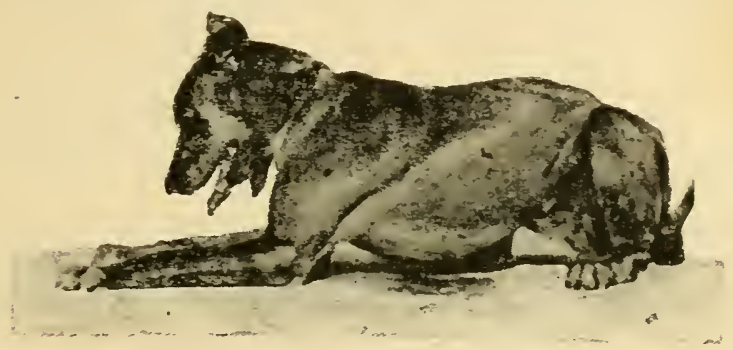

Fig. 52. - Contracture du train antérieur.

de seusation de fatigue et sa tempéralure ne s'élère pas.

Dans les intoxications par les essences, les mourements sont quelquefois rendus impossibles par la contracture (ig. ว้2).

\section{IXVII. - SPASHES.}

Définition. - Les spasmes sont des contractions anormales des muscles de la rie or ranique. Parmi ces muscles soustraits à l'influence le la volonté, les uns sont à fibres lisses, les autres à fibres slriées; les premiers se contractent lentement, les seconds rapidement.

Les spasmes se produisent rapidement sans prodromes; leur durée est très variable; ils sont souvent douloureux ténesme vésirul, nophagisme, ele.).

Pathogénie. - Ils sont gènéralement dus à une irıitation anormale: les lésions traumatiques ou ulcérations de l'œsophage, du vagin ou de l'urètre, ou de la bouche déterminant des spasmes plus ou moins prononcés de l'osophage ipsophagisme), du ragin (raginisme) ou de l'urètre, spasme urétral, ou de la bouche (constriction permanente les machoires), ou du col de la vessie (spusme du col le lu ressir). 
On les observe dans les appareils digestif, respiratoire, circulatoire, urinaire. Le spasme de l'apsophage ou a'sopha. gisme rend la déglutition inpossible; celui de l'intestin amène en quelque sorte son obstruction, puis des coliques; le spasme de la glotte provoque des phénomènes asphyxiques; le spasme des artérioles détermine des stases, des infiltrations, le refroidissement, la diminution de la sensibilité et des échanges nutritifs.

Les calculs des urelères, ceux de la vessie, les inflammations, les tumeurs de ce viscère, provoquent des spasmes de ces organes, ce qui entraîne la rétention d'urine ou gène l'exécution du cathétérisme.

Le raginisme ou hyperesthésie des parties génitales externes, peut arriver à produire, dans le spasme, des constrictions intenses du vagin el des autres muscles du plancher du bassin.

Sous cette influence, certaines chiennes ne peuvent ètre couvertes malgré leur élat de rut el le désir qu'elles peuvent en avoir.

Au moment de l'introduction du pénis, elles fuient, poussent des cris aigus, attaquent le chien el courent comme folles et parfois on observe presque imnédiatement des convulsions. L'exploration du vagin nécessile quelquefois l'anesthésie, car la contraction des sphincters est tellement intense que le doigt ne peut y pénétrer.

On a conseillé de pratiquer la résection du nerf honteux interne; cette opération a été pratiquée avec succès par Parascoudolo.

\section{XXVII. - MAGHONXEMENT ET GIINCEMENTS I) DENTS.}

Définition. - Ces deux signes se traduisent par des mouvements spasmodiques de mastication, généralement déterıninés par des doulęrs intenses.

Ils sont symptomuliques de coliques, d'inflammation céri- 
Lrale, diempoisommements, de maladies fibriles graves; on les rencontre dans le rouget "du pore, dans l'onterite chro-

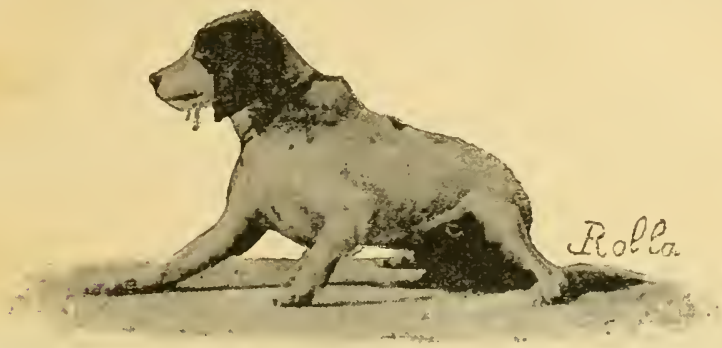

Fïg. 33. - Mâchonnement arec écoulement de bave chez le chien.

mique du bouf, dans la fière typhoüde du cheval el dans diverses intoxications du porc, du chien et du cheral.

\section{XXIX. - TREMIBLMENT.}

Le tremblement est un trouble de la motilité caraclérisé par des oscillations involontaires du corps, des membres,

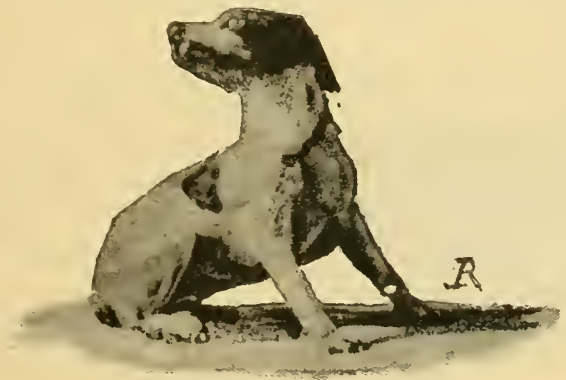

Fig. 54. - Physionomie du chien iutoxiqué par i essence de fenouił au moment où la tète est violemment agitée par un tremblement simulant la paralysie agitante.

ou d'une région, compatibles avcc l'exéculion des mouvements volontaires.

Canéac. - Sémiologie, จ" édit.

II. -14 
242 SEMHOLOGIE DE L APPAREIL DE I."INNERVATIOA.

Caractères cliniques. - Le tremblement détermine le plissement et l'agitation de la pean ou ne provoque que des mouvements invisibles, sensibles seulement au toucher : ce sont alors des contractions fibrillaires.

L'agitation affecte parfuis les muscles d'une région senlement (con, grasset), cenx d'un membre, des lèvres, des paupières (nystagmus), de la langue (paralysie labioglosso-laryngée commencrante), du cou, du Irain postérieur ou de tout le corps.

Quand les muscles du cou sont pris, la tête est agitée d'un balancement vertical ou latéral (tremblement affirmatif ou négatif); si ce sont ceux de tout le corps, celui-ci est animé de mouvements alternatifs de propulsion, de rétropulsion ou de latéralité.

Parfois, au repos, les tremblements font défaul, mais les coutractions nécessaires à la station suffisent à les produire. Le plus souvent, les trémulations persistent au repos, mais elles sont renforcées par les excitations extérieures, pucements, mouvements violents, par exemple. La marche est rendue hésilante; tous les mourements s'accompagnent de trémulations et perdent ainsi de lenr jrécision.

Le nombre des secousses musculaires est de 8 a 12 par

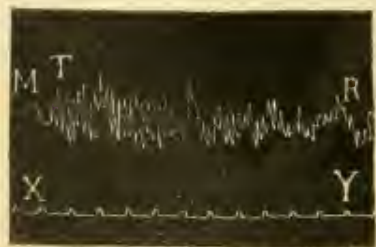

Hig. 5.. - Tremb!ements délerminés chez le chien par l'essence d'hysope. seconde; elles se produisent par séries, séparées par de courtes intermittences, leur amplitude, toujours faible, est peu variahle.

Pathogénie. - Le tremhement consiste dans une altération de la contraction musculaire dueà un trouble nerveux. La contraction normale est produite par une lusion de secousses commandées par un nombre équiva. 
lent dexcitations; les trémulations apparais-ent quand ces excitalions ne sont ni assez nombreuses ni assez rapprochées pour se fusionner 12 au lieu de 30 environl.

Tout ce qui diminue l'aclivilé cérébrale engendre le tremblement. Les maladies infectieuses, fébriles (charton, fievre typhö̈le, septicémie, corysa gangreneux, pneumonir infectiense), les maladies du cerveau, de la moelle, la paralyxie latio-glosso-laryngẻe sont susceptibles de le délerminer.

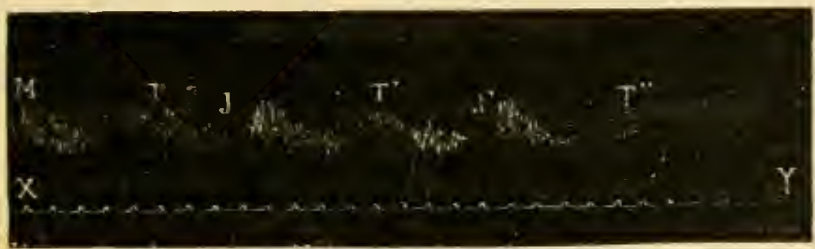

Fig. 36. - Tremblements en série.

$\mathrm{TT}^{\prime} . \mathrm{T}^{\prime \prime}$, séries de ribralions musculaires. - JJ'. périodes de repos relatif.

Divers poisons, le plomb, le mercure, le tabac, l'opium, les alcaloïdes, la cicutine, la colchicine, l'aconitine, les essences romarin, feıouil, sarriette, origan, etc.)(Cadéac et Ileunier peuvent lui donner naissance.

On l'observe encore dans l'hémiplégic, dans l'épilepsic à la suite de l'atlaque courulsire, dans les maladies de la moelle.

L'expérimentation plysiologique et l'étude des essences ont permis de préciser la partie du système nerveux qui préside aux tremblements toxiques.

Charcot el Vulpian ont constaté que le crrure de méme que la destruction du centré céretro-spinal, enpèchent la production du tremblement nicolinique, tandis que, si l'on empoisonne, par la nicotine, une grenouille à laquelle on a extirpé l'encéphale, il persiste tant que le bulbe reste intact. Celte partie du système nerveux règle donc 
244 SEMIOLOGIE DE L'AI'IAREIL DE L'INNERIATION.

les trémulations nicotiniques. Les recherches de Cadéac et Meunier ont également démontré que, dans l'iutoxication par les essences, c'est encore le bulbe qui lance le tremblement et la moclle qui le propage.

Ce tremblement toxique est une manifestation convulsive et uon paralytique. Il sert de pont entre la contraction musculaire normale et la convulsion el non, comme l'a cru Romberg, entre la parilysie et l'état convulsif.

\section{XXX. - NYSTAGMUS.}

Définition. - On désigue sous le nom de nystugmus un tremblement associé des muscles nculaires.

Ce phénomène est șımptomatique de lésions traumatiques porlant sur le cràne, de lésions auriculaires(caisse du tympan ou labyrinthe), d'altérations des centres nerveux (tubercules quadrijumeaux, couches optiques, corps restiformes, cervelet) sans qu'on puisse déterminer le véritable mécanisme de ces troubles oculaires.

Le nystagmus est quelquefois accompagné d'un tremblement continuel des yeux (Storch) (1). On constate quelquefois chez le cheval un état convulsif caractérisé par la projection continuelle du corps clignotant sur le globe oculaire, de telle sorte qu'en entrant dans l'écuric on croit avoir affaire à un animal tétanique. Cet étal s'accompaśne d'ailleurs quelquefois d'un pen de raideur des oreilles. Le corps clignotant recouvre principalement le globe oculaire quand l'animal baisse la tête ou quand on ouvre brusquement l'écurie ou qu'on soumet l'animal à une excitation quelconque. La guérison survient ordinairement au bout de quelques semaines; mais nous avons vu ce trouble persister plus de deux mois.

1) Storch, Nystagmus oscillatoricus chez une vache. Progrès vélérinaire, 1895. 


\section{XXXI. - FRISSON.}

Définition. - Le frisson est une sorle de convulsion caractérisée par une contraction clonique violente des muscles généralisée, mais passagère et se répétant à intervalles rythmiques, réguliers, correspondant ordinairement aux pauses de la respiration.

On reconnait trois espèces de frissons : le frisson thermique, le frisson psychique et le frisson toxique.

Le frisson therMlQue constitue pour l'animal un puissant moyen de se réchauffer; la contraction musculaire produit de la chaleur en exagérant l'activité du chimisme respiratoire. Les auimaux anesthésiés par le chloral, etc. présentent à leur réveil un frisson thermique, car leur température est basse et si on les réchauffe, ils ne frissonnent point.

Le frisson tosıQue qui intéresse parliculièrement le clinicien se manifeste très neltement et diffère du frisson thermique en ce qu'il se manifeste dans des affections fíbriles marquées par une température liès élevée. Les toxines produites par les microbes dans l'organisme infecté sont la source de ces frissons. Ces mèmes poisons sont susceptibles de déterminer suivant la dose loutes les formes d'hyperkinésie des centres moteurs encéphalo-médullaires : frisson, tremblement, contracture, conculsion.

Le frissox occupe, en somme, l'échelon le plus inférieur de la convulsion; le tremblement accompagne ordinairement les mouvements volontaires, mais il constitue souvent un frisson exagéré, un véritable état convulsif. Il se produit alors dans le repos arec des redoublements et des rémissions qui le rendent très analogue aux convulsions.

La contracture est un épisode de la convulsion; on l'observe souvent au débul et à la fin des grandes convulsions; mais ce phénomène est généralement passager ; la 
contracture généralisée ne peut se prolonger; l'aninal mourrait asphyxié ; il n'y a que les contractures localisées qui résultent de traumatismes ou de lésions du système nerveux engendrées par destoxines qui puissent subsister. C'est pour ces motifs qu'il y a des poisons conculsivants el qu'il n'y a pas à proprement parler de poisons contracturants. La convulsion vérilable envalit tous les muscles soit de la vie animale, soit de la vie organique; le frisson intéresse superficiellement ot exclusivement les muscles de la vie animale.

Signification. - Lefrison se manifeste principalement dans les maladies infectieuses, particulièrement dans les septicimies, les pusteurelloses, les formes graves du coryza gangreneux, dans le rou!get du porr, daus les formes aiguës du charbon bacléridien, dans l'infection puerpérale de la varhe, dans le type aigu du rharbon symptomatique.

On constate des frissons très intenses dans la peste hovine et dans la morve aiguë. Ils amoncent généralement une petite élévation le température et coexistent avec un grand nombre de symptômes grénrírax.

\section{XXXII. - PARAISSIES.}

Définition. - Labolition ou la diminution de la contractilité musculaire r'ésultant d'une perturbation dans l'innervation motrice, portent le nom de paralysic ou d'atinésie.

l'une manière plus générale, le terme paralysic indique l'abolition d'une fonction motrice, sensilive, sensorielle, vaso-motrice. Quand on l'applique à la molilité, il indique la suppression ou la diminution de la motricité des muscles striés ou des muscles lisses.

La paralysie molrice est la conséquence d'une lésion matérielle du neurone moteur (paralysic motrice orgunique) ou résulte de troubles purement dynamiques daus le fonctionnement de ce neurone, comme]l'inhi- 
bition, les influences l'arrèt (paralysie motrice fonctionnelle).

C'est une monoplegic yuand la paralysie est localisée ¿ un membre, un groupe musculaire ou un muscle (monoplégic faciale, brachiale, etc.), unc lémiplégic quand elle envahit tout un còté du corps; elle est dite transverse ou croisée lorsqu'un bipède diagonal est frappé; elle est ulterne quand une moitié đH la lace est paralysée en mème lemps que le lipède laléral du coté opposé; c'est une paraplégie quand la paralysic est limilée au train postérieur; c'est une liplégic quand la praralysie est bilatérale ou intéresse les quatre membres. Tantòt les muscles paralysés sont mous, llasques, relàcliés; on peut llechir ou étendre les membres sans effort (paralysie flasque ou fluccule), tantòt ils sont raides, contracturés (paralysie spusmodique ou spastique).

On désigne sous le nom d'unalyésie et d'anesthésie la disparition de la sensibilité ì la douleur et au tact.

La faiblesse musculaire genérale, la parsisie et la paralysic complite expriment les divers degrís de la paralysie motrice.

1"Faiblessemunculaire on parésie. - La fuiblesse musculaire yenerale on la purésie se traduisent par une grande fatigue, par une démarche chancelaute souvent accompagnée de clıutes répétées et de décubitus prolonøéé

C'est uı des symptòmes des maladies les plus diverses: maladies le la moelle et du cerveau, maladies fébriles on général, muluties infectieuses et tout particulièrement les affections lyphoüles des solipèdes, preumonies infcctieuses, coryza gangreneux, clarbon bacteridien, tuberculose, rouget, peste bovine, coryza parasituire, tournis du mouton, liarhee du rean, muladies typhö̈des des rolailles, animic, hydrohémie, diabítc insipide, ictêr', voire mème le simple catarihe stomacul du chcral et celui des rolailles.

La faiblesse musculaire est également symptomatique 
248 SÉMHOLOGIE DE L'APPAREIL DE L'INNERVATION.

d'un grand nombre d'empoisonnements (arsenic, phosphore, mercure, ptomaines, etc.). Elle peut être provoquée expérimentalement par des injections de substances diverses (urine, acide lactique, essences).

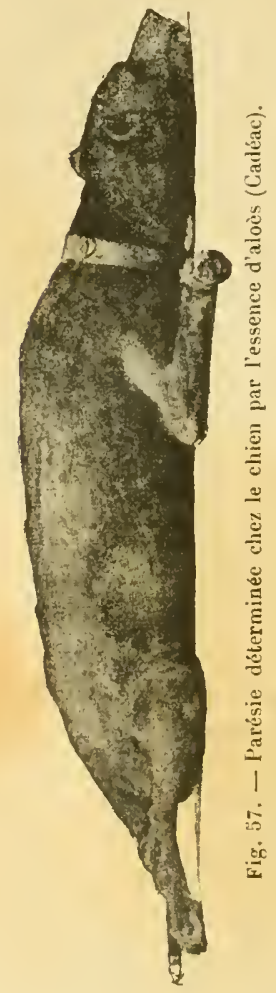

Dans les cas de maladies infectieuses, et dans toules les maladies fébriles, il n'est pas douleux que la faiblesse musculaire est la conséquence d'une véritable intoxication par les produits de sécrétion microbienne ou par les produits de dénutrition.

L'expérience a montré, en effet, que l'inoculation des produits de culture filtrés et stérilisés provoque non seulement de l'hyperthermie, mais encore une faiblesse musculaire très marquée chez tous les aninaux. On trouve encore un argument en faveur de lintoxication dans les résultals obtenus par Charrin, à la suite de l'inoculation du produit de cullure du microbe pyocyanique. Charrin a constaté, en elfet, qu'il se produit chez le cobayc, deux à trois mois après l'inoculation, une monoplégie ou une paralysie spasmodique, sans lésion de la moelle, des nerfs, ni des muscles.

Ces accidents tardifs sont la conséquence du trouble nutritif causé dans les cellules par le poison qui les a imprégnées.

20 Paralysies. - Les paralysies sont d'origine cérébrale, médullaire ou periphérique. 
१. Paralysies d'origine cérébrale. - Le cerveau, qui est le centre des excitations volontaires, est une cause de paralysie quand les circonvolutions dites motrices ne lancent plus d'excilations initiales ou quand celles-ci ne peuvent plus gagner par la portion sous-jacente du centre ovale et de la capsule interne, le faisceau moteur qui constitue le faisceau latéral de la moelle.

Ces paralysies consistent en des monoplégies, des hémiplégies aflectant très souvent le type alterne, parce qu'elles atteignent les noyaux des nerfs craniens.

Les MONOPLÉgies résultent de lésions circonscrites de la couche corticale du cerveau ou de I altération de quelques tibres motrices (monoplégie brachiale, crurale, faciale, lingruale, etc.). Ces monoplégies se distinguent des monoplégies spinales ou périphèriques (paralysie brachio-faciaíc) parce qu'elles sont très souvent alter"nes; la face est paralysée du côté de la lésion, les membres du còté opposé; il y a souvent en même temps inégalité des pupilles, myosis ou mydriase, quelques troubles psychiques malgré l'indépendance des centres psycho-moteurs.

L'HÉMIPLÉGIE consiste dans l'abolition plus ou moins complète des mouvements volontaires dans une moitié du corps.

Elle relève toujours soit d'une lésion des neurones moteurs corticaux, soit des fibres qui partent de ces neurones et coustituent en se réunissant le fuisceau dit pyramidal.

Quelques considérations anatomiques sur la disposition du faisceau pyramidal ou faisceau volontaire sont indispensables pour comprendre la production de l'hemiplégie et ses caractères.

Le faisceau pyramidal occupe les deux tiers antérieurs du segment postérieur de la capsule interne. A ce niveau, il peut ètre subdivisé en trois faisceaux secondaires qui sont, d'avant en arrière et de dedans en dehors : le fais- 
ceau de la face, celui de la langue et celui des membres. Poursuivant son trajet, ce faisceau pyramidal occupe li partie moyenme de l'étage inférieur du pédoncule, puis, dans la protubérance, éparpille ses fibres pour les ramasser de nouveau dans la pyramide bulbaire. Dans la prostubérance et dans le bulbe, il perd une partie de ses libres, qui se mettent vraisemblablement en rapport avec les noyaux des nerfs moteurs cèrébraux (apı ès décussation) particulièrement arec les noyaux du facinl et de l'hypoylosse.

Au niveau du collel du lulbe, le faisceau pyramidal se divise en deux faisceaux secondaires. L'un, faisceau pyramiclal direct ou de Türch, lescend dans la moelle, du même còté, le long du sillon médian antérieur, et peut étre suivi jusqu'à la partie moyenne de la moelle dorsale. Chemin faisant, il s'entre-croise avec le faisceau pyramidal lirect situé dans le còté opposé, ses fibres se terminent en réalité dans la moitié opposée de la moelle. liautre faisceau pyramidal croisé passe d'emblée dans le cordon latéral opposé et se place près de la corne postérieure.

On le suit jusquà la partie inférieure le l'axe spinal. L'étude des dégénérations descendantes el l'embryologie ont parfaitement établi le trajet et la topographiede ces faisceaux pyraınidaux direct et croisé.

Le faisceau pyramidal direct peut faire défaul et la décussation être totale; il peut, par contre, avoir un volıme éral ou supérieur à celui đu faisceau croisé (Flechsig).

Enlin, le faisceau pyramidal croisé peut manquer, faute de décussation, et on concoit que, dans ces cas, l'hémiplégie siège du mème còté que la lésion cérébrale.

Le faisceiu pyramidal, dans la capsule interue, est contigu au faisceau sensilif. Au niveau de la protuberance et du bulbe, il touche le tronc de plusieurs nerfs craniens (moteur oculuire commun et externe, facial et hypoglosse). Ces rapports expliquent la possibilité d'une lésion simultanée du faisceau pyramidal el d'un ou plusieurs de ces 
organes et, par suite, la coexistence d'une hémiplégrie croisée des membres arec une paralysie directe d’un nerf cranien. L'hémiplégie d'origine enciphalique frappe le côté du corps oppose al l'hemisphère malade; c'est une hémiplégie rroisée.

Si le faisceau pyramidal est détruit ou comprimé dans son trajet encéplılique, l'hémiplégie est dite cérébrale; si la lésion siège au-dessous de l'entrecroisementdes pyramides, dans la région cervicale, l'hémiplégie est dite spimale.

Caractères. - On reconnait l'hemiplegie cérebrale à la participation plus ou moins prononcée des nerfs craniens, aux phẻnomènes psychiques observés et à l'exploration électrique; la contractilité Gectro-musculaire est conservée à la faradisation et à la galvanisation.

L.es affections du cerveau capables de produire l'hémiplégie sont: l'h's-

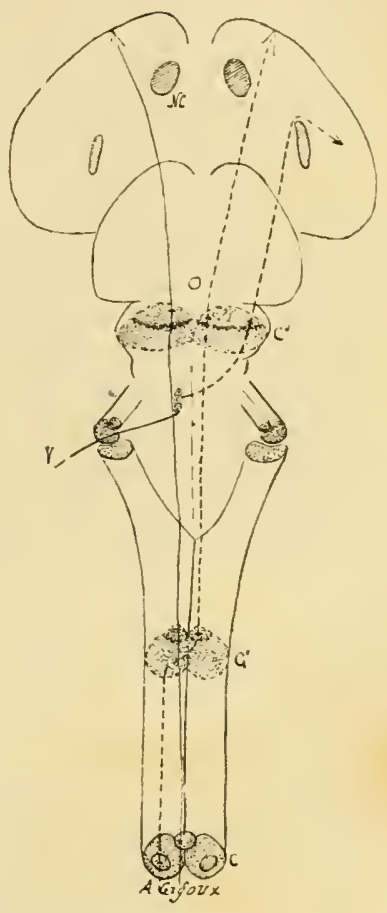

Fig. "is.

Disposition du faisceau pyramidal. - $c^{\prime}, c^{\prime}, c^{\prime \prime}$, coupes au niveau de la moelle, du collet du bulhe, des lubercules quadrijumeaux, $-V$, nerf eranien trijumeau. La ligne pointillée repressente le faisceau pyramidal croise, et le Irait plein le faisceau pyramiulal direert.

morragie cérébrale, les ernbolies, les tumeurs, les abrès, les tubercules, les purusites 
(commes, trichines, etc.), les affections inflammatoires.

Toutes les fois que le faisceau moteur est lésé l'bémiplégie en est la conséquence.

Les maladies infecticuses et toxiques produisent l'hémiplégie en engrendrant des embolies, des thromboses ou des hémorragıes (hémorragies méningées, corticales, etc.).

C'est par un mécanisıne analogue qu'on voit l'hémiplésie compliquer chez le chat et le chien la maladie du geune âye et des maladies des voies respiratoires (catarrhe infectieux du chat et surtont les maladies du cœur et en particulier les lésious de l'orifice mitral).

Tantòt l'hémiplégie survient brusquement sous forme l'altaque apoplectiforme, avec ou sans perte de connaissance, tantòt elle débute par de la parésie, les vortiges et des troubles variés. Elle est somvent précédée chez le rhien de manifestations épileptiques. Ine fois constituée, elle présente d’alıord une période de paralysie flasyue suivie plus ou moins rapidement d'une période de contracture.

la piralysie rasque est totale ou partielle. Si la face est. paralysée, elle est asymétrique ; le còté paralysé est tirí du côté sain (Voy. Parulysie du facial). Aux membres, les muscles sont llasques et sans consistance; les divers mouvements sont abolis.

Ces paralysies ne sont jamais flaccides d'une manière permanente; elles peuvent devenir spastiques sous l'influence de la moindre excilation.

Pour expliquer la contracture permanente, on admet que le faisceau pyramidal, dans les mouvements voulus, exerce une action excito-notrice sur les cellules des cornes antéripures; f'altération de ce faisceau exciterait ces cellules en permanence. On accorde aussi au faisceau pyramidal un ròle d'arrết sur la machine motrice médullaire " toujours sous pression ". Quand ce faisceau est délruil, il n'exerce plus son rỏle d'inhibition. La machine privée de son frein fonctionne indéfiniment, la contraction muscu- 
saire, due à ce foncliomnement, est comme lui ininterrompue, la contracture survient et persiste.

En outre, dans l'hémiplégie, il $\mathrm{y}$ a parésie des membres sains; les réflexes tendineux sont exagérés, la percussion de la région rotulienne produit le soulèvement brusque et énergịue du memlire; la température s'élève quelquefois du còté paralysé par suite de la participalion du sympathique à l'hèmiplègic; il n'y a pas de réaction de dégénérescence tant qu'il n'y a pas d'umyotrophie. On constate fréquemment chez le chicn des mourements chorëryues, des tremblements, de l'atrophie musculaire, osseuse, des troubles vaso-moteurs caractérisés par l'oedème de l'extrémité de la patle et sourent accompagnés de la formation d'escarres.

Les hémiplégies motrices évoluent seules ou s'accompagnent d'hemianesthésie sensitiro-sensorielle qui consiste dans la perte de la sensibilité, de la totalité de

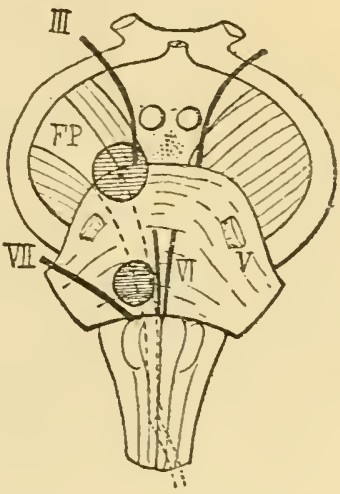

Fig. :̈9. - Faceinfèrieure de la région pédonculo-protubérantielle d’aprẻs Eonchard el Charcol).

III. troisiime paire ou moteur oculaire commun. - $\zeta$. trijumeau. II, sixiome paire ou moteur oculaire esterne. - VIl, septième paire ou nerf facial. - FP, faisceau pyramidal daus le pied du pédoncule. - La lésion sup rieure (cercle strié transrersalement) est celle qui correspoud au ssndrome de Weber ou paralssic alterne supérieure. La lésion inférieure est celle du șnd rome de MillardGubler. la moitié du corps, ordinairement la mème qui est déjà paralysée; la limite de l'insensibilité occupe la ligne médiane. Elle intéresse lit rue, l'ouie, la langne, la peau, etc.

L'hemianesthisie sensorielle indirue presque toujours une lésion capsulaire et non un foyer cortical. L'hémiaCADÉac. - Sémiologie, àe édit. II. $-1:$ 


\section{2วั4 SÉMIOLOGIE DE LAPPAREIL DE L'INNERVATION.}

nesthésie d'origine corticale se borne ordinairement à la semsibilité générale et respecte la sensibilité sensorielle.

L'hyperalgésie est généralement bilatèrale et régionale.

b. Paralysie d'origine pédonculaire, etc. - Les altérations pélonculaires, bulbaires ou protuberunticlles déterminent une paralysie alterne caractérisée par une hémiplégie faciale et une liémiplégie des membres du còté opposé; d'abord la prolubérance est un lieu de passage pour les fibres nerveuses réunissant les noyaux bulbaires et médullaires aux centres supérieurs. Les cordons se rendant aux centres butbaires s'y entre-croisent, tandis que les cordons motenrs des nembres s'entre-croisent plus en arrière. Il s'ensuit que toute lésion protubéran. tielle unilatérale entraine une paralysie du même còté dans le territoire innervé par des uerfs craniens et une paralysie du còté opposé dans les régions innervées par les nerfs rachidieus. C'est ce qui constilue le syndrome de Millard-Gubler ou hémiplégie alterne.

Cette hémiplégie alterne consiste quclquefois exclusivement dans la paralysie de l'oculaire moleur commun du còté de la lésion avec hémiplégie du còté opposé. Ce type de paralysie alterue constitue le syndrome de Weber.

Le syndrome de Weber consiste donc dans la combinaison d'une paralysie du moteur oculaire commun du còté de la lésion avec une hémiplégie croisce, totalc ou non, motrice et ıuelquefois sensitive. Les lésions bulbaires symétriques donnent naissance à la paralysie labio-glossolaryngée (Voy. Pathologie interne).

c. Paralysies d'origine spinale. - Elles affectent fiéquemment le type paruplègique intéressant les membres postérieurs et quelquefois les membres antérieurs.

On peut constater del'hémiplégie ou de la monoplégie; la motricité est toujours supprimée du côté de la lésion. 
Ces paralysies se manifestent loutes les fois quil y a interruption dans les rapports du faisceau latéral de la moelle avec la substance grise des cornes antéI ieures.

Les paralysies qui ı’ésultent de la compression ou de la destruclion dine moitie latérale de la moelle sont caractérisées par une donleur milatérale qui peut cesser spontanément sans qu'on puisse préciser la cause de sa disparisition. L'ensemble des troubles motenrs et sensilifs qui résultent de cetle compression constituent le syndrome de Broun-Séquard.

Ce syndrome est caractérisé de la manière suivante :

Iu còlé correspondant à la lésion, on observe :

$1^{\circ}$ Lne paralysie motrice plus ou moins complète comprenant à l'occasion tous les muscles du tronc lu còté malade, et s'accompagnant, dans certains cas, d'exagération des réflexes tendineux el cutanés.

$2^{\circ}$ Dans certains cas une absence complète de troubles de la sensibilite. qui reste absolument normale; dans d'autres, au contraire, il existe dans tout le colé paralysé une hyperesthesie quelquefois assez considérable.

$3^{\circ}$ Une zone étroite d'anesthésie, plus ou moins marquée, juste au-dessus du terriloire lıyperesthésique. Celte anesthésie est due à la lésiou des racines raclidiennes postérieures du còté lésé qui sont en rapport avec l'agent de la compression.

$4^{\circ}$ Lne autre zone, supérieure à la précédente, dans laquelle on constale un certain degré d'hyperesthésic généralement bilatérale el sous forme d'une ceiuture à peu près complète.

" "3o Dans les parties paralysées, des troubles trophiques de la peau, des muscles (atrophie musculaire), des articulations, etc., et enfin des troubles vaso-moteurs: rougeur de la peau, élévation de la température locale.

Uu còté opposé à la lésion, on trouve :

$1^{\circ}$ L'intégrité absolue de la motilité, des reflexes, etc. ; 
250 SÉMIOLOGIE DE L'APPAREIL DE L'INNERVATION.

20 Une anesthésie géuéralement complète, portant sur tous les modes de la sensibilité.

Ces sortes de paralysies sont les plus communes et les plus faciles à constaler chez nos animaux: elles ne saccompagnent janais d'altérations psychiques, ni de lésions des nerfs craniens, el sont toujours suivies de l'atrophie des membres qui cessent d'ètre innervés.

L'atrophie musculaire consécutive s'explique facilement par l'influence trophique exercée sur les muscles par les cellules ganglionnaires des cornes motrices antérieures de la moelle. De plus, ces parilysies sont sensitives et motrices, parce que le processus alteint toute la muelle, et qu'elles s'accompagrnent généralement de la paralysie de la vessie et du rectum.

Les troubles de la sensibilité offrent de grandes variations; on peut constater des zones d'anesthésie el des zones d'hyperesthesie el d'hyperalgésie.

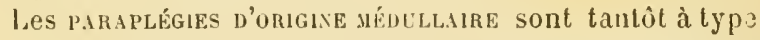
flusque, tantôt à type spusmodique. Eilles revètent le premier lype quand la moelle est clétruite dans toute son épaisseur; elles affectent le second yuand les racines el les nerfs rachidiens sont comprimés ou irrités. H. Bouley a décrit chez le chich un cas d'hémiplégie spasmodique déteruninée par une balle qui avait atteint la moelle au niveau de la septicme vertèbre cervicale; elle avait supprimé la motricité mais elle avait respecté la sensibilité (1 .

l.es troubles de la nélicéctivité sont fréquents dans les paralysies : les réflexes sont exagérés, diminués ou supprimés. Leur conservation est subordonnée à l'intégrité de l'axe spinal réllexe, c'est-à-dire au passage des excitations conduites de la substance grise de la moelle aux cellules motrices des cornes antérieures qui les trans. mettent aux muscles.

Les réflexes tendincux (réllexe rotulien, réllexe du ten-

(1) 11. Bouley, Recueil de mél. vél., 187\%, p. 13is. 
don d'Achille) sont exagérés toutes les fois que les rapjorts d'une portion de moelle comprimée ou détruite ont rossé arec le cerreau.

Le réflexe rotulien est connu sous le nom de signe de liestphal, parce que cet auteur a établi que les fibres par Ir zquelles passent les impressions centripètes nécessaires jour la production de ce réflexe se trouvent situées dans I. zone d'entrée des racines (bandeleltes externes de ('harcol et Pierret).

Les reflexes tendineux comme les réllexes cutanés et l" tellaires son considérablement exagérés dans la pachy"iningite ossifiante et dans tous les cas de compression lente de la moelle.

Le réflexe pluntaire est quelquefois si vif qu'il suffit de soufller énergiquement sur l'extrémité de la patte pour provoquer une extension énergique du membre. Les frictions cutanies de la face interne d'un membre provoquent quelquefois des mouvements cloniques de la partie symétrique du membre opposé. La percussion du jaırel peut provoquer de l’épilepisie spinale.

On peut constater en même temps l'exagéralion du riflexe bulbo-cacerneux et de celui du sphincter urétral.

Les réflex€s sont supprimés si les fibres nerveuses cont détruites ou s'il n'existe plus de relations entre la substance grise et les cornes antérieures. Les muscles qui ont cessé de recevoir des excilations motrices dégénèrent : la contractilité faradique de ces muscles est abolie ou bien ils ne réagissent que par une secousse lente : la contractilité voltcïque est au contraire conservée et même exallée ; c'est ainsi que, du còté paralysé, la contraction minima est obtenne arec un comrant d'une intensilé insuffisante pour faire contracter les unuscles du còté sain. La secousse musculaire, au lieu d'ètre rapide comme à l'état normal, est, dans les muscles paralysés, lente, paresseuse, et se transforme avec un courant relativement faible, en une contraction tétanique qui persisle 
tant que le courant passe. Cette lenteur de la secousse constitue peut-èlre le caractère le plus important de la réaction de dégénérescence.

Les paralysies d'origine médullaire sont généralement ascendantes, c'est-à-dire qu'elles se propagent d'arrière en avant.

Les puralysies spinales sont symplomatiques des uffections: paclıyméningite ossifiante, mèningite spinale, myélite, hemorragie, écrasement de la moelle, compression par des tumeurs, tubercules, canures. On les observe encore dans la rage du chien, la domine du chevil, daus la maladie du ienne aye chez le chien.

La moclle lombaire est alteinte quand il y a paralysie de la vessie et du rectum; la moelle dorsale et la moelle cervicale sont intéressées quand il y a paralysie des membres.

La paralysie d'origine spinale débute soudainement quand elle est la conséquence d'un traumatisme on l'une inflammation aiguë; son développement est graduel quand elle résulte d'une tumeur.

c. Paralysies d'origine périphérique. - Ce sont ঐles monopléyies; elles se différencient des paralysies d'origine cérébrale par l'absence d'altérations psychiques quand elles n'atteignent aucun nerf cranien. Ue plus, elles s'accompagnent habituellenent d'anesthésie, d'atrophie musculaire, de paralysie vaso-motrice, de troulles trophiques. L'exploration électrique donne la réaction de dégénérescence.

Les paralysies périphériques frappent des territoires plus ou moins vastes, suivant que la lésion siège à l'orighine du plexus ou seulement sur les cordons nerveux qui en émanent.

Les causes les plus diverses peuvent engendrer ces sortes de paralysies. Il faut mentionner : les contusions, les compressions, les distensions et les inflammations des uerfs. 
On sait d'ailleurs que certaines paralysies périphériques sont d'origine réflexe. D'autre part, il n'est pas absolument rare de constater des paralysies musculaircs périphériques en l’absence de toute lésion apparente.

Dans ce dernier cas, les modificalions matérielles sont vraisemblalılement peı importantes, car la paralysie peut disparaitre instantanément comme elle avait apparu.

Dans les paralysies récurventiclles, on doit chercher les altérations du nerf récurrent à sa partie périphérique, notamment à la rérrion laryngienne dans 99 p. 100 de lous les cas de cornage nerveux. Ces altérations ne dépendent vraisemblablement pas d'une névrite, mais d'un simple processus secondaire de dėgénérescence (Thomassen) (1).

\section{XXXII. - VERTIGES ET MOUVEMENTS FORCÉS.}

Définition. - Le rertige est un trouble de l'innervation encéphalique, uno erreur de sensation sous l'influence de laquelle l'homme croit que sa personne ou les objets enviromnants sont animés d'un mouvement giratoire ou oscillatoire. Sous l'influence de la mème cause les animaux sont incités à tourner en cercle ou en manège.

Les troubles consécutifs aux lésions irritatives du cervelet, à la section des canaux demi-circulaires, à la piqùre des péloncules cérébelleux, ou des pédoncules cérébraux, à la galvanisation de la région cérébelleuse chez l'homme sont des phénomènes vertigineux. On peut même affirmer que toutes les lésions asymétriques des centres nerveux qui troublent l'équilibre sont des causes de vertige. Au point de vue expérimental, on distingue le vertige rotatoire et le vertige galranique.

Vertige rotatoire. - Quand on subit un mouvernent de rotation rapide; s'il y a arrèt brusque, les objets envirounants ou notre corps nous semblent animés d'un

(1) Tluomassen, Revue rèt., 1902. p. 296. 
mouvement giraloire de direction opposée à celle du premier mouvement.

Les animaux eliectuen! des mouvements de rotation caractéristiques; ils tournent en cercle, en manèye (fig. 60), en rayon de roue (fig. 61). Cheranx, brinfs, chiens, et lapins marchent quelquefois pendant des heures entières

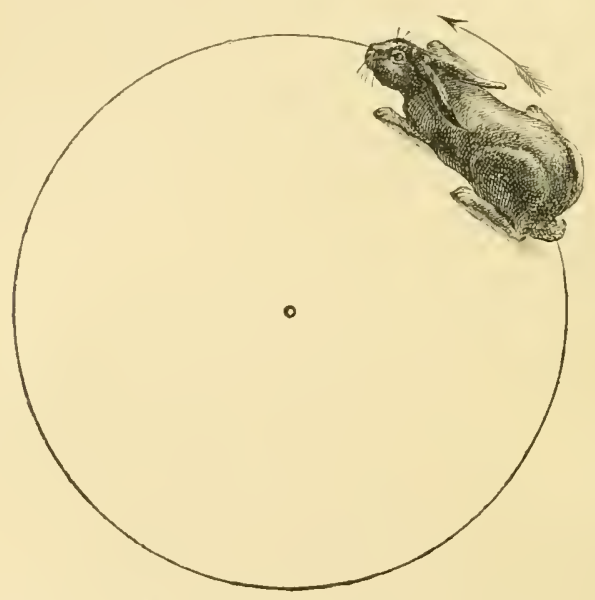

Fig. 60. - Mouvements de manège (d'après Beaunis).

antour du même point. Tantòt les cercles décrits se rétrúcissent de plus en plus, de telle sorte que l'animal finit par tomber, tantòt le sujet décrit un cercle de plus ou moins graud rayon clans le sens des aiguilles d'une montre ou en sens inverse. Quelquefois les mouvements de rotation s'effectuent autour du train postérieur qui sert d'axe. Il peut mêue $y$ avoir roulement ou rotation sur l'axe.

Dans le mouvement de rotation sur l'axe, l'animal tourne aulour de laxe longitudinal du corps. Il lombe sur un cóté, puis il exécute, très rapidement, ce monve- 
mrnt, il semble mù par une force intérieure irrésistible. Ce phínomène est symptomatique d’une lésion des pédonculles cérébelleut moyens (1), d'une altération de la partic

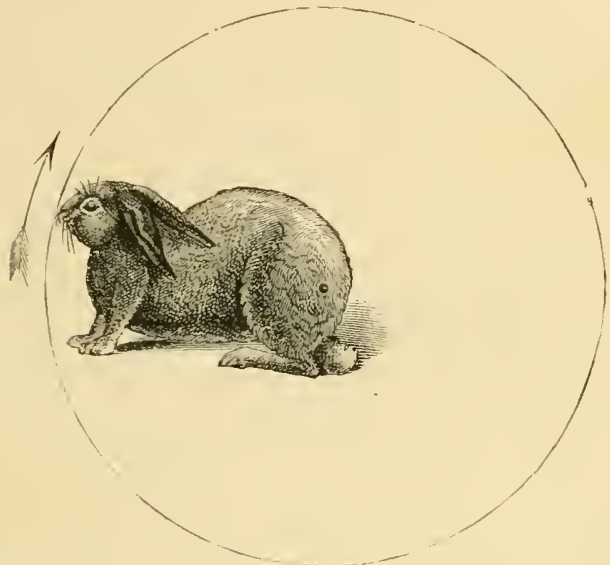

Fig. 61. - Mourement de rolation en rayon de roue (d'après Beaunis,

supérieure et externe des fúduncules cérétranta, des lésions de la partie posterieure de l'encéphale et même des lésions lis plus diverses des himisphires.

T ertige galvanique. - Quand les deux électrodes d'in courant galvanique sont appliquées sur les apophyses 1:'astoïles, il semble au sujet en expérience, pendant que Is courant est fermé, que les objets environnants se 'ćplacent du pôle négalif ou positif ; c'est un mouvement rumparable à celui d'une roue se déplaçant parallèlement an visage; lecorps semble tourner dans le mème sens.

(1) Mauri, Du monvemient de rotation (roulıment); lésions des pédoncules rircbellevx; diagnostic daprès le mode et le sens du roulement (Revue vélínaire, 1893, p. 1). 
Mécanisme. - La cause iuline des vertiges parait résider dans les contractures plus ou moins intenses qui déforment le colps et déplacent le cenlre de gravilé, provołuant aiusi l'iustalilité de l'équilibre. Le sujet atleint de verlige éprouve à la fois des illusions sensorielles se traduisint par le mouvement apparent des objets et du sol et une sensation subjective le pesantenr qui fait pencher le corps daus le sens de la rolation.

" L'ètat vertigineux comporte donc deux éléments : $1^{\circ}$ un élément subjertif, se traduisant par des hallucinations et des jüements faus sur la situation tes objets euvironnants; 2o un élément ol,jectif el mécanique consistant dans des conlractures réllexes et déformantes. Ces deux éléments procedent de la mème cause póriphérique ou centrale, et, pour ne parler que du verlige de Purkinje, ils sont causés par des impressions nées dans les canaux demicirculaires. Ces impressions excessives et importantes atteignent, d'une paıl, l'écorce cérébrale où elles suscitent des images fausses, et, d'autre part, les centres moleurs qu'elles sollicitent d'une nanière asymétrique et inégale" (Laulanié).

Le vertige pathologique ue cousiste pas toujours en un mouvement illusoire de rotation soit du corps, soit des oljjets; il s'agit patfois d'une illusion qui sollicite les animaux à se porter en avant ou à reculer.

lia plupart des animaux atteints de maladies vertigineuses marchent habituellement la tête basse; ils sout liébétés, se heurtent à tous les obslacles, prennent des attitudes impulsives; ils poussent au mur. Les mouvements verligineux se produisent fréquemment.par acces yui reviennent quelquefois à intervalles très rapprochés, car ils funissent par déterminer l'épuisement et la mort.

Causes du vertige. - Le vertige est une manil'estation symptomatique :

$1^{\circ}$ D'affections des mininges et de l'cncepphale (congestion, 
anémie, inflammatious, hydrocéphalie, liémorragie, anémie, tumeurs, abcès, œlèmes), c’est alors le vertige cérébral.

2. De molaties de loreille (lésions des canaux demicirculaires, gale psoroptique du lapin, otite interne, corps itrangers, simulies) qui déterminent des mouvements de rotation, de culbute, de manège, dont la direclion est constamment la mème que celle du canal sectionné, ou des accideuts nerveux désignés sous le nom de verliges, d'étourdissements.

Les cheraux attelés ou montés préseutent une sudation intense, lèvent la tête, l'agritent d'un côté à l'autre; ils se livrent aux monvements les plus désordonnés, s'emporteut, se cabreut, se défendent, tombent ou s'arrètent brusquement el restent insensibles à toutes les excitafions pendant les quelques inslants que dure l'accès.

Il suffit sourent d'examiner loreille pour constater l'existence d'un bouchon de cermmen refoulant le tympan; mais chez le chien ce sontsurtout les affections suppuratives de l'oreille moyenne et de l'oreille interne qui provoquent le vertige labyrinthique.

$3^{\circ}$ Des maludies de l'appureil digestif (indigestions, altérations du foie, corps étranger's, obstructions, parasites, gastro-entérites); c'est alors le vertige abdominal.

$4^{\circ}$ Les maladies lu cœur (insuffisance valvulaire chez le chicn peuvent égalemant le produire par congestion, par embolie, etc.

$\tilde{3}^{\circ}$ Les maladies des cavités nasales (larves d'œestres chez le mouton, linguatules chez le chicn).

6o Le vertige peut ètre eufiı observé daus un grand nombre d'empoisonnements (essences, alcool, solanine, uxyde de carbone).

$\tau^{\circ}$ Dans certaines affections de lixil (amaurose, rayons solaices trop intenses, éclats de la neige, reflets des œillères), on observe des vertiges désignés sous le nom d'éblouissements. 


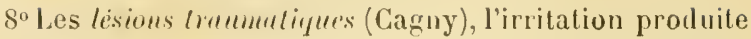
par la litiere sur la partie inférienre des membres (linibert) provoquent aussi des plénomènes vertigineux apyelés faux vertiges.

$9^{\circ}$ Le rertige conslitue une allection spéciale chez l'homme et clıe le chit. Cet animal présente en effet un ensemble de troublés nerveux qui ont été décrits par Cieslier sous le nom de vertige paralysunt. Il s'observe particulièrement chez les "halles pendant l'hiver et se traduit par des alternatives d'exacerlation et d'amélioration. Les accès sont tantòt courplets, lantòt incounplets. Ils se bornent parfois à une titubation sur les jambes avec fixité du regard et immobilité passagère; mais le plus souvent la parésie motrice est poussée jusqu’à la paralysie.

La plus commune de ces paralysies est celle des extenseurs de la nuque qui prodıit la chute de la tête sur le sol. Cette chute est précédée de salutations de la tète exprimant la lutte de l'animal contre la parésie.

Dans les accès complets, le clıat paralysé de la nuque et des quatre membres tombe le ventre sur le sol et simule le chat crevé.

Le cliat comme l'homme affecté de cette maladie peut présenter le faux trismus et l'impossibilité de manger. Bien qu'il ne soit pas atteiut de ptosis, il at des troubles de la vision reconuaissables à la fixité des regards et à l'immohilité des paupières.

l.a durée de la maladie est géuéralement moindre chez le cliat que chez l'bomme.

\section{XXXIV. - ATAXIL.}

Définition. - On désigue sous le nom d'ataxie un syndrome caractérisé par l'incoordination des mouvements.

(1) Gerlier, Journal vétérinaive de Lyon, 1891, p. 510. 
Ciest un trouble de la coordination des mouvements. Gertains actes, tels que la marche ou la station debout, penvent ètre envisagés comme la combinaison de plusicur's mouvements ou de plusieurs coordinations musculaires; daus l'un et l'autre cas, les coordinations musculaires se manifestent par une répartition proportionnelle et régulière de l'énergie dans cerlains groupes musculaires et daıs un temps défini.

Quand la Iransmission des impressions est uormale, les réactions sont bien adaptées au but et sont exćcutées llans le temps nécessaire; si la transmission est ralentic, incomplète, on nulle, les réactions le sont aussi, d'où les troubles profonds de la coordination : l'ataxie.

Ailleurs, la transmission des impressions périphériques est normale, mais les centres qui l'emmagasinent sont atteints dans leur fouctionnement et les réactions auxquelles ils président font défaut ou sont incomplètes; il y a encore ataxie.

En réalité, l'ataxie peul donc ètre d'origine périphèrique, ou d'origine centrale.

$1^{\circ}$ Ataxies d’origrine périphérique. - Les ataxies périphériques conprennent toules les interruptions dans les impressions qui interviennent dans le réflexe locomoleur; c'est-à-dire les troubles du sens tactile, du sens muscalaires, de la vision, de l'orientation (canaux demi-circulaires).

L’ataxie périphérique reconnait aussi des degrés et des formes différentes suivant que l'interruption des excitations périphériques a lieu au niveaı du premier neurone ou des neurones du deuxième ou du troisième ordre.

Lorsque le premier neurone (ganglion rachidien avec ses deux prolongements : la racine postérieure et le nerf périphéri(que) est atteint dans son fonctionnement, l'activité de tous los centres coordinateurs est suspendue ou modifiée, l'alaxie s'associe en outre à des troubles très 
intenses des divers modes de la sensibilité. Ceci s’applique non seulenent aux neurones périphériques médıllaires, mais aussi aux neurones périphériquues bulbaires (racine labyrinthique, trijumeur, etc.).

Si la lésion est localisée sur un neurone de deuxième ordre qui transmet à un centre coordinateur les impressions recueillies à la périphérie, les fonctions de ce centre seront forcément suspendues ou altérées, mais l'activilé Jes autres centres continuant à s'exercer normalement, la sensibilité sous tous ses modes sera intacte, à moins que le neurone interrompu ue soit affecté aux voies le perception des impressions périphériques. Il importe de passer en revue les diverses formes d'ataxie périphéripue en examinant ses diverses sources établies par la clinique ou par l'expérimenlation.

a. Ataxie par altération de la sensibilité cutanée. On suit qu'une grenoulle écorchée est incapable de se nouvoir ; elle ne peut ni sauter ni nager, ni se retourner quand on la met sur le dos.

L'atrophie ou la section des racines postéricures provoque l'atuxie tabélique qu'on observe quelquefois chez le chien, affecté de pachyméninģite ossifiante. Elle débute généralement par une vive douleur résultant de la compression des racines nerveuses; les animaux poussent des cris ou manifestent des envies de mordre dès qu'on eflleure l'épjderme; jls refusent de mouler ou de descendre les escaliers. Plus tard, les membres au lieu de se mouvoir dans l'axe du corps se portent en dedans ou en dehors, fortement en avant ou en arrière; ils se heurtent les uns contre les autres, il y en a qui eifectuent de véritables sauts de pie; ils galopent du devant et trottent du derrière. Ces ataxies sont à la fois locomotrices et musculaires.

b. Ataxie par altération du sens musculaire. - La sclérose des cordors postérieur's chez l'homme jatuxie 
locomotrice) ou la section de ces cordons chez les mammifères fait ressortil l'importance du sens musculaire dars la coordination des mourements.

Si l'on sectionne les nerfs sous-orbitaires, le nerf mentomnier et le nerf temporal surperficiel des deux cỏtés chez un cheval, on constate que l'animal saisit exclusivement les aliments avec les deuts et ne se sert plus des lèvres conıme organes de préhension; mais dès que la mastica. tion commence, les lèvres retrouvent leur mobilité et

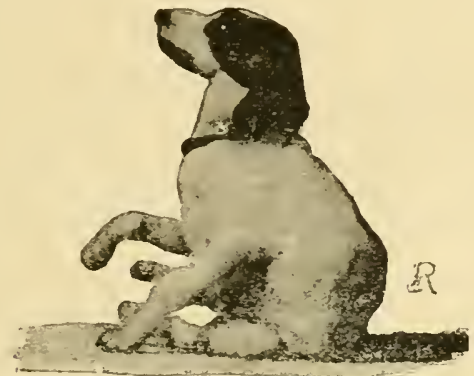

Fig. 62. - Clien alfecté d'incoordinalion motrice.

exécutent les mouvements associés à ceux des muscles masticateurs. C'est que, dans ce deruier acle, les noranx moleurs de l'orbiculaire, du releveur de la lèvre supérienre, du sous-maxillo-labial sont excités en même lempıs que ceux du nasséter, du crotaphyle et des ptérygoidiens.

L'incoordination motrice périphérique accompagne fréquemment les hémorragies spinales. La station et la progression deviennent difficiles, mème impossibles. L aninal " projelle ses membres en avant, en arrière, à droite, à gauche, salıs pouvoir parvenir à coordonner les mouvements, et, s̈il réussit à faire quelques pas, c'est en prenant appui, non sur la face plantaire, nais sur le carpe "(SaintCyr).

Cette démarche, que l’on a fiéquemment décrite en 
médecine vétérinaire sous le nom d’ataxie locomolrice, ne se rapproche de celte maladie de l'homme que par sa physionomie symptomatique. Elle en diffère complètement par la cause : l'ataxie locomotrice de l'homme étant due à une sclérose des eorlons postérieurs de la moelle. Parfois la démarche est vacillante: l'animal tríbuche, entortillant sesjambes l'une dans l'autre ou tombe à chayue pas, plutòt d'un côté que de l'autre, suivant l'étendue et le siège des foyers hrimorragịues. Les membres sont tantôt flasifues, tantòt raides; les riflexes sont conservés.

L'inflammation plus ou moins aiguë ou la sclérose simultanée des cordons-postéricurs et des corlons latérauce que nous avous décrite sous le nom de sclérose pscudo-systématique du cheval, observée par Barrier, Dexler, elc. (1), est celle qui réalise le mieux l'ataxie ou la démarche tabéto-spasmodique parce qu'elle se rapproche le plus du tabes dorsulis de l'lomme, connue encore sous le nom d'ataxie locomotrice progressive, de leucomyélite postérieure, de dégénération grise des cordons postèricurs.

Au pas,les membres postérieur's se lèvent brusquement; ils sont projetés en avant sans souplesse; l'animal fauche du derrière; au trot, l'allure semble se raffermir, l'allure est moins dégingandée que celle du pas; le galop parait être l'allure préférée.

Les mouvements ont cessé d'ètre automatiques; jls sout conscients, voulus, exigreant une attention soutenue; ils sont toujours brusyues, disproportionnés avec le but à alteindre; les membres antérieurs éprouvent à chaque pas une flexion brusque des genoux suivic d'une extension saccadée, de telle sorte que l'animal perd l'équilibre et est menacé de lomber.

L'homme frappe le sol du talon, avec une grande énergie; on dit qu'il talonne. L'animal comme l'homme

(1) Pathologie interne. Tome VIII, p. 307. 
projette ses membres en dehors afin d'élargir la base de sustentation et conserver l'équilibre.

Cel alaxique a plus de peine à marcher an départ qu’au bout de quelques instauts; le cheval reprend pen à peu l'habitude de se déplacer, mais si on l'arrète, il risque de tomber; il lui est presque impossible de tourner, chaque mouvement nouveau exige une éducation nouvelle; c'est toujours la volonté qui s'efforce de suppléer à l'automatisme.

En descendant, l'animal retient la voiture avec peine; clıez l'homme, on désigne cette aggravation des troubles à la descente sous le nom de signe de l'esealier.

l'incoordination tubétique est corrigée en partie par la vue. L'occlusion des yeux chez l'ataxique provoque de l'iustabilité, des oscillations du corps, de la titubation ou une chute immédiale. Cet ensemble de troubles qui accompagne l'occlusion des yeux constitue le signe de Romberg.

Ce signe s'observe également clıcz le cheral. La marche, déjà difficile, devient impossible lorsqu'on vient à aveugler l'animal en lui mettant une couverture sur la tète. Ghez l'ataxique, les impressions visuelles suppléent les impressions musculaires absentes ou émoussées.

L'ataxie coexiste avec un certain nombre d'autres symptômes : abolition des réflexes tendineux et cutanés, troubles de la sensibilité cutanée et profonde, perte de la notion de position, altération du sens musculaire.

"Les troubles de la coordination sont principalement dus à la difficulté qu'éprouvent dans leur transmission les impressions provenaut des parties profondes des membres : muscles, articulations, aponévroses. Les cellules molrices de la moelle ne peuvent alors régler la décharge motrice daus l'exécution du mouvement projeté. Peut-èlre la difficulté de trausmission tient-elle aux lésious qu'éprouveraient les corpuscules de Golgi, petits dynamomètres aunexés aux tendons, près de leur union avec les 
muscles; ces corpuscules présentent en effet un chevelu nerveux très riche, 'gui doit servir dans l'appréciation des eflorts de chaque organe. "

Dans la trenblaxte du moutou, l'utuxie est liée principalement à une nérite intense des nerfs moteurs. La démarche est vacillante; elle se fait par bonds; le train postërieur galope, pendant que l'avant-train trolte; les ongles raclent le sol.

'Toutes les névrites périphèriyues peuvent, indépendamment de tonte altération médullaìe, engendrer l'incoordination motrico. Les ataxies qui accompagnent les intoxications sutumine, ursenicule, etc. relèvent d'une névrite périphérique localisée surlout dans les rameaux sensitifs.

L'alaxie complique quelquefois les névrites ascendantes du plexus brachial (Trasbot) ou des nerfs plantaires (Cadéac).

\section{$2^{\circ}$ Ataxies atorigzine centrale. - $a$. Ataxie par alté-} ration des canaux demi-circulaires ou labyrinthiques.-On sail que la section bilatérale d'un canal demi-circulaire détermine une tendance à la rotation en rapport avec la direction des canaux sectiomnés.

" lour les canaux horizontaux, c'est une rotation ou une tendance à la rotation autour de l'axe vertical. Après la section des canauxverticaux postérieurs, l'animal manifeste une tendance Irès vive à culbuter en arrière. Il se penche en avant et tend à faire la culbute dans la mème direction après la section des canaux verticuux antérieurs.

Après quelques minutes de repos, ces troubles s'apaisent en parlie; le pigcon vole régulièrement; mais il ne se pose pas sur le sol; il le rencontre et parait y tomber comme une masse.

La section bilaterale des trois canaux demi-circulaires, ou mème seulement de deux, provoque des troubles indescriptibles. Abandonné à Ini-mème après l'opération, l'animal fuit égaré, roulant dans tontes les directions, cul- 
butaut plusieurs fois en avant ou en arrière, d'un còté ou de l'autre, irappant le sol et agitant désespérément ses ailes.

Quand il parvient à s'arrêter, il reste assis et titubant, imprimant à sa tète de vives oscillations, qui saufla rapidité et l'étendue, rappellent les oscillations séniles de la tète des vieillards (Laulanié).

Comext wTER préter ces troubles ? C'est que les canaux demi-circulaires sont des organes chargés de nous renseigner sur l'orientalion de la tète et du corps et sur la direction des mouvements de la lête.

- Les troubles moleurs conséculifs aux lésions des canaıx demi-circulaires comprennent à la fois des phénomènes de délicit et des phénonènes d'excitation.

En effet, si l'on enlève les hémisphères cérébraux et si l'on excite, comme l'a fait Laulanié, deux canaux symétriques à l'aide d'excitateur's portant des électrodes capillaires, on obtient les résultats suivants :

Les excitalions électriques dirigées isolément sur chaque paire de canaux demi-circulaires, à l'aide de cour ants continus, déterminent immédiatement des contractures réllexes qui figent l'animal dans une attitude ou sollicitent de sa part une rotation dont le sens est en rapport avec la direction mème du canal excité.

L'excitation des canaux horizontaux détermine un mourement de valse très régulier et lellement précipité yue les fils du circuil s'enroulent rapidement l'un sur l'autre.

L'excitation des canaux postérieurs enlraine constamment lopisthotonos et rarement une culbute en arrière.

L'excitation uniluterale de chaque canal provoque des résultats identiques.

Il résulte de ces expériences que les canaux demi-circulaires font partie d'un appareil sensitivo-moteur dont le fonctionnement entraine des attitudes définies, attitudes dont l'effet est de rétablir l'épuilibre menacé par 
272 SÉMIOLOGIE DE L'APPAREIL DE L'INNERVATION.

les déviations de la tète et les illusions sensorielles qui en pourraient résulter (Laulanié). Ces divers troubles produits dans les expérieuces qui précèdent se retrouvent chez le chicn daus diverses maladies suppuratives qui atteignent les canaux demi-circulaires (Voy. Maladies de lorcille in Pathologie chirurgicale.

b. Ataxie cérébelleuse. - Les recherches de Thomas ont démontré que le cervelet est un centre d'énergie du tonus musculaire, mais cette énergie est affectée au maintien de l'équilibre dans toutes les attitudes et dans tous les nouvements; le cervelet exerce dans chayue altitude, dans chaque mouvement une coordination musculaire spéciale; c'est l'organe de la coordination musculaire.

Le syndrome cérébelleux est surtout caractérisé par la difficulté qu'éprouve l'animal à se tenir debout; la tête pend entre les membres; puis la station debout devient impossible; la démarche devieut étricuse, il reste enfin couché loujours sur le côté correspondant à la lésion ; la tête se trouve reportée sur l'épaule. Excité, il se déplace sur son axe longitudinal en faisant une série de tours; il peut parcourí ainsi jusqu'à 10 mètres. Ce phénomène de roulement s'accompagne d'une déformation du corps de l'opéré par du pleurotlıotonos. Celte alaxie est symptomatique de lésions cérébelleuses (abcès, tumeurs, hémorJ'ugies. Dès que ce centre de l'équilibre est alléré, l'ataxic cirébelleuse, la titubation font leur apparition.

Ataxie dans les empoisonnements aigus. - Il existe de nornbreuses ressemblances entre l'ataxie déterminée par l'extirpation du cervelet et l'ivresse déterminée par l'alcool et diverses essences. On constate de la tilubation, tes oscillations latériles, une démarche irrégulière, incoordonnée, en zigzag. On a vu des chevaux, boire quatre litres de moût de raisin en fermentation et s'enivrer ainsi 
à tel point quón a cru avoir affaire à la congestion cérébrale (1).

Liivresse provoquée par les essences présente un grand

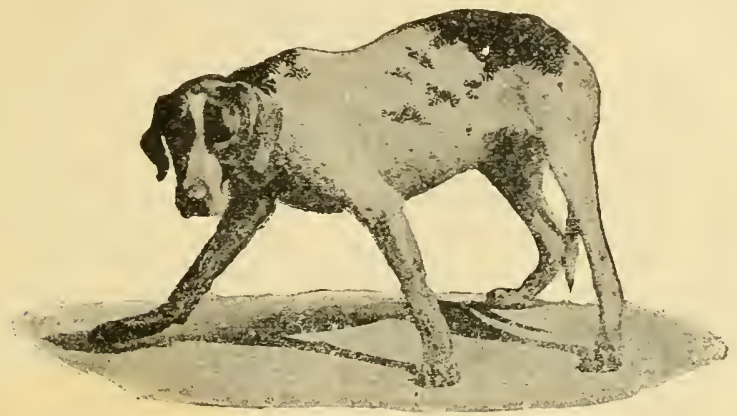

Fig. 63, - Ivresse delerminée par lessence de thym.

nombre de modalités. Toutes les formes d'ivresse sont reproduites par elles. La menthe, l'angélique, le cala-

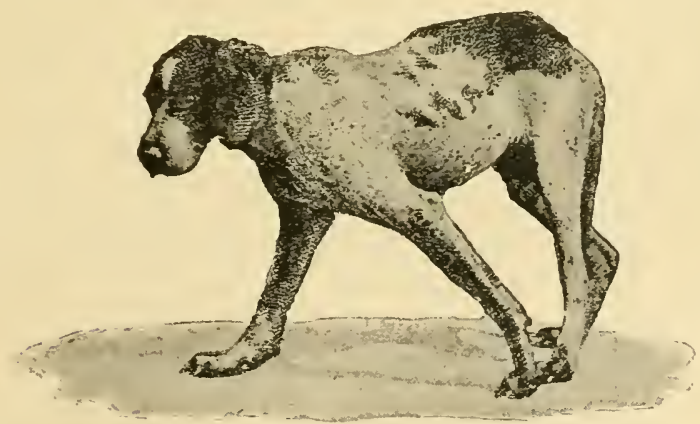

Fig. 64. - Ivresse délerminée par l'essence de serpolet.

ment, etc., produisent l'ivresse saie; la sarriette, le romarin, etc., l'ivresse triste; le fenouil, le basilic, etc.

(1) Mestre, Recueil de méd. vét., 18:2. 
27.' SÉmiologie dE L'APPAREIL DE L'INNERVATION.

l'ivresse folle; la rue et la lavande, l'ivresse stupide; le thym, le serpolet, la mélisse, l’irresse anesthésique; la sauge, l'hysope, etc., l'ivresse batailleuse.

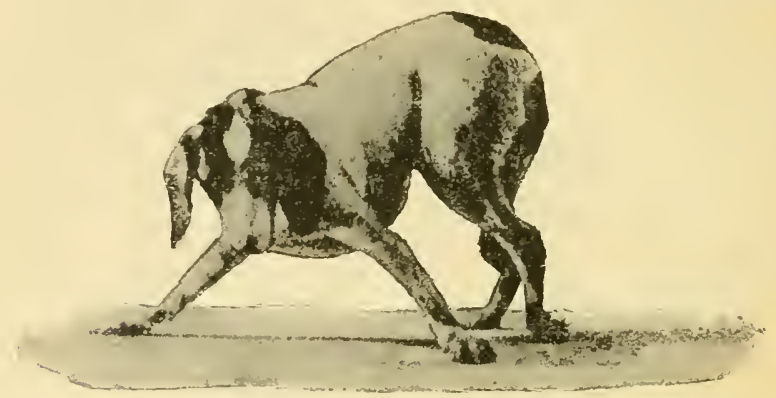

Fig. 6.̈. - Irresse extrème ditermmée par l essence do rue.

Ataxie dans les lésions protubérantielles et du cerveau. - Certains anteurs ont siınalé une ataxie simple des membres associée à des troubles dı sens musculaire dans les lésions prolubèrantielles.

l'ataxie qu'on peut obstrver dans certaines affections cŕćbrales est difficile à ètudier à cause de l'hémìlégie et de lit contracture des membres.

L'ataxie s'ohserve fréquemment chez le chich el chez le cheral saus qu'on puisse en léterminer la cause. Pauchenne a rencontré ce Irouble de la locomotion chez deux jeunes chils; il élait caractérisé par un hochement de la tête et par un tremblement négatif.

\section{XXYV. - ALTÉIATIONS UE LA SENSIBILITÉ.}

Définition. - La sensibilité est la propriété que possèdent lesanimaux d'éprouver des sensations. C'est elle qui est le point le départ des actes réflexes conscients ou inconscients de la vie organique et de la vie de relation. 
11 existe en effel dans tous les organes des appareils récepteurs formés de terminaisons nerveuses plus on moins différenciées et reliées aux centres cérébraux où réside la perception consciente par des fibres conductrices sensitives. Toules les modifications qui intéressent l'une de ces parties provoquent des troubles de la sensibilite.

On peut distinguer des troublessensoriels qui sont l'expression de modifications de la vue, de l'ouie, de l'odorat et di goùt, et des troulles de la sensibilité générale qui est caractérisée par la douleur. Ladouleur peut semanifesterdanstontes les parties de l'organisme pourvues de nerfs sensibles.

La sensibilite ginérale
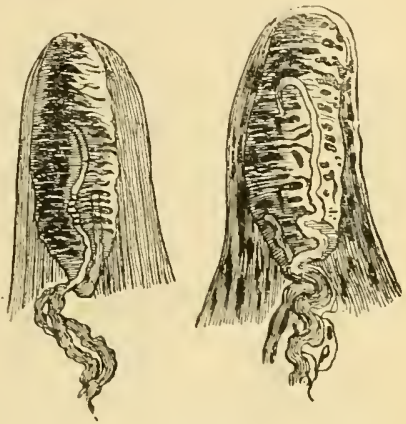

Fig. 66. - Corpusicules de Meissner (d'apres Frey).

$a$, nerf afférent ; $b$, sa gaine de Henle; $d$, ramificalions du cylinilraxe entre les cellules $d, e$, du corpuscule.

comprend anssi les sensibilités supērficielles (sensibilite tactile, sensibilité douloureuse, sensitilité thermique, sensibilités électrique cutanée et musculaire) et les sensibilités mrofondes comme la sensibilité musculaire et la sensibilité articulaire qui ne penvent guère ètre étudiées avec fruit chez les animanx.

I. Sensibilité tactile. - Chez les animaux, le louche est moins parfait que chez l'homme; il se localise généralement aı niveau de la lèvre supérieure et des poils tactiles qu'elle renferme. Ces organes délicats se rencontrent principalement dans le groin du porce, les lèvres du cheval, les moustaches des lélins et des rongeurs. Ces poils sont pourvus d'un bourrelet qui embrasse la région 
moyenne et qui est rempli de terminaisons nerveuses. La disposition de cet anneau ressemble beaucoup aux corpuscules de Meissner (fig. 66).

Les oiscaur se servent de leur bec pour recueillir les impressious tactiles. Le bec et la langue du canard

D

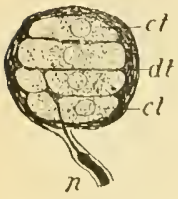

C

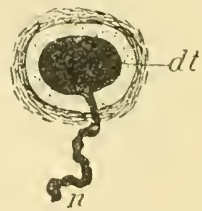

$B$

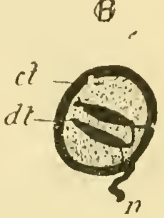

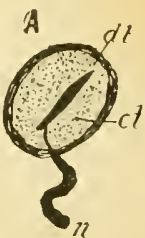

Fig. 67. - Corpuscules de Grandry dans le bec et la laugue du canard (il’après Rauvier).

A, B, C, corpuscules traités par le chlorure d'or: A, corpuscule formé d'uu seul disque tactile; $B$, corpuscule formé de denx disques et vu de trois quarts: C, le même vu de face; 1 , corpuscule du ticl de la langue du canard pourvu de trois disques tacliles $\left(d^{\prime} t\right)$.

renferment en effet des corpuscules analogues à ceux de Meissner, et connus sous le nom de corpuscules de Grandry (fig.)

En outre, les plumes qui couvrent le corps des oiseaux transmetlent les impressions de contact aussi fidèlement que le sabot du chevial.

Sensibilité thermique. - Elle nous renseigne sur la température des corps mis en contact avec le légument cutané ou muqueux. Ce sont des terminaisons nerveuses libres intra ipidermiques étudices par Ranvier qui recoivent les impressions thermiques. Répandues dans les couches profondes de l'épiderme, ces arborescences nerveuses dépourvues de myéline se terminent par des ménisques concavo-convexes, dans les espaces intracellulaires.

On mesure la sensibilité thermique par l'écart minimum 
de tempéralure entre deux corps; l'écart minimum perceplible serail de un cinquième de degré.

Sensibilité électrique cutanée et musculaire. - Les courants électriques permettent d'apprécier le degré de

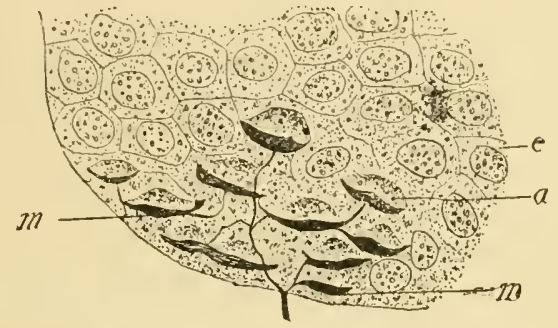

Fig. 6s. - Terminaisons sensilives intra-épidermiques dans la pean du groin du porc (d'après Ranvier).

$n$, fibre nerveuse afférente; $m$, ménisques tactiles appliqués sur des celtules tactiles (a); $e$, cellules épithéliales ordinaires.

sensibililé à la douleur. On peut mesurer le minimum de sensation produit par un courant direct ou indirect et le minimum de douleur.

Le courant électrique appliqué sur les muscles provoque également en dehors de la contraction une sensation particulière qui devient douloureuse avec une certaine intensité de courant; on la désigne sous le nom de sensibilité électro-musculaire.

Sensibilité douloureuse. - La sensibilité douloureuse se manifeste chaque fois que l'excitation d'un des modes de la sensibilité générale dépasse un certain degré. Si, en effet, la sensibilité douloureuse est particulièrement déreloppée dans les régrions mêmes où existe la sensibilité tactile, elle existe égaleınent dans les organes qui en sont dépourvus comme les os, les tendons, les muscles, les viscères et tous les tissus de l'organisme.

$$
\text { Cadéac. - Sémiologie, 2e édit. II. - } 16
$$


278 SÉMIOLOGIE DE L'APHAREIL DE L'INNERVATION.

La douleur peut ètre éveillée par des excitations d'urdre mécanique, thermique, électrique ou chimique. Habituellement, on se sert d'une épingle el l'on pique la peau plus ou moins profondément jusqu'à ce qu'on éveille de la douleur.

Modifications pathologrinues. - - Les modifications pathologiques de la sensibilité observées chez les animaux consistent dans une exaltation, une diminution ou une abolition de la sensibililé.

Hyperesthésie. - L'liyperesthésie ou exaltation de la sensibilité consisle en une transformation rapide les sensations tactiles en sensations douloureuses et en une exagération de la sensibilité douloureuse; c'est une hyperalyisie.

Les maladies itrulentes comme le tétanos, la ra!yc s'accompagnent d’une hyperesthésie cutanée extrème; le moindre attouchement de la peau suffit à provoquer des crises convulsives généralisées et des phénomènes spasmodiques. On constate une susceptibilité extrème de la réflectivité spinale.

Les empoisonnements par la strychuine, le phénol, les narcoliques, les essences convulsivantes (sauge, absinthe, fenouil, hysope, romarin) sont également une source d'hyperesthésie et de souffrances.

L’hyperesthésie tégumentaire est un signe précieux pour le diagnostic des maladies de peau (eczéma, gale sarcoptique on psoroptique, etc.). On l'observe dans la eicatrisation des plaies, dans la rage, au point inoculé; dans certains cas d'épilepsic réflexe où la sensibilité excessive se localise en certaines régions.

Les diverses formes de la méningite sont généralement caractérisées au début par une hyperesthesie prononcée de l'encolure exagérée au niveau de l'atlas; on observe en même temps de l'hyperesthésie sensorielle. 
Anesthésie. - L'anesthésie consiste dans l'abolition de la sensililité ; l'hypoesthésie signifie diminution de la sensibilité dans tous ses modes; l'tmalgésie exprime la suppression de la sensibilité à la douleur.

L'anesthésie est totale ou partielle; on dit dans ce cas quelle est dissociée; cest-à-dire n'intéressant qu'une ou plusienrs des formes de sensibilité que nous avons énumérées.

Elle est produile par les agents dils anesthésiques; on l'observe dans l'intoxication déterminée par le thymol, par l'eugénol; dans les paralysies, qu'elles procèdent d'une altération nerveuse ou d'une obstruction vasculaire, comme on le remarque dans les membres postérieurs du cheval dont les artères iliaques sont oblitérees. Elle est considérablement diminuée dans l'anasarque du bauf, dans les indurations: de la peau, dins les engargements chroniques des membres postérieurs.

Les parèsies cérébrales et spinales, les paralysies, les inflanmations de la moelle épinière parvenues au dernier stade, l'hydrocéphalie chronique, l'encéphalite etronique, l'épilepsie, le vertige, l'évanouissement, déterminent l'abolition plus ou moins complète de la sensibilité.

Dans la compression de l'extrémité terminale de la moelle, on constate fréquemment l'anesthésie de la peau du périnée et d'une parlie de la surface de la croupe; la sensibilité de la muqueuse de la vulve et du rectum est éleinte.

On remarque une asymétrie dans le contour de la croupe. En dedans de la ligne pointillée, la seusibilité cutanée était notablement diminuée, et tout à fait conservée dans la partie stratifiée.

La puchyméningite ossifiante du chien s'accuse simultanément par des douleurs névralgiques et par des zones l'anesthésie, disposées en plaques dans le domaine des nerfs affectés; mais ces zones hyperesthisiées ne sont jamais bien délimitées. Les territoires unesthesiés sont 
habituellement entourés au niveau des épaules d'une zone de sensibilité amoindrie, à peitıe défnissable en forme

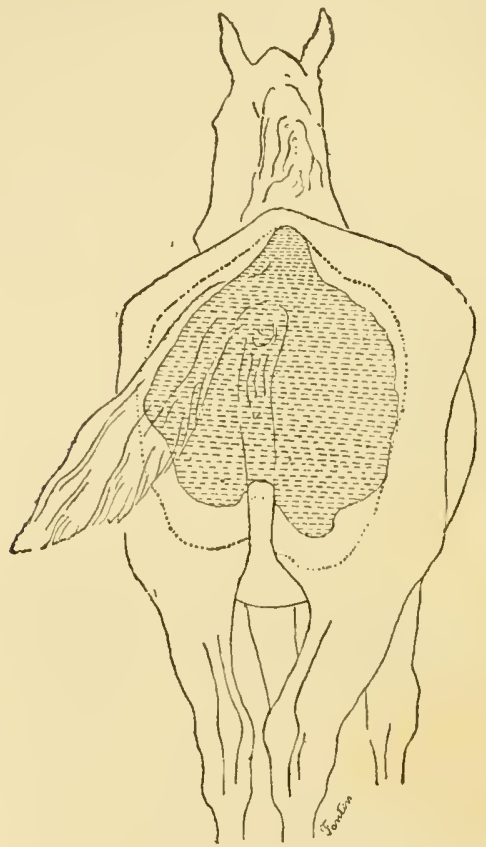

Fig. 69, - Aspect d'un cheral atteint dune paralysic de la queue. On' remarque une assmétrie dans le contour de la croupe. En dedans de la ligne pointil'éc, la sensibilité cutanép était nolablement dimimuée, tout it fait conservée daus la pattlic stratifićc; anus et vulve entrouverts (Dexler).

de bande ou d'anneau qui se confond insensiblement avec la peau normale.

La disparition de la sensibilité est partielle au début, plus tard l'anesthésie s'étend beaucoup et devient bilatérale. Parfois lout le corps, hormis la tète et l'encolure, cesse de réagir à la piquire, au pincement, à l'arrachement 
des poils par toulles; on peut placer les pattes dans l'eau chaude jusqu'à la brùlure, ou les empaqueter dans la glace salss que l'animal cherche à les retirer.

La sensibilité musculaire parait seule persister. L'anesthésie qui a compagne l'ictus apoplectique de l'hémorragie cérébrale est habituellement passagère. C'est ordinairement au niveau de l'aile du nez et des lèvres du côté paralysé que l'anesthésie pent être mise facilement en évidence; elle peut s'étendre à la moitié de la lêle ou demeurer localisée au naseau.

Cliez nos animaux domestiques, on peut observer une diminution de la sensibilité tactile par suite de la perte des poils tactiles.

On pent constaler des anesthésies viscérales.

Les teslicules présentent sourent une insensibilité comulète à la pression dansla pachyméningite ossifiante. Dexler a altilé l'attention sur l'importance de ce signe. Celte analgésie testiculaire n'a pas de rapport avec l'état de la sensibilité cutanée; le testicule peul être insensible à la pression alors que la peau possède intégralement toute sa sensibilité.

\section{XXXVI. - DOULEUR.}

Définition. - La clouleur est une manifestation psychique, transmise par les nerfs sensitifs et provoquée par la plupart des lésions de l'organisme.

Elle se traduil par des gémissements, des soupirs, des cris plaintifs, des beuglements, des cris stridents. Les animaux sont dans l'inquiétude, la tète est tournée du côté dı ventre; ils piétiuent, trépignent, battent le sol avec les pieds, se roulent, premnent des attiludes hizarres, courent, s'agilent, grincent des dents, roussent le dos, remuent la queue, boitent, etc.

Ses caractères varient suivant la cause de l'excitation, le siège du mal, l'espèce animale et le degré de sensibilité du sujet. 
282 SÉMHOLOGL DF L'APPAREIL DE L'INNERVATION.

Les lésions vistirales amènent chez les animaux des douleur's d'une acuité parfois excessive (coliques), des grincements de dents (fiére lyphoüde), de la raideur et de la courbature (pleurésie, péricarilite, etc.).

Les maladies de l'uppareil locomoteur déterminent des crampes, des raidens et des lancinations.

Le brut' parait souffrir moins que le cheval. Chatque espèce manifeste sa douleur d'une maniere diflérente; le rhien se gratte avec les paltes el se roule; le monton se mordille, les soliperles se déchirent avec les deuls ou se frottent avec une telle violence qu'il se produit souvent des plaies.

Pathogénie. - L'origine le la douleur peut ètre cérébrale, spinale, nerveuse ou périphérique.

La douleur l'origine cérebrale se voit laus les lésions méningics, elle se caractérise alors par une hébétude marquuée, de l'abaltenrent, de la prostration.

La douleur d'origine spinale a sensiblement les caractères de la précédente, surlout quand elle provient des méninges. Ses effets sont le plus souvent instantanés.

La douleur provenant des corlons neveux se traduit par des élancements. Elle peut déterniner, par action réllexe, des contraclures durables suivies de déformation persistante, ce qui pourrait expliquer la production de la houleture chez nos animaux domestiques, dans les cas où celle-ci ne provient pas de causes tangibles.

Les violences extérieures telles que les coups de fouct déterminent des souffrances très intenses.

Il résulte des recherches d'Anchald que le fout dit manille procure une pression totale de 30 kilos; le fouet perpignan, une de 5 t kilos, quand la lanière est carréc, une pression de 66 kilos avec une lanière ronde, whe de 3 kilos avec une laniere rectangulaire, et, enfin, avec la lanière conique, dite queue de ral, une pression de 142 kilos. 
Ce sont là vraiment des coups effroyables et qui peurent cependant encore ètre dépassés par un homme en colère en maniant son fouet, ou le nuanche qui domne alors une pression de $\ddot{3}$ lilos par centimètre linéuire de contact.

Comme comparaison, un coup de règle appliqué sır la paume de la main, fail venir les larmes lor:que la pression totale ressentie est égale seulement à 2 kil. $\{30$.

Quand la lanière est surchargée d'un cerlain nombre de nœuds daus lesquels s'accumulent, comme dans une pointe, les pressions voisines des parties de lanière ne pouvant pas entrer en contact direct arec la peal, les pressions peuvent atteindre 6 à 7 kilos par centimètre cirré de surface.

Quant à la mèche, elle est plus bruyante que méchante; elle pénètre très peu, en présentant une ondulation gracieuse, car elle arrive en retard par suite de sa grande léŁèreté.

Si ou ramène les pressious constatées à l'unité de surface, on trouve que les lanières rondes et coniques sont beancoup plus douloureuses que les lanières carrées et rectanguluires. Ceci provient de ce que les premières entrent en contact par une ligne et les secondes par une surface.

La lanière rectangulaire présentant la surface la plus large, puisqu'elle pirote toujour's sur son épaisseur, est ce!le qui est la nooins cruelle, $t$ celle qu'il faudrait voir montée sur tons les fonts.

Une lanière de 7 millimètres sur $\dot{x}$ avec une longueur conforme à la nature des manches, mais ne dépassanl pas $1^{\mathrm{m}}, 20$, serait plus que sulfisanle pour tenir éveillé n"importe quel cheral paresseux, tout en procurant un claquement qui satisferail le charretier faisant parade de sa force.

Dans la compression de la moelle par des tumeurs et dans la pachyméningite ossifiante du chien, on constate la nerralgie.

La méningite cèrébrale el cérébro-spinule sont fréquemment accompagnées d'irritation des nerfs basilaires, 
28' SÉMIOLOGIE DE L'APPAREIL DE L'INNERVATION.

principalement du facial, du trijumeau, du spinal, des nerfs oculaires.

\section{XXXVII. - NEVIRILGIES.}

La névalgic est le type de la douleur nerveuse; elle est caractérisée par une douleur plus on moins intense siégeant sur le trajet des nerfs.

Définition. - Caractères. - Chez l'homme, la névralgie est mu syndrome bien connu et la douleur qui en est la manifestation dominante est Irès variable. Elle peut simuler une piqûre, mue déchirure, un arrachement, une brùlure vive; les névralgies du sciatique el du trijumeau sont les plus douloureuses. Cette douleur est continue; mais sujelte à des exacerbations; parfois elle est nettement intermittente; elle s'exagère sous l'influence du mouvement; elle dure quelques minutes, une heure, rarement davantage et occupe toute la sphère de distribution du nerf ou seulement une branche principale.

Les névralgies résultent fréquemment des nécrites à altérations médullaires diverses.

Chez les animanx, les douleurs névralgiques beaucoup moins connues sont également très intenses.

Parmi les divers types de névralgies signalés, citons :

La nívralgie faciale, caractérisée par de la photophobie, du larmoiement, la rougeur de l'œil, l'hypersécrétion nasale et le ptyalisme.

La schatique ou NÉvralgie fÉMORO-POPLitée est caraclérisée par des tremblements musculaires, de la louleur et une boiterie du membre affecté.

La nérralgic peut se localiser encore à la nuque, au garrot, se confondre avec le torticolis signalé chez le buenf, le cheval el caractérisé par la raideur musculaire, l'inclinaison de l'encolure, la difficulté de prendre les aliments à terre, le port anormal de la tête (1).

(1) Bedel, Suciete centrale, 1901. 
Outre la névralgie cervico-occipitale, on a également recounu une névralgie dorso-intercostale, dénoncée par une vive louleur à chaque inspiration; ume névralgie lomboabdominale, etc.

Les pserido-névralgies sont communes chez le chien affecté de compression de la moelle et surtout de pachyméningite ossifiante.

Dans ces affections de la moelle, la douleur est due à la compression et à l'irritalion des racines et des nerfs rachidiens; elle peut ètre due à la compression de nerfs dans leur trajet périphérique.

Pendant très longtemps, ces névralgies constituent le signe exclusif de l'ossification de la dure-mère ou de la compression exercée par les exostoses et les végétations intervertébrales.

Les douleurs nevolyiques sont très intenses; elles arrachent aux chiens des plaintes presque continues ou des cris déchirants. Elles sont habituellement nocturnes, intermittentes, elles sont eufin exagérées par la marche, les pressions el les moindres mouvements.

L'hyperalyesie est généralement bilutirale et résionale; elle intéresse la peau, mais jamais les muscles.

Chez le bruf, Furlanetto a signalé la nevralgie rhumatismale diffuse: l'animal levait tour à tour les quatre membres. Lorsqu'une des extrémités postérienres était levée à son maximum de hauteur, la llexion était brusque et le pied venait loucher la paroi abdominale; la face externe des membres ne présentait aucune sensibilité anormale; mais la main appuyée sur la face interne de lacuisse provoquait une vive douleur; la pression exercée sur le trajet du nerf snphine ou du nédian était particulièrement douloureuse 1 .

I.es névralgies sont combaltues par les injections de morphine, d'anlipyrine et de cocaïne, pratiquées sur le trajel des nerfs, ou par la névotomic. 


\title{
SEMIOLOGIE DES ORGANES DES SENS
}

\author{
PREMIERE SECTION.
}

\section{YEUX}

L'wil comprend des parties accessoires comme les paupières, l'appareil lacrymal et les muscles, et des parties essentielles dont la réunion forme le globe oculaire.

\section{I. - PICPIÈRS.}

Exploration. - ANNExES DE L'EIL. - Quand on constale une irritation de l'oil, il faut examiner la face externe, le bord des paupières et les voies lacrymales à la lumière solaire; puis, on doit explorer la cornée, la conjonctive bullsaire et palpélırale, les culs-de-sac conjonctivaux par le procédé Rolland :

$1^{\circ}$ On met en place l'écarteur d'exploration (figg. 33, $\left.n^{\circ} 1\right)$;

$2^{\circ}$ On fait tomber sur la partie supérienre de la cornée, à l'aide d'un compte.gouttes, dix gouttes d'un collyre à la cocaüne (chlorhydrate de cocaine, 20 centigrammes, eau bonillie, 4 grammes);

30 Après cinq minutes d'attente de l'effet anesthésique de la cocainne, on explore à l'éclairage latéral ces diverses parties ;

4. Enlever l'écarteur et prendre la curette d'exploralion $\left(1^{\circ} 3\right)$ enduite de vaseline qu'on introduit et qu'on 
fait glisser dans le cul-de-sac conjonctival pour s'assurer qu'il n’y a pas de corps étranger. Ce procédé est encore

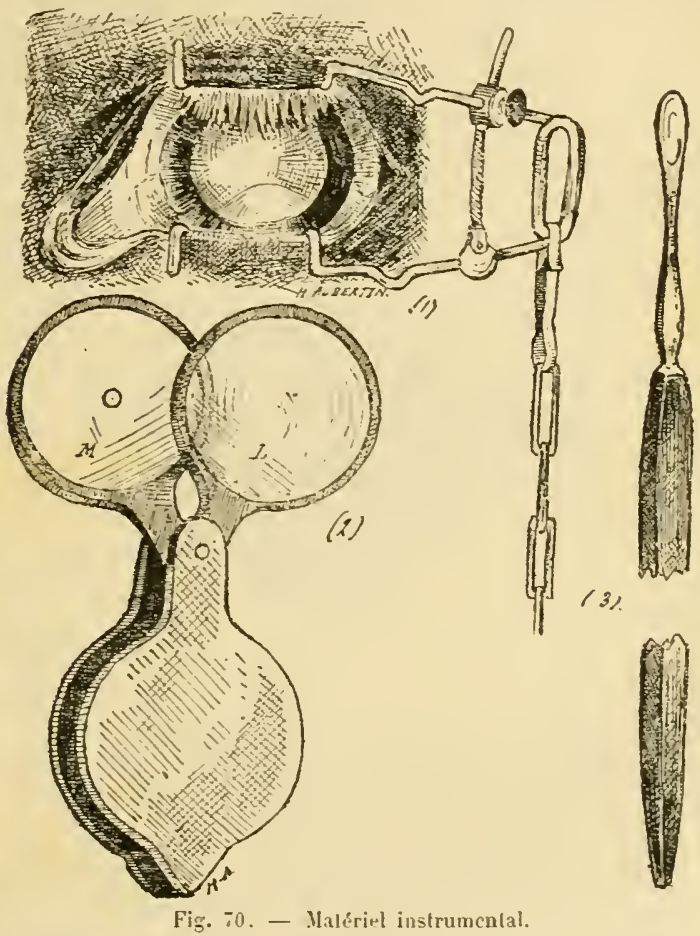

(1). écarteur en place. - (2), ophtalmoscope. - (3), curelle à exploration du cul-de-sac (d'après le Dr Rolland).

plus utile chez le bruf, dont la conjonctive est très développée, que chez le cheral.

Iodifications pathologiques. - Les paupières sout des voiles membraneux comprenant : une muqucuse, une couche fibreuse, une musculeuse, le cartilage tarse et 
des glaudes de Mcibomius, recouverts par la peau; ces organes peuveut ĉtre le siège de lésions tranmatiques, d'inflammations, de tumeurs et de vices de conformation.

Les lésıons traunatıues consistent dans des piqûres, des plaies, des contusions, des déchirures superficielles ou profondes, des tuméfaclions (fièvre typhoïde), des wedèmes, de l'emphysème, déterminé intentionmellement clez les vieux cheraux par insufflation d'air dans les salières, ou consécutif à des traumatismes, à l'extension de l'emphysème pulmonuire ou à l'évolution de la seplicémic.

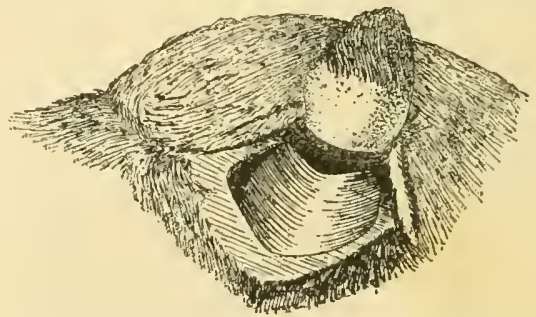

Fig. 71. - Dermoïde de la conjonclive avee atrophie du globe oculaire.

Les inflawatioxs intéressent le bord libre des paupières (bléplıarite ciliaire), la partie superficielle de la peau (eczèma, gale folliculaire) ou toute l'épaisseur des paupières (phlegmon ou abcès).

Les tumfurs des paupières sout fréquemment des verrues, quelquefois des sarcomes, des mélanomes, des épithéliomes.

Les vices de conformation sont variés: les bords libres des paupières peuvent être renversés en dehors (cetropion) ou en dedaus (entropion); ils peuvent ètre soudés (anliyloblipharon); enfin les paupières peuvenl adhérer au globe oculaire (symbléphuron), à la suite d'ulcères, de brûlures de la conjonctive. Elles forment un angle droil du còté interne quand les yeux sont atleints de fluxion périodique.

Les cils peuvent se diriger ver's le globe oculaire (tri- 
chiasis); on peut constaler des dermoïdes de la conjonctive.

Dans l'inflammation du cerveau, la parésie génitale et l'empoisonnement par les ptomaïnes (botulisme), dans la fievre typhoüde, pendant l'hydrocéphalie chronique et autres affections du cerveau, les yeux sont mi-clos.

\section{II. - CORPS CLIGNOTANT.}

Pour explorer le corps clignotant, il est nécessaire de le faire saillir de l'angle interne de l'œil; il faut se placer à côté du sujet, mettre la main droite sur le chanfrein de l'animal quand on explore à gauche, puis, avec le pouce et l'index de la main opposée, on exerce une légère pression sur les paupières, le corps clignolant vient alors recouvrir une partie du globe oculaire el l'on peut voir s'il est le siège de lésions.

Modifications pathologiques. - Le corps clignotant peut être le siège d'une inflammation aiguë, qui n'est qu'une forme de conjonctivite ou d'une inflammation chronique, accompagnée de l'hypertrophie de cet organe qui est fréquente chez le chien.

On peut observer aussi la nécrose du cartilage, consécutive à des traumatismes ou à une éruption de lıorsepox, ou des tumeurs de cet organe (onglet chez le chien, sarcomes et épithéliomes chez lous les animaux).

Dans le tétanos, le corps clignotant recouvre le globe oculaire à la suite de la moindre excitation.

\section{III. - APPAREIL LACRYMAL.}

Exploratiou. - L'appareil lacrymal comprend : la glande lacrymale, sécrétant Jes larmes, située eutre la partie supérieure du globe de l'œil et la cavilé orbitaire ; les points lacrymaux, situés à peu de distance de la commissure nasale, et par lesquels les larmes passent de la surface oculo-palpébrale dans les conduits lacrymaux; ces deux conduits déversent leurs produits dans le sac

CADÉac. - Sémiologie, $2^{\mathrm{e}}$ édit.

II. -17 
lacrymal, réservoir logé dans l'infundibulum de los lacrymal; enfin le canal lacrymal, prenant naissance dans le sac lacrymal et aboutissant à l'égout nasal. En plus de cet appareil, on trouve la caronculc lacrymale, petil corps arrondi situé dans l'angle nasal de l'œil et qui a probablement pour but de diriger les larmes vers les points lacrymaux.

Modifications pathologiques. - La glande lacrymale peut être le siège de plaics accidentelles, d'inflammations (dacryoadenite), de fistules, de tumeurs. L'inflammation glandulaire est suivie d'une sécrétion abondante de larmes (épiphora); cette lyypersécrétion s'observe dans les conjonctirites, la peste bovine, la fierre typhoïde, etc.

La CARONCULE LACRYMALE peuts'ly pertrophier (encanthis), présenter des tumeurs (sarcomes, épithéliomes et mélauomes).

Les points et les conduits lacryaud peuvent s'enflammer; ils sont fréquemment obstrués sous l'influence de l'inflammation ou de corps étrangers; ils peuvent subir une déviation en dedans (incersion) dans l'entropion, ou en dehors (eversion) dans l'ectropion.

Le sac lacryal s'enllamme, comme la conjonctive ou la muqueuse nasale; cette inflammation porte le nom de dacryocystite. Il peut être obstrué, il y a alors écoulement des larmes sur le chanfrein.

Le CONDUIT NASal s'obstrue sous l'influence des mêmes causes; il est nécessaire de pratiquer les injections pour amener l'évacuation des bouchons muqueux qui provoquent cetle obstruction.

\section{IV. - GLOBE OCULAIRE.}

Le globe oculaire, appelé encore bulbe de l'œil, est une coque sphéroïdale, renfermant les parties liquides ou sem:-fluides formant les milieux de l'œil. 
Exploration. - Ce globe oculaire (fig. 72) comprend: une membrane externe fibreuse. la sclérotique; une membrane moyenne vasculaire et pigmentaire, la choroìde; une membrane interne, la rétine. Ėn outre, la cornée, l'humeur aqueuse, le cristallin et le corps vitré constituent un appareil dioptrique destiné à faire converger les rayons lumineux sur la rétine.

Linspection du sujet et de l'œil permet d'effectuer uు examen d'ensemble et de juger sommairenent de l'état de la vue, du volume des yeux, de leurs mouvements et de leur expression.

\section{$1^{\circ}$ Exameu du sujet.}

La demarche, l'attilude et l'expression du sujet peuvent faire soupcouner une maladie d'yeux. Si la vue est faible, l'animal, en mourement, relève fortement les membres comme pour ériter des obstacles; il est peureux, ombrageux, fait de fréquents écarts, dresse les oreilles, les porte dans tous les sens pour recueillir les bruits; il cherche à suppléer à la rue par l'ouie.

On peut juger aussi de l'intégrité de la rue par une petite manœurre, qui consisle $\dot{a}$ frap-

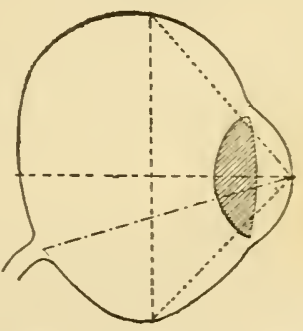

Fig. :ə. - Schémalique du globe oculaire. per légèrement sur le bout du nez de l'animal arec la main: il se retire brusquement; si l'on simule une nouvelle lape el s'il ne voit pas, il ne cherche pas à s'y soustraire (1).

a. Volume des yeux. - Le mulet a normalement un œil plus volumineux que le cheral, avec un rebord orbitaire plus saillant.

(1) Niculas et Fromaget. 
Pathologiquement, on peut constater une augmentalion ou une diminution du globe oculaire.

$1^{\circ}$ Augmentation. - Les yeux peuvent augmenter et cette modification peut se produire sur un seul ou sur les deux yeux. Parfois, on observe une sorte d'hydropisie de la chambre antérieure de l'œil (hydrophtalmie, buphtalmie ou exophtalmie). L'œil est saillanl, proéminent, lorsqu'une tumeur se développe dans les parties profondes de l'orbite, il peut en résulter quelıues Iroubles nerveux dus à la compression du nerf optique. Lícil peut faire saillie en dehors,de la cavité orbitaire (luxation du globe oculaire).

$2^{\circ}$ Diminution. - L'oil diminue de volume, s'atrophie à la suite de blessures ou de perforations, qui ont intéressé profondément les parties conslituantes de cet organe, et lésé le nerf optique, de certaines affections graves telles que la fluxion perriodique et la tuberculose de l'œil.

Dans les maladies graves, l'œil est chassieux et enfoncé dans l'orbite par suite de la résorption du coussinet adipeux; c'est toujours un signe fàcheux.

\section{b. Mouvements et expression des yeux. - Les} yeux sont fixes, immobilcs dais les affections graves des centres nerveux (télanos); hagards, égarès, pirouttants, dans la rage, peudant les attaques d'épilepsie et pendant les paroxysmes des affections vertiyineuses.

Chez le chien affecté d'altéralions cérébrales, on observe fréquemment un tremblement plus ou moins accusé ( $r y s-$ tagmus); souvent aussi les axes visuels sont deviés (tournis du mouton, altérations cérébrales).

Les yeux sont étincelants, menaçants dans la rage furieuse, pendant la durée des paroxysmes des maladies vertigineuses.

Pendant le tétanos, on observe une forte rétraction du bulbe par suite de la contraction télanique des muscles droils de l'œil. Il eı est de même dans l'empoisonnement 
par la nicotine et la strychnine. On peut voir le bulbe rouler ou trembler dans l'orbite (empoisonnement par l'eau salée et nystagmus).

\section{$2^{\circ}$ Examen de l'oil.}

L'examen de l'cil peut avoir lieu: $1^{\circ}$ à l'œil nu; $2^{\circ}$ à l’éclairage oblique ou latéral; $3^{\circ}$ à l'ophtalmoscope.

$1^{\circ}$ A l'oeil inu et à la lumière naturelle. - Cette méthode consiste à examiner les milieux visuels en plaçant convenablement l'animal de façon que l'œil soil modérément éclairé et que l'on puisse se rendre comple de son état.

On dispose ordinairement le sujet sous une porte, à l'entrée d'une écurie ou d'un local dont le fond n'est pas éclairé. Oı a soin d'éviter qu'en fae de l'animal se trouvent des surfaces brillantes ou un mur blanc susceptible de réfléchir la lumière et de nuire à l'obserration. Il faut éviler aussi d'examiner à la lumière solaire, car la lumiere trop vive fait contracter l'iris et empèche de juger de l'étal des parties profondes de l'œil.

L'examen á l'œil nu permet d'explorer les culs-de-sac conjonctivaux, c'est-à-dire les replis de la conjonctive; on examine d'abord la paupière inférieure, on la saisit avec le pouce et l'index et on l'abaisse de manière à bien apercevoir son cul-de-sac. Pour la supérieure, on se sert des deux mains et on relève la paupière en dessus.

Avant de procéder à cetle opération, il est quelquefois nécessaire de cocainniser l'cil avec une solution à 1 p. 50 . Quand la région est insensibilisée, on peut faire usage pour explorer la conjonclive, de la curette imaginée par le $D^{r}$ Rolland.

L'exploration du corps clignotant est des plus faciles. Arec le pouce ét l'index, le premier étant appliqué sur la paupière inférieure, le second sur la supérieure, on effectue une pression modéréequi faitsaillir la troisième paupière. 
Il faut examiner comparativement les deux yeux, s'assurer s'ils sont du même volume ou si l'un est plus grand que l'autre, apprécier également la tension du globe oculaire à l'aide de l'index et du médius en exerçant une pression à travers la paupière supérieure el s'assurer si cette pression ne détermine pas de douleur; on peul apprécier enfin l'atrophie du bulbe, ses modifications de volume, de forme, et sa disposition dans l'orbile.

Pour juger ensuite de l'état des milieux, l'opérateur doit se placer d'abord un peu en avant de l'animal, puis sur le côté. Il est quelquefois utile de se porter un peu en arrière pour examiner la situation de lésions diverses, intéressant l'épaisseur de la cornée (corps etrangers, etc.).

Les petits animaux sont placés sur une table en face d'une fenêtre, de manière que la tête du sujet soit tournée vers le jour.

En opérant ainsi, on peut juger de l'état des organes de protection, des annexes de l'xil, de la conjonctive, de la cornée : taches et opacités symptomatiques de la maladie du jeune âge du clich, de la fière catarrhale maligne du bouf, du diabète; ulcêrations de la rage, de la kératite ulceireuse; phlyctènes et abcès des keratites infectieuses, de la malıdie du jeune age, de la fievre aphteuse, du horsepox ; perforations produites par ces maladies.

On peut constater aussi une courbure plus accentuée de la cornée, comme dans l'exophtalmie ou cerlaines formes de myopies.

On se rend compte en même temps des opacités et des troubles de la chambre antérieure (hémorragies de la fière pétéchiale, scorbut, influenza, épilepsie, septicémie, anémie yornicieuse).

Cet examen permet en outre de faire l'Épreuve de la pupılle. Lorsque l'aninıal est placé sous une porte, la pupille est assez dilatée et, chez le cheral, elle affecte une forme elliplique particulièrement dans le jeune âge. Dans les àges plus avancés, elle tend de plus en plus à prendre 
la forme rectangulaire, car les contours inférieur et supérieur sont moins incurvés. L'ourerture pupillaire doit être de mème dimension dans les deux yeux, régulièremenl dilalée si l'œil est sain; les bords peuvent ètre garnis de grains de suie, provenant de la membrane uiéale et ne gènant en rien la vision.

L'observateur doit s'assurer de la régularité d mouvements pupillaires; on peut pour cela fermerl'œil opposé à celui que l'on examine et au bout de quelques instants on voit la pupille se dilater. Si l'on enlève la main de l'œil que l'on vient de fermer, elle se resserre. On procède de la même façon pour examiner l'autre œil. En passant à la lumière plus vive, on la voil encore se resserrer davantage et l'on peut en conclure que l'iris n'est pas altéré et que l'animal voit.

Cette épreuve ainsi faite peut fournir des indications sur l'état de l'œil du sujet examiné, sur les altérations de liris inflammation avec hypopyon comme dans la pneumonie infectieuse, l'influenza, la pyohemie), sur ses opacités (cataractes du diabète sucré); sur ses teintes anormales (œil vairon); immobilite de l'iris, la pupille élant dilatée (amaurose); contraclures de l'iris dans des maladies nombreuses (fluxion périodique); adhérences contraclées par l'iris (synéchies anterieures et postérieures).

La pupille peut ètre RÉTrÉcIE anormalement (inflammation cérébrale au début, empoisonnement par la morphine); diLAtéE à l'excès (amaurose, accès épileptiques, empoisonnements divers ou par le colchique, tétanos, parèsie génitale, deuxiènie stade du tournis, etc.). Mais ces indications sont très incomplètes, quelquefois insuffisanles.

Cependant dans les conditions ordinaires de la pratique, on s'en contente. Mais lorsqu'on veut faire l'acquisition d'un cheral de prix, ou lorsqu'il fait l'objet d'une contestation légale, il faut alors faire l'épreute dE LA pUPILLE d'une façon plus complète.

On instille dans l'œil une solution de sulfate d'atropine 
à 1 p. 200, c'est-à-dire une solulion composée de un gramme de sulfate d'atropine ponr 200 grammes d'eau distillée. Au bout de trois quarts d'heure, même au bout d'une demi-heure, la pupille se dilate dans toute son élendue. Son ouverlure, de rectangulaire devient presque circulaire; on voit alors si les contours en sont réguliers ou bien, festonnés, déchiquelés. Si l'ouverture pupillaire est déformée, c’est qu'il existe des adhérences, des synéchies postérieures qui réunissent l'iris avec la cristalloüde anterieure el qui témoignent d'une ancienne inflammation de l'œil ayant intéressé le corps ciliaire et les parties avoisinantes.

Si l'on veut encore obtenir des indications plus netles, il est alors utile de recourir à la deuxième méthode.

$2^{\circ}$ Examen à l'éclairage latéral ou oblique. Cette méthode ccnsiste à éclairer obliquement la cornée et
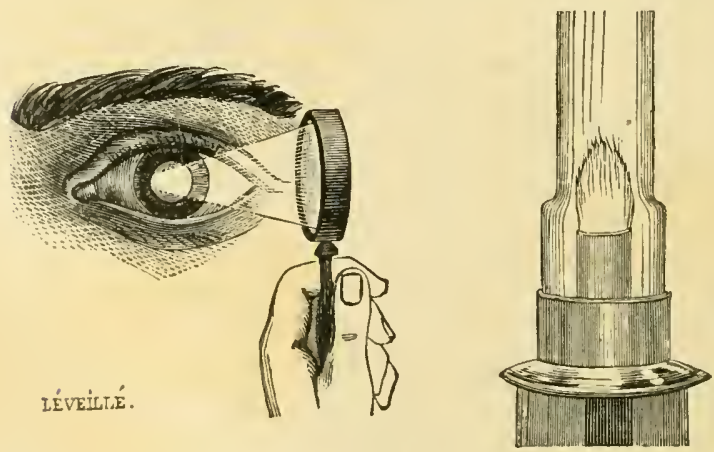

Fig. 73. - Eclairage latèral.

les parties situées en arrière de celle-ci au moyen d'une source lumineuse artificielle. Ce moyen est fort ancien; il a élé conseillé par Solleysel, qui eslime qu'en exáminant l'cil dans un lieu sombre et en l'éclairant latéralement 
avec une chandelle, on peut arriver à bien connaître l'état de la cornée (fig. 73).

La source lumineuse peut être une bougie, une lampe à huile ou à pétrole simple ou munie d'un réflecteur.

Il faut placer l'animal à l'obscurité dans une écurie, ou seulement dans un coin obscur, si l'on ne dispose pas d'un local bien sombre. Un aide tient le cheval et l'observateur place la loupe en arrièr'e de l'œil à une distance de 20 à 30 centimètres, de façon que la chambre antérieure soit bien éclairée. Il peutégalement placer la lampe en avant; alors, il se tient en arrière.

Раг ce simple examen, on peut apercevoir très distinctement les lésions de la cornée et de la chambre antérieure de l'oil.

Pour rendre encore les altéralions plus évidentes, on concentre les rayons lumineux au moyen d'une loupe ordinaire ou d'une lentille biconvexe de quinze dioptries environ, on place alors la loupe entre la source lumineuse et l'œil. C'est l'observateur qui tient dans ce cas la loupe et l'aide qui tient la lampe. Celle-ci, placée à la hauteur de l'ceil un peu en arrière du côlé de l'épaule, doit êlre soutenue par un aide intelligent, qui, suivant les indications de l'explorateur, l'avancera, la reculera, l'élèvera, l'abaissera, en diminuera ou en augmentera la flamme, se préoccupera en un mot, étant donné qu'il ne faut pas compter sur la bonne volonté du palient, de maintenir la source lumineuse, tangente à la cornée, pour l'examen de cette membrane, de la chambre antérieure, de l'humeur aqueuse, et moins oblique pour l'examen de la pupille et du cristallin.

Dans les salles spécialement aménagées pour ces modes d'exploration, il est préférable de suspendre la source lumineuse à l'une des extrémités d'une chaîne armée d'un crochet, enroulée sur deux poulies horizonlalement placées au plafond et porlant à l'autre extrémité un contrepoids. Ce mode de suspension, très facile á établir, ne 
dispense pas de l'aide, mais il évite sa fatigue, il n'a plus quà diriger la lampe soutenue par le contre-poids, et donne par suite plus de fixité à la source lumineuse.

L'examen à l'éclairage latéral ou oblique fournit des résultats d'une importance capitale en médecine vétérinaire. Il permet d'apercevoir les plus petites altérations de la cornée, de la chanbre antérieure, de l'iris et du cristallin.

\section{CORNÉE.}

Caractères. - Nonmalement, la cornée est une membrane trausparente, en forme de calotte ovoïde, qui fait saillie en avant de la sclérotique, d'environ

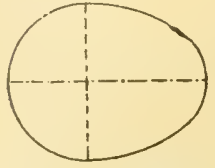

Fig. 74. - Forme de la cornće (Nicolas).

7 millimètres (fig. 7is).

Elle comprend une couche cxterne épithéliale, une couche moyenne, formée de faisceaux consécutifs réunis en lamelles superposées; une couche interne, également épithéliale qui appartient à la membrane de Descemet, et elle est dépourvue de vaisseaux, dont l'existence a toujours une signification pathologique. Ils accompagnent les taies, les ulcerrations, les infiltrations communes, les licratites diffuses, traumatiques ou infectieuses.

\section{V1. CILMBRE ANTÉRIEULE.}

Modifications. - La chambre antérieure, remplie d'humeur aqueuse, liquide, fluide et incolore, absolument transparente, peut renfermer des dépôts séreux, sanguins ou purulents, qui sont l'expression d'irilis ou d'iridochoroüdite.

Tantòt, en effet, on y observe des flocons plus ou moins volumineux de fibrine et de leucocytes formant de véritables dépòts, en forme de croissant, à bord concave en 
haul, connus sous le nom d'hypopyon; lanlôt des amas de sang liquide coagulé (hypoéma); tantôt, entin, des grains de suie, qui se sont détachés et jonent le ròle de corps étrangers qu'il ne faut pas confondre avec les résidus de la fluxion pèriodique.

On peut juger aussi par le mème examen de la profondeur de la chambre antérieure, qui est augmentée par luxation ou disparilion du cristallin, et diminuée à la suite de la perforation de la cornée, de la hernie de l'iris ou de son refoulement en avant, consécutivement à une irido-choroïdite. Quand l'bumeur aqueuse passe dans la chambre postérieure, on a un processus glaucomateux.

\section{VII. - IRIS.}

Caractères. - L'iris est un diaphragme situé au-devant du cristallin el percé en son centre d'une ouverlure qui est la pupille. Il divise l'espace situé entre la cornée et le cristallin, en deux compartiments inégaux ou chambres de l'œil. Sa face anlérieure, plane ou très légèrement convexe, présente des sillons circulaires, très prononcés et des stries rayonnantes, sensibles seulement vers la grande circonférence de la membrane. Elle reflète une teinte brune, plus ou moins jaunàtre, quelquefois blanc plombé, quand elle est dépourvue de pigment (yeux vairons).

La face postérieure en rapport avec le cristallin et les procès ciliaires est recouverte de pigment noir connu sous le nom d'zvée. Ce pigment ou grain de suie obstrue une plus ou moins grande étendue de l'ouverture pupillaire el peut être une cause d'amblyopie.

Modifications pathologiques. - L'éclairage latéral oblique permet de constater les déformations de l'ouverture pupillaire et les adhérences qui relient l'iris à la cristalloïde antérieure. Les adhérences qui brident l'iris, connues sous le nom de synéchies, sont des traces irrécu- 
sables d'iridocyclite, d'iritis ou d'irillo-choroüditc ancicnne ou présente (Nicolas et Fromanget).

Lessynéchies donnent à la pupille des formes diverses: forme de huit, d'une haltère, d'un pied de cheval, d'un chapelet; elle peut être festomnée, irrégulière, etc.; elle peut demeurer immobile malgré l’emploi des mydriatiques. Ce phénomene résulte de la solidité des exsudats, qui fixent le bord libre del'iris, sinon la pupille se dilate sous l'influence de l'atropine dans tous les points oì elle n'est pas immobilisée; de là proviennent toutes ces formes bizarres de l'ouverture pupillaire (fig. 73 à 77).

Quand les synechies sont trop faibles pour résister à l'action des nydriatiques, la pupille reprend sa forme régulière et arrondie, mais la face antérieure du cristallin présente des stiğmates indélébiles, des adhérences ọui se

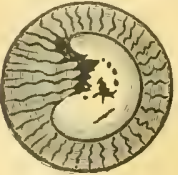

Fig. 75, 76. - Sỷnéchies postérieures.

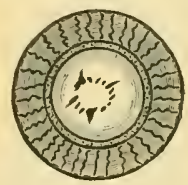

Fig. 77. - Synćchies.

sont rompues. Ces synéclies permetlent de distinguer l'immobililé de l'iris provenant de l'iritis, des lésions de la rétine, de la choroüde qui rendent également l'iris inerte comme dans l'amaurose.

On peut observer aussi une anomalie conginitale qui consiste dans la persistance de la membrane pupillaire; ce qui constitue une rareté.

\section{VII. - CIISTALLIN.}

Caractẻres. - Cetle leulille biconvexe, située derrière l'iris, au milieu de la couromne des procès ciliaires, sépare l'humeur aqueuse de l'humeur vilrée, se compose d'une membrane d'enveloppe et d'un tissu propre. 
La membrane d'enceloppe ou capsulaire comprend la cristalloüde antérieure et la cristalloüde postérieure, indépendante du tissu propre et présente partout la même épaisseur.

Le tISSU PROPRE esl composé de couches concentriques, dont la résistance augmente de la périphėrie au centre.

Quand la pupille est dilatée par l'atropine, on peut explorer facilement la cristalloïde antérieure par l'éclairage latéral ou oblique et $y$ constater les résidus d'une

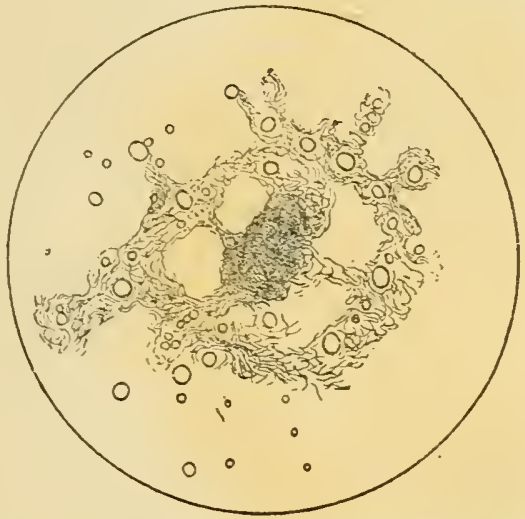

Fig. 78. - Lésions de la cataracte commençante (Nicolas).

iritis ancienne ou récente. On y observe de petits points qui ne laissent pas passer les rayons lumineux, de légères stries opaques, dont la disposition rappel,e parfois celle des rayous d'une roue et qui s'étendent de la périphérie au centre.

On peut localiser enfin le siège exact de ces opacités, comme les points de cataracte dans les parties superficielles ou dans les parties profondes du cristallin (fig. 78 .

Mais dans quelques circonstances, l'examen Jatéral oblique est insuffisant, les parties antérieures de l'wil paraissent normales, alors qu'il existe des allérations 
profondes qui peuvent nuire considérablement à la vision. On ne peut apercevoir ces dernières que par l'examen ophtalmoscopique.

\section{IX. - EXAMEN A LOPITALMOSCOPE.}

Historique. - L'ophtalmoscope a été inventé en 1831 par llelrnholız; il a été employé pour la première fois en vétérinaire, en 1838, par Reynhal.

Son usage devait demeurer longtemps limité, car le procédé à l'inıage renversée était difficile ả appliquer aux animaux en raison de leurindocilité. En 1864, Guérineau publie un opuscule sur l'emploi de l'ophtalmoscope dans l'examen des yeux de nos animaux domestiques.

Depuis, son emploi s'est répandı dans tous les pays, gràce aux travaux de Schlampp, de Möller en Allemagne, de Bayer en Autriche, de Zorawshi en Bulgarie oì son ophtalmoscope est réglementaire dans l'armée, de Ilocquart et Bernard, de Carrère, du Dr Rolland, d'Ablaire, de Nicolas et Fromaget en France, de Vachelta en Italie et de Smilh en Angleterre. Le Précis d'ophtalmoscopie vétérinaire de Nicolas et Fromaget renferme les données les plus précises relatives à l'ophtalmoscopie appliquée à nos animaux domestiques.

Principe de I'ophtalmoscope. - Normalement, le fond de I'œil apparaît avec une teinte bleuâtre ou noir bleuàtre, qui ne permet pas d'apercevoir les membranes situées au delà du cristallin. Pour les rendre apparentes, il faut les éclairer, transformer la rétine en un écran lumineux dont les rayons seront perçus par l'observateur.

Le problème à résoudre consistait à trouver un moyen pratique d'éclairage du fond de l'œil. Ce problème a été résolu à l'aide de l'oplıtalmoscope. On peut employer celui de Follin ou celui du Dr Rolland.

L'ophtalmoscope de Follin se compose d'un miroir con- 
cave percé dans son centre d'une ouverture laissant passer les rayons lumineux qui doivent impressionner l'œil de l'observateur. Derrière le miroir, se trouve un disque muni de quatre verres correcteurs servant à corriger les aberrations visuelles de l'observateur et de l'animal. En face des verres concaves se trouve gravé le signe moins (-), en face des verres converes le signe plus $(+)$, ainsi que les dioptries ou puissance de ces verres.

La pioptrie, ou unité dioptrique, est la puissance réfringente d'une lentille dout la distance focale est à un mètre. Le dioptre oculaire a une dioptrie lorsque son remotum est à un mètre. La puissance réfringente d'une lentille et la longueur de son foyer sont en raison inverse. Ainsi une lentille a 4 dioptries lorsque la distance est $\frac{100}{4}$. Donc une lentille de 4 dioptries aura son foyer à $0 \mathrm{~m}, 20$. Étant donnée la distance fo-

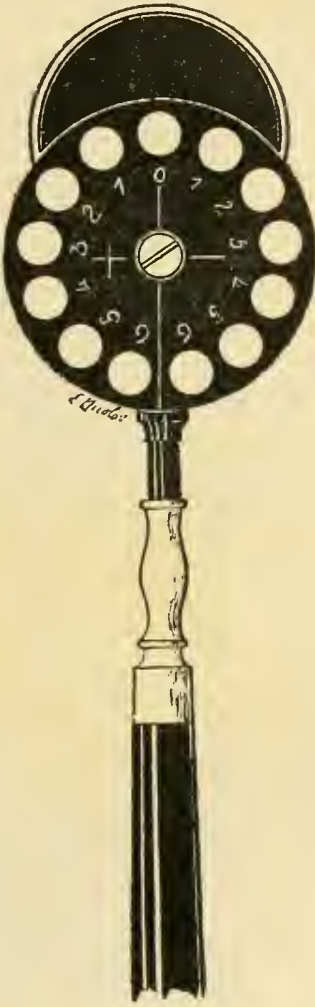

Fig. 79. - Ophtalmoscope à réfraction modifie du professeur Badal. cale d'une lentille, il est facile d'obtenir sa puissance en dioptries d'après le principe énoncé plus haut.

Ainsi ces verres placés sur le disque et qui peuvent, Iorsqu'on le fait tourner, passer à tour de rôle devant 
l'ouverture du miroir sont faits pour rendre emmétropes l'wil de l'animal et de l'observateur et que ni l'un ni l'autre n'accommodent. Celui de Nicolas et Fromaget et celui de Badal (fig. 79) présentent, au lieu de quatre verres correcteurs, treize verres dont six marqués du signe moins (-), six narqués du signe plus (+) et un neutre. Avec cet oplitalmoscope, l'observateur peut mieux corriger les aberrations visuelles inhérentes à ses yeux et à ceux de l'animal.

L'ophtalmoscope domme à l'œul de l'observateur le moyen de recueillir les rayous qui émergent du fond éclairé de l'oeil du patient. D’une part, grâce au miroir, l'ail explorateur est lui-mème une source lumineuse, car le miroir ophtalmoscopique placé devant son ceil projette dans l'œil observé un faisceau de lumière, émanant d'une lampe placée latéralement. Si une leutille convexe est interposée entıe l'œil observé el le miroir, les rayons émis par le fond de l'œil du patient forment au delà de la lentille une ima re réelle, renversée, d'autant plus pelite yue la lentille est plus puissante (cxamen ál'imagerencersée).

Si au lien d'une lentille comrexe, on interpose une lentille biconcave, cetle lentille redresse l'image aérienne renversée du fond de l'ueil (examen a l'image droite).

L'oplilalmoscope permet ainsi: $1^{\circ}$ l'examen du fond de l'wil à l'éclairage direct; $2^{\circ}$ l'examen à l'image droite ; $3^{\circ}$ l'examen à l'image renverséc.

1. Examen à l'éclairage 'direct. - L'olsservateur, placé à 30 ou 60 centimètres de l'animal ou plus près, exanine la transparence des milieux réfringents de l'wil. On peut constater ainsi :

$1^{\circ}$ Des opacités frxes, c'est-à-dire ne se déplaçant que lorsque l' œil se déplace, dues à des troubles du cristallin ou à des taies de la cornée;

$2^{\circ}$ Des opacités MoвıLes, dues à des troubles de l'humeur aqueuse ou du corps vitré. 
L'éclairage direct permet aussi de juger de la forme de la pupille après l'action de l'atropine, et de constater les irrégularités provenant des synéchies.

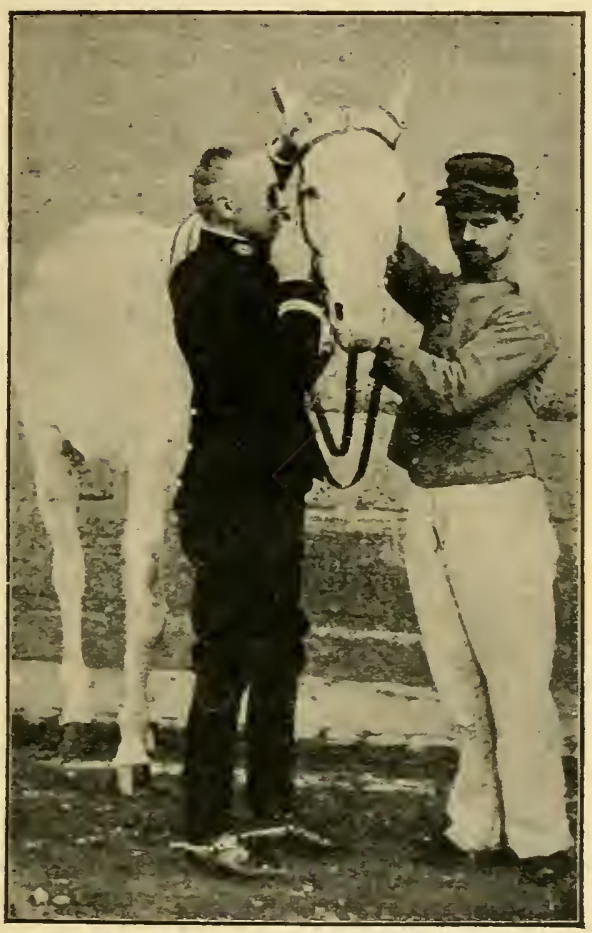

Fig. 80. - Esamen ophtalmoscopique (Nicolas et Fromaget).

L'exploration des membranes profondes de l'œil nécessite l'examen à l'imagge droite ou à l'image renversée. Les diverses méthodes se complètent mutuellement et doivent être appliquées conjointement arec l'examen à l'œil nu 
et l'éclairage latéral quand il s'agrit de faire un examen méthodique de l'œil.

II. Examen à l'image droite. - Dans le procédé à l'image droite, on peut examiner l'œil à la lumiere naturelle ou à la lumière artificielle. Nicolas et Fromaget recommandent particulièrement l'examen à la lumière naturelle.

On place l'animal dans une écurie parallèlement à la porte, l'aide tient l'animal par le bridon et l'oreille du côté opposé; l'observaleur avec son miroir cherche à renvoyer les rayons lumineux, qu'il reçoit de la croisée, dans l'vil à examiner (fig. 80). L'œil étant ainsi éclairé, on dëcouvre très bien le fond de l'œil, qui montre nettement son tapis clair (tapetrum lucidum) et son tapis sombre.

Chez le cheval, le tapis clair présente desteintes multicolores où dominent le jaune, le vert ou le bleu ; il s'illumine sous l'inlluence des rayons lumineux. La couleur varie un peu suivant la robe : chez les chevaux de robe alezane ou baie, le jaune domine; chez les chevaux à robe foncée, c'est le noir qui a la prépondérance.

Au-dessous du tapis clair, on voit le lapis sombre qui s'unil au premier par une ligne á peu près droite, d'où l'on voil émerger les vaisseaux qui se distribuent dans le tapis clair. Un peu au-dessous de celle ligne, et à peu près au centre, on aperçoit la papille du nerf optique, qui apparaîl avec une teinte claire.

On y distingue nettement trois couches : une externe ou corticale, enveloppe celluleuse du nerf optique ; une centrale, jaunâtre, où l'on distingne une vague disposition aréolaire correspondant à la lame fenêtrée; enfin, entre ces deux zones, on constate une zone rouge, formée par un lacis de capillaires. En dehors de la papille, on conslate aussi l'anneau sclérotical qui revêt une teiste foncée. Dans la papille, on aperçit les vaisseaux rétiniens qui la sillonnent.

Chez le chicn, la papille est nettement triangulaire et 
se trouve située un peu au-dessous de la ligne qui sépare le tapis sombre du tapis clair. Le tapis sombre est peu pignıenté et le pigment choroïdien, groupé autour des vaisseaux, leur forme une bordure qui les rend bien apparents.

Chez le chat, la papille est ronde et le tapis sombre présente des ilots de pigmentalion qui çà et là laissent apparaitre le tissu sous-jacent. Les vaisseaux rétiniens, comme ceux du chien, sont volumineux et partent en divergeant de la papille.

L'exaınen à limage droite peut êlre fait à la lumière artificielle, mais c'est une complication inutile, la loupe n'est pas nécessaire pour faire l'examen ophlalmoscopique. Dans l'examen à la lumière naturelle, il n'est pas même nécessaire de dilater la pupille; cependant on pourra embrasser une plus grande étendue de la rétine en se servant du collyre mydriatique à l'atropine à $/ p .200$.

On a aussi conservé le blépharostat, qui a l'inconvénient d'irriter les animaux si l'on n'a pas le soin d'insensibiliser l'œil.

Le tord-nez ne sera pas non plus employé autant que possible.

L'animal sera examiné à la lumière diffuse dans une écurie, sous une porte ou sous un arbre à la rigueur.

III. Examen à l'image renversée. - Dans ce procẻdé, on obtient une image renversée du fond de l'œil, image réelle mais plus petite que l'objet, de telle sorte que l'on peut embrasser une plus grande élendue de la rétine. Cet avantage qui parait très important, disparait devant les difficultés d'un pareil procédé chez nos animaux. Pour examiner l'œil à l'image renversée, il faut se servir non seulement de l'ophtalmoscope, mais encore d'une lentille de 14, 15, 16 dioptries. Il faut ensuile se munir d'une loupe, placer l'animal dans un local obscur; il peut être nécessaire d'appliquer le blépharostat, d'où la nécessité 
d'insensibiliser l'œil avec une solution de cocaïne à 1 p. 50 . Il faut aussi dilater la pupille avec le sulfate d'atropine à 1 p. 200. Ces instillations doivent ètre failes environ une heure avant l'examen.

Deux aides sont nécessaires pour maintenir le cheval, l'un saisit l'oreille opposée au côté à examiner et le bridon de façon àimmobiliser le plus possible la tête ; l'autre aide tient la lampe de facon à envoyer les rayons lumineux vers Je miroir qui les projette sur l'œil que l'on examine.

L'observaleur se place environ à 60 centimètres de l'animal, puis tenant la loupe de la main gauche, il applique l'auriculaire et l'annulaire sur la paupière supérieure et il cherche à éclairer le fond de l'cil avec le faisceau convergent qui émerge de la lentille. Les mouvements de la lentille el de l'ophtaimoscope sont absolument solidaires et l'on peıtéprouver de grandes difficultés pour explorer l'œil en raison des mouvements de l'animal, qui est plus ou moins gèné par une lumière aussi intense.

Celle méthode d'examen de l'oeil exige un apprentissage beaucoup plus long ; ce n’est point le procédé pratique à adopter en ophtalmoscopie vétérinaire. Néanmoins, on s'accorde à dire que celte méthode peut être une méthode descriptive permettant d'éclairer une plus grande élendue du fond de l'cil.

Que comprend l'examen ophtalmoscopidue? - L'examen à l'ophlalmoscope comprend : 10 la détermination objective de la réfraction statique de l'œil ; $2^{\circ}$ l'examen ophtalmoscopique proprement dit ou l'inspection des membranes du fond de l'œil.

$1^{\circ}$ Dètermination de la réfraction statique. - Il est nécessaire de déterminer les réfractions statiques de l'œil examiné, autrement dit le degré de myopie, d'hypermetropie ou d'emmetropic dont un cheval ou un petit animal peut être affecté.

Les irrégularités dans le degré de réfraction des mi- 
lieux visuels peuvent résulter d'un excès de courbure des méridiens des dioptries oculaires, de variations dans l'indice de réfraction de ces milieux (humeur aqueuse, humear vitrée, cristallin).

Lorsque l'œil présente un excès de courbure, qu'il est allongé dans le sens antéro-postérieur, on dit qu'il y a myopie; dans ce cas, le foyer cristallinien posterieur se trouve placé en avant de la rétine.

S'il y a hypermetropie, le foyer est en arrière de la rétine el il est virtuel puisque les images ne peuvent pas aller s'y former.

S`il y a emmetropie, le foyer cristallinien postèrieur coüncide avec la rétine.

S'il y a des inégalités de courbure dans les différents méridiens des dioptries oculaires, on dit que l'œil est astigmate. Que l'animal soit myope, hypermetrope, astigmate, il n'est plus dans les conditions normales pour percevoir les objets lumineux; n'apercevant plus que des images diffuses, il devient peureux, ombrageux, il fait de fréquents écarts et, dès lors, il

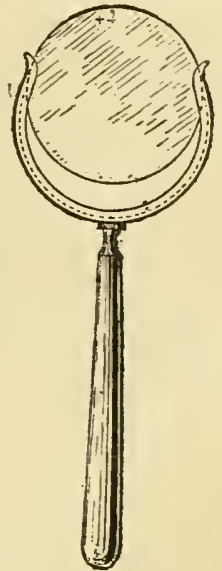

Fig. 81. - Porteverre pour l'étude de la réfraction. est important que l'on puisse découvrir ces affections.

Pour faire cette détermination chez nos animaux domestiques, on ne peut pas employer les méthodes objectives qui, rapidement, permettent de porter un diagnostic précis; nous en sommes réduits à l'emploi des méthodes subjectives.

Les auteurs en indiquent deux principales: $1^{\circ} \mathrm{La} m e^{3}$ thode de la determination de la réfraction statique de l'œil par image droite; $2^{\circ}$ La kératoscopie ou méthode de Cuignet.

$1^{\circ}$ Image droite. - Voy. p. 306. 
$2^{\circ}$ Kératoscopie ou méthode de Cuignet. - Cette dernière méthode seule est à employer chez le cheval.

Elle esı basée sur la marche des ombres que l'on olstient au moyen d'un miroir ophtalmoscopique (fig. 82).

Pour l'appliquer, il faul done se munir d'un miroir ophtalmoscopique, puis d'une série de lentilles concaves

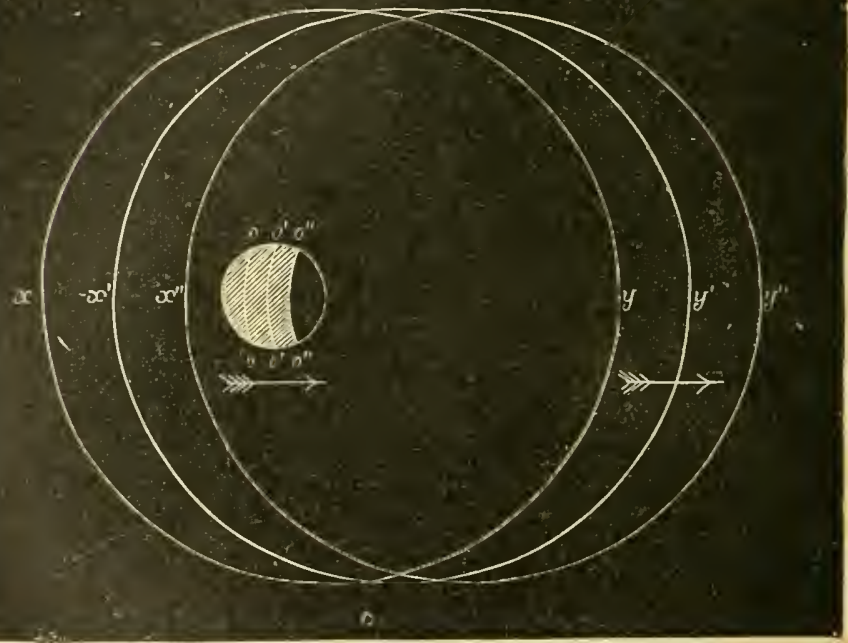

Fig. 82. - Marche de l'ombre direcle.

et convexes montées sur manche, environ six à huit lentilles de chaque sorte. Il n'est pas nécessaire d'employer d'autre source lumineuse que la lumière solaire; le cheval élant placé parallèlement à une fenètre et après avoir dilaté la pupille par le sulfate d'atropine, l'observateur se place ordinairement à un mèlre de l'animal, et s'arrange de façon à éclairer convenablement l'oeil du sujet et à obtenir une ombre de la pupille (fig. 83). 
En faisant mouvoir l'ophtalmoscope à droite ou à gauche, il obtient un déplacement de l'ombre vers la droite ou vers la gauche, ou bien il n'obtient aucun déplacement de l'ombre. Ainsi, nous avons trois cas principaux à envisager : $1^{\circ}$ l'ombre marche dans le sens du déplacement du miroir (ombre directe); $2^{\circ}$ l'ombre marche en sens inverse du miroir (ombre inverse); $3^{\circ}$ l'ombre ne se déplace pas (point neutre).

Marehe le lombre llirecte. - Quand l'ombre marche dans le sens du miroir, l'animal est myope de plus d'une dioptrie; la distance entre le punctum remolum el l'oil est deplus d'un mètre. Après cette constatation, on fait placer devant l'wil, un verre concave dont les dioptries sont numérotées. Quand on obtient le point neutre, ce numéro augmenté d'une dioptrie donne le degré de myopie $-n \mathrm{D}+(-\mathrm{D})=(n+1) \mathrm{I}$.

Si le verre a - une dioptrie, l'œil sera myope de deux dioptries.

Marche de lombre inverse. - Trois cas peuvent se présenter: on a un cil myope de moins d'une dioptrie, ou un ceil emmetrope, ou un eil hypermetrope.

Premer cas. - Si l'on place devant l'œil un verre convexe de + une dioptrie, on peut observer que l'ombre devient directe, alors on remplace ce verre par un verre moins fort de $+0,73$ dioptrie, puis de $+0,25$ dioptrie, jusqu'à ce que l'on ait obtenı le point neutre.

Si l'on obtient le point neutre avec le verre de trois quarts de dioptrie, l'oeil est myope de $+0,75 \mathrm{D}+(-\mathrm{D})$, soit - 0,23 D, c'est-à-dire de un quart de dioptrie.

Deuxiène cas. - Avec le verre convexe de 1 dioptrie, on a le point neutre; c'est que l'œil est normal.

Trolsième cas. - La marche de l'ombre est loujours inverse (fig. 84́); c'est qu'alors l'œil est loujours hypermétrope; on remplace ce verre par celui qui a un nombre de dioptries suffisant pour obtenir le point neutre, soit un verre ayant +3 dioptries, le degré d'hypermétropie sera 
exprimé par $3-1=2$ dioptries. Dans ce cas, pour rendre la vue normale, il faudrait donner à l'animal des verres ayant une puissance de 2 dioptries.

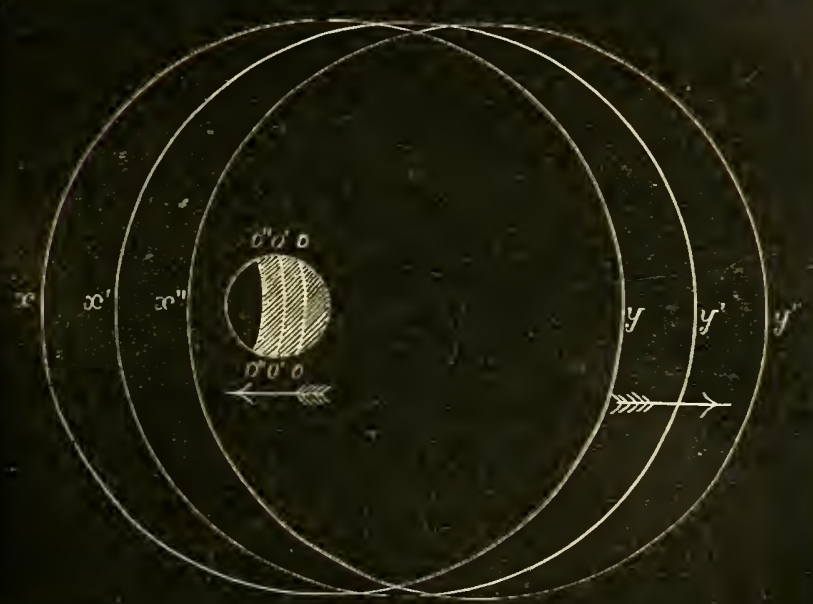

Fig. 84. - Marche de lombre inverse.

Point neutre. - La myopie égale une dioptrie, attendu qu'un verre concave ou convexe interposé entre l'œil et le miroir a pour effet de faire déplacer l'ombre dans le sens direct ou indirect. Ainsi, cette détermination de la réfraction statique de l'œil fournit des indications sur l'état normal ou anormal de la vue.

Si l'animal est myope de plus d'une dioptrie, de deux, trois, quatre dioptries, par exemple, il y a lieu de craindre qu'il soit ombrageux. S'il est hypermétrope de deux, Irois, quatre dioptries, les mèmes craintes sont justifiées.

$1 l$ résulte des recherches faites en France par Nicolas CanÉac. - Sémiologie, ze édit. $\quad$ II. - 18 
que bon nombre de chevaux sont hypermétropes à un faible degré, une demi-dioptrie. Quand ce degré dépasse une dioptrie et demie, ils sont presque tous peureux.

La myopie est moins fréquente que l'hypermétropic et les animaux myopes ne sont pas conslamment peureux. Cependant Tondeur a trouvé, sur 17 chevaux peureux, 9 myopes ì un haut degré.

La myopie peut être soupçonnée par l'examen extérieur de l'œil; le bombement de la cornée accuse cette fàcheuse conformation.

Ce mème examen extérieur peut donıer une idée de l astigmalisme du sujet.

Un ril est astigmate quand les différentes dioptries de l'oil ne sont pas des surfaces de révolution; c'est-à-dire quand il n'offre pas le mème degré de courbure dans tous les méridiens.

"La détermination de l'astigmatisme montre que c'est le méridien vertical de l'ail qui est le plus réfringent : c'est lui qui est emmétrope dans l'astigmatisme hypermétropique simple; c'est lui qui est myope dans l'astigmatisme simple.

"L'astigmatisme conforme à la règle est celui dans lequel le méridien vertical est le plus réfringent; c'est celui quon rencontre le plus fréquemment.

"L'astigmatisme contraire à la règle a son méridien horizontal plus réfringent que le vertical ; c'est l'exception. " (Nicolas.)

L'astigmatisme, chez le cheval comme chez l'homme, peut ètre congénital ou acquis. On distingue aussi un astigmatisme cornéen déterminé par les irrégularités de courlıre de la cornée, un astigmatisme cristallinien anterieur, eristallinien postériéur.

L'astigmatisme peut èlre encore la conséquence de brûlures de la cormée ayant déterminé des pertes de substance.

La réfraction statique de l'œil permet de se rendre 
compte de l'état d'astigmalisme des deux méridiens principaux de l'œil.

La différence de réfraction entre les deux principaux méridiens de l'veil donne l'astigmatisme total.

Le cheval est fréquemment astigmate; la conformation de ses yeux est telle que l'astigmatisme léger est un phénomène physiologique.

Quand il dépasse une dioptrie, le cheval peut ètre peureux.

Il reste encore à préciser dans quelles conditions d'emmétropie et d'astigmatisme les animaux sont dangereux.

Cetle question est difficile à résoudre, car il faut tenir comple des susceptibilités individuelles.

$2^{\circ}$ Examen ophtalmoscopique des membranes $d u$ fond de l'œil. - Le corps vitré, le nerf optique, la rétine, la choroïde sont sujets à diverses altérations que l'examen ophtalmoscopique peut révéler.

\section{CORPS VItré.}

Modifications. - Le corps vitré est une masse gélatineuse et transparente siluée entre la face postérieure du cristallin et la réline. La portion de membrane d'enveloppe qui s'étend de la papille à l'ora serrata porte le nom de membrane hyaloüde. On trouve dans l'humeur vitrée une substance amorphe et des cellules embryonnaires.

L'inflammation du corps vitré ou hyalitis peut affecter une forme suppurative qui aboutit à la perte de l'œil, ou une forme membraneuse caractérisée par des flocons et des filaments qui empêchent de voir le fond de l'œil, ou une forme chronique, scléreuse, accompagnée de décollement de la rétine. Dans d'autres cas, il y a synchisis ou ramollissement du corps vitré, caractérisé par la rapidité de déplacement des opacilés flotlantes, ou des cristaux 
de cholestérine et de tyrosine (synchisis étincelant). Les opacités elles-mêmes sont formées par des poussières, des filamenls, des membranes ou des produits lıémorragiques, reliquats de caillots provenant d'hémorragies ciliaires, de la rétine, du nerf oplique (Nicolas et Fromaget), conséculives à des chutes ou à des traumatismes.

\section{$2^{\circ}$ NERF OPTIQUE.}

Modifications. - On désigne sous le nom de papille optique, frunctum cxcum, ou de tache aveugle, le lien d'épanouissement da nerf optique. Sa forme est celle d'une ellipse, et ses dimensions de 4 à 5 millimètres.

Cette région peut êtrele siège de papillite ou d'inflammation de l'extrémité terminale du nerf, de névrites rétrobulbaires ou névrites ascendantes. Ces névrites succèdent à des inflammalions intraoculaires telles que les rétinites, les chorcüdites, les irido-choroüdiles, les phlegmons de l'urbite, les ostétes, les périostites ou les tumeurs qui compriment le nerf optique.

Dans les tumeurs cérébrales, l'expansion du nerf optique est étranglée par suite de l'étroitesse du trou sclérotical, Ja circulation est entravée ou arrêtée: il y a névite étranglée.

La névrite étranglée est symptomatique non seulement des tumeurs cérébrales, mais aussi de l'hydrocéphalie et de toutes les causes qui déterminent l'hydropisie des gaines du nerf optique (crenure, échinocoque, gliome, exostose, etc.).

L'atrophie du nerf optique peut ètre congenitale, succéder à une papillite, à une névrite étranglée, à une inflammation cérébrale chronique. La fonction visuelle est abolie et la pupille conserve souvent sa forme normale. Ces affections se traduisent par l'amblyopie ou l'allaiblissement nolable de la vision ou par l'amaurose, c’est-à-dire par la cécilé. 


\section{3' RÉTINE.}

Anatomie. - La rétine ou membrane sensible de l'ceil est une circonvolution cérébrale portéesur un pédicule épanouie à l'extrémité périphérique du nerf optique.

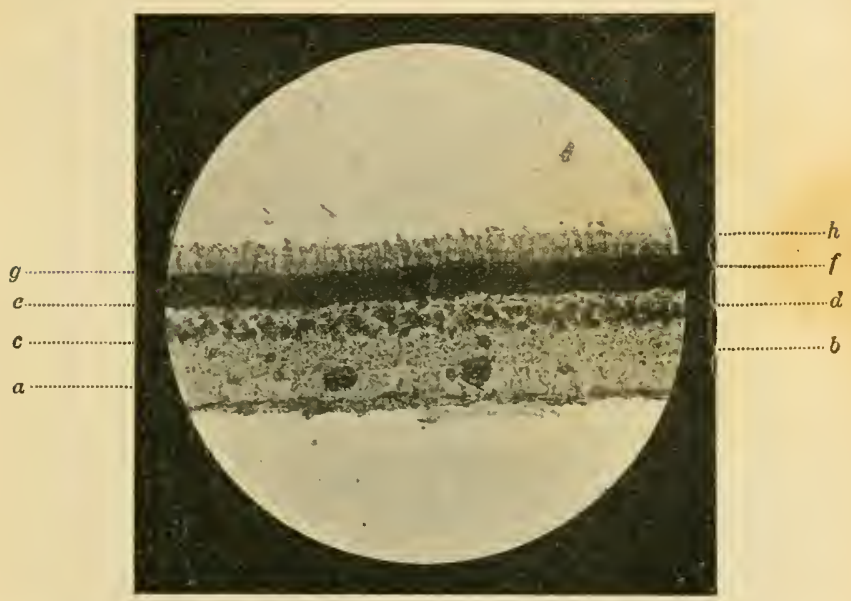

Fig. 8s̃. - Rètine du cheval.

$a$, couche des fibres optiques; $b$, cellules ganglionnaires; $c$, plexus réti. culairc interue; $d$, couche des grains internes; $e$, plexus réticulaire externe; $f$. couche des grains exlernes (sur trois rangs); $g$, limitante externe; $h$, bâtonnets et cônes (Nicolas et Fromagel).

Elle possède : $1^{\circ}$ Une membrane limitante interne, en contact avec le corps vitré;

$2^{\circ}$ Une couche de fibres optiques à plusieurs étages de fibres; son épaisseur augmente graduellement de la périphérie à la papille (fig. 83̈);

$3^{\circ}$ Une couche unique de cellules ganglionnaires volumineuses, en relation avec les fibres optiques et avec la couche sus-jacente par un prolongement qui s'étale dans 
le plexus réticulaire interne (Nicolas et Fromaget) ;

$4^{\circ}$ Le plexus réticulaire interne ou plexus cérébral, qui est mal conuu ;

$5^{\circ}$ La couche des grains internes, constituée par des cellules unipolaires ou bipolaires ;

$6^{\circ}$ Le plexus réticulaire externe, où viennent aboutir les terminaisons des cellules bipolaires et des cellules des cònes ou bâtonnets ;

7० La couche des grains externes, formée de nombreux noyaux colorés disposés régulièrement sur les trois élages ;

$8^{\circ}$ La membrane limitante externe;

$9^{\circ}$ Les bâtonnets et les cônes, zone formée de corps réfringents qui surmontent la couche précédente;

$10^{\circ} \mathrm{La}$ couche pigmentaire, composée de cellules épithéliales à noyaux en relation avec la choroïde.

Modifications pathologiques. - La rétine est le siège d'altérations variées; on peut y observer des inflammations (rétinites), produites par des névrites, des tumeurs, des cysticerques, des corps étrangers, ou des maladies organiques comméles néphrites ou des maladies infectieuses comme la gourme, la pneumonie, l'anasarque, la leucémie.

La rétine est sujette à des hẻmorragies consécutives à des névrites étranglées, à des maladies infectieuses, à des empoisonnements, à l'anémie pernicieuse, à des embolies; elle peut présenter des décollements, succédant à une altération du corps vitré, à l'augmentation de caillots sanguins ou à des exsudals extrachoroïdiens épancliés entre la membrane vasculaire et les bàtonnets, dans l'irido-choroülite ou pendant l'évolution de tumeurs malignes.

La rétine est enfin exposée à subir la dégénérescence kystique ou cystoüde, particulièrement clıez les vieux animaux (rhevaux, houfs, chiens), ou à ètre envahie par des sarcomes ou des tumeurs indéterminées. 


\section{$4^{\circ}$ CHOROIDE.}

La chorsïde comprise eutre la rétine et la sclérotique présente à sa face interne une teinte brune due au pigment rétinien et une teinte bleu verdàtre, qui constitue ce qu'on appelle le tapis. Cette membrane est très vascu-

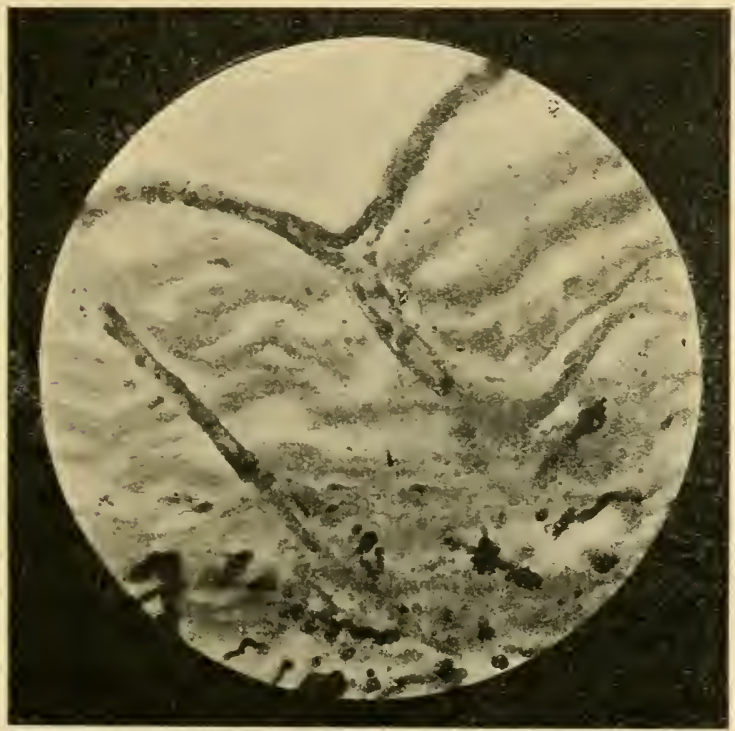

Fig. 86. - Choroïde du cheval. Chorio-capillaire et vaisseaux du tapis. (Nicolas el Fromaget.)

laire et entretient des rapports de continuité avec le corps ciliaire et l'iris et de contiguïlé avec la rétine et la sclérotique (lìg. 86).

Modifications pathologiques. - la choroïde peut s'enflammer d'une manière indépendante (irido-choroüdite); elle prend alors une teinte jaune sale et présente des 
plaques grisàlres ou blanchâtres de diverses dimensions témoignant d'me atrophie partielle ou tolale. L'inflammation se propage d'arrière en avant, envalivi tout le tractus uvéal et se traduit par Jes symptomes d'uue irido-cyclite ; c’est-i-dire par les symptòmes de la fluxion périodique : "Le cristallin devient npaque, le globe oculaire se ramollil, le vitré se rétracle, la rétine se décolle et on assiste progressivement à la plitisie de l'œil atteint. ) (Nicolas et Fromaget.)

La choroüdite disséminée ou diffuse peut résulter de l'infection tubcrculeuse; elle est consécutive à l'omphalite des jeunes animaux el à l'urthrite purulente, à la pleuropncumonic infectieuse, ou bien elle résulte de traumatismes ou d'inflammations de voisinage.

La choroïde peut ètre enfin le siège de tumeurs (sarcomes, elc.) qui offrent tomjours une pigmentalion intense.

\section{X. - MMIBIOPIE. - IMAUROSE.}

Définition. - On désigne sous le nom d'amblyopie et d'amaurose un syndrome caractérisé par l'affaiblissement. ou laholition de la faculté visuelle à la suite de l'inflammation ou de l'altération des parties profondes de l'ceil.

Les parties antérieures conservent ainsi leur parfaile trausparence.

Symptômes. - On observe des symptòmes objectifs et les symplômes rationnels.

Les plúnomènes obsectifs consistent dans une immobilité complète ou incomplète de la pupille chez le cheral amaurotique; elle est largement dilatée lorsque la lésion siène en avant ou dius les centres gangliomaires de la hase du cerveau.

Dans le cas d'amblyopie tenant à des lésions profondes, les mouvements iricus sont lents, paresseux et s'effectuent daus des limites moindres qu'à l'élat normal. 
Les plaies, les contusions de la tète et particulièrement des arcades sourcilières qui sont saillantes, sont quelquefois des indices d'une mauraise rue (Nicolas et Fromaget).

Symptones Ratioxiels. - L'animal aveugle révèle sa cécité par les atlitudes de sa tète et de ses membres dans la locomotion.

Le cheral trotteur 'porte la tête élerée, stargazing (regardant les étoiles), comme disent les Anglais, le nez au vent, les oreilles droites et altentives; aux allures vives ou lentes, il lère haut les membres dans la peur instinctire de rencontrer des obstacles sur le sol.

Le cheral de trait marche arec ure lenteur pour ainsi dire calculée, la tête en avant et un peu inclinée de côté, ayec les oreilles érigées dans l'attitude de l'attention.

Le bœuf est lent à se mouroir.

Le chien marche le nez près du sol, le cou tendu, se guidant par le flair, cherchant à voir par le nez.

Le cheval affecté d'amaurose n’a plus l'expression du regard fière el résolue; l'àme s'est enfuie de ses yeux (H. Bouley).

La main agitce devant ses yeux ne produit aucun m uvement de défense.

S'il est affecté seulement d'amblyopie, sa physionomie n’a pas perdu toute son expression, mais elle offre quelque chose d'insolite. Ses oreilles sont Irès mobiles, l'animal est craintif et ombrageux, de telle sorte qu'un cheral aveugle est sourent préférable à un cheval dont lit rue est affaiblie. Son pas est mal assuré et il commet des fautes grossières.

Pour reconnailre l'œil qui présente la diminution de rision, Nicolas préconise le moyen suivant : "Mettre l’animal à la longe, sur une haie, après lui aroir courert la tête arec une capote, dont l'œilleton correspondant à l'œil suspect est libre. Dans ces conditions, si après deux ou trois passages, l'animal retombe dans les mêmes 
fautes, renverse l'obstacle avec les genoux ou les avantbras, par exemple, sans avoir fait aucun effort ou un effort manifestement insuffisant pour la tâche à accomplir, la présomption d'amblyopie passe à l'élat de certilude. on peut varier l'expérience en opérant tantôt à une lumière vive, tantót à une lumière crépusculaire.

Signification. - L'amblyopie et l'amaurose sont symptomatiques de lésions risibles à l'ophtalmoscope, telles que : le défaut de cohérence ou l'ossification de la rétine, les altérations du nerf optique par des tumeurs osscuses, des carcinomes, des épaississements de la dure-mère, de la pie-mère, par des foyers purulents ou hémorragiques, des tumeurs ou des conures du cerveau.

Elle peut succéder à des contusions de l'orbite, à un coup de corne sur le front, ou à des commotions produites par une chute.

L'amaurose et l'amblyopie sont symptomatiques de l'intoxication par le plomb, la belladone, le pavot, l'arsenic, l'ivraie, la viande pourrie et le chlorhydrate de quinine.

l)es signes d'amaurose peuvent succéder à l'influence d'une vive lumière, à des hémorrayies, à l'iudigestion vertigineuse ou coïncider avec la gestation ou la diminution sapide de la sécrétion lactée.

L'amaurose, comme la cataracte, est quelquefois hèréditrive; des parents aveugles ont engendré des poulains unlaurotiques.

\section{DEUXIĖME SECTION}

\section{AUDITION.}

L'appareil de l'audition comprend (rois parties: l'oreille externe, l'oreille moyenne et l'oreille in terne ou labyrinthe, avec les terminaisons du nerf acoustique. 


\section{1. - OREILLE EXTERXE.}

L'oreille externe comprend le parillon et le conduit auditif.

\section{A. - PaVilloN.}

Ròle du pavillon. - Le pavillon, très mobile chez la plupart des animaux, notamment chez les solipèdes, recueille les ondes sonores.

Modifications pathologiques. - Les oreilles peuvent èlre modifiées.

$1{ }^{\circ}$ Dans leur atritude; les oreilles couchées indiquent un cheral méchant; les oreilles inquiètes, portées alternativement dans diverses directions, sont le signe d'un trouble

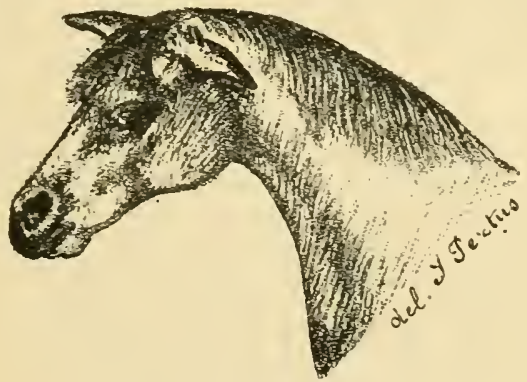

Fig. 8. . - Oreilles de cochon.

de la vision (cécité, amaurose); les oreilles pendantes (oreilles de cochon) sont un indice de vieillesse, de lymphatisme, parfois de paralysie (fig. 8i); les oreilles droites, immobiles, se redressant brusquement quand on les plie, se remarquent dans le tétanos et dans le cas de surdité.

$2^{\circ}$ Dans leur température; elles sont très chaudes quand la circulation centrifuge est suractivée, l'action du cœur renforcée, les artérioles et les capillaires dilatés (stade de 
chaleur de la fievre, coliques, etc.); elles sont froides quand la circulation centripète est aclivée ou qu'il y a une vasoconstriction (collapsus), frissons de la fievre, empoisonnements, hemorragies internes).

$3^{\circ}$ Dans leur colonation: pâles dans l'anémie du chien; tachetées de blanc, chez le cheval, à la suite de piqùres de simulies; rouges dans l'eczéma, le calarrhe, les affections fébriles intenses; jaunes dans l'ictère; violacées et ecchymosees dans le rouget du porc.

$4^{\circ}$ Dans leur humidité : sèches dans les maladies chro. niques, cachecliques, humides dans l'eczema, le calarrhe, la gale chorioptique (chien et chat), la gale psoroptique (lapin).

$5^{\circ}$ Les or eilles peuvent présenter des ALTÉRA TIONS DIVERSES: insectes, corps étrangers, qui déterminent des accès de rage et d'épilepsie ; blessures et ulcérations de la conque auriculaire; écoulement sanguin, abcès, tumeurs, papillomes, etc.

\section{B. - CONIUUT AUDITIF.}

Rôle du conduit auditif. - Le conduit auditif externe transmet les ondes sonores jusqu'à la membrane du tympan; son obstruction eutraine la surdité.

Il protège les parlies profondes et délicates de l'appareil auditif, gràce à sa sensibilité, aux poils dont il est garni et au cérumen sécrété à sa surface.

Exploration. - Le conduit auditif peut ètre exploré directement ou indirectement.

Directesent, à la lumière solaire, on saisit la conque des deux mains et, en la tournant légèrement, on peut voir le conduit auditif externe.

Il est indispensable d'assujettir les animaux qui souffrent de cet appareil; le tord-nez peul suffire pour le cheval; mais il est ordinairement plus commode el plus avantage ux de coucher les solipèdes en plein air et de fixer le chien, muselé, sur une table. 
L'introluction dı loigt dans le conduit auditif permet de constatrr la présence de mutiere cérumineuse, te pus, de sung, de corpsétranger's, de parasites ou de tumeurs.

La M TTHODE INDIRECTE consiste à recourir aux spéculum auris pour dilater el rendre plus rectiligne la partse externe du couduil. Mans ce but, on utilise le spéculım unicalve on tubulaire rt le spéculum bivalie.

Les spéculums tubulaires ont la furme d'un entonnnir à long col quon introduit arec précaution dans le fond de l'orrille; on ṕlarre ensuite le conduit audilif à l'ai te d'un miruir réflectenr qui concentre łans la cavilé du spéculum, les rayons lumineux d'une la'ıpe à pérrole ousimplement ceux se la lumière naturelle.

Chrz le chien, on utilise les spéculums bivules tel que celui d'Itard à ralves longues, arrondies l'un còté ì l'autre el fixṕes sur deıx branches articulées.

Cel examen peut réréler des plıies, des hémorrugies, des ulcérations, les tumeurs, des accumulations de rírumn, quelquefois de pus et des parasites (malarlie épileptifurne des chiens de meute).

La maladie de Meniere a éte signalée chez le chat par Wilıried Lellıanı; elle se traduit par de fréquents accès de verlige arec impossibililé dr la station après l-s crises el vomıssements; parfois l'auimal tourne et secoue la tète en tous les sens, en poussant des cris; il semble, en outre, que l'auimal percoit de forts liruits anormaux, dont il cherche la provenance. La surdité complète peut être la terminaison de ces troubles.

\section{II. - OREHLLE MOYENNE.}

L'oreille moyenne ou caisse dı tympan (fig. 88) possède : 10 Une face externe coistituée par la nembrane du tympan;

20 Une face interne prósentant la fenétre ovule formée par l'ètrier $(e t)$ et la fenètre ronde pourvue d'une munce CADÉAC. - Sémiologie. əe édit. 
membrane circulaire $(f \circ)$. Entre ces deux ouvertures, on trouve une pelite saillie osseuse, le promontoire $(p)$ et en bas, on observe l'orimine de la trompe d'Eustache (T F) qui va s'ouvrir dans le pharynx; il faut y signaler enfin la chaîne des osselels, le mårleau $(m)$, l'enchume $(e n)$, le lenticulaire non figuré et l'étrier $(e t)$.

I, a membraue du tympan protège les parties internes le l'oreille et vibre à l'unisson de tous les sous extérieurs qui viennent l'impressionner. Puis les ondes sonores se propicgent dans l'oreille moyenue par la chaine des osselets, les os du cràne ou l’air enfermé dans la caisse du l) mpin.

Les us-ELEts de l'oreille conslituent en s'articulant une chaîne brisée, mais rigide, tendue entrela membrane dı tympan et la fenètre cvale. Les vibrations des osselets ont été constatées sur le calavre; mais elles ne deviennent perceptibles que lorsque les sons offrent une grande intensité.

La destruclion de la chaine des osselets ne provoque pas surremeut une sırdité complèle, pas plus que la perforation de la membrane du tympan, mais a la condilion que l'strier soit conservé. La suppression de cet osselet abolic l'oü̈e, parce que la périlymphe s'écoule librement dans l'oreille moyenne et ne peut plus transmettre les ondes sonores au labyrinthe membraneux.

Les os DU Cr.Ne sont de bons conducteurs des sons : ils permettent d'entendre sa propre voix quand on a les oreilles bouchées, de percevoir les vibrations d'un diapason tenu entre les dents ou le tic-tac d'une montre qui touche les dents ou qu'on place sur le sommet de la tête.

l'alr exflermé daxs La CAIsse du trupan propage les vibrations sonores quand la chaine des osselets a été détruile de la membrane du tympan a la membrane èlastique de la fenétre ronde.

Lit trompe d'Eustache a pour fonction de maintenir en équilibre de pression l'air renfermé dans l'oreille moyenne 
avec l'air extérieur gràce à la communication intermittente qui s'élablitentre la caisse du tympan et le pharynx. A ce uiveau, lorifice de la trompe d'Eustaches'ouvre a clıaque déglutition sous l'iufluence du muscle péristaphylin interne. Quand la trompe d'Eustache est oblitérée, la surdité résulte de la raréfaction de l'air dans la caisse,

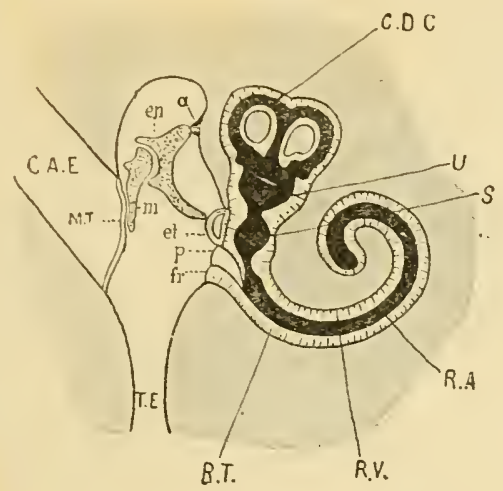

Fig. 8s. - Scluéna de l'urcille.

- C.AE, conduil audilif externe, MT, membràne du lympan; TE, orifice de la trompe d'Eustaclie; $m$, marteau; en, enclume: et, étrier, formant la fenètre ovile par ou l'oreille moyenne communique avec le labyrinthe. Le labyrinthe memhraneux rempli par l'endolymphe occupe toute la région colorée en noir; $R A$, rampe auditive (limaçon membraneux); $\mathrm{HV}$, rampe veslibulaire: RT, rampe tympanique séparéc de lorcille moyenne par la menbrane tendue sur la fenètre ronde $\mathrm{fr}$.

de la dépression consécutive de la membrane du tympan qui comprend et immobilise la clıaine des osselels.

Les déglutitions qui ouvrent la trompe d'Eustache sont aiusi nettement associées à l'audition.

Les cellules yastoipiexises sont des espaces remplis d'air ; elles augmentent la masse gazeuse de l'oreille moyenne et diminuent en les répartissant sur un plus grand volume d'air, les effets dus aux changements de la pression intra-tympanique. 


\section{III. - OREILLE INTERAE.}

L'oreille interne comprand le lahyrinthe osseux et membraneux et les terminaisons du nerf acoustique.

Labyrinthe. - Le Labyrinthe ossuux creusé dans le roclser, comprend le vestibule, Irs canaux demi-circulaites et le limacou; ce deruier est pourvu a si lace interue d'une lame osseuse, la lame spirule, qui se délache de sa petite courbure ou courbure interue et pínètre jusqu'iu centre ou elle se termue par un bord libre.

Le Labyrixthe membraneux alfecle la disposition d'un sac formant dans son ensemble un systeme de carités concentıiques a celles du lalyrinthe osseux. II est rempli par un liquide appelé l'endolymplic et forme un sac clos de toutes parts.

L'espace coronaire compris entre les deux labyrinthes est rempli par la perrilymphe.

Dams la ligure 88, on penł voir les diverses parties du lahyrinlue membraneux dessinées en noir:

L.e vestibule comprend deux régions liées entre elles par une itroite communication :

$1^{\circ}$ L'utricule (U), dans laquelle s'ourrent les canaux demi-circuliares;

Et $2^{\circ}$ la saccule (S), qui communi,jue avec le limaçn membranes appele rampe cochlícirc ou rampe andilive du limacon RA).

Cie dernier compartiment est limité en bas parla membrane basilaire, en luaut par la membrane de Heissuer (lig. 89 CC).

An nivean du vestibule, l'espace perilymphatique (dessiné en blanc dans la ligure 88) s'ouvre dans l'oreille moyenne par la lenêtre ovale; mais la pirilymphe ne peut s'écouler dans la caisse du lympan gràce à l'étrier qui bouche la fenètre ovale. C'est elle qui est l'agent des 
relations physịues qui peuvent s'établir entre l'oreille moyenne.

"Les ondes sonores Iransmises par la chaine des osselets parviemment à la fenèrre ovale et retentissent sur la

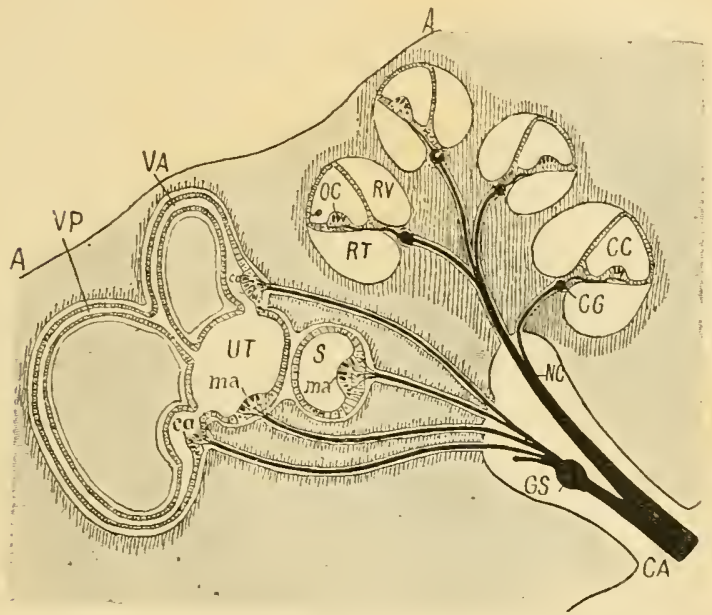

Fig. 89. - Sclíma de la distribution du nerf acoustique dans les direrses parlies de l'oreille interne (d'après Mathias Duval).

RT, ramçe tympanique du limaçon; RV. rampe vestibulaire; CC, rampe auditive ou cochléaire; OC, organe de Corti; GC, ganglion de Corti; $S$, sacculc; [T, utricule; VA, VP, canaux demi-circulaires vertical antérieur et vertical postérieur); ma, ma, macult's audilives; $r a, c a$, crètes auditives dans les ampunles des canaux dem-circulaires; CA, orifice du conduit auditif interne livrant passage au nerf acouslique; GS, ganglion de Scarpa, placé sur la branche veslibulaire du nerf audiof: GC, ganglion de Corti ou ganglion spiral, placé dans le canal de Rosentlıal, à la base de la lame spirale.

périlymphe. Les demi-oscillations internes de la membrane dutympan entrainenl une demi-uscillation de mème sens dans l'élrier qui presse sur la pèrilymphe. Les demioscillations internes produisent un effet inverse el appellent l'étrier en dehol's. Celui-ci agjt done à la manière 
d'm piston qui, tour à tour, refoule et appelle la péritymphe. Or, celle-ci, incompressible comme tous les liquiles, remplit un espace qui n’est léformable qu'en un seul point : la membrane élastique qui ferme la fenêtre ronde.

Il en résulte que les vibrations de l'étrier entraînent dans la périlymphe des nouvements de lotalité, des oscillations qui intéressent simultanément toute sa masse et se reflètent sur la memhane de la fenètre ronde » (I.aulanié).

Nerf acoustique. - Le nerf acouslique se divise en deux branches, la branche restibulaire et la branche cochlénire.

La branche vesiibulaire présente sur son trajet un renflement gangliomnaire (iS, fig. 89), le ganglion de Scarpa, au dela duquel il émet ses hranches terminales qui se rendent sur les appareils récepteurs, les macules audilives (ma) pour la saceule et l'utricule et les crétes aulitives (ca) pour les canaux demi-circulaires.

La branche cochléaire (NC) pénètre dans l'axe solide et osseux du limaçon et répand ses branches terminales en éventail; celles-ci présentent à lenr tour un renllement ganglionnaire (ganglion de Corti).

Au delà du ganglion, les fibres auditives trouvent la lame spirale et se terminent sur l'organe de Corli (OC).

l.es vibrations de la périlymphe se répercutent sur les diverses parties du labyrinthe membraneux et atteignent les terminaisons sensitives. Les cellules auditives éprourent en réalité un ébranlement mécanique, dans lequel l'endolymphe, les crins auditils, les otolithes (sorte de poussière eristalline microscopi(yue) et la membrane basilaire out chacun leur part.

L'impression unditive est recueillie par le linacon, les canaux demi-circulaircs, et le vestibule. L'intérieur du limacon est garni de nombreuses pelites cordes tendues 
comme les corles d'un piano et qui sont en relation avec les terminaisons du nerf acoustique.

L'oreille moyenne et l'oreille interne ne sont gruère explorables.

Les otoscopes n'ont pas recu d'application chez nos animaux domestiques.

Pourtant, les altérations de l'ouie sont assez fréquentes.

La surdité survient dans l'épilepsie avec hyılrocéphalie (cliens et chats); la pharyngite, en oblitérant lts trompes d'Eustache et en empèchant l'entrée de l'air dans l'oreile moyenne, peut la produire. La diphterie des volailles, la tuberculose de l'oreille moyenne, la pneumonie unfectieuse peuvent aussi amener celte infirmité. 


\section{SÉMIOLOGIE DE L'APPAREIL LOCOHOTEUR}

On peut observer des changements dans le port de la tite, dr la quewe, daus l'atlitule du corps, des modifications atrophiques ou lype:trophiques des musil..s, des altératious des os, des a ticulations, des tendons et des pieds, des troubles des allures (boilerie, harper).

\section{I. - TETE ET FICIES.}

Modifications. - La région de la tèle peut être le siège de praies provenant d'accillents récents, dr fi:lules conséculives à đes nécroses, a la trépanation les sinus, d'excoriations, de cicatrices déterminées par des chutes.

Eu oulre, la tète présente diverses ATtitudis qui ont une grande signification pathologique : ellt est haute dans le tétmos qui provoyue la contraclure des inuscles releveurs d: l'encolmre et dans toutes les maladirs accompagnées de dyspnée (gourme, bronchite, pnrumonie, pleurésie, em hysieme pulmonare); elle est busse. daus les inflammations graves (périlonile, pleurésie au délunl), maladırs daus lesquelles l'animal esl stupélié par la lièvre, dans les arrectious typhoïles où le sujet est accahlé, comalcux, a l'ail terme, fixe et la conjonctive jammâtre.

D'autres attiludes lle la tête aident le clinicien à apprécier le siège et la gravité d'une maladie. La tète est 
penchée, c'est-à-dire inclinée latératement à droile ou à wuche dans les affections des contres encephaliques (mèningite, meningo-encéphulite, tumeurs, conures, parasites divers), et dans diverses affections catarrhales ou parasilaires des oreilles.

La tète est appuyée sur la mangeoire, dans la méningo-encéphalite chronique, dans la méningo-encéphalite

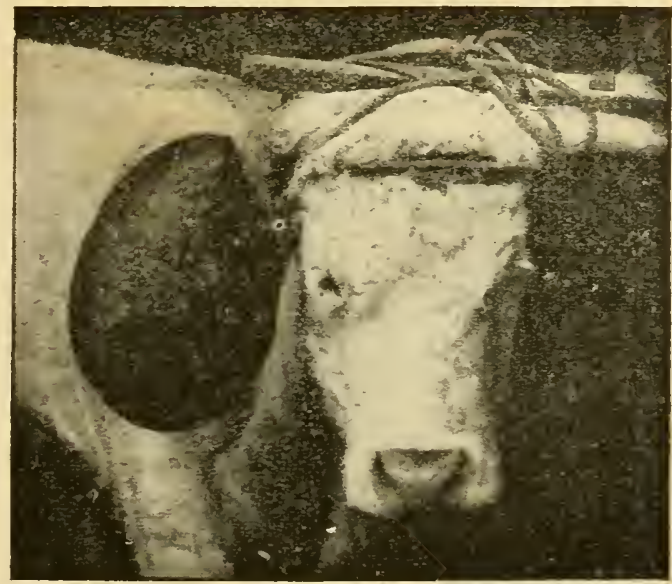

Fig. 90. - Tumeur myxomaleuse développée sur une corne à la suile d'une fraclure (Desaintmartin).

riguë, pendant la périole de rémission et dans divers empoisonnements.

I.a tête est souvent porlée du côté du flanc, l"œil regardant l'abdomen dans les coliques; elle est agitée en sens divers dans les rertiges, le typlus, la firre charbonneuse (Labat); elle est agitie de haut en bas dans la hernie inguinale étranglèe.

La tète chandc, accompagnée de rougeur des conjonctives, témoigne d'une méningo-encéphalite, do douleurs 
très aiguës ou d’une affection grave fébrile; la tète est. froide el les conjonclives sont pàles dans l'anémie.

Les sinnes fournis par la tète font reconnaîlre que l'animal est malade, mais ils n'ont qu'une valeur relative pour établir le diagnostic de la malarlie.

Chez les ruminants, les corues préseutent d'importantes modilications :

$1^{\circ}$ Au point de vue dela temperature, elles sont cliaudes ou froides sous l'influence des mèmes causes que les oreilles ;

$2^{\circ} \mathrm{Au}$ point de vue de leur intégrité; on peut constater l'inflammation du tissu réticulaire qui unit l'élui corné à la cheville osseuse (étonnement des comes analogue à la fourbure du cheral), la fructure sans avulsion ou le détachement complet d'une corne ou des tumeurs volumineuses fibreuses ou myxumateuses, développées à l'extrémité des comillous (lig. 90 ).

Il arrive fréquemment qu’il est nécessaire de pratiquer l'amputation descornes en raison de la mauvaise direction qu'elles ont prise, ce qui rend lapplication du joug impossibie.

\section{II. - ISYMETRIES.}

Caractères. - En médecine humaine, l'asymétrie du cràne, de la face, lu thorax, de l'abdomen, des membres, etc., est considérée comme la révélation extérieure d'un développement défectueux de l'organisme. Toules les malformations anatomiıues de ces rémions constituent des stigmutes physiques de déginerescence.

Chez les animaux, l'élude des formes asymétriques du corps est à peine ébauchée. On peut cependant les constater:

$1^{\circ}$ Dans le tic de l'ours et certaines stéréotypies du léchaure, comme en témoignent les inléressants travaux de Chomel et de Rudle (Voy. Tic de l'ours).

$2^{\circ}$ Dans les arthrites des membres; .I. Joly a iusisté sur 
l'asymétrie de la hanche comme simne important d'une lésion ancienne du membre postŕrieur correspondant.

$3^{\circ}$ Dans l'épilepsie, Bassi a montré que l’asymitrie du cràne jone un rôle causal important; Bertetti a remarqué aussi que I'asymétrie cranienne exisle chez Ies chevaux atteints de manie périodique, sujets à reculer ou à se

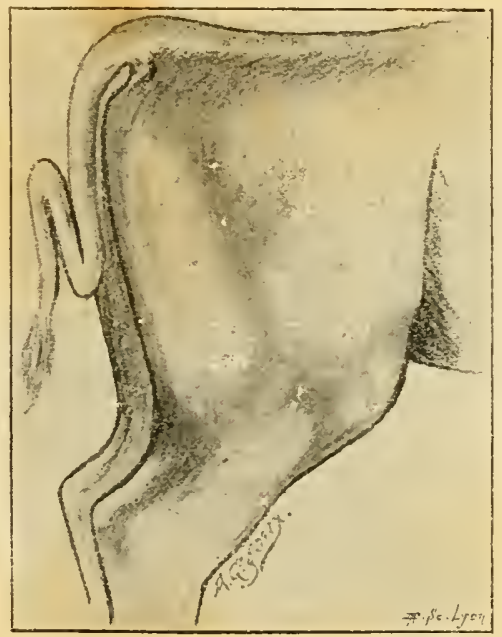

Fig. 91. - Double luxation de la queue.

cabrer, ou à faire des écarts sans molifs, comme chezles chevaux épileptiques.

$4^{\circ}$ En marechalerie, on fait ressortir l'importance de l'asymétrie dles extrémités.

$\check{3}^{\circ}$ Il n'est pas rare de constaler une asymétrie prononcée dans la pousse et l'évolution de l'arcade dentaire (Joly).

\section{III. - SIGNES FOURNIS PAR LA QUEUE.}

Modifications. - Les positions de la queue sont peu expressives : 
La queue est flasque et tombanle pendant l'évolution des maladies aiguës et pendant la période de convalescence lles maladies très graves; dans la paraplégie, la queue, incapable de tout monvement, pent inerte el insensible. Par contre, dans le tétanos, la contraction énergique de tous les muscles de l'organisme maintient la queue droile, raide.

l.es helminthes (oxyures, ascarides, tenias, sclerostomes), l'OEstrus hemorrhoülalis, les hippohosques lixés à la marge de l'anus ou à la partie inlérieure de la quene déterminent des mouvements désordonués de cet appendice.

On peut constator une atrophic congénitale de cet organe chez le veau; la quewe n'a pas plus de 10 a 12 centimètres (Bedel). Exceptionnellement, on penl observer une luxalion simple ou double le cet appendive (lig. 91).

La quene pent s'abcider, se nicroser à la suile d'inoculations, de vaccinatirns chez les bovides; elle es। frérfuemment le siège de naıifeslations eczémateuses clız le chien.

\section{IV. - ATTITUDES.}

Station. - L'arimal debout est supporté par ses quatre memlires ou seulement par trois d'entre eux, l'un, élant porté en avant et appuyant seulement par la pince, est dit en slation.

Quaud le sujet se tient sur ses qualre membres, les hanches sont à la mème hauteur; s'il est seulement supporté par trois d'entre eux, la lianclie correspondante au nembre fléchi est légèrement abaissée yuand il s'agit, conme c'est le cas ordinaire, d'un membre postérieur.

Au bout d'un certain temps, l'animal change de support de sorte, qu'allernativement, le membre gauche remplace le mombre droit et réciproquement, dans le soulèrement du corps.

Les membres antérieurs ne cluangent pas de place; à 
peine observe-t-on, quand l'animal est fatigué, un piétinement assez rígulier qui soulage les muscles.

Le cheval est de tous nos quadrupèles domestiques celui yui peut rester le plus lougtemps debout; souvent il dort dans cette attitude.

Quanıl il est bien portant, la station est aisée, facile, calme, les aplombs sont normaux.

L. bout, le cliten et le porc sont assez rarement observés en station; ils se couchent presque toujours. Les sigues que l'ou peut tirer de leur examen en station sont du reste Inin d'ètre aussi nombreux el aussi importants que ceux fouruis par le cheral.

Modifications pathologiques. - Dans les cas pathologiques, l'auimal loune à ses membres des posilious souvent caractérictiques; il pointe dans les malalies des pieds; il soulève convulsivem $n t$ le membre et racle le sol laus le cas d'arthrite, te javirt, de clou de rue; il tient le membre inerte dans le cas de paralysie. Souffre-t-il des talons (bleimes), l'appui s'effectue surtout par la pince.

Est-il atleint de maladie naviculaire? le membre sera portien avant pour éviler la clouleur prołuite par le perforant. La lésion siège-t-elle au còlś interne on au còté externe de la boîte cornée? l'appui se fait surlout par la branche externe ou interne du ler; autant d'indications. utiles pour le praticien.

Daus la péritonite, le cheval tient les membres daus l'extension, railes; quand il est menacé d'asphyxie, il écarte les membres autérieurs du thorax comme pour amplifier sa poilrine: daus les coliques dues à la hemic inguinule, il fléchit les jarrets et prend la position du chen assis.

Décubitus. - Quand les solipèles se conchent, ils sont en décubitus sterno-costal, ou en décubitus latéral, conplet ou incomplet. En bonne santé, ils preınent généra- 
lement la posilion sterno-costale. Le déculitus latéral complet ne s'observe que dans les maladies graves. Parfois l'animal se place en décubitus dorsal; dans ce cas, il est atteint de coliques graves pouvant procéder de la hernie inguinale ou d'un étranglenent intestinal.

\section{V. - MUSGLES.}

Les modifications le volume éprouvées par les muscles sont firciles à constater par l'inspection et la palpation.

Inspection. - L'inspection permet de juger de la différence de volume de deux régions symétriques : épaules, bras, avant-bras, cuisses, etc. On constate ainsi des atrophies ou des hypertrophies.

Palpation. - Elle permet dapprécier les variations de températıre, de sensibilité, de consistance des muscles.

La température est surélevée dans le rhumatisme aigu, le tétanos, abaissée daus la paralysie musculaire.

La sensibilite est très vive dans le cas de rbumalisme musculaire (cheval et chien), d'lıémoglobinémie (cheral et boull), de lumbago, de fatigue oa surmenage musculaire. Le saturnisme est caractérisé aussi par une douleur très vive des muscles. La sensibilité est annulee dans la paralysie, la paraplégie.

la consistunce est dure dans l'hémoglobinémie, le titanos; certains muscles sont rigides, tendus comme une corde.

Cette dureté s'accentue encore dans la myosite fibreuse lucalisée à certains groupes musculaires, comme ceux de l'épaule, de l'avaut-bras ou de la cuisse. La myosite devient quelquefois ossifiante au voisinage des aponévroses qui semblent êlre le point de départ ou des foyers d'exten sion des phénomènes d'ossification. 


\section{VI. - AMYOTROPHES.}

Caractères. - Les amyotrophies sont les lésions musculaires les plus communes. L'atrophie musculaire revêt tantòt une forme générale, tantôt une forme locale.

L'appareil musculaire entier peut être atrophie a la

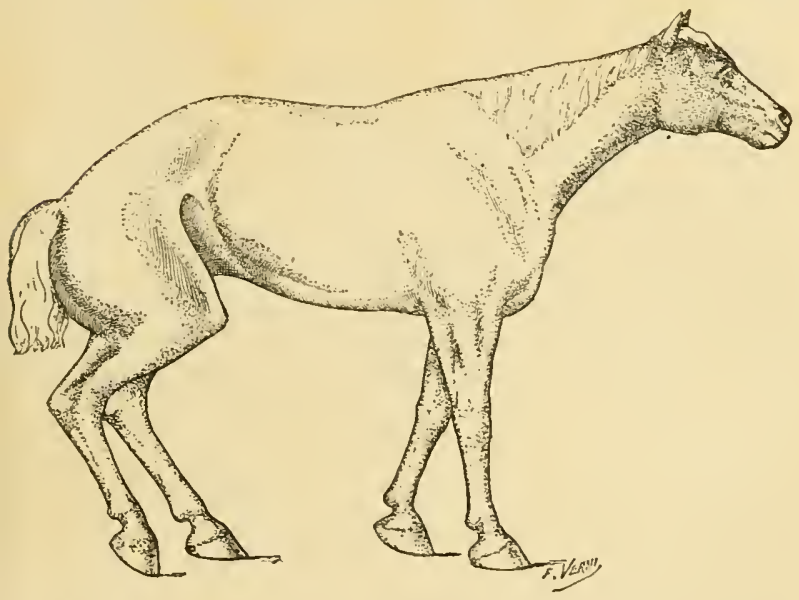

Fig. 92. - Alrophie du triceps crural dans la paralysie du nerf fémoral antérieur.

suite de maladies graves (épilepsie du chien, tuberculose du bacif, inlluenza du (heval).

Toules les maladies chroniques sont susceptibles de déterminer une sorte de fonte musculaire; citons i'anémie, la leucémie, le diabète sucré, l'hydrohémie, la distomatose, la carcinomatose, etc.

Quelquefois les atrophies sont limitées à certaius groupes musculaires comme dans l'hémoglobinemie du cheral, les empoisonuements par le plomb, le phosphore, l'arsenic, l'oxyde de carbone, l'iode. A la suite de lésions 
nerveuses, on voit toule la région de distribution de ces nerfs s’atruphier: sus-éfineux, Iriceps crural, masséter.

Diver-es maladies infecticuses sunt susceptibles de produire cer amyotrophes, soit en altérant la nutrition du muscle, soit eu produisaul des myélites et des nerrites infeclienses romparables à celles qui résultent de l'intoxication par le plomb. L'alrophie dn triceps crural fig. 92), dans lhèmoylobinémie, celle des muscles aryténoïdiens postérieurs ou de divers muscles daus la gourme paraissent résulter de névriles infectieuses.

Les umyolrophies, qui se manifestent en quelyues jours daus les arthrutes, sont plus difficilement explicables. Les muscles foudent et s'émacient en quelyues jours jlus rapilement guaprès la seclion des nerfs moteurs; on prétend que ces amyontrophies résulıent de l'iuflammalion des hranches nerveuses qui se distribuent aux tissus malades; mais daus larthrite du jarret, on les vort se manifester indistimctement dans tous les muscles de la cuisse, de sorte (ju'(u) dehors de limpotence fonctionnelle ou de la nature réllexe de ces allérations, il $y$ a place pour une intoxication des neris et des muscles par les produits morbidus élaborés dans le foyer articulaire.

Les alrophies myélopalhiques consécutives à une altération des cellules des cornes antérieures de la moelle allectent nue forme disséminée inléressaut des groupes de muscles ou seulement cerlaines parties des muscles (Voy. Myélites).

Les atrophies musculaires myopathiques, c'est-à-dire indéprndantes des altéralions de la moelle et des nerfs, consistent dans une dégémérescence primitive des muscles 'arartérisée par une fragnentation des fibres musculaires accompagnée de proliféralion conjonctive el d'infiltralion. graisseuse.

Les atophies d'origine vasculaire résultent d'une irrigalion sanguine insultisante consécutive à une thrombose 
artiriclle; les muscles, insuffisamment nourris, s'alrophient progressivement. On a rattaché à celle cause l'atrophie des fessiers, des pectoraux; mais cette étiogénie exclusive n’a pas été établie d’une manjère indiscutable.

\section{VII. - AFFAISSEMENT DE LA IIANCHE.}

L'allaissement de la hanche a été étudié en 1890 chez les bovins par Fullanelto (1).

"Dans beaucoup de maladies des memlires postérieurs, de nalıre différente et de sièıes divers, on peut ub-erver chez le bout l'atrophie de toute l'étendne du membre qui cependant est toujours plus apparente à la hanche.

L'affaissement peut aussi ètre limite ex.lusivemunt à celte région, à la suite de maladies locales aig̨uës ou chroniques telles que les forles contusions sur !'articulation coxo-fémorale, la distension des muscles de la cuisse, l'osteite traumatique ou rhumatismale de cel os, le déplacement du muscle ischio-tibial externe, l'ygromia aigu au niveau du trochanter, la fracture du fimmr, la luxation coxo-fémorale, les phlegmons aigus de la cuisse el la fracture de l'ilium.

L'affaissement de la Lanche se produit d'autant plus vite et est d'aulant plus prononcé que le membre malade demeure plus longtemps inactif; il fait defant dans certaines mynsites traumatiques el est très apparent au bout de huit à dix jours daus d'autres maladies.

A la suile de cette atrophie musculaire, on remarque une déformation de la croupe el de la hanche; les os du côté de l’atrophie, c'esı-à-dire les angles antérieurs de l'ilium, l'üminence du trochanler et la pointe de l'ischium se moutrent plus saillants que ceux tu còté sain.

Latrophie musculaire persiste un temps plus ou moins

(1) Progrès vét., 1890, p. 231 . 
long; elle disparaît après la cessation de l'état morbide qui l'a produite ou après que l'animal ne ressent plus de douleurs à la partie malade. " (Furlanetto.)

\section{VIII. - IFFAISEEMENT IIIMQIE.}

On connaissait l'asymétrie de la croupe chez les chevaux qui looitent depuis longtemps d'un membre postérieur. Joly s'est attaché à démontrer que le dénivellement des éminences osseuses constilutives de l'angle de la croupe est un signe important pour le diagnostic des boiteries du membre postérieur. Cette déformation est un témoignagge irrécusable de l'ancienuelé de la boiterie.

Caractères. - L'émaciation des muscles se produit taulòt lentement, tantòt rapidement, suivant la cause provocatrice. Quand elle résulte d'une simple impotence fonclionnelle, elle se produit lentement et n'est jamais très accusée; elle est rapide et très prononcée quand elle r'ésulte d'une arllurite. Dans ce cas, l'émaciation desmuscles est suivie de l'atrophie des os; mais l'affássement de lilium est plus appréciable que son atroplie.

On reconnaît facilement cet affaissement quand on fait marcher le cheval devant soi. On constate que l'angle interne de l'ilium du membre boiteux est alfaissé, son anıle externe exhaussé, désincurvé dans sou bord antérieur (Joly). Il est vrai que cette déformation u'est pas absolument pathognomonique d'une boiterie; elle pent ètre congénitale (Belli); nous l'avons observée sur deux chevaux qui n'ont jamais hoité; ces déformations ne sont souvent accompagnées l'aucune alrophie osseuse.

Pathogénie. - Le mécanisme qui préside aux déformatıons acquises a été cliversement interprélé. Autrefois on altribuait l'atrophic musculuire de la croupe à une simple impolence fonctionnelle du membre procédant 
d'une altération quelconque de l'un des rayons de ce membre.

Selon Joly, l'affaissement iliaque est dû à une autre façon de marcher et de se teuir consécutive à l'ankylose, voulue ou mécanique, des articulations inférieures du tarse, à une nouvelle adaptation de l'ilium et des muscles qui s'y insèrent.

Liénaux soutient que l'affaissement iliaque est uniquement dù à une atrophic régressive. Les phénomènes se succèdent de la manière suivante :

La musculature de la croupe s'affaisse sous l'influence de la douleur déterminée par l'iparvin : "le repos forcé qui en est la conséquence entraine la dénutrition des unsiles de la croupe, ainsi que colle plus lente et plus tardive des os; puis la douleur s'atténue, par suile de l'ankylose qui immobilise les jointures tarsiennes inférieures, le cheval est remis en service; ses muscles repremnent rapidement leur volume primilif, tandis que persiste l'inégalité des os, plus lents à récupérer leurs dimensions normales. Ici, comme dans toute autre boiterie, l'émaciation musculaire précède l'atrophie des os; mais celle-ci se répare moins vite et persiste alors que la première a déjà cessé d'exister.

"L'observation de sujets dejà porleurs l'un éparvin avec exostose et chez lesquels la mème tare se produit ultérieurement au jarret jusque-là indemne, est particulièrement intéressante à ce point de vue. Dans ce cas, le premicr eparrin, bien sorti et ankylosé par l'exostose, est habiluellement devenu indolore, tandis que le seconul, tout à son début et parlois invisible encore, s'accuse par une boiterie plus ou moins intense.

11 n'est pas rare alors de tronver une asymétrie croisée de la croupe; l'angle interne de l'ilium lu còté primilivement entrepris est en contre-bas du mème angle du côté de l'éparvin récent, tandis que les muscles de la croupe de ce dernier côté sont notablement amaigris par 
rapport à ceux de la région correspondante du membre opposé.

Sur le membre atteint depuis longtemps et devenu indılore, e'est l'atrophie ossemse qui dumme; sur celui qui soulfre depuis peu, l'atrophie musculaire atlire seule l'attention.

Quand la lésion sera plus avancée sır ce dernier membre, les deux éminen'es de l'angle de la croupe reprendront le mêne niveau.

Ces fails démontrent a l'évidence la marche plus rapide de l'alrophie el de la répraration dans les musiles ¡ur daus les os, ce que la structure, la vascularité et la fouclion differente de ces paslies de l'appareillocomoteur faisilent d'ailleurs prévoir."

S'i est vrai que l’atrophie musculaire se trouve liée à l'iparcin, il est rertain aussi, romme l'a élabli Belli, que l'alrophie des os ou, pour parlerplus exacteme:ı, l'asymétrie d" squelette cararlérisée par l’affaissenent te l'ilium avec diminution d'épaisseur el de poid-drs os, s'observent nornalem. ut chez des animaux non bniteux.

A nolre avis, la déformation iliaque est beaucoup moins significative que l'atrophie musculaire. Les musiles de la croupe s'atrophient et fondent dans toutes les inflammalions articulaires aiguës ou chroniques.

lieparin-arthrite est la cause la plus Iriquente de ces atruphies; ou peut soupçomer l'existence d'un éparvin chaque lois quon constate cetle alrophie, parce que l’inflanmation des autres articulations est relativement tıès rare. Cette atrophie musculare élant le phénomène promitif, essentiel, lié à l'iullammation articulaire, comment se pruduit l'atlaissement iliaque? Ce phénomène est. simplement la couséquence de l'auliude de l'auimal qui soustrait ce m mbre à l'appıi, el ıe me paraît avoir aucme rulation directe avec le mal qui nous occupe. Ce n'est pas en raison de l'existence d'un éparvin ou d'une boiterie quelconque que se produil laffaissementiliaque; 
mais uniugement parce que l'animal, par soullrance cu habitule, ne se sert pas de ce memtire

On peut dire, il est vrai, que la soutfrance entrainant généralement cette impotence functionmelle, la déformation iliaqne est la conséquence, la caractéristipue de toules les boitıries des membres postérieurs. Elle sera au même titre l'expression d'un habitude viciense qu'olfrent certaius chevaux nou boiteux de reposer pour ainsi dıre constamment el. exclusivement sur un memlire postérieur, pendaut que l'autre est tenu plus ou moins fléchi; la dé ormalion iliaque est la conséquence de cette habitule, an même titre que la dilormation des épaules observée chez les hommes de bureau dont l'épilule droule parait to jours remontée par rapport à l'épaule gauche qui parait abaissée.

D'aillenrs nous avons vu mainles fois des personnes qui avaient subi dans leur enfance une résection de 12 à 1 ä centimètres de tibia pour eulever un foyer tubrrculeux et ¡jui à l'àge adulte présentaient de ce còté un abaissement graduel du bassin d'une hanteur presyue équivalente à la résection ossouse, à tel point qu'on ne roustalait aucune boiterie (1). Il se produit quelque chuse d'aıalogue ithez les chevaux, l'ilium s'abaisse du còté qui correspond au minimum de fonctionnenent. Il est probable que le cheral, comme l'homme, est très souvent asymétrique même en lehor's de tout éparvii et de toute tare lointaıne ou récente.

L'amyotrophie musculaire a une signification beaucoup plus nette : elle témoigne d'une altération articulaire détermmant un trouble nerveux réflexe ou une utoxication el des effets beaucoup plus prononcés que ceux qui résultent de la section des nerfs moteurs $(2)$.

(1) Ollier, Les résections osseuses. Présentation des opèrès à la Societé de médecine de Lyon.

(2) Joly, Revue vëterinaire, 1897, p. 5 -S. Bulletin de la Sociële centrale,

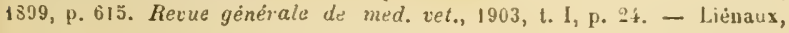


En résumé, l'amyotrophie musculaire a une graude importance pour le diagnostic; elle est généralement symptomatique de l'éparvin et est d'antant plus prononcée que le mal est plus grave; l'affaissement iliaque per. toute signification précise, parce qu'il est tantot conycinital, tautòt l'expression d'une habilule, tautôt consécutif à un défaut ou à l'inégalité d'appui des membres postérieurs résultant d'une boiteric.

\section{IX. - HYPEIRTOPIIES.}

Les hypertrophies musculaires sont tantôt vraies, tantòt fausses.

Les hypertrophies vraies, qui résultent d'une exagération du fonctionnement, se manifestent quand certains muscles doivent suppléer à d'autres qui sont atrophiés ou impuissanls; c'est ainsi que les muscles du plat de la cuisse s'hypertrophient quand il y a paralysie du netf fémoral et atrophie du triceps crural (tim. 93).

L'exagération du fonctionnement suffit pour amener cette hypertrophie; les sterno-maxiltaires s'hypertrophient chez les chevaux affectés du lic aèrophagique.

Les muscles sont le siège d'une hypertrophie fausse consistant dans une congestion accompagnée de tuméfaction: $1^{\circ}$ dans le rhumatisme, où les muscles le la croupe, le l'encolure, etc., sont luméfiés et volumineux; 20 daus l'hémoglobinémie, où ils devienuent en même temps durs comme du bois. Cette tuméfaction est quelquefois tres prononcée dans les myosites aiguës.

\section{X. - os.}

Modifications. - Les os sont susceptibles de subir de nombreuses modifications; ils ollrent très fréquemment Annales de méd. rét., 190?, p. 150. - Francesco Belli, Il nufvo Ercolane, 15 oclobre 190 .. 
des déformations dues à des incur'ations (rachitisme) ou à des régétations (Dériostoses); ils sont susceptibles de résorber lessels calcaires et de préparer ainsi les deformations, les fiactures comme daus l'ostémalacie; ils peuvent mème résorber les os morts.

Billroth et hölliker ont inséré dans un os virant une

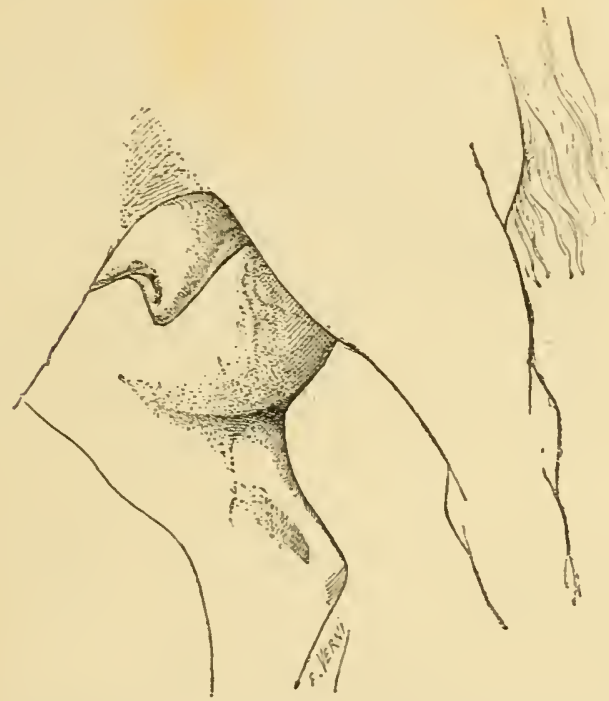

Fig. 93. - Hỵpertrophie compensatrice des muscles du plat de la cuisse chez le cheval affecté de paral! sie et d'atrophie du Iriceps crural (Cadéac).

cherille d'iroire et en ont constaté la résorption partielle. Lannelongue et Vignal, implantant des clous d'ivoire ou d'os mort dans le tibia des lapins, ont ru l'ivoire rester presque inaltéré, tandis que l'os morl, au bout de soixantetrois jours, était en voie de désag̣régation el envahi par une néoformation osseuse tendant à se substituer à lui. Barth, après trépanation du cràne chez le chien et le lapin, 
remplace la rondelle enlevée par une rowlelle d'os mort; il olsserve la résurption de l'os nort et son remplacement par un os nouveau suivant un processus identique a celui qu'on observe dans la rísorption d'une rondelle vivante.

Gornil et Gundray on constaté, au contraire, dans six expériences faites chez les chicus, que l'os mort demeure à peu près tel qu'il a èté implantr; il s'entouse seulement d'une capsule fihreuse; il se comporte comme un corps ptranı̆er et mème au bont de six mois sa résorptıon est insignifiante.

Si la pathogénie du rachitisme n'a pas fait de grands progrès, celle de l'ostromalncie vient d'entrer dnus une voie nouvelle grâce aux recherches de Charrin et Moussu, qui onl démontré la contagiosilé et linoculabilité de cette maladie.

Les os d'un liapin inoculé avec une émulsion de moelle osseuse d'un por'c ostéomalacique deviennent mous et dépressibles, surtout ‘eux de la tèle; on imprime aisément an museau, ainsi qu'aux maxillaires, les torsions les plus prononcées.

Si on analyse l’affection décrite sous la dénomination de cachesie osseuse, qui atteint le porc, la chèrre, le lapin, etc., on voit que celte allection, au point de vue anatomo-pathologique, symptomatique et étiologịue secondaire, est identique à l'ostéomalacie counue en pathologie humaine et considérée comme le type des processus purement humuraux. Or, par des injections sonscutanées d'émulion cle moelte usseuse, ils ont reproduit l'alfection chez le porc, la chèvre, le lapin, etc. De plus, en tai-ant rivre des sujets sains a côté de por'es contaminés, ils ont parfois réussi à propager le mal; les aliments, la voie digrotive, dans ces conlitions, paraissent entrer en jeu.

Dans la phase chronique, ils n'ont pas découvert de microbes; mais, au cours de la phase aiguë, ils ont aperçu dans la moelle osseuse ou la synovie articulaire, des 
microcoques et un bacille que des cultnres ont reproduit. Inoculées, crs cullures, clıez deux petils lapins, ont jruvoqué de la phosphaturip, une augmentation des acides, mais famaıs l'alfection typique. Toutelois, quand les milieux ensemencés demeurent stériles, les inoculations de moelle osseuse sont sans effet.

Culte réluction de la période d'activité du virus, à certaiues époques, la rareté du mal capable à d’autres moments d'exercer d'importants ravagrs, la lenleur de l'érolıtion (deux à sept nois), et quelyues autres condilions renilent ces recherches laborieuses. D’ailleurs, si on met à part les fausses ostíomalacies, au point de vue de l'anatomie pathologique, des symptòmes, etc., cette affectiou pent ètre l'wuvre de différents agreuts, pourvu que ceux-ci soient aptes à engendrer en excès des acides capables, en solubilisant des phosphates, de déminéraliser le squelelte. Or, c'est le cas de divers microhes, de cortaims champiguons (Oospora Guignardi), c'est également celui de nos cellules surmenérs ou iusuftisamment actives au cours des mutations nutritives. Toutefois, on conçit que, suivant lagent en cause (lıactérı ou cellıle), la maladie est ou n'est pas contagieuse, facilement inoculable (1). Sur les os se développent un grand nombre d'exostoses.

\section{M. - Exostoses.}

Les exostoses ont fait l'objet d'un grand nombre de travaux et elles ont une telle inıportance, tn médecine vétérinaire, qu'il est nécessaire de résumer ici succinctement les données pathogréniques acquises.

Pathogénie des exostoses. - La palhogénie les exostoscs indépendanles d'un tranmatisme, ou d'une in'ection microbienne (streptocoques de la gourme) ou mycosique, est actuellement fortement controversée.

(1) Charrin et Moussu, Académie des sciences, 190 :

$$
\text { Cauéac. - Séuiologie, } 2 \text { édit. } 11 \text {. }-20
$$


Nous croyons qu'on peut les rattacher à deux origines: $1^{\circ}$ à une hyperextension ligamenteuse; 20 à une altération osseuse ostéitique.

A. Théorie de l'hyperextension ligamenteuse. - C'est un principe que, l'hyperextension d'un ligament, d'une aponévrose on d'un tendon des rayous inférieurs des membres est susceptible de délerminer par liraillement ou arrachement une exostose au niveau de leur point d'insertion. Il existe autant de variélés d'exostoses que de points d'insertion.

Ces tares osseuses sont localisées dans les rayons inférieurs à partir du genou et du jarret; elles sont presque inconnues dans les régions supérieures. C'est que l'amortissement des efforts locomoteurs est beaucoup moins parfait pour les parties basses des membres, dont les jointures deviennent d'autant plus rigides qu'on descend davantage. Les glissades, les torsions, les percussions répétées se font particulièrement sentir vers les parties inférieures (1).

Un grand nombre de suros se produisent par ce mécanisme.

Le suros intermétacarpien profono lésulte d'un tiraillement excessif du ligament suspenseur du boulet, comme Barrier en a fonrni la démonstration.

" Le jeu phalangien aux diverses phases de l'appui consiste dius une modification des rapports angulaires que le paturon entretient, l'une part avec le sabor, d'autre part avec le canon. An moment du poser, canon, paturon et sabot sont en ligne droile, dans le prolongement les uns des autres. Iès que l'appui commence, la rectitude le ces régious disparait; deux angles se forment simultanément: l'un au niveau du sabot, l'autre au niveau du

(1) Hrouin, Etiologie et pathogénie des tares osseuses (Revue gënérale de méd. vit., 15 avril 1903). 
bonlet. C'est aı nilieu de l'appui que ces deux angles semblent atteindre le minimum de lermeture.

L'un d'eux, linférieur, a pour conséquence immédiate d'opérer le relâchement du perforant el la chule du boulet, et aussi de faire supporter au ligament suspenseur la plus grande somme des traclions causées par cetle chute. Si l'abaissement est excessif par rapport à la résisłance du ligament, cet organe se rupture (1).

Les scros post-MÉTACARPIExs sont ploduits par la dilacération ou l'arrachement de l'arcale aponérrotique carpienne qui se relàche quand le pied est levé et se tend vigzoureusement à l’appui (Joly).

Les SUROS INTERMÉTACARPIExs résultent du tiraillement du ligament interossenx par la descente de l'os rudimentaire; nais, selon Joly, cette descenle aurait plutôt ponr effet de relàcher les fihres du ligament qui sont obliques de haut en bas et d'arrière en avant, de sorte que ces exostoses auraient la même origine que les postmétacarpiennes; l'hyperextension des fibres inférieures de l'arcade carpienne exerce son effort de bas en haut el d’avanı en arrière. Ces petites périosloses restent localisées à la scissure inlernétacarpienne et s'élendent un peu sur la face poslérieure du métacarpien principal le long du bord du péroné.

Elles ne représentent, hien sourent, que la soudura hàtive et un peı amplifíe du mélacarpien rudimentaire. Cette soudure quasi-physiologique explique peut-ètre la grande fréquence de ces périostoses eı mème temps que leur bénignilé, car elles rétrocèdent presque toujours avec le temps (2).

Ces périostoses se produisent surtout du côté interne en raison de l’inégalité des pressions que supportent les deux métacarpiens rudimentaires. En effet, " a॥ còté externe

(1) Barrier, Nerf-ferure (Bulletin de la Societte centrale, 1891).

(2) Berton, Étiologie et pathogénie tes tares osseuses du cheral. Toulouse, $190 \pm$. 
l'os crochu repose à la fois sur le métacarpien principal et sur le rudimentaire; son corresponlant interne, le trapéze, s'appuie exclusivement sur le métacalpien rullimentaire. Il en résulte que, de ce côté de l'articulation, les pressions sont transmises intégralement au métacarpien rudimentaire, d'où tiraillements du ligament qui l'unit a l'os pruncipal, particulièrement clıez les jeunes, a cause du peu de solidité de l'appareil ligamenteux". (Klingber.t.)

l.es suros carpuens qui siègent tout aulonr du genou, c'està-dire près des arliculations iutercarpienues el carpomélararpieunes, résultent plutòl de l'ostéo-arthrite du genou que d'uu tiraillement ligamenteux.

Les suros sus-carblins résultent les tratrtions excessives exercées par les tendons des fléchisscurs oblique et externe du melacurpe au nivean de leurs points dialticlse; ils peuvent garner les articulations du carpe; mais ortinairement, ils remontent le long des tendons, envahissent la partie supérieure de la gaine carpienne, s'étalent en plafues et immobilisent en graude partie la région.

Le suros du higament lateral du gexou on de la tète d" métacarpirn rudimentaire est du anx eflorts du ligament latéral commun du carpe au niveau de son insertion infériemre.

Les suros métatarsiens sont très rares, ils paraissent résulter ainsidusurmplage des gaines le contention; mais ils sont intiniment plus rares quaux membres antérieurs.

Le suros hu Ligamext lateral du boulit ou suros inférieur, suros duboulet, est dù aux efforts ilu ligament latéral superficiel de l'articulation du houlet an niveau de sou insertion supérieure.

Les suros d'origine traumatique intéressent particulièrement le métacarpien principal; ils ont une torme plus ronde et ils sont le plus souvent ébumís; tanilis que les suros spontanés, apparaissant sur le métacarpien rullimen- 
taire, ont la forme d'ostcophytes et sout accompagnés de lésions d'ostëite raréfante.

Les Foryes ont une origine analowne selon les partisans de l'hyperextension, éest-a-dire de ceux qui prétendent comme Barier, Berlon, etr., que daıs les exostoses le processus osseux évolue concentriquement, de la périphérie des os vers les parties centialus.

Les exostoses phalangiennes ou formes résultent d'un travail outré; elles se développent au niveau des champs d'insertion desbrides d'attache de divers appareils fibreux du boulet et des phalanges.

On les rencontre pll effet vers la moitié inférieure des bords latéraux de la première el de la deuxième phalange ou au sommet de l'éminence pyramidale de la troisième. Pour légilumer encore davanlage celle origine externe, Berton soutient quel'unflammation est lucalisée a la couche plus superficielle des os, ou periostique sans relentissement sur les couches prolondes du tissu ossenx. En faveur de celte opinion, on invaque enfin l’état des brides d'inscrtion, qui, an niveau des formes, sont généralement épaissies, indurées, frappées d'inflammation chronique.

La courbe, la jarle et le jarlon n'existant pas comne tumeurs osseuses, la yuestion de leur origine est tranchée d'emblée.

L'ÉP.ırvix est d'origrine périphériøue, c'est-à-dire procède d'une hyperextension ligamenteuse, telle est l'opinion soutenue par Barrier. Le phénomène initial consiste dans un tiraillement des courts ligaments imlerosseux, d'oì résulte une ostéile périplérique, qui moditie la nutrilion du cartilage et lait apparâtre les lésious ałthritiques. Ces altérations débutent par la partie inféro-interne du jarret, parce que cest la rangrée sous-astragalienne du tarse (scaphoüde et grand cuneiforme) qui a le plus à soull'rir pendant les eflorts de la locemotion. 
B. Théorie de l'ostéite primitive. - Les parlisans de la théorie osseuse soutiennent au contraire que toutes les exosloses (suros, formes) et les éparvins eux-mèmes ont une origine osseuse. Toules ces altérations procèdent

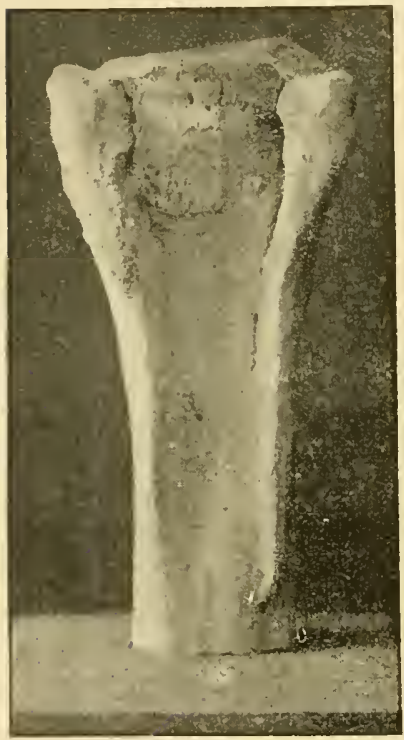

Fig. 9i. - Osléo-arlhrite carp̧o-métacarpienne. - Ostéles de la marge articulaire de l'insertion du suspenseur el des métacarpiens latéraux (Lefebrre et Thary). d'une ostéite deformante susceptible de frapper les diverses parties dı squelelte, particulièrement celles qui jouent un rôle prépondérant dans la locomotion comme les membres, ou qui doivent supporter le poids du cavalier comme la région dorso-lombaire (fig. 9't à 98).

Celte "ostéite raréliante lébute profoudémen t dans les systèmes de Havers el transforme progressivement les couches osseuses sous-périostiques, le périoste lıi-même et les attaches ligamenleuses qu'elle ossifie (ostéite moliférante), ou bien, dirigeant sa marche vers les cartilages articulaires, elle les mine par la base, les détruil et produit l'ankylose articulaire (ostéite ankylosante), ou bien l'osteite condensante." (Joly.)

l.es suros intermétacarpiens ou post-mitacarpiens, les eparvins avec leurs diverses formes d'arthriles sèches, cerLaires formes coronaires qui jaillissent en quelque sorte des arliculations interplatangiennes, les osteo-arthrites 
déformantes du genou étudiées par Lefebvre et Thary en sont autant de manifestations.

Ces lésions osseuses se retrouvent dans la fourbure, dans l'encastclure el dans diverses formes de cet eusemble

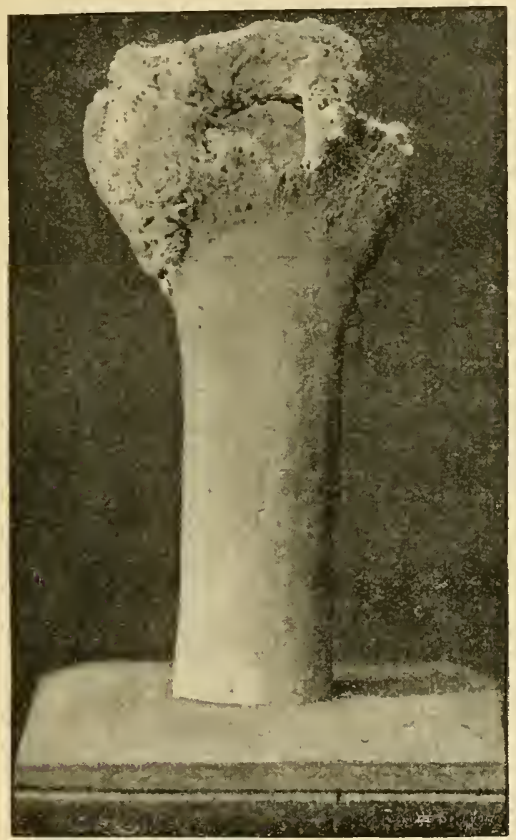

Fig. 93̈. - Ostéo-arthrite déformante ou ankylosante carpo-métacarpienne. Face antírieure. - Soudure des os de la rangée inférieure du catrpe entıe eux el avec le métacarpe et les métacarpiens latéraux (Lefebvre et Thary)

d'altérations, auquel on donne le nom de maludie naviculaire. C'est l'ostèile raréfiante ancienne qui explique les fractures qui se produisent chez les animaux couchés sur le lit de paille arec toutes les prícautions, comme les fractures consécutives à des contractions musculaires 
violentes, les fractures des phalanges qui surviennent aprés une injection de cocaïn ligaments et des tentons, les difformités de la tète (1).

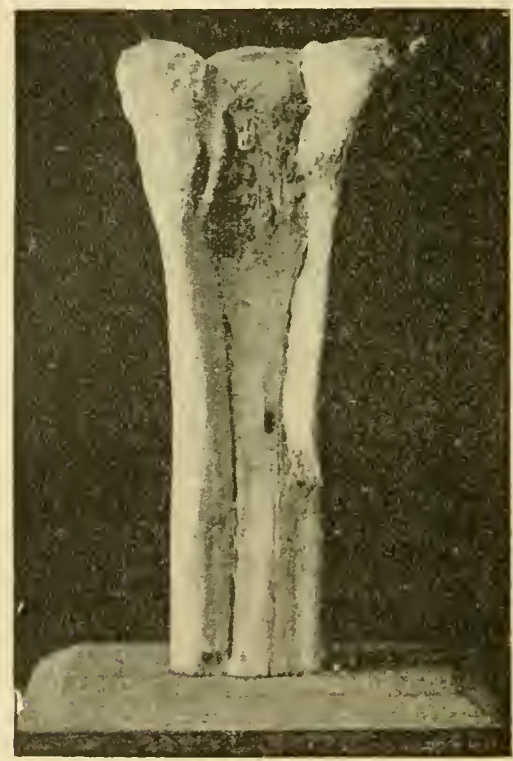

Fig. 96. - Ostéo-arthrite tarso-métalarsienne. - Face po-tícieure du métatarse dont la surface arlicularre est ulcérée. - Ostéophyles le l'altache du suspenseur et des métatarsiens latéruux (Lefebrre et Thary).

Les recherches histologiques de Vivien ont mis en éridence la fréquence de ces alıéralions ossenses et leur dissémination sur les diverses parties du sıuelette.

Lin Vaveur de l'ostéitisme, on peut invoquer un certain nombre d'arguments importants qui sout tirés du rôle de

(1) Voy. Maladie du son (Pathologic chirurg cale, t. I, p. 303, in Encyclopédic vétérinaire). 
la prédisposition indivirluelle, de la bilatéralilé ou de la

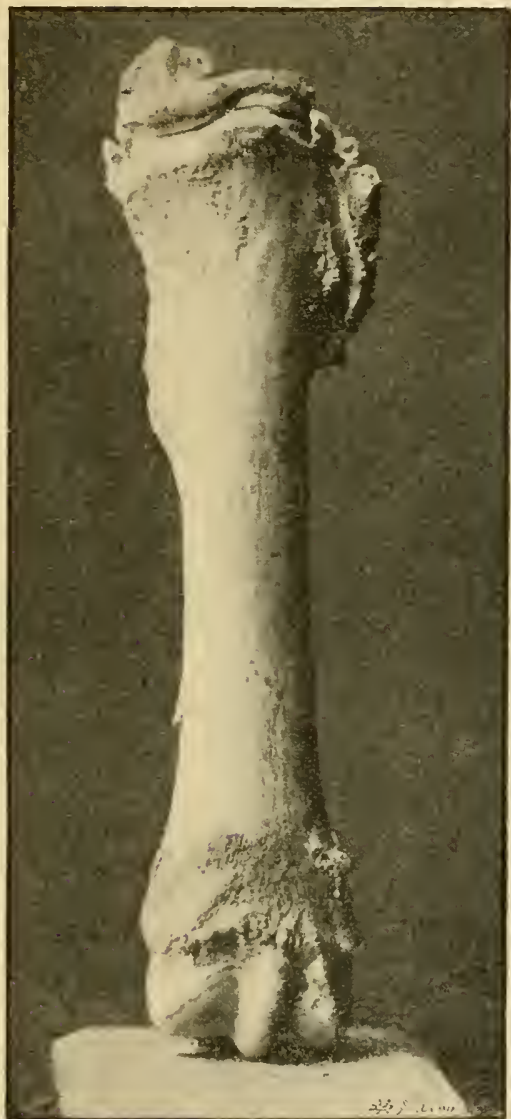

Fig. 97. - Osted-anthrte disormante ou ankylonante tars ,-métalarsienne et lésions ostéot ques, de mème nature, des mé:atarsieıs latéraux et de l'extrémité infèrieure du corps de los. - Face antérieure (Lefebvre et Tharỳ).

dissémination des lésions osseuses, de l'influence exercée 


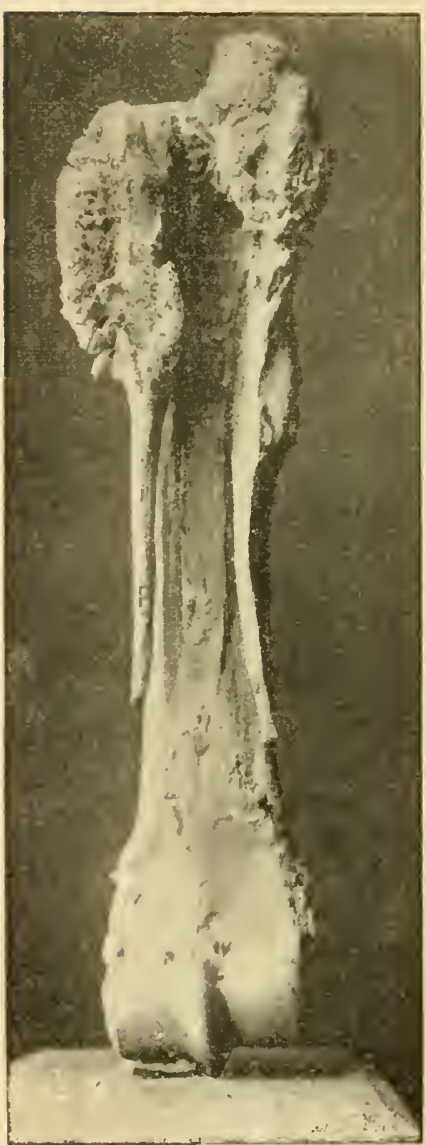

Fig. 98. - Ustio-arthrile déformaute, conforme à la précédente. Face postérieure (Lefebrre et Thary). par la fatigue du syslème osseux, par l'alimentation.

10 Prédisposition individuelle. - Les exosloses font à peu près défaut chez l'anc, sauf l'iparvin; elles sont rares chez le mulet, frèquentes chez les chevallx. Dans cette espèce, il existe encore de profondes différences qui tiennent à la race, et surtout à la nature des aliments.

Dans toutes les races, ce sont les jemnes animaux qui sont le plus prédisposés aux exosloses, parce que les insertions ligamenteuses et les os eux-mèmes manquent de solidité; mais en dehors de cette influence, il existe " une réceptivité et une inmunilé individuelles indiscutables. Des chevaux d'apparence grèle peuvent fournir pendant des anuées une tàche effrayante, sans que la plus petite tare ossense 
en apparence, quine résistent pasaux premières éprouves ; leurs membres se couvrent de tares osseuses. On peut retrouver toute la gamme entre ces types extrèmes II semble logique de rechercher la cause de ces varialions dans une différence de structure de la trame osseuse, entraìnant sans aucun doute le défaut d'adhérence des insertions " (Drouiu).

Joly a constaté, en effet, que le phénomene d'ossification des canons chez les cheraux de sang commence souvent avant tout travail, d'où il conclul que l'osteite te fatigue est un phénomène non diathésique, non spécilique, mais au contraire, normal, physiologrque. Les manilestations ostéiliques sont "I'aboulissant pathologifue l'une évolution mille fois séculaire " vers la solipédisation.

D'autre part, l'ostéile de soufure intermétucarvienne se prolonge, dit-il, sous le carpe el le tarse pour donuer naissance aux soudures intereurpiennes el larsımmes, d'où i) résulle que la pallıologie des tares osseuses ne prut. se scinder de la solipédisation. Et comme celle-ci est fonction de l'hérédi té, celle-là aussi ne le serait pas moins. L'hérédité fatale des tares osseuses est ainsi une résultante inévilable de ce processus paléontologique quj lend à spécialiser le cheval comme animal de vitesse. La pathogénie des suros intermétacarpiens et la soudure ṕrolutive interuétacarpienne ne peuvent ètre sépaıées Joly) (1).

Au point de vue de la patholosie comparée, il semble qu'on peut rapprocher les osteites de fatigue de l'homme, de l'ostéite de fatigue du cheval et qu'ıl soit pussihle d'établir une analogrie entre les fractures du métalarse des lantassins et les fractures énigmatiques dı paturon par ostéite raréfiante; entre les périostiles non traumaliques de la jambe de l'homme ou encore les exostoses de croissance (exostoses osteogriniques) et ce que l'on observe de similaire chez le cheral.

(1) Chomel, Réperloire de police sanitaire, 13 mai 1903. 
20 Bilatéralitê et dissémination des lésions osseuses. La multiplicite des tares ossenses cljez certains animaux n’a pas manqué de frapper les oliservateurs. On constate toujours l'existenre simultanée des suros intcrmélacarpiens et post-mitacarpicns: les formes se manilestent qéuéralement aux deux membres et offrentunedisposition parfaitement symetrique.

Daus les forues curonares, la subslance osseuse est le plus sonvent la première alteinte, comme en lémoignent les lecherihes anatomo-pathologiques d'Udriski. C'est une osteite centrale primitive, débutant dans la partie compacte de l'os, caractérisfe par la raréfartion du tissu osseux avec lacunes de Howship, suivie d'une phase d'ostéite condensante, d'ostéosilérose réparatrice; mais on peut constater aussi dles formes coronaires par ostéite périphérique ligamenteuse.

Antérieurement, ell 1897, Joly rattachait les formes coronaires à une ostér-arthrite à tros slades: $1^{\circ}$ sans exostose et sans ankylose: $2^{\circ}$ avec exostose et sans ankylose; $3^{\circ}$ avec exostose el ankylose, la lésion primtive étant une altération źénérale de l'os, mais lébutant toujours au niveau des atlaches ligamenteuses el tendineuses.

Les Formes de LA DEUxième articulation iNTERPHaLANGIENNE peuvent avoir, selon Eherlein et Karbach. une triple origine $: 1^{0}$ la lusion débute par une ostéite raréliante primitive qui determme secondairement une arthrite, avec hyperostose périarticuliare;

20 La lésion débute par une ostéite de la couche non périostée, arec formation d'exosloses pririarticulaires et tardivement il y a arthrite chronique du pied.

$3^{0}$ L'altération commence dans le voismage de l'os, daus les tissus périphériques.

Les folmes cartuagineuses elles-mèmes résultent d'un accroissement latèal dela troisiène plıalange, le tissu osseux ayant une tendance naturelle à envahir le carti- 
lage complémentaire. Ce sont les causes susceptibles de

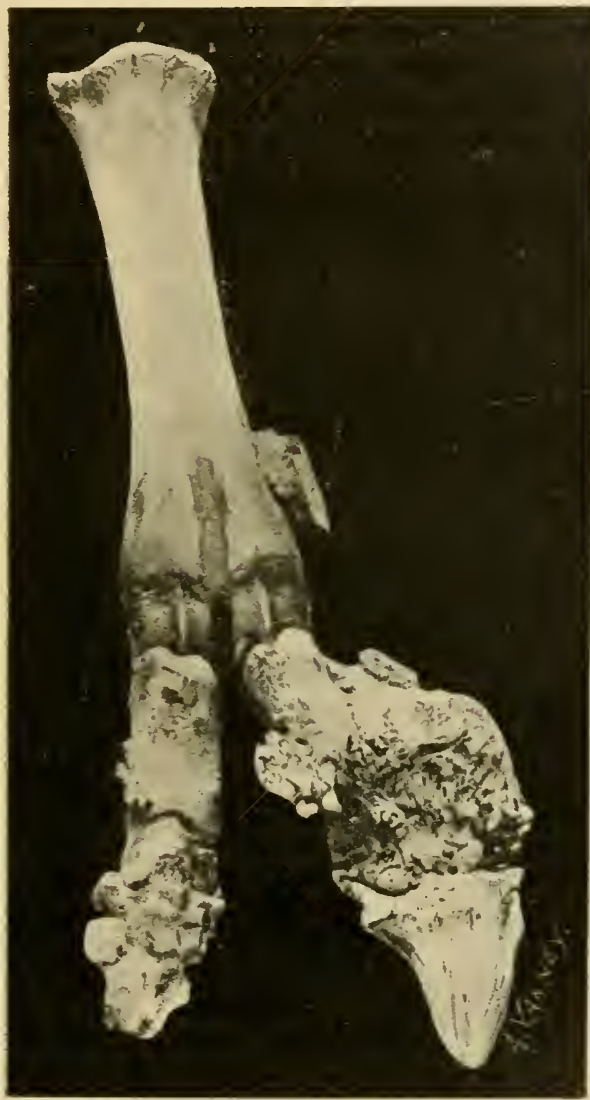

Fig. 99. - Furmes phalangıtues chez le beul' (Cadeat et Worot).

congestionner la phalange unguéale qui aclivent ce processus d'ossification. On peut constiter chez le bouf, CADÉ.Ac. - Sẻmiologie, ?e édit. 11. - 21 
comme chez le cheval, des formes phalangiennes déterminées par une ostéite qui envahit les divers os (fig. 99).

L'ÉParvin est presque constamment bilatéral. Son développement, exclusivement osseux selon Joly, comprend un processus complexe, qui correspond successivement:

"En première phase : à une arthrite sèche des articulations tarsiennes inférieures, d'où l'éparvin-arthrite;

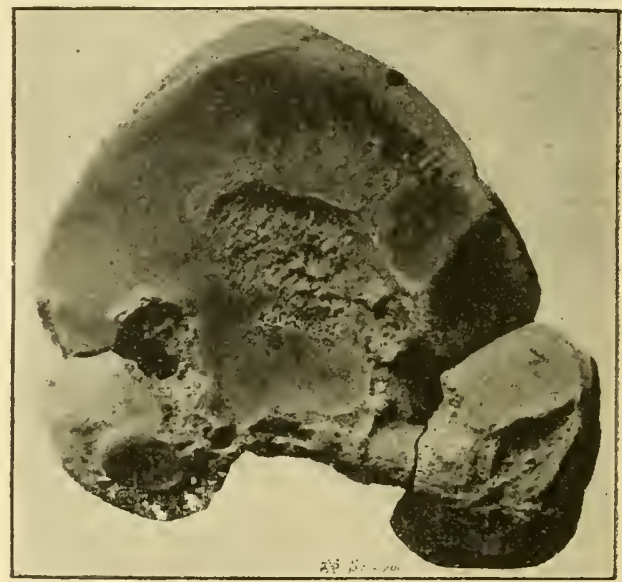

Fig. 100. - Ostéo-arllırile tarso-mélatarsienne. - Ulcéralion élendue de la lable articulaire. - Sondure du métatarsien latéral interne. - Ostéop̣orose et ostéphyles (Lefebrre et Thary).

En deuxième phase : à une ankylose des articulations entlammées; d'où l'éparvin-anlylose;

En troisième phase : à une exostose localisée, de par la constilution anatomique du jarret, au cóté interne de la base de l'arliculation, d'oì l'éparvin-exostose;

En quatrième phase : la maladie a débordé les articulations tarsiennes inférieures el envahi le pourtour des articulations tarso-métatarsiennes et tarsiennes supérieures, d'où l'sparvin cerclant." 


$$
\mathbb{I}
$$


Dans tous les cas, la lésion articulaire esı, pour Joly, le prélude dr loutes les autres. Celte opinion est partıgée par Eberlein, qui reconnaît fue l'alléralion primitive, c'est une osléite raréliante qui apparait au seiı de l'os, doù elle gagne l'articulation el en dernier lien la surface exlérienue lizampnteuse. Cette sorte d'ostioporose ne demenre pas localisér à la jointure atteinte; rlle se retrouvedans l'antre farret et sur d'autres poinıs du sque. lette dont elle prépire les déformations et les lractur's. - Il est clair que la mauvaise conformation des jarrets ou des autres régłons, les aplomis défertueux lavorisent singulièrement l'apparition de toutes ces tares osseuses.

$3^{\circ}$ Influence de la fatigue. - Les efforts locomoteurs, les causr, mécani,jues engendreut l'ostéite de fatiıue, qui est la malarlie profissimmelle du cheval. Cette osıéite de fatigue a individuellement acquise est accumılée par les ancètres. Lors du travail, la fatiærue individuelle hàte tonjours lasoudure iutermétacarpienne etprovoıue lesuros; le jeume àıedu sujet, sit vigneur el son énergie au Iravail ont une grande iulluence sur la précocité et l'irrégularité des phénomèues.

La débilité oryanique, les vices de nutrition et de conformation dı squelelle ne soul pas évilemment sans influence sur la résistance du tissu osseux à l'apparition d'une ostéite de fat rue morbile, provocatrice de suros pathologiques " (Joly).

L'ostérte de fatigue met le tis u osseux dans un tel état que le tirallement ligranenteux le plus discret levient le point de dépalt d'une exostose, et comme tout le tissu osseux offre cette altération, les exostoses tendent à se produire ou se prodıisent effectivement dans lous les points où ces tiraillements peuvent s'exercer.

Cetle fatigue morbile provocalrice setransmet à la descendance; l'éparvin n’est pas plus héréditaire que les 
autres tares osseuses; ce qui se transmetc'est cetle faiblesse native, cette vicieu-e nutrition du lissu osseux qui conduira la race ou mème l'espèce à une déchéance progressive. "Ln canon ou une articulation frappés par l'ostéite de ṫatigue héréditaire reste grêle et vicié daus ses aplombs par le faitmème de son état palhologique» (Joly).

Celle ostéite de fatigue joue un ròle prépondérant dans l'apparition des éparvins et dans toutes les altérátions de la troisième phalange, notamment dans la falblesse chronique et lans les boiteries dorigine ossense que préseuteut souvent les clipvaux de course (1).

Elle prend naissance dans la profondeur de la trame osseuse et envahit lout. "Rencontre-t-elle un lizament interosseux : elle le remanie, l'absorbe et provoque une symphyse. Est-ce une articulation qui se trouve sur son passàe : elle la détruit et la remplace par une ankylose. Les carlilages, elle lestrausformeen os et s'il n'y devant elle aucun tissu ayant des rapports intimes avec l'os et dans lequel elle puissepénélrer par replation, elle aboutit à une poussée nćoformative " (Vivien).

$4^{\circ}$ Influence de lalimentation. - L'alimentation, c'est un fait reconmu par tout le moule, joure un rôle considérable dans la formation et l'évolution du tissu osseux. Il y a ainsi des pays à sol pauvre en phosphates où tous les animaux out les os grèles; comme il y a des pays où le tissu osseux acquiert un grand déveloi'pement.

Partout où l'os ne prend pas dis consistance, il est éminemment prérlisposé aux exostoses. Drouin a justement attiré l'attention sur cette panrelé de l'alimentation : "Les inserlinns ligamenteuses et aponérrotiques ne peuvent contracter une adhérence suffisante dans ce tissu trop vasculaire qui garde trop Iongtemps les caractères de

(1) Cagny, Bulletin de la Socièté centrale, 1900, p. 132. 
l'ètat fœtal. Cespays à tares osseuses paraissent être surtout des régrions marécageuses, à terrains acides, dépourvus de phosphates terreux, dont les marais de Rochefort et de la Basse-Vendée nous fournissent le type.

D'ailleurs, certaines substances alimentaires acides peuvent déterminer une dénutrilion osseuse générale, laquelle se traduit bientòt dans une colleclivité, par la fréquence des fructures, des arrachements ligamenteux et des végétutions osscuses. C'est ainsi qu'on observe des types d'osteite métacarpienne générale, presque toujours symétriques sur le mème sujel et se traduisant clinịuement par de larges periostoses de toute la région dı canon. Ces périostites sont produites par des tractions aponévrotiques et ligamentenses; larticulation elle-même est presıue toujours indemne; mais l'os est atteiut dans toute son épaisseur; très fréquemment, l'évolution se termine parune fracture consécutive au traumatisme le plusléger. Lefebvrè et Thary ont décrit une ostén-arthrite du genou, qui, débutant par une pointe synoviale, détermine rapidement l'ankylose de la rangée inlérieure " (Drouin).

Des recherches diverses témoignent de l'influence exercée par l'alimentation sur le développement des exostoses. Marcone a consiaté de la phosphaturie chez les animaux affectés de cette ostéite ráéfinte. Reul(1) a fait ressortir l'utilité des phosphates dans l'élevage des animaux domestiques. Les animaux nourris d'aliments riches en phosphales oflrent des os plus développés et plus résistants que ceux qui sont nourris d'aliments acides. - Néanmoins, il faut reconnaitre que le rôle des aliments dans la production des exostoses est loin d'ètre élucidé : il y a encore bien des points obscurs et des faits contradictoires.

lle ces diverses considérations, on peut conclure que les solipèdes sont fréquemment affectés d'un état constitulionnel désigné sous le nom d'ostéisme ou d'ostéitisme qui 
les prédispose aux diverses tares osseuses qui se développent sous l’influence d'efforts locomriteurs qui déterminent des tiraillements ligamenteux. - D'ailleurs, ces derniers peuvent aussi faire développer des tares indépendamment de toute altération préalable, c'est-à-dire de toute ostéite de fatigue. Il n'en est pas moins vrai que l'ostéice de fatigue constitue un lien entre les diverses exostoses qui évoluent souvent sous l'influence de causes bénignes de l'hérédité ou de la faligue.

L'ostirite de fatigue est une maladie professionnelle du cheval a comme l'anthracose des mineurs, la chalicose des verriers, la furonculose des cavaliers, à cette dilférence près que "l'ostéite de fatiggue " n'est pas 'imitée à l'individu, ni à certains organes, mais tient aussi sous sa dépendance loules les maladies essentielles du cheval: encastehre, fourbure, maladic naviculaire, etc. (Chomel). Il fitut reconnaitre cependant que l'ostéite de fatigue ne fait pastoute la lésion, que celle-ci se déreloppe à la faveur d'autres causes et que lostéite de fatigue joue surtout le ròle d'une cause prédispusante importante, de mème que dans l'anthracose, la chalicose, etc., les corps étrangers inbalés ne font qu'assurer l'inoculation des germes de la tuberculose el que, sans bacilles de Koch, les corps étrangers ne produisent aucune lésion, mais simplement une pigmentalion.

\section{XII. - ARTICULATIONS.}

Anatomie. - Les articulations sont composées d'un cartilage recouvrant les épiphyses et d'une séreuse réunissaut les deux têtes osseuses.

Le c.artilage est formé de capsules primitives qui se poursuivent jusque dans la couche la plus profonde infiltrée de sels calcaires et unissant le cartilage hyulin au tissu ossenx. Toutes les cellules de la surface et de la couche moyemne renferment des granulations et des gouttelettes de graisse (fig. 102). 
Pour parvenir jusqu'au cartilage hyalin, le plasma des vaisseaux de l'os doil traverser les couches osseuses et la counhe de cartilage calcilié. Or cette dernière couche ne paraît pas perméable, de telle sorte que les matérianx

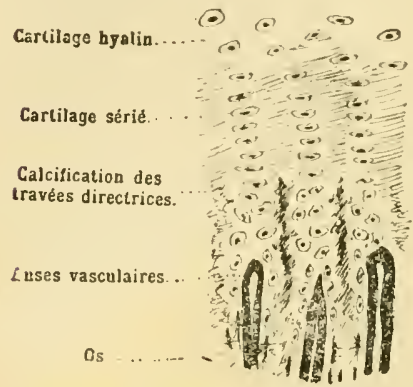

Fig. 102. - Cartilage de conjugaison. untrilils doivenl lui arriver par une autre voie. Ces sucs nutritifs virnuent probahlement. du liquide qui baigne les surfaces articulaires et qui est exhalé par les vaisseaux de la synoviale.

La synoviste présente des surlitces planes et des surfaces villeuses. Ces dernières sont connues sous le nom de framyes symoxiales.

Les franges synorinles sont constimees par doux feuillels adossés te la symoviale. Entre les deux fenillels, il y a un tis:u conjonctiflàche, des cellules adipeuses, disposées en îlo's et de nombieux vaisseaux.

Si les vaissenux sont congestionnés, on es। frappé du volume des artères et les veines, eu égard à la faible quantité de tissus qu'elles semblent alimenter.

De l'extrémité des tranges partent des corps, bien décrits par Kölliker. Ce sont tantòt des prolongements filiformes, constitués par un axe de tissu conjonctif et revètus par deux ou trois couches de cellules épithéliales.

Parfuis ces prolongements sont en forme de massue donl l'axe de tissu conjonctif peut contenil des capsules de cartulage. Ces diver's prolongements ne coutiemuent pas de vaisseaux.

Les lrames symoviales ont un ròle physiologique important. Les cellules qui recouvient les prolongements 
villeux ou en massue de la synoviale sont les véritables organes de la sécrétion de la synovie.

La synoviale ne recouvre pas la surface des cartilages diarthrodiaux là où ils glissent les uns sur les autres.

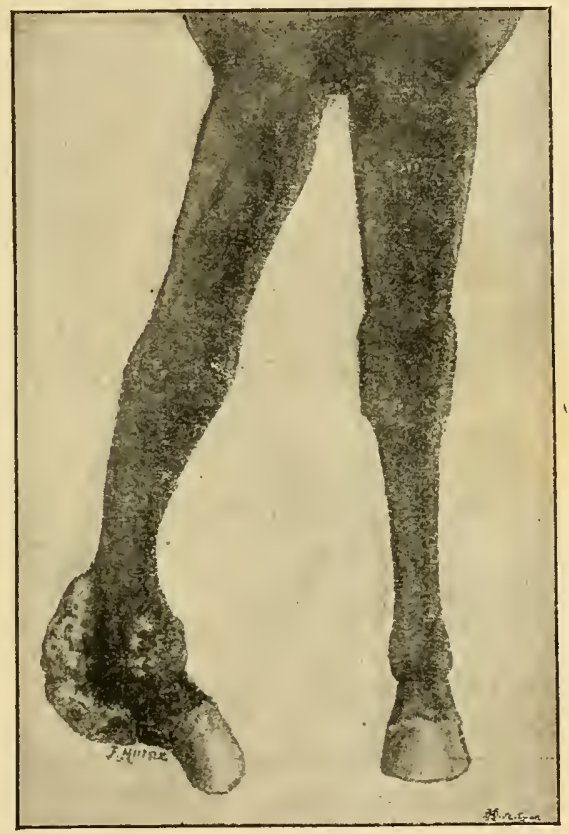

Fig. 103. - Ankylose de l'arliculation métacarpo-phalangienne chez un poulain.

La synovie contient de l'albumine, de la mucine en forle proportion, des traces de matières grasses, des cellules épithéliales et des leucocytes.

Exploration. - Lisspection permel de reconnaitre des augmentations de volume (arthriles, éparvins, vessi- 
gons, molctes, hydarthroses, entorses) ou des changements de direction: Iuxution, bouleture, arret de la rotule, chez le cheval et cliez le builf.

La MENSURATION comparative des articulations homologues fait apprécier l'intensité de ces changements; les variations de volume des liydarthroses, sous l'unfluence du travail ou d'un traitement approprié.

La I'ALPation révèle une sensibilité el une chaleur anormales daus l'arthrite, les entorses, les luxutions; elle met en évidence la fluctuation des molettes et des hydarthroscs, la dureté des tissus dans l'éparvin, l'ankylose, etc.

Modifications pathologiques. - Les articulations peuvent présenter :

10 Des plajes périarticulaires, consécutives à des cliutes, à des blessures superficielles, à des démangeaisons; elles sont sujelles à de nombreuses complications du côté des ligamenls, des tendons, des os et des synoviales el aboutissent quelquefois à l'aukylose (tig. 103).

$2^{\circ}$ Des PLAIES PÉNÉrRaNTEs, dont les conséquences sont variables, suivant quelles sont aseptiques ou septiques ou plus ou moins infectées et suivant que la synoviale est cloisonuée, divisée en plusieurs loges ou quelle est exposée à une infection grinéralisée.

$3^{\circ}$ Des ARthrites closes à forme séreuse, pseudo-membraneuse, mais rarement purulente (fig.10\%), et des arturites TR.Aumatiques, caractérisées par la pénétration de corps étrangers, de germes iufectieux qui déterminent une suppuration abondante ef une inflammation très grave.

$4^{\circ}$ Des hydartunoses, c'est-à-dire des lydropisies des symoviales artıculaires résultant de contusions, d'entorses, de luxations, de plaies periurticulaires et d'artluriles, de lil gourme, de divers états infectreux et de la grestalion.

Elles se léveloppent lentement sans provoquer des troubles bien appréciables ou seulement une boiterie 
déterminée par l'obstacle apporté par la synovie aux mouvements de l'articulalion.

Les hydarthloses prononcées s'accompagnent d'écartement des surfaces articulaires et quelquefois de luxation et de hernie des culs-de-sac symoviaux.

$5^{\circ}$ Des corps ÉTRANGERs, venus du dehors (projectiles,

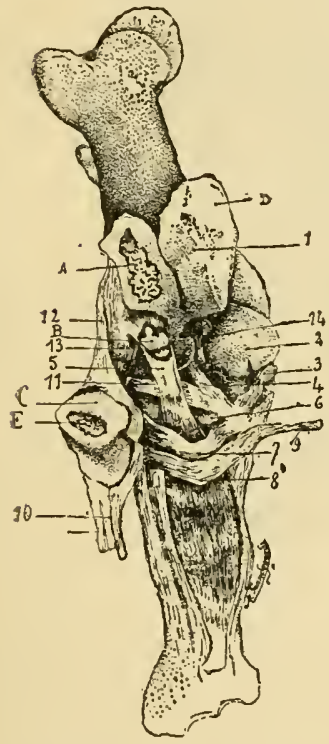

Fig. 10\%. - Arthrite chrouique du grasset (Cadéac).

1, lèvre inlerne de la trochlée fémorale;

2, condyle interue dı fémur;

3, ménisque interarticulaire interne :

4, ligament interosseux croisé antérieur ;

5 , tendon d'origine de la corde du flíchissenr du mélalarse;

¿, tubérosité antérieure du tibia ;

7 , ligament rotulien externe;

8, - - médian;

9, - - interne:

10, attache rotulienne du ligament rolulien interne;

11, ménisque interarticulairc exlerue :

12, bride fibreuse renforcant le ligament capsulaire fémoro-rotulitn ;

13, tendon du poplité;

14, ligament interossenx croisé interne;

A, ulcération de la lève externe de la trochlice;

B, proluction cartilagincuse siégeanl sur J'origine de la corde du llè-

C, rotule : chisseur du neétatarse;

'E, ulcérations.

échardes) ou développés dans la synoviale ou dans les tissus parasynoviaux.

Ce sont des grains riziformes de nalure fibreuse, graisseuse, cartilagineuse, ustéo-carlilagineuse ou osseuse résultant de l'exsudat inflammatoirt déposé à la sturface de la synoviale; ce sont aussi quelquefois des arthrophytes d'origine traumatique, c'est-á-dire des parcelles de tissu osseux arrachées des épiphyses, par des lıeurts, 
des coutusions violentes. Tous ces corps étrangers peuvent séjourner impunément dans les articulations ou provoquer des boiteries intenses en s'engageant sous un ligament ou un tendon; les débris d'os peuvent enfin se fixer

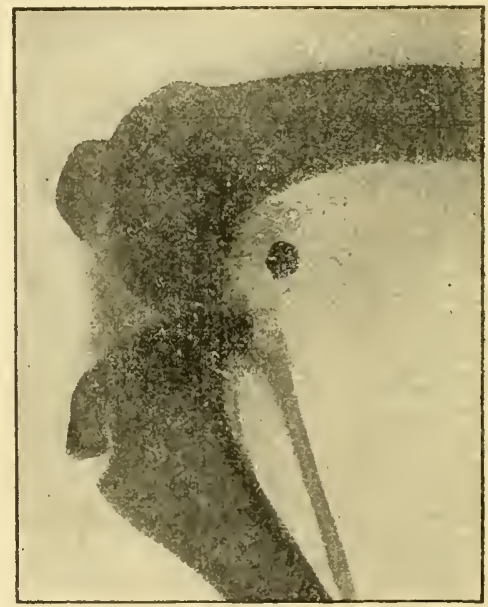

Fig. 103. - Balle de revolver, fisée chez un chien, en arric̀re des condyles fémoraux (radiographie).

et se souder aux tissus avoisinants et gèner les mouvements articulaires (fig. 10:3).

$6^{\circ}$ I) ES ARTHRopathies consícutives à diverses affections de la molle épinirre (myclites, contusions, trummatismes, ou nivrites tranmatiques.

\section{XII. - ARTHRITES.}

Définition. - Caractères. - On désigne sous le nom génériyue d'arthrites les diverses formes d'inflammation des articulations dont les caractères anatomo-patholo- 
giques sont la reproduction des phénomènes qui accompagnen I linflammation des séreuses.

La syorale s'injecte, les capillaires se dilatent, une exsudation se produit; elle trouble la synovie et distend la synoviale, sauf les cas où l'articulation est ouverte. Alors, la plaie laisse écouler de la synovie louche ou purulente.

Le cartilage subit de profondes modifications; il y a hypernutrition et prolifération des cellules cartilagineuses. Sur des coupes perpendiculaires à la surface, les capsules lenticulaires, qui renferment à l'étal normal un élément cellulaire peu distiuct, se gronflent, les noyaux augrmentent de volume et deviennent vésiculeux, un nucléole très net apparait ; les cellules aplaties premuent une forme globuleuse. A un moment donné, une capsule peut contenir plusieurs cellules; mais bientòt chaıue cellule s'eutoure d'une capsule secondaire. Cette multiplication affecte la forme d'ilots.

Quand les couches profondes sont atleintes, les lésions sont visibles sous le microscope : elles consistent dans des soulevements du cartilage, qui est mou en ces points, ou dans des ulcérations provenant de la fonte rapide de la substance cartilagineuse. Dans les points où le cartilage est tuméfié, on observe l'envahissement des couches profondes et mème de la couche calcifiée par une néoformation aclive. Le phénomène profond est le même que

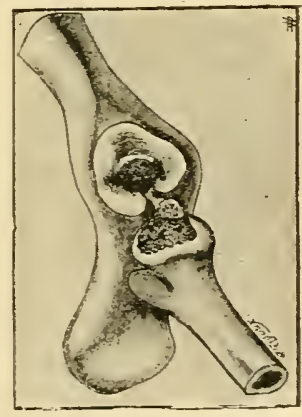

Fig. 106. - Décorlications de surfaces articulaires. Nécrove du ligament inlerarti-ulaire dans l'arthrite pyobémique du veau (Cadéac et Morot). celui de la surface (fig. 106).

La prolifération cellulaire s'accompagne toujours d'une segmentation de la substance fondamentale, et cette seg- 
mentation est suivie de la lormation de stries parallèles augrand axe descapsules primitives, et quand le proressus est très avancé, le cartilage présente des fentes comme si on l'avait incisé.

Quand il y a ulcération, la subsłance fondamentale se ramollit et subit une sorte de liquéfaclion. Les lésions du cartilage commencant par la surfice, il faut admettre que c'est le liquide de l'articulation qui joue le principal rôle dans la production de ces altérations.

Les arthrites sont exsudatives on purulentes, aiguës on chroniques. Les cellules cartilagineuses participent à la formation dı pus daus les atticulations.

Les inflammations articulaires s'accompagnent d'umyotrophies et de lésions des vaisseaux et des nerfs. Elles s'accusent par des phénomènes inllammatoires Irès accusés : chaleur, douleur, tum faction prononcées; les animaux présentent souvent des lancinatious très intenses.

Pathogénie. - L'arthrile est l'expression de tons les traumatismes qui intéressent la synoviale articulaire (arthrites traumatiques) ou les tissus avoisinants (urthrites closes). Dans les deux cas, il y a infection de la synoviale; toute arthrite traumatique a une origine microbienne.

Les arthrites secondaires relèvent de la même cause. Dans loute articulation enflammée, il y a, ou il y a eu, à un moment donmi, des germes infectieux qui ont pu disparaître à la longue, ce qui fait croire à l'existence d'arthrites amicrobiemnes.

$1^{\circ}$ Les arthrites rhumatismales sont aussi des arthrites nicrobiennes. Meyer a, en dix-lıuit mois, étudié 25 cas de rhumatisme articulaire aigu; il a trouvé le streptocoque dans la plupart des cas et a pu reprodnire avec ce microbe des arthrites et des endocardites. Oppenheim et Lipmann ont obtenu 6 fois sur 9 des cultures dans le rhumatisme arti- 
culaire aigu. Poynton et Paine ont rencontré dans celte forme de rhumatisme un strepto-diplocoque arec lequel ils sont parvenus à reproduire, par injection intraveineuse, des polyarthrites chez le lapiu.

20 l.es synovites les plus simples dapparence rhumatismale et les arthrites et périarthrites suppurées très graves, qui compliquent la gourme catarrhale, ont également une origine streptococcique.

Dans la gourme septicémique, les séreuses articulaires contiennent une sérosité sanguinolente, liquide ou coagulée; les synoviales tendineuses sont recouvertes de bourgeons charnus qui soudent les feuillets et accolent les tendons entre eux et avec les parois de la gaine.

Dans le cours de la morve aiguë, on rencontre également des arthrites et des synovites d'apparence rhumatismale ; ou peut mème constater, chez quelques malades, l'apparition de claudications subiles, continues ou intermittentes, en l'absence de loute lésion appréciable.

D’une manière générale, toutes les infections atténuées ont une grande tendance à se localiser dans les articulations. On peut produire expérimentalement des arthrites à pneumocoques avec des microbes très atténués.

L.es arthrites à pneumocoques du lapin sont généralement monoarliculaires el frappent les grandes articulations, les os et les tissus périarticulaires. A l'aide du streptocogue puerpuéral atténué, Dor a fait développer des ostéo-arthrites chroniques chez le lapin. Les nisrobes atténués, susceptibles de produire encore des lésions, ont des localisations différentes de ces mêmes microbes virulents. Le staphylocoque, introduil dans le système veineux du lapin adulle, et atténué à un degré suffisant pour qu'il soit incapable de produire des lésions rénales et musculaires, tue, à longrue échéance, en déterminant des arthrites suppuries mulliples.

Les arthrites infectieuses à staphylocoques et à strepto- 
coyues sont communes; il en est d'autres qui se rattachent. à des microbes spécifíques.

Les arthrites infectieuses peuvent être en effet l'expression de la preumonie, de la fievre typhoüde, de la tuberculose.

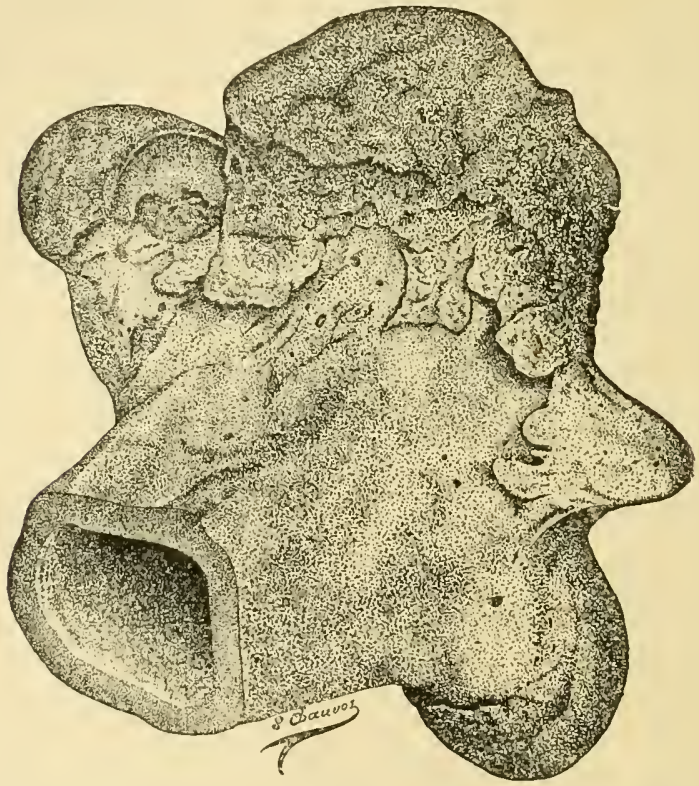

Fig. 107. - Arthrite fémoro-tiliale des ruminants. - Végétalions et déformations de l'extrémité inférieure du fémur. (P'ièce recueillie par Repiquet.)

chez tous les animaux, et particulièrement chez les ruminants et chez les gallinacés; 230 p. 100 des perroquets tuberculeux sont arthritiques (Eberlein).

Le choléra des poules à forme chronique se traduit fréłuemment par des manifestatious arliculaires.

Les arthrites post-partum ou arthrites des vaches laitières, appelées encore psendo-rhumatismales, sont également des arthrites infectieuses (fig. 107). 
L'arthrite pyohimique des jelınes animanx est fréquemment liée ì une phlébite du cordon ombilical (1) qui est également l'expression d'une infoction par celte voie (llorot) (fig. 108).

Ces diverses formes d'arthrites peuvent aboutir à l'usure des cartilages diarthroliaux, a la production d'osteophytes et à la délormation des surtaces articulaires. Celte affection, monoarticulaire ou polyarliculaire porte le nom d'arthrite sèche, ou d'arthrite deformante.

Quand les jointures ont perilu la totalité ou une partie de leurs mouvemenls nurmaux, on dit qu'il y a ankylose.

Les ankyluses sontvraies quand elles résultent de la fusion les surfaces articulaires; fausses, quand

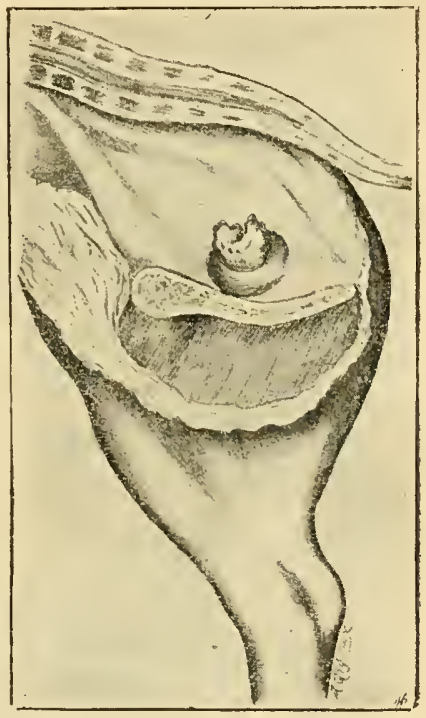

Fig. 108. - Arthrile pyohémique du veau. - Nérrose du ligament coxofémora (Cadéac et Morot). les mouvements sout eurpêchés par des altérations des tissus périarliculaires. Elles sont complètes quand tout mouvement est aboli; incompletes, quand il y a seulement dıminution des mouvements normanx.

\section{XIV. - TENDONS.}

Anatomie. - A. Membranes péritendineuses. - Chaque tendon possède une envelopve particuliere composée de

(1) Cadéac et Morol, Arthrite pyohémique du veau (Société des sciences vét., 1900, p. 23). 
deux couches principales entre lesquelles sont disposés les vaisseaux et les nerfs (1).

Cette enveloppe des tendons subit diverses modifications

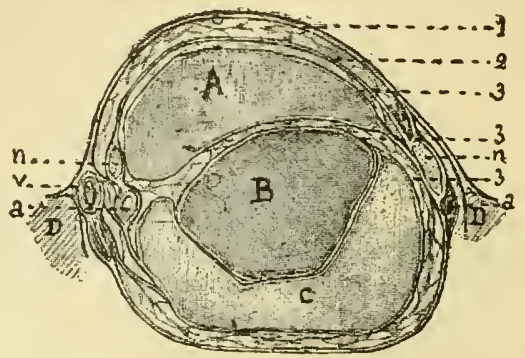

Fig. 109. - Membranes péritendineuses. - La conpe lígèrement schémalisće passe immediatement au-dessons de la gaine carpienne (Pader).

A, perforé; B, perforant; C, bride carpienne; DD, métacarpiens laléraux ; 1 , premic̀re membrane péritendıneuse ; 2 , deuxiene menbrane périlendineuse ; 33 , péritendineuse propre at chaque tendon; $a$ a, artères collatérales; $\vartheta n$, nerfs plantaires; $v$, veine collatérale ıuterne.

suivant les fonctions qu'elle doit remplir (fig. 109, 110,111); elle forme des culs-cle-sac, au niveau des replis synoviaux;

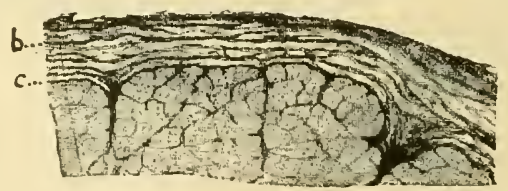

Fig. 110. - Péritendinense propre au perforant. (Les fenillels se sont lègèrement écarlés les uns des antres sous la pression de la lamelle de recouvrement.)

elle se dissocie au niveau des poulies sésamoïdiennes et devient hyalo-cartilagineuse dans les parties où elle doit

(1) Pader, Considerations sur l'anntomie des tentons fléchisseurs du pied chez le cheval (Societi des sciences rit., 1304, 1. 204). 
subir de nombreux frottements, comme au niveau des poulies sésamoïdiennes; elle contribue enfin à l'accroissemenl de dimension des tendons dans loutes les lendinites. Tuus ces faits sont uettement mis en lumière et déreloppés par M. Pader à propos des maladies des tendons (1).

$B$. Corps des tendons. - Les tendons sonl constitués par des faisceaux tendineux séparés par des cloisnns conjonctives issues de la couche périlendineuse (fig. 112). "l.es cloisons principales fournissent par dichotomie toutes Ies cloi-

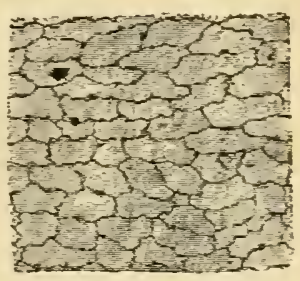

Fig. 111. - Endothẻlium limité à certaines régions des membranes péritendineuses (Pader). sons secondaires; elles servent de support aux troncs nerveux et aux vaisseaux qui se

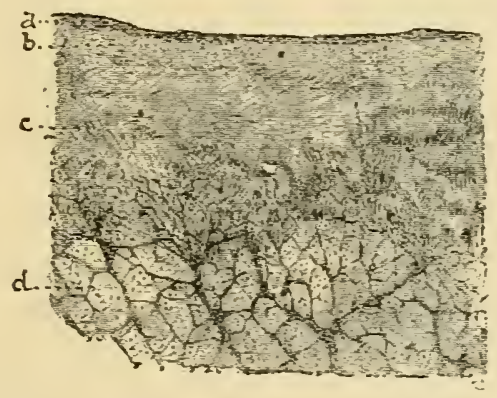

Fig. 112. - Coupe transrersale de la parlie périphérique du perforant ris-àris la rẻgion sésamoîdienne (Pader).

$a$, couche cartilagineuse hyaline; $b$, couche fibro-cartilagineuse $: c$, couche fibreuse diffuse ; $d$, faisceau tendineus.

Jivisent de plus en plus, de façon à assurer la nutrition des faisceaux du tendon.

(1) Pader, Pathologie chirurgicale, t. IV. 
Modifications pathologiques. - Les tendons sont susceptibles de présenter des luxalıons, des plaies, des distensions, des ruptures, des inflanmations, des raccourcissements suivis de bouleture.

a. Les luxations tendineuses intéressent le perforé el se produisent au niveau de la calotte calcuméenne ou to tendon du sous-épinłux à son passage sur la convexité dus Irochanter. Elles se traduisent par une sortede déclenchement du trndon accompagné d'une douleur, l'une gène extrème des mouvements, d'une tuméfaclion plus ou moins prononcée.

b. Les plates tenineuses sont simples on contuses, superficiell's ou profondes, complètes ou mcomplèles, aseptipues ou septiques; elles succèdent à des traumatisme divers, à les chutes, à les contusions, à des bleimes; elles sont sonvent compliquées de sinovites. Leur cicatrisation s'effectue plus on moins rapidement, et siaccompagne d'une déformation yni diminue souvent avec le temps (Voy. Plaies tendineuses).

Les plaies tendineuses s'observent presque exclısivement au niveau les tendons lléchisseurs ou des extenseurs des membres.

c. Les distensions texdineuses, connues sous le nom d'efforts de tendons, de nerf-férure, consistent dans la rupture par distension d'un certain nombre de faisceaux fibreux et dans l'eusemble des troubles inflammatoires qui accompagnent celte rupture.

Ces allections intéressent particulièrement les brides carpiennes et tarsiennes, le perforé, le perforant, el le suspenseur du boulet, qui est souvent envahi par des filaires. On peul constater les efforts du perforé au niveau de la régıon sous-carpienne ou de la région moyemne; ils sont communs chez les chevaux de course (tendons chauffis ou cluquess.

d. Les ruptures tendineuses ne sont accompagnées d'aucune plaie extérieure; ce sont des distensions exa- 
gérées plus qraves. Selon leur ordre de fréquence, on cite: la cord ${ }^{\circ}$ d" tléchissenr du mé atarse, lfs fléchisieurs du pied, le ligament suspenseur du boulet, le tendon d'Arhile, l'extenseur antririeur des phalanges. Le plis snuvent, il ne s'agit que de désinsertiun des tendons, ale telle sorle que ce n'est pas le lemion qui est l'urgane le plus altéré, mais le périoste et l’os sur lesıurls le tendon prend son iuserriun.

e. Les texnivites peuvent résulter de certaines infections localisees au nivean des syuovales, et les tendons (gourme, anasaryur, etc.) ; elles sont conséculives aussi à l'inflammation purulente des sinoviales temdineuses ou a tes invasions parasitaires des lendons par les filaires reliculees qu creusent des galeries an sein du lissu fibrenx

Ces inflanmations parasitaires atteigurnt rarticulièrement le susuenseur. du toulet (fig.113); le-inflammations trutumatiques du perloré se manifrstent au niveau de la rézion sous-carpirune ou ife la régiou moyenne.

$f$. La bouleture esı caraclérisée par la déviation en avint des rayons osseux qui construent larticulation du boulet. Ell est dite du premier deyré, quand le canon et le paturou sont sur la mème ligne; 'ln deuxième degré, quand le boulet, porté en avant, forme un anyl ouvert en arrière; du Iroisième degré, quand le bo't. let, fortement projelí en arant, dépasse la verticale élevie de la pince du sabol. La bouleture peut

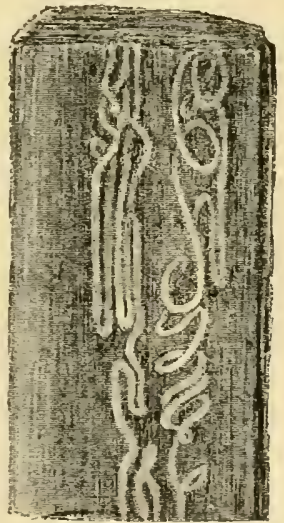

Fig. 113. - Filaire dins le ligament suspenseur du boulet (Pader). être l'expression, chez les poulains, de myosites, de symovites, de myélites, d'intlinmmation chronique du perforant, d'encastelure, etc. 
g. LARqure consiste dans la projection des genoux en avant de la ligne d'aplomb des membres. L'animal est dit brassicourt, quand ce défaut est congénital, et arqué, quand il est déterminé par l'usure. l'arqure résulte d'une létraction des lléchisseurs dı mélacarpe : l'externe, l'oblique et l'interne.

\section{XV. - PIED.}

Exploration. - Il fant toujours explorer le pied dans le cas de boiterie ou de position anormale des membres. Le pied malade reposant sur le sol, un aide lève le membre homologue du còlé opposé pendant que l'opérateur percute avec un brochoir ou un marteau ad hoc les ditférentes régions de la paroi. Existe-l-il une sensibilité anornale en un point donné ? l'animal manifeste la douleur qu'il éprouve par les mouvements qu'il exécute.

Si l'exploration de la paroi n'a rien douné, le pied est déferré, paré, et le praticien comprime, à l'aide de Iricoises ou de pinces spéciales, les diverses parties de la sole. S’il y a altération des lissus sous-jacents, l'animal cherche à dérober son pied aux attouchements qu'on lui fait subir.

I a fourchelle elle-mème donne d'utiles renseignements dans le cas de maladie naviculaire. Percutée, elle décèle quelquefois une sensibilité anormale des tissus sousjacents.

"Lorsqu'à l'aide des tricoises, du brochoir, de la renette, on s'est assuré ıju'il n'existe ni point douloureux sur le Irajet des clous, ni blessures ou traumatismes, que la sole est exemple de bleimes, la paroi, de seimes en barre, de kéraphyllocèles, on doil comparer le pied du membre malade ả son congénère, juger la température respective des deux sabots, leur volume, leur direction générale, la hauteur et l'écartement des talons, l'inclinaison des barres, le degré de souplesse des cartilages complémentaires du pied. 
L'exploralion se continue en preuant le sabot à pleines mains, les ponces rapprochés sur les talons. On exerce une forte pression sur toute la boîte cornée, étroitement enveloppée, particnlièrement au-dessous du bourrelet. Il n'est pas rare de provoquer le retrait du membre lorsifue la pression se porte sur un point douloureux, assez commun au niveau du quartier interne. Si la boiterie est intense, le pied chand, el yuaucune autre douleur n'existe dans toute l'étendue du membre, il y a de tortes présomptions pour que le siège du mal réside à cet endroit. L'application d'un cataplasme chaud, ou l'emploi d'un bain local d'eau simple à la tumpérature de 40 à 4 öo aide à préciser le diagnostic. Si le cheral supporte difficilementle topique mucilagineux, ou s'il refuse de laisser le pied malade dans le bain, on peut affirmer qu'il existe de la suppuration dans la boite cornée; une intervention chirurgicale s'impose.") (Chenot.)

Modifications pathologiques. - Le pied est susceptilule de présenter un grand nombre de défectuosités (1); il pent ètre grand, petit, étroit, haut, has, long, droit, évasé, plat, comble, à oignons, dévié en arrière (pied talus), en dedans (pied cagneux), en dehors (pied panard); il peul présentr encore un grand nombre d'autres délectuosités d'aplomb (pied pinçard, rampin, bot, etc.); il est sujet à de nombreuses défectuosités d'épaisseur t de qualité de la corne (pied cerclé, pied tendre, sec, fendillé, dérobé, etc.) (fig. 114).

On peut constater le rétrécissement général ou parliel ilu sabot avec atrophie des parties serrées du pied (encustelure).

Chez les carnassiers comme le furet, les ongles peuvent comme les sabots, acquérir une longueur démesurée. Ce

(1) Voy. Bournay et Sendrail, Chirurgie du pied in Encyclopédie vétérinaire. 
phénomène se produit quanıl l'animal est immobilisé par la gale sarcoptique des pattes (fig. 113).

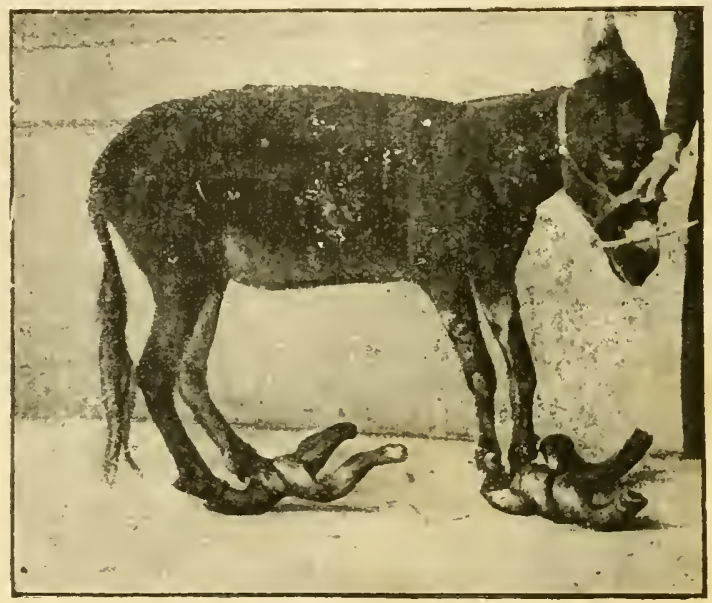

Fig. 114. - Pieds longs, hélicoïdaux (Cadẻac).

Le pied est exposé à tous les accidents truumatiqucs

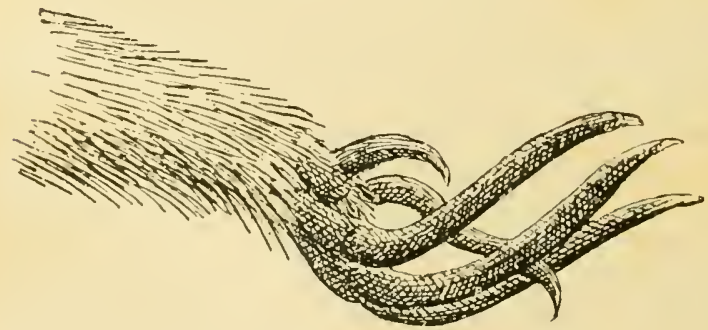

Fig. 113̆. - Palte malade du furet (Mégnin).

(contusions, plaies, ierasements), aux brûlures et aux gelures; on peut constater l'exongulation sous l'influence 
de canses mécaniques, d'inflammations nécrosanles, du crapaud, ou à la suile de troubles trophipues consécutifs à la néviotomie. On constate des atteiutes, le phlegmon coronaire, la dermatite gangreneuse coronaire, le javart cartilagineux, le clou de rue, les bleimes. La paroi peut se fissurer (-eimes), se décoller el une nouvelle production cornée recouvre bientòt le tissu podophylleux, c'est le faux-yuartier, qui est l'expres-ion de la fourbure. On lui donne le nom de kérrphyllocèle quand cette production, anormalement développée à la surface du tissu podophylleux, affecte la forme conique, cylindique ou pyramidiale.

On appelle kieracèle la tumeur cornée développée entre le tiŝsu velouté et la sole; crapaudine, la poussée irrégulière de la cornée liée au psoriasis do la courome ; intertrigo et eczéma de la fourchelte, la maladie qu'on désigne encore sous le nom de fourchette échauffée et de fourchette pourrie; crapaud, l'eczémä végétant sous-ongulé.

\section{XVI. - ALLURES.}

L'allu re peut ètre irrégulière (boiterie), s'accompagner de mourements succales, quasi-convulsifs de l'un des membres postérieurs (harper) ou d'une incoortlinition motrice (ataxie); elle peut ètre hésitante, tilutjante (maladies du cerreau et de la moelle, maladies infectieuses wraves comme le charbon, l'intluenza); elle peut être raide (rhumatisme musculaire et tétanos; elle est trottinante, dans le tournis du mouton; elle est incertaine, dans l'immobilité.

\section{XVII. - BOITERIE.}

Définition. - La boiterie ou claudication est une irrégrularité daus les allures, due ì l'inégalité d'action ou a l'impuissance d'un ou de plusieurs membres.

Les claudications sont l'expression d'une douleur plus CADÉAC. - Sémiologie, ?e édit.

II. -22 
ou moins vive produite par une contusion ou de lésions inflammatoires, néoplasiques ou parasitaires, de déchirures ou de distensions ligamenteuses, tendineuses ou musculaires ou résultent d'une altération des fonctions mécaniques des organes de la locomotion.

S'il est facıle de reconnaître l'existence d'une boiterie, il est sourent très difficile de reconnaitre le siège et la cause de la boilerie.

Les principaux moyens utilisés pour établir ce diagnoslic sont : l'uspection, la palpation, la mensuration, la percussion, l'auscultation, les injectious de cocaine, le coin de Lungritz, l'élude des caractères de la boiterie.

10 Inspection. - L“inspection fait souvent découvrir une atrophie musculaire, indice d'une paralysie ancienne, une forme, un éparvin on une hydarthrose ou toute autre lésion susceptible d'expliquer par son siège, son étendue, son importance, la production de la boiterie.

2o Palpation. - La palpation fait apprécier la consistance, la souplesse, la résislance, la mollesse, le glissement, les adhérences on les connexions des tissus d'une rigion. L'application de ce moyen a fait l'objet d'une ilude spéciale de $\mathrm{M}$. Chenot auquel nous faisons de nombreux emprunts (fig. 116).

Les moyens à l'aide desquels sexerce la palpation chiruracicale sont : la pression, le pincemeut, le glissemeut, le frôlement.

A) Palpation par pression. - Elle sert aux explorations qui se funt dans la profonleur des régions (muscles de

1) Chenot, Exploration du membre boiteux. Sémiologie, diagnostic, pronostic et traitement de quelques affections peu connues de l'appareil locnmoteur. (Bulletin de la Soriété des Sçiences vélérinaires, 1902). 
utilisée doit être proportionnelle à la résistance des tissus, mais toujours modérée.

Les olıstacles à la pénétration profonde des mains ou des doigts viennent de l'épaisseur des parties molles, des contractions musculaires. Ils sont passifs ou actifs. Ces derniers peuvent ètro éludés par la position des malades et par la mession en mesure.

"Suivant les circonstanres. on a recours à la pression verticule, á la pession horizontale, ou aux deux combinées (exploration des rayous osseux, de l'alticulation coxo-femorale, de la rolule, de l'épaule, rtc.) au travers des masses musculaires gui les reconvrent.

(B) Palpation par pincement. - Elle est destinée aux explorations superficielles (leudons fléchisseurs du pied, briles lizamenteuses, a ponévroses, expansions fibreuses). Elle se fait en saisissant, entre les doigts, la partie à explorer. C'est ainsi que l'on se rend comple de la souplesse t du degré de sensibilité de la peau, de son élasticité, de ses connexions avec le tissu cellulaire sous-cutané et avec les parties qu'elle recouvre. On reconuait, de cetle facon, les moindres modilications de l'état normal, les points donloureux, voire les plus minimes produchons pathologiques existant sur les tendons, les branches du suspenseur, les hrides ligamenteuses, etc.

"C'est encore par le pincement qu'on peut apprécier la température le ces lissus, leur consistance, leur expansibilité, leur état de rétraction, etc.

"C Palpation par glissement. - Encore plus que celle par pincement a laquelle on l'associe sourent, elle est destinue à la recherche des sensations délicales. C'est en faisant glisser la pean sur les tissus yu'elle recouvre: aponérroses, couches lamineuses, revètements osseux, articulaires, tondinenx, (ju'un reconnait lenrs connexions, leurs points d'adhérences normales on pathologiøues. 
"Combinée à la pression verticale ou horizontale, la palpation par glissement fournit $n$ des éléments les plus utilisables de l'exploration des plans osseux, pour la détermination des points douloureux siégeant à l'apophyse olécranienne, au paluron, à l'épiphyse supérieure dı mélatarse, au pourtour des marges articulaires.

“ D) Palpation par frỏlement. - Utilisable sur les chevaux de sang, d'une grande irritabilité nerveuse, elle lournit des sensations d'une extrême tinesse. Pour la pratiquer, on fait usage de la pulpe des doigts qu'on promène, très légèrement, sur la surlace des régions à explorer.

Le frôlement permet de reconnaitre, à travers la peau fine des chevaux de race distinguée, des saillies superficielles qui ne sont pas distincles à la vue. Ou arrive, lorsque la sensation tactile est suffisamment développée, à percevoir les plus légers reliefs sous-cutanés, les plus petites exostoses, les boursouflements périostiques invisibles à l'œil le plus pénétrant. C'est par le frôlement qu'on peut apprécier, délimiter les névromes commençants, les plus légères dermites, les petites masses ganglionnaires souvent très douloureuses qui parsèment la peau au-dessus des crevasses, des psoriasis du paturon, et qui. parfois, causent une grande gêne de la locomotion attribuée, souvent à torí, au traumatisme épidermique.

La palpation chiruruicale est, en médecine vétérinaire, le seul moyen qui permette de faire l'étude clinique de la douleur.

C'est par elle que l'on mesure l'étendue des zones sensibles palhologiques, qu'on peut établir le siège anatomique des desmodynies, des ostéalgies, des myosites de fatigue, causes si fiéquentes des claudications.

La palpation peut révéler l'existence d'un point douloureux silué au fond du sillon transversal du pli dı paturon, et qui est symptomatique de la malaclie naviculaire (fig. 117). Il existe souvent d'autres points douloureux sur la face 
antérieure ou latérule de la première phalangre qui font reconnaitre des boiteries du paturon (fig.118). Violet avait attiré l'attention sur la fréquence des cutorses et des ostiopériostites de la région phalangienne; Abadie avait également signalé des points douloureux à la partie superro-

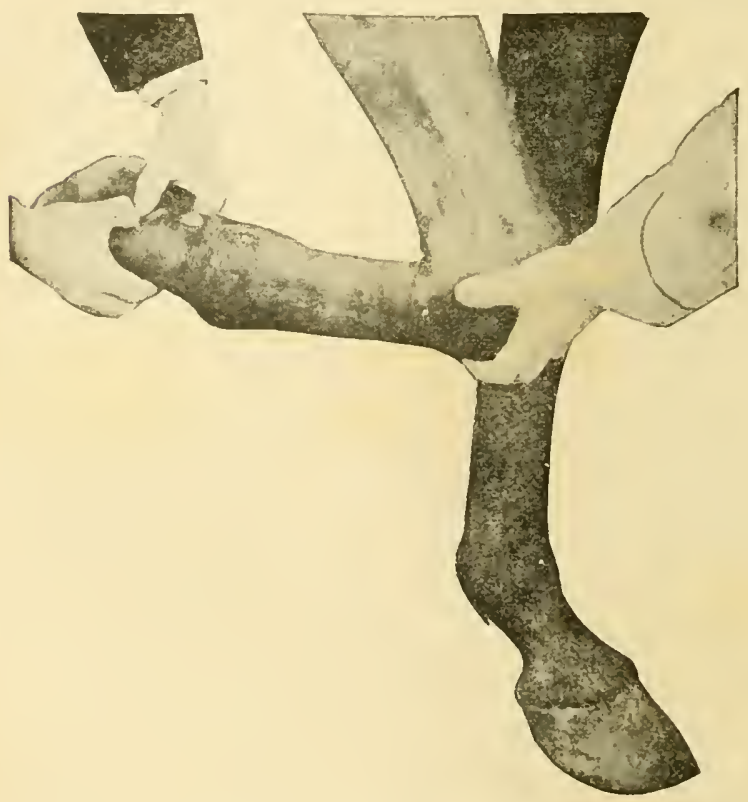

Fig. 117. - Exploration du pli du paturon d'un membre anterieur (maladie naviculaire) (Chenol).

anteriemre de la phalange, ainsi qu'au borl interne du tendun cxtensenr du pied. Pour bien explorer la région plialangienne, il faut placer le pied sur un billot de ıоyenue hauteur, faire maintenir l'avant-bras par uı aide dans un état de demi-relâclıement, comme l'indique la figure 118. 
Il est d'autres points douloureux qui occupent la face postérieure du canon au-dessous du pli de l'articulation carpo-métacarpienne à l'extrémité supérieure des méta-

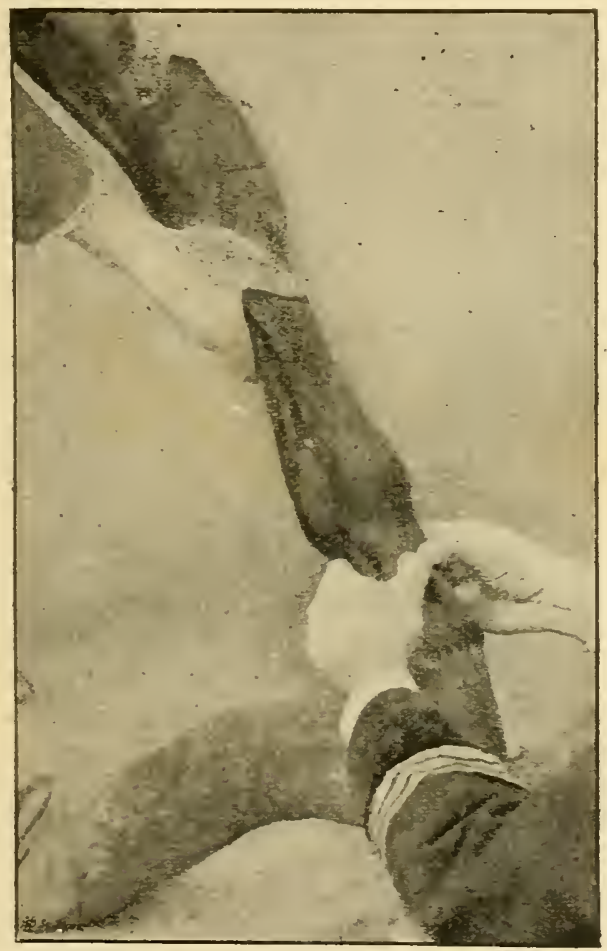

Fig. 118. - Exploralion de la face antérieure du praluron (ostéalgie pré-phalangienne ou boıterie du puturon) (Lhenol).

carpiens rudimentaires en avant des tendons, de chaque còté du suspenseur du boulet; ils sont faciles à ınetıre en évidence en maintenant à demi fléchi le membre qu'on explore. 
"La base du genou offre des vessigons, des exosloses (osselets), u u'Abadie avait indiqués.

"Le pincement du suspenseur du boulet et des tendons flechisseurs du pied fait reconnaitre l'effort de la bride carpienne, ta distension, la déchirure ou la nollesse des tendous du ligament suspenseur du boulet et de ses branches terminales; la ralpation par glissement met en évidence les ténosites cripitantes, les témosynovites aiguës sèches ou une alıération de l'épiphyse supérieure du métacarpirn mincipal à la lmuite de son bord exlerne et de sa face antérieure.

"Exceptionnellement, on peцt constater ainsi une douleur olécranienne, ou une douleur sciatique; le médıan enfoncé perpendiculairement dans la nasse les muscles fessiers détermine l'affaissement du membre lout entier sous la pression des doigts. Ce signe est particulièremeut nel au niveau des points d'émersence des nerl's ilio- et ischio-musculaires (petit sciatique) " (Chenot).

$3^{\circ}$ Mensuration. - La mensuration peut révéler une atrnphie du sabot, in resserrement des talons, symptomatiques de la maladie naviculaire ou d'une altération ancienne qui réduit l’appui du membre sur le sol; on pent suivre les déformations eutrainées par les artlırites et les synovites ou les lymphangites.

$4^{\circ}$ Percussion. - I.a percussion est journellement employée pour diagnostiquer les maladies du nied, lelles que les blessures, les enclourres, etc.; on met ainsi en évidence l'exagération de sensibilité d'une partie du pied comme, à l'aide de la pression des doigts, on révèle un suros ou un commencement de périostite.

$3^{\circ}$ Auscultation. - L'auscultation est un moyen de diaguostic important des luxutions et des fractures; l'oreille perçoit nettement et bien mieux que la main qui la palpe le bruit dèterniné par le frottement des deux abouts osseux 
irréguliers qui sont déplacés; elle percoit aussi le choc détermin par la rentrée ou la sortie le la tête du fémur qui glisse daıs la carité colyloïde ilu coxal chez le chien.

$6^{\circ}$ Injections de cocaïne. - Las injections de cocaïne sur le trajet des nerls constitnent un excellent moyen pour préciser le siège de la boiterje.

G r Inoyen a été hien étudié par Dassonville, Deysine,

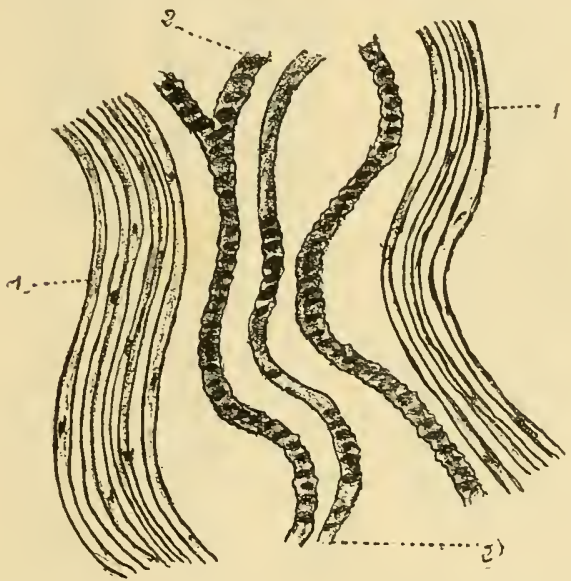

Fig. 119. - Nerfs après immersion dans la cocaïne à 3 p. 100 (acide osmiquc).

1, fibres restẻes normales; fibres granulcuses (Pécus).

Vidron el Pécus. On fail dissoudre 20 à 40 ceutigrammes de cocaïne dans 20 grammrs d'eau bouillie, et après avoir savonué et désinlecté l'extrémité du membre, on pratique une injection de 10 centimètres cubes de cha jue còté du memhre au-dessus ou aı-dessous du boulet sur le trajet des nerfs plantaires, et l'on fait ensuite un peu de massage sur la région pour faciliter la diffusion du lıquide anesthésique, puis au bout de dix à quinze minutes, on fait trotter l'animal. 
Ia boiterie a disparu si la douleur siège au-dessous des points d'injection; elle persiste dans le cas contraire. On peut différencier ainsi très facilement les boiteries des

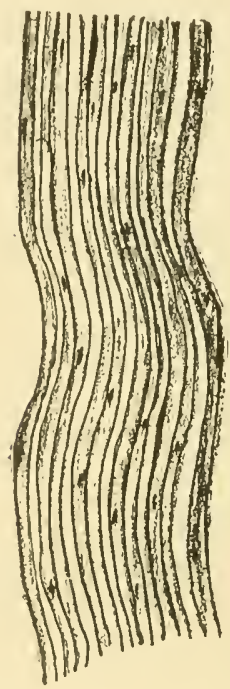

Fig. 120. - Nerfs après inmersion dans l'eau distillée (acide osmique) (Pécus).

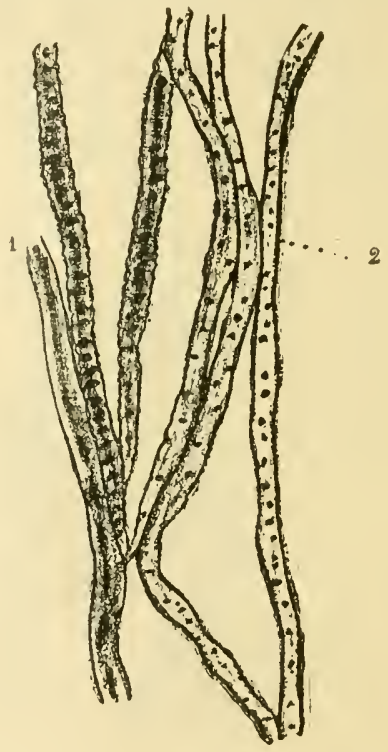

F g. 121. - Nerfs après imnersion daus la morphine à 4 p. 100 et soumis ensuite à l'action de l'acide osmique.

1, fibre à grosses granulalions; 2 , fibre ponetuée.

pieds des boiteries de l'epaule, des muscles, des os, des endons, etc.

La durée de l'insensibilité varie depuis quuarante minutes à deux heures, suivant que l'injection a été plus ou moins exactemınt faite sur le trajet des nerfs.

Les sels de cocaïne altèrent lemporairement les propriétés physiques du protoplasma des éléments nerveux terminaux et fibrillaires; ils deviennent brun jaunâtre; 
les fibres paraissent remplies de granulations (fig. 119 et 120$)$. Cette altération est la cause de l'aclion anesthésiante si remarquable de ces substances.

Quand on utilise ce moyen de diagnostic pour les boiteries des membres postérieurs, on commence par injecter d'abord la solution de cocaine à un travers de main

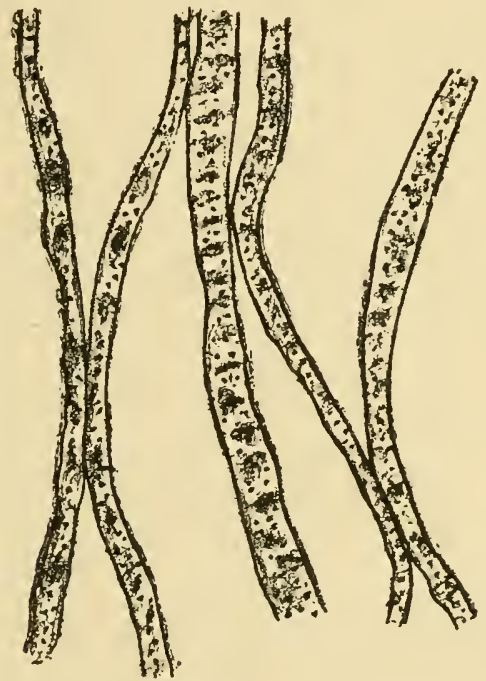

Fig. 122. - Nerf après immersion dans la solution cocaïnc-morphine ou dans la solution de Schleich renforcée (acide osmique) (Pécus).

au-dessus du jarret sur le trajet des nerfs grand sciatique et tibial antérieur, afin de s'assurer d'abord que le jarret n'est pas le siège de la douleur. On renouvelle le lendemain la même injection au-dessous du boulel. Grâce à ce moyen, on élablit un diagnostic certain par élimination; et ces névrotomies temporaires font counaître immédiatęment les résulıats des névrolomies définitives.

Quand on substitue la morphine à la coccöme, on peut constater la disparition prolongée de la boiterie. 
Les nerfs immergés quarante-huit heures dans une solution a 4 p. 100 de morphine reviennint forlement jaunâtres th présentént sous le microsıop le même a pect gramuleux yue sous l'influpnce de la coraine. Cerıaius lubes offrent drs goutteiettes le myéline isolées ou ađgglnmérées. rappelanı à s'y méprendre l'aspect des lubes dégémérís lu bout périphérịne des nerl's à la suite d'une section expérimentale (fig. 121).

Celte modification analımique profonde est peut-être la cause de l'action a alıésiante spéciale de longue durẻe des injections de morphine (Pécus).

On peut associer la morphine à la cocaüne. Cette association a été laite chez l'humıe pour atlénuer l'action dangereuse de la cocaïne sur certaius organismes. D'autre part, l’adjonction de la morphine à la cocaïne a l'avantage de renfor'er l'action analgésique de ce dernier medicament; elle prolonge l'insensibulisation nerveuse hien au delà des limites ordinaires, ce yui permet d'en retirer le bènéfre d'une vérıtałle aıtion curative.

Les nerfs plougés daus uı mélange de cocaine à 3 p. 100 et de morphine à 4 p. 100 uffrent les nıêmes lésions qu'avec la morphine purr. Ellrs sont plus accentuées en ce sens que le nombre des lilıres nerveuses à myéline fragmentée est plus considérable que sur les preparations taites avec des nerls trempés dans la solution de morphine simple (fig. 122).

Linjection de ce mélange sur le traję des nerfs plantaires provuque une analgésic prolongée et assez complète pour quon puisse la consilérer comme ayant une véritable action thérapeutique (Pécus) (1).

$7^{\circ}$ Procédé Lungwitz, - On peul utiliser le procédé de Lungwil\%, recommandé par Joly, pour le diagnostic de la muludie naviculare.

(1) Pécus, Journal de mid. vit. de Lyon, 1901, p. 526. L'injection mixte de coeaïne et d'adrénaline pruduit les etfets analogues (Domadieu of Thiriel). 
Un coin de bois dur, long de 20 centimètres et large de 15 , est taillé suivant un angle de $18^{\circ}$; on le place sous le pied suspect de maladie naviculaire, la pointe du coin vers les talons et sa base sous la pince; on lève alors le pied opposé et l'on constate que l'auimal manifeste une grande douleur s'il est affecté de maladie naviculaire. Il s'appuie sur le tenseur du pied, porte ses membres postérieurs en avant ou se dérobe.

Immédiatemert après le poser du pied soustrait à l'appui, il soulage le membre en le portant en avant ou en arrière. Au contraire, le coin étant placé de façon que les talons du pied suspect soient exhaussés, le cheval reste tranquille quand on lui lère le pied sain et change á peine de place lors du poser. Si le résultat parait douteux, on fait l'épreuve comparative du coin sous le pied sain.

Gràce à ce procédé, Joly constate qu’à Saumur sur 10 chevaux affectés de boiteries non iraumatiques du pied, huit on neuf ont de l'ostëite phalangienne et un ou deux seulement de l'osteite naviculaire.

Les autopsies confirment absolument les indications fouraies par le coin de Lungwitz.

"Ces indications montrent immédiatement le degré d'utilité de la ferrure à éponges nourries, pour le soulagement des boiteries non traumatiques du pied ". (Joly.)

Nous croyons que dans la plupart des cas, il est inutile de recourir à ce procédé de diagnostic qui peut ètre dangereux et susceptible d'occasionner des accidents.

Caractères de la boiterie. -- La boiterie peut se mon. trer dans les diverses allures ou se traduire au repos par diverses atlitudes; ses caractères varient suivant son degré d'intensité ; l'animal feint quand elle est très légère ; toite quand elle est nettement perceptible; on dit que l'animal boite tout bas ou marcheà trois jambes, quand elle est très intense; elle est alors visible au pas.

C.ADÉ.Ac. - Sémiologie, əe édit.

II. -23 
La boiterie peut varier dans son type : être intermittente, rémiltente ou continue, s'alténuer ou augmenter, comme dans la boiterie résultant d'oblitérations artérielles.

La boiterie est le symptôme d'une douleur plus ou moins intense ou d'un déraugement fonctionnel des organes de la locomolion.

10 ON RECONNAit LE MEMBRE Boltecx en examinant l'animal au repos et en mouvement.

Au repos, le sujet cherche instinctivement à diminuer l'appui du membre malade, soit en le portant en avant, fléchi au genou ou au boulet, soit en le portant en dehors, c'est-à-dire en l'éloignnant toujours de la base de sustentation, en rapprochant les membres sains pour augmenter leur charge el diminuer d'autant celle du membre malade.

Quand la douleur est très vive, on constate des lancinations, c'est-à-dire des oscillations synclirones aux baltements artériels. Si l'animal soulfre de plusieurs membres à la fois, il se tient couché le plus longtemps possible et quand il est debout, il piéline sur place, changeant constamment d'appui; il porte alternativement les membres antérieurs en avant et ramẻne la litière en arrière.

Pendant le mouvement, la durée de l'appui du membre malade est plus courte, le lever plus rapide, l'extension en avant raccourcie et le poser plus hésité; l'animal allège le plus qu'il peut la charge de ce membre : il reporte ainsi sur le membre opposé diagonalement la plus grande partie du poids, de telle sorte que ce dernier a une période d'appui plus longue, un lever plus haut, un mouvement en avant plus étendu et un poser plus ferme.

La téte et la croupe, par des mouvements alternalifs d'élévation el d'abaissement, permettent à l'animal de diminuer ou d'augmenter la charge supportée par les menubres.

Quand l'animal boite d'un membre antérieur, il élève la tête lors de l'appui du membre malade, rejetant aiusi 
le centre de gravité en arrière et la plus grande partie du poids du corps sur le membre postérieur opposé en diagonale; la tête s'abaisse, au contraire, lors de l'appui du membre sain.

Quand l'animal boite d'un membre postérieur, la tête s'abaisse à chaque battue sur le membre antérieur opposé en diagonale.

La croupe éprouve aussi des mourements inégaux d'abaissement et d'élévation sous l'inlluence de l'inégralité d'action du membre boileux, mais ces mouvements sont moins prononcés, moins importants que ceux de la tête.

L'abaissement est heaucoup moins marqué qu'à l'état normal lors de l'appui du nembre boiteux; il est plus accusé à l'appui du membre sain, lors de la boiterie postérieure. On peut rencontrer des exceptions: dans la paralysie du nerf fémoral antérieur, le libia ne pouvant plus ètre étendu sur le fémur, l'abaissement de la croupe est exagéré lor's du poser du membre malade.

Les boiteries des membres antérieurs ont aussi une influence sur les mouvements de la croupe; un cheval étant boiteux d'un membre antérieur, le membre postérieur qui lui est opposé en diagonale doit raccourcir son appui comme son congénère antérieur, conséquemment, la croupe s'élève; elle s'abaisse lors du poser de l'autre membre postërieur.

Ainsi, lors de boiterie du membre antérieur gauche, par exemple. tête et croupe s'élèvent lors du poser du bipède diagonal gauche; lors de boiterie postérieure gauche, la tète s'alaisse et la croupe s'élève lors de l'appui du bipède diagonal gauche, de là, une cause d'erreur possible, qui consiste à placer la boiterie dans le membre antérieur gauche. On confondra plus facilement une boiterie postericure avec une antericure qu'inversement, car le coup de tête indiquant la boiterie postérieure est plus accusé que le mouvement de la croupe indiquant une boiterie antérieure. 
Si l'animal boite de plusieurs membres, l'irrégularilé d'allure est plus manifeste; les mouvements de la tête et de la croupe sont plus accusés.

Quand la boiterie est très peu accusée, il est quelquefois difficile de reconnaître le membre boiteux, il est nécessaire d'exercer l'animal au trot sur un terrain dur, tenu en main, mais la tête aussi libre que possible; il est nécessaire de le monter pour augnenter et surtout pour prolonger la durée de l'exercice quand la boiterie se manifeste senlement à chaud. On fait tourner brusquement l'animal pour meltre en évidence une boiterie d'éparvin ou d'un effort du paturon des membres antérieurs.

20 LE SIÈGE DE LA BOITERIE est souvent dénoncé par les caractères de la boiterie : les chevaux affectés de maladie naviculaire ou de fourbure chronique ont un trot extrêmement raccourci; on dit qu'ils out les épaules chevillées ; la boiterie qui procède d'une thrombose arterielle ne se manifeste qu'à chaud, celle qui provient de la maladie naviculaire ou de l'eparion diminue au contraire sous l'influence de l'exercice. Le termin meuble exagère les boiteries d'origine musculaire; un terrain dur exagère les boiteries d'origine osseuse.

Les boileries provenant des régions supérieures sont caractérisées par les mouvements limités da nembre boileux et le poser normal du pied (luxation scapulo-humérale, déchirure du ligament interosseux de l'articulation coxo-fimorale, fructure de la téte du fémur), mais dans la plupart des cas, il n'en est pas ainsi : il existe des relations intimes entre les divers rayons locomoteurs et toute lésion de l'un d'eux retentit plus ou moins sur les autres. Ainsi, dans la maladie naviculaire, l'animal marche à pas raccourci, il rase le lapis, le poser est très hésité, on dirail qu'il a les épaules froides ou chevillées.

Les altérations de l'épaule se traduisent par un mouvement d'abduction de l'angle scapulo-huméral quelquefois si prononcé que la région semble se détacher du tronc 
ou bien l'extrémité supérieure fauche d'une manière très prononcée. Ces boiteries résultent de myosites, de chutes ou de chocs contre des obstacles.

L'allure au pus fait nettement reconnaitre la rupture de la corle du fléchisseur du métalarse, parce que la corde calcanéenne demeure relàchée et le cánon ne se fléchit plus sur la jambe; l'extension du membre postérieur en arrière avec impossibilité de flexion des articulations est caractéristique de l'accrochement de la rolule; la flexion des rayons inférieurs du membre avec ouverture exagérée de l'épaule s'observe dans la paralysie du radial, enfin l'articulation fémoro-tibiale se ferme brusquement chaque fois que l'animal pose le pied sur le sol dans la paralysie du nerf fémoral, parce que les muscles fémoraux antérieurs ne soutiennent plus la rotule.

Les aulécédents du sujet peuvent renseigner sur le siège de la boiterie; on sait que l'animal a déjả boité du boulet, du jarret ou d'une autre région; l'affection (entorse, nerf-férure, etc.) récidive fréquemment.

Les circonstances étiologiques qui ont présidé au développement de la boiterie (traumatisme, chute, course, etc.) peuvent faire soupconner le siège de la boiterie et permettent d'éliminer certaines tares préexistantes comme des formes, des suros ou des éparvins. Les lésions observées sout de nature variable; elles sont aiguës ou chroniques, congestives, inflammatoires, néoplasiques, parasitaires, musculaires, osseuses, tendineuses, ligamenteuses; elles intéressent les synoviales articulaires ou tendineuses.

Dans tous les cas de boiterie des membres antérieurs, il faut commencer par explorer le pied,l'examiner et quand cet examen demeure sans résultat, on examine successivement le paturon, le boulet, le canon, le genou, l'avantbras et l'épaule en procédant de bas en haut, car le siège des boiteries est relativement rare dans les parties supérieures.

L'exploration des membres postérieurs doit se con- 
centrer plus spécialement sur les jarrets, le grasset, et le boulet, car le canon et le pied sont, beaucoup plus rarement qu'aıx membres antérieurs, le siège d'altérations productrices de boiteries.

Signification. - Les boiteries expriment une douleur variable comme siège et comme intensité, diminuant la durée d'appui du membre souffrant, prolongeant celle des membres sains; elles sont un signe de faiblesse ou d'inerlie musculaire ralentissant ou empèchant les mouvemenls d'un ou de plusieurs membres; d'un dérangement mécanique des ressorts locomoteurs entravant ou empêchant leurs fonctions; enfin d'une inégalité accidentelle ou congénitale des rayons locomoteurs.

Les maladies susceptibles de produire la boiterie sont du domaine: $1^{\circ}$ de la pathologie externe (maladies du pied, des tendons, des os, des ligaments, des articulations, des muscles); $2^{\circ}$ de la pathologie interne (rhumatisme musculaire et articulaire, rachitisme ou ostéomalacie, polyarthrite pyohémique des reaux et des poulains, arthrites tuberculeuses, morveuses, gourmeuses, synoviles et arthrites déterminées par la fièvre aphteuse, la clavelée).

Des boiteries succèdent aussi à l'oblitération des artères iliaques et humérales, aux adénites des membres.

Les jeunes animuux sont prédisposés aux boiteries osseuses procédant de suros, de formes et d'éparvins; on les désigne souvent sous le nom de boiterie des jeunes chevaux.

Les animaux qui travaillent dans les villes sont exposés à des clous de rue el à des traumatismes.

\section{IVIII. - IIARERR.}

Définition. - On désigne sous le nom de harper, l'hyper. flexion du jarret. Ce trouble de la locomotion est carac- 
térisé par une sorte d'anachronisme des mouvements des membres postérieurs donnant lieu auormalement à des variations angulaires normales du jarret.

C'est un acte involontaire, non douloureux, une incoordiuation purement mécanique, une hyperflexion automatique, souvent discontinue, d'intensité variable, des divers rayons osseux du membre postérieur pendant le soutien.

Quand l'animal est affecté de cette défecluosité de la locomotion, on dit qu'il harpe, eprrvine, tronsse, qu'il a la marche du coq, ou encore yu'il est affecté d’éparvin sec.

Le cheral, et quelquefois l'aine, le mulet et le bouf en sont affectés.

Le harper a été étudié spécialement par Berton (1), auquel nous ferons de fréquents emprunts.

Etiologie. - A l'état physiologique, l'exagération du raccourcissement du membre simulant le harper se manifesle quand l'animal doit éviter des obstacles inaccoutumés comme quand il marche dans certains terrains irréguliers, semés de pierres, creusés d'ornières. Une entrave placée au paturon, un pansement un peu serré, produisent ce mouvement en provoquant la contraction volontaire des masses nusculaires crurales ou du voisinage de l'articulation du grasset, sans qu’on puisse préciser le siège et la nature de la lésion déterminante du harper (Berton).

Ce signe est d'ailleurs l'expression d'altérations très diverses : contusions du bourrelet, seimes, fourbure, kéraphyllocèle, compression du podophylle, rétraction de l'aponévrose jambière, dilacération des muscles de la fesse et de la région jambière postérieure, rayures des surfaces arliculaires libio-tarsienne, fémoro-libiale, fémoro-rotulienne el tibio-astragalienne, éparvin calleux, accrochement du ligament fémoro-rotulien interne au-

(1) Berton, Contribution à leitude du harper (Bulletin de la Société des Sciences vétérinaires, 1003, p. 181). 
dessus du rebord correspondant de la trochée fémorale, tension des releveurs de la cuisse.

Ce sont alors des actes réllexes, provoqués par une soulfrance qui s'accentue pendant l'appui; ils sont ordinairement accompagnés de boiterie qui fait défaut dans le barper habituel. Chez des clievaux harpant du devant, Goubaux el Barrier ont constalé aussi des rayures de l'articulation huméro-radiale.

En dehors des lésions musculaires, tendineuses, aponévroliques, ou osseuses, on a fait intervenir des lésions nerveuses. On a incriminé les lésions du grand scialique, la névrile de ce nerf ou des altérations médullaires.

Chez beaucoup de harpeurs, on ne constale rien d'anormal, soit dans les articulalions, soit dans les muscles, les tendons, les aponévroses, les synoviales, les vaisseaux el les nerfs.

Caractères. - $1^{\circ}$ Le cheval affecté d'éparvin sec fléchit d'une manière brusque et saccadée le caunon qui ferme dans une proportion excessive la jointure tibio-tarsienne, et le sabol touche quelquefois la paroi abdominale; puis le membre s'allonge avec la même brusquerie et le pieł atleint le sol dans un poser brutal et bruyant, sans qu'il y ait à proprement parler de boilerie. Tous les rayous participent au raccourcissement exagéré du membre; mais c'est au niveau du jarret que l'jrrégularité présente son maximum d'intensité.

Quand les chevaux à éparvin sec reculent, la plupart cessent de harper, d'autres présentent au contraire cette flexion convulsive du jarret. Selon Berton, la disparition du harper pendant le reculer semble indiquer une lésion siégeant dans les régions supérieures, c'est-à-dire des troubles angulaires de la lianche ou du grasset, tandis que la persistance de l'hyperflexion parait résulter de l'altération de l'articulation tibio-tarsienue elle-mème.

L'éparvin sec se manifeste au pas, particulièrement 
quand le sujet tourne sur le membre atteint; il se produit seulement au début du travail ou persiste en diminuant d'intensité, il s'attémue pendant les allures vives comme celle du trot et disparait pendant le galop, parce que l'automatisme tarsien n'a pas le temps matériel de se produire ou est combattu par cerlaines contractions musculaires antagonistes; de plus, le cheval harpeur peut fournir de nombreuses foulées régulières même au pas; c'est donc un pliénomène intermittent.

$2^{\circ}$ Chez le bouf, le harper n'est pas très rare; il se manifeste particulièrement, chez les animaux énergiques et pleins d'ardeur, sur un seul membre, ou exceptionnellement sur tous les deux à la fois. Quand le harper est prononcé, l'animal éprouve de la difficullé à soulever le membre affecté; " il le porte en abduction d'une façon remarquable et parfois très surprenante, et il montre un véritable arrêt de brève durée dans la flexion. Presque dans tous les cas, on percoit à chaque llexion du membre affecté, un bruit pareil à celui des têtes articulaires qui se heurtent entre elles; ce bruil se produit a l'articulation du tibia avec l'astragale.

Quand le pas s'effectue, le jarret se porte un peu en dedans et le pied en dehors. Le mouvement anormal est mieux marqué aux premiers pas que fait l'animal; la marche continuant, il devient moins apparent; il est généralement peu visible.

Le harper disparait quandl'animal est échauffé; parfois il ne se manifeste que lorsqu'on fait tires l'animal, qu'on le fait marcher rapidementsur un sol inégal, mou, comme un champ labouré ou un terrain marécageux. Ce trouble de la locomotion est généralement incurable.

On l'a vu se manifester à la suite de contusions, de foulures siégeant particulièrement au-dessous des talons; c'est une manifestation clinique du tournis (Gilotti).

Pathogénie. - Le harper ne parait ètre que l'exagéra23. 
tion des mouvements automatiques d'extension et de flexion de l'articulalion du jarret. L'arliculation semble mue par un ressort, et il suffit d'une pression exercée sur l'un ou l'autre des rayons tibio-tarsiens, pour que le mouvement commencé se lermine de lui-mème dans une course spontanée, précipitée el violente comme celle qui caractérise le harper. D'un còté, le plan iucliné trochléen favorise le glissement des surfaces articulail'es l'une sur l'autre; de l'autre, les ligaments latéraux constituent de clıaque còté de l'articulation des plastrons fibreux d'une grande puissance contentive, composés de couches superposées, d'aulaut plus puissantes, d'autant plus étendues qu'on les considère plus superficiellement. Chacun de ces gros ligaments se trouve constilué par l'adossenent de faisceaux d'inégale longueur, en grande partie indépendants, qui montrent, selon les phases de l'extension ou de la flexion, des étals variables et successifs de tension, ne pouvant jamais les conduire, dans leur ensemble, au relàchement complet. Ces organes disposent donc ainsi d'une force contentive toujours en éveil, toujours agissante, quel que soil le plan du mouvenent, comparable en somme à l'action impulsive de contractions musculaires alternées. Eneffet, sur une pièce disséquée, l'automatisme tarsien se manifeste encore avec une grande intensilé (Berton).

L'excursion lotale du métatarse comporte deux phases successives: une flexion passive réduisant l'angle du jarret à $130^{\circ}$ environ, commandée par la corde tibiotarsienne, une flexion spontanée sous la dépendance des ligaments latéraux tibio-larsiens. Dès lors, le harper résulte de l'exagération de la flexion passive ou de la flexion spontanée, et est sous la dépendance étroite de la corde fémoro-tarsienne ou des ligaments latéraux tibio-tarsiens.

En dernière analyse, on pent invoquer à la fois les lésions directes de la corde du jarret et celles des ligaments latéraux comme causes du harper. 


\section{SÉMIOLOGIE DE LA TEMPÉRATURE}

\section{I. - FIÈYRE}

Définition. - La fièvre est un syndrome caractérisé essentiellement par l'exagération des processus chimiques qui engendrent la chaleur animale. L'élévation anormale de la température est donc le signe principal; les autres troubles fonctionnels (caruiaques, circulatoires, digestifs, cutanés, urinaires, cérébraux) n'ont pas la tnème importance pour le diagnostic.

Les mots ficre et hyperthermie ne sont pourtant pas synonymes.

Un animal fail une course ou est placé dans une étuve, sa température s'élève de 1 à 2 degrés; il a de l'hyperthermie, mais il n'a pas de fièvre.

Dans la fière, l'augmentation dans la production de la chaleur animale résulte de l'action des toxines microbiennes ou des toxines cellulaires.

Température normale. - La chaleur animale résulte de la combustion des principes immédiats dépensés; les pertes de calorique de l'organisme résultent surtout de l'éraporation pulmonaire et cutanée.

Dans l'organisme sain, une production plus abondante de chaleur est compensée par de plus grandes pertes. De cel équilibre entre la chaleur produite et la chaleur perdue résulte l'uniformité de la tempéralure normale des êtres supérieurs.

Cette température varie un peu suivant chaque espèce : 


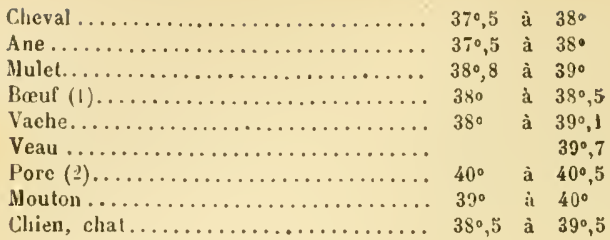

Variations physiologiques. - Les variations de température sont trés fréquentes et les causes en sont nombreuses :

Travail musculaire. - Sous l'influence du travail, les combustions augmentenl d'intensité et la température s'élève. Après un travail pénible ou une course rapide la température angmente souvent de plus de un degré chez le cheval.

Les chiens qui se débattent dans leurs liens, sur la table de vivisection, s'échauffent notablement et leur température peu( s'élever jusqu'à $41^{\circ}$ (IRichet); les excitations électriques déterminent infailliblement une élévation croissante de la température qui peut atleindre $44^{\circ}$, et même $40^{\circ}$; mais généralement les animaux succombent quand leur température centrale atteint $\dot{1}^{\circ}$ (Richet).

L'élévation de température n'est pas uniforme dans tout le corps; elle est toujours plus marquée au niveau des muscles qui travaillent; l'accroissement de la thermogénèse est lié au travail musculaire.

Heure de la journée. - La température est tous les soirs élevée de quelques disièmes de degré et même de

(1) D’après Krabbe et Müller la température normal du bœuf est de $38^{\circ}, 8$; Siedamgrolzky l'a trouvée de $38^{\circ}, 9$; Zundel de $39^{\circ}$; Lydtin de $38^{\circ}, 1$ à $38^{\circ}, 5$; llajnal a constaté qu'elle varie de $38^{\circ}$ à $39^{\circ}$. La température des bovidés audessus d'un an est en mojenne de $38^{\circ}, 6$; celle des bovidés au-dessous d'un an varie gènéralement entre $39^{\circ}$ et $40^{\circ}$; elle dépasse quelquefois $40^{\circ}$. Penlant les premières semaines de la vie, la température se maiutient autour de $40^{\circ}$ : vers six mois elle oscille enlre 39 et 40 degrés.

(2) Aruch l'a trouvée de $39^{\circ}, 1$ à $39 \circ, 3$. 
un degré de plus que le malin. L'animal présente le maximum de température vers cinq heures $\mathrm{du}$ soir, puis la température s'abaisse de neuf heures à une heure du matin où elle atteint son minimum. Elle augmente jusqu'au malin pour décroître jusqu'à midi et augmenter enfin jusqu'à cinq heures. Ces variations physiologiques sont encore plus prononcées pendant la fièvre.

Saisons. - Pendant l'été la température des animaux est plus élevée que pendant l'hiver.

Manotsliow a constaté, pendant l'été, une grande augmentation de la température chez des animaux soumis à un travail pénible. Sur deux cheraux venant de fournir une course rapide en élé, la température a monté de $37^{\circ}, 5$ à $41^{\circ}$; c'est-à-dire de $3^{\circ}, 5$ au-dessus de la normale. Exposé au soleil, la température du cheral peut s'élever de 1 à 2 degrés; exposé à la pluie, elle baisse d'autant.

Sur des boufs conduits à 10 kilomètres par une chaleur extérieure de $30^{\circ}$, l'auteur observa la température de $38^{\circ}, 4$ au départ, puis $39^{\circ}, 2 ; 39^{\circ}, 9 ; 40^{\circ}, 9$.

- Les chiens à poil ras ont une température de $38^{\circ}, 8$; les chiens à poil long ont $39^{\circ}, 6$.

Chez les por'cs gras, on observe d'énormes élévations de température pendant les fortes chaleur's.

On constate également que la température des animaux est plus élevée dans une étable chaude que dehors. Les bœufs à l'étable ou au pâturage en été offrent uı degré de plus, les chevaux au bivouac présentent un abaissement de un demi-degré.

La température moyenne de l'homme et des animaux varie avec chaque climat; elle est toujours plus élevée dans les pays chauds.

Influence de l'alimentation. - Les animaux produisent d'autant plus de chaleur que leur ration est plus forte, 
surtout si elle est ricle en principes aptes à la combustion (principes carbonés et bydrogénés).

Pendant la digrestion, la température peul s'élever de $0^{\circ}, 0$ cette élévation se produit peu d'instants après le repas.

Après l'ingestion de boissons froides, en assez grande 'quantité, on voit la température s'abaisser.

La privation absolue d'aliments détermine une chute plus ou moins rapide et plus ou moins profonde de la température. Le refroidissement coincide avec la disparition du glycose lans le saug.

Influence de l'àge. - La température normale n'est pas la même aux diverses époques de la vie: d'abord, chez les animaux très jeunes, eile est bien moins élevée que chez. lälulte; chez les nouveau-nés, la température s'abaisse dẻs que la mère s'éloigne d'eux.

Chez les animaux un peu plus ìgés, elle est au contraire plus élevée et atteint $0^{\circ}, 5$ au-dessus de la température de l'adulte. I.es sujels àgés retombent dans le même cas que les sujets très jeunes et leur température peut descendre jusqừa $0^{\circ}, \breve{b}$ au-dessous de la température normale.

Sexe. - Chez la femelle, la température normale est en général moins élevée de $0^{\circ}, \ddot{3}$ que chez le mâle; elle peut même varier suivant l'épouque; pendant les chaleurs, la température est bien plus élevée; après le part, elle descend beaucoup.

Race. - L'inlluence de la race se fait aussi sentir, la température est en général un peu plus élevée dans les races distinguées.

Sommeil. - Pendant le sommeil, la circulation, la respiration se ralentissent. Le débit de l'oxygène diminue et la chaleur animale de mème. En général, on trouve, pendant le sommeil, un abaissement de température de $0^{\circ}, 3$ cliez tous les animaux (Coliu). 
Tonte. - Chez les animaux comme le cheval ou le mouton, on roit de grandes variations de température au moment de la tonte, surtout en hirer : la température du cheral descend de $3 S^{\circ}, 3$ à $37^{\circ}, \bar{i}$ immédiatement après la tonte, mais la température s'élève peu de temps après en se rapprochant de la normale.

Ajoutons à toutes ces influences celle des opéralions, des douleurs intenses; les sujets présentent dans ces cas un abaissement de température assez variable, mais plus ou moins considérable.

Les rariations pathologiques de la température se traduisent par une élération (fière) ou par un abaissement de la température normale collapsus).

Gestation. - Saint-Cyr et Chapelle avaient déjà montré que la température des raches en étal de gestation arancée peul alleindre $40^{\circ}$ cinq ou six jours avant le part. Hobday avait trouvé, un muis avant le part, des températures variant entre $38^{\circ}, 9$ et $39^{\circ}$ sur 30 raches tuberculinées sans résultal positif. Lenz. sur \& vaches examinées deux a trois semaines arant l’époque de la parturition, arait observé des rariations extrèmes de $3 \aleph^{\circ} .1$ à $39^{\circ}, \pi$.

Post a fait les constatations suirantes, sur six bovidés (3 raches et 3 génisses) la tempéralure a été prise, matin et soir, quelques jours arant el quelques jours après la parturition : deux vaches ont servi de témoins. Celles-ci avaient une température rectale qui oscillait entre 38 et $39^{\circ}$, au roisinagre de $38^{\circ}, \dot{4}$ ou $38^{\circ}, 5$, tandis que les vaches en état de festation arancée présentaient de fortes variations. Ces variations neuvent atteindre $1^{\circ}, \breve{3}$. Tous les Jiagrammes établissent que peu de temps arant le part la température subit un abaissement assez considérable.

Influence de la respiration. Polypnée thermique. Dès que la température extérieure ou la température du sang s’élère, le chien se met à respirer la 
gueule ouverte, la langue pendante et accélère son rythme respiratoire au point de produire 300 à 400 mouvements par minute.

Cette polypnée thermique est un réflexe respiratoire d'origine périphérique ou d'origine centrale; elle se manifeste chez les animanx exposés au soleil ou placés dans une étuve; elle se produit sous l'influence de l'exercice ou du tétanos électrique et a pour effet d'amener la saturation du sang eu oxygène et de réaliser ainsi la condition essentielle de l'apnée.

Cette polypnée ne peut se produire chez les chicns pourvus d'une muselière, de sorte qu'ils meureut très vite par hyperthermie quand ils sout placés dans une étuve.

Influence de la sudation. - Le réflexe vaso-dilatateur qui détermine la sudation el la réfrigération du corps se produit sous l'influence de l'échauffement des extrémités des nert's cutanés et sous l'influence des causes internes qui tendent à élever la température centrale.

Régulation périphérique et régulation centrale. l'organisme est un thermostat à fonctions multiples unies et réglées automaliquenent de manière à le maintenir à une température moyenne à peu près constante. Les actions régrulatrices consistent d'une part à graduer l'intensité de la production de chaleur (réflexes thermogẻnes) et d'autre part à assurer des déperditions de la chaleur produile en excès (réflexes dépcrditeurs).

Le réflexe cutané vaso-constricteur qui chasse le sang de la périphérie vers les parties internes pour le soustraire à l'infuence réfrigéranle du milien extérieur est le premier moyen dont dispose l'organisme pour se préserver du froid.

Le frisson thermique qui exagère la tonicité et l'activité musculaire est une source de chaleur. Le frisson s'observe chez les chiens à poils ras, comme les lévrieıs, les che. 
vaux tondus ou chez les animaux refroidis par un bain d'eau froide. Il y a en même tenips cxagéralion des combustions respiratoires.

Les variations de température intérieure ou des centres nerveux nous défendent plus vite contre le chaud intérieur que contre le froid extérieur. En effet, il faut un abaissement de température centrale de 3 degrés (Richet) pour provoquer le frisson réchauffant, tandis qu'ıl suffit d'une élévation de 0,4 pour amener la sudation réfrigérante.

" Le système nerveux périphérique se clarge surtout de maintenir la température constante; le système central a surtout pour fonction de ramener le corps à la lempérature constante quand elle a élé modifiée dans un sens ou dans l'autre.

"Le système périphérique est outillé surtout pour empècher le corps de se refroidir; le système central agit dans les deux sens. ") (Guinon.)

Oscillations fébriles. - De toutes les manifestations de la fièvre, l'élévation anormale de la température constitue le sigue le plus frappant et le plus exactement. perceptible. C'est lui du reste qui sert de base à l'étude de ce syndrome, à la distinction d'un certain nombre de types de fièvre.

On peut reconnaître quatre degrés de fièvre:

$1^{\circ}$ Fièvre peu intense $\left(39^{\circ}, 3\right.$ chez le cheval et le chien);

20 Fièvre moyennement intense $\left(40^{\circ}, 5\right)$;

$3^{\circ}$ Fièvre inlense $\left(41^{\circ}, 00\right)$;

$4^{0}$ Fièvre très intense ou hyperpyrétique (au-dessus de $\left.41^{\circ}, 5\right)$ (fig. 123).

L'hyperthermie est par elle-mème une source de symptômes graves, comme en témoignent les phénomènes observés dans l'échaullement artificiel. Un animal plongé dans un bain un peu au-dessous de la tempéralure rectale, atteint une lempérature de $45^{\circ}$. Avant de mourir, il donne les signes de la plus vive agitation; les battements 
du creur el les mouvements respiratoires s'accélèrent; les combustions respiratoires s'exagèrent, puis l'agitalion s'apaise et l'animal entre dans une période de coma et d'anesthésie interrompue par des crises de spasmes convulsifs et de contractures, enfin l'animal pousse un cri et meurt. On Irouve le cœur inmobile, rigide, le sang fluide, des muscles rigides et inexcitables.

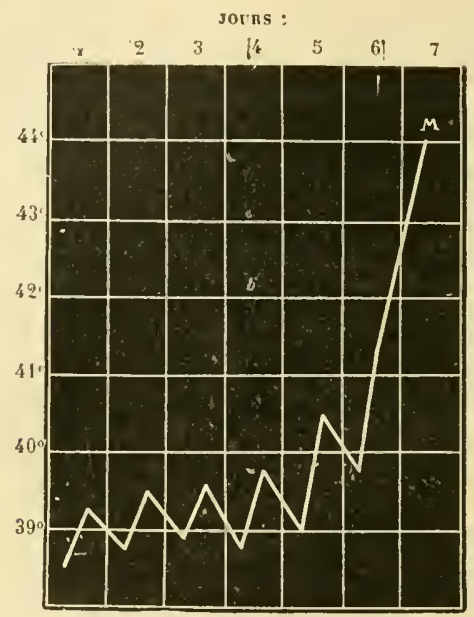

Hig. 123. - Tracé thermométrique d'un cas mortel de tétanos.

Les modifications que subit la température chez le fébricitant peurent être divisées en trois périodes: $1^{\circ}$ ascension; $2^{\circ}$ état; $3^{\circ}$ déclin; elles nont jamais rien d'uniforme dans leurs manifestations.

Ainsi la période ascenlante est tantòt courle (fig. 124, tantòt Iongue (fig. 12\%), tantòt elle dure quelques heures, tantôt plusieurs jours. Et, non seulement, elle varie dans sa durée, mais aussi dans le mode d'ascension de la tempéralure. 
Habituellement, lorsque cette période est courte, l'élévation de la température est brusque; elle commence par des frissons.

Le frisson est calaclérisé par des secousses, des trem-

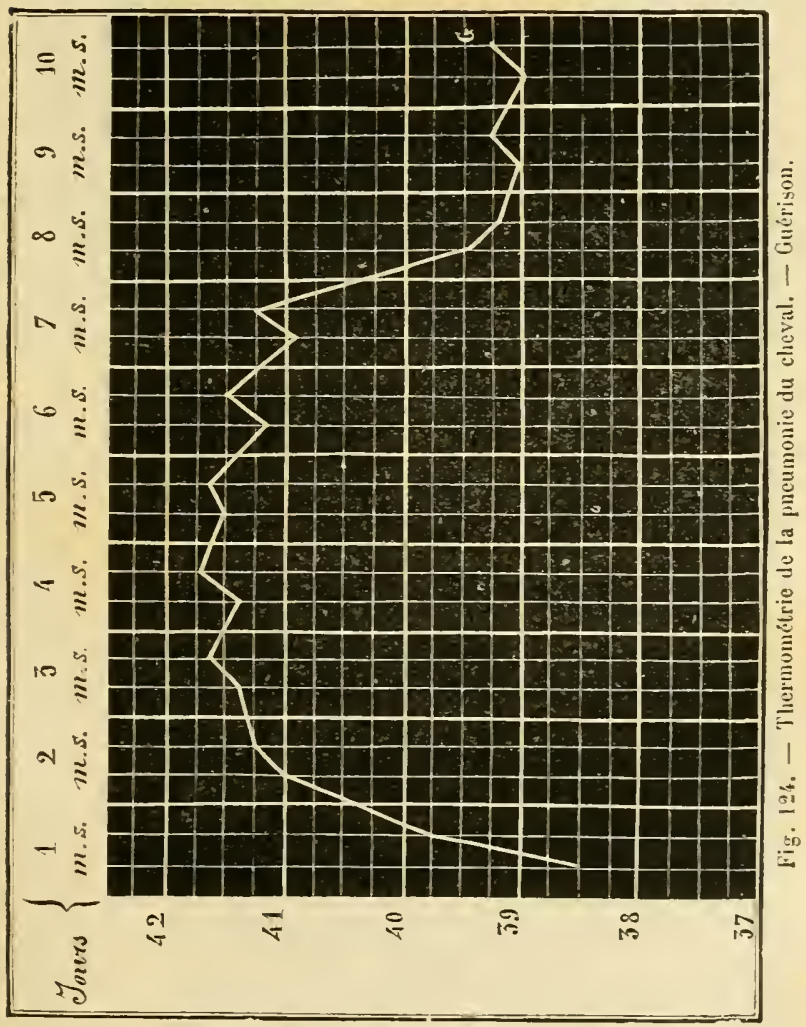

blements généraux qu'on peut surtout remarquer, chez nos animaux, aux muscles du grasset et de l'olécrùne, en même temps qu'on percoit un manifeste refroidissement des extrémités. 
Le frisson fébrile est le résultal d'une loxine spéciale qui agit sur les centres nerveux. La toxine produit le spasme vasculaire, l'ischémie cutanée, d'où résultent le refroidissement el la sensation de froid et le tremblement réflexe.

La prenmonie, la septicimie, la bronchite aiguë, le charbon, la pleuresie, la pericurdite tranmatique du boent sont marquées par la brusque apparition de l'hyperthermie et des frissons.

Lorsque la période ascendante dure plusieurs jours, l'élévation de la température est plus ou moins régulière et se fait sans frisson. Chaque matin la température subit un abaissemen!, qui de jour en jour diminue d'am- . pleur. La période d'état est caractérisée par l'élévation maxima.

Elle peut ètre de faible ou de longue durée. Ainsi dans la pneumonie du cheval, elle dure ordinairenuent trois à quatre jours, et pendant tout ce temps, la température reste à peu de clıose près la mème. D’autres fois, elle dure à peine quelques heures, comme dans le charbon. symptomatique.

Parfois, au contraire, elle est de longue durée, et la température subit de nombreuses modifications : il en est ainsi dans la plupart des maladies infectieuses qui passent à l'état chronique. Quelquefois la période d'état est marquée par des oscillations brusques et sans cause appréciable; on donne alors à celte période le nom de stade amplibole.

La période de déclin est caractérisée par l'abaissement de la température qui tantôt se fait graduellement, tantòt. brusquement. Ordinailement, il se fait suivant le même type que l'ascension.

Quand la mort doit survenir, la température peut s'élever brusquement (fig. 1:6) ou bien s'abaisser (fig. 127) pour remonter au moment de la mort. L'élévation de la température peut mème continuer après la mort, ce qui 


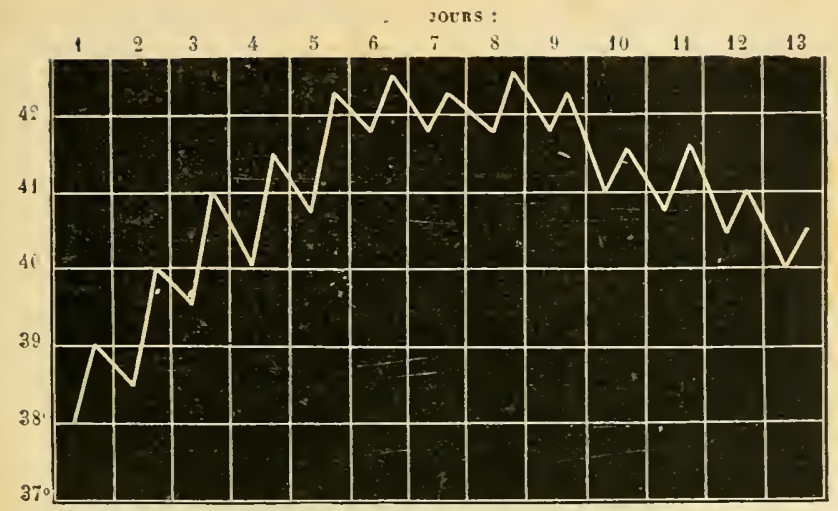

Fig. 125. - Tracé thermomètrique d'une pneumonie tı̣phö̈de du cheval (d'après Schmidt).

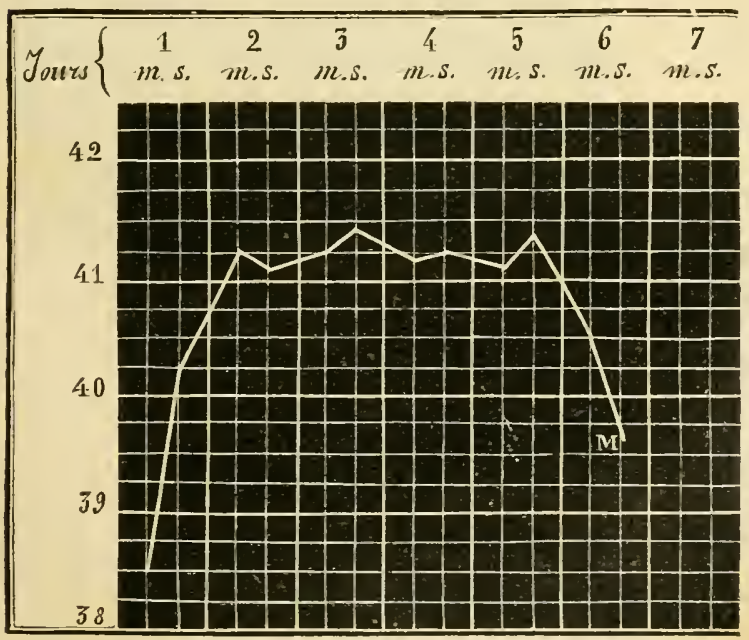

Fig. 120. -- Pneumonic du cheval. - Mort daus la déferiescence. 
lient à la suppression de l'évaporation pulmonaire et de la circulation cutanée.

Rien n'est donc fixe dans la marche de la température fébrile, qui se trouve sous la dépendance du processus morbide qui la produit. On a cependant tenté de grouper les divers lypes de fièvre en se basant uniquement sur les

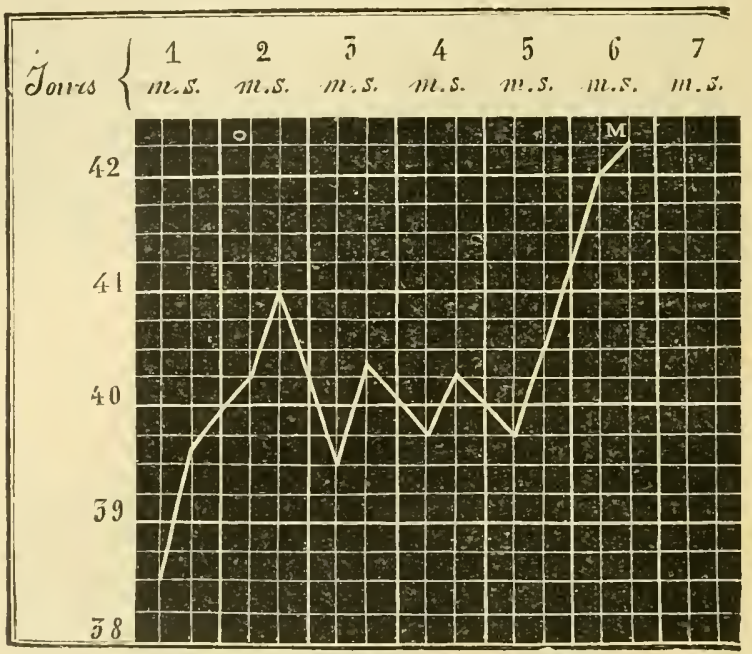

Fig. 127. - Pneumonie du cheval. - Mort en élévalion de la température.

modifications de la lempérature, et l'on a distingué des fièvres continues, rémiltentes, intermittentes, récurrentes et atypiques.

Dans le type continu, la température suit une marche à peu près régulière; ses oscillations ne dépassent pas $1^{\circ}$; on la rencontre le plus habituellement dans les grandes pinlegmasies aiguës, dans la pneumonie infectieuse des solipides, la peste bovine, la pleurésie, elc.

Dans la fièvre rémittente, la température subit des 
oscillations de plus de $1^{\circ}$; ce sonl surtout les maladies infeclieuses qui nous en donnent des exemples.

La fièvre intermittente se caractérise par des accès fébriles plus ou moins longs alternant avec des périodes presque apyrétiques. Chez nos animaux, on ne l'observe guère que dans la pyolémie; parfois aussi dans la septicemie. C'est surtout chez l'homme quon recontre ce type de fièvre (fièvres intermiltentes, fièrres paludéennes). Ia fièvre récurrente, qu’on n’observe que chez l'homme, est caractérisée par des accès de fièvre de cinq à six jours alternant avec des périodes non fébriles de mème durée.

Eıfiu, dans la fièvre atypique, les oscillations de la température sont irrégulières. C'est le type le plus fréquent cliez nos animaux domestiques (gourme, maladie du jeune age, fière pétéchiale, elc.).

Tels sont les différents types de fièvre.

Comment se produit l'élévation de température? Quelles sont les causes et le mécanisme de cette hyperthermie?

Y a.t-il, dans la fièvre, production anormalede calorique et par suite augmentation des combustions, ou bien simplement rétention plus ou moins complète de la chaleur que produil habituellement l'organisme?

Les recherches récentes démontrent pẻremptoirement que les dépenses chimiques de l'organisme sout augmentées dans la fièvre.

Cet accroissement est dénoncé : $1^{\circ}$ pal' l'exagération des combustions respiratoires; $2^{\circ}$ par l'exa gération du rayonnement calorifique; $3^{\circ}$ par l'accroissement de l'excrétion azotée; $4^{\circ}$ par la perte de poids des malades; $5^{\circ}$ par la chute rapide et profonde du quotient respiratoire consécutive à la dénutrition.

Cet ensemble de faits établit l'accroissement des combustions et de la production de chaleur. Pourtant divers auteurs se sont attachés à montrer que, dans la fiẻvre, il y 
a une vaso-constriction de la peau el par suite rétention d'une parlie de la chaleur.

Pour Traube, e'est la rétention de la chaleur qui eause 'hyperthermie.

Liebermeister et Kiœnig ont cependant démontré qu'un rébricitant plongé dans un bain perd plus de chaleur que normalement et Leyden, en employant le calorimètre, a fait la mème constatation. Les pertes de calorique augmentent done dans la fièvre, mais l'exagération de cette déperdition serail loin d'être en rapport avec l'hyperthermie; du reste, les sueurs font défaut pendant la période d'ascension et d'état et, comme l'a montré Senator, les capillaires de la peau sont plus souvent contractés que dilatés chez le fébricitant.

Au contraire de Traube, qui n’a vu que le frisson, Marey n'a considéré que la dilatation des vaisscaux cutanés chez les fébricilants et la chaleur des téguments. D'où cette conclusion que l'hyperthermie résulte d'une nouvelle répartition de la clıaleur qui se répand dans tous les points; mais cette théorie ne saurait intirmer les faits qui préeèdent.

En résumé, il y a dans la fièvre augmentation des combustions; mais il faut qu'il y ait rétention d'une partie de la chaleur produite pour expliquer l'hyperthermie.

Effectivement, l’organisme lutle victorieusement à l'état normal contre toutes les causes d'echaullement. Il y a donc dans la fièvre un trouble de la régulation. Le fébricitant est un malade qui règle sa température à un degré plus élevé que le degré normal (Löwil). Ce phénomène résulte ineontestablement d'une action spéciale des poisons thermogènes sur le système nerveux.

Comment la fière disparaît-clle? - L'acè̀s fébrile eesse quand le poison a été éliminé ou détruit par les tissus; alors les centres thermogènes n'étant plus sollieités à produire les troubles nutritifs qui élèvent la température, la chaleur se produit suivant le type normal, la fièvre s'éleint. 
Dans les fièrresinfectieuses, la guérison exige la destruction des bactéries, l'affranchissement de l'organisme à l'égard des poisons; et le retour de la nutrition à l'état normal. La fin de la fièrre, dit Bouchard, c'est le retour à la consommation normale de la matière.

Troubles des divers appareils. - Mais la fièvre ne se traduil pas seulement par l'hy perthermie; d'autres manifestations lui font cortège.

L'ACCÉlÉratiox dC pools est manifeste et on la considère comme l'un des signes les plus importants de la fièrre. Cette accéléralion résulterait de l'élévation de la température, car elle se produit presque tonjours, ainsi que l'ont démontré plusieurs auteurs, quand on élève artificiellement la température d'un animal.

On a admis qu'une élévation de température de un degré entraiuait une augmentation de huit pulsalions par minute. Il n'y a rien d'absolu à ce sujet, car le pouls peut rester lent malgré une élération considérable de température; chaque fois que le pneumogastrique est excité, le pouls est ralenti.

Du reste, la fréquence du pouls est liée à la tension artérielle à laquelle elle est inversement proportionnelle. Or. M. Marey a démontré que parfois, dans le cours de la fievre, la teusion augmente el le nombre des pulsations diminue. Il y a accélération tant que le cœur n'a pas éprouvé de dégénérescence; il esiste cependant des poisons microbiens qui ralenlissent le cœur, comme ceux du streptocoque de l'érysipéle, du bacille diphtéritique, du bacillus coli.

Tantòt le pouls est fort, dur; tantòt, au contraire, il est petit, filiforme, ce qui tient à l'état du cœur. A la fin d'une longue fièvre, le pouls sera toujours petit, faible, car le cœur, dégénéré, est épuisé. Pendant la fièrre, on remarque aussi l'accélération des mourements respiratoires; cette modification est la conséquence de l'élévation thermique.

Cadéac. - Sémiologie, 2 e édit.

11. -2 it 
La tension artérielle est généralement diminuée dans la fièvre. Il existe cependant des produits vaso-constricteurs comme les toxines pyocyaniques qui empêchent la dilatation ıasculaire et la diapédèse et augmentent la tension artérielle; mais la plupart des toxines, comme la maléine, dilatent les vaisseaux, abaissent la tension et accélèrent le cceur.

Le SANG est généralement fluide, il se coagule mal et plus lentement, il prend quelquefois une teinte noire et asphyxique; l'albumine est diminuée, la fibrine augmentée; il offre des plaques phlegmasiques (fibrilles réticulaires nombreuses et épaisses) quand on le fait coaguler sous le microscope; il y a leucocytose. La capacité respiratoire du sang est diminuée, mème avant l'élévation de la teınpérature; les globules rouges diminuent de nombre pendant la fièvre et augmentent la défervescence ; il y a alors une véritable poussée hématoblastique.

La respiration et le chimisme respiratoire sont également modifiés dans la fièvre. I'après Kaufmaun, "chez l'animal féhricitant, en étal d'abstinence, les phénemènes chimiøues intraorganiques sont simplement exagérés, mais non modifiés dans leur nature; le quotient respiratoire couserve à peu près sa valeur normale et la clıaleur produite en excès est sensiblement proportionnelle à l'excès de l'oxygène absorbé; la totalité de la chaleur produite peut être rapportée à un simple processus d'oxydation faisant passer l'albumine et la graisse à l'état de sucre et le sucre à l'élat d'acide carbonique et d'eau ". Mais dans les maladies, il faut distinguer à cet égard :

10 La période d'activité ou de lutte, caractérisée par une exagération de la ventilation, c'est-à-dire parl'accélération des mouvements respiratoires el l'augrnentation de l'oxygène absorlué et de l'acide carbonique rejeté;

2 La période de défaillance ou de défaite dans laquelle lous les échanges tombent à des chillres très inférieurs.

En somme, les oxydations sont accrues proportionnelle- 
ment à l'élévation de la température chez les malades qui guérissent.

L'apPareil digestif présente également des modifications. La bouche devient sèche, chaude, fuligineuse; la soif est vive; le fébricitant a perdu l'appétit; il est toujours constipé quand l'iutestin n'est pas lésé. Dans le cours de la fièvre, le bout du nez devient toujours sec; ce signe est très important chez le chien et les bovins. Chez ces derniers aninaux, les cornes sont le siègre d'une chaleur anormale, ou présentent des alteruatives de chaud el de froid.

La SÉcrétıox urixaIre subil de notables changements. A début, la tension artérielle étant forte, les urines sont augnentées ; elles sont ordinairement plus colorées, plus denses, plus riches en matières extractives. Il y a une plus grande quantité d'urée, quoique les fébricitants soient habituellement à la diète.

Dans la fièvre, il y a destruction de la matière et fermentations intracellulaires, l'oxydation vient ensuite, comme en témoignent les urines des fébricitants.

L'urine fébrile est plus chargée en produits toxiques et en matières colorantes; elle renferme enfin une quantilé double ou triple de potasse, mais la soude, au contraire, qui domine dans les tumeurs et particulièrement dans les exsudats, tombe parfois au huitième de l'état normal.

Les MusCles el la Graisse fournissent la plus grande quantité de matériaux à la destruction fébrile; les muscles perdent leur glycogène et subissent diverses dégénérescences, la graisse est résorbée et brûlée ; ces deux tissus perdent de leur poids; ils fondent pour ainsi dire dans cerlaines maladies aiğuës, comme la pneumonie.

Les REINs sont congestionnés et souvent hémorragiques; les cellules éprouvent la tuméfaction trouble ou subissent la dégénérescence graisseuse.

Le fole perd son glycogène et subit diverses dégénérescences par action toxique et par hyperthermie.

A ces troubles anatomiques et fouctionnels s'ajoutent 
souvent des troubles de l'innervation : l'animal est triste, abattu, daus le coma. Chez l'homme, ces troubles nerveux offrent parfois une grande intensité; leur forme la plus ordinaire est le délive.

Les sécrétroxs sont diminuées. La salive est plus rare et la bouche est sèche, excepté chez le chien, dont la salivation joue le même rôle modérateur que la sudation chez les autres animaux.

Le suc gastrique est moins abondant, dépourvu d'acide $\mathrm{HCl}$ el, partant, de puissance digestive.

La bile diminue ou est supprimée; la sudation seule est augmentée sous l'inlluence de l'élévation de la température et de l'intoxication.

Certaines toxines comme la maléine provoquent chez le cheral et le chat une sudation très nette trois minutes après linjection veineuse en produisant une vaso-dilatation intense, car la seclion du sciatique supprime la sueur dans le membre énervé.

Pathogénie. - Au point de vue pathogénique, on peut distinguer des fièves fonctionnelles, produiles par le fonctionnement exagéré d'un tissu ou d'un appareil dont l'activité normale est source de chaleur, et des fières nutritives qui résultent de la vie exagérée de tous les tissus (Bouclıard).

Fièvres fonctionnelles. - Parmi les fièvres fouctionnelles, il faut citer la fièvre musculaire, et la fièvre de digestion.

La fièvre uusculaire résulte d'une contraction musculaire prolongée (télanos); la température s'élève d'un degré et quelquefois même davantage chez le cheval; mais cette élévation est fugace; la compensation entre la production de chaleur et sa déperdition par la sudation se fait vite; au bout d'une heure environ de repos, la température redevient normale. 
La FIÈVEe de DIGESTION est peu appréciable; elle provoque seulement une élévation de tempéralure de quelques dixièmes de degré.

Fièvres nutritives. - Les fièvres nutrilives comprennent des fièrres nerveuses et des fièvres toxiques.

Les' fièvres d'origine NERveuse résultent d'un trouble dans l'innervation.

Les lésions des centres nerveux (hémorragies cérébrales, écrasement de la moelie), les affections convulsivantes comme l'epilepsie, J'iclampsie, le tétanos l'engendrent.

Dans ces ditférents cas, la tempéralure peut dépasser $\mathbf{1 2}^{\circ}$. C'est particulièrement dans le télanos qu’on a observé les températures les plus élevées; le thermomètre peut marquer $40^{\circ}$ après la morl.

Les fièvres toxiques sont les plus communes : les intoxications quelle que soit leur origine peuvent modifier la tempéralure; mais les effels des poisons varient suivant la dose et le mode d'introduction du poison, le sujet choisi pour l'expérience et l'agitation de l'animal ou son immobilité.

Les recherches des physiologistes ont démontré l'existence, à la base du cerveau, de centres thermogénétiques, dont l'excitation élève la température.

Les poisons médullaires, comme la strychnine, la thébaïne, la picrotoxine, la caféine, la spartéine, la piridine, etc., élèvent la température, en exaltant le pouvoir excito-moleur de la moelle et en exagérant les contraclions musculaires.

Les poisons périphériques, comme le curare, produisent une vérilable fièvre.

La pathogénie des autres fièvres d'intoxication se précise de jour en jour. En 188't, Weber élablit que la fièvre traumatique résulte de la résorption des produits pjrétogènes élaborés dans le foyer lésé; des expérieuces innombrables ont démontré la véracité de celle opinion. 
L.es poisons animanis pyrétogènes sont très nombreux. la plupart des tumeurs et des tissus d'un animal, infectés à un individu de la même espèce et surtout d'une espèce dillérente, élèvent la température. le bouillon de viande est pyrétogène; l'extrait alcoolique de rate est hyperthermisant; le ferment de la coagulation du sang injecté dans les veines peut produire une élévation de température qui va jusquà $4^{40}$; le sang, le sérum sanguin, les extraits d'organes, les urines son pyrétiques; les plénomènes de la vie cellulaire, hydratation, oxydation de la molécule albuminoïde, produisent de la chaleur et toutes les cellules vivantes de l'organisme produisent des toxines pyrétogènes susceptibles d'engendrer la fièvre. Il en est aiusi dans la goutte; daus le surmenage, il y a également accumulation de produits de déchet quicausent la fièvre.

Ce sout des fièvres par unto-intoxication, comme la fièvre parnecrose aseptique comne celle qui succède à l'oblitèration artériclle, spontanée ou expérimentale. Gangolphe, Courmont, etc., ont démontré que la fièvre diminue en pareil cas, á mesure qu'augrmente la mortification des tissus; elle cesse quand la nécrose est complèle; la matière pyrélogène rúsulte done de la désintégration des cellules en voie de mortification. De mème la nérose testiculuire froduite chez le hélier par bistournage est apyrétique, mais si l'on produit la nécrose an moyen d'une ligature jetee en masse sur le cordon et ses enveloppes, tout ell gardant la facilité de rétablir la cirrulation, on voit apparatre une hyperthermie considérable quand on enlève la ligature; à ce moment, on jette dans la circulation les produits de la nécrose et la fièvre cesse quand la résorplion est complète. " (Courmont.)

D'ailleurs les recherches expérimentales ont démontré que lis produits de destruction des flobules blanes et l'hémogluhiue sont pyrétogènes; enfun la mutrition viciẻe des lissus traumatisés engrendre une substance pyrétogène. 
Rogger ayant remarqué que le sang veineux contient une substance thermogène volatile qui s'élimine par les poumons et que le sang artériel contient une substance hypolhermisante qui s'élimine par le rein et qu'il y a enfin une autre substance qui se forme dans le rein aux dépens du sang, attribue à ces substances une action régulatrice de la températıre.

"Le froid, en activant la sécrétion rénale, favorise l'élimination de la matière hypottsermisante ei eutrave l'exbalation de la substance volatile thermogène, ce qui permet à l'animal de résister au froid. La chaleur a l'etfet inverse, en favorisant l'élimination de la substance volatile thermogène, elle diminue la sécrétion rénale et par conséquent l'éliminatiou de la substance hypothermisante." (Roger.)

Les fièrres d'origine tosigue sont infiniment plus importantes. Elles procèdent de substances toxiques ou médicamenteuses ou de toxines.

Toutes lesintoxications peuvent modifier la température, mais les résultats varient avec la dose et le mode d'introduction du poison, avec l'agitation de l'animal, etc.

Les poisons élèveut la température par trois procédés différents : en meltant en jeu les centres de calorification, en activant les combustions organiques, et en provoquant des convulsions. Les matières pyrétogènes sont d'origines très diverses.

Le règne minèral ne fournit pas de poisons franchement thernıgènes. Cependant l'eau distillée injectée en grande quantité dans les veines produit la fièvre.

Les poisons vézétaux pyrétogènes sont trẻs nombreux; ils agissent sur le cerveau, le bulbe, la moelle ou les nerfs.

Les poisons cérébraux (anesthésiques, morphine, quinine) après a voir élevéla température ne tardent pas à ètendie leur action sur la moelle el à produire la résolution musculaire et l'hypothermie, conséquence de l'inerlie musculaire. 
Les poisons bulbaires (aconitine, vératrine, digitaline) atteignent directement la fonction thernique des centres nerveux; ces poisons élèvent la température périphérique, mais diminuent la température centrale.

l.es fièvres infectieuses sont les plus communes; mais la maladie infectieuse n'est fébrile que parce qu'elle est toxique. Les microbes ont en effet la propriété de créer des substances pyrétogènes.

Weber a démontré que les produits de la putréfaction sont phlogogènes el hyperthermisants; Chauveaı a produit l'élévation de température par l'injection de pus ou de liquides putrides; Brieger a découvert la mydaléine, qui produit une grandelyyperthermie. Les toxines renfermées dans les cultures donnent les mèmes effets.

Serafini (1887) a produit la fièvre chez le chien en injectanldes cultures stérilisées du bacilie de Friedländer.

Depuis, de nombreux expérimentateurs ont prouvé ıjue celle propriété appartient à de nombreuses loxines microbiennes et au protoplasma des microbes.

La clinique est venue aussi apporter ses preuves : ce sont les maladies microbiennes qui produisent les fièrres les plus intenses.

Par quel mécanisme les toxines produisent-elles la fièvre? - On prétend que toute substance altérant le sang ou rendant les globules rouges incapables de fixer une portion normale d'oxygène fait éclater la fièvre. Pillon attribue la fièvre à la mise en liberté des matériaux constituants des leucocytes et des hématies détruits par les microbes ou leurs loxines.

Les loxines aq̣issent-clles sur chaque élément cellulaire et, le reudant moins stable, favorisent-elles ainsi les oxydations? Ou bien est-ce en agissant sur les centres nerveux qu'elles produisent un accroissement de la calorilication?

Quoi qu'il en soit, on peut regarder la fièvre comme la consétuence d'un trouble nerveux primitif ou bien, sui- 
vant les cas, comme le résultat d'une infection de l'organisme par des produils de dénutrition, soit végétaux, soit microbiens ou élaborés pal l'organisme lui-même.

Signification. - La lièvre est une manifestation symptomatique d'une grande importance au point de vue clinique. Dans la plupart des cas, elle témoigne d'une infection de l'organisme.

Lorsqu'à la suite d'une operation, une fièvre intense s'allume, c'est que la plaie a été infectée et la fièvre devient en quelque sorte un ordre de pratiquer la désinfection de la plaie. Ala suite de l'opération du javart, dı clou de rue, etc., on peut à volonté produire la fièvre, l'exagérer, la diminuer ou la supprimer en entretenant des germes à la surface des plaies ou en maintenant celles-ci parfaitement aseptiques. La désinfection, l'emploi des antiseptiques tarissent la source de la lièvre en détruisant à la fois les poisons qui l'allument et les germes qui sécrètent ces poisons.

La valeur sémiologique de la fièvre varie avec les types. Une ascension régulière est un signe favorable, une ascension irrégulière est un signe défavorable. Un abaissement brusque, une longue période d'état avec des oscil'ations de Ja température sont également des signes fâcheux (fig. 126).

Cependant la fièvre peut être regardée comme un moyen de défense de l'organisme qui lutte pour se débarrasser des produits de dénutrition et de leur canse. La fièvre n'est pourtanı pas un syndrome indispensable pour qu'il y ail guérison.

Celle-ci peut se produire malgré l'abaissement extrème de la température (fig. 128).

Il est exceptionnel de rencontrer la température de $42^{\circ}$; la septicémie et la pneumonie infecticuse sont à peu près les seules maladies qui présentent celte élévation de la température. 
Par contre, la température de $41^{\circ}$ se rencontre dans beaucoup de maladies : fievre typhoüde, pneumonie gourmense, pneumonie lobaire, ficre puerpérale, scplicèmic, peritonite, pericardite traumatique, charbon.

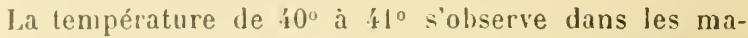
ladies précédentes el dans les suivantes : maladie du jeune âge, endocarlite aigü̈, cucephalite aiguë, catarrhe ai!ju bronchique.

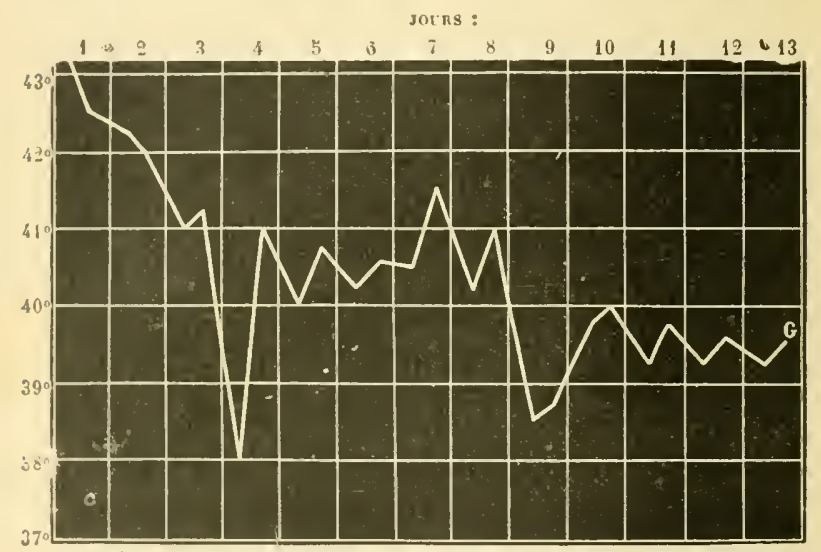

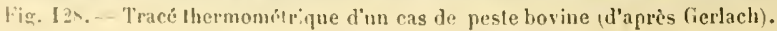
Malgré le collajpsus, il y a eu guérison.

l.es tempriratures hyperpyrétiques sont réalisées par le coup de chaleur (cheral, mouton, buint, porc), par le lougget du pore et le titanos, maladie dans laquelle la enpérature peut alteindre $4 i^{\circ}$ au moment de la mort Bayer) par la piroplasmose (maluria, fière du Texas, verlurter, elc.).

Dans l'espece borinc, on constate que les boufs égyplieus qui ont succombé à cetle dernière maladie prisentent, dans les premières heures qui suivent la mort, une lempérature intra-abdominale qui alteint et 
dépasse mème $\dot{q}^{\circ} \dot{q}^{\circ}$ centigrades (la température ambiante variant de 23 à $2 \tau$ degrés). A l'autopsie de ces animaux, pratiquée de la première à la quatrième heure après la mort, on est immédiatement surpris par la sensation de chaleur intense que produit le contact des viscères abdominaux. La température rectale de ces animaux avant la mort était pour les uns de $37^{\circ}, 5$ à $38^{\circ}$; pour les autres de $40^{\circ}, 3$ à $41^{\circ}, \dot{4}$; la température s'élève après la mort sous l'influence d'une fermentation secondaire dont l'agent actif est inconnu et auquel la piroplasmose préparerait le terrain farorable à son érolution.

La fièrre, considérée par les anciens comme un bienfait, conme un phénomène réactionnel et curatif, comme un effort de la nature médicatıice, est regardée par les modernes comme un mal, un danger constant et quelquefois une cause de mort. Mais la gravité de beaucoup de maladies est indépendante de la température qui préserve les animaux de certaiues infections, comme la poule du charbon.

L'évolution du pneumocoque est retardée chez le lapin infecté, soumis au chauffage; l’érysipèle expérimental de l'oreille du lapin a une marche plus rapide et plus restreinte quand on chauffe l'animal. L'invertine, aux doses qui sont mortelles en quelques semaines, ne tue pas les animaux dont on élère la température à $41^{\circ}$.

Les températures élevées sont quelquefois nuisibles ì la vie des microbes, et favorables à l'aclivité des phagrocytes et au pouvoir bactéricide du sérum. Cependant, les substances antitoxiques se développent anssi bien chez leś animaux maintenus artiliciellement apyrétiques que chez ceux qui ont de la fièvre.

Diagnostic raisonné. - L’ẻlération de la température permet d'apprécier la gravité et la nature des maladies, quand on interroge en même tenıps les signes fournis par les divers appareils. 
La septicrimie, qui complique fréquemment les maladies nternes el les maladies chirurgicales, commence par des frissons viulenis, une température de $42^{\circ}$, des battements cardiaques Irès violents et un pouls très faible sans signes locaux. La stupéfaction des animaux est très grande; l'évolution morbide est tıès courte; ces caractères permettent de différencier cliniquement la septicémie des inflammations locales et mème, au début, des maladies contagieuses èruptives (tièvre aptheuse, horsepox, clavelie, etc.), qui s'annoncent par une forte fièvre, mais qui ne tardent pas à être accompagnées de leurs signes caractéristiques.

La pyohemie et toutes les affections marquées par la formation de pus ou d'alıcès superficiels ou internes (abcès pulmonaires, abcès mésentériques intestinaux, etc.), sont dénoncées par une fièrre rémittente, changeante, irrégulière, accompagnée de frissons fréquents; Jeur durée est longue; l'amaigrissement de l'animal est progressif et continu.

Ia gourme et la maladie dı jeune âge, qui débulent comme les maladies infectieuses, se comportent ensuite comme des maladies purulentes, la fievre est atypique, irréguliere: la gourme est bientôt suivie du développement de ses symptòmes et de ses lésions pathognomoniques (pharyngite, adénite, lymphangite, etc.); la maladie du jeune àge, de ses manifestations catarrhales : catarrle nasal, bronchique, pulmonaire; catarrhe gastrointestinal, ou de troubles nerveux (convulsions épileptiques, chorée, etc.), ou enfin d'une éruption significative.

Le coryza gangreneux se distingue des autres affections du bouf par une température très élevée accompagnée de larmoiement, de lératite, de stomatite, de néphrite, de cystite, de vertige el de coma.

Jans la fierre charbonneuse, l'élévation de la température, qui atteint fréquemment $42^{\circ}$, est rendue significa- 
tive par le pissement de sang, le jetage sanguinolent, la dyspnée, la cyanose, l'excitation cérébrale, la marche foudroyante de l'affection chez le mouton et sa forme subaiguë chez le cheval.

Le rouget du porc débute par une température de $42^{\circ}$ à $43^{\circ}$; on observe ensuite des taches rougeàtres ou bleuâtres dans les points où la peau est froide.

Dans le choléra el la pneumo-entérite du porc, la fièvre est moins intense. Quand les porcs sont très fiévreux, on doit toujours soupconner une maladie infectieuse et pratiquer immédialement leur isolement.

En temps d'épizootie, l'apparition brusque de la fiève chez un animal fait soupçonner le développement de la maladie infectieuse et permet de prendre toutes les mesures capables d'en empècher l'extension.

\section{II. - HYPOTIERMIE.}

Définition. - On désigne sous le nom d'hypothermie, de collapsus ou de réaction algide, un syndrome caractérisé par un abaissement anormal de la température organique.

Normalement, l'animal, ayant une température plus élevée que celle du milieu dans lequel il vit, lutte continuellement contre le refroidissement par le réflexe culané, vaso-constricteur, par l'excitation réflexe des centres thermogènes et par une production plus grande de chaleur.

Causes de l'hypothermie. - Les causes déterminantes de l'hypothermie sont : le froid, l'inanilion, les traumatismes, les intoxications et aulo-intoxications et les infections.

$1^{\circ}$ Le FroId extérıeur produit l'hypothermie, chaque fois que son intensité dépasse les limites et les ressources du pouvoir de régulation. Les animaux sont incapables de

Cadéac. - Sémiologie, حe édit.

II. - 25 
se réchauffer quand la t’mpérature centrale est descendue a $20^{\circ}$, parce que les extrémités cutanées des nerfs sensitifs sont paralysées et par conséquent incapables de régler la production de chaleur d'après l'intensité du rayonmement périphérique.

2. L'iñरitiox est suivie de la destruction progressive des réserves de matériaux bydrocarbonés qui produisent la chaleur. Au premier jour de l'inanition, à l'avantdernier jour de la vie, la température décroît ainsi très lentement de $0^{\circ}, 3$ par jour, puis, le jour de la mort, la chute s'accentue brusquement et atteint $14^{\circ}$ en moins de vingt-quatre heures. D'ailleurs, on constate mème pendant la première période, un trouble considérable de la régulation; la température est pendant le sommeil de $3 \circ$ au-dessous de la température de la veille; ces différences témoignent de l'influence excitatrice qu'exerce le système nerveux sur la calorification et qui est plus intense pendant la veille que pendant le sommeil (Chossat). L’animal inanitié meurt en partie de froid ; il a perdu la propriété de conserver sa chaleur normale, il subit l'influence du milieu ambiant; il est devenu animal à sang froid. Ce phénomène s'observe dans le rélrécissement de l'œsophage chez les cancéreux.

$3^{\circ}$ Les traudatisues graves entrainent l'hypothermie ; cet abaissement de température est lié au choc iraumatique qui ralentit les échanges et produit les syncopes cardiaque et respiratoire. La douleur est hypothermisante; l'excitation des nerfs sensitifs est toujours suivie d'un abaissement plus ou moins prononcé de la température centrale, de sorte que les traumatismes les plus douloureux (lésions de la maelle, du bulbe, du cerveau, de l'épigastre, du testicule, traumatisnıes des organes thoraciques, brûlures) sont ceux qui produisent l'lypothermie la plus accusée.

Cette dépression est particulièrement prononcée dans les traumatismes des riscères abdominaux et les trauma- 
tismes cutanés; les uns et les autres s'accompagnent de phénomènes cardiaques, respiratoires et vasculaires identiques qui résultent du choc nerveux ou qui sont consécutifs à l'intoxication.

$4^{\circ}$ Les ixtoricatioxs sont généralement suivies d'hypothermie, qui est la véritable cause de la morl. A faible dose, presque tous les Ioisons (alcool, atropine, morpline, elc. élèrent la température; à forte dose, presque tous l'abaissent.

L'intoxication chronique par la digitaline, fait tomber la tempéralure du chien de $38^{\circ}, 9$ à $36^{\circ}, \bar{\imath}$. Les hadigeonnages de la surface cutanée arec du gaïacol, produisent l'hypotlıeımie ; la cocaine, l'ellél orine, la spartéine, la solanine, ont une influence aualogue.

Les agents anlipyrétiques, administrés à des sujets affaiblis ou malades, produisent quelquefois une hypothermie dangereuse; ce sont d'ailleurs de véritables poisons des globules et bcaucoup produisent la rasodilatation périphérique.

Certains poisons comme le curare, la morphine et le chloral diminuent la thermogenèse.

$5^{\circ}$ Les atтo-nтолісатіокs, comme celles qui résultent de l'arrêt de la sécrétion uriuaire, produisent l'hypothermie. On peut l'obtenir facilement par la ligature des uretères.

Le sérum du Joeuf, de la chèrre, du mouton, le sang artériel, les exlraits musculaires des sujets sains, la bile, sont également lyypothermisants.

La résorption des produits engendrés par la fermentation des matières fécales engendre l'hypothermie dans la deuxième pluase de l'obstruction intestinale.

$6^{\circ}$ L.es INFECTIONS MICROBIENNES engendrent ordinairement l'bypothermie; l'hypothermie est exceptionnelle au début de l'infection; mais elle succède soureut à une phase d'hypothermie plus ou moins prononcée.

L'injeçtion sous-cutanée des cultures du bacille pyocya- 
nique produit l'lyypothermie immédiate; lempoisonnement par les toxines diphléritiques est caractérisé à la période terminale par une hypothermie considérable. Dans le stade secondaire de la fierre charbonneuse, on observe également de l'hypothermie correspondant à la pullulation des bactéridies dans le sang.

L'abaissement de température se produit d'autant plus rapidement que la quantité de toxines déversée dans le sang est plus importante. On peut ainsi observer, tantôt de l'hypertbermie, tantôt de l'hy pothermie, suivant la quantité de toxines élaborées par les microbes et suivant le degré de virulence et la porte d'entrée de ces derniers.

L'hypothermie fait défaut chez les animaux réfractaires; l'hyperthermie se manifeste seule comme signe de la réaction de l'organisme contre les effets des toxines; les formes les plus graves des maladies aiguës sont celles qui sont marquées par une période fébrile plus courte.

L'hyputhermie se manifeste quelquefois chez le bœut sous l'inlluence des maladies cachectisantes comme la lymphudenie, les tumenrs, la tuberculose; on voit quelquefois la température descendre à $36^{\circ}$ après l'injection de tuberculine à la suite de troubles digestifs mal définis (Cantiget).

Chez l'âne, l'hypothermie est un phénomène consécutif it la coprostase, à l'anèmie déterminée par une maladie "énérale ou par une opération comme la castration ; j’ai vu la température descendre à $32^{\circ}$ sous l'influence de la diète accompagnée d'une constipation opiniàtre.

\section{II. - GOLlapsus.}

Définition. - Le collapsus algide est caractérisé à la fois par l'abaissement anornal de la température orgauique, lc ralentissement du pouls et de la respiration.

La tempéralure indique le collapsus quand elle est inférieure on ne dépasse pas $36^{\circ}$ eq que cette hypolher- 
mie s'accompagne de troubles graves des autres appareils de l'orgauisme. Cet état qui précède l'agonies'accompagne d'une grande diminution de l'activité cardiaque el respiratoire. C'est donc toujours un signe défavorable.

Le collapsus précède la mort dans les empoisonnements graves, dans l'ictère el dans diverses maladies aiguës ou chroniques; il peut se manifester aussi dans les maladies infectieuses suivies d'intoxication et de la production de substances hypothermisantes.

Le collapsus porte aussi le nom de réaction algide.

Ce trouble saccompagne toujours d'une grande prostration des forces el fait partie des manifestations du choc traumatique.

Pathogénie. - Il parait dépendre d'une contracture des petits vaisseaux liée à une excilation des vaso-constricteurs (Marey). Le calibre des arlères se trourant rétréci, le sang ne circule plus qu'en quantité insuffisante dans les capillaires et s'accumule dans les troncs veineux, d'où l'insuffisance de l'hématose par suite du trouble de la circulation pulmonaire Hallopeau), l'abaissement de la température, l'asphyxie, l'anurie, l'anémie encéphalique.

On a également attribué le collapsus à un épuisement du système nerveux (Richet), à la paralysie du cœur déterminée par l'excitalion des nerfs centripètes et suivie de troubles des ganglions aulomoteurs ou du pneumogastrique.

La pathogénie de ce syndrome n'est pas encore complètement élucidée. 


\title{
ÉVOLUTION DES MALADIES
}

\author{
CHAPITRE PREMIER
}

MARCHE DES MALADIES

\section{I. - PROPAGATION DES TROCBLES MORBIDES.}

Circonstances qui font varier l'évolution de la maladie. - L'évolulion de la maladie dépend de la cause provocatrice el du torrain.

La cause de l'altération irritative ou dégénérative, qu'elle agisse par sa qualité (mécanique, physique, chimique ou dynamique), par sa quantité (microles, parasites), influence l'ensemble de l'organisme.

Elle impressionne le système nerveux, modifie la répartition des humeurs en créant des congestions, des inflammations, des liémorragries, etc., etc., change la température et la composition des humeur's (fievre, lcusocylose, etc.), retarde la composition du sucre ou accélère la destruction des albuminoïdes, détermine l'accumulation dans le sang de produits de léchet qui surchargent le rein et nuisent au bon fonctionnement de lous les organes et principalement du système nerveux, le régulateur universel.

Par son interméliaire, la lésion primitive propaje ses effets dans tous les appareils : digestif, respir it jire, cir- 
culatoire, calorificatiou, sécrétion, etc. Le degré de participation de chacun de ces appareils à la scène morbide locale est en rapport avec la nature et l'intensité de la cause morbide. Il n'y a rien de fixe dans ces effets, la virılence peut varier de zéro jusqu'à 1000 ; les agents toxiques, les traumatismes eux-mèmes peuvent, suivant les doses et les degrés, déterminer le maximum ou le minimum de troubles.

A cette propagation de la réaction s'ajoute fréquemment l'extension de la lésion primitive. Et la propagation de la lésion, c'est la propagation de la maladie.

L'inflammation des premières voies respiratoires s'étend aux bronches, au poumon; l'inflammation de la bouche et de l'arrière-bouche se communique à l'estomac et à l'intestin; dans la tuberculose pharyngée, les bacilles de la tuberculose du pharynx vont se fixer dans l'intestin; c'est la propagation par continuité de tissu, ou par des infections secondaires et successives (pleurésies, péritonites).

Les vaisseaux sanguins et lymphatiques charrient les microbes, les toxiues, les solides et les liquides altérés et tous ces agents élaborés par l'altération primitive peuvent s'arrèter dans le voisinage ou dans des organes similaires où ils font souche de nouvelles altérations qui s'ajoutent à la maladie primitive ou créent des maladies nouvelles (embolies bactériennes, parasitaires, organiques, etc.).

L'évolution morbide, profondément modifiée par ces divers accidents, cliange encore davantage quand lc système nerveux est altéré dans ses dépendances ou dans ses centres.

Ses éléments sont si fragiles que le contact d'un sang altéré par la putréfaction d'un foyer traumatique circonscrit, ou par une lésion rénale, etc., peut rapidement compromettre la vie. Le danger d'une toxine, d'un produit qui a pénétré dans le sanğ, est proportionnel à son influence sur le bulbe. 
L'exaltation, l'affaiblissement de la cause sont une source indéfinie de variations dans la marche des maladies chez le sujet qui en est affecté. Ces modifications expliquent aussi l'extension et les propagations de la maladie aux divers animaux placés dans le voisinage du sujet infecté. Cette propagation au dehors engendre les enzooties et les épizooties.

\section{II. - ENZOOTIES.}

Définition. - On désigne sous le nom d'enzootie ou d'endèmie, la maladie qui sévit à l'état permanent ou périodique dans une contrée.

La fievre charbonneuse chez les animaux, la fievre cyphoïde, la fièvre intermittente chez l'homme, sont des maladies endémiques. Elles apparaissent généralement aux mémes époques de l'année, sous l'influence des mêmes conditions extérieures. En Auvergne, en Beauce, les spores charbonneuses qui proviennent de l'enfouis. sement des cadavres charbonneux, sont ramenées à la surfacedu sol par les vers de terre et déterminent des enzooties charbonneuses. Les bactéries de la fierre typhoïde, les germes du choléra se régénèrent ou conservent leır vitalité dans les eaux de puits, de mares, elc., et font développer des enzooties.

La cause de l'enzootie se trouve renfermée dans les aliments, les boissons d'une ou de plusieurs localités. Les maladies parasilaires revêtent le type enzootique comme les maladies microbiennes. Les bronchites vermineuses, la distomalose, l'helminthiase, le tomnis, sont essentiellement des maladies des pays marécageux.

Chaque pays a ses maladies endémiques. La Bresse, la puxion périodique; la Beauce, la fièrre charbonneuse; la Bresse, la Sologne, la cachexie aqueuse, la fievre intermittenle; la Savoie et les Pyrénées, le goitre; la Forêt-Noire, la llaute-Loire, l'ostéomalacic el le pica, etc. 
La cause d'une maladie endémique ne se trouve pas également répartie dans tous les endroits de la contrée ou de la localité où clle règne; les germes infectieux semblent concentrés dans certains points : champs maudits pour la fièvre charbonneuse, puits spéciaux pour la fièrre. typhoüde.

Les maladies endémiques, indépendantes des microbes, récidivent fréquemment ou passent à l'état chronique; les maladies microbiennes tuent rapidement ou confèrent l'immunité; le déplacement des sujets malades est pour toutes le seul remède salutaire. L'émigration du troupeau de moutons ou de boufs ravagé par le charbon bactéridien arrête brusquement l'endémie. On ne constate plus de malades quand on supprime les aliments ou les boissons infectés.

\section{III. - ÉPIZOOTIES.}

Définition. - Les épizooties ou épilémies sont des maladies caractérisées par leur apparition rapide sur un grand nombre de sujets à la fois et par leur exlension aux animaux de divers pays. L'idée d'épizootie implique l'idée de contagion. Les microbes infectieux sont propagés par les déplacements, les transporls en chemin de fer, les rassemblements d'animaux malades. La clavelée, la fière aphteuse, la péripneumonie contagieuse, la peste bovine sont des maladies épizootiques.

Toutes les affections dont les germes se conservent ou se multiplient dans le sol sont des endémies. Toutes celles dont les germes se propagent par contact médiat ou immédiat des animaux malades avec les animaux sains peuvent revêtir le caraclère épizootique. Les épizooties ne renaissent jamais de leurs cendres comme les endémies.

" La marche d'une épidémie de quelque gravité se traduit généralement par une courbe dont la partie descendante est plus rapide que la portion ascendante. En 
langrge ordinaire, cette courbe signifie que la contagion, propagée d'abord avec une grande intensité, s'esl aballue sur beaucoup de malades en peu de lemps, puis s'est brusıurment limitée à un petit nombre de sujets.

"Théoriquement, il semblerait que le nombre croissant des malades dut entraîner une production et une dissémination plus abondantes de virus et consé [uemment une augmentation loujours plus grande des cas nouveaux, de soste ufue la marche d'une épidémie devrait ètre régie par des lois analogues à celles du mouvement sur un plan incliné. Il y a donc entre les faits et la théorie une sorte de contradiction, frappante surtout dans les épidémies trausporlées hors lu lieu où elles sévissent habituellement, épidémies qui seéteignent tout à coup, après avoir fait rage. Mais sil'on prend la peine de l'examiner à la lueur desnotions acquises sur la płysiologie générale des virus, elle cesse d'exister.

"Les épidémies doivent s'éteindre, et s'éteindre rapidement, à un moment donné, sans l'interrention de moyens artificiels, parce que les facleurs d'une épidénie, les sujels et les virus se modifient peu à peu forcément et naturellement.

"Tous les individus d'une espèce ne possèdent pas le mème clegré de réceptivité pour un virus détermiué. L'expérimentation démontre qu'on peut les diviser, à ce point de vue, en trois groupes l'une importance inégale : $1{ }^{\circ}$ celui des sujets doués d'une grande réceplivité nalurelle; $2{ }^{\circ}$ celui des sujets pourvus d'une réceptivité moyenne; $3^{\circ}$ enfin, celui des individus dépourvus ou presque dépourvus de réceptivilé. Vers les points de conlact, ces groupes se confondent par transition insensible.

L'épidimie sévira l'abord sur les sujels du premier groupe oì le contage ne rencontre aucun olsslacle à se transmettre d'un indivilu à l'autre. Chaque malade constituant un milieu de culture aussi farorable que possible émettra une grande quantite le virus très actif; on verra 
donc le nombre des malades augmenter et atteindre rapidement le maximum.

"Les individus de réceptivité moyenne, qui avaient échappé à la contagion au moment de l'invasion, plongés maintenant dans un milieu plus riche d'un virus plus aclif, vont ètre atteints à leur tour, dans une certaine proportion, bien inférieure toutefois à celle des sujets du premier groupe. L'épidémie entrera dans sa période de déclin, laquelle se prononcera avec une grande rapidilé, allendu que la quantité de virus disséminée dans le milieu ambiant diminuera en raison du nombre des malades. La gravité de l'épidémie suivra une marche analogue, vu que, dans cette période, elle s'attaque à des sujets de moindre réceptivilé.

"Le contage finira par se trouver en présence des individus du troisième groupe. Là, il ne pourra faire qu'un bien petit nombre de victimes. En effet, ces sujets étaient préparés à la résistance avant l'apparition de l'épidémie; ils le sont encore mieux à la fin, car, vivant dans un milieu virulifère, ils ont probablement subi une ou plusieurs infections légères qui élèvent le degré de leur immunité naturelle.

“Ainsi, à n'envisager que les sujets, l'extinction des épidénies se concoit; il s'impose, si l'on envisage le devenir des agents virulents (1). " (Arloing.)

Pendant qu'il y a augmentation des résistances opposées par les sujets à la contagion, il y a diminution de l'activité pathogène des microbes infeclieux.

Cette atténuation est due à des causes multiples qui surajoutent leurs effets : les microbes répandus dans le sol, dans l'air, sont détruits en grand nombre par l'oxygène, la lunière, l'électricité, etc., leur virulence est affaiblie quand ils évoluent dans des organismes de faible réceptivité; leur nombre est diminué à la fin de la période

(1) Arloing, Les virus, 1891, p. 214. 
d'augment de l'épidémie par la limitation du chiffre des malades.

\section{IV. - DULÉE DES MALADIES.}

Le terrain qui favorise ou qui combat l'influence morbide préside encore plus que la cause à cette évolution.

A part quelques microbes extrêmement virulents, ou des traumatismes excessirement violents, la complicité de l'organisme est indispensable à la cause pour réaliser la maladie.

Ce consentement n'est jamais absolu; l'organisme se défend, nous l'avons vu, par divers moyens : le tube digestif alimente la circulation, le sang bactéricide maintient les éléments daus leur intégrité, les barrières épithéliales, la peitu, le tube digestif et les autres muqueuses s'opposent aux infections; la salive, le suc gastrique sont antiseptiques; la sécrétion parotidienne joue un rỏle mécanique et protège les conduits sécréteurs et les acini contre les inrasions microbiennes; les amygdales opèrent une abondante destruction de parasites par le processus de la phagocytose; la bile prête sa puissance antiseptique: les voies respiratoires, leur épithélium, leurs qualités bactéricicles, leur phagocytisme, leur puissance éliminatrice; la peau, sa résistance, ses acides gras; le système nerreux toutes ses forces dynamiques; tous les orranes el toutes les lumeurs, leur activité phagocytaire ou bactéricide; le sang agit sur les microbes par l'oxygène, l'acide carbonique, le mouvement, la pressiou, la vitesse.

Tous ces moyens de prolection agissent par des procédés différents ou avec une puissance très inégale : aussi la marche des processus est-elle considérablement influencée par la porte d'entrée des microbes (charton symptomatique); le bacille de kioch, qui ulcère ou sclérose le parenchyme pulmonaire, stéatose le foie. Ces diverses influences peurent fléchir plus ou moins de telle 
sorte que l'érolution morbide, rapide ou lente, est en rapport aussi avec les secours fournis par les divers organes à la réaction de l'économie.

\section{La durée des maladies est essentiellement variable.}

Les unes ont une marche très rapide, foudroyante même; on les désigne sous le nom de suraiguës; telles sont la septicémie, le charbon, etc.; d'autres éroluent plus régulièrement et plus lentement, se terminent dans un laps de temps qui ne dépasse pas six jours; ce sont les maladies aiguës. La pneumonie infectieuse, les affections typhoüdes, la clarelée, la fièrre aphtheuse sont des maladies aiguës.

Les affections éruptives et la plupart des maladies microbiennes qui rentrent dans ce groupe sont des affections cycliques dont la durée est généralement fixe pour tous les êtres d'une mème espèce. Quand ces maladies se sont développées, les symptòmes progressent jusqu'au moment où l'état bactéricide, la phagocytose, l'épuisement du milieu, les réactions organiques, l'affaiblissement des parasites par suite de leur usure propre, de l'hyperthermie ou des conditions de culture dans lesquelles ils se trouvent, amènent une amélioration qui se produit lentement ou rapidement.

Les animaux alteints de fierre aphtheuse, de clavelée, de pneumonie, sont malades pendant un nombre de jours qui varie peu pour la même espèce.

Expérimentalement, on peut cependant modifier le cycle connu en introduisant chez ces espèces des microbes plus actifs ou plus nombreux. La nature de l'agent pathogénique modifie également ce cycle; il y a autant de modes d'évolution que d'espèces microbiennes.

Certaines maladies dont l'érolution est plus prolongée que les précédentes, s'accusent par des symptômes qui sont également moins tranchés; on les qualifie de SUBAIGZEes.

L'état du terrain, la débilité d'un organe, d'un ṣ̣s- 
tème, les maladies générales diathésiques, les maladies anciennes, les influences extérieures favorisent l'acclimatement et prolongent leséjour des microbes dans l'économie; elles affaiblissent en mème temps les forces organiques et dyıamiıjues et rendent les réactions moins violentes.

Les MALADIES CHRONOUES sont primitives ou consécutives à des maladies aiguës; leur seule particularité c'est d'évoluer lentement, de persister longtemps ou indéfiniment. En dehors de ce fait, elles forment un groupe essenliellement disparate.

Leur nature est très variable. Les unes sont dues à des micrabes ou a des parasites qui trouvent toujours dansl'organisme les conditions favorables à leur développement (morve); d'autres présentent des poussées successives et envaluissent un numbre plus ou moins considérable d'organes (tuberculose). Certaines ont peu de tendance à guérir en raison du peu de vitalité des tissus lésés; les ulcères mélaniques se cicalrisent difficilement.

I.es maladies entretenues par mue diatḥèse (diathèse herpétique) affectent une chronicité désespérante (eauxaux-jambes, crapaud).

La plupart des néoplasies grossissent indéfiniment; les tumeurs malignes se propagent par la voie veineuse ou lymphatique et vont former des dépôts secondaires; l'évolution de ces maladies est aussi illimitée que leur propagation.

Certaines maladies aiguës peuvent enfin engendrer une lésion indélébile qui devient à son tour la cause d'accidents secondaires: les lésions du foie, du rein créent des troubles persistants de la circulation; l'excitation qui part d'une cicatrice étcndue, d'une tumeur, peut influencer le bulbe et produire l'épilepsic.

\section{V. - TYPE IDES MALIDIES.}

L'évolution des maladies peut ètre intermiltente, rémittente ou continue. 
$1^{\circ}$ L'intermittexce ou l'interruption des symplòmes se manifeste d'une manière régulière ou irrégulière; c'està-dire à des périodes égales ou inégales suivant les maladies. La fièvre paludéenne offre fréquemment le type régulier; l'épilepsie, la fluxion périodique se manifestent par des accès irréguliers, survenant après des rémissions d'un mois, trois mois, six mois, et même davantage. Dans l'intervaile des accès d'épilepsie et de fluxion périodique, l'animal présente tous les caractères de la santé.

$2^{\circ}$ La RÉMtTExce est caractérisée par des alternatives d'exagération et d'atténuation des symptômes. Ces variations sont physiologiques tant qu'elles sont légères; la température du soir dépasse toujours de quelques dixièmes de degré celle du matin; mais la maladie mérite d'ètre appelée rémittente quand la rémission est très marquée et se renouvelle périodiquement. Les chevaux frappés de vertige sont sujets à une excitation intense, suivie de coma.

L'accroissement d'intensité des symptômes est dési. gnée sous le nom d'exacerbation, d'accès (accès de fluxion periodique des yeux ) ou de paroxysme (congestion et inflammation des meninges et de l'encéphale).

Le mot ATTAQue sert à désigner les phénomènes instantanés procédant d'un trouble profond du système nerveux (attaque d'apoplexie, d'épilepsie, de paralysie).

L'exacerbation se manifeste dans la plupart des malidies à heure fixe; le soir, pour les maladies de l'appareil respiratoire, la dyspnée s'exagère et si la gêne respiratoire est très grande pendant le jour, c'est presque toll jours pendant la nuit que l'asphyxie derient imminente ou se produit.

La rémittence et l'intermittence des maladies microbiennes n'est pas nettement expliquée. Dans la malariu, on pense que l'hematozocire finit par rendre le sang impropre à son développement, il se replie alors dans la 
rate pour s'y régénérer el reparaître quand les toxines ont été détruiles ou éliminées.

Les matières empêchantes sont en effet brùlées, dédoublées, eniportées par les émonctoires, de telle sorte que si le nicrobe n’a pas conféré l'immunité la première fois, il se met à pulluler et à fonctionner de nouveau.

$3^{\circ}$ Dans le TYPE continu, les symptòmes s'accroissent graduellemeut sans interruption jusquan moment où ils atteignent leur maximum d'intensité, puis ils décroissent avec la mème régularité. La plupart des maladies aiğüs présentent celte marche.

\section{VI. - PEIRIODES DE LA MALADIE.}

L'évolution morbide se traduit ainsi par une courbe régulière ou irrégulière dans laquelle on peut recomnaitre trois périodes : invasion, élat el déclin.

$1^{\circ}$ Invasion. - L'invasion peut ètre brusque et soudaine ou lente et progressive. Elle est brusque dans la plupart des maladies infectieuses (clavelée, péripneumonie, pneumonie), elle est graduelle dans la tuberculose. Tantòt les poisons sécrétés par les microbes agissent à mesure qu'ils sc produisent, et les accidents suivent une marche progressive; lantôt, ils ne manifestent leur action que yuand l'organisme en est sursaturé : les signes qui marquent l'invasion morbide sont les preniers effets des doses accumulés; il se produit une décharge violente, imprévue, qui s'accuse par une fièvre intense et des troubles qénéraux impétueux.

La connaissance de ce fail est très importante en temps d'épizootie où l'élévation de la température fait reconnaître le début de la maladie. Il y a souveut un profond désaccord cntre les manifestations locales et les manjfostations générales; les réactions locales évoluent lentement el sont éclipsées par la violence des troubles grinéraux. 
La période d'invasion fait défaut ou est peu appréciable dans les maladies subaiguës ou chroniques.

Celte période s'étend depuis le début des premiers symptômes locaux jusqu'au moment où ils ont leur maximum d'intensité; elle embrasse tous les progrès de la maladie; très brève dans les maladies suraiguës, elle peut se prolonger plus d'une semaine dans les maladies subaiguës; elle est illimitée dans les maladies chroniques.

$2{ }^{\circ}$ État. - C'est la période où les symptòmes ont toute la gravité qn'ils peuvent acquérir.

Au début, l'organisme envahi par les microbes se tient sur la défensive; à la période d'état, la lutte offre son maximum d'intensité; les symptômes demeurent stationnaires sans s'accroiltre, ni rétrograder, ou présentent une série d'oscillations qui sont en rapport avec les diverses péripéties de la lutte.

La plupart des maladies cycliques (pneumonie, maladies éruptives) présentent ce caractère; la fièvre se maintient au voisinage de son chiffre le plus élevé pendant un temps déterminé et à peu près fixe; les sécrétions sont généralement diminuées, les urines sont rares.

Les symptômes locaux et généraux suirent une marche parallèle; ils s'aggravent ou s'atténuent simultanément; ils déclinent et disparaissent à peu près en mème temps. Parfois, la lésion locale rétrocède tandis que les phénomènes généraux s'aggravent; c'est presque toujours l'indice d'une complication ou d'une généralisation du processus microbien primitivement local. Dans les cas de septicémie, de pyémie consécutives à des lésions locales, les changements dans les phénomènes généraux font reconnaitre l'enrahissement de l'organisme.

La période d'élat est abrégée dans les maladies suraiguës ou foudroyantes qui progressent sans arrèt jusqu'à la mort ou affectent une marche abortice. La pneumonie commençante s'arrète quelquefois brusque- 
ment et la défervescence se produit dès le troisième ou le quatrième jour.

La période d'état est quelquefois prolongcie par suite de poussées successives. Au moment où l'infection doit guérir, un nouveau foyer se produit. La pneumonie, les nécroses cartilagineuses présentent fréquemment ce caractère particulier.

Quand la réaction de la période d'étal est énergique el rapide, elle parvient à détruire les microbes envahisseurs; bénigne ou taldive, elle arrète seulement les progrès des agents microbiens, l'infection passe à l'état chronique.

$3^{\circ}$ Déclin. - La durée est variable; el!e s'étend du moment où il y a diminution dans l'intensité des symptòmes jusqu'à la disparition complète de la maladie.

Pendant toute l'évolution de la malarlie, il se produit des modifications de la nutrition cellulaire qui transforment le sang, les humeurs, les tissus et en font de mauvais milieux de culture pour l'agent pathogèns.

Deux éventualités deviennent alors possibles :

Tantòt la terminaison est brusque, soudaine; il se produit une crise;

Tantòt, l’organisme se débarrasse, lentement, progressi vement des germes qui le souillent et des toxines qui l'imprègnent; la terminaison s'effectue par lysis.

Crises. - Ce sont des modifications sécrétoires et sanguines qui annoncent une guérison prochaine. Elles se produisent pendant la période d'état ou de déclin des maladies aiguës et sont caractérisées par l'élimination des poisous accumulés daus l'économie (décharge urinaire, sudorule), par la chule de la fièvre et par la régénération du sang (crisc hématique).

la CRISE sudorale est marquée par des sueurs abonlantes qui précèdent l'abaissement de la température; 
elles sont dues à des troubles vaso-moteurs cutanés qu1 modifient la répartition du sang.

La décharge urivalre se traduit par une élimination abondante d'urine et de produits toxiques accumulés dans l'organisme. On a observé un rapport étroit entre l'abaissement thermique el l'élimination des poisons; mais l'expulsion des poisons est souvent tardive. Pendant la maladie (pneumonie), le sujet élimine par l'urine deux à trois fois moins de poison qu'à l'état normal; l'élimination atteint ou dépasse le taux normal au moment de la défervescence; la décharge urotoxique s'effectue ordinairement le lendemain ou le jour de la chute de la fièvre.

La crise thermique est caractérisée par la chute rapide de la température, le thermomètre peut descendre en quelques heures, ou en une journée, au chiffre normal.

La crise hématique s'accuse par une abondante production d'hématoblastes qui se transforment en globules rouges. Cette régénération des globules débute au moment de la défervescence et atteint son maximum au moment où la chaleur est redevenue normale. Ce phénomène représente un effort réparateur.

La crise est le résultal et non la cause de la guérison; elle n'est qu'une exagération des phénomènes normaux due au retard de l'élimination des produits toxiques fabriqués par l'organisme. La terminaison de la maladie résulte d'un changement survenu dans l'aclivité de l'agent infectieux.

Les microbes succombent ou s'atténuent; il se produit des antitoxines et les microbes cessent de fabriquer des toxines. Les troubles disparaissent, le fonctionnement cellulaire se rétablit et l'élimination des poisons s'effectue.

Lysis. - Dans la terminaison par lysis, les symptòmes et les lésions décroissent graduellementsans perturbation critique. 


\section{VII. - GLÉRISON.}

Définition. - La disparition lente de la maladie (lysis) ou rapide (crises) constitue la guérison.

Celle-ci est facilitée par l'évacuation des produits solubles par le rein, des produits volatils par le poumon, par leur destruction par le foie, par les propriétés microbicides des humeurs, par l'ensemble des fonctions digeslive, respiratoire, circulatoire et nerveuse et par l'activité des éléments qui détruisent les parasites, les microbes, isolent les corps étrangers, réparent les tissus détruits, etc.

Dans la vacine, le horse-pox, la clavelée, elc., la variole, les microbes sont éliminés avec les éléments mortifiés de l'épiderme ou du derme; ils sont détruits par les phagocytes dans les affections microbiennes locales; ils sont rejetés par la salive dans la rage, par le rein dans toutes les affections du saug; ils sont isolés du reste de l'organisme par un tissu de sclérose quilimite l'exten. sion des lésions dans la morve et la tuberculose; ils sont lréquemment détruits par les dégénérescences (graisseuse, calcaire), qu'ils ont provoquées.

Les produits solubles sécrétés par les microbes sont également éliminés; leur formation est ralentie par la diminution des matériaux qui conviennentà leur développement et par l'augmentation de l'état bactéricide des humeurs. La fièvre elle-mème, quand elle est très intense, ralentit la pullulation des microbes, la production des toxiues, etc., et contribue à la guérison.

Celle ci est absolue ou relative; elle est ABsolue, quand il y a disparition complète des symptòmes, comme dans la maladie du jeune âge, la vaccine, etc.; elle esl Relative, quand le sujet guéri présente des infirmités qui sont la suite de la maladie (cécité, claudication, arthrites, etc.), 
ou quand des inflammations secondaires chroniques poursuivent leur érolution.

Beaucoup de maladies ne présentent qu'une guérison apparente; le microbe qui les a fail évoluer une première fois s'est replié dans certains organes ou s'est confiné dans les lésions qu'il a fait dérelopper (tuberculose, péripneumonie contagieuse); la maladie, devenue momentanément latente, peut reparaître promptement.

D'autre part, l'action locale des loxines se traduit par des dégénérescences ou des nécroses cellulaires dont la réparation est toujours imparfaite. La cicatrisalion d'un os, elc., reproduit le tissu osseux, le tissu conjonctif, etc., la destruction des cellules hépaliques, rénales et est suirie d'une sclérose de remplacement. La sclérose est l'aboutissant de tous les processus inflammatoires profonds, élendus ou prolongés.

\section{VIII. - RECIIUTE ET RÉCIDIVE.}

Définition. - La RECHUTE est l'aggravation subite des symptòmes morbides d'une affection en roie de guérison. On l'obserre dans toutes les maladies.

Dans les maladies microtiennes, une infection secondaire peut reproduire les symplòmes de la maladie primilive ou ceux d'ure maladie analogue. C'est ainsi que les microbes de lá suppuration se greffent sur la pneumonie, ceux de la septicémie sur la plupart des maladies.

Les affections microbiennes chroniques peurent aussi, à la faveur de causes débilitantes, présenter des exacertations dans leur marche et passer brusquement de l'étal chronique à l'état aigru; telles sont la morve, la dourine, etc. La cause débilitanle a transformé l’organisme en un milieu plus farorable au développement des gernies morbides; ils se sont multipliés, ont sécrété plus de poisons, d'où résulte une agg̣ravation subite des symptònes. La fierre paludienne offre des exemples de rechute qui 
paraissent dus à cette cause. Les accès se dissipent petil à pelit, gràce à lélimination des poisons par les égouts de l'économie (bile, urine, etc.) pour reparaitre ensuite.

Dans la clavelée, ainsi que dans toutes les affections éruptives, les causes débilitantes amènent très souvent des rechutes. Dans les affections non microbiennes, les rechutes s'observent fréquemment. Dans l'indigestion chronique des ruminants, l'inobservation d'une diète sévère fait apparaitre des symptòmes plus graves que ceux de la maladie primitive.

La RÉCIDIVe se distingue de la rechute en ce qu'elle apparait longtemps après une première guérison.

C'est ainsi qu'une arthrite, une pleurésie, peuvent récidiver. I.es maladies eezémateuses, rhumatismules, diathésiques prísfntent surtout ce phénomène, qui devient, chez elles, leur caractéristique.

La maladie réapparait très souvent au point le moins résistant de l'organisme. Dans les maladies microbiennes, les récidives ne s'observent pas tonjours, car très souvent ces maladies confèrent une immunité plus ou moins grande à l'organisme, et la récidive n'apparaît qu'autant que l'organisme n'est plus entrainé à la lutte microbienne.

\section{I.I. - COMILICATIONS.}

Définition. - Caractères. - Les complications sont des maladies sccondaiıs préparées ou engendrées par la maladie primitive.

Un sujet affecté d'anasarque ou de tétanos meurt de pneumonie gangreneuse; rette dernière maladie est une complication parce qu'elle est étroitement liée aux troubles circulaloires et digestifs, à la difficulté de la déglutition déterminée par l'œdème ou ‘au spasme du pharyux et de la glotte, à la pénétration de salive ou de corps étrangers dans la trachée et les bronches, tous 
accidents qui se produisent fréquemment dans le tétanos et l'unasarque.

Chaque maladie modifie l'ensemble de l'organisme, change la composition du bouillon de culture, diminue la résistance des tissus, favorise les intoxications, trouble la nutrition, engendre des désordres trophiques. Rien n'est difficile comme de guérir quand on est malade. Les germes qui habitent notre appareil digestif, respiratoire, etc., habituellement inoffensifs, s'implantent et végètent activement dans un organisme débilité par une maladie primitive, produisent des infections secondaires, c'est-à-dire des complications.

Les microbes du pus, des gangrènes, des septicémies, des inflummations des séreuses sont placés au-dessus des saprophytes mais au-dessous des bactéries hautement pathogènes tactéridie charbonneuse, etc.), ils sont les causes ordinaires des complications et penvent se développer à la faveur d'un changement dans la composition chimique de l'organisme, d'une maladie qui a affaibli l'état bactéricide et le phagocytisme, d'un traumalisme qui leur a ouvert une porte d'entrée et de toutes les influences qui facilitent leur multiplication et l'éducation de leur virulence.

Un traumatisme déchire les tissus, diminue leur résistance, rupture les vaisseaux, ouvre les voies d'absorption. Celte maladie locale se complique de toutes les maladies déterminées par les germes que l'air et les autres véhicules viennent $y$ dépnser : les complications chirurgicales n'ont pas d'autre origine.

Le bacille de lioch se développe facilement chez le diabétique, parce qu'il y trouve les mèmes éléments que dans son habitat naturel, la vache laitière (Charrin). La suppuration et la gangrène sont des complications fréquentes chez les diabétiques, parce que les germes de ces infections secondaires trouvent également dans les milieux sucrés des conditions qui favorisent leur érolution. 
La plupart des maladies infectieuses primitives se compliyuent d'infections secondaires : la suppuration, la gangrène, les ulcérations accompagnent la clavelée, la peripneumonie, la fièvre aphtheuse, etc.; les maladies du système nerveux créent des congestions, des anémies; la paraplégie du chien trouble la miction, la défécation, provoque des infections secondaires de l'appareil urinaire.

Chaque appareil organiıue altéré est une source de complications.

Les stomatites ulcéreuses engendrent souvent la septicémie chez le cheval et le chien; l'entérite déterminée par des caustiques entraine la chute de l'épithélium et permet à tous les microbes de l'intestin d’infecter le sang, le périloine; c’est-à-dire de déterminer des complications qui sont des infections secondailes comparables à celles qui succèdent aux accidents traumatiques.

L'appareil respiratoire est ouvert à tous les germes. l'animal qui a contracté les germes d'une bronchite, d'une pneumonie lobaire, est exposé sans cesse aux complications de gan nrène, de suppuration déterminées par les germes qui pénètrent dans l'arbre aérien avec l'air iuspiré. Ces microbes sont normalement détruits ou atténués dans les voies respiratoires; ils deviennent le point de départ d'infections secondaires, et engendrent des bronchites, desbroncho-pneumonies, des abcès des poumons, quand une maladie générale ou une pneumonie lobaire franche leur a créé un milieu favorable. Ce sont ces microbes surajoutés qui engendrent des maladies secondaires souvent plus graves que la première.

Dans l'appareil genito-zrinaire les complications sont égralement faciles à suivre. l'u chien présente une hypertrophie de la prostate, ou'un calcul urétral; il se produit de la rétention urinaire et des infections secondaires. Liurine qui stagne est rapidement infectée par le sang ou le canal urétral; elle fermente dans la vessie, 
les germes qu'elle renferme s'associent, provoquent uue cystite, plus tard une pyélite et finalement une néphrite secondaire. Cette dernière complication se produit toutes les fois que la vessie est infectée et que la sécrétion urinaire est momentanément suspendue par la fièvre, etc., les germes gagnent alors le rein el y produisen des inflammations et des abcès.

La parturition est chez toutes les femelles un acte physiologique; mais le décollement du placenta, les contusions de la vulve, les manipulations nécessaires pour l'extraction du fœetus entrainent desinfections secondaires (septicémie, fièrre puerpérale, ałcès).

Les pustules de vaccin, de clavelée sont envahies par les germes pyogènes qui changent la phỹsionomie primitive de l'éruption; l'actinomycose se complique également de phénomènes de suppuration.

L'existence des complications est souvent masquée par la maladie primilive; la formation d'abcès pulmonaires, la production de broncho-pneumonies passent iuaperçues quand le sujet est affecté de tétanos; les sýmptòmes de l'indigestion intestinale dissimulent la péritonite par perforation.

Pour décourrir les complications qui surviennent dans le cours d'une maladie primitive, il faut les rechercher sans cesse p̣ar l'examen répété de tous les organes de l'économie.

Les affections secondaires qui surviennent pendant la convalescence ont toujours une symptomatologie plus nelte; la fièvre qui était tombée se rallume et lon voit reparaitre des signes analogues ou différents de ceux de la première maladie. On peut alors soupconner une rechute ou une complication. C'est l'érolution irrégulière et toujours fébrile qui indique la nature pyogénique ou gangreneuse des derniers accidents et les différencie des rechules.

D'ailleurs, la plupart des maladies infectieuses ne peurent

CAdéac. - Sémiologie, ¿̨e édit.

II. -26 
recommencer immédiatement leur évolution; mais elles peuvent, il est vrai, se localiser dans d'autres organes et ces localisations prennent souvent la touruure des complications. Une synovite qui apparaît au niveau d'une gaine articulaire ou tendineuse, à la suite d'une preumonie, est-elle le résultat d'une infection secondaire greffée sur l'infection primitive ou une conséquence de l'extension primitive?

On sait que des microbes peuvent acquérir des propriétés différentes suivant le terrain dans lequel ils sont ensemencés; le pneumocoque, les microbes de la septicémie, le bacille de lioch, peuvent engendrer la suppuration.

Il peut donc y avoir simple propagation du microbe qui a déterminé l'infection primitive; il n'y a pas alors complication. Celle-ci existe quand une maladie secondaire s'ajoute à la première, quand un deaxième microbe distinct du premier s'additionne au premier et multiplie ses effets. Ainsi l'apparition d'une tuberculose pulmonaire, hépatique, elc., à la suite d'une tuberculose ganglionnaire, ne constitue pas une complication, le bacille de Koch étend simplement ses foyers; les généralisatious ne sont que des lésions secondaires d'un mème processus.

Un microbe qui envahit lesang, le poumon, les plèvres, le rein détermine partout la même infection: les microbes septiques peuvent, chez le mème animal, engendrer une arthrite traumatique, de la gangrène cutanée, de l'endocarlite, de la pleurésie, de la péricartite; ces divers états morbides ne sont pas des complications, parce qu ils sont l'œuvre du nième germe patlıgène.

D'uu autre côté, il y a quelquefois infection simultanée et non secondaire; deux microbes peuvent pénétrer en même temps dans l'économie, par exemple le bacille de la tuberculose et le microbe de la péripneumonie. Les complications sont donc des maladies qui se greffent sur une maladie primitive d'origine dyscrasique, nerveuse ou mi- 
crobienne. Ce sont le plus sourent des maladies infectieuses qui compliquent les affections primitives, car les microbes du dedansou du dehors trouvent dans l'infection primitive les raisons principales de leur évolution secondaire.

Il imporle aussi de distinguer les complications de la COINCIDEXCE YORBIDE. Plusieurs maladies peurent exister chez le mème sujet sans avoir de relation directe entre elles. Un cheral peut présenter une endocardite et les eaux-aux-jambes; on a une coïncidence morbide ; chacune d'elles peut se compliquer indépendamment de l'autre ; elles peurent également s'influencer réciproquement. L'endocardite favorise les engorgements des extrémités el exagère les lésions déterninées par les eaux-aux-jambes; les eaux-aux-jambes à leur tour entravent les circulations de retour et augmentent l'anasarque engendrée par l'endocardite.

Les complications aggravent toujours le pronostic. Elles sont une preuve de l'insuffisance des moyens de résistance de l'organisme. Elles accusent son état de débilité et de réceptivité pour les agents infectieux. Les abcès, les traumalismes déterminent des poussées aiguës chezles animaux morveux ou tuberculeux. On doit toujours chercher à les prévenir en obstétrique, en chirurgie, à l'aide de l'antisepsie, en pathologie interne à l'aide des toniques, des antiseptiques du tube digestif, etc.

\section{X. - CONVALESGEXCE.}

Définition. - On appelle convalescence, la période qui s'écoule depuis l'achèvement de l'évolution morbide jusqu'au rétablissement complet de la santé. La conralescence n'est pas constatée dans toutes les maladies. Les affections locales en sont dépourvues quand elles n'ont déterminé aucune perturbation nutritive; elle succède toujours aux maladies graves qui ont porté une atteinte sérieuse à l'organisme. 
La convalescence est de peu de durée, courte, longue, traînante, languissante, suivant l'étal du sujet au terme de la maladie. Les cellules de l'organisme ont été blessées physiquement ou chimiquement; elles ont souvent beaucoup de peine à revenir à l'état normal. Quand elles sont déviées de leur nutrition, elles poursuivent souvent leur évolution dans un sens pathologique; elles peuvent même subir diverses dégénérescences. La convalescence dure autant que ces altérations secondaires; elle finit quand chaque organe a pu réparer les dommages qu'il a éprouvés, renouveler les aliments perdus et rétablir sa nutrition compromise.

Beaucoup de sujets frappés d'une maladie ne se rétablissent jamais d'une manière complète; certaines altérations persistent et la nutrition demeure toujours au-dessous de son taux normal ; les cellules ne peuvent plus reconquérir leur puissance d'assimilation et de désassimilation. Ainsi, tout n'est pas terminé avec la disparition des symptòmes caractéristiques de l'affection ; les lésions rétrogradent, mais leurs effets locaux persistent encore.

État des tissus et organes au moment de la convalescence. - L'orgune qui a supporlé le choc microbien se trouve déprimé, débilité; il est au-dessous de son fonctionnement normal ; ses éléments essentiels sont plus ou moins altérés, dégénérés; des cellules nouvelles ou des éléments de lutte ont pris leur place; le territoire infecté s'est dépouillé des microbes qui ont joué le rôle prépondérant pendant toute la phase aiguë; mais les toxines sécrétées par ces microbes ont engendré des troubles cellulaires. Ces troubles survivent à la disparition des microbes.

L'organisme se trouve ainsi privé d'une partie du travail fourni par un collaborateur indispensable; c'est le foie dont les fonctions biligénique, glycogénique, uréogénique 
et antitoxique sont insuffisantes; c'est le poumon, qui n'assure qu'une hématose incomplète du sang et des tissus ; c'est le rein dont la fonction éliminatrice s'effectue d'une manière très imparfaite; c'est enfin le système nerceux qui ne réglant plus les échanges d'une manière régulière, compromet la vie du malade aprèsla guérison apparente de la maladie.

Pendant la convalescence, chaque organe malade est, à un degré plus faible, il est vrai, que pendant la maladie, une cause de privations et une source de dangers pour le reste de l'organisme. Il continue à affamer les tissus en ne leur procurant pas, comıne le foie, l'appareil digestif, le poumon, le système nerreux, les aliments nécessaires à leur entretien; il les menace aussi d'une intoxication en s'opposant à l'élimination ou à la destruction de leurs matériaux de déchet. Tous les organes de l'économie souffrent de l'imperméabilité relative du rein à la fin des néphrites, du défaut de neutralisation des poisons par le foie dont les doures ont disparu en laissant un parenchyme altéré. En outre, le rein et le foie menacent toujours les autres organes d'une intoxication directe: le foie de l'intoxication biliaire, le rein de l'intoxication urinaire, le poumon de l'asphyxie générale, le système nerveux de l'arrêt de toutes les fonctions.

L'organe malade, qui n'a pu jouer son rôle d'une manière nornale, a été pendant toute la durée de la maladie une charge, une cause de dépense pour l'ensemble de lorganisme. Toute affection microbienne produit la fièvre. Or, la fièvre modifie les globules, la fibrine, les sels, les gaz du sang, exagère les combustions, produit une élévation de la température, entraine des dégénérescences dues à l'hyperthermie et à l'inanition; le cœur, le foie, les reins, le tube digestif sont plus ou moins blessés par la fièrre.

Le poumon doit se surmener pendant toute la durée de la maladie pour fournir l'oxygène nécessaire à ces com- 
bustions exagérées. L'animal brùle davantage, il reçoit moins de principes nutritifs, car le premier effet de la fièvre, c'est de supprimer l'appétit. Tous les organes ont ainsi plus de dépenses et moins de receltes.

L'organe maiade est aussi une cause directe d'intoxication pour le reste de l'organisme. L'affection microbiemne, localisée dans un lissu, transforme ce tissu en bouillon de culture; le système nerveux vaso-moteur excité se dilate; il se produit des congestions, des anémies, des thromboses, des embolies, des hemorragies, des æclèmes.

Les produits issus des fermentations microbiennes peuvent altérer le sang, l'endocarde, les parenchymes, délerniner des altérations chroniques secondaires, engendrer des dégénérescences cellulaires, frapper de mort les cellules des tubes urinifères, exciler le systènıe nerveux et provoquer des convilsions, ou le déprimer et le paralyser. Les chieus affectés de la maladie du jeune age sont souvent atteints de paraplégie, de chorée, d'epilepsie, au moment de la convalescence. Ces troubles nervenx paraissent relever d'une action spéciale exercée par les toxines sur le système nerveux.

Les microbes eux-mêmes peuvent passer, de leur foyer primitif de développement, dans le sanğ, contribuer à produire des embolies, se greffer sur l'endocarde et déterminer des effractions vasculaires et épithéliales au niveau des reins.

Tous les appareils, tous les viscères et tous les tissus doivent subir des guérisons individuelles pour que la guérison soit complète et défnitive.

Au début de la convalescence, ils sont tous dans un état particulier très favorable pour les rechutes, les complications et les récidives.

Etat des fonctions au moment de la convalescence. Cet état dépend le la gravité, de la durée des maladies, 
de l'importance de l'organe atteiıt, de l'intensité des lésions et des symplòmes. On observe toujours une modification de l'ensemble des fonctions organiques.

L'appétit, supprimé ou considérablement diminué pendant la maladie, reparaît au début de la convalescence. Il augmente progressivement d'intensilé; le malade devient quelquefois rorace; on est souvent obligé de le ralionner pour l'empècher de surmener son appareil digestif dont les sécrétions n'ont pas encore repris leur actirité.

L'appareil digestif débilité par la diète forcée, parésié parfois, par suite d'une nutrition insuffisante, se paralyse souvent, ce qui engendre des obstructions alimentaires. Les aliments sont ingérés en grande quautité quand on ne surveille pas attentivement les sujets; on remarque des alternatires de coustipation et de diarrhée.

L'absorption très aclive emprunte aux tissus adipeux et musculaire la graisse qu'ils renferment pour hàter la restauration des autres tissus; l'animal continue pariois de maigrir au commencement de la conralescence, puis le poids du corps, considérablemeut dininué, angmente peu à peu, l'embonpoint reparait avec toutes les autres fonctions physiologiques.

Le système musculaire conserve longtemps un certain degré de faiblesse; les animaux doivent ètre laissés au repos; le moindre travail leur est pénible, dangereux mème; il provoque une trop graude accélération de la circulition et de la respiration. Les troubles respiratoires persistent longtemps, surtout après les maladies aiguës de poitrine; le flanc est soubresautant, quelquefois discordant; les chevaux ne peuvent travailler; les chiens s'essouffleut rapidement et s'arrètent haletants.

Les battements cardiaques, très accélérés, sont tumultueux, le pouls est petit, dépressible en raison de l'anémie.

Les sécrétions, peu abondantes au début de cette période par suite de l'exagération de l'absorption, 
reviennent rapidement à l'état normal. L'urine est rejetée en plus grande quantité ; mais elle est pauvre en principes dérivés des substances azotées; sa densilé est faible; elle renferme une grande proportion de carbonates et de phosphates terreux.

La peau présente des altérations diverses dues à l'insuffisance de la nutrilion. Les poils tombent pour repousser rapidement, la sécrétion épidermique et la sécrélion sébacée s'exagèrent, la peau se couvre rapidement de squames épidermiques; elle s'assouplit et reprend sou brillant.

Marche et durée de la convalescence. - La marche et la durée de la convalescence sont plus variables: l'àge, l'énergie des sujets, la durée de la maladie, l'importance de l'organe atteint, l'intensité des allérations nécessitant un temps plus ou moins long pour la restauration, le régime auquel les malades ont été soụmis, les médications employées sont les principales causes de ces variations.

Les animaux nourris très substantiellement durant la maladie sont dans un état d'adynamie el d'amaigrissement moins prononcé que ceux que l'on n'a pu entretenir ; aussi la durée de la convalescence est-elle plus courte chez les premiers; elle est plus longue chez les sujets très jeunes ou vieux que chez les animaux adultes et vigoureux.

Lihygienc exerce une action importante sur la convalescence; il faut éviter aux sujets convalescents les intempéries atmosphériques, réveiller leur activité par un exercice modéré, leur donner des aliments très uutritifs en proportion convenable pour éviter les indigestions, combattre les différents troubles passagers ou les complicalions qui surviennent.

Les organes les plus direclement en rapport avec l'organe malade sont dans un état défavorable pour se 
défendre : ils peuvent, sous une influence légère, être atteints d'une infection quelconque susceptible de déterminer la mort du sujet. Ainsi, à la suite d'une maladie de cœur, le rein est toujours plus ou moins altéré, l'atlection primitive peut guérir, mais sous la moindre influence, il peut se déclarer, pendant la convalescence, une néphrite albumineuse. l.es diurétiques, administrés pendant l'érolution de la maladie et pendant la convalescence, peuvent empècher l'apparition de celte complication. Il ne faut pas remettre l'animal immédiatement au travail ; mais on doit toujours opérer graduellement, commencer par lui faire effectuer des promenades dont on augmente progressivement la durée; on doit éviter pendant longtemps de le soumettre à un travail trop pénible.

Maladies et accidents de la convalescence. - L'action débilitante exercée sur l'organisme par la maladie et la diète crée une imminence morbide dont nous avons déjà étudié les effets (1). Le sujet convalescent offre une vulnérabilité exceptionnelle. Il présente l'ensemble des prédispositions individuelles et se trouve prèt à se laisser influencer par toutes les causes extérieures. Tous les appareils de l'économie peuvent présenter des manifestations morbides dont le siège varie suivant les maladies et suivant les malades.

Les fonctions digestives sont fréquemment troublées chez les convalescents; les cheraux sont atteints de gastro-entérite, avec constipation ; les chiens offrent des alternatives de constipation et de diarrhée.

Certains animaux sont affectés d'une entérite chronique, de sorte que leur guérison est toujours incomplète.

La circulation présente des troubles caractérisés par des palpitations, des souftles anémiques ou parfois des

(1) Voy. Inanition, in Pathologie générale de l'Encyclopédie vétérinaire, 2e édition, $190 \%$, p. $15 \%$. 
souffles organiques. Les valvules cardiaques, altérées par les toxines et les microbes qui s'y sont implantés, deviennent insuffisantes; une endocardite sourde évolue à la suile de lésions articulaires, de maladies du poumon, etc. Le cocur, toujours modifié par l'hyperthernie, manifeste des signes de dégénérescence graisseuse ou de ramollissement de ses fibres; il peut s'arrêter à la suite d'une course; les syncopes chez le chien, les ruptures cardiaques chez le cheval, peuvent se produire pendant la convalescence.

L'insuffisance des contractions cardiaques détermine des stases dans les parties déclives; on voit apparaitre des adèmes sous le ventre et la poitrine, des engorgements des membres postérieurs chez le cheval, ou du fanon chez le bouf.

L'apparcil respiratoire est pent-ètre celui qui reprend le plus lentement son fonctionnement physiologique; la respiration demeure longtemps irrégulière; elle est souvent soubresautante ou légèrement discordante chez le cheval après la disparition de tous les troubles morbides. Le poumon reste facilement altérable; l'emphysème pulmonaire se déclare fréquemment pendant $l_{\text {a }}$ convalescence. Les voies respiratoires offrent une grande réceptivité pour les microbes : le coryza, les angines, les bronchites surviennent. On observe très souvent chez les jeunes chevanx une hemiplégie luryngienne; les chevaux qu'on croit complètement guéris montrent les signes d'un cornage intense dès qu'on les remet au travail.

La peau est souvent envahie pardes parasites (poux, puces, gales), des atfeclions eczémateuses, dont le pityriasis cst la forme la plus commune.

Le foie, souvent ramolli par la dégénérescence amyloïde, se rupture et les animanx meurent presque sulitement d'une hémorragie interne.

L'uppareil locomoteur est sujet à divers accidents; l'animal, affaibli par la maladie, est exposéà tomber, à se 
couronner, à se contusionner. Les synoviales articulaires et ten lineusus s'enflamnent assez souvent chez le cheval, quilıze à ringt-cinq jours apı ès la résolution d'une pneumonie, d'une endocardite, elc.

Le systeme nerveux se montre généralement déprimé. A la suite d'affections encéphaliques, on constale du coma, de l'immobilité; les sujets semılent aroir complètenient perdu Jeurs facultés. Ils se déplacent lentement, pénililement et manifestent une lourdeur extraordiuaire. La maladie du jeune âge des chiens s'accompagne souvent de plénomènes nerveax inaltendus qui surviennent pendant la convalescence et qui rerètent le type choréique ou épileptiforme. On voit aussi des paralysies ou des paraplégies apparaître; les sens s'émoussent quelquefois pendant la conralescence; des chiens perdent l'ouie et l'odorat.

Ces accidents nerveux paraissent résulter des modifications dégénératives imprimées aux centres nerveux par les toxines microbiennes ou par les microbes déveJoppés tar divement dans ces organes.

Les sujets affectés de maladies contagieuses transmettent ces maladies pendant la cunvalescence; la clavelée, le horse-pox, la peste torine, elc., sont fréquemment propagés par des animaux paraissant complètement guéris; les microbes survivent à l'évolution morbide : ils sont lentement expulsés de l'organisme par les poussières épidermiques, l'urine, le jetage et tous les produils d'élimination.

\section{II. - MÉTASTASE.}

Définition. - La métastase exprime le déplacement d'une maladie et son transport dans un point plus ou moins éloigné de son siège primilif.

Le transport de la maladie est fréquent, banal; il se produit des maladies secondaires par infection, par intoxication, par altération de la nutrition. Ces affections 
secondaires résultent de localisations de la maladie primitive, d'une continuité dans l'infection ou de complications. En dehors de l'apparition de la maladie nouvelle, la métastase véritable ne peut exister s'il n'y a pas disparition totale de tous les phénomènes morbides primitifs avant la manifestation des troubles secondaires. Ce déplacement total ne peut se produire; les lésions primitives caractéristiques de la maladie ne peuvent se dissiper instantanément. Le not métastase ne répond plus aux doctrines médicales actuelles; il n'en existe pas un seul exemple qui soit authentique.

\section{XII. - PASSAge a L'état CHRONIQUE.}

Définition. - Quand l'évolution de la maladie se prolonge au delà des limites habituelles, on dit quelle passe à l'état chronique. On considère comme chronique toute. maladie aiguë qui se prolonge au delà du quarantième jour.

L'organisme qui n'a pu détruire entièrement les microbes ou expulser les poisons s'efforce de circonscrire la lutte; mais il lutte sans énergie.

Les réactions reprennent une certaine acuité quand sous l'influence d'un coup de froid, d'un traumatisme, etc., il se produit des poussées aiguës. La tuberculine de Koch donne un coup de fouet à l'évolution torpide de la tuberculose.

L'évolution chronique est caractérisée par la disparition de la plupart des symptômes observés pendant l'évolution aiguë; elle est quelquefois même un simulacre de guérison; certains foyers de nécrose se ferment pendant des mois ou plus longtemps encore, puis le foyer qui semblait éteint se rallume, une nouvelle poussée morbide se produit avec formation d'un nouvel abcès et d'une nouvelle fistule.

L'état chronique aboutit fréquenment à l'induration; 
l'organisme a détruil l'agent pathogène, mais l'effort pour l'anéantir a été si considérable que le tissu produit en excès ne peut disparaitre entièrement : il se produit une néoformation fibrense. C'est le sort des inflammations nodulaires et interstitielles.

\section{XII. - MORT.}

Définition. - La vie élant la résultante des activités élémentaires, la mort est la conséquence de leur indépendance ou de leur anarchie.

La mort apparente se produil dès que le système nerreux cesse de régler les rapports des appareils, des organes, des tissus et des élénents entre eux. Mais ceuxci continuent à vivre isolément pendant un temps variable.

La mort est complète quand l'activitẻ phrsique et chimique de tous les éléments est détruite. A leétat normal, on constate des morts partielles dans l'économie ; c'est, par exemple, la cellule épitbéliale kératinisée qui tombe. c'est la cellule de rerètement d'une muqueuse qui, après aroir dégénéré, est entrainée hors de l'organisme. Mais la rie cénérale de tous éléments anatomiques ne peut se conserver qu'autant que les grandes fonctions innerration, respiration, circulation) peurent s'accomplir; la suppression de l'une ou de l'autre amène la mort.

On peut mourir par le cœur, le poumon ou l'encéphale.

La mort survient par le cœul quand cet organe se rupture, s’atrophie, s'altère;

Elle survient par le poumon qui détermine l'asphyxie (maladies de l'appareil respiratoire);

Elle survient par les centres nerveux et le bulbe quand ils sont annihilés par des traumatismes, des hémorragies, des thromboses, des embolies, des poisons.

C'est toujours le système nerveux qui est la cause essentielle de la mort; l'arrêt du cœur entraine la paralysie

CAdÉac. - Sémiolugie. حe édit.

II. - 27 
du bulbe qui, à son tour, arrête les mouvements respiratoires; l'arrêt ou l'insuffisance de la respiration supprime aussi les fonctions bulbaires. Cet organe est toujours touché directement, indirectement ou par voie réllexe par les traumatismes, les hémorragies, les sécrétions des agents pathogènes, les sécrétions des éléments anatomiques; d'une manière générale, la mort est la conséquence d'un empoisonnement.

Le rein altéré par les produits toxiques n'est plus qu'un émonctoire imparfail, le foie stéatosé cesse de détruire les poisons, le sang surchargé d'acide carbonique, de toxines, pauvre en oxygène, intoxique les centres nerveux et spécialement les centres bulbaires; de là arrèt de la respiration, arrêt de la circulation.

A part les grands traumatismes qui tuent instantanément, mourir par le cœur, par l'encéphale ou le bulbe, c'est mourir empoisonné.

La inort est dite naturelle quand elle est amenée par un affaiblissement sénile de l'organisme et qu'elle met un terme à l'évolution normale de l'individu. On peut comparer l'état de l'animal placé dans de semblables conditions à celui d'une lampe qui s'éteint faute de combustille.

Dans ce cas, la mort arrive insensiblement. Il est rare d'observer une pareille fin chez nos animaux domestiques. Le plus souvent, chez eux, la mort est accidentelle.

b)ans les maladies aiguës, la mort peut survenir brusquement, d'une manière inopinée ou arriver progressivement, lentement, précédée d'une agonie plus ou moins longue.

On appelle agonie, le moment qui précède la perte complèle des manifestations vitales : respiration, pulsations cardiaques. A ce moment, le système nerveux fonctionne encore, mais faiblement. Le sujet est plongé dans le cona: la vie végétative persiste seule. On en a déduit 
MORT.

que, de tous les tissus de l'économie, le système nerveux meurt le premier.

Signes de la mort. - Les manifestations symptomatiques de la mort sont frappantes:

L’animal est ćnuché, les membres allongés sur la litière, en état de résolution.

Parfois il y a des mouvements convulsifs fugaces; la sensibilité et l'intelligence disparaissent. La respiration et la circulation, après avoir été accélérées, se ralentissent, deviennent irrégulières, présentent des intermittences de plus en plus grandes. 


\section{DIAGNOSTIC ET PRONOSTIC}

\section{$-\cdots$ \\ CHAPITRE PREMIER}

DIAGNOSTIC

Définition. - Le diagnostic est l'art de recomnaitre les maladies el de les différencier entre elles.

Le clinicien appelé auprès d'un animal malade doil s'appliıuer à rechercher tout ce qui peut le conduire à une counaissance complète de la nualadie dont cet animal est affecté. P'oser le diagnostic précis de la maladie qu'on doit combattre est la première obligation du praticien qui veut instituer un traitement rationnel, efficace ou conseiller des mesures propres à empẻcher l'extension d'une alfection contagieuse.

Quand le diagnostic est inexact, le traitement est empirique, dangereux, le pronostic est livré au hasard, l'étiologie est inconnue et les mesures sanitaires les plus utiles ne peurent être ui recomınandées, ni appliqueées. Qu'un cheval morveux s'introduise dans l'écurie d'un régiment: la contagion dissémine la maladie méconnue; qu'un tronpeau de burufs affectés de peste bovine franchisse les frontières: c'est un fléau qui s'abat sur notre sol quand le diagnostic, rapidement formulé, n'est pas sanctionné par la destruction immédiale de tous les animaux malades ou contaminés. 
L'importance du diagnostic est indiscutahle; toutes les études médicales n'out pas d'autre but que de nous donner le tact médical, c'est-à-dire la faculté de reconnaitre sûrement et promptement la valeur des signes les plus cachés qui demeurent impénétrables pour le vulgaire, pour tous ceur qui sont mal doués ou qui ont fait des études médicales incomplètes. Le tact médical résulte de l'intelligence naturelle qui fait rapidement démèler les signes caractéristiques d'une maladie et de l'éducation scientifique secondée par une grande expérience clinique.

\section{I. - IOYENS ET DIFFICULTÉS DU DIAGNOSTIC.}

Moyens de diagnostic. - Les moyens de diagnostic sont théoriques et pratiques.

Les moyens théoriques se résument dans la connaissance approfondie des maladies et de leurs formes variées. Le praticien doit évidemment posséder les principaux signes de chaque maladie afin de savoir, quand il les observe, les rattacher à la maladie qui les produit.

Les signes objectifs caractéristiques des maladies comprennent : $1^{\circ}$ des signes apparents facilement reconnaissables; $2^{\circ}$ des signes cachès, qui ne peuvent ètre décelés que par une exploration minutieuse ou par des procédés spéciaux.

L'isspection nous indique des modifications anatomiques et dynamiques; la pressior nous renseigne sur les changements de résistance de la peau et des parties sous-jacentes, précise le siège de la douleur et permet de la localiser; la palpatrox multiplie les contacts et les froltements, fait connaitre la configuration, le volume, la consistance, la situation, les rapports réciproques des différentes parties.

la succussion peut rendre des services chez les petits 
animaux; la percussion el l'auscultation, la bascule pour juger des progrès de l'amaigrissement sont d'un emploi quotidien pour l'examen des sujets atteints de maladies de la poitrine, de la cavité abdominale, etc.

Des sens bien exercés, servis par un esprit patient, réfléchi, par un jugement droit, permettent d'interpréler logiquement les symptômes, d’apprécier la signification des lésions, d'en expliquer la production, de remonter anx causes, d'en déduire le début de la maladie et de fixer, au moment de l'examen, les phases accomplies par l'évolution morbide.

Outre ces moyens que le clinicien a toujours à sa disposition, il doit toujours emporter avec lui les instruments qui peuvent lui donner des indications immédiates. Le thermomitre, les sondes, un trocurt, un plessimètre et son marteau, un stéthoscope, des spéculums pour les conduits dilatables, complètent les renseignements qu'il peut recueillir à l'aide des sens.

Parfois, il devra, avant de se prononcer, recourir ì l'examen microscopique, à l'analyse chimique, aux inoculations révélutrices (produits virulents ou substances thernogènes comme la tuberculine et la maléine), aux cultures, à l'autopsie des malades, quand une affection parasitaire est soupconnée.

Quand il s'agit d'une lumeur, on peut rechercher la quantité d'eau qu'elle renferme. Ce dosage a une grande importance pour déterminer immédiatement si elle est bénigne ou maligne. Plus la quantité d'eau est considérable, plus la tumeur est grave.

C'est en s'aidant de tous les procédés qui peuvent révéler les troubles morbides qu'on parvient à établir un diagnostic légitime. Le praticien qui commet la moindre négligence est exposé à de fréquentes erreurs, très préjudiciables à sa réputation. Il doit toujours être exempt d'entêtenent; son esprit doit être suffisamment souple pour renoncer à un jugemeut inexact dès qu'il a la 
preuve de son erreur. Tant qu'il est éloigné du malade, il doit être prudent dans ses réponses, et il doit éviter de questionner. Le vétérinaire s'expose à se fatiguer inulilement l'esprit en accompagnant la personne qui est venue le chercher. Celle-ci émet souvent les opinions les plus variées et les plus contradictoires sur les causes et les symptômes de la maladie et jette souvent le jeune praticien dans un grand embarras. Il est préférable de se rendre seul auprès du malade; là, le propriétaire peut renseigner utilement; il ne peut plus égarer.

Le clinicien, en présence du malade, sans préoccupation, sans crainte, sans idées préconcues, peut reclifier les avis erronés ou les renseignements inexacts qui lui sont donnés. Pour arriver à établir le diagnostic, il doit recueillir sur la maladie et le malade toutes les données qui lui sont utiles.

Difficultés du diagnostic. - L'impossibilité dans laquelle se trouvent les animaux de parler place le vétérinaire dans la même position que le médecin des enfants et le prive de renseignements précieux pour le diagnostic.

Cette ignorance du passé et de l'histoire de nos malades empêche souvent le diagnostic d'aroir une précision absolue. Le rhumatisme musculaire ne peut être reconnu si l'on n'a pas de renseignements; on ue sait jamảis a quelle date remonte l'épilepsie, et l'on ne peut reconnaitre si l'animal voit les objets tourner aulour de lui (vertige) que lorsque des accidents se sont produits.

Le vétérinaire est obligé de recourir sans cesse à tous les procédés d'investigation pour établir le siège du mal. Cette localisation présente fréquemment des difficultés insurmontables; dans le cas de boiterie, on hésite sourent au sujet d'une altération de l'épaule, du boulet, ou du pied. La coexistence de lésions morbides sur un membre rend le diagnostic incertain; un cheval "qui 
boite depuis deux jours présente une forme, un éparvin ou une autre exostose depuis des années. Les lésions anciennes dissimulent les lésions récentes; les lésions superficielles qui frappent l'attention de l'observateur empêchent souvent de découvrir des lésions profondes.

L'albuminurie du chien, qui est fréquemment chronique, n'est reconnue qu'à la suite d'une néphrite ou d'une endocardite, ce qui la fait attribuer à ces maladies. C'est faute d'une investigation minutieuse, incessante que beaucoup de maladies de nos animaux sont complètement ignorées et que beaucoup d'altérations du poumon, du foie, de la rate, des reins, voire mème du cœur, demeurent loujours latentes.

Certaines maladies sont difficiles à reconnaitre parce qu'elles ne s'expriment que par des symplômes trop vagues et trop rares. La morve et la tuberculose sont souvent méconnues; la maléine et la tuberculine ont nettement fait ressortir l'insuffisance des moyens de diagnostic utilisés jusqu'à présent.

Le praticien peul éprouver de grands embarras quand il s'agit de diagnostiquer une maladie très rare, inconnue dans une localité et survenant à la suite d'une importation d'animaux. La dourine, la peste bovine sont très difficiles à reconnaître tant qu'il n'y a pas eu contagion. La transmission de la maladie en révèle la nature contagieuse; elle est d'un grand secours pour le diagnostic.

Il faut toujours être prudent et circonspect quand il s'agit d'affirmer l'existence d'une maladie contagieuse prévue par la loi sanilaire; car le diagnostic de la maIadie est suivi de l'abatage de l'animal et de l'application de mesures onéreuses. C'est dans les cas douleux se rapportant à ces maladies que l'inoculation, l'examen microscopique, les cultures, etc., rendent de grands services.

D'autres difficultés tiennent à. l'insuffisance des moyens de diagnostic qui sont à la disposition du praticien. L'af- 
faiblissement de la vue, de l'ouie, elc., est une cause de jugements erronés. Un examen précipité de l'animal malade, placé dans de mauvaises conditions, conduit à des conclusions hâtives et inexactes. On se trompe souvent sur la cause d'une claudication quand on néglige d'explorer et de déferrer le pied.

La percussion el l'auscultation d'un seul côté de la poitrine peuvent faire méconnaitre une pneumonie placée du côté opposé, qu'on a oublié d'examiner.

Procédés seientifiques. - Il est souvent nécessaire de compléter les premiers examens des malades par l'application de procédés scientifiques précis, mais souvent très délicats.

On peut recourir à l'exayen spectroscopique du salıg dans certains empoisonnements pour les distinguer de l'intoxication par l'oxyde de carbone.

Les appareils enregistreurs (pneumographe, sphygmographe), permettent d'étudier dans tous leurs détails certains troubles passagers de la respiration, comme les troubles nerveux dans les muscles, pendant les crises convulsives.

Il est d'autres moyens de diagnostic, comme la radingraphie, les sérums précipitants, la méthode du sérodiagnostic, du cyto-diagnostic qu'il faut passer en revue.

10 Radiographie. - Ce moyen de diagnostic peut être utilisé pour reconnaître les corps étrangers (projectiles, clous) logés dans les tissus ou avalés accidentellement par les animaux (pièces de monnaies, etc.).

La radiographie est une application des rayons $X$ ou rayons de Röntgen, découverts en 1895 par ce physicien. Pour donner une idée de la nature de ces rayons, il est indispensable de résumer les divers types de lumière solair'e.

On sait que cette lumière est formée de sept groupes 
principaux : rouge, orangé, jaune, vert, bleu, indigo, violet.

"Ces diverses lumières simples ou radialions lumineuses ne sont pas la seule partie constitutive du spectre solaire et il existe en deçà du rouge, dans l'infra-rouge, un groupe de radiations caractérisées par leurs effets calorifques, et au delà du violet, un autre groupe ćgalement invisible, doué de propriétés chimiques très actives. Ces radiations infra-rouges et ultra-violettes sout de nuème nature; elles ne diffèrent que par leur longueur d'onde qui décıoîl progressivement de l'infra-rouge à l'ultra-violet.

Certains corps comme le sulfure de calcium, de baryum et de zinc deviennent phosphorescents à l'obscurité quand jls ont préalablement subi l'action des rayons violets ou ultra-violets; il en est d'autres, comme les sels et les verres d'uranium, les solutions de sulfate de quinine, le platinocyanure de baryum qui cessent d'être lumineux quand on les soustrait à l'action de ces radiations : on dil qu'ils sont fluorescents. Ces phénomènes de phosphorescence et de fluorescence se manifestent encore sous l'influence d'autres rayons spéciaux d'origine électrique : les rayons cathodiques et les rayons $\mathrm{X}$.

Considérons uı tube de verre, traversé à chacune de ses extrémités pal deux tiges de platine, qui peuvent être reliées extérieurement avec les pôles d'une bobine Ruhmkorff et sont terminées à l'intérieur du tube par deux plaques du même métal. La tige par laquelle le courant pénètre dans le tube est l'électrode positive ou anode; celle qui sert à son échappement est l'électrode négative ou cathode. Si un pareil tube est rempli d'air à la pression ordinaire et si la bobine d'induction est mise en marche (fig. 129), le courant ne passe pas, aucune étin-

(t) La longueur d'onde est l'espace parcouru par le mouvement vibratıire pendaut une période; la période est la durée d'une vibration complète; la vibralion complète correspond à l'oscillation double : aller et retour du pendule (Nicolas). 
MOYENS ET DIFFICULTÉS DU DIAGNOSTIC.

celle n'éclate entre l'anode et la cathode, l'air étant mauvais conducteur. Si le vide est fait dans le tube jusqu’a ce que la pression du gaz soit réduile à quelques millimètres

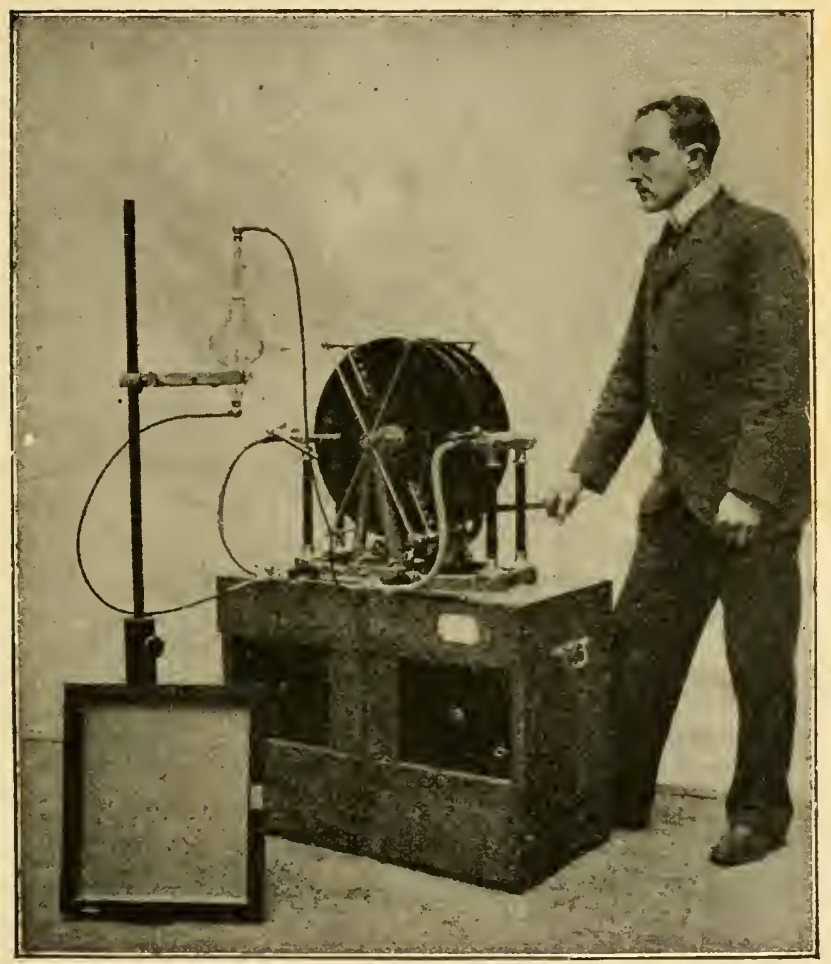

Fig. 129. - Dispositif pour la radiographie.

de mercure, le courant passe, une lueur rouge violacé part de l'anode pour gagner, en s'affaiblissant, la cathode qui, elle, est entourée d'un espace obscur (Nicolas).

$\mathrm{Au}$ fur et à mesure que la raréfaction du gaz augmenle, 
l'espace obscur péricathodique s'accroit; lorsque le vide est à un millionième d'atmosphère (la pression est alors de $0^{\mathrm{mm}}, 00076$ de mercure), cet espace envahit presque tout le tube; aucune lueur ne s'échappe de l'anode et la cathode devient le siège d'un rayonnement spécial invisible, le rayonnement cathodique, découvert par llittorff en 1868. Le tube où prend naissance ce rayonnement est appelé tube de Crookes.

Rayons cathodiques. - Les rayons cathodiques ne sont pas visibles; ils peuvent cependant ètre manifestés, gràce à la remarquable propriété qu'ils possèdent de rendre lumineuses les substances phosphorescentes placées sur leurs parcours: les sulfures de calcium et de zinc, la craie, les pierres précieuses (diamant, rubis), le cristal, s'illuminent sous leur influence.

Les rayons cathodiques sont déviés par l'aimant, ce qui prouve qu'ils convoient avec eux des charges électriques à la façon d'un courant; on a démontré, en ellet, que le transport des charges d'électricité négative était inséparable des rayons cathodiques et l'on pense que les rayous sont les trajectoires de particules matérielles (d'liydrogène sans doute) chargées négativement et lancées dans l'espace par la calhode, avec une vitesse de 40000 kilomètres à la seconde. Ces rayous ne sont pas de véritables radiations, et ne peuvent ètre comparés aux radiations lumineuses, qui consistent en de pures transmissions de mouvements vibratoires sans transport de matière, depuis le centre d'ébranlement, qu'est la source lumineuse, jusqu'à l'œil, organe récepteur. Les rayons cathodiques rendent les gaz conducteurs de l'électricité, traversent une faible épaisseur d'aluminium, impressionnent la plaque photographique. Ils sont arrèlés par les obstacles solides, auxquuels ils conımuniquent la remarquable et inattendue propriété d'émettre de nouvelles radiations appelées rayons $X$ (Nicolas).

Rayons X. - Les rayons X se propagent en ligne droite, 
traversent les corps avec plus ou moins de facilité suivant leur nature ou leur épaisseur; les matières organiques, les tissus animaux se laissent aisément pénétrer; les substances minérales sont au contraire moins tıansparentes, et les os qui renferment de fortes proportions de ces substances (phosphate et carbonate de calcium) sont, pour cetle raison, plus opaques que les autres tissus; les métaux sont, à l'égard de ces rayons, les plus opaques de tous les corps.

Les rayons I provoquent la phosphorescence et la lluorescence, possèdent des propriétés chimiques, notamment celle d'impressionner la plaque photographique, ce quil les rapproche encore des rayous ultra-violets. C'est à ces derniers effets qu'ils doivent leur application et, en particulier, leurs applications médicales; ces rayons constituent un précieus moyen d'investigation, qui pent être adapté aux études anatomiques et au diagnostic clinique (corps étrangers, fraclure de rayons osseux).

Veut-on examiner un objet quelconque an moyen des rayons $\mathrm{X}$, il suffit de placer cet objet sur le trajet de ces rayons, entre l'ampoule où ils prennent naissance et un écran fluorescent (au platinocyanure de baryum par exemple); l'objet projette, sur l'écran illuminé, son ombre, véritable ombre chinoise, qui dessine les contours sans leur faire subir la moindre déformation et dont l'intensité varie avec l'opacité de l'objet aux rayons X. Un pareil examen est dit examen radioscopique. Veut-on conserver l'image de l'objet, il suffit de remplacer l'écran lluorescent par une plaque sensible : cette inage alors fixée sur la plaque pholographique est une radiographie.

Les rayons $\mathrm{X}$ possèdent encore des propriétés électriques, ils rendent l'air et les gaz conducteurs de l'électricité, et déchargent, par suite, les corps électrisés placés à une certaine distance.

Ces rayons exercent sur le tégument une action irritante et provoquent des lésions pouvant aller de l'érythème 
simple jusqu'à la mortification de la peau (rudio-dermites). Au point de vue thérapeutique, leur action et l'influence élective, qu'ils semblent avoir sur les éléments cellulaires jeunes, les ont fait employer dans le traitement des tumeurs, du cancer notamment. Dans bon nombre de cas, ils ont donné d'excellents résultats, mais on ignore encore quel est exactement leur mode d'action.

Rayons $\mathbf{N}$. - Le tube de Crookes émet encore les rayons N, découverts en 1903 par Bloudlot. Ces rayons invisibles traversent la plupart des corps opaques, les mélaux sous de faibles épaisseurs, excepté le plomb et le platine; l'eau pure les arrête; mais l'eau légèrement salée est transparente.

Ils diffèrent des rayons $X$ en ce qu'ils se réfléchissent, se réfractent et interfèrent; ils peuvent ètre concentrés au foyer d'une lentille d'aluminium et sont incapables d'impressionner directement la plaque photographique. Ils ne provoquent pas la phosphorescence, mais peuvent accroître l'intensité lumineuse d'un écran phosphorescent, ils augmentent aussi l'éclat d'une source lumineuse faible, comme l'étincelle électrique, ou une très petite flamme de gaz; ils accroissent l'activité visuelle et permettent de voir nettement dans l'obscurité des objets que l'on ne dislingue autrement que peu ou pas du tout.

Les rayons $N$ sont émis non seulement par le tube de Crookes, mais encore par le soleil, la lampe à pétrole, le bec Auer, les sources lumineuses les plus direrses, les corps phosphorescents et la généralité des corps exposés à la lumière de ces sources qui après les avoir emmagasi nés (cailloux, briques de muraille) les dégagent.

Ces radiations sont émises indéfiniment par les corps qui se trouvent dans un état moléculaire contraint, comme les sabres gallo-romains qui en dégagent encore actuellement.

Le corps humain dégage des radiations analogues. Charpeutier a démontré que l'émission des rayons $\mathbf{N}$ par 
l'organisme est mise en évidence au moyen d'un objet d'épreuve phosphorescent ou fluorescent dont l'intensité lumineuse augmente lorsqu'on l'approche du corps.

On peut se servir comme objet d'épreuve d'un écran áu platinocyanure de baryum dont l'intensité lumineuse est réglée au moyen d'un morceau de radium recouvert d'un papier noir et placé à une distance variable. On peut se servir tout simplement du sulfure de calcium qui a été modérément insolé.

Ces objets phosphorescents augmentent d'intensité lumineuse lorsqu'on les approche d'un organisme vivant. Cette augmentation est surtout nette au voisinage d'un muscle, d'un nerfou d'un centre nerveux, elle est encore beaucoup plus considérable lorsque ces organes sont en activité. L'intensité de la phosphorence est en rapport avec la force decontraction du muscle, de même qu'arec le degré de fonctionnement du nerf ou du centre.

Tous les animaux comme le corps humain émettent des rayons $\mathbf{X}$, et ce sont les tissus dont le fonctionnement est le plus intense qui en émettent le plus.

Les éléments musculaires et nerveux émettent constamment des radiations pendant l'état de repos et cette émission est augmentée pendant l'état de travail.

Le trajet de la moelle est marqué par une luminosité plus forte qui augmente au niveau des renflements cervical et lombaire. Grâce à cette méthode d'exploration, on peut reconnaitre la présence d'un nerf superficiel et en suivre toutes les ramifications.

On peut faire également, à l'aide de ces mêmes tubes phosphorescents, une étude très curieuse sur la topographie des centres psycho-moteurs de l'écorce cérébrale.

C'est ainsi qu'en promenant l'objet d'épreuve sur le còlé gauche du crâne, pendant que le sujet parle soit à luaute voix, soit à voix basse, la luminosité du sulfure de calcium augmenle lorsqu'on se trouve dans la région roisine du centre de Broca. Du còté droit, rien de pareil, 
si ce n'est une trẻs légère augrnentation de phosphorescence, lorsque l'axe du tube se trouve daus la direction du centre opposé.

On croit que la pensée non exprimée, l'attention, l'effort mental, donnent lieu aussi à l'émission de radiations. Par conséquent, tout travail cérébral, volitiou, idéation, s'accompagnerait de l'émission au dehors d'une certaine quantilé d'énergie, sous forme derayons $\mathrm{N}$ ou d'autres radiations; rien d'impossible alors à ce que celte énergie soit capable d'impressionner un autre cerveaı. N'est-ce point là l'explication des phénomèues de suggestion, de transmission de pensée et de télépathie?

En résumé, tout centre nerveux qui fonctionne ajoute à son émission de repos, de nouveaux rayons en proportion de son degré d'activité. Ces rayons se transmeltent el divergeant suivant les lois de l'optique, traversant avec plus ou moins de réfraction les divers milieux, et se manifestant par une augmentation de luminescence de l'objet d'épreuve, augmentation variable avec l'intensité de l'émission et avec la distance.

Les radiations musculaires ne sont donc pas identiques aux radiations nerveuses, par conséquent le muscle rayonne bien par lui-même et non pas seulement par les terminaisons nerveuses qu'il renferme.

Il existe encore d'autres caractères différentiels entre les radiations musculaires et nerveuses. Le nerf est beaucoup plus sensible à la compression que le muscle, la radiation est beaucoup plus exagérée dans le premier cas que dans le second.

$2^{\circ}$ Sèrums précipitants. - Les sérums précipitants peuvent servir de moyens de diagnoslic pour reconnaitre l'existence dans les produits de l'économie d'une matière provenant d'une espèce différente.

Ainsi quand on inocule à plusieurs reprises à un animal A, une matière albumineuse (sang, sérum, 
sperme, liquide d'ascite) prorenant d'une espèce animale différente, B, le sérum de l'animal A acquiert la propriété de précipiter in vitro les solutions albumineuses provenant de l'espèce $B$.

Qu'ou injecte plusieurs fois dn sérum d'anguille à un lapin; il est facile de conslater que le sérum recueilli chez cel animal, possède la curieuse propriété de précipiter fortement le sérum d'anguille.

Propriétés. - Les sérums précipitants peuvent ètre chaulfés impunément pendant une heure à วัo sans subir la moindre altération; mais leurs propriétés disparaissent après un chauffage à $70^{\circ}$. Ils se distinguent ainsi des sérums cytolytiques, bactériolytiques ou toxiques, très sensibles au chauffage et se rapprochent au contraire des sérums agglutinants, antiloxiques ou anti-infectieux.

On a proposé pour leur substance active, qui est précipitable par le sulfate de magnésie, le nom de coaguline, qui a été rejeté et remplacé par celui de précipitine, seul exact, puisque le trouble que provoque, dans une solution albumineuse, l'addition du sérum précipitant correspondanl, est le résultat de la précipitation de l'albumine et non d'une coagulation de cette substance (Vallée).

Préparatiox. - On inocule dans le péritoine d'un lapin ou d'un chien du sérum sanguin obtenu par des saignées répétées, du liquide d'ascite ou de l'épancliement pleural; le pouvoir précipitant du sérunı du lapin augmente à mesure qu'on multiplie les inoculations; il est actif à 1 pour 1000 au bout de six jours; il agit à 1 pour 50000 au bout de plusienrs mois. Pour fortifier le pouvoir précipitant, il faut renouveler ces inoculations tous les deux jours au moins et l'on attend cinq à six jours pour récolter le sérum de l'animal traité; on le saigne à jeun pour l'avoir bien limpide et parfaitement aseptique. Dans ces conditions, les sérums tenus au frais et dans l'obcurité conservent leurs propriétés précipi- 
tantes pendant plusieurs semaines. On peut également les conserver en les desséchaut rapidement dans le vide.

Eyploi des sérums préclpitants. - On mélange un volume de solution albumineuse limpide, à un volume légèrement supérieur du sérum préparé. La sensibilité de la réaction est remarquable; on peut utiliser ces sérums précipitants pour déceler des traces d'albumine dans les urines; ils sont utilisés avec succès pour le diagnostic des taches de sang, pour distinguer des taches sanguines d'origine hnmaine de celles d'origine animale; ils sont utilisés avec succès pour déceler des mélanges de lait et pour différencier les diverses espèces de viandes fraîches, salées oı fumées, mais non cuiles.

Mélanges de Lait. - Bordet a commencé par démontrer que si l'on pratique sur des lapins, des iıjections intra-péritonéales de lait de vache, on obtient chez ces animaux un séruın qui, mélangé au lait de vache, " y fait naître rapidement des grains, d'abord fins, qui grossissent bientôt et forment des flocons épais. Le liquide se sépare ensuite en deux parties. On a même obtenu un sérum précipitant l'albumine du lait de vache dont la dilution à 1 pour 100000 était active à la dose de quelques gouttes seulement, tandis que les réactifs chimiques ne seraient sensibles qu'à 1 pour 1000 . Ce sérum précipitant est d'autant plus pratique que le sérum des lapins inoculés avec le lait de vache ne précipite que les albumines du lait de vache; de même les lapins traités par le lait de chèvre fournissent un sérum précipitant seulement le lait de chèvre. On peut reconnaître facilemeut si le lait de brebis utilisé pour certains fromages a été additionné frauduleusement de lait de vache.

3० Séro-diagnostic. - Cetle méthode repose sur l’immobilisation et l'agglutination de certains bacilles sous l'influence du sérum, de la sérositè des sujets affectés d'une maladie infectieuse. 
Ce procédé extemporané est utilisé chez l'homme pour reconnaitre la fierre typhoüde. Il suffit de piquer le doigt pour recueillir un peu de sang et de sérum.

Ce sérum peut se conserver plusieurs jours, mème sinfecter sans que pour cela le pouvoir agglutinant soil sensiblement moditié. La persislance de globules rouges ou la dissolution de l'hémoglobine dans le sérum ne nuit en rien à la réaction. On mélange une goutte de sérum avec 10 goultes d'une culture de 24 heures.

Lorsque le sérum provient d'un malade atteint de fièvre typhoỉde, on aperçoit immédiatement des amas de bacilles agglutinés; il se forme des centres agglutinatifs; ils se fondent par pression réciproque et ne sont plus distincts au centre de l'amas. On peut mesurer ce pouroir agglutinatif en faisant des dilutions à 1 p. ว̈0, à 1 p. 100 , etc.

Le séro-diagnostic a été utilisé dans le choléra, la diphtérie, la pneumococcie, la colibacillose, la tuberculose, le tétanos; mais c'est particulièrement dans la fievre typhoide de l'homme qu'il fournit les meilleures indications. Il a été utilisé en médecine vétérinaire dans le diagnostic de la morve (Rabiaux), mais ses résultats sont peu précis.

$4^{\circ}$ Cyto-diagnostic. - La méthode du cylo-diagnostic ou diagnostic par lescellules est basée sur la recherche dans le liquide épanché dans les séreuses des diverses formes de cellules qu'on y trouve en suspension. On sait qu'on rencontre au sein des épanchements les plus limpides, en apparence, des globules blancs et des cellules endothéliales. Ces éléments diffèrent avec la nature des agents provocateurs.

Pour les rechercher, il faut commencer par la défibrination et la centrifugation des liquides fibrineux; on décante ensuite et on mélange complètement éléments cellulaires et liquide en les agitant ensemble et en les 
aspirant à plusieurs reprises dans une pipelle, et on l'étale ensuite sur une lame, on fixe à l'alcool-éther pour les colorations à l'hématéine-éosine et au bleu de Unna ou à la thionine; à la chaleur pour les préparatious au triacide d'Ehrlich, et on examine les préparations avec l'objectif à immersion.

Ce procédé de diagnostic, trouvé en 1900 par Widal et Ravaut, a été appliqué avec succès aux épanchements de la méningite tuberculeuse, aux hydrocèles, aux hydarthroses, aux épanchemenls séro-fibrineux de la plèvre.

Les élémeuts dont il faut rechercher l'existence et la proportion dans ces liquides sont: les leucocyles polynucléaires dérivant de la moelle des os, les lymphocytes et les leucocytes mononucléaires qui prennent naissance dans les organes lymphoïdes.

Les leucocytes polynucléaires, qui sont les agents essentiels de la phagocytose, s'observent dans les affections aiguës; les mononucléaires luttent plus parliculièrement contre les cellules et les microbes des affeclions chroniques comme la tuberculose et les lymphocytes ne jouissent d'aucuse action phagocytaire. Puisque chaque type d'affection a ses réactifs cellulaires; le nombre et la nature des cellules qu'on rencontre sont autant d'indications importantes pour le diagnostic.

Dieulafoy a pu, grâce à ce moyeu, reconnaître dans les pleurésies séro-fibrineuses trois types bien caractérisés :

$1^{0}$ Les grands placards endothéliaux indiquent une pleurésie d'origine mécanique; $2^{\circ}$ les polynucléaires accompagués de quelques mononuctéaires sont le signe d'un épanchement de uature infectieuse indépendante du bacille de Koch; $3^{\circ}$ enfin la présence des leucocytes est la preuve certaine d'une pleurésie tuberculeuse. Ces résultats sont confirmés par tous les moyens dont la science dispose aujourd'bui : inoculation de liquide pleurétique aux cobayes; injections de tuberculine (non sans danger chez l'homme), cultures sur le sang gélosé 
et glycériné, séro-diagnostic par le procédé d'Arloing, etc.

MM. Widal, Sicard et Ravaut ont appliqué leur méthode à l'élude des néningites avec les mèmes résultats. Le cyto-diagnostic permet de formuler cette équation : polyıucléose = méningite bactérienne; lymphocytose $=$ méningite tuberculeuse.

Enfin même formule cellulaire du liquide épanché quand il s'agit des hyulrocèles.

Il y a cependant quelques exceptions à la l'ègle, ce qui laisse, suivant la nature présumée de l’affection, une large place à d'autres moyens d'investigation : cryoscopie, sérodiagnostic, elc.

Néanmoins les résultats obtenus en pédiatrie pour le diagnostic différenliel des méningites tuberculeuses sont des plus précieux (1).

Toutes ces données amènent à penser que les applications du cyto-diagnostic à la médecine vétérinaire peuvent avoir la plus haute importance clinique.

Les animaux les plus exposés à la pleurésie, tels le cheval, le chien, le mouton, sont précisément les plus réfractaires à la tuberculose et on peut se demander si les affections de ce genre chez le cheval sont toujours franches, idiopalhiques ou entachées de tuberculose.

$5^{0}$ Épreuve du vésicatoire. - On applique, chez l'homme, un vésicatoire de 4 à ö centimètres de côté et douze heures plus tard, quand la bulle est bien formée, le liquide est recueilli, centrifugé et décanté el puis examiné.

On constate que la formule leucocytaire se modifie dans toutes les infections. Habituellement, on observe une diminution ou une disparition des éosinophiles yui reparaissent progressivement à la convalescence; à ce moment les polynucleaires neutrophiles diminuent, tandis que les mononucléaires augmentent (Roger).

(1) Lhomel, Répertoire de Police sanitaire, 1903. 


\section{II. - ÉLÉMENTS DU DIAGNOSTIC.}

L'interrogatoire des personnes qui ont soigné le malade et l'examen du sujet permettent de recueillir les signes diagnostiques.

\section{$1^{\circ}$ Interrogatoire des personnes auxquelles a} été contié l'animal malade. - Afin de bien préciser les questions qu'il doit poser, le vétérinaire doit toujours commencer par se faire une idée de la maladie en jetant. un coup d'œil rapide sur le malade. Il apprécie son individualité; il considère son espèce, son sexe, son àge, sa taille, sa race, son usage; il est même nécessaire d'en faire le signalement complet quand il s'agit d'une maladie contagieuse. Le praticien puise dans ces premières données des renseignements importants.

Au sujet de l'espèce, il se rappelle que la périlonite estrapidement mortelle chez le cheval; les boufs la supportent plus ou moins longtemps; les chèvres y sont très sensibles.

Les femelles sont exposées à la fièvre puerpérale, à l'iclumpsie; les chevaux entiers à la hernie inguinale ètranglée, les vieux chiens a l'hypertrophie de la prostate, les baufs aux calculs urétraux.

L'âge fait soupconner diverses maladies : les jeunes chiens sont affectés de la maladie du jeune àge, les poulains de la gourme, les vieıx chevaux de la morve, de l'emphysème pulmonaire. Dans cet examen d'ensemble, le vétérinaire apprécie les modifications extérienres que la maladie peut avoir imprimées à l'organisme; il note le degrré relatif de résistance que l'animal peut opposer à la maladie et aux moyens thérapeutiques qu'il sera nécessaire d'employer.

La maigreur du sujet, son altitude, sa physionomie trahissent l'ancienneté de la maladie et l'intensité de ses souffrances. La tuméfaclion des paupières, les lancina- 
tions d'un membre, la raideur tétanique du corps, les éruptions et les dépilations cutanées, le jetage mettent sur la voie du diagnostic et permettent de donner à l'interrogatoire une direction déterminée.

Après cet examen rapide et silencieux, l'interrogatoire commence; il doit être court, méthodique et complet; il faut éviter les questions inutiles, oiseuses, ou qui ne peuvent ètre comprises; les demandes doivent ètre formulées en termes clairs, précis; elles varient suivant les soupçons qu'on a sur le siège et la nature de la maladie. Généralement, on débute par la question suivante : "Depuis quand l'animal est-il malade? Mange-t-il? Se contche-t-il ? "Par une série de questions tendant à déterminer les caractères des symptòmes observés, le praticien parvient souvent à déterminer le siège de la maladie. On peut écouter le récit fait par le ccnducteur de l'animal qui narre les premiers troubles qu'il a observés, leur succession, leurs modifications et fail souvent une histoire inexacte de la maladie. Les points obscurs peuvent ètre éclaircis par d'autres questions habilement posées; on peut savoir si l'animal a été malade antérieurement et s'il est-resté longtemps sans travailler. Quand il saisit des contradictions dans les réponses qui lui sont faites, il interroge de nouveau en se servant d'expressions plus vulgaires et plus intelligibles. Dans cel interrogatoire, il faut passer tous les appareils en revue et s'attacher à mettre en évidence tous les troubles fonctionnels observés.

Au sujet de l'étiologie de la maladie, le pralicien obtient les réponses les plus variées et souvent les plus erronées. On ne peut espérer obtenir des propriétaires des données étiologiques bien sérieuses. On peut demander des renseignements au sujet de la nourriture, des boissons, des conditions hygiéniques dans lesquelles l'animal a vécu jusqu'à l'apparition de la maladie. La contagiosité de la maladie, son extension rapide dans un troupeau 
précisent son origine endémique ou épizootique. Ces renseignements peuvent modifier l'opinion du clinicien et l'obliger à donner une autre direction à ses recherches.

Tous les faits énoncés par cet interrogatoire doivent être contròlés; ils ne peuvent ètre acceptés comme vrais yu'autant qu'ils cadrent avec les signes recueillis à l'examen du malade. Il faut se défier des préjugés des propriélaires, de l'imagination des conducteurs el squvent de leurs avis intéressés. Ils ont souvent contribué au développement de la maladie par leur négligence, par l'inobservation des règies d'hygiène; ils mentent quelquefois par habitude ou se livrent à de telles interprétations au sujet de la maladie qu'il est impossible de tirer parti des faits précis qu'ils ont recueillis. Les vices rédhibitoires, les maladies contagieuses sont souvent dissimulés et il est impossible d'avoir le moindre renseignement sur les symptỏmes observés, sur l'ancienneté et l'origine du mal.

20 Examen du malade. - Ou le soumet à l'action de tous les sens et de tous les instruments spéciaux qui peuvent étendre le domaine de nos investigations. L'examen doit être rapide et complet; il doit porter d'abord sur l'organe soupçonné d'être le siège du mal, puis sur les autres appareils par ordre d'importance et de rapports avec le premier, sans en oublier aucun (Labat). On peut passer en revue tous les organes d'une région ou explorer appareil par appareıl.

L'examen du malade région par région est simple et commode quand on en a l'habitude. Le vétérinaire passe en revue la tête, la gorge, la poitrine, le ventre, les membres; il fixe son altention sur le facies, l'état des yeux, de la conjonctive, des naseaux, de la bouche, du pouls, de l'auge; il comprime le pharynx, le larynx, constate les déformations présentées par ces régions, fait glisser la main le long de la gouttière jugulaire pour s'assurer qu'il n'y a pas de corps étrangers et passe à l'examen de la 
poitrine qu'il explore par l'inspection, la palpation, la percussion, l'auscultation.

L'abdomen fail l'objet d'une investigation minutieuse quand les troubles fonctionnels (ictère, coliques, etc.), dénoncent l'existence d'une affection de l'un des organes contenus dans celle cavité.

Le fourreau, la rerge, la ressie sont examinés arec le plus grand soin; il faut toujours recueillir de l'urine, y rechercher des matières anormales (sucre, albumine, matières colorantes de la bile) et déterniner, quand on est outillé couvenablement pour ces recherches, les variations subies par les matières qui y sont contenues normalement. On interroge la sensibilité cutanée, on exerce des pressions sur la colonne vertébrale pour juger du degré de flexibilité des reins; on fail marcher l'animal pour apprécier ses forces et les troubles engendrés par la fierre et les intoxications.

Ce procédé, très expéditif, évite de revenir plusieurs fois au mème point pour examiner des,appareils différents.

L'examen du malade, appareil par appareil, est plus en rapport arec l'étude sémiologique qui précède.

On explore successirement tous les appareils de l'économie : appareil digestif, respiratoire, circulatoire, génital, système nerreux, peau, etc.; on tire une mèche de crins pour juger de la nutrition du malade; on prend la température pour mesurer l'iutensité de la fièrre; on résume les principaux faits observés et l'on constate s'ils s'éloignent ou se rapprochent de l'idée qui a présidé à ces investigations. Quand les signes recueillis sont caractéristiques d'une maladie différente de celle qu'on avait supposée, il faut renoncer immédiatement, à la première opinion, pour adopter celle qui est conforme aux faits observés.

En possession de tontes les données qu'il a puisées dans l'interrogatoire du propriétaire el dans l'examen du malale, le praticien est suffisamment renseigné pour résoudre successivement les problèmes suirants : $1^{\circ}$ reconCAADÉAC. - Sémiologie, 2e édit. Il. -28 
naitre les symptòmes; 20 reconnaître les lésions et l'alfection; $3^{\circ}$ reconnaitre la maladie.

\section{III. - DIAGNOSTIC DES SYMPTOMES.}

En comparant le fonctionnement morbide avec le fonctionnement physiologique, on reconnail les symptômes.

Linspection fait diagnostiquer le jetage, les chancres, les boiteries, etc.; la palpation fait reconnaitre la sensibilité, la flucluation, elc.; la percussion met en évidence la malité ou l'excès de sonorité du poumon, l'auscultation fait conslater des bruits et des souffles anormaux.

Les symptômes recueillis se rapportent ordinairement à un grand nombre de maladies; il faut les étudier, en préciser la nuance et l'origine. Quand on a reconnu qu'un cheval boite, il faut rechercher si la boiterie provient d'une allération des os, les tendons, des ligaments, des symoviales, des muscles ou des nerfs; il faut examiner minutieusement le pied, le boulet, le canon, les articulations et les rayous des parties supérieures. S'il s'agit d'un animal qui jette, on étudie les caractères de ce jelage ; on regarde s'il provient du nez, du sinus, du pharynx, du larynx, des poches gutturales, des bronches, du poumon, de l'resophage, de l'estomac, ou s'il procède d'une carie dentaire, d'une maladie spécifique comme la morve ou la tuberculose. Le diagnostic des symptòmes n'est complètement fait que lorsque leur signification est suffisamment précise pour pouvoir les transformer en signes et les faire servir au diagnostic de la lésion et de l'affection.

\section{V. - DIAGNOSTIC DE LORGANE MALADE ET DE LAFFEC'TION.}

L'organe malade est le problème le plus important à résoudre; il découle de l'observation des syıptòmes et de la détermination des lésions. 
Celles-ci doivent êlre spécifiées quant à leur siège et à leur nature. Le diagnostic local indique l'existence d'une fracture intra-articulaire ou épiphysaire, d'une pleurésie simple ou double, d'une pneumonie lobaire ou lobulaire, générale ou circonscrite à une partie du poumon. Cette détermination a une importance capitale au point de vue du pronostic et du Irailement.

Pour reconnaître le siège d'une altération, il suffit quelquefois d’analyser les symplòmes observés. Certains d'entre eux ont un caractère général, secondaire, d'autres sont l'expression d'une altération organique dont le trouble fonctionnel a été la conséquence.

L'ictère indique toujours une rétention biliaire ou une hypercholie, le cornage chronique est 99 fois sur 100 le symptôme d'une hémiplérgie laryugienne; d'autres symptòmes ont une origine très variable; le soubresaut du flanc est un symptòme de toutes les affections de l'appareil respiratoire, el de diverses maladies de l'appareil circulatoire; le jetage n'a jamais une signification univoque; il peut provenir d'une maladie des cavités nasales, des sinus, des bronches, du poumon. A défaut de troubles fonctionnels caractéristiques, on percoit des modifications physiques qui sont pathognomoniques des altérations que l'organe a subies. La percussion, l'auscultation dénoncent les épanchements pleuraux et pulmonaires; la mensuration, les changements de volume; la palpation, les altérations de consistance ou de sensibililé.

La déterminalion de l'organe malade doit être complétée par la localisation du mal dans cet organe. Le poumon étant reconnu altéré, il faut savoir quelle est l'étendue de l'altération; il faul rechercher si elle est bornée à un lobe antérieur, à la parlie moyenne ou postérieure. S'agit-il du tube digestif: on doit s'efforcer de préciser le point qui est le siège du mal et reconnaittre si l'altération est bornée à l'estomac, au duodénum, à l'inteslin grêle, au cæcum, au côlon ou au rectum. 
La nature des altérations que présente l'organe est indispensable à connaitre quand on veut caractériser un cas clinique. L'absence de murmure respiratoire et la matité de la poitrine sont deux signes qui relèvent d'un épanchement liquide dans la plèvre, d'un exsudat séreux, hémorragique, fibrineux, dans les alvéoles du poumon ou de tubercules conglomérés ou même de tumeurs de cet organe. Il importe de spécifier la nature de cette lésion pulmonaire ou pleurale.

Les symplômes généraux sont d'un graud secours dans cette détermination. La fièvre intense sans oscillations prononcées dénonce une affection inflammatoire ou une infection locale; la maigreur du sujel sans réaction fébrile est l'indice d'une néoplasie maligne. La prostration du sujet ou l'excitation du système nerveux, le rapport ou la disproportion qui existent entre les symptômes généraux et les lésions locales doivent égalemeut entrer en ligne de compte pour établir ce diagnostic. Parfois les signes locaux sont eux-mèmes très siønificatifs : ils peuvent révéler l'existence de cavernes qui font soupçonner la tuberculose ou la péripneumonie contagieuse chez le bauf, et des abcès chez le cheval.

L'examen des produits rejetés peut faire recueillir des signes caractéristiques : on peut trouver du sang dans les matières fécales, des débris de tumeurs dans l'urine, du sang, du pus, des moules fibrineux dans le jetage. Le clinicien peut réunir ainsi les éléments d'une affection inflammatoire, hydropique, reconnaitre une tumeur, mais il ne doit pas s'en tenir là; il doit rechercher la maladie.

\section{V. - DIAgNostic DE LA MaLADIE.}

Le diagnostic de la maladie comporte la détermination de la cause, de l'intensité, de la marche et de la période de la maladie. 
Le diagnostic étiologique met en évidence la cause déterminante de la maladie.

Quand un troupeau de moutons maigrit et présente de la cachexie, l'examen des matières fécales y fait découvrir des œufs de distomes qui sont la cause de la cachexie. L'examen anatomique d'un animal sacrifié fournit les mèmes renseignements et fait différencier la distomatose de la bronchite vermineuse; l'examen microscopique du jetage nasal y fait reconnaître la présence d'œufs et d'embryons de strongles.

On ne peut combattre la maladie avec quelque chance de succès que lorsque sa cause est nettement connue. 11 est souvent impossible de distinguer l'eczéma de la gale; la déconverte du parasite de cette dernière maladie guide le pronostic et le traitement. La tuberculine et la maléine parviennent à dépister les bacilles de la tuberculose et de la morve qui se cachent derrière une bronchite, une entérite, etc. Une arthrite peut être traumatique, morveuse, tuberculeuse, pyohémique, rhumatismale; il faut loujours en préciser la nature.

La plupart des parasites qui occupent les cavités en rapport avec l'extérieur peuvent ètre facilement décelés; ceux qui sont inclus dans les viscères (kystes hydatiques, etc.) ne sont reconnus (qu'à l'autopsie.

L'intensité de la maladie est établie par l'élendue des lésions et par les symptômes généraux qui l'accompagnent. On reconnait ainsi le degré de gravité de la maladie et l'on proportionne le traitement à son intensité. Une angine simple n'est pas traitée comme une angine phlegmoneuse accompagnée de cornage; un clou de rue simple ne réclame pas les mêmes moyens chirurgicaux qu'un clou de rue compliqué de synovite, de signes de nécrose de l'aponévrose plantaire.

La marche de la maludlie est aiguë, suraiguë ou chronique. Celte détermination préside au pronostic, au trai_ tement du malade; les moyens d'enrayer l'affection aiguë 
sont différents de ceux qui doivent être employés contre la maladie chronique. En outre, la forme chronique est toujours plus grave que la forme aiguë.

La période à laquelle la maladie est arrivée peut être établie en tenant compte de l'évolution des lésions et des symptômes qui en découlent. Dans le cas de pneumonie, la matité et le souffle tubaire indiquent la période d'élat; des rougeurs et des macules disséminées à la surface de la peau dénoncent une éruption commençante. la chute de la fièvre et l'expulsion d'urines critiques appartiennent à la défervescence et annoncent la fin de la maladie.

Ainsi, il ne suffit pas de reconnaître une pneumonie, une pleurésie, il faut savoir si la maladie diagnostiquée est à la période de début, d'état ou de déclin. Quand elle présente une cause et des symptômes bien caractérisés et une marche régulière, le diagnostic est direct, simple et relativement facile; il est difficile, indirect et comparutif quand les signes sont cachés, obscurs ou trop vagues; on doit procéder alors par élimination de toutes les maladies qui se trahissent par des signes caractéristiques et aboutir par voie d'exclusion à l'élat morbide qui n'a pas de physionomie spéciale.

Dans ce diagnostic différentiel, il faut s'attacher à mettre en évidence tous les signes positifs ou négatifs par lesquels une maladie donnée se distingue de loutes les aulres avec lesquelles, en raison des signes communs ou analogues qu'elle présente, on pourrait être tenté de la confondre (Hecht). Le diagnostic doit être réservé quand la maladie n'a pas sa symptomatologie habituelle ou présente une marche inaccoutumée; il est préférable d'attendre que de porter un pronostic hypothétique et d'instituer un traitement irrationnel. 


\title{
CHAPITRE II
}

\author{
PRONOSTIC
}

Apprécier la gravité ou la bénignité des maladies, prévoir leur marche, leur durée, leur terminaison, leurs complications, c'est-à-dire prédire le sort de chaque malade pris en particulier, tel est le but du pronostic. Ce jugement porté d'arance résulte d'une connaissance complèle de la maladie et du malade. Le pronostic est le corollaire du diagnostic.

\section{I. - PRonostic tiré de La Maladie.}

Les signes tirés de la maladie embrassent ses causes, ses symptỏmes el ses lésions.

$1^{\circ}$ Causes. - Les causes influent sur la bénignité ou la gravité des maladies par leur nature, leur nombre (germes et parasites), leur voie d'introduction, et leur degré d'énergie.

a. Nature. - Tous les germes n'ont pas la mème faculté d'infection. Certains ne peuvent s'installer dans l'organisme que d'une manière passagère; ils produisent des maladies cycliques à marche prérue, toujours facile à prévoir et que rien ne peut modifier. Les maladies éruptives (clavelée, fièvre aphteuse), ont une érolution toujours à peu près identique; la vaccine engendre toujours les mêmes pustules; elles arrivent à la période de dessiccation et guérissent dans le mème temps. Le pronostic 
individuel est bénin; il est relativement grave par suite de la conlagiosité de ces maladies et du chiffre considérable d'animaux infeclés. Celte gravilé résulte aussi de la diminution de lait et de poids des animaux qui en sont affectés. A côté de ces germes infectieux qui président au développement de maladies aiguës, il en est qui n'ont habituellement qu'une action locale et qui sont incapables de se propager. Les germes de la suppuration demeurent cantonnés au point où ils ont été inoculés; les affections qu'ils déterminent ont un pronostic bénin quand ils n'intéressent pas un organe essentiel à la vie, comme le cerveau, le bulbe, le cœur, le rein, ou quand le pus dont ils ont déterminé la production ne contamine pas d'autres organes importants inaccessibles aux moyens thérapeutiques.

Les germes septiques et souvent les germes de la suppuration ne produisent chez les solipèdes et chez le bœuf des maladies graves que lorsqu'ils s'associent à d'autres grermes qui ont préparé, dans l'organisme, un milieu favorable à leur action pathogène.

Il est des microbes, comme celui de la peste bovine, qui tuent très vite; d'autres peuvent végéter indéfiniment dans l'économie el infecler d'autres animaux pendant toute la vie de l'animal qui les porte : le microbe de la morve chez le cheval, le bacille de la tuberculose chez le bœuf déterminent toujours des maladies très graves pour les animaux affectés et très dangereuses pour les hommes et les animaux qui se trouvent à proximité des sujets infectés.

Le pronostic est d'aulant plus bénin qu'on a affaire à un germe plus inoffensif; il est d'autant plus redoutable que le microbe appartient à une espèce plus active, plus prolifique et plus rebelle à tous les moyens thérapeutiques. Les formes les plus graves de tumeurs sont également celles qui se reproduisent et qui grossissent avec le plus de rapidité; ce sont celles qui possèdent le plus de 
malignité dans les tissus qu'elles occupent el qui risquent le plus de se généraliser.

Les parasites logés dans le tube digestif produisent des affections moins graves que ceux qui recherchent un habilat dans le cerveau, le cœur, le foie, le poumon; les ténias sont ainsi moins redoutables d'une manière générale que les cysticerques, les cœnures, les échinocoques, etc.

Les traumatismes onl une action bénigne ou grave suivant leur nature; les contusions ont un pronostic beallcoup plus favorable que les plaies pénétrantes. Toutes les fois qu'un organe ou une cavité normale est mis en communication avec l'extérieur par suite de la perforation de la peau, le pronostic esl très grave. S'agil-il d'une inloxication, la gravité du pronostic est en rapport avec le degré de causticité, de toxicilé du poison et de son affinilé pour cerlains éléments analomiques. L'empoisonnement par l'oxyde de carbone n'est si dangereux que parce que ce gaz forme une combinaison slable avec l'hémoglobine. Certaines maladies ne guérissent jamais quand elles sont confirmées: la rage parmi les maladies aiguës, la tuberculose pulmonaire parmi les maladies chroniques se comportent ainsi.

b. Nombre. - La quantité de germes infectieux décide généralement de l'issue d'une maladie bactérienne. Quelques microbes sont généralement peu à craindre quelle que soil leur virulence; une masse de microbes peu pathogènes ou mème habituellement saprogènes tue. Les expériences de Watson-Cheynes, de Bollinger, de Gebhardt, etc., sont très démonstratives de l'influence excercée par le nombre sur la gravité ou la bénignité d'une infection. Les moutons algériens contractent la fièvre charbonneuse, mais ils guérissent si la quantité de bactéries inoculées ou ingérées n'est pas très considérable.

Les parasites ont une action proportionnelle à leur 
nombre. Quelques trichines peuvent laisser l'animal indemne; une infection complète comme celle qui est produite expérimentalement peut faire périr les animaux de péritonite; un seul cœnure cérébral provoque une inaladie chronique qui permel l'utilisalion du sujet; la mort est rapide et résulte d'une encéphalile quand il y a beaucoup de ces parasites dans la masse cérébrale. Le foie qui n'est envahi que par quelques douves, le poumon des ruminants qui ne présente que quelques strongles continuent à fonctionner normalement.

Les parasites superficiels comme ceux des gales modifient le pronostic par leur nombre; les piqûres isolées d'abeilles n'ont qu'une action locale insignifiante; un essaim d’abeilles qui s'abat sur un cheval détermine des troubles locaux et généraux capables d'entraîner promptement la mort.

On trouve cependant des causes dont l'action isolée est assez énergique pour permettre de prévoir une issue défavorable ou des complications mortelles dès qu'elles ont agi: un corps étranger dans le cœur, un calcul dans l'urètre du bœuf suffisent pour compromettre la vie.

c. Voies d'introduction. - La gravité des intoxications est subordonnée à la quantité de poison ingérée. Les maladies les plus graves deviennent parfois les plus bénignes quand la cause provocatrice se trouve éloignée de son terrain de prédilection. Le bacille du charbon symptomatique est inoffensif s'il ne peut atleindre le muscle; il en est de même des microbes de la vaccine, de la clavelée, maintenus éloignés de l'épiderme, du microbe de la rage injecté dans la circulation des ruminants. Qu'il s'agisse d'une affection contractée par inoculation, par ingestion, par inhalation, il faut que le microbe infectieux parvienne dans l'organe qui lui convient pour faire évoluer sous sa forme la plus grave la maladie contagieuse qu'il engendre. Le pneumocoque, le microbe de la péripueumonie contagieuse réclament le poumon; le bacille 
de Koch, le système lymphatique; l'acné contagieuse, le derme, sinon ces microbes ne peurent produire que des maladies insignifiantes.

Les parasites qui ne penvent arriver dans le milieu qu'ils réclament s'atrophient et meurent : le cœnure qui s'arrète dans le poumon, dans le foie du mouton ne provoque que des troubles passagers; l'animal guérit conıplètement par la mortification et la disparition des parasites.

Les corps étranger's introduits dans les voies digestives engendrent des maladies (entérites, gastrites, etc.) dont le pronostic est très grave en raison des nombreuses complications qui sont les conséquences de leurs migrations. Il convient d'être toujours réservé sur l'issue des accidents qu'ils déterminent.

Ils peuvent obstruer complètement l'intestin, le perforer, engendrer une péritonite mortelle, des abcès hépatiques, rénaux, spléniques, abdominaux, une pneumonie, une pleurésie, une péricardite, une myocardite, et une endocardile mortelles. On ne peut se prononcersur l'issue des troubles consécutifs à l'ingestion de corps étrangers que lorsque ceux-ci sont éliminés.

Pareille réserve est commandée par les intoxications déterminées par l'ingestion de caustiques acides. Quand les phénomènes toxiques sont conjurés, on doit redouter les septicémies; les germes contenus dans l'intestin se répandent facilement dans le sang quand la protection épithéliale et phagocytaire est supprimée.

Les morsures de chiens enragrés intéressant la tête sont plus graves que dans les autres parties du corps; les microbes pathogènes, en général, sont moins dangereux quand ils sont ingérés que lorsqu'ils sont inoculés. Les corps étrangers de l'œil, de la conjonctive, produisent des effets plus graves que ceux de la peau, du tissu cellulaire sous-cutané et des muscles.

d. Intensité d'action. - La virulence des agents micro- 
biens varie constamment; elle est le principal élément d'appréciation de la gravité des maladies contagieuses. Le pronostic est favorable ou défavorable, suivant que le virus est fort ou faible.

Les renseignements fournis par les autres malades guéris ou morts, l'extension plus ou moins rapide de la maladie contagieuse fortifient le jugement porté sur le malade et sur l'épizootie. Les morsures du loup enragé sont plus graves que celles du chien; la morve aiguë est plus facilement transmissible et plus rapidement mortelle que la morve chronique. Le microbe de la septicémie est parfois si affaibli qu'il ne détermine qu'un phlegmon; il peut devenir si aclif qu'il tue d'une manière foudroyante. On voit parfois des épidémies de pneumonie contagieuse déterminer la mort de la plupart des animaux atteints; il en est d'autres, au contraire, dans lesquelles ils sont peu malades et rapidement guéris.

En dehors des conditions individuelles et susceptibles d'exagérer les effels des microbes, il faut tenir un grand compte du degré de virulence propre à chacun d'eux et des nombreuses variations que cette virulence peut subir dans chaque localité, dans chaque contrée, dans chaque climat. Si le pronostic de la clavelée, du charbon est moins grave en Algérie qu'en France, c'est que les microbes de ces maladies ont une virulence plus faible ou sont moins aptes à se développer dans l'organisme d'animaux doués également d'une résistance physiologique plus considérable.

Les caustiques ont une action proportionnelle à leur degréde concentration. Les acides sulfurique, chlorhydrique, l'ammoniaque, la potasse diluée n'offrent aucun danger; ce sont des poisons mortels quand ils sont purs ou insuffisamment dilués. Leurs effels varient également, comme nous l'avons ru, suivant leur point d'application. Relativement moins dangereux quand ils sont déposés sur la peau ou sur une plaie extérieure, ils produisent toute 
leur action nocive quand ils sont répandus sur une muqueuse.

Les traumalismes sont d'autant plus grares qu'ils sont plus énergiques; ils peurent, suivant leur degré d'intensité, contusionner les tissus, produire des ecchymoses, des épanclıements séreux, des déchirures, des fractures, des mortifications et les complications les plus variées.

20 Symptòmes. - Les symptômes dénoncent le siège, l'intensité et la gravité des infections, des maladies locales et des maladies générales. Le diagnostic précède toujours le pronostic. Les signes diagnostiques sont très souvent différents des signes pronostiques. Si le râle crépitant, le jetage rouillé et la plainte fout reconnaitre une pueumonie, ces signes ne permettent pas de porter un jugement sur sa durée, sa marche, sa terminaison.

Les symptòmes rationnels (fièvre. etc.) ont souvent une signnification prépondérante. On pent les interroger pendant le cours des maladies aiguës et des maladies chroniques en tenant compte de leur marche, le leur durée, de leurs complications, de leurs récidives ou rechntes.

a. Maladies aiguës. - Les maladies aiguës ont des symptòmes communs qui expriment imnédiatement leur gravité relative.

La fievre est l'un des symptomes les plus importants pour le prouostic. Les températures extrèmes sont suivies de combustions trop intenses el de dégénérescences trop profondes pour ètre curables; une température de 43", chez le cheval, précède généralement la mort. L'abaissement considérable de la température donne une indication défavorable; une température de 2 t à $30^{\circ} \mathrm{chez}$ le chien appartient à la période agonique; uue température de $32^{\circ}$ peut persister pendant cinq jours chez les âues affectis de pelotes stercorales sans annoncer une terminaiso: mortelle (Cadéac).

Tous les appareils organiques fournissent des renseiCadíac. - Sémiologie, $2^{\circ}$ edit.

II. -29 
gnements imporlants pour le promostic, quel que soit le siègre de la maladie.

L'appareil circulatoire doit être exploré altentivement au niveau du cœur, des artères, des veines, des capillaires.

Des battements cardiaques précipités, tumultueux sont l'indice d'une inloxication par des produits végétaux ou microbiens; la septicémie, l'endocardite infectieuse, deux complicatious d'un grand nombre d'autres maladies, ont un pronostic également redoutable.

Le pronostic est très grave quand les battements sont imperceptibles à la palpation, comme dans le cas d'épanchement péricardique ou de myocardite. In pouls petil, filiforme, exprime la vacuté de l'appareil circulatoire a la suite d'une hémorragie, ou l'iusuftisance de l'activité cardiaque. L'absence de pulsations sur le trajet d'une artère est le résultat d'une thrombose et doit toujours faire craindre une embolie.

La réplétion excessive des veines périphériques, qui résulte de la compression des vaisseanx internes par les viscères abdominaux distendus, est un siğne défavorable; il indique l'anémie des organes comprimés el précède souvent leur rupture. la lividilé des muyueuses témoigne d'un défaut de circulation et d'oxyénénation du sang; c'est-à-dire l'un état plus ou moins prononcé d'asphyxie et d'inloxication.

L'apparcil respiratoire présente, du commencement à la fin de chaque maladie aigné, une série de modilicalious caractéristiques. Li dilatation extrème des naseaux cliez les solipèdes, l'agitation les lèvres chez les carnivores trahissent une dyspnée intense, loujours proportionnée ¿ l'étendue des lésions respiratoires, à l'allération du sang et à l'iusuffisance de l'hématose.

Le pronostic est toujours extrèmement grave quand l'animal a de la dyspnée sans lésions bien appréciables de la poitrine; les troubles respiratoires sont fréquemment déterminés par une destruction globulaire rapide 
et irréparable, qui est le fait d'affections microbiennes. L'odeur gangreneuse de l'air expiré est, dans toules les maladies aiguës, le prélude d'une mort prochaine. Lne respiration dyspnéique, soubresautante ou discordante, siflante, stertoreuse, appartient aux maladies graves de l'appareil respiratoire qui gènent l'entrée de l'ail dans le poumon, aux affections des nerfs qui imnervent le larynx ou des centres nerveux (congestions, inflammalions) qui suppriment l'action du pneumogastrique. L'animal peut à lout instant périr asphyxié.

L'appareil digestif fournit les signes pronostiques les plus frappants sinon les plus exacts. Toute maladie qui supprime complètement l'appétit, la soif. le tic, chez les animaux alteints de ce vice, qui détermine des máchonuements, des grincements de dents, des vomissemenls opiniàtres chez les carnivores, de la diarrhée ou de la dysenterie chez lous les animaux, avec coliques et relàchement de l'anus, est grave. Cerlains signes lels que l'expulsion involontaire d'excréments et de sang amnoncent une fin pruchaine.

L'absence de lous ces signes doil faire émeltre un avis favorable, malecré l'imporlance Je l'organe malade et la gravilé apparente de la malarlie.

L'appareil génito-urinaire donne une mesure précise de la bénignité et de la gravité des maladies. Un étalon qui ne hennit pas, qui n’entre plus en érection, qui se montre indifférent à l'approche d'une jument est gravement malade. L'urine chargée, riche en pus, en sang, l'urine rare, la suppression complète de la miction sont des signes défavorables.

Il en est de mème de l'arrèt brusque des exsudations et les sécrétions pathologiques des plaies, des résicatoires, des sétons, le la mauraise odeur répandue par les malades. Les chiens affectés de la maladie du jeune àge répandent une odeur cadavérique avant de mourir.

La physionomie et l'attitude du sujet sont les meilleurs 
éléments pour se faire une opinion sur la maladie. La face grippée, les lèvres rélractées, laissant voir les dents, le rire sardonique, les yeux enfoncés dans les orbites, le décubitus prolongé, l'agitalion continuelle, la station debout forcée sont des signes défavorables.

Le systeme nerveur el l'apparcil locomoleur fournissent souvent des symplómes d'une haute gravité tels que : douleurs violentes, vertige, épilepsie, convulsions, coma, proslration, insensibilité, raideur, tremblements partiels ou généraux, soubresauts, épuisement des forces, collapsus, blessures, excoriations, paralysies, paraplégie.

Quelques signes précèdent et ammoneent une terminaison mortelle prochaine : décubitus latéral complet, rire sardonique, sueurs froides, verge pendante, respiralion stertoreuse, intermittente, arrachement ficile des crins et poils, relìchement des sphincters.

Parmi les signes facorables yui atlirent plus particu. lièrement l'attention dans le cours des maladies aiguës, il faut signaler : la conservation de l'appétit et de la soif, lattention du malade, la souplesse des reins du cheval, les pandiculations des bètes bovines, la disparition des plaintes el des cris, le retour de la rumination el des habitudes contractées parles animaux, les hemnissements chez les miles (chevaux), la conservation des forces, chez tous les animaux.

b. Maladies chroniques. - Ie pronostic des maladies chroniques est très variable. Un certain nombre de ces affections sont compatibles avec la vie el l'utilisation des animaux (emphysème pulmonaire, cornage); d'autres ne sont dangerenses que lorsqu'elles sont généralisées (mélanose, tumeurs malignes) ou qu'elles nuisent au fonctionnement d'un organc indispensable a la vis (cour, foir, moelle, etc.). La tuberculose, la péripneumonie contagieuse, la morve, sont des maladies funestes pour le sujet aflecté de l'une d'elles; les exostoses, les lésions articulaires sont une cause de soulfronces 
continues ou intermittenles nécessitant le repos du sujet.

La plupart des affections chroniques doivent faire émettre un maurais pronostic parce qu'elles sont sourent incurables, qu'elles rendent l'utilisation du sujet impossible ou qu'elles s'accompagnent de diverses conplications graves ou mortelles. L'endocardile chronique impose au cheval et au chien une allure lente, un repos fréqueut et provoque des troubles et des lésions secondaires de l'appareil digestif, du foie, du rein et du poumon.

La gravité des maladies chroniques dépend de la complexilé ou de l'importance des fonclions de l'orģane malade.

Les maladies du cerveau, du cœur, du foie, des reins, doivent toujours faire craindre une terminaison fatale.

Des inflammations aiguës se grelfent fréquemment sur les maladies chroniques; elles tuent promptement le sujet (pleurésie, péritonite) ou favorisent la disparition des lésions, la destruction des tissus de nouvelle formation.

c. Marche. - La marche régulière de la maladie; sa décroissance progressive quand elle est arrivée à la période d'état, les crises (sueurs, miction abondante), la rareté des accès dans les affections nerveuses, l'abaissement graduel de la température annoucent une terminaison heureuse.

La narche anormale de la maladie; les oscillations brusques de la température, la périodicité ou la multiplicité des attaques, la disparition instantanée de la douleur doirent inspirer des craintes sérieuses.

d. Durèe. - La durée prolongée d'une maladie est toujours un signe fàcheux: elle est l'indice de l'exlension des lésions, d'infections secondaires, de complications ou du passage de la maladie à l"étal clıronique ou de l'impuissance de tous les moyens thérapeutiques employés. 
e. Complications. - Quand l'organisme est endonmagé par un processus morbide, les autres organes sont plus vulnérables. Les complications qui se produisent aggrarent le pronostic parce qu'elles comprometlent la défense et qu'elles témoignent de l'exagération de la réceptivité organique pour toutes les influences pathogènes.

$f$. Rechute et récidive. - La rechute frappe l'organisme débilité et triomphe facilement de ses résistances. Les rechutes sont généralement produites par des microbes secondaires qui évoluent dans l'organe altéré par le microbe de l'infection primitive. Les rechutes prennent souvent un caractère septique et emportent rapidement le malade.

A cet égard, les récidives sont beaucoup moins à craindre que les rechutes. La première alteinte a souvent renforcé l'état bactéricide et le phagocytisme du sujel, de sorte que la maladie qui récidive revèt une forme plus bénigne que la maladie primilive. La vaccine, la clavelée, la fièvre aphteuse el toutes les affections bactériennes sont dépourvues de gravité quand elles récidivent.

\%. Coïncidences morbides. - L'existence de leux maladies, clıez le même sujet, aggrave toujours le pronostic.

Certaines maladies bénignes fout comrir de grants dangers quand elles so développent chez des animaux atteints de niphlute, d'endocardite en raison de l'insuffisance d'ólimination et des troubles circulatoires délerminés par ces maladies.

La suppuralion entraine la gangrene cliez les diabétiques; la pneumonie entraine l'asphyxie des animaux alteints de péricardite, de myocardite et d'endocarlite.

h. Effets du traitement. - Les ellets obtenus avec le Iraitement employe indiguent une terminaison favorable quand le mal a éte murayé, qu'il y a cu une amélioration dans l'état général du malade.

Le pronostic est fàcheux quand les médicaments ne pro- 
duisent pas leurs effets accoutumés, quand les révulsifs, les dérivatifs n'exercent aucune action sur l'organisme.

$3^{\circ}$ Lésions. - Le siège, l'étendue et les caractères des lésions morbides font prévoir l'issue funeste ou heureuse de la maladie.

a. Siège. - Le bulbe, le cœur, le poumon, sont les trois organes les plus indispensables à la vie (Voy. Mort, p. (669).

Des lésions insignifiantes dans les autres organes suffisent pour supprimer les fonctions du bulbe.

Le cour et le poumon sont beaucoup plus tolérants.

Tous les organes pairs ou divisés en lobes se suppléent fréquemment. Les fonclions hépatique, rénale, pulmo. naire ne sont pas supprimées par suite de l'atrophie d'un lolse du foie, du poumon on d'un rein.

Les lésions du cœur sont toujours graves, parce que cet organe ne se repose jamais.

Une nécrose du cartilage complémentaire de l'os du pied, de l'aponévrose plantaire tire sa gravité du peu de vitalité de ces tissus.

La destruction d'une portion de tissu osseux, les fractures diaphysaires, les sections nerveuses ont un pronostic moins fächeux en raison de la puissance de régénération de ces tissus.

L.es obstructions de la portion thoracique de l'œsophage, de l'intestin grèle ont un pronostic fàcheus; les occlusions rectales dans des parties accessibles ì la main ont lin pronostic bénin.

Les corps étrangers qui pénètrent dans le coeur déterminent une maladie mortelle chez les rmminants; cenx qui séjournent dans la panse on le réseau ne produisent souvent aucun accident.

Les plaies situées dans des parties peu vasculaires s'opposant à l'écoulement du pus ou se prêtant à $d \mathbf{s}$ mouvemenls très étenlus out un pronostic fàcheux; celles 
qui intéressent des tissus immobiles, très vasculaires et à l'abri de tout frottement guérissent rapidement.

b. Étendue. - L'étendue des lésions a une signification prépondéranle dans les prévisions cliniques.

Une pleurésie simple est souvent curable; une pleurésie double est extrênement grave.

L'inflammation de tous les organes pairs est relativement bénigne quand elle n'intéresse que l'un d'eux; elle doit toujours faire craindre la mort quand les deux sont alteints.

Les lésions peu redoutables par leurs effets deviennent alarmantes quand elles sont très étendues ou généralisées. L'œdème des membres postérieurs est fréquent el bénin; il devient grave quand il se convertit en anasarque; un phlegmon superficiel qui ne contient qu'une petite quantité de pus peut passer inaperçu; un phlegmon volumineux de l'aine détermine des troubles généraux el quelquefois la mort des jeunes chevaux atleints de gourme.

Le décollement d'une partie du sabot n'empèche pas l'appui du pied sur le sol; le détachement complet de l'ongle entraine toujours la mort des chevaux lourds.

c. Caractères. - Les caractères des lésions tiennent une grande place dans le jugement à porter sur les événements futurs.

Lne pneumonic exsudative, fibineuse a une terminaison ordinaitement favorable; une pueumonie gangreneuse, médicamenteuse ou par corps étrangers est, règle générale, une maladie mortelle.

In phlegmon pyogène se limite, s’abcède et guérit; un phlegmon septique se diffuse et détermine souvent l'infection générale et la mort.

\section{II. - PRONOSTIG TIRÉ DI MALADE.}

Le terrain de la maladie fournit de nombreux renseignements pronostiques. 
a. Hérédité. - L'hérédité crée les bons comme les mauvais terrains pour les germes morbides. Elle communique l'immunité complète ou incomplète, les prédispositions ou les maladies.

Les affections lıéréditaires sont rebelles à tous les moyens thérapeutiques; elles sont combattues par l'hygiène qui modifie l'hérédité.

$b$. Age. - On sait que la clavelée congénitale est plus grave que la clavelée acquise.

Quand on inocule le virus de la pneumonie à deux veaux, l'un à la mamelle, l'autre plus ìgé, il se développe, chez le premier, une maladie générale frappant les séreuses articulaires et viscérales; il se produit, chez le second, une lésion locale œuémateuse au niveau du médiastin.

Le microbe du charbon symplomatique détermine une maladie moins grave chez les veaux que chez les adultes.

En général, cependant, le pronostic est d'autant plus mauvais que les animaux sont plus jeunes.

c. Race. - Les moutons africains sont moins sensibles à la fièvre charbonneuse el à la clavelée que les moutons de nos contrées.

d. Sexe. - Les femelles pleines avortent sous l'influence de la plupart des maladies infectieuses et contractent souvent la fièvre puerpérale.

$e$. Tempérament, constitution, diathèses. - Les tempéraments, la conslitution débile, les maladies générales diathésiques aggravent le pronostic des maladies dartreuses, et expliquent leur incurabilité.

$f$. États pathologiques. - Tous les états pathologiques assombrissent le pronostic des maladies locales comme des maladies générales.

Quand l'appareil digestif est troublé dans son fonction. nement, ses sécrétions sont disséminées et moins antiseptiques; il se produit des infections locales et des 
infections générales; la nutrition est insuffisante, la phagrocytose et tous les autres moyens de défense sont supprimés.

Les maladies de l'appareil respiratoire, du foie, des reins, du système nerveux préparent la défaite de l'organisme.

Quand le rein est altéré, il y a souvent urémie, adème, infection du poumon et mort.

Les tumeurs maligrnes sont une source d'intoxication de l'organisme et d'aggravation pour toutes les maladies.

g. Intoxications. - Les agents toxiques, les antiseptiques eux-mêmes dépriment les forces du sujel, suppriment les résistances cellulaires ct aggravent le pronostic.

h. Convalescence, travaux pénibles. - La convalescence, les travaux pénibles contribuent également à diminuer les résistances du sujet, à préparer la mulliplication des germes, à favoriser les complications.

j. Influences extérieures. - Les influences extérieures peurent actionner la virulence, préparer le terrain, faire prospérer les microbes ou entraver leur développement. Tantòt elles exercent une influence làcheuse, lantòt elles ont une influence favorable.

Les agents microbiens, qui séjournent dans les voies digestives, les voies respiratoires ne deviennent pathogènes que gràce à un changement du terrain yui est le point de départ de leur multiplication.

L'air, l'eau, le sol, le climat, les localités, la température, la saison, la lumière, l'électricité, l'humidilé, la sécheresse peuvenl, suivant les cas, angmenter ou diminuer la virulence et rendre les maladies luénignes on graves (1).

L'encombrement facilite les infections, exalte la virulence, rend les complications plus fréquentes.

(1) Voy. Étiologie in l'athologie générale dle l'Encyclopédie vétérinaire. 


\section{II. - MITHODE A SLIVRE POTR ÉTABLIR LE PRONOSTIC.}

On commence par s'aider de tous les moyens employés pour établir le diagnoslic, afin d'arriver à une connaissance complèle de la maladie.

S'agit-il d'une pneumonie, on en pèse les causes, les symptònes, l'étendue, on tient compte des complications qui se sont produites, et on envisage la gravité de celte pneumonie par rapport à une autre pneumonie.

Ce pronostic général est incomplet el inexact. Le jugement porté sur la maladie est sourent modifié par l'ensemble des renseignements tirés du malade.

Le pronostic individuel est le complément obligé, indispensable dı pronostic général (Labat). Il faut mettre eı présence la maladie et la résistance du malade.

Le pronostic est favorable quand le terrain est relativement peu modifié par la maladie, quand le sujet parait devoir l'emporler. Ce jugement n'a rien d'alisolu, lous les éléments qui servent à le former peuvent changer et rendre très grave un pronostic bénin. Certains cas paraissant désespérés, guérissent, tels autres dont on escomptait la guérison out une terminaison mortelle.

Le pronostic doil suivre loutes les oscillalions de la maladie; il doit tenir compte de toules les probabilités du moment, il doil être agggravé, alténué, corrigé ou complété à chałpue visite.

Assurément, on peut se prononcer d'une manière plus affirmative pour cerlaines maladies.

La morve, la tuljerculose sont des maladies mortelles à bref délai, de sorte qu'on ne peul hésiter qu'au sujel de celle dernière éventualité.

La rage détermine si sùrement la mort qu'on ne court aucun risque à annoncer ce dénouemenl.

Les affections aiguës se terminent par la résolution qui s'opère ou commence à s'opérer avaut le dixième jour. 
Passé ce délai, le pronoslic devient grave, car les termimaisons qui se produisent ensuite (induration, etc.) ne permeltent qu'une restauration incomplète de l'organe malade.

Le pronoslic est plus dilficile à poser quand les signes sont vagues el insuffisauts. On doit alors s'inspirer des résultats fournis par les stalistiques et surtout par l'expérience personnelle. I.e pronostic doit être réservé chaque fois que le diagnostic est donteux, que la maladie est obscure ou que le malade présente des troubles organiques importants ou un étal général défeclueux.

Quand le pronostic médical est favorable, c'est-à-dire quand la guérison peut être envisagée comme certaine, il fint se préoccuper du pronostic économique.

" Nos malades n'ont de valeur que par les services qu'ils rendent ou l'agrément yu’ils procusent. Leur vie est une clarge du moment qu'ils deviennent inutiles. S'ils menrent, ils occassionneront une perte d'argent et rien de plus.

"Par ces motifs, le vétérinaire, arrivé en présence de son malade, doit débrouiller une siluation assez compliquée. Il lui sera demandé de se prononcer sur les points suivants: la curabilité et surtout la prompte curabilité de la maladie; le quantum de la dépense qu'occasionnera la maladie; la dépréciation subie par le malade, conservera-t-il ou perdra-t-il (et alors, dansquelle mesure) ses aplitudes au travail ou telles aulres qualilés qui en font le prix? Et, s'il s'agit d'un animal de bouclserie, ne vaul-il pas nieux le mener tout de suite à l'ahalloir?

"Ces questions résolues favorablement, le malade sera soumis au traitement qui conviendra.

"Dans les cas contraires, il y anra intérêt à le sacrifier sais retard.

"Il est de loute évidence que la solution de ces questions dépend de la rigueur avec laquelle le pronostic médical aura été tout d'abord institué. 
“ On comprend également qu’un pronostic, médicalement parlant favorable, peut devenir, dans le sens économique, un pronostic ficheux. Où est en effet le résultat pratique, utile, de la guérisun d'un animal, si cet animal, quoique guéri, doit rester incapable de servir, si sa valeur doit ètre moindre que celle qu'il possède actuellement, si enfin il doit couter, en soins divers et en perte de temps, une somme supérieure à celle qu'il vaut présentement ou qu'il vaudra plus tard?

"Le vétérinaire doit, avant tout, regarder aux intérêts pécuniaires de son client. Le malade et la maladie ne viennent qu'en second lieu. C'est une nécessité à laquelle il ne peut se soustraire.

" Au point de vue du client, on ne saurait meltre en doute les avantages d'un pronostic judicieusement établi.

" Mais il y a profit aussi pour le vétérinaire. Plus encore que le diagnostic el la thérapeutique qui éclıapent au contròle des gens du monde, le pronostic assure au vétérinaire de l'estime el de la réputation.

"Si le malade guérit, comme il aura prévu cette solution, il montrera aux yeux de tous la solidité de son savoir et de son jugement.

"Si le malade meurt, on ne peut le rendre responsable de cette terminaison, puisqu'il l'avait prédite.

"Toute personne étant à mème de vérifier les prévisions annoncées, d'un pronostic qui se réalise ou qui se trouve en défaut naît la considération ou le discrédit pour le vétériuaire. Celui-ci ne saurait donc être trop prudent ni trop circonspect, en formulant un pronostic (Labat) " (1).

(1) Labal, arlicle Pronoslic, t. XVIII, du Dictionnaire pratique de méde. cine vétérinaire. 


\section{TABLE DES MATIĖRES}

SÉMIOLOGIE DE L'APPAREIL GÉNITAL

PREMIĖRE SECTION

Organes génitaux du mâle.

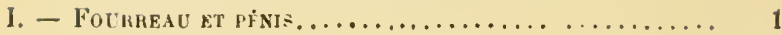

Topographie............................

Exploration, 1. - Modifications pathologiques..... 2

II. - Testicule et gaine vagivale............... 3

Exploralion .............................. 3

\section{DEUYIÈIE SECTION}

Organes génitaux de la femelle.

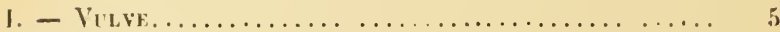

Topographie............................. 5

Exploration............................. 5

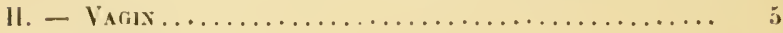

Topographie............................. 5

Exploration............................ 6

III. — Urénus ............................. 10

Topographie............................. 10

Exploration, 10. - Modifications pathologirques.... 11

$1^{\circ}$ Accidents pendant la gestation, 11. - $2^{\circ}$ Accidents consécutifs à l'açcouchement........... 
IV. - Castration..........................

Effets de la castration...................... 1i

10 Caractère de l'animal, 17. - 2o Forme du corps, 18. - $3^{\circ}$ Prostate, 20. - 40 Utérus, 21. - 50 Les instincts génésiques sont-ils constamment supprimés par la castration? 22. - $6^{\circ}$ Inlluence de la castration sur la sécrétion lactée, 27. - 70 Influence de la castration sur la nutrition............. 28

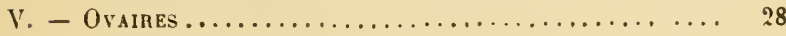

Topographie.......................... 98

Exploration ........................ 28

VI. - NYMPhonanie...................... . 29

Définition, 29. - Pathogénie, จ9. - Garactères...... 30

V'll. - SatYRLAsis ........................ 30

Définition, 30. - Caractères............... 30

VIII. - OXanis.HE........................ 31

Définition, 31. - Caractires................ 31

IX. - Perversion génitale................. 32

Х. - Impuissance........................ 33

XI. - StÉRILITẺ........................ 33

XII. - Attentats chininels.................... 35

10 Bestialité, 35. - 2" Salisme, 36. - Symptòmrs... 39

\section{SÉMIOLOGIE DES MAMELLES} ET IJE LA LACTATION

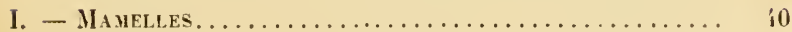

I. Topographie $\ldots \ldots \ldots \ldots \ldots \ldots \ldots \ldots \ldots \ldots \ldots \ldots$ i0

II. Exploration ........................ if

a. Inspection....................... it

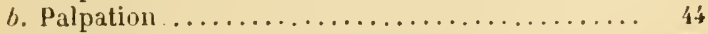

c. Sondage............................ it

II. - Oblitéuhtion des thayons .............. 45

III. - Galactose axomiale.................. 16

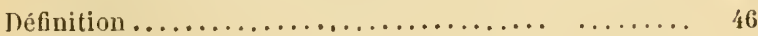

IV. - AgAlaxie....................... 46

péfinition.......................... 
V. - Polygalactie ou polyg.llaxie............. 47

Définition. 4i. - Siguification............... 47

V'l. - Galactorruée oll fill icturhie............ 48

Définition........................... 48

Vil. - Perversion le la sícnétrox lactí........... 49

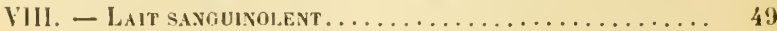

Caractères, 43. - Significalinı... . . . . . . . . 49

IX. - Lait puRUlext. .................... 49

Caracteres, 49. - Signification............. 引)

X. - Lait grateleux ...................... . 50

XI. - Lait visquei'X................... 51

Définition ......................... 51

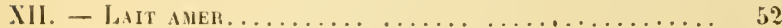

XItI. - LaIt aCIDE........................ 52

XIV. - LAIT VIRULENT. . ................. 52

\section{SËHOLOCIE DE LAPPAREIL.} DE L'INNERYATION

Topographie, 54. - Physiologie, 54. - Élėments du système nerveux, 55. - Modifications des neurones.

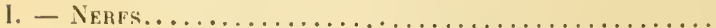

Division, 61. - Fonetions, 61. - Sensibilití récurrente, 61. - Modifications pathologiques..........

I. - Altérations primilives.................. 63

Dégénération, 6i. - Régénéralinu des neıf:..... 66 Signification ......................... 68

It. Lisions secondaires ...................... 70

Lésions de la moelle conséentives a la section des racines postérieures, 70 . - Lésions centrales consécutives á certaines altérations des nerfs craniens et raclidiens, 70 - Névromes, ít. - Lésions des muscles, 78. - Lésions des os, is. - Comment se comportent les néoformations osseuses en présence de la névrotomie? 81. - Comment se produisent ces lésions trophiques? 83. - Lésions des vaisseaux et du tissu conjonclif, 8't. - Lésions de la cornée 
et des téguments, \$5. - Lésions pulmonaires, 91. Lésions cardiaques................... 9 ?

II. - Muelle. . . . . . . . . . . . . . . . . . . 92

Fonctions, 92. - Rủle conducteur............ 93

Joyens utilisés pour dillérencier ces voies........ 96

$1^{\circ}$ Méthode vallérienue, 96. - " Méthode cmbryologique, 96. - $3^{\circ}$.léthode expérimentale, 97. Injections auesthésiques, 100 . - La moelle est un centre nerreux, 100. - Modifications pathologiques........................ 102

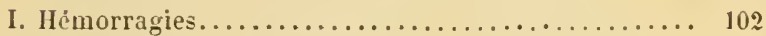

Définitiun, 10?. - Signification............. 103

II. - Iyélites......................... 103

Définition, 103. - Caractères, 103. - Pathogénie... 111'

111. Dégẻnẻrations secondaires............... 105

l’athogénie, 105. - Caractèrcs............. 106

I. Dégénẻrations descendantcs.............. 106

A. Lésions cérébrales, 106. - B. Lésions médullaires, 107. $-1{ }^{\circ}$ Cordon antéro-latéral. 10s. 20 Cordon postérieur...... .......... 108

1I. Dégénérations ascendantes.............. 109

Cordons postérieurs, 109. - Cordon antéra-latéral. 110

IlI. - BllBe.

Conduclibilité, 111. - Connexions du bulbe et de la moelle arec le cerrelet, 112. - Du bulbe envisagé comme centre, $11 \%$ - $1^{\circ}$ Centre respiratoire, 113. 20 Centre de la deglutition, 114. - $3^{\circ}$ Centre de la mastication et de la succion, 114. - $4^{\circ}$ Centre du clignement, 114. $-5^{0}$ Centre de la phonation.......

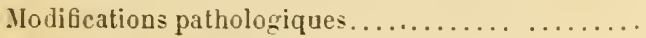

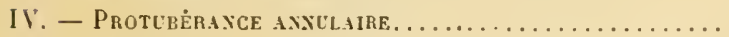

Conductibilité, 115. - Excitabilité, 116. - Centre nerveux.......................... 117

Modifications pathologiques............. 117

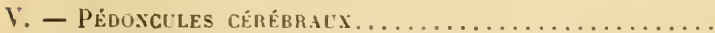

Excitabilité, 118. - Conductibilité, 118. - Modificalions pathologiques.................... 11 s 
V1. - Tuberclles gladrijugeat............... 119

Couches optiques......................... 119

VIl. - Conps striés....................... 121

Corps striés, 121. - Capsule interne.......... 122

VIII. - Cenvelet........................ 123

Rapports, 123. - Le cervelet est un centre, 124. Excilabilité, 126. - Modifications patholngiques....

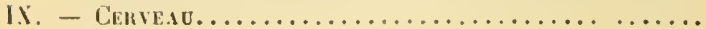

Fonctions, 126. - Circonvolutions, 127. - Localisations cérébrales........................ 127

10 Centres psycho-moteurs............... 128

Effets de l'excitation des rentres psycho-moteurs........................ 132

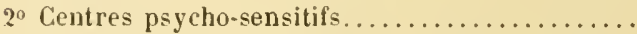

Centre de la vision, 133. - Centre psychoacoustique, 135. - Centres gustatifs et olfactifs, 135. - Modifications pathologiques, 136.

- Suppression totale.................. 136

Exploration................... 137

Inspection, 137. - Palpatiou, 138, - Percussion.

X. - Rỏle ju systène nerveux................ 138

Réaction nerveuse, 138. - Congestions........... 140

Y a-t-il antagonisme entre les deux groupes d'extraits vaso-constricteurs et raso-dilatateurs......

llémorragies, 143. - Obièmes, 143. - Intlammations, 143. - Manifestations dystrophiques, 14. - Choc nerveux, 144, - liéactions nerveuses dans les intoxications, 145. - Réations nerveuses dans les infections........... 146

XI. - Intrh.hience.................... 148

Considérations qénérates, lís. - Facultés intellectuclles............................. 148

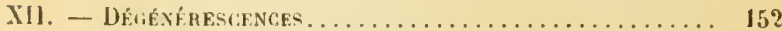

Défunition, 152. - Dégénérescence exprimentale.... 153 Stigmates physiques..................... 156 
10 Cràne, 15i. - 20 Face, 15i. - Oreilles, 158. leux, 158. - Nez, 158. - Bouche, 158. - Tronc et membres.

Symptômes somatiques, 159. - Stignates psychiques

XIII. - DÉlihe.

Pathogẻnie, 161. - Caractères.

XIY. - Hallcinatioxs.

164

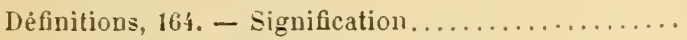

Xl. - Accés Rabiforues.

$16{ }^{\prime}$

Définition, 167. - Pathogénie, 16\%. - Caractères, 169. - Comment reconnaître si l'animal qui présente ces synuptômes est enragé?

XVI. - Hypnotisme, - Catalepsie.

Définition. 171. - Animaux susceptibles d'être tı̣̣notisés.

XYII. - PeCr

Définition

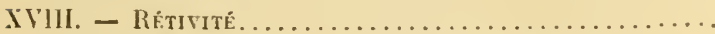

Caractères, 181. - Pathogénie

XIX. - JÉchaxcetÉ.

183

Caractères, 184. - Pathogénic.

184

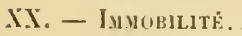

186

Pathogénie, 186. - Caractères, 187. - Conditions susceptibles de modifier l'intensité des symptômes, 190.

- Diagunstic.

XXI. - APOPLEXIE

Définition, 193. - Siguification.

XXII. - Сом.

Pathogénie, 196. - Caractères.

XXHI. - Tics notecks.

Définition

I. Tics actifs, 198. $-1^{\circ}$ Tics par habitude ou habitudes vicipuses, 198. - ?o Tics conrulsifs........ 
Définition

B. - TICS DE LA LAXGUE................. 20I

Caracteres....................... 201

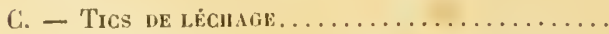

Caractères, 201. - 10 Phénomènes moieurs, :201. -20 Troubles psychiques, 202. - 30 Troubles de la sensibilité, :02. - Stigmates physiques.

D. - Tics DIvers.

a. Tic de frotter les dents contre la mangeoire, 203. - $b$. Tie d'agiter la lèvre inférieure, 203. $-c$. Tie du menton, 203. - $l$. Tic de grincer des dents, d'ouvrir et de fermer alternative ment la bouche. 203. - e. Tic de saisir l'une desbranclıes du mors avec la lévre inférieure ou avec les dents, 203. - $f$. Tic d'encenser.

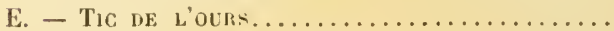

Jéfinition, 20i. - lo Phénomèues molcurs, 20 s.

- 20 Phénomènes psychiques, 206. - $3^{\circ}$ Troubles de réflectivité rt de sensibilité, 207. $4^{0}$ Stigmates physiques.............. 208

F. - Tús des Meurres................ 208

Caractères......................... 208

1I. Tics passifs. . . . . . . . . . . . . . . . . . 208

XXIV. - Cosvussioxs........................ 209

Définition............................. 209

Pathogénie.............................. 210

a. Muscle, 210. - b. Système nerveux, 211. $1^{\circ}$ Nerfs, 211. - $2^{\circ}$ Centres nerveux, 215. fo Moelle, 215. - $4^{\circ}$ Bulbe el prolubérance, $2 i 6$. - $5^{0}$ Cerveau, 216. - Caracteres des couvulsions, 219. - siguification...............

XXY. - Convulsions nu porcel.ET . . . . . . . . . . . . 229

Détinition, 2:29. - Historique, 229. - Caractires..... 229

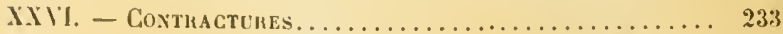

Définitiou, 233. - Caractères, 234. - Pathogénie.... 236

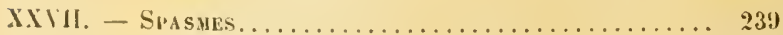

Définition, 239. - Pathogénie.............. 239 
XXVIII. - MACHONEMENT ET GRINCENENTS DE NEXTS...... 240

Défiuition .......................... $2: 0$

X.XIX. - Trembleyext .................. $2 \mathbf{i l}$

Caractères cliniques, 242. Pathogenie........ 212

X.X. - Nistagues ..................... 244

Définition. . . . . . . . . .

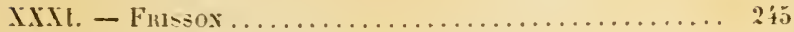

Définition, 245. - Signification............ 2i6

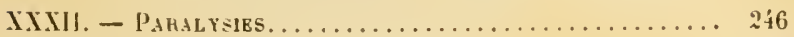

béfinition.......................... 2 i6

$1^{0}$ Faiblesse musculaire ou parésie, 247. - 20 Para-

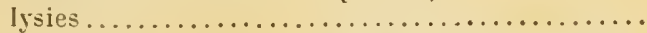

๔. Paralysies d'origine cérébrale, 249. - Caractères, 251. - b. Paralysie d'origine pédonculaire, 25\%. - c. Paralysies d'origine spinale,

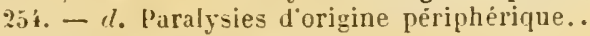

XXXIII. - Vektjges et Mouvenests foncés..........

Définition............................ \$59

Vertige rotatoire, 259. - Vertige galvanique...... 261

Mécanisme, 262. - Causes du vertige.......... 262

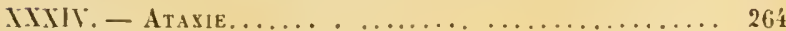

Définition ............................. $26 \dot{q}$

$1^{\circ}$ Ataxies d'origine périphérique............. 265

थ. Itaxie par altération de la sensibilité cutanée, 266. - b. Ataxie par altération du sens musc 1laire.

$2^{\circ}$ Ataxies d'origine centrale..

266

a. Ataxic par altération des canaux demi-circulaires ou Jabyrinthiques, 270.- -6 . Ataxic cérébelleuse, 9?. - Ataxie dans les empoisonvements aigus, 272. - Ataxic dans les lésions protubérantielles et du cerveau........................ 2 i

X.XY. - ALtẺRATIONS DE LA SEXSIDILITÉ........... 27 í

Définition, 27\%. - 1. Sensibilité tactile, 275. - Sensibilitẻ thermique, 2i6. - Sensibilité électrique cutanẻe et musculaire, 27\%. - Sensibilite dou'ourense......

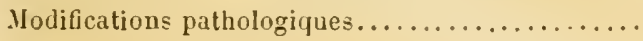


IIyperestlıésie, 278. - Anesthésie.

XXXVI. - Douleur...................... 281

Définition, 281. - Pathogéuie.............. 282

XXXVII. - NéVhalgles................... 284

Délinition, 281. - Caracteres............. $28 \mathrm{i}$

\section{SEMIOLOGIE DES OLGANES DES SLYS}

\section{PREMIĖRE SECTION}

\section{Yeux.}

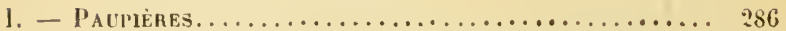

Exploration, 286. - Modilications pathologiques..... 287'

11. - Colips Clignotast................... 289

Modifications pathologiques................ 289

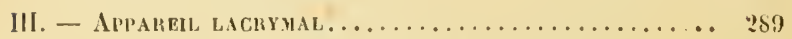

Exploration, 289. - Molifications pathologinues..... 290

IV. - Globe oculaine....................... 290

Exploration, 291. - $1^{\circ}$ Examen du sujet, s91. - «. Volinme des yeux....................... 291

10 Augmentition, 292. - 90 Diminution........ 292

b. Mouvements et expression des yeux, 29: 20 Examen de l'oxil, 293. - $1^{\circ} \mathrm{A}$ l'ceil nu et it la lumière naturelle, 293. - $2^{\circ}$ Examen d l'éclairago latéral ou oblique............ 296

V. - ConNÉ............................ 298

Caractères......................... 298

VI. - CHanbRe ANTÉHeURE.................. 298

Modifications........................ 298

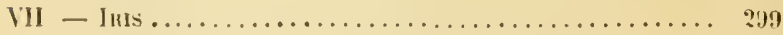

Caractères, :99. - Modılications pathologiques...... 299

VIII. - Caistallin........................ 300

Caractives.... ........................ 300

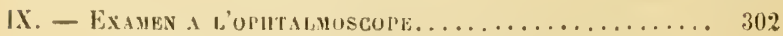

Historique, 302. - Principe de l'ophtalmoseupe, 302.

- 1. Examen it l'éclairage direct, 30't. - II. Examen 
a l'image droite, 306. - III. Examen á l'image renversée.

Que comprend l'examen ophtalmoscopique?.... 308

10 Détermination de la réfraction statique.... 308 20 Kératoscopie ou mėthode de Cuignet, 309. - Marche de l'ombre directe, 312. - Marche de l'ombre inverse, 312. - Point neutre. . .

20 Examcn ophtalmoscopique des membraues du fond de l'oil..................... 315

10 Corps vitré.................. 315

Modifications................ 315

20 Nerf optique................. 316

Modifications.................. 316

3० RÉTINE..................... 31 \%

Analomie, 317. - Modifications palhologiques ................. 318

4o Chonolde... .................. 319

Modifications palhologiques......... 319

X. - Ambrlople. Anavrose. . . . . . . . . . . 320

Dėfinition, 3:0. - Symptômes, 3:0. - Siguiticalion... 322

\section{DELXIEMME SECTION}

\section{Audition.}

1. - Oreill.e externe...................... 323

A. - Payllow....................... 323

Rôle du pavillon..................... 323

Modifications pathologiques.............. 323

B. - Condurt audrir .................... 324

Rôle du conduit auditif................ 32 í

Exploration ....................... 324

11. - Oneille yoyenNe.................... 325

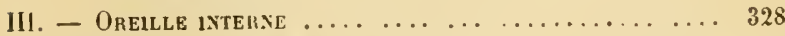

Labyrinthe, 328. - Nerf acouslique.......... 330 


\section{SESMIOLOGIE DE LAPPAREIL LOCOMOTEUK}

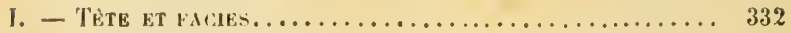

Modifications........................... 332

11. - Astuétries....................... 334

Caractères..............................

111. - Signes rolknis p.Al la queve................ 335

Modifications............................ 335

IV. - AtTITUES...................... 336

Station, 336. - Modifications pathologiques, 337. - Dicubitus............................ 337

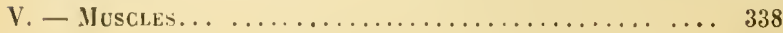

luspection, 338. - Palpatiou............... 338

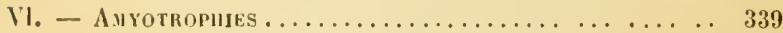

Caractères.......................... 339

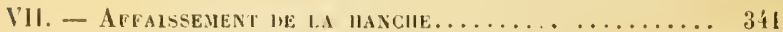

VIII. - ATFAISSEMENT ILIAQLE................ 342

Caractères, 342. - Pathogénje.............. 342

IX. - Hrpenthormes...................... 346

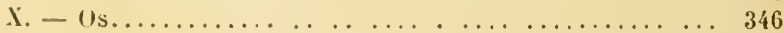

Modifications... ..................... 316

X1. - Exostoses.......................... 399

P'athogénie des exostoses, 349. - 1 . Théorie de l'hyperextension ligamenteuse, 350. - B. Théorie de l'ostéite primitive, 351. - $\mathbf{1}^{0} \mathrm{I}^{3} \mathrm{rédisposition} \mathrm{individuelle,} 358$. - $2^{\circ}$ Bilatéralité et disséminatiou des lésions usseuses, 360. - 3" Influence de la fatigne, 36'́. Influence de l'alimentation.................. 365

Xil. - Articulatioxs ........................ . 367

Anatomie........................... 367

Exploratiou ......................... 369

Modifications palliologinues............. 370

Xill. - Artuhtes....................... 372

Détinition, 3i2. - Canactéres, 372. - Pathonenie.... 374

XIV. - TENHNS ....................... 377 
Anatomic, 37\%. - A. Membraues péritendineuses, 37 .

- B. Corps des tendons................... 379

Modificalions pathologiques . ............ 380

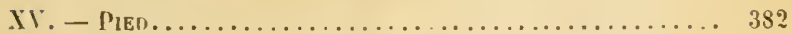

Exploration, 38\%. - Mudilicatious pathologiques..... 383

XVI. - Allthes........................ 395

XVII. - Bolterie....................... 385

léfinition, 385. - $1^{0}$ L'iuspection, 386. - 20 Palpa-

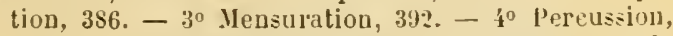
$39 \%$ - $5^{\circ}$ Auscultation, 392. - $6^{\circ}$ Injections de cocaïne, 393. - Caractères de la boiterie, 397. - Signification

XVIII. - HARPER...................... 4 . 2

Définition, 402. - Éliologie, f113. - Caraclères, 401. -

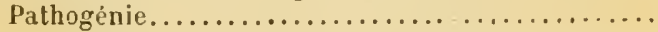

\section{SEMMIOLOGIE DE LA TEMPERATURE}

1. - FIEVRE......................... $400^{\circ}$

Définition .......................... 407

Température normale.................. 407

Variations physiologiques, 408. - Travail musculaire, 408. - Heure de la journee, 408. - Saisons, 409. - Influence de l'alimentation, 409. Inlluence de l'àge. 410. - Sexe, 410. - Race, 410. - Sommeil, ilo. - Toute, ill. - Gestalion, 411. - Inlluence de la respiration, $\mathbf{1 1 1}$. Polypnée thermique, 411. - Influence de la sudation, 412. - Régulation phériphérique et régu-

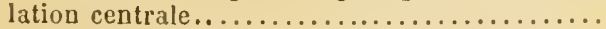

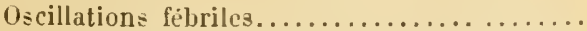

Comment se produit l'élévation de température? Quelles sont les causes et le mécanisme de celte hyperthermie?.

Troubles des divers appareils. ........

Pathogénie, \{24. - Fièvres fonctionnelles, 424. - Fièvres uutritives, 425.

CADÉAC. - Sémiologic, 2 édit. II. - 30 
- l'ar quel mécanisme les toxines produisent-elles la fière? 428 . - Signification, iथ9. - Diagnostic raisonné .......................

H. - Ilтpothermie......................... $\$ 33$

Définition, 133. - Causes de l'hypothermie........ 433

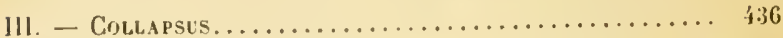

Definition, 436. - Pathoginic.............. 437

\section{Évolution des maladies.}

1. - P'ropagation des tholbles morbines............. $43 \delta$ Circonstances qui font varier l'évolution de la maladie. 438

II. - Enzooties ......................... 440

béfiniliou ....................

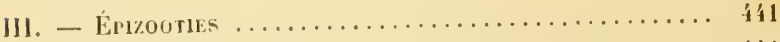

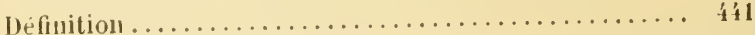

IY. - DURÉE JES MALAHES.................. 414

V. - TYPE IES MALADIES.................... 446

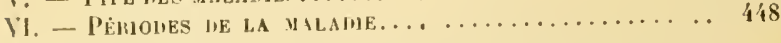

10 Invasion, 448. - 20 Etat, 49. - 30 Déclin, 450. -

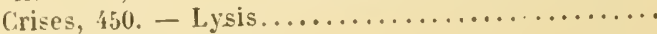

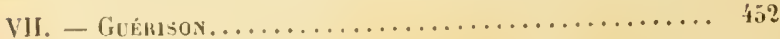

Définition ......................... 452

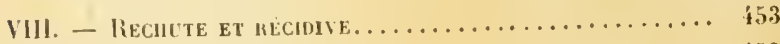

béfintion......................... 453

IX. - Complicationa

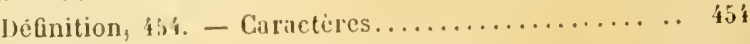

X. - Gonvalescence. ...

Definition, 459. - Ktat des tissus et organt's all woment de la convalescence, 460. - Etat des fouctious aul moment de la convalescence, 462. - Narche et Inrée de la convalescence, 464. - Maladies et accicents de la convalescence. 
XI. - Métistafe................... 467

Déftuition ........................... 467

Xil. - Passage a l'état chlmonig̨ a................ 468

Défiuition......................... 168

XIII. - 11 onт .......................... 469

Définition, 469. - Signes de la mort.. ......... 171

\section{Diagnostic et pronostic.}

\section{CIIAPITRE PREMIER}

\section{Diagnostic.}

Définition ............................... 4i?

I. - Motens ef difficultés ue magnostic........... 173

[0 Moyens de diagnostic, 473. - ?" Ditlicultés du diaguostic, $475 .-$ Procédés scientifiques, $477 .-1^{\circ} \mathrm{Ra}-$ diographie, 477. - liayons calhodiques, 480. Rayons X, 480. - Rayous N, 48?. - 20 Sérums précipitants, 48i. - $3^{\circ}$ Séro-llagnostic. 486 . 4́ Cyto-diagnostic, 487. - 50 Épreuve do résicatoire. 48?

11. - Élénenté de dilgnostic . . . . . . . . . . . . . . 490

10 Interrogatoire des per: onnes auxquelles a été confié I'auimal malade, 490. - $2^{\circ}$ Examen clu malade...

III. - Diafinustic des sinitines................ 49 i

IV. - Diafinostic de l'Obgaxe malade et IE L'aliection. 49 '

V. - DI.IGXOSTIC DE L.I MALAHIE.............. 496

\section{CIIAIPITRE II}

\section{Pronostic.}

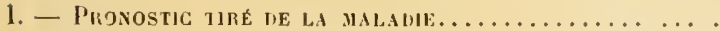

10 Causes, 499. - $a$. Nature, $499 .-b$. Nombre, 501. $c$. Voie d'introduction, $502 .-d$. Intensité d'action. 20 Symptòmes.... 503

a. Maladies aiguës, 505 . - b. Maladies chroniques, 508. - c. Marche, 509- - $l$. Durée, 509. - e. Complications, 510. $-f$. Reclute et réci $=$ 
dive, 510. - \%. Coïneidences morbides, 510.ん. Effets du traitement................ 510 $3^{\circ}$ Lésions, 511. - $a$. Siège, 511. - b. Étendue, 512 - $c$. Caractères............... 512

II. - Plionostic tiré du hal.doe................. 512

a. Hérédité, 513. - b. Age, 513. - c. Race, 513. d. Sexe, 513. - e. Tempérament, constitution. diathèses, 513. - $f$. États pathologiques, 513. - g. Intoxications, 514. - h. Convaleseence, travaux pénibles, 514. - $j$. Influeuces extérieures .......... 514

111. - Méthode a suvre pouh étabi.ı le pronostic .... 515 



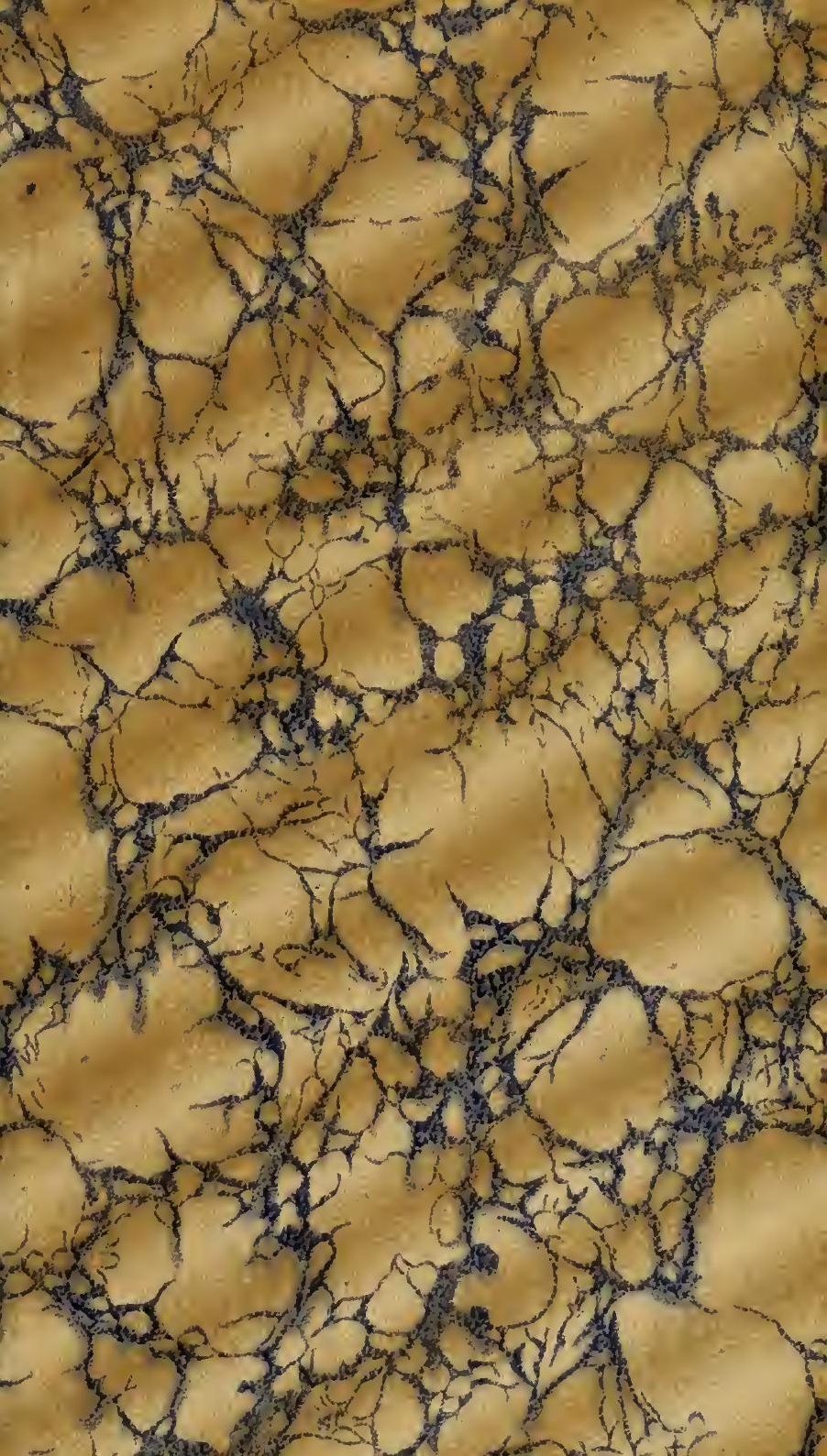




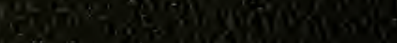

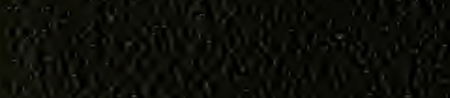
(⿻)

wheng

Lithe

int 3

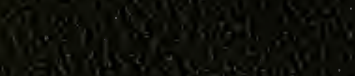

6.

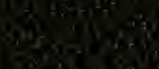

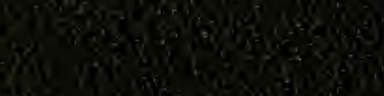

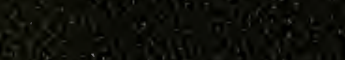

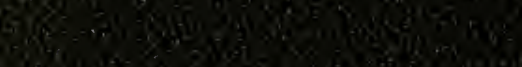
$x^{2} x^{2}$
3.
28
(2).

Whet

Sil 3

$x^{2}=2$

2

"sans

A.

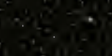

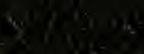

